Florida International University

FIU Digital Commons

FIU Electronic Theses and Dissertations

University Graduate School

$6-23-2020$

\title{
Singularities and Global Solutions in the Schrodinger-Hartree Equation
}

Anudeep Kumar

Florida International University, ana001@fiu.edu

Follow this and additional works at: https://digitalcommons.fiu.edu/etd

Part of the Analysis Commons, Non-linear Dynamics Commons, and the Partial Differential Equations Commons

\section{Recommended Citation}

Anudeep Kumar, "Singularities and Global Solutions in the Schrodinger-Hartree Equation" (2020). FIU Electronic Theses and Dissertations. 4505.

https://digitalcommons.fiu.edu/etd/4505

This work is brought to you for free and open access by the University Graduate School at FIU Digital Commons. It has been accepted for inclusion in FIU Electronic Theses and Dissertations by an authorized administrator of FIU Digital Commons. For more information, please contact dcc@fiu.edu. 


\section{FLORIDA INTERNATIONAL UNIVERSITY \\ Miami, Florida}

\section{SINGULARITIES AND GLOBAL SOLUTIONS IN THE SCHRÖDINGER-HARTREE EQUATION}

A dissertation submitted in partial fulfillment of the requirements for the degree of DOCTOR OF PHILOSOPHY in APPLIED MATHEMATICAL SCIENCES

by

FNU Anudeep Kumar 
To: Dean Michael R. Heithaus

College of Arts, Sciences and Education

This dissertation, written by FNU Anudeep Kumar, and entitled Singularities and Global Solutions in the Schrödinger-Hartree Equation, having been approved in respect to style and intellectual content, is referred to you for judgment.

We have read this dissertation and recommend that it be approved.

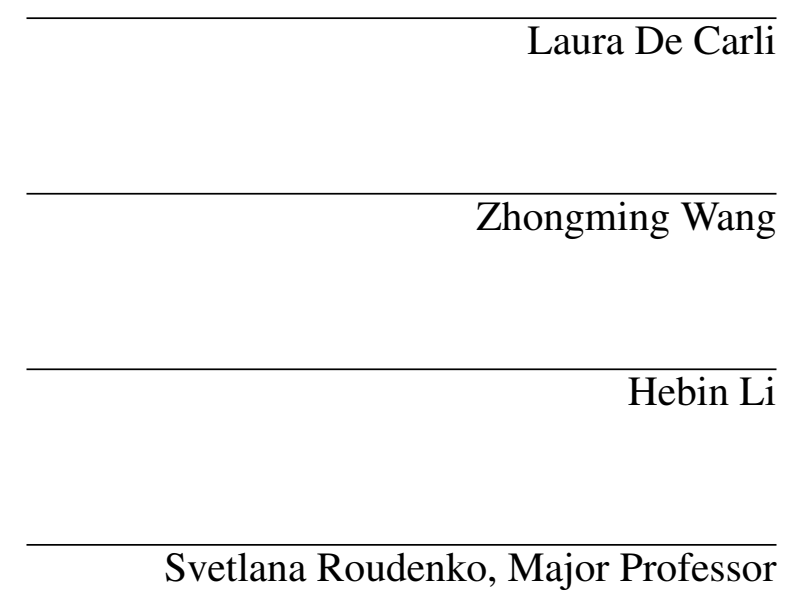

Date of Defense: June 23, 2020

The dissertation of FNU Anudeep Kumar is approved.

Dean Michael R. Heithaus

College of Arts, Sciences and Education

Andrés G. Gil

Vice President for Research and Economic Development and Dean of the University Graduate School

Florida International University, 2020 
(C) Copyright 2020 by FNU Anudeep Kumar

All rights reserved. 


\section{DEDICATION}

I dedicate this dissertation to my late grandparents, especially to my grandfather Khairati Lal who passed away recently and could not see my graduation. This is for him. 


\section{ACKNOWLEDGMENTS}

First and foremost, I would like to thank my advisor, mentor and guru Svetlana Roudenko for her undaunting support and inspiring me to always achieve a level higher that I thought possible. Her expertise, explicit directional guidance and continuous encouragement was invaluable to the dissertation. I could not have asked for a better

$\mathrm{PhD}$ adviser.

I am grateful to my parents for their unwavering patience over the last 10 years and for supporting in my great and small adventures and experiences of life. To my brothers

Prateek, Shailesh, Karan, Rhythm and Siddharth for their love and unbreakable bond. I am also grateful to my other family members for their support, love and blessings along the journey.

I would also like to express my gratitude towards Laura De Carli, my Master's adviser, for giving me the valuable exposure of research in the field of mathematics during my early years of graduate studies. She has constantly encouraged and supported me during my transition to the field of Mathematics with her immense knowledge and patience.

Thank you for always believing in me.

Last but in no means the least, I convey my sincere and deepest gratitude to my committee members for agreeing to be a part for my dissertation committee and dedicating their time to provide valuable inputs to enhance my dissertation research. 


\title{
ABSTRACT OF THE DISSERTATION \\ SINGULARITIES AND GLOBAL SOLUTIONS IN THE \\ SCHRÖDINGER-HARTREE EQUATION
}

\author{
by \\ FNU Anudeep Kumar \\ Florida International University, 2020 \\ Miami, Florida \\ Professor Svetlana Roudenko, Major Professor
}

In 1922, Louis de Broglie proposed wave-particle duality and introduced the idea of matter waves. In 1925, Erwin Schrödinger, proposed a wave equation for de Broglie's matter waves. The Schrödinger equation is described using the de Broglie's matter wave, which takes the wave function, and describes its quantum state over time.

Herein, we study the generalized Hartree (gHartree) equation, which is a nonlinear Schrödinger type equation except now the nonlinearities are a nonlocal (convolution) type. In the gHartree equation, the influence on the behavior of the solutions is global as opposed to the case of local (power type) nonlinearities.

Our first goal is to understand the behavior of finite energy solutions. We start with proving the local existence and then extend to the global existence for small data. We then, in the energy-subcritical critical regime, classify the behavior of finite energy solutions under the mass-energy assumption identifying the sharp threshold (depending on the size of the initial mass and gradient) for global (scattering) versus finite time (blow-up) solutions.

Next, we revisit the problem of scattering and give an alternative proof of scattering, for both NLS and gHartree equations in the radial setting. The alternative approach provides a simpler proof of scattering, which might also be useful for other contexts. 
Our next aim is to understand the phenomenon of wave collapse (blow-up, the sudden energy transfer from higher levels to lower ones), i.e., solutions with finite time of existence. We first give a sufficient condition for finite time blow-up for the large data and give examples of the various thresholds available in a variety of cases (energy-subcritical, critical and supercritical) for Gaussian data.

We then investigate stable singularity formations in the mass-critical gHartree equation, and in particular, rigorously prove a stable blow-up formation in dimension 3 . We observe that the nonlocal nonlinearity does not destroy the blow-up dynamics, similar to the local nonlinearities. On the other hand, one of the necessary properties, namely the spectral property required for the blow-up analysis, is modified remarkably. Nevertheless, we are able to prove that stable blow-up occurs with a self-similar profile at the square root rate with a logarithmic correction.

Finally, we present the reader with the conclusion and possible future research directions, wrapping up the dissertation. 


\section{TABLE OF CONTENTS}

CHAPTER

PAGE

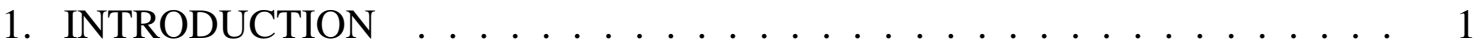

1.1 Prelude: Physical world . . . . . . . . . . . . . . . . . . . . 1

1.1.1 Enter uncertainty . . . . . . . . . . . . . . . . . . 6

1.2 World of mathematics . . . . . . . . . . . . . . . . . 8

2. PRELIMINARIES . . . . . . . . . . . . . . . . . . . . . . . . . 14

2.1 Notation . . . . . . . . . . . . . . . . . . . . . . . . . . . . 14

2.2 Symmetries . . . . . . . . . . . . . . . . . . . . 16

2.3 Embeddings . . . . . . . . . . . . . . . . . . . . . . . 17

2.4 Strichartz estimates . . . . . . . . . . . . . . . . . . . . . . . 18

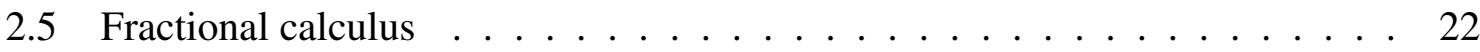

2.6 The local theory . . . . . . . . . . . . . . . . . . . . . . . . 23

2.6 .1 Local well-posedness in $H^{1} \ldots \ldots \ldots$. . . . . . . . . . . . . . . 24

2.6.2 Local well-posedness at the critical regularity . . . . . . . . . . . 30

2.7 Local well-posedness in $\dot{H}^{s}$ for $0<s<1 \ldots \ldots \ldots \ldots$. . . . . . . . 51

2.8 Ground state solutions . . . . . . . . . . . . . . . . . . . . 54

2.8 .1 Properties of the ground state . . . . . . . . . . . . . . 56

2.8.2 Uniqueness of the ground state for $p=2, \gamma=2 \ldots \ldots \ldots$. . . . . 60

3. GLOBAL BEHAVIOR OF SOLUTIONS TO THE FOCUSING GENERALIZED

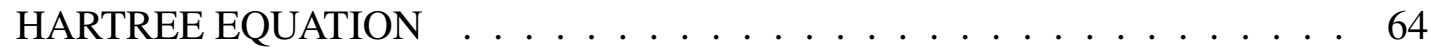

3.1 Background and statement of main result . . . . . . . . . . . . . . 64

3.2 Small data theory . . . . . . . . . . . . . . . . . . . . . 68

3.3 Dichotomy: Global vs blow up solutions . . . . . . . . . . . . . . . 93

3.4 Compactness . . . . . . . . . . . . . . . . . . . . . 103

$3.4 .1 \quad$ Roadmap . . . . . . . . . . . . . . . . . . . . . . . . . 103

3.4.2 Profile decomposition . . . . . . . . . . . . . . . . . . 105

3.4 .3 Critical solution . . . . . . . . . . . . . . . . . . . . . . . . 113

3.5 Rigidity Theorem . . . . . . . . . . . . . . . . . . . . . . . . . . 128

3.6 Divergence to infinity (Theorem 3.1.1 (2) part (b)) . . . . . . . . . . . . 131

4. SCATTERING IN THE NLS AND GHARTREE EQUATIONS: AN ALTERNA-

TIVE APPROACH . . . . . . . . . . . . . . . . . . 135

4.1 Setting up the problem . . . . . . . . . . . . . . . . . . 135

4.2 Scattering criterion . . . . . . . . . . . . . . . . . . . . . . 139

4.3 Variational analysis . . . . . . . . . . . . . . . . . . . . . . . . . . . 148

4.3 .1 The NLS equation . . . . . . . . . . . . . . . . . . . . . . . . . . . 149

4.3.2 The gHartree equation . . . . . . . . . . . . . . . . . . . . 152

4.4 Proof of Theorem $4.1 .4 \ldots \ldots \ldots$

4.5 Proof of Theorem $4.1 .5 \ldots \ldots \ldots$ 
5. SINGULARITY FORMATION IN THE GHARTREE EQUATION . . . . . 165

5.1 Blow-up criterion . . . . . . . . . . . . . . . . 166

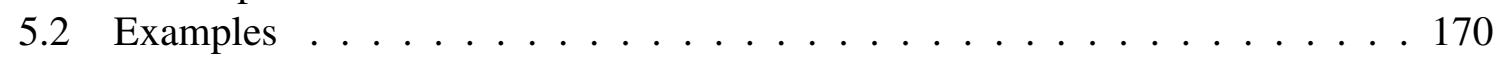

5.2 .1 Gaussian initial data . . . . . . . . . . . . . . . . . . . . . . . . . . . . . . . . . .

5.3 Blow-up analysis . . . . . . . . . . . . . . . . 178

$5.3 .1 \quad$ Linearized equation around $Q \ldots \ldots \ldots \ldots$. . . . . . . . . . . . . . . . . . . . . . . 182

5.3 .2 Equation for $\varepsilon \ldots \ldots \ldots \ldots \ldots$

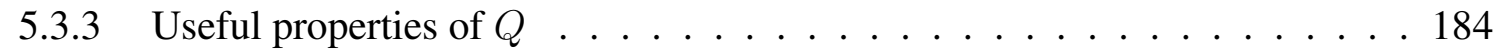

5.3.4 Spectral properties of $L_{+}$and $L_{-}$in $N=3 \ldots \ldots \ldots$. . . . . . . 185

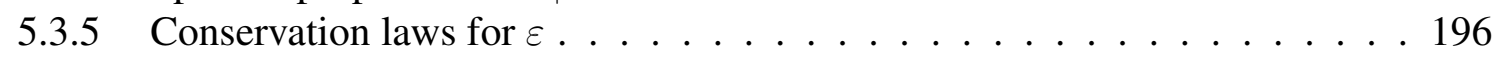

5.3.6 Choice of modulation parameters . . . . . . . . . . . . . 198

5.3 .7 Control of parameters . . . . . . . . . . . . . . . . . . . . . . . . . . . . . . . . . . . . . . . . . . .

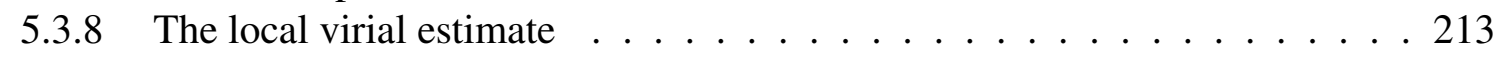

5.3 .9 Spectral property . . . . . . . . . . . . . . . 217

5.3.10 The local virial inequality: Application of orthogonality conditions . . . 220

5.3 .11 Refined virial inequality . . . . . . . . . . . . . . . . . 222

5.3.12 Monotonicity: Control on scaling parameter . . . . . . . . . . . . . 224

5.3.13 Finite time blow-up and preliminary control (upper bound) on blow-up rate 228

5.3 .14 Refined upper bound on the blow-up rate. . . . . . . . . . . . . . . . 234

5.3 .15 Climax: Log speed . . . . . . . . . . . . . . . 236

6. CONCLUSIONS AND FUTURE RESEARCH . . . . . . . . . . . . 238

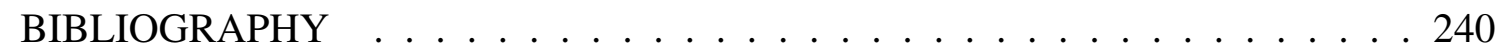

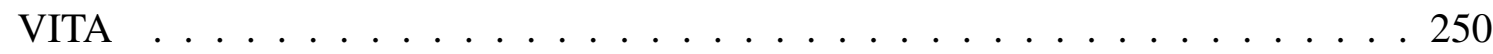




\section{LIST OF FIGURES}

FIGURE

PAGE

1.1 Blackbody radiation, taken from $[\mathrm{CU}] . \ldots \ldots \ldots \ldots$

1.2 Are we inside of an atom which is part of a giant brain? [Ima], [Wal], [WID],

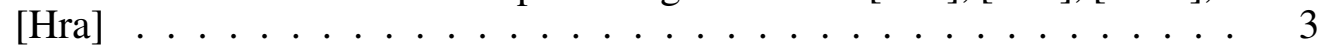

1.3 Wave-particle duality. The wave nature is exhibited by the upper part (wavy bit), while the particle nature is demonstrated below (perpendicular to the wave), see $\left[\mathrm{PLQ}^{+} 15\right]$ for explanation and details on duality. Image source: Fabrizio Carbone/EPFL. .............. 5

1.4 Heisenberg gets pulled over! (taken from $[$ fon $]$ ) . . . . . . . . . . . . 7

1.5 Dispersive equations are everywhere. [Bok], [lad], [Orn17], [Fla] . . . . . 9

1.6 Where does NLS come from?, appeared in [DK98]. . . . . . . . . . . . 10

1.7 Black hole. At its center, the mass of a black hole is compressed into a single, zero-dimensional point, taken from [Hoo]. . . . . . . . . . . . . 12

5.1 Thresholds for the Gaussian data $u_{0}(x)=\beta e^{-|x|^{2}}$ in the energy-subcritical case, see (5.20)-(5.23) . . . . . . . . . . . . . . 173

5.2 Thresholds for Gaussian data $u_{0}=\beta e^{-|x|^{2}}$ in the energy-critical case,see (5.25)-(5.28) and (5.30)-(5.33). . . . . . . . . . . . . 176

5.3 Thresholds for Gaussian data $u_{0}=\beta e^{-|x|^{2}}$ in the energy-supercritical case,

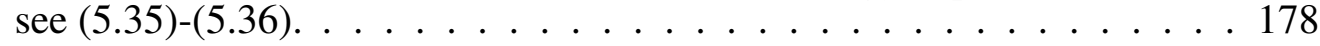




\section{CHAPTER 1}

\section{INTRODUCTION}

Politics is for the present, but an equation is for eternity. (Albert Einstein)

\subsection{Prelude: Physical world}

A partial differential equation (PDE) is an equation involving derivatives in space and, possibly, in time. Motivated by the problems in mathematical physics, Schrödinger-type partial differential equations are one of the standard equations studied in mathematics. The Schrödinger equation integrates both classical and quantum mechanics. It uses conservation of energy from classical mechanics written in terms of its wave function from quantum mechanics. It has its roots at the very start of 20th century and that is where we begin with. The main sources of the present section are lecture notes $[\mathrm{mbSP}]$ and an article on Noble Laureates [Zur].

At the end of 19th century Max Planck began working on the problem of black body radiation (see Figure 1.1, [CU] for the two best examples of black body radiation). He tried to explain the dependence of electromagnetic energy on the frequency of the radiation and the temperature of the body. When heated, the molecules comprising a black body vibrate and emit light of the same wavelength as their vibration. In 1900, Planck introduced an assumption that the energy of electromagnetic wave is quantized and given by the relation

$$
E=n h \nu
$$

where $n=1,2,3, \ldots, h=6.6 \times 10^{-34} \mathrm{~m}^{2} \mathrm{~kg} / \mathrm{s}$ is Planck's constant and $\nu$ is the frequency of electromagnetic wave. This assumption helped Planck to derive a formula (now known as Planck's law), which accurately describes the spectrum of a black body radiation.

In 1905, Albert Einstein proposed that the light is not a wave but is made up of separate wave packets (photons, termed by Gilbert N. Lewis in 1926), which allowed 

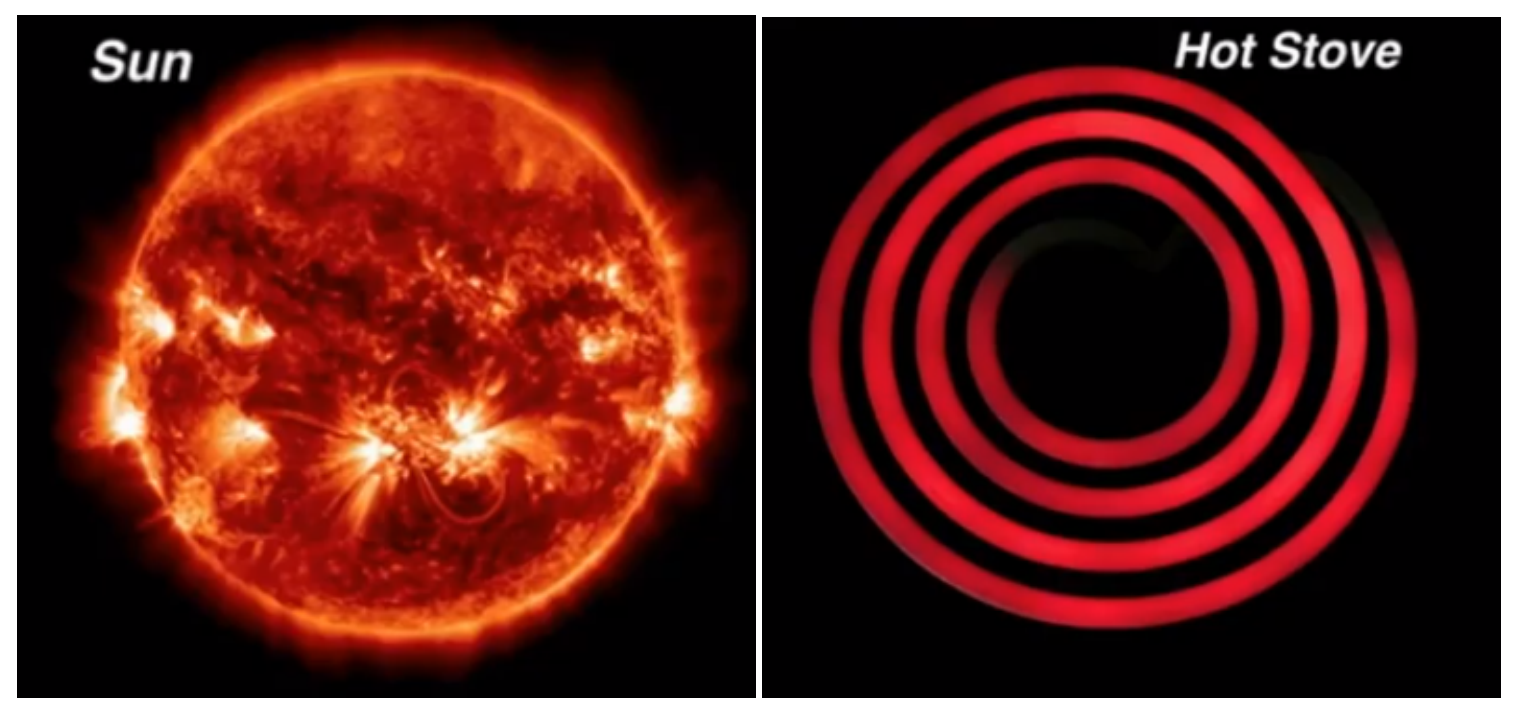

Figure 1.1: Blackbody radiation, taken from [CU].

Einstein to write an equation for the Photoelectric effect

$$
E=h \nu=\hbar \omega
$$

Here, $\hbar=\frac{h}{2 \pi}=1.05 \times 10^{-34} \mathrm{Js}$ and $\omega=2 \pi \nu$ is the angular frequency. It provided the explanation that the energy received by an electron, allowing it to leave the surface) is dependent on the frequency (not intensity) of a photon. This lead Einstein to deduce the particle-like behavior of the photon.

In 1913, Ernest Rutherford and Neils Bohr gave a model for an atom, which consisted of a small and densely concentrated region (called nucleus) of positive charge (protons) with electrons orbiting around it. The model was an improved extension of the Rutherford model (1911) that had a drawback that an electron, orbiting around a nucleus, accelerates, and thus, it would loose energy in the form of electromagnetic radiation. Hence, it should collapse by spiraling into the nucleus, which suggests that all atoms are unstable, a devastating suggestion. To eliminate the problem, Bohr (1913) added the following assumptions to Rutherford's previous model, he suggested that the electron must be treated as a particle having a definitive position and momentum: 
- The angular momentum of an electron orbiting around a nucleus is an integral multiple of Planck's constant, $L=m v r=n \hbar$. Bohr calculated that each allowed orbit has a specific energy level and there are no other orbits in between.

- These orbits with definite energies are stable and an electron revolving in a particular (stable) orbit does not loose or gain any energy.

- The lose or gain of energy can only happen when an electron jumps from one orbit to another, $\Delta E=h \nu$, where $\nu$ is the frequency of the electromagnetic radiation emitted (energy lose) or absorbed (energy gain).
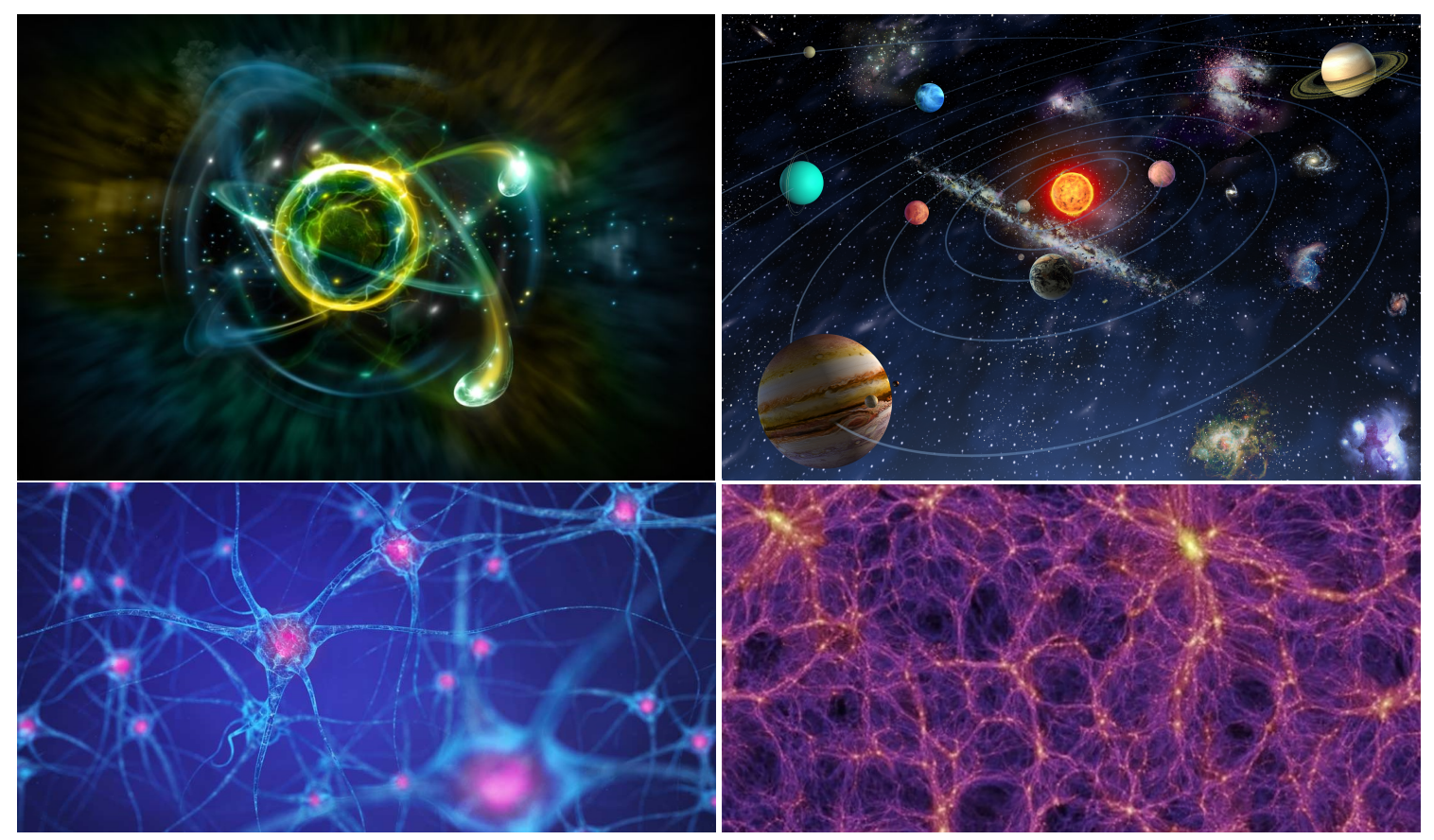

Figure 1.2: Are we inside of an atom which is part of a giant brain? [Ima], [Wal], [WID], [Hra]

The idea depicted in Figure 1.2 has been floating for decades, which depicts two interesting analogies: one represents that an atom (top left) is like the solar system (top right), where the gravity is replaced by electrostatic forces and other draws a comparison of a brain cell (bottom left) with the universe (bottom right). 
Louis De Broglie (1924) proposed in his $\mathrm{PhD}$ thesis the theory of electron waves. We already know that while describing the spectrum of black body radiation, Planck assumed that the energy of electromagnetic waves is quantized and Einstein explained the photoelectric effect by proposing that the waves carrying electromagnetic energy are also quantized, which led to the existence of photons (a particle-like behavior). The energy of photon is given by the Planck-Einstein relation, $E=h \nu$. Louis de Broglie posed a question, refer [Nig]: "If a photon, which has no mass, can behave as a particle; does it follow that, an electron, which has mass, can behave as a wave?" He suggested that the wave-like nature of an electron should be demonstrated by the relation $\lambda=\frac{h}{p}$, which generalizes the Planck-Einstein equation by rearranging the momentum of a photon given by $p=\frac{E}{c}$, where $c$ is speed of light and the wavelength $\lambda=\frac{c}{\nu}$. Furthermore, since the electron revolves in a stable orbit, now according to de Broglies's hypothesis if it behaves as a wave, it would be a stationary wave. Thus, we may assume from the geometric condition for a standing wave that the circumference of the orbit would only admit an integer number of wavelengths. Therefore, we have

$$
\begin{aligned}
& 2 \pi r=n \lambda \Longrightarrow r=\frac{n \lambda}{2 \pi}, \\
& L=r p=r \frac{h}{\lambda}=\frac{n \lambda}{2 \pi} \frac{h}{\lambda}=n \hbar,
\end{aligned}
$$

which is Bohr's first assumption that the angular momentum of an electron is quantized. This introduced the concept of wave-particle duality, i.e., just like a wave is made up of particles, a particle can behave as a wave (see Figure 1.3, the first ever peek of light behaving as both a wave and a particle at the same time).

"In the summer of 1925, Erwin Schrödinger, read the doctoral thesis of Louis de Broglie, who proposed that matter, for instance, electrons (assumed to have particle-like behavior) - also have wave properties. Schrödinger devoted his Christmas and New Year 1925/26 break with all of his focus in constructing the equation for de Broglie's matter 


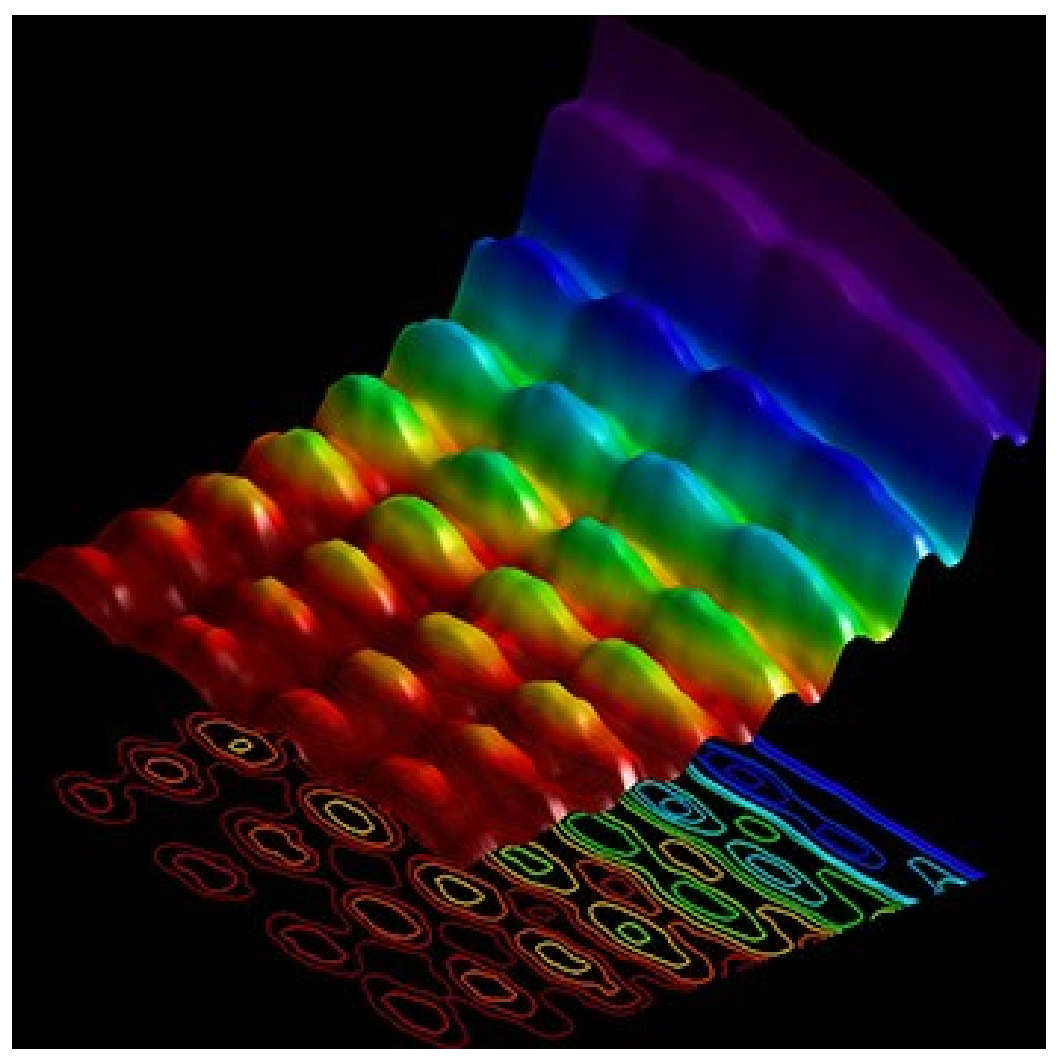

Figure 1.3: Wave-particle duality. The wave nature is exhibited by the upper part (wavy bit), while the particle nature is demonstrated below (perpendicular to the wave), see $\left[\mathrm{PLQ}^{+} 15\right]$ for explanation and details on duality. Image source: Fabrizio Carbone/EPFL.

waves. On December 27, he wrote to his physicist colleague, Wilhelm Wien: "At the moment I am bothered by a new atomic theory. If only I were better at mathematics! I'm very optimistic about this, and hope that it will turn out beautifully as long as I can manage the calculations." (source: [Zur])

On January 26, 1926, Erwin Schrödinger published his paper in which he constructed the wave equation for de Broglie's matter waves, which now bears his name, the Schrödinger equation. He treated the electron as a wave represented by a wave function,

$$
\psi(x, t)=A e^{i(k x-\omega t)},
$$

where $A$ is the amplitude, $k=\frac{2 \pi}{\lambda}$ is the wave number (number of waves per unit distance), and $\omega$ is the angular frequency of the wave. 
The total energy of a free particle is the sum of kinetic and potential energy $V$, thus, $E=\frac{1}{2} m v^{2}+V=\frac{p^{2}}{2 m}+V$. Now observe that Planck-Einstein relation yields that $E=h \nu=\hbar \omega$ and de Broglie's equation implies that $p=\frac{h}{\lambda}=\hbar k$. Substituting the energy and momentum into the energy conservation equation, one obtains the relation, $\hbar \omega=\frac{(\hbar k)^{2}}{2 m}+V$. Schrödinger then tried to express the wave function using this energy relation. He realized that taking the partial derivative with respect to time gives

$$
\frac{\partial \psi}{\partial t}=-i \omega \psi
$$

and the second order partial derivative with respect to space yields

$$
\frac{\partial \psi}{\partial t}=-i \omega \psi \frac{\partial^{2} \psi}{\partial x^{2}}=-k^{2} \psi
$$

which led to the equation

$$
i \hbar \frac{\partial \psi}{\partial t}=-\frac{\hbar^{2}}{2 m} \frac{\partial^{2} \psi}{\partial x^{2}}+V(x) \psi
$$

known as the Schrödinger equation. It is probably one of the most important breakthroughs in the history of physics.

\subsubsection{Enter uncertainty}

Schrödinger could not give a successful meaning to the wave function $\psi$ related to the physical world applications.

Max Born in 1926 suggested that the wave function is not associated with any definite physical state, he mentioned that it gives the probability of the different states of particles. He postulated that the probability of finding the particle at a position $x$ in the region $R$ at time $t$ is given by the following integral

$$
\int_{R}|\psi(x, t)|^{2} d x
$$


The probabilistic explanation is related to a fascinating discovery by Werner Heisenberg in 1927, in which he generalized the de Broglie's formula for the wavelength and momentum of a particle. Heisenberg realized that there is a fundamental limit to the precision (given by a mathematical inequality) in measuring any two quantities of a system, for instance, the position and momentum of a moving particle. The more accurately you measure one physical attribute, the more uncertain the other becomes (see Figure 1.4 for a fun interpretation). This result (for which Heisenberg won the Nobel prize in 1932) is now called Heisenberg's uncertainty principle. The uncertainty principle suggests the unpredictability of nature around us.

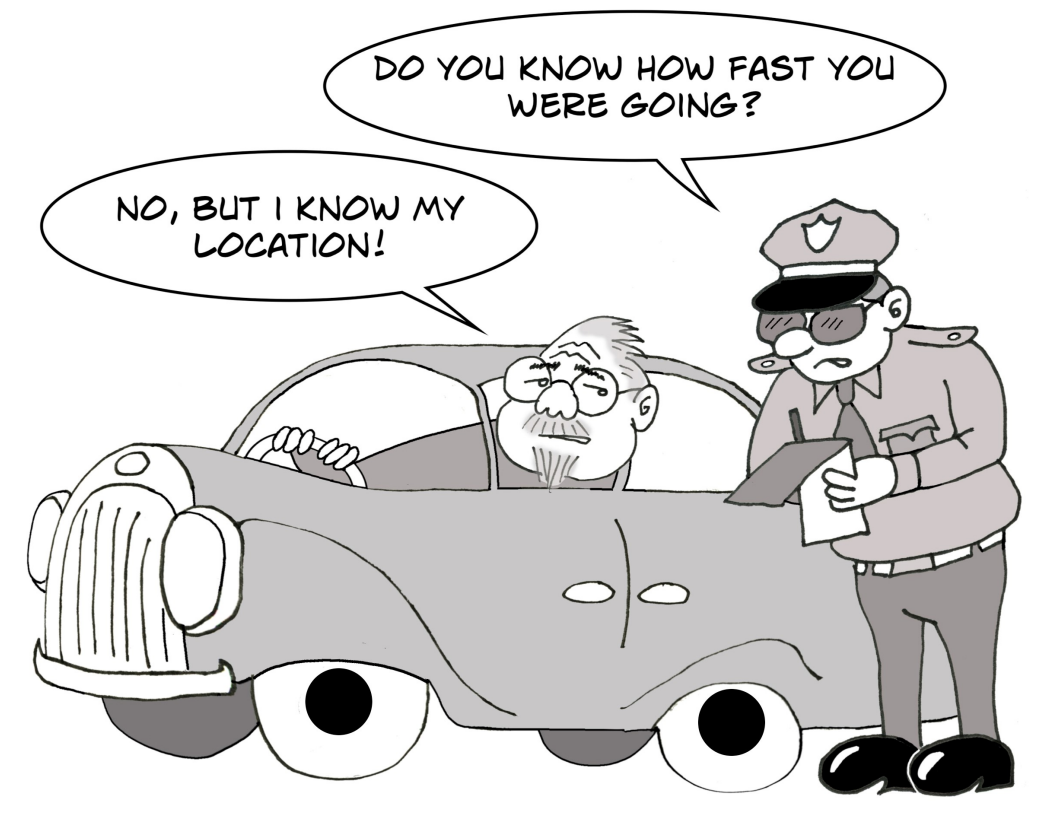

Figure 1.4: Heisenberg gets pulled over! (taken from [fon])

Schrödinger, Einstein, de Broglie and some of their other contemporaries were not happy with this view where physics fate was left to chance. Albert Einstein reacted with the remark that "God does not play dice." To which Niels Bohr reportedly replied to Einstein's expression of this sentiment by advising him to "stop telling God what to do."1

\footnotetext{
${ }^{1}$ source: https://en.wikipedia.org/wiki/Hidden-variable_theory
} 


\subsection{World of mathematics}

Schrödinger equations are partial differential equation of dispersive category. Intuitively, this means that something, initially concentrated, spreads out spatially. In mathematics, a partial differential equation is called dispersive, if the speed of wave propagation depends on its frequency, i.e., waves of different wavelength travel at different phase velocities.

The linear Schrödinger equation,

$$
i u_{t}+\Delta u=0
$$

has one derivative in time, $u_{t}, u=u(x, t)$ is a complex-valued function, and $\Delta$-stands for a Laplacian, which is two spatial derivative, having the property to diffuse the function $u$. Introducing a nonlinear term, $F(u)$ to the linear equation, we get

$$
i u_{t}+\Delta u \pm F(u)=0,
$$

a nonlinear Schrödinger type equation. Here, - sign indicates that the equation is called defocusing, this means that the nonlinear effects work in favor of the Laplacian resulting in faster dispersion. On the other hand, with + sign, the nonlinear term acts in an opposing manner to Laplacian causing a battle between dispersion and nonlinear effects. The battle opens the door to the questions we address in this research

- If the Laplacian is asymptotically larger, the solutions last forever.

- If the Laplacian and the nonlinear term are asymptotically equal, we get a borderline case, in which solutions become the solitary waves (or solitons).

- If the nonlinear term is larger, then a "blow up" (collapse or singularity) occurs.

The Schrödinger-type equations are one of the simplest models in a dispersive equations category, yet are still in their infancy for understanding the solutions. Dispersive equations provide descriptions of certain real life time-evolving phenomena in nature (see 
Figure 1.5). Examples in the Figure 1.5 include laser beams - propogation in nonlinear medium, rainbow (scattering phenomenon), Bose-Einstein condensate (bottom left) image by NIST/JILA/CU-Boulder, reappeared in [Orn17]) - velocity distribution data for a gas of rubidium atoms, the discovery of the Bose-Einstein condensate of 1920 only confirmed after 75 year, in $1995^{2}$ : atoms cooled to near absolute zero (273.15 degree Celsius) condensed from less dense areas on the left (red, yellow, and green) to very dense areas at the center and the right (blue and white), water waves. More often these phenomena are nonlinear and their study require special methods and techniques.
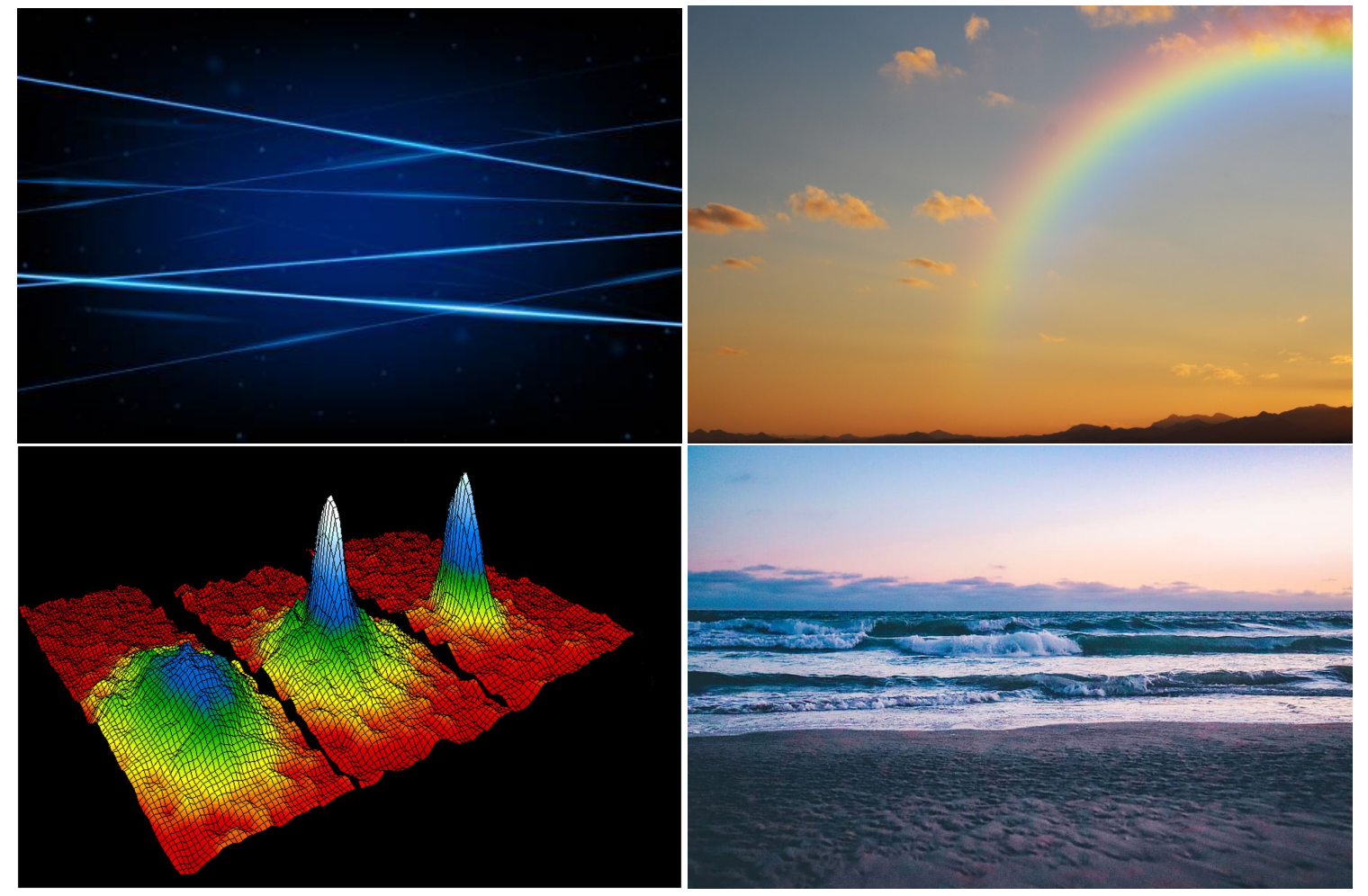

Figure 1.5: Dispersive equations are everywhere. [Bok], [lad], [Orn17], [Fla]

To summarize, various nonlinear phenomena give rise to nonlinear Schrödinger-type equation

$$
i u_{t}+\Delta u+F(u)=0,
$$

\footnotetext{
${ }^{2}$ Two labs received 2001 Nobel prize in Physics for their achievement.
} 
with $F(u)$ depending on a specific model or application. Some of the simplest types:

- Local (power type): $F(u)=|u|^{p-1} u$, nonlinear Schrödinger equation (NLS), see Figure 1.6 for an important application (here, $p$ is the total power of nonlinear term)

- Non-local (convolutions type):

- $F(u)=\left(|x|^{-b} *|u|^{2}\right) u$, Hartree equation $(b>0)$.

- $F(u)=\left(|x|^{-b} *|u|^{p}\right)|u|^{p-2} u$, generalized Hartree (abbreviated gHartree) equation.
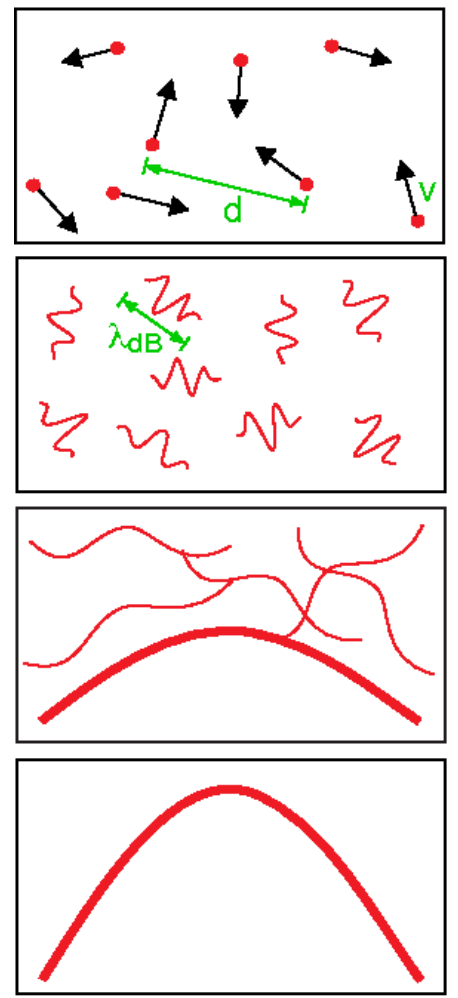

High Temperature T:

thermal velocity $v$

density $\mathrm{d}^{-3}$

"Billiard balls"

\section{Low}

Temperature T:

De Broglie wavelength

$\lambda \cdot d B=h / m v x T^{-1 / 2}$

"Wave packets"

$$
\mathrm{T}=\mathrm{T}_{\text {crit: }}
$$

Bose-Einstein

\section{Condensation}

$\lambda_{\mathrm{dB}} \approx \mathrm{d}$

"Matter wave overlap"

$$
\mathrm{T}=0 \text { : }
$$

Pure Bose

\section{condensate}

"Giant matter wave"

Figure 1.6: Where does NLS come from?, appeared in [DK98].

In this dissertation, we study the focusing generalized Hartree, or Schrödinger - Hartree, equation in $N$ spatial dimensions of the form

$$
i u_{t}+\Delta u+\left(\frac{1}{|x|^{N-\gamma}} *|u|^{p}\right)|u|^{p-2} u=0, \quad x \in \mathbb{R}^{N}, t \in \mathbb{R},
$$


where $0<\gamma<N, p \geq 2$, the function $u(x, t)$ is complex-valued and $*$ denotes the convolution operator in $\mathbb{R}^{N}$.

The equation (1.1) is a generalization of the standard Hartree equation with $p=2$,

$$
i u_{t}+\Delta u+\left(\frac{1}{|x|^{N-\gamma}} *|u|^{2}\right) u=0, \quad x \in \mathbb{R}^{N},
$$

which can be considered as a classical limit of a field equation describing a quantum mechanical non-relativistic many-boson system, interacting through a two-body potential $V(x)=\frac{1}{|x|^{N-\gamma}}$, see [GV80]. How it arises as an effective evolution equation in the meanfield limit of many-body quantum systems, can be traced to work of Hepp [Hep74], see also [GV80], [Spo80], [BGM00], [BEG ${ }^{+}$02], [FGS07]. Lieb and Yau [LY87] mention it in a context of developing theory for stellar collapse. The stellar remnants can take one of three forms (depending on the mass of a star): neutron stars, white dwarfs and black hole (see Figure 1.7, for collapse of larger stars, [Hoo]). Lieb and Thirring [LT84] conjectured that the collapse for boson stars can be predicted by a Hartree-type equation. A special case of the convolution with $\frac{1}{|x|}$ in $\mathbb{R}^{3}$ is referred to as the Coulomb potential, which goes back to the work of Lieb [Lie77] and has been intensively studied since then, see reviews [FTY02], [FTY00]. With $\gamma=2$ and $N=3$, a pseudo-relativistic version of this equation arises in the mean field limit of weakly interacting molecules and bosonic atoms (for example, see [FJL07], [FL04]), taking the form

$$
i u_{t}-\sqrt{-\Delta+m^{2}} u+\left(|x|^{-1} *|u|^{2}\right) u=0, \quad x \in \mathbb{R}^{3} .
$$

Unlike the standard nonlinear Schrödinger equation with pure nonlinearity $|u|^{p-1} u$, the distinct feature of the Hartree equation (1.2) is that it models systems with longrange interactions. Possible experimental realizations of such interactions, where the power in the convolution changes, include the interaction of ultracold Rydberg atoms that have large principal quantum numbers [Lus10]. The interactions between atoms in highly excited Rydberg levels are long range and dominated by dipole-dipole-type forces 


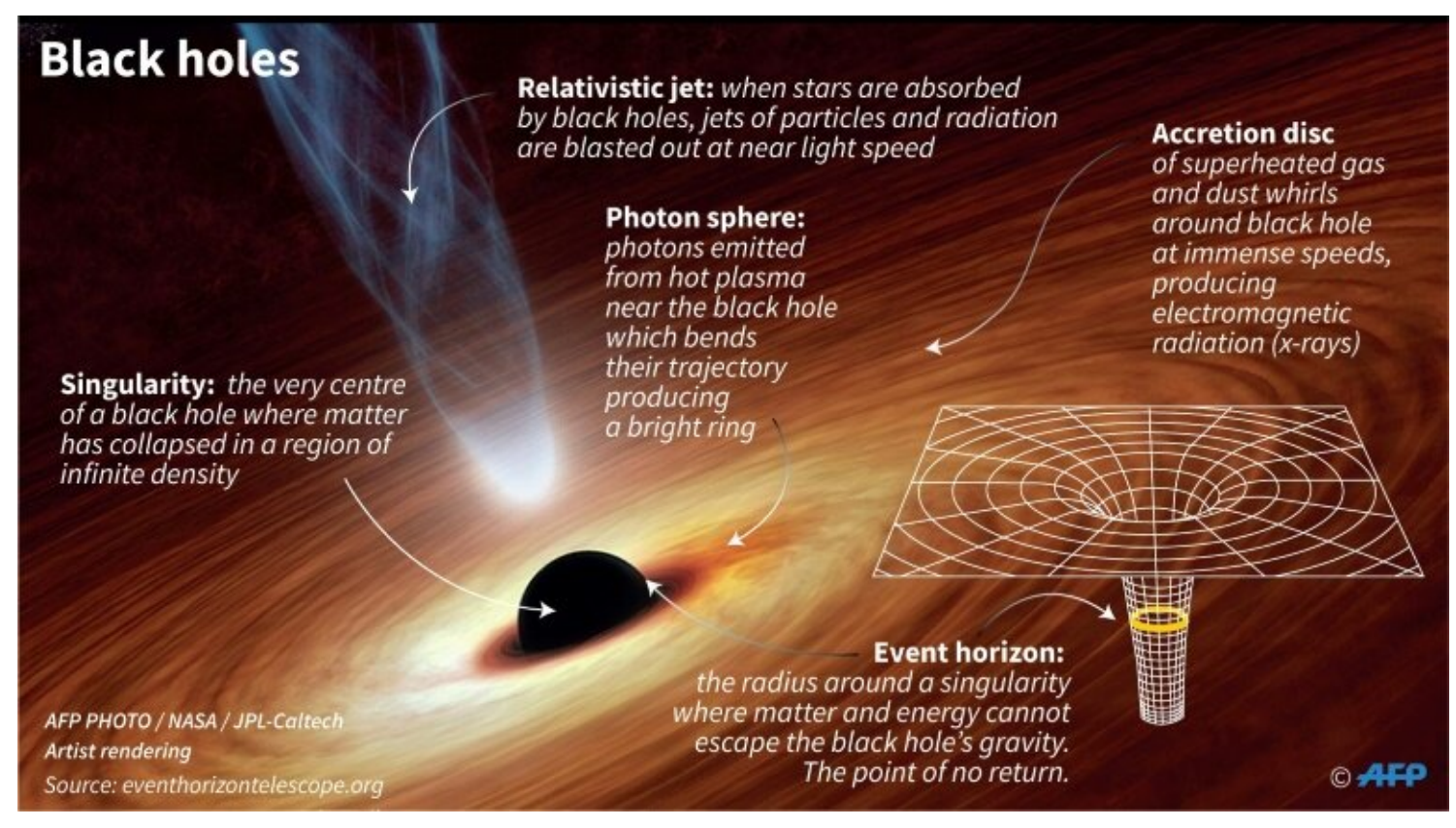

Figure 1.7: Black hole. At its center, the mass of a black hole is compressed into a single, zero-dimensional point, taken from [Hoo].

(the strength of the interaction between $\mathrm{Rb}$ atoms is about $10^{12}$ times stronger than that between $\mathrm{Rb}$ atoms in the ground state [SWM10]). The spatial dependence of interactions may be $1 /|x|^{3}$ for small $|x|$ and $1 /|x|^{6}$ for larger $|x|$. Other powers such as $1 /|x|^{2}$ are also possible, see [OGKA00]. Even more general, the potential can incorporate not only radial dependence, but also angular dependence $\frac{\theta(x)}{|x|^{N-\gamma}}$ [Lus10], but we do not consider the case here.

The equation (1.1) can be written (in terms of the wave function $u$ and the potential $V)$ as the Schrödinger - Poisson system of the form

$$
\left\{\begin{array}{l}
i u_{t}+\Delta u+V|u|^{p-2} u=0 \\
-\Delta V=(N-2)\left|\mathbb{S}^{N-1}\right||u|^{p}
\end{array}\right.
$$

where $\mathbb{S}^{N-1}$ stands for the surface area of $(N-1)$-dimensional unit sphere. This can be thought of as an electrostatic version of the Maxwell-Schrödinger system, which de- 
scribes the interaction between the electromagnetic field and the wave function related to a quantum non-relativistic charged particle (see, for example, [CG04] and [Lie03]).

With numerous applications, it makes sense to develop a unified mathematical theory of solutions behavior for the generalized Hartree equation (1.1). The thesis is devoted to that goal.

However, the nonlocal (convolution-type) nonlinearity offers certain complications to the extension of the theory, which was originally developed for the nonlinear Schrödinger equation with local nonlinearity. For instance, the presence of convolution makes it harder to study the solutions in spaces with higher regularity, since the convolution kernel in the gHartree equation can be written as an inverse (fractional) Laplacian, suggesting an inbuilt restriction. Another challenge is related to the ODE techniques, which are used to prove the uniqueness of ground state solutions; those methods are not compatible with the Hartree-type equations (see Section 2.8). Another challenge arises because of the slow decay of convolution kernels $\frac{1}{|x|^{b}}$ (related to the long-range interactions), which directly affects the blow-up analysis in the Hartree-type equations (see Remark 5.3.22, Remark 5.3.24 and Lemma 5.3.26 in Section 5.3). The study of certain spectral properties of linearized operators also require a different approach because of the presence of convolution kernel (see Proposition 5.3.7 in Section 5.3). These challenges serve as a motivation to change (if feasible) the techniques available for the nonlinear Scrödinger equation (with local nonlinearity) and, at the same time create new approaches to study the Hartree-type equations. 


\section{CHAPTER 2}

\section{PRELIMINARIES}

The greatest challenge to any thinker is stating the problem in a way that will allow a solution. (Bertrand Russell)

In Chapter 2 we establish some notation, initial definitions and basic estimates, which are used throughout the work.

\subsection{Notation}

For a given $1 \leq p<\infty$, we define the space

$$
L^{p}\left(\mathbb{R}^{N}\right)=\left\{f: \mathbb{R}^{N} \rightarrow \mathbb{R}: \int_{\mathbb{R}^{N}}|f(x)|^{p} d x<\infty\right\}
$$

equipped with the norm

$$
\|f\|_{L^{p}\left(\mathbb{R}^{N}\right)}=\left(\int_{\mathbb{R}^{N}}|f(x)|^{p} d x\right)^{\frac{1}{p}} .
$$

If $p=\infty$, we denote by $L^{\infty}\left(\mathbb{R}^{N}\right)$ the space of essentially bounded functions. Define the Schwartz space $\mathcal{S}\left(\mathbb{R}^{N}\right)$, the space of the $C^{\infty}$-functions decaying at infinity, i.e.,

$$
\mathcal{S}\left(\mathbb{R}^{N}\right)=\left\{f \in C^{\infty}\left(\mathbb{R}^{N}\right): x^{\alpha} \partial^{\beta} f \in L^{\infty}\left(\mathbb{R}^{N}\right) \text { for every } \alpha, \beta \in \mathbb{Z}_{+}^{N}\right\}
$$

The Fourier transform on $\mathbb{R}^{N}$ for $f \in \mathcal{S}\left(\mathbb{R}^{N}\right)$ is given by

$$
\hat{f}(\xi)=(2 \pi)^{-N / 2} \int_{\mathbb{R}^{N}} e^{-i x \xi} f(x) d x .
$$

One can also define (by duality) the space of tempered distributions $\mathcal{S}^{\prime}\left(\mathbb{R}^{N}\right)$

$$
\mathcal{S}^{\prime}\left(\mathbb{R}^{N}\right)=\left\{\mathcal{T}: \mathcal{S}\left(\mathbb{R}^{N}\right) \rightarrow \mathbb{C}: \mathcal{T} \text { is linear and continuous }\right\}
$$


and given $\mathcal{T} \in \mathcal{S}^{\prime}\left(\mathbb{R}^{N}\right)$ the Fourier transform $\widehat{\mathcal{T}} \in \mathcal{S}^{\prime}\left(\mathbb{R}^{N}\right)$ is defined as

$$
\widehat{\mathcal{T}}(f)=\mathcal{T}(\hat{f}), \text { for any } f \in \mathcal{S}\left(\mathbb{R}^{N}\right)
$$

Define the operator $|\nabla|^{s}$ for $s \geq 0$

$$
\widehat{|\nabla|^{s} f}(\xi)=|\xi|^{s} \hat{f}(\xi)
$$

which is also known as the Riesz operator of order $-s$, where $s>0$. This allows us to define the homogeneous Sobolev space

$$
\dot{W}^{s, p}\left(\mathbb{R}^{N}\right)=\left\{f \in \mathcal{S}^{\prime}\left(\mathbb{R}^{N}\right):|\nabla|^{s} f \in L^{p}\left(\mathbb{R}^{N}\right)\right\}
$$

and the associated norm

$$
\|f\|_{\dot{W}^{s, p}\left(\mathbb{R}^{N}\right)}=\left\||\nabla|^{s} f\right\|_{L^{p}\left(\mathbb{R}^{N}\right)}
$$

for $p \geq 1$. The inhomogeneous Sobolev space is defined by

$$
W^{s, p}\left(\mathbb{R}^{N}\right)=\left\{f \in \mathcal{S}^{\prime}\left(\mathbb{R}^{N}\right):\left(1+|\nabla|^{2}\right)^{\frac{s}{2}} f \in L^{p}\left(\mathbb{R}^{N}\right)\right\},
$$

equipped with the norm

$$
\|f\|_{W^{s, p}\left(\mathbb{R}^{N}\right)}=\left\|\left(1+|\nabla|^{2}\right)^{\frac{s}{2}} f\right\|_{L^{p}\left(\mathbb{R}^{N}\right)}
$$

where

$$
\left(\left(1+|\nabla|^{2}\right)^{\frac{s}{2}} f\right)^{\wedge}=\langle\xi\rangle^{s} \hat{f}
$$

Here, $\langle\xi\rangle=\left(1+|\xi|^{2}\right)^{1 / 2}$, often called the Japanese bracket. If $p=2$, we denote

$$
\dot{H}^{s}\left(\mathbb{R}^{N}\right)=\dot{W}^{s, 2}\left(\mathbb{R}^{N}\right) \quad \text { and } \quad H^{s}\left(\mathbb{R}^{N}\right)=W^{s, 2}\left(\mathbb{R}^{N}\right)
$$

For any spacetime slab $I \times \mathbb{R}^{N}$, we use $L_{t}^{q} L_{x}^{r}\left(I \times \mathbb{R}^{N}\right)$ to denote the space of functions $u: I \times \mathbb{R}^{N} \rightarrow \mathbb{C}$, whose norm is

$$
\|u\|_{L_{t}^{q} L_{x}^{r}\left(I \times \mathbb{R}^{N}\right)}=\left(\int_{I}\left(\int_{\mathbb{R}^{N}}|f(x, t)|^{r} d x\right)^{\frac{q}{r}} d t\right)^{\frac{1}{q}}<\infty .
$$


Lastly, we write $X \lesssim Y$ or $Y \gtrsim X$ whenever $X \leq C Y$ for some constant $C>0$ depending upon dimension $N$ or powers $p$ and $\gamma$. In case $C$ depends upon some additional parameters, we will indicate this as follows: for example, $X \lesssim_{u} Y$ denotes that $X \leq C_{u} Y$ for some $C_{u}$ depending on $u$. Similarly, we will write $X \sim Y$ if $X \lesssim Y \lesssim X$.

\subsection{Symmetries}

We consider initial data in the $H^{1}$ space, $u_{0} \in H^{1}\left(\mathbb{R}^{N}\right)$, so that we can study finite Hamiltonian or finite energy solutions (definition below). During their lifespans the solution $u(x, t)$ to $(1.1)$ satisfy the following mass, energy and momentum conservation laws:

$$
\begin{aligned}
& M[u(t)] \stackrel{\text { def }}{=} \int|u(x, t)|^{2} d x=\int\left|u_{0}(x)\right|^{2} d x=M\left[u_{0}\right], \\
& E[u(t)] \stackrel{\text { def }}{=} \frac{1}{2} \int|\nabla u(x, t)|^{2} d x-\frac{1}{2 p} \int\left(\frac{1}{|x|^{N-\gamma}} *|u(\cdot, t)|^{p}\right)|u(x, t)|^{p} d x=E\left[u_{0}\right], \\
& P[u(t)] \stackrel{\text { def }}{=} \operatorname{Im} \int \bar{u}(x, t) \nabla u(x, t) d x=\operatorname{Im} \int \bar{u}_{0}(x) \nabla u_{0}(x) d x=P\left[u_{0}\right] .
\end{aligned}
$$

The equation (1.1) has several invariances: if $u(x, t)$ is a solution to $(1.1)$, so is $\tilde{u}(x, t)$ :

- Spatial translation: for a fixed $x_{0} \in \mathbb{R}^{N}, \tilde{u}(x, t)=u\left(x-x_{0}, t\right)$.

- Time translation: for a fixed $\tau \in \mathbb{R}, \tilde{u}(x, t)=u(x, t+\tau)$.

- Time reversal: $\tilde{u}(x, t)=\bar{u}(x,-t)$.

- Phase rotation: for a fixed $\theta \in[0,2 \pi), \tilde{u}(x, t)=e^{i \theta} u(x, t)$.

- Spatial rotation: for a fixed $R \in S O(N), \tilde{u}(x, t)=u\left(R^{-1} x, t\right)$.

- Galilean transformation: for a fixed $\xi_{0} \in \mathbb{R}^{N}, \tilde{u}(x, t)=e^{i\left(x \cdot \xi_{0}-t\left|\xi_{0}\right|^{2}\right)} u\left(x-\xi_{0} t, t\right)$.

- Scaling: for a fixed $\lambda \in(0, \infty), \tilde{u}(x, t)=\lambda^{\frac{\gamma+2}{2(p-1)}} u\left(\lambda x, \lambda^{2} t\right)$.

The equation (1.1) is referred to as the $\dot{H}^{s_{c}}$-critical, if the $\dot{H}^{s_{c}}$ norm of the solution is invariant under the scaling. The critical scaling index $s_{c}$, which comes from the scaling 
invariance, is defined as

$$
s_{c}=\frac{N}{2}-\frac{\gamma+2}{2(p-1)} .
$$

- If $s_{c}=0$, or $p=1+\frac{\gamma+2}{N}$, the equation (1.1) is referred to as the mass-critical (or $L^{2}$-critical).

- If $s_{c}=1$, or $p=1+\frac{\gamma+2}{N-2}$, the problem is called the energy-critical (or $\dot{H}^{1}$ critical).

- If $0<s_{c}<1$, the equation is intercritical.

- If $s_{c}>1$, the equation is said to be energy-supercritical (or $\dot{H}^{1}$-supercritical).

\subsection{Embeddings}

Here we state embeddings and inequalities used later in the document. The first one is most useful to deal with the convolution (nonlocal) term in the gHartree equation (1.1).

Lemma 2.3.1 (Hardy-Littlewood-Sobolev inequality, [Lie83]) For $0<\gamma<N$ and $1<p, q<\infty$, there exists a sharp constant $c_{N, p, \gamma}>0$ such that

$$
\left\|\int_{\mathbb{R}^{N}} \frac{u(y)}{|x-y|^{N-\gamma}} d y\right\|_{L^{q}\left(\mathbb{R}^{N}\right)} \leq c_{N, p, \gamma}\|u\|_{L^{p}\left(\mathbb{R}^{N}\right)},
$$

where $\frac{1}{q}=\frac{1}{p}-\frac{\gamma}{N}$ and $p<\frac{N}{\gamma}$.

Lemma 2.3.2 (Sobolev inequality, [Ste70]) Let $0 \leq s_{1}<s_{2}, 1<r_{2}<r_{1}<\infty$. Then

$$
\left\||\nabla|^{s_{1}} f\right\|_{L^{r_{1}\left(\mathbb{R}^{N}\right)}} \leq C\left|\left\|\left.\nabla\right|^{s_{2}} f\right\|_{L^{r_{2}\left(\mathbb{R}^{N}\right)}}\right.
$$

where $\frac{N}{r_{1}}-s_{1}=\frac{N}{r_{2}}-s_{2}$.

Lemma 2.3.3 (Radial Sobolev inequality, [Str77a]) Let $u \in H^{1}\left(\mathbb{R}^{N}\right)$ be radially symmetric and $N \geq 2$. Then, for any $R>0$,

$$
\|u\|_{L^{\infty}(|x| \geq R)} \leq \frac{C}{R^{\frac{N-1}{2}}}\|u\|_{H^{1}\left(\mathbb{R}^{N}\right)} .
$$




\subsection{Strichartz estimates}

We define the linear Schrödinger evolution from initial data $f_{0}(x)$ as follows

$$
f(x, t)=e^{i t \Delta} f_{0}(x)=\frac{1}{(4 \pi i t)^{N / 2}} \int_{\mathbb{R}^{N}} e^{i \frac{|x-y|^{2}}{4 t}} f_{0}(y) d y
$$

Then by the $L^{2}$-isometry and $L^{\infty}-L^{1}$ estimate, one can obtain the time decay estimate (see, for example [LP09, Lemma 4.1]) for $2 \leq r \leq \infty$ and $\frac{1}{r}+\frac{1}{r^{\prime}}=1$,

$$
\left\|e^{i t \Delta} f_{0}(x)\right\|_{L^{r}\left(\mathbb{R}^{N}\right)} \lesssim|t|^{-\frac{N}{2}}\left\|f_{0}\right\|_{L^{r^{\prime}\left(\mathbb{R}^{N}\right)}}
$$

for all $t \neq 0$. In some PDE applications, one needs to have estimates both in space and time, for example, in the study of well-posedness. This space-time integrability is demonstrated by Strichartz estimates. In what follows, we will always consider the case $0 \leq s<\frac{N}{2}$

Definition 2.4.1 The pair $(q, r)$ is called $L^{2}$-admissible pair, if $N \geq 1$, and

$$
\frac{2}{q}+\frac{N}{r}=\frac{N}{2}, \quad 2 \leq q, r \leq \infty \text { provided }(q, r, N) \neq(2, \infty, 2) .
$$

Remark 2.4.2 One can also define the $\dot{H}^{s}$-admissibility for $N \geq 1$ and $s \geq-1$ by

$$
\frac{2}{q}+\frac{N}{r}=\frac{N}{2}-s
$$

Definition 2.4.3 (see [Fos05]) The pair $(q, r)$ is called acceptable, if $N \geq 1$ and

$$
1 \leq q, r \leq \infty \quad \text { and } \quad \frac{1}{q}<N\left(\frac{1}{2}-\frac{1}{r}\right), \quad \text { or }(q, r)=(\infty, 2)
$$

Remark 2.4.4 For $s \geq 0$, every $\dot{H}^{s}$-admissible pair is acceptable.

We now recall the well-known Strichartz estimates (see [Str77b], [KT98], [Caz03]). 
Lemma 2.4.5 If $(q, r)$ is an $\dot{H}^{s}$-admissible pair with $s \geq 0$, then the following linear estimate holds

$$
\left\|e^{i t \Delta} f\right\|_{L_{t}^{q} L_{x}^{r}\left(\mathbb{R} \times \mathbb{R}^{N}\right)} \lesssim\|f\|_{\dot{H}_{x}^{s}\left(\mathbb{R}^{N}\right)}
$$

We next consider the inhomogeneous estimate, see [Fos05].

Lemma 2.4.6 Let $1 \leq q, \tilde{q}, r, \tilde{r} \leq \infty$. Suppose the pairs $(q, r)$ and $(\tilde{q}, \tilde{r})$ are acceptable, satisfy

$$
\frac{1}{q}+\frac{1}{\tilde{q}}=\frac{N}{2}\left(1-\frac{1}{r}-\frac{1}{\tilde{r}}\right)
$$

and verify the following conditions:

- $N=2$, we require that $r, \tilde{r}<\infty$,

- $N>2$, we classify two cases;

- non sharp case:

$$
\begin{gathered}
\frac{1}{q}+\frac{1}{\tilde{q}}<1, \\
\frac{N-2}{N} \leq \frac{r}{\tilde{r}} \leq \frac{N}{N-2}
\end{gathered}
$$

- sharp case:

$$
\begin{gathered}
\frac{1}{q}+\frac{1}{\tilde{q}}=1, \\
\frac{N-2}{N}<\frac{r}{\tilde{r}}<\frac{N}{N-2}, \\
\frac{1}{r} \leq \frac{1}{q}, \quad \frac{1}{\tilde{r}} \leq \frac{1}{\tilde{q}} .
\end{gathered}
$$

Then the following estimate holds

$$
\left\|\int_{0}^{t} e^{i\left(t-t^{\prime}\right) \Delta} F\left(t^{\prime}\right) d t^{\prime}\right\|_{L_{t}^{q} L_{x}^{r}}+\left\|\int_{t}^{\infty} e^{i\left(t-t^{\prime}\right) \Delta} F\left(t^{\prime}\right) d t^{\prime}\right\|_{L_{t}^{q} L_{x}^{r}} \lesssim\|F\|_{L_{t}^{\tilde{q}^{\prime}} L_{x}^{\tilde{r}^{\prime}}}
$$


For the next notation, we would like to have a uniform control of the constants in Strichartz estimates (in the intercritical case, i.e., $0<s_{c}<1$ ) and for that we need the following restriction on the range of pairs $(q, r)$ (as introduced in [Gue14], also see [HR08]):

$$
\left\{\begin{array}{l}
\left(\frac{2}{1-s}\right)^{+} \leq q \leq \infty, \quad \frac{2 N}{N-2 s} \leq r \leq\left(\frac{2 N}{N-2}\right)^{-}, \text {if } N \geq 3 \\
\left(\frac{2}{1-s}\right)^{+} \leq q \leq \infty, \quad \frac{2}{1-s} \leq r \leq\left(\left(\frac{2}{1-s}\right)^{+}\right)^{\prime}, \text { if } N=2 \\
\frac{4}{1-2 s} \leq q \leq \infty, \quad \frac{2}{1-2 s} \leq r \leq \infty, \text { if } N=1 .
\end{array}\right.
$$

Here, $n^{+}$is a fixed number (slightly) greater than $n$ such that $\frac{1}{n}=\frac{1}{n^{+}}+\frac{1}{\left(n^{+}\right)^{\prime}}$. Respectively, $n^{-}$is a fixed number (slightly) less than $n$. We now introduce the $S\left(\dot{H}^{s}\right)$ notation:

$$
\|u\|_{S\left(\dot{H}^{s}\right)}=\sup \left\{\|u\|_{L_{t}^{q} L_{x}^{r}}:(q, r) \text { as in Remark 2.4.2 and (2.10) }\right\} .
$$

Similarly, in order to define the dual Strichartz norm (in the inter-critical case), we set the following restrictions:

$$
\left\{\begin{array}{l}
\left(\frac{2}{1+s}\right)^{+} \leq q \leq\left(\frac{1}{s}\right)^{-}, \quad\left(\frac{2 N}{N-2 s}\right)^{+} \leq r \leq\left(\frac{2 N}{N-2}\right)^{-}, \text {if } N \geq 3 \\
\left(\frac{2}{1+s}\right)^{+} \leq q \leq\left(\frac{1}{s}\right)^{-}, \quad\left(\frac{2}{1-s}\right)^{+} \leq r \leq\left(\left(\frac{2}{1+s}\right)^{+}\right)^{\prime}, \text { if } N=2 \\
\frac{2}{1+2 s} \leq q \leq\left(\frac{1}{s}\right)^{-}, \quad\left(\frac{2}{1-s}\right)^{+} \leq r \leq \infty, \text { if } N=1,
\end{array}\right.
$$

and define the dual Strichartz norm as

$$
\|u\|_{S^{\prime}\left(\dot{H}^{-s}\right)}=\inf \left\{\|u\|_{L_{t}^{q^{\prime}} L_{x}^{r^{\prime}}}: \text { with }(q, r) \text { as in Remark 2.4.2 and (2.11) }\right\},
$$

where $\frac{1}{q^{\prime}}+\frac{1}{q}=1, \frac{1}{r^{\prime}}+\frac{1}{r}=1$. Note that $S\left(\dot{H}^{0}\right)=S\left(L^{2}\right)$ and $S^{\prime}\left(\dot{H}^{-0}\right)=S^{\prime}\left(L^{2}\right)$. Using the Lemma 2.3.2 (since $e^{i t \Delta}$ commutes with derivatives), we obtain

Corollary 2.4.7 For any $\dot{H}^{s}$-admissible pair $\left(q, r_{s}\right)$ with $s \geq 0$, if the pairs $(q, r)$ and $(\tilde{q}, \tilde{r})$ are $L^{2}$-admissible and satisfy the assumptions in Lemma 2.4.6, then

$$
\left\|\int_{0}^{t} e^{i\left(t-t^{\prime}\right) \Delta} F\left(\cdot, t^{\prime}\right) d t^{\prime}\right\|_{L_{t}^{q} L_{x}^{r_{s}}} \lesssim\left\||\nabla|^{s} \int_{0}^{t} e^{i\left(t-t^{\prime}\right) \Delta} F\left(\cdot, t^{\prime}\right) d t^{\prime}\right\|_{L_{t}^{q} L_{x}^{r}} \lesssim\left\||\nabla|^{s} F\right\|_{L_{t}^{\tilde{q}^{\prime}} L_{x}^{\tilde{r}^{\prime}}} .
$$


Remark 2.4.8 Although, we can have a wider range for $(\tilde{q}, \tilde{r})$ (from Lemma 2.4.6), for the well-posedness in the energy-supercritical cases it suffices to use Corollary 2.4.7.

We next consider a function $\eta \in C_{c}^{\infty}\left(\mathbb{R}^{N}\right)$ such that

$$
\eta(\xi)= \begin{cases}1 & \text { if }|\xi| \leq 1 \\ 0 & \text { if }|\xi| \geq 2\end{cases}
$$

For each dyadic number $\mathcal{N} \in 2^{\mathbb{Z}}$ we define the Littlewood-Paley operators

$$
\widehat{P_{\leq \mathcal{N}} f}(\xi):=\eta\left(\frac{\xi}{\mathcal{N}}\right) \widehat{f}(\xi), \quad \widehat{P_{>\mathcal{N}} f}(\xi):=\left(1-\eta\left(\frac{\xi}{\mathcal{N}}\right)\right) \widehat{f}(\xi)
$$

and

$$
\widehat{P_{\mathcal{N}} f}(\xi):=\left(\eta\left(\frac{\xi}{\mathcal{N}}\right)-\eta\left(\frac{2 \xi}{\mathcal{N}}\right)\right) \widehat{f}(\xi)
$$

We now define the Besov space $\dot{B}_{r, 2}^{s}\left(\mathbb{R}^{N}\right)$ for $1 \leq r \leq \infty$ and $s \in \mathbb{R}$ (see [Caz03, Section $1.4],[$ Gue14])

$$
\|u\|_{\dot{B}_{r, 2}^{s}}^{2}=\sum_{j=-\infty}^{\infty}\left(2^{s j}\left\|P_{2^{j}} u\right\|_{L_{x}^{r}}\right)^{2}=\sum_{\mathcal{N} \in 2^{\mathbb{Z}}}\left(\mathcal{N}^{s}\left\|P_{\mathcal{N}} u\right\|_{L_{x}^{r}}\right)^{2} .
$$

We point out that the estimates in Lemma 2.4.6 can also be stated in Besov spaces, see [Caz03, Corollary 2.3.9].

Lemma 2.4.9 Given any $s \geq 0$, if $(q, r)$ and $(\tilde{q}, \tilde{r})$ are admissible pairs satisfying the assumptions in Lemma 2.4.6, then

$$
\left\|e^{i t \Delta} f\right\|_{L_{t}^{q} \dot{B}_{r, 2}^{s}\left(\mathbb{R} \times \mathbb{R}^{N}\right)} \lesssim\|f\|_{\dot{B}_{2,2}^{s}\left(\mathbb{R}^{N}\right)}=\|f\|_{\dot{H}^{s}\left(\mathbb{R}^{N}\right)}
$$

and

$$
\left\|\int_{0}^{t} e^{i\left(t-t^{\prime}\right) \Delta} F\left(\cdot, t^{\prime}\right) d t^{\prime}\right\|_{L_{t}^{q} \dot{B}_{r, 2}^{s}\left(\mathbb{R} \times \mathbb{R}^{N}\right)} \lesssim\|F\|_{L_{t}^{\tilde{q}^{\prime}} \dot{B}_{\tilde{r}^{\prime}, 2}^{s}\left(\mathbb{R} \times \mathbb{R}^{N}\right)} .
$$

We also record the following two inequalities (see Remark 1.4.3.(i) and (ii) in [Caz03]) that if $2 \leq r<\infty$, then

$$
\left\||\nabla|^{s} u\right\|_{L_{x}^{r}} \lesssim\|u\|_{\dot{B}_{r, 2}^{s}}^{2}
$$


and if $1<r^{\prime}<2$, then

$$
\|u\|_{L_{t}^{q^{\prime} \dot{B}_{r^{\prime}, 2}^{s}}}^{2} \lesssim\left\||\nabla|^{s} u\right\|_{L_{t}^{q^{\prime}} L_{x}^{r^{\prime}}}
$$

Lastly, we give a Sobolev embedding in Besov space (see Remark 1.4.2.(v) in [Caz03])

$$
\|u\|_{\dot{B}_{r_{1}, 2}^{s_{1}}} \lesssim\|u\|_{\dot{B}_{r_{2}, 2}^{s_{2}}}
$$

where $\frac{1}{r_{1}}-\frac{s_{1}}{N}=\frac{1}{r_{2}}-\frac{s_{2}}{N}$ and $1 \leq r_{2} \leq r_{1} \leq \infty, s_{1}, s_{2} \in \mathbb{R}$.

\subsection{Fractional calculus}

Lemma 2.5.1 (Product rule, see [FGO18] and references therein) Let $s>0$ and $1<$ $r, p_{1}, p_{2}, q_{1}, q_{2}<\infty$ such that $\frac{1}{r}=\frac{1}{p_{i}}+\frac{1}{q_{i}}$ for $i=1,2$. Then,

$$
\left\||\nabla|^{s}(f g)\right\|_{L^{r}\left(\mathbb{R}^{N}\right)} \lesssim\|f\|_{L^{p_{1}\left(\mathbb{R}^{N}\right)}}\left\||\nabla|^{s} g\right\|_{L^{q_{1}\left(\mathbb{R}^{N}\right)}}+\left\||\nabla|^{s} f\right\|_{L^{p_{2}\left(\mathbb{R}^{N}\right)}}\|g\|_{L^{q_{2}\left(\mathbb{R}^{N}\right)}}
$$

We also record the following fractional chain rule.

Lemma 2.5.2 (Fractional chain rule, [CW91]) Suppose $G \in C^{1}(\mathbb{C})$ and $s \in(0,1]$. Let $1<q, q_{1}, q_{2}<\infty$ are such that $\frac{1}{q}=\frac{1}{q_{1}}+\frac{1}{q_{2}}$. Then,

$$
\left\||\nabla|^{s} G(u)\right\|_{L^{q}\left(\mathbb{R}^{N}\right)} \lesssim\left\|G^{\prime}(u)\right\|_{L^{q_{1}\left(\mathbb{R}^{N}\right)}}\left\||\nabla|^{s} u\right\|_{L^{q_{2}\left(\mathbb{R}^{N}\right)}} .
$$

When the function $G$ is no longer $C^{1}$, though it is Hölder continuous, we have the following chain rule:

Lemma 2.5.3 (Fractional chain rule for Hölder continuous functions, [Vis06]) Let $G$ be a Hölder continuous function of order $0<\rho<1$. Then, for every $0<s<\rho$, $1<q<\infty$, and $s / \rho<\sigma<1$, we have

$$
\left\||\nabla|^{s} G(u)\right\|_{L^{q}\left(\mathbb{R}^{N}\right)} \lesssim\left\||u|^{\rho-\frac{s}{\sigma}}\right\|_{L^{q_{1}\left(\mathbb{R}^{N}\right)}}\left\||\nabla|^{\sigma} u\right\|_{L^{\frac{s}{\sigma} q_{2}}}^{\frac{s}{\sigma}}
$$

provided $\frac{1}{q}=\frac{1}{q_{1}}+\frac{1}{q_{2}}$ and $\left(1-\frac{s}{\rho \sigma}\right) q_{1}>1$. 
We also have the following corollary as a consequence of Lemma 2.5.2 and Lemma 2.5.3 along with interpolation.

Corollary 2.5.4 (Corollary 2.7 in [KV10]) Let $F(u)=|u|^{p-2} u$ with $p \geq 2$ and let $s>$ 1 if $p$ is an even integer or $1<s<p-1$ otherwise. Then

$$
\left\||\nabla|^{s} F(u)\right\|_{S^{\prime}\left(L^{2}\right)} \lesssim\left\||\nabla|^{s} u\right\|_{S\left(L^{2}\right)}\|u\|_{\frac{(p-2)(N+2)}{L_{t, x}^{\gamma+2}}}^{p-2} .
$$

\subsection{The local theory}

We first observe that by the Duhamel formulation (for example, see [Tao06]), the solution $u: I \times \mathbb{R}^{N} \rightarrow \mathbb{C}$ to the equation (1.1) can be written in the integral form

$$
u(t)=e^{i t \Delta} u_{0}+i \int_{0}^{t} e^{i\left(t-t^{\prime}\right) \Delta}\left(|x|^{-(N-\gamma)} *|u|^{p}\right)|u|^{p-2} u\left(t^{\prime}\right) d t^{\prime}
$$

for all $t \in I \subset \mathbb{R}$. The interval $I$ is known as the lifespan of $u$. If $I=\mathbb{R}$, the solution $u$ is said to be global. We want to prove that the equation (1.1) is locally well-posed, i.e., there exists a unique local-in time solution satisfying (2.16) that lies in $C_{t}^{0} H_{x}^{s_{c}} \cap L_{t}^{q} W_{x}^{s_{c}, r}$ and the map from the initial data to the solution (in some cases) is uniformly continuous in these norms.

Remark 2.6.1 Let $f(z)=|z|^{p-2} z$. The complex derivative of $f$ is given by

$$
f_{z}(z)=\frac{p}{2}|z|^{p-2} \quad \text { and } \quad f_{\bar{z}}(z)=\frac{p-2}{2}|z|^{p-4} z^{2}
$$

For $z_{1}, z_{2} \in \mathbb{C}$ we get

$f\left(z_{1}\right)-f\left(z_{2}\right)=\int_{0}^{1}\left[f_{z_{1}}\left(z_{2}+\theta\left(z_{1}-z_{2}\right)\right)\left(z_{1}-z_{2}\right)+f_{\bar{z}_{1}}\left(z_{2}+\theta\left(z_{1}-z_{2}\right)\right)\left(\overline{z_{1}-z_{2}}\right)\right] d \theta$

Hence,

$$
\left|f\left(z_{1}\right)-f\left(z_{2}\right)\right| \lesssim\left(\left|z_{1}\right|^{p-2}+\left|z_{2}\right|^{p-2}\right)\left|z_{1}-z_{2}\right|
$$


Also, observe that for $p \geq 1$ (e.g., see [CFH11])

$$
\left.|| z_{1}\right|^{p}-\left|z_{2}\right|^{p}\left|\lesssim\left(\left|z_{1}\right|^{p-1}+\left|z_{2}\right|^{p-1}\right)\right| z_{1}-z_{2} \mid
$$

\subsubsection{Local well-posedness in $H^{1}$}

We consider the integral equation (2.16) with $u_{0} \in H^{1}\left(\mathbb{R}^{N}\right)$ and $0<\gamma<N$ with

$$
\begin{cases}2 \leq p \leq 1+\frac{\gamma+2}{N-2}, & \text { if } N \geq 3 \\ 2 \leq p<\infty, & \text { if } N=1,2\end{cases}
$$

Proposition 2.6.2 If $p$ satisfies (2.20), then for $u_{0} \in H^{1}\left(\mathbb{R}^{N}\right)$ there exists $T>0$ and $a$ unique solution $u(x, t)$ of the integral equation (2.16) in the time interval $[0, T]$ with

$$
u \in C\left([0, T] ; H^{1}\left(\mathbb{R}^{N}\right)\right) \cap L^{q_{1}}\left([0, T] ; W^{1, r_{1}}\left(\mathbb{R}^{N}\right)\right),
$$

where $\left(q_{1}, r_{1}\right)$ is an $L^{2}$-admissible pair given by

$$
\left(q_{1}, r_{1}\right)=\left(\frac{2 p}{1+s_{c}(p-1)}, \frac{2 N p}{N+\gamma}\right)
$$

In the energy-critical case $p=1+\frac{\gamma+2}{N-2}\left(\right.$ or $\left.s_{c}=1\right)$ we require an additional assumption of smallness of $\left\|u_{0}\right\|_{H_{x}^{1}}$. In any energy-subcritical case $p<1+\frac{\gamma+2}{N-2}$ the time $T=$ $T\left(\left\|u_{0}\right\|_{H^{1}}, N, p, \gamma\right)>0$

Proof. For $T>0$, specified later, define $\nu(u)=\max \left\{\sup _{t \in[0, T]}\|u\|_{H_{x}^{1}},\|u\|_{L_{t}^{q_{1}} W_{x}^{1, r_{1}}}\right\}$ and for an appropriately defined constant $M>0$, also specified later, let

$$
\mathcal{S}=\left\{u \in C\left([0, T] ; H_{x}^{1}\left(\mathbb{R}^{N}\right)\right) \cap L_{t}^{q_{1}}\left([0, T] ; W_{x}^{1, r_{1}}\left(\mathbb{R}^{N}\right)\right): \nu(u) \leq M\right\}
$$

We prove that the following operator

$$
\Phi(u(t))=e^{i t \Delta} u_{0}+i \int_{0}^{t} e^{i\left(t-t^{\prime}\right) \Delta} F\left(u\left(t^{\prime}\right)\right) d t^{\prime}
$$


is a contraction on the set $\mathcal{S}$, where

$$
F\left(u\left(t^{\prime}\right)\right)=\left(|x|^{-(N-\gamma)} *|u|^{p}\right)|u|^{p-2} u\left(t^{\prime}\right)
$$

Using Lemma 2.4.6, we obtain

$$
\|\Phi(u(t))\|_{L_{t}^{q_{1}} L_{x}^{r_{1}}} \lesssim\left\|u_{0}\right\|_{L_{x}^{2}}+\|F(u)\|_{L_{t}^{q_{1}^{\prime}} L_{x}^{r_{1}^{\prime}}}
$$

and

$$
\|\nabla \Phi(u(t))\|_{L_{t}^{q_{1}} L_{x}^{r_{1}}} \lesssim\left\|\nabla u_{0}\right\|_{L_{x}^{2}}+\|\nabla F(u)\|_{L_{t}^{q_{1}^{\prime}} L_{x}^{r_{1}^{\prime}}}
$$

Using Hölder's inequality in time on the second term in (2.25) and (2.26), we have

$$
\|F(u)\|_{L_{t}^{q_{1}^{\prime}} L_{x}^{r_{1}^{\prime}}} \lesssim T^{\theta}\|F(u)\|_{L_{t}^{q_{1}} L_{x}^{r_{1}^{\prime}}} \text { and }\|\nabla F(u)\|_{L_{t}^{q_{1}^{\prime}} L_{x}^{r_{1}^{\prime}}} \lesssim T^{\theta}\|\nabla F(u)\|_{L_{t}^{q_{1}} L_{x}^{r_{1}^{\prime}}}
$$

where $\theta=\frac{\left(1-s_{c}\right)(p-1)}{p}$. Using Hölder's inequality, Lemma 2.3.1 and Lemma 2.3.2, we estimate

$$
\begin{aligned}
\|F(u)\|_{L_{t}^{q_{1}} L_{x}^{r_{1}^{\prime}}} & \lesssim\left\|\left(|x|^{-(N-\gamma)} *|u|^{p}\right)\right\|_{L_{t}^{q_{1}} L_{x}^{\frac{2 N}{N-\gamma}}}\left\||u|^{p-2} u\right\|_{L_{t}^{\infty} L_{x}^{\frac{2 N p}{(N+\gamma)(p-1)}}} \\
& \lesssim\|u\|_{L_{t}^{q_{1} p} L_{x}^{r_{1}}}^{p}\|u\|_{L_{t}^{\infty} L_{x}^{r_{1}}}^{p-1} \\
& \lesssim\|u\|_{L_{t}^{\infty(p-1)} L_{x}^{r_{1}}}^{2}\|u\|_{L_{t}^{q_{1}} L_{x}^{r_{1}}} \lesssim\|u\|_{L_{t}^{\infty} H_{x}^{1}}^{2(p-1)}\|u\|_{L_{t}^{q_{1}} L_{x}^{r_{1}}}
\end{aligned}
$$

and (noting that the gradient lands on two different terms)

$$
\begin{aligned}
\|\nabla F(u)\|_{L_{t}^{q_{1}} L_{x}^{r_{1}^{\prime}} \lesssim} & \left\|\left(|x|^{-(N-\gamma)} * \nabla\left(|u|^{p}\right)\right)\right\|_{L_{t}^{q_{1}} L_{x}^{\frac{2 N}{N-\gamma}}}\left\||u|^{p-2} u\right\|_{L_{t}^{\infty} L_{x}^{\frac{2 N p}{(N+\gamma)(p-1)}}} \\
& +\left\|\left(|x|^{-(N-\gamma)} *|u|^{p}\right)\right\|_{L_{t}^{\infty} L_{x}^{\frac{2 N}{N-\gamma}}}\left\||u|^{p-2} \nabla u\right\|_{L_{t}^{q_{1}} L_{x}^{\frac{2 N p}{(N+\gamma)(p-1)}}} \\
\lesssim & \left\|\nabla\left(|u|^{p}\right)\right\|_{L_{t}^{q_{1}} \frac{2 N}{L_{x}^{N+\gamma}}}\|u\|_{L_{t}^{\infty} L_{x}^{r_{1}}}^{p-1}+\|u\|_{L_{t}^{\infty} L_{x}^{r_{1}}}^{p}\|u\|_{L_{t}^{\infty} L_{x}^{r_{1}}}^{p-2}\|\nabla u\|_{L_{t}^{q_{1}} L_{x}^{r_{1}}} \\
\lesssim & \|\nabla u\|_{L_{t}^{q_{1}} L_{x}^{r_{1}}}\|u\|_{L_{t}^{\infty} L_{x}^{r_{1}}}^{2(p-1)}+\|u\|_{L_{t}^{\infty} L_{x}^{r_{1}}}^{2(p-1)}\|\nabla u\|_{L_{t}^{q_{1}} L_{x}^{r_{1}}} \\
& \lesssim\|u\|_{L_{t}^{\infty} H_{x}^{1}}^{2(p-1)}\|\nabla u\|_{L_{t}^{q_{1}} L_{x}^{r_{1}}}
\end{aligned}
$$


where in the first inequality we have used the product rule, second inequality is a consequence of Lemma 2.3.1 and Hölder's inequality, in the second to last line we have used Hölder's inequality in the first term and lastly we use Lemma 2.3.2 to get the last estimate. Combining (2.25) and (2.26), respectively, with (2.27) and (2.28), we obtain

$$
\|\Phi(u(t))\|_{L_{t}^{q_{1}} W_{x}^{1, r_{1}}} \lesssim\left\|u_{0}\right\|_{H_{x}^{1}}+T^{\theta}\|u\|_{L_{t}^{\infty} H_{x}^{1}}^{2(p-1)}\|u\|_{L_{t}^{q_{1}} W_{x}^{1, r_{1}}}
$$

Following a similar argument, we also have

$$
\|\Phi(u(t))\|_{L_{t}^{\infty} H_{x}^{1}} \lesssim\left\|u_{0}\right\|_{H_{x}^{1}}+T^{\theta}\|u\|_{L_{t}^{\infty} H_{x}^{1}}^{2(p-1)}\|u\|_{L_{t}^{q_{1}} W_{x}^{1, r_{1}}}
$$

Adding the last two lines, we get that for $u \in \mathcal{S}$

$$
\|\Phi(u(t))\|_{L_{t}^{q_{1}} W_{x}^{1, r_{1}}}+\|\Phi(u(t))\|_{L_{t}^{\infty} H_{x}^{1}} \leq C\left\|u_{0}\right\|_{H_{x}^{1}}+C T^{\theta} M^{2 p-1}
$$

Set $M=2 C\left\|u_{0}\right\|_{H_{x}^{1}}$ and take $T$ so that

$$
C T^{\theta} M^{2(p-1)} \leq \frac{1}{2}
$$

yielding that the right-hand side of (2.29) is bounded by $M$. Therefore, for $T \lesssim\left\|u_{0}\right\|_{H_{x}^{1}}^{-\frac{2 p}{1-s_{c}}}$, we obtain $\Phi: \mathcal{S} \rightarrow \mathcal{S}$. Note that the above estimate works for any $s_{c}<1$. In the energycritical case, $s_{c}=1$, we have $\theta=0$, and thus, there is no time dependence in (2.29),

$$
\|\Phi(u(t))\|_{L_{t}^{q_{1}} W_{x}^{1, r_{1}}}+\|\Phi(u(t))\|_{L_{t}^{\infty} H_{x}^{1}} \leq C\left\|u_{0}\right\|_{H_{x}^{1}}+C M^{2 p-1} .
$$

Hence, we can proceed only if $\left\|u_{0}\right\|_{H_{x}^{1}}$ is small enough, namely, if

$$
C\left\|u_{0}\right\|_{H_{x}^{1}}^{2(p-1)}<\frac{1}{2}
$$

which then bounds the right-hand side of (2.31) by $M: C\left\|u_{0}\right\|_{H_{x}^{1}}+C M^{2 p-1}<M$, yielding $\Phi$ mapping $\mathcal{S}$ into itself.

To complete the proof we need to show that the operator $\Phi$ is a contraction. This is achieved by running the same argument as above on the difference

$$
d(\Phi(u(t)), \Phi(v(t))) \stackrel{\text { def }}{=}\|\Phi(u(t))-\Phi(v(t))\|_{L_{t}^{q_{1}} W_{x}^{1, r_{1}}}+\|\Phi(u(t))-\Phi(v(t))\|_{L_{t}^{\infty} H_{x}^{1}}
$$


for $u, v \in \mathcal{S}$. We again note that because of the convolution and estimating at the $H^{1}$ level, we end up with extra terms to work unlike the proof for the mapping $\Phi$ into itself above.

We first apply Hölder's inequality in time to get

$d(\Phi(u(t)), \Phi(v(t))) \lesssim T^{\theta}\left(\|\Phi(u(t))-\Phi(v(t))\|_{L_{t}^{q_{1}} L_{x}^{r_{1}^{\prime}}}+\|\nabla(\Phi(u(t))-\Phi(v(t)))\|_{L_{t}^{q_{1}} L_{x}^{r_{1}^{\prime}}}\right)$

where

$$
\begin{aligned}
&\|\Phi(u(t))-\Phi(v(t))\|_{L_{t}^{q_{1}} L_{x}^{r_{1}^{\prime}} \lesssim}\left\|\left(|x|^{-(N-\gamma)} *|u|^{p}\right)\left(|u|^{p-2} u-|v|^{p-2} v\right)\right\|_{L_{t}^{q_{1}} L_{x}^{r_{1}^{\prime}}} \\
&+\left\|\left(|x|^{-(N-\gamma)} *\left(|u|^{p}-|v|^{p}\right)\right)|v|^{p-2} v\right\|_{L_{t}^{q_{1}} L_{x}^{r_{1}^{\prime}}} \\
& \stackrel{\text { def }}{=} A_{1}+A_{2}
\end{aligned}
$$

and

$$
\begin{aligned}
&\|\nabla(\Phi(u(t))-\Phi(v(t)))\|_{L_{t}^{q_{1}} L_{x}^{r_{1}^{\prime}} \lesssim}\left\|\nabla\left[\left(|x|^{-(N-\gamma)} *|u|^{p}\right)\left(|u|^{p-2} u-|v|^{p-2} v\right)\right]\right\|_{L_{t}^{q_{1}} L_{x}^{r_{1}^{\prime}}} \\
&+\left\|\nabla\left[\left(|x|^{-(N-\gamma)} *\left(|u|^{p}-|v|^{p}\right)\right)|v|^{p-2} v\right]\right\|_{L_{t}^{q_{1}} L_{x}^{r_{1}^{\prime}}} \\
& \stackrel{\text { def }}{=} B_{1}+B_{2} .
\end{aligned}
$$

Here, we have added and subtracted the term $\left(|x|^{-(N-\gamma)} *|u|^{p}\right)|v|^{p-2} v$. For $A_{1}$, we use Hölder's inequality, Lemma 2.3.1 and (2.18) to obtain

$$
\begin{aligned}
A_{1} & \lesssim\left\|\left(|x|^{-(N-\gamma)} *|u|^{p}\right)\right\|_{L_{t}^{q_{1}} L_{x}^{N-\gamma}}^{\frac{2 N}{N-\gamma}}\left\||u|^{p-2} u-|v|^{p-2} v\right\|_{L_{t}^{\infty} L_{x}^{(N+\gamma)(p-1)}}{ }^{\frac{2 N p}{(N+)}} \\
& \lesssim\|u\|_{L_{t}^{q_{1} p} L_{x}^{r_{1}}}^{p}\left(\|u\|_{L_{t}^{\infty} L_{x}^{r_{1}}}^{p-2}+\|v\|_{L_{t}^{\infty} L_{x}^{r_{1}}}^{p-2}\right)\|u-v\|_{L_{t}^{\infty} L_{x}^{r_{1}}} \\
& \lesssim\|u\|_{L_{t}^{\infty} L_{x}^{r_{1}}}^{p-1}\|u\|_{L_{t}^{q_{1}} L_{x}^{r_{1}}}\left(\|u\|_{L_{t}^{\infty} L_{x}^{r_{1}}}^{p-2}+\|v\|_{L_{t}^{\infty} L_{x}^{r_{1}}}^{p-2}\right)\|u-v\|_{L_{t}^{\infty} L_{x}^{r_{1}}} \\
& \lesssim\|u\|_{L_{t}^{\infty} H_{x}^{1}}^{p-1}\|u\|_{L_{t}^{q_{1}} L_{x}^{r_{1}}}\left(\|u\|_{L_{t}^{\infty} H_{x}^{1}}^{p-2}+\|v\|_{L_{t}^{\infty} H_{x}^{1}}^{p-2}\right)\|u-v\|_{L_{t}^{\infty} H_{x}^{1}} .
\end{aligned}
$$


We again use Hölder's, Lemma 2.3.1 and (2.19) to estimate $A_{2}$

$$
\begin{aligned}
A_{2} & \lesssim\left\||x|^{-(N-\gamma)} *\left(|u|^{p}-|v|^{p}\right)\right\|_{L_{t}^{q_{1}} L_{x}^{\frac{2 N}{N-\gamma}}}\left\||v|^{p-2} v\right\|_{L_{t}^{\infty} L_{x}^{\frac{2 N p}{(N+\gamma)(p-1)}}} \\
& \lesssim\left\||u|^{p}-|v|^{p}\right\|_{L_{t}^{q_{1}} L_{x}^{\frac{2 N}{N+\gamma}}\|v\|_{L_{t}^{\infty} L_{x}^{r_{1}}}^{p-1}} \\
& \lesssim\left(\|u\|_{L_{t}^{\infty} L_{x}^{r_{1}}}^{p-1}+\|v\|_{L_{t}^{\infty} L_{x}^{r_{1}}}^{p-1}\right)\|u-v\|_{L_{t}^{q_{1}} L_{x}^{r_{1}}\|v\|_{L_{t}^{\infty} L_{x}^{r_{1}}}^{p-1}} \\
& \lesssim\left(\|u\|_{L_{t}^{\infty} H_{x}^{1}}^{p-1}+\|v\|_{L_{t}^{\infty} H_{x}^{1}}^{p-1}\right)\|u-v\|_{L_{t}^{q_{1}} L_{x}^{r_{1}}\|v\|_{L_{t}^{\infty} H_{x}^{1}}^{p-1} .}
\end{aligned}
$$

For $B_{1}$ we first use the product rule

$$
\begin{aligned}
B_{1} \lesssim & \left\|\left(|x|^{-(N-\gamma)} * \nabla\left(|u|^{p}\right)\right)\right\|_{L_{t}^{q_{1}} \frac{2 N}{L_{x}^{N-\gamma}}}\left\||u|^{p-2} u-|v|^{p-2} v\right\|_{L_{t}^{\infty} L_{x}^{\frac{2 N p}{(N+\gamma)(p-1)}}} \\
& +\left\|\left(|x|^{-(N-\gamma)} *|u|^{p}\right)\right\|_{L_{t}^{\infty} L_{x}^{\frac{2 N}{N-\gamma}}}\left\|\nabla\left(|u|^{p-2} u-|v|^{p-2} v\right)\right\|_{L_{t}^{q_{1}} \frac{2 N p}{L_{x}^{(N+\gamma)(p-1)}}}
\end{aligned}
$$

then applying Hölder's inequality, Lemma 2.3.1, Lemma 2.3.2 and (2.18), yields

$$
\begin{aligned}
B_{1} \lesssim & \left\|\nabla\left(|u|^{p}\right)\right\|_{L_{t}^{q_{1}} L_{x}^{N+\gamma}}^{2 N}\left(\|u\|_{L_{t}^{\infty} L_{x}^{r_{1}}}^{p-2}+\|v\|_{L_{t}^{\infty} L_{x}^{r_{1}}}^{p-2}\right)\|u-v\|_{L_{t}^{\infty} L_{x}^{r_{1}}} \\
& +\|u\|_{L_{t}^{\infty} L_{x}^{r_{1}}}^{p}\left(\|u\|_{L_{t}^{\infty} L_{x}^{r_{1}}}^{p-2}+\|v\|_{L_{t}^{\infty} L_{x}^{r_{1}}}^{p-2}\right)\|\nabla(u-v)\|_{L_{t}^{q_{1}} L_{x}^{r_{1}}} \\
\lesssim & \|\nabla u\|_{L_{t}^{q_{1}} L_{x}^{r_{1}}}\|u\|_{L_{t}^{\infty} L_{x}^{r_{1}}}^{p-1}\left(\|u\|_{L_{t}^{\infty} L_{x}^{r_{1}}}^{p-2}+\|v\|_{L_{t}^{\infty} L_{x}^{r_{1}}}^{p-2}\right)\|u-v\|_{L_{t}^{\infty} L_{x}^{r_{1}}} \\
& +\|u\|_{L_{t}^{\infty} L_{x}^{r_{1}}}^{p}\left(\|u\|_{L_{t}^{\infty} L_{x}^{r_{1}}}^{p-2}+\|v\|_{L_{t}^{\infty} L_{x}^{r_{1}}}^{p-2}\right)\|\nabla(u-v)\|_{L_{t}^{q_{1}} L_{x}^{r_{1}}} \\
\lesssim & \|\nabla u\|_{L_{t}^{q_{1}} L_{x}^{r_{1}}}\|u\|_{L_{t}^{\infty} H_{x}^{1}}^{p-1}\left(\|u\|_{L_{t}^{\infty} H_{x}^{1}}^{p-2}+\|v\|_{L_{t}^{\infty} H_{x}^{1}}^{p-2}\right)\|u-v\|_{L_{t}^{\infty} H_{x}^{1}} \\
& +\|u\|_{L_{t}^{\infty} H_{x}^{1}}^{p}\left(\|u\|_{L_{t}^{\infty} H_{x}^{1}}^{p-2}+\|v\|_{L_{t}^{\infty} H_{x}^{1}}^{p-2}\right)\|\nabla(u-v)\|_{L_{t}^{q_{1}} L_{x}^{r_{1}}}
\end{aligned}
$$

Again using the product rule and Lemma 2.3.1 to estimate $B_{2}$, we get

$$
\begin{aligned}
B_{2} \lesssim & \left\||x|^{-(N-\gamma)} * \nabla\left(|u|^{p}-|v|^{p}\right)\right\|_{L_{t}^{q_{1}} \frac{2 N}{L_{x}^{N-\gamma}}}\left\||v|^{p-2} v\right\|_{L_{t}^{\infty} \frac{2 N p}{L_{x}^{(N+\gamma)(p-1)}}} \\
& +\left\||x|^{-(N-\gamma)} *\left(|u|^{p}-|v|^{p}\right)\right\|_{L_{t}^{\infty} L_{x}^{\frac{2 N}{N-\gamma}}}\left\|\nabla\left(|v|^{p-2} v\right)\right\|_{L_{t}^{q_{1}} \frac{2 N p}{L_{x}^{(N+\gamma)(p-1)}}} \\
& \lesssim\left\|\nabla\left(|u|^{p}-|v|^{p}\right)\right\|_{L_{t}^{q_{1}} \frac{2 N}{L_{x}^{N+\gamma}}}\|v\|_{L_{t}^{\infty} L_{x}^{r_{1}}}^{p-1}+\left\||u|^{p}-|v|^{p}\right\|_{L_{t}^{\infty} L_{x}^{N+\gamma}}^{\frac{2 N}{N+\gamma}}\|v\|_{L_{t}^{\infty} L_{x}^{r_{1}}}^{p-2}\|\nabla v\|_{L_{t}^{q_{1}} L_{x}^{r_{1}}}
\end{aligned}
$$


Using (2.19) and Lemma 2.3.2, we obtain

$$
\begin{aligned}
B_{2} \lesssim & \left(\|u\|_{L_{t}^{\infty} L_{x}^{r_{1}}}^{p-1}+\|v\|_{L_{t}^{\infty} L_{x}^{r_{1}}}^{p-1}\right)\|\nabla(u-v)\|_{L_{t}^{q_{1}} L_{x}^{r_{1}}}\|v\|_{L_{t}^{\infty} L_{x}^{r_{1}}}^{p-1} \\
& +\left(\|u\|_{L_{t}^{\infty} L_{x}^{r_{1}}}^{p-1}+\|v\|_{L_{t}^{\infty} L_{x}^{r_{1}}}^{p-1}\right)\|u-v\|_{L_{t}^{\infty} L_{x}^{r_{1}}}\|v\|_{L_{t}^{\infty} L_{x}^{r_{1}}}^{p-2}\|\nabla v\|_{L_{t}^{q_{1}} L_{x}^{r_{1}}} \\
\lesssim & \left(\|u\|_{L_{t}^{\infty} H_{x}^{1}}^{p-1}+\|u\|_{L_{t}^{\infty} H_{x}^{1}}^{p-1}\right)\left(\|\nabla(u-v)\|_{L_{t}^{q_{1}} L_{x}^{r_{1}}\|v\|_{L_{t}^{\infty} H_{x}^{1}}^{p-1}}\right. \\
& \left.+\|u-v\|_{L_{t}^{\infty} H_{x}^{1}}\|v\|_{L_{t}^{\infty} H_{x}^{1}}^{p-2}\|\nabla v\|_{L_{t}^{q_{1}} L_{x}^{r_{1}}}\right) .
\end{aligned}
$$

Combining (2.33), (2.34), (2.35) and (2.36), we obtain that for $u, v \in \mathcal{S}$

$$
d(\Phi(u(t)), \Phi(v(t))) \lesssim T^{\theta} M^{2(p-1)} d(u, v)
$$

This together with (2.30), the bound on time $T$, implies that $\Phi$ is a contraction on $\mathcal{S}$ for the energy-subcritical case. Similarly, for the energy-critical case, we have that for $u, v \in \mathcal{S}$

$$
d(\Phi(u(t)), \Phi(v(t))) \lesssim M^{2(p-1)} d(u, v)
$$

which with the smallness of (2.32) implies that $\Phi$ is again a contraction on $\mathcal{S}$. To prove the continuous dependence with respect to $u_{0}$, we note that if $u$ and $v$ are the corresponding solutions of (2.16) with initial data $u_{0}$ and $v_{0}$, respectively, then

$$
u(t)-v(t)=e^{i t \Delta}\left(u_{0}-v_{0}\right)+i \int_{0}^{t} e^{i\left(t-t^{\prime}\right) \Delta}(F(u)-F(v))\left(t^{\prime}\right) d t^{\prime} .
$$

Thus, the same argument as in (2.33), (2.34), (2.35) and (2.36) (and the appropriate modifications when $s_{c}=1$ ) yields

$$
\begin{aligned}
d(u(t), v(t)) & \stackrel{\text { def }}{=}\|u(t)-v(t)\|_{L_{t}^{q_{1} W_{x}^{1, r_{1}}}}+\|u(t)-v(t)\|_{L_{t}^{\infty} H_{x}^{1}} \\
& \lesssim\left\|u_{0}-v_{0}\right\|_{H^{1}}+C_{N, p \gamma} T^{\theta} M^{2(p-1)} d(u(t), v(t)) .
\end{aligned}
$$

This implies that if $\left\|u_{0}-v_{0}\right\|_{H^{1}}$ is small enough (see (2.30) or (2.32)), we have that

$$
d\left(u(t), v(t) \leq \widetilde{C}\left\|u_{0}-v_{0}\right\|_{H^{1}}\right.
$$

which completes the proof. 


\subsubsection{Local well-posedness at the critical regularity}

We now obtain the local well-posedness at the critical regularity $\dot{H}^{s_{c}}, s_{c} \geq 0$, for which we again consider the integral representation (2.16) of (1.1) with $u_{0} \in \dot{H}^{s_{c}}\left(\mathbb{R}^{N}\right), 0<$ $\gamma<N$ and $p \geq 2$. We also require that the nonlinearity power $p$ satisfies an additional constraint, $s_{c}<p-1$ if $p$ is not an even integer. This ensures that one can take the derivative of $|u|^{p-2} u$ term $s_{c}$ times. The following proposition was proved in [AR19].

Proposition 2.6.3 Let $0<\gamma<N$ and $p \geq 2$, so that $s_{c} \geq 0$. Assume in addition that if $p$ is not an even integer, then $s_{c}<p-1$. Let $u_{0} \in \dot{H}^{s_{c}}\left(\mathbb{R}^{N}\right)$. Then there exists a unique solution $u(x, t)$ of the equation (1.1) with data $u_{0}$ defined on $[0, T]$ for some $T>0$, and such that

(1.) for $s_{c}=0$ and $N \geq 1, u \in C\left([0, T] ; L_{x}^{2}\right) \cap L^{q}\left([0, T] ; L_{x}^{r}\right)$, where $(q, r)=$ $\left(2 p, \frac{2 N p}{N+\gamma}\right)$ is the $L^{2}$-admissible pair and $x \in \mathbb{R}^{N}$,

(2.) for $0<s_{c}<1$ and $N \geq 1, u \in C\left([0, T] ; \dot{H}_{x}^{s_{c}}\right) \cap L^{q_{1}}\left([0, T] ; \dot{W}_{x}^{s_{c}, r_{1}}\right) \cap L^{q_{2}}\left([0, T] ; \dot{W}_{x}^{s_{c}, r_{2}}\right)$, where $\left(q_{1}, r_{1}\right)=\left(\frac{2 p}{1+s_{c}(p-1)}, \frac{2 N p}{N+\gamma}\right),\left(q_{2}, r_{2}\right)=\left(\frac{2 p}{1-s_{c}}, \frac{2 N p}{N+\gamma+2 s_{c} p}\right)$ are the $L^{2}$-admissible pairs and $x \in \mathbb{R}^{N}$,

(3.) for $s_{c}=1$ and $N \geq 3, u \in C\left([0, T] ; \dot{H}_{x}^{1}\right) \cap L^{q}\left([0, T] ; \dot{W}_{x}^{1, r}\right)$, where $(q, r)=$ $\left(2, \frac{2 N}{N-2}\right)$ is the $L^{2}$-admissible and $x \in \mathbb{R}^{N}$,

(4.) for $s_{c}>1, u \in C\left([0, T] ; \dot{H}_{x}^{s_{c}}\right) \cap L^{q}\left([0, T] ; \dot{W}_{x}^{s_{c}, r}\right)$, where $x \in \mathbb{R}^{N}$ and

- for $p=2($ thus, $N \geq 5),(q, r)=\left(3, \frac{6 N}{3 N-4}\right)$ is the $L^{2}$-admissible pair,

- for $p>2$ (thus, $N \geq 3)$ and $0<\gamma<\min \left(N, \frac{2 p}{p-2}\right)$, the $L^{2}$-admissible pair is $(q, r)=\left(2, \frac{2 N}{N-2}\right)$

Moreover, for all $0<\widetilde{T}<T$, the continuous dependence upon the initial data holds. 
Proof. (1.) $s_{c}=0$.

For $T>0$ and $M_{0}>0$ determined later, let

$$
X_{0}=C\left([0, T] ; L^{2}\left(\mathbb{R}^{N}\right)\right) \cap L^{q}\left([0, T] ; L^{r}\left(\mathbb{R}^{N}\right)\right),
$$

and

$$
B_{0}=\left\{u \in X_{0}:\|u\|_{L_{t}^{q} L_{x}^{r}\left([0, T] \times \mathbb{R}^{N}\right)} \leq M_{0}\right\}
$$

We prove that the operator defined in (2.23) is a contraction on the set $B_{0}$ for some $T>0$. Denoting by $I=[0, T]$ and using Lemma 2.4.6, we obtain

$$
\|\Phi(u(t))\|_{L_{I}^{q} L_{x}^{r}}+\|\Phi(u(t))\|_{L_{I}^{\infty} L_{x}^{2}} \leq 2 c_{1}\left\|u_{0}\right\|_{L_{x}^{2}}+2 c_{1}\left(\int_{0}^{T}\|F(u)\|_{L_{x}^{r^{\prime}}}^{q^{\prime}} d t\right)^{1 / q^{\prime}}
$$

where $F(u)$ is defined in (2.24). Using Hölder's inequality and Lemma 2.3.1, we get

$$
\|F(u)\|_{L_{I}^{q^{\prime}} L_{x}^{r^{\prime}}} \leq\left\||x|^{-(N-\gamma)} *|u|^{p}\right\|_{L_{t}^{2} L_{x}^{\frac{2 N}{N-\gamma}}}\|u\|_{L_{I}^{q} L_{x}^{r}}^{p-1} \leq c_{N, \gamma}\|u\|_{L_{I}^{q} L_{x}^{r}}^{2 p-1}
$$

Substituting (2.38) into (2.37), we have that for $u \in B_{0}$,

$$
\|\Phi(u(t))\|_{L_{I}^{q} L_{x}^{r}}+\|\Phi(u(t))\|_{L_{I}^{\infty} L_{x}^{2}} \leq 2 c_{1}\left\|u_{0}\right\|_{L_{x}^{2}}+2 c_{1} c_{N, \gamma} M_{0}^{2 p-1} .
$$

Take $\left\|u_{0}\right\|_{L_{x}^{2}}$ small enough so that

$$
\left\|u_{0}\right\|_{L_{x}^{2}}^{2(p-1)} \leq \frac{1}{\left(8 c_{1}\right)^{2 p-1} c_{N, \gamma}} .
$$

Set $M_{0}=8 c_{1}\left\|u_{0}\right\|_{L_{x}^{2}}$. Thus,

$$
M_{0}^{2(p-1)} \leq \frac{1}{8 c_{1} c_{N, \gamma}}
$$

Take $T>0$ such that $\left\|e^{i t \Delta} u_{0}\right\|_{S\left(L^{2} ;[0, T]\right)} \leq \frac{M_{0}}{4}$. Thus, for $u \in B_{0}$ with $T$ as above, (2.39) becomes

$$
\|\Phi(u(t))\|_{L_{I}^{q} L_{x}^{r}}+\|\Phi(u(t))\|_{L_{I}^{\infty} L_{x}^{2}} \leq \frac{M_{0}}{4}+2 c_{1} c_{N, \gamma} \frac{M_{0}}{8 c_{1} c_{N, \gamma}}<M_{0},
$$


yielding $\Phi$ mapping $B_{0}$ into itself. To see that $\Phi$ is a contraction, we carry similar calculations as above on the difference

$$
d(\Phi(u(t)), \Phi(v(t))) \stackrel{\text { def }}{=}\|\Phi(u(t))-\Phi(v(t))\|_{L_{I}^{q} L_{x}^{r}},
$$

for $u, v \in B_{0}$. We first apply Lemma 2.4.6 to get

$$
\begin{aligned}
& d(\Phi(u(t)), \Phi(v(t))) \leq c_{1}\left\|\left(|x|^{-(N-\gamma)} *|u|^{2}\right)\left(|u|^{p-2} u-|v|^{p-2} v\right)\right\|_{L_{I}^{q^{\prime}} L_{x}^{r^{\prime}}} \\
&+c_{1}\left\|\left(|x|^{-(N-\gamma)} *\left(|u|^{2}-|v|^{2}\right)\right)|v|^{p-2} v\right\|_{L_{I}^{q^{\prime}} L_{x}^{r^{\prime}}} \\
& \stackrel{\text { def }}{=} \\
& c_{1}\left(D_{1}+D_{2}\right) .
\end{aligned}
$$

For $D_{1}$, we use Hölder's inequality, Lemma 2.3.1 and (2.18) to obtain

$$
D_{1} \leq c_{N, \gamma}\|u\|_{L_{I}^{q} L_{x}^{r}}^{p}\left(\|u\|_{L_{I}^{L_{x}^{r}}}^{p-2}+\|v\|_{L_{I}^{q} L_{x}^{r}}^{p-2}\right)\|u-v\|_{L_{I}^{q} L_{x}^{r}} .
$$

We again use Hölder's, Lemma 2.3.1 and (2.19) to estimate $D_{2}$

$$
D_{2} \leq c_{N, \gamma}\left(\|u\|_{L_{I}^{q} L_{x}^{r}}^{p-1}+\|v\|_{L_{I}^{q} L_{x}^{r}}^{p-1}\right)\|u-v\|_{L_{I}^{q} L_{x}^{r}}\|v\|_{L_{I}^{q} L_{x}^{r}}^{p-1} .
$$

Combining (2.42) and (2.43), we obtain that for $u, v \in B_{0}$

$$
d(\Phi(u(t)), \Phi(v(t))) \leq 4 c_{1} c_{N, \gamma} M_{0}^{2(p-1)} d(u, v) .
$$

Taking $M_{0}$ and $T$ as in (2.41) together with (2.40) implies that $\Phi$ is a contraction on $B_{0}$.

(2.) $0<s_{c}<1$.

For $T>0$ and $M_{\text {in }}>0$ determined later, let

$u \in X_{i n} \stackrel{\text { def }}{=} C\left([0, T] ; \dot{H}^{s_{c}}\left(\mathbb{R}^{N}\right)\right) \cap L^{q_{1}}\left([0, T] ; \dot{W}^{s_{c}, r_{1}}\left(\mathbb{R}^{N}\right)\right) \cap L^{q_{2}}\left([0, T] ; \dot{W}^{s_{c}, r_{2}}\left(\mathbb{R}^{N}\right)\right)$.

Define

$$
B_{\text {in }}=\left\{u \in X_{\text {in }}: \max \left(\left\||\nabla|^{s_{c}} u\right\|_{L_{t}^{q_{1}} L_{x}^{r_{1}}\left(\mathbb{R}^{N}\right)},\left\||\nabla|^{s_{c}} u\right\|_{L_{t}^{q_{2}} L_{x}^{r_{2}}\left(\mathbb{R}^{N}\right)}\right) \leq M_{\text {in }}\right\},
$$


where $t \in[0, T]$ for some $T>0$. We again prove that the operator defined in (2.23) is a contraction on the set $B_{\text {in }}$ for some $T>0$. Denoting by $I=[0, T]$ and using Lemma 2.4.6, we obtain

$$
\begin{aligned}
&\left\||\nabla|^{s_{c}} \Phi(u(t))\right\|_{L_{I}^{q_{1}} L_{x}^{r_{1}}+}\left\||\nabla|^{s_{c}} \Phi(u(t))\right\|_{L_{I}^{q_{2}} L_{x}^{r_{2}}}+\|\Phi(u(t))\|_{L_{I}^{\infty} \dot{H}_{x}^{s_{c}}} \\
& \leq 3 c_{1}\left\|u_{0}\right\|_{\dot{H}_{x}^{s_{c}}}+3 c_{1}\left\||\nabla|^{s_{c}} F(u)\right\|_{L_{I}^{q_{1}^{\prime}} L_{x}^{r_{1}^{\prime}}} .
\end{aligned}
$$

Using product rule, we have

$$
\begin{aligned}
\left\||\nabla|^{s_{c}} F(u)\right\|_{L_{I}^{q_{1}^{\prime}} L_{x}^{r_{1}^{\prime}}} \leq & \left\|\left(|x|^{-(N-\gamma)} *|\nabla|^{s_{c}}\left(|u|^{p}\right)\right)|u|^{p-2} u\right\|_{L_{I}^{q_{1}^{\prime}} L_{x}^{r_{1}^{\prime}}} \\
& +\left\|\left(|x|^{-(N-\gamma)} *|u|^{p}\right)|\nabla|^{s_{c}}\left(|u|^{p-2} u\right)\right\|_{L_{I}^{q_{1}^{\prime}} L_{x}^{r_{1}^{\prime}}} \\
& \stackrel{\text { def }}{=} A_{1}+A_{2},
\end{aligned}
$$

where

$$
\begin{aligned}
& A_{1} \leq\left\||x|^{-(N-\gamma)} *|\nabla|^{s_{c}}\left(|u|^{p}\right)\right\|_{L_{I}^{2} L_{x}^{N-\gamma}}\|u\|_{L_{I}^{q_{2}} L_{x}^{r_{1}}}^{p-1} \\
& \leq c_{N, \gamma}\left\||u|^{p-1}|\nabla|^{s_{c}} u\right\|_{L_{I}^{2} L_{x}^{\frac{2 N}{N+\gamma}}}\left\||\nabla|^{s_{c}} u\right\|_{L_{I}^{q_{2}} L_{x}^{r_{2}}}^{p-1} \\
& \leq c_{N, \gamma}\|u\|_{L_{I}^{q_{2}} L_{x}^{r_{1}}}^{p-1}\left\||\nabla|^{s_{c}} u\right\|_{L_{I}^{q_{1}} L_{x}^{r_{1}}}\left\||\nabla|^{s_{c}} u\right\|_{L_{I}^{q_{2}} L_{x}^{r_{2}}}^{p-1} \\
& \leq c_{N, \gamma}\left\||\nabla|^{s_{c}} u\right\|_{L_{I}^{q_{2}} L_{x}^{r_{2}}}^{2(p-1)}\left\||\nabla|^{s_{c}} u\right\|_{L_{I}^{q_{1}} L_{x}^{r_{1}}}
\end{aligned}
$$

and

$$
\begin{aligned}
A_{2} & \leq\left\||x|^{-(N-\gamma)} *|u|^{p}\right\|_{L_{I}^{1-s_{c}}} \frac{2 N}{L_{x}^{N-\gamma}}\|u\|_{L_{I}^{q_{2}} L_{x}^{r_{1}}}^{p-2}\left\||\nabla|^{s_{c}} u\right\|_{L_{I}^{q_{1}} L_{x}^{r_{1}}} \\
& \leq c_{N, \gamma}\|u\|_{L_{I}^{q_{2}} L_{x}^{r_{1}}}^{p}\left\||\nabla|^{s_{c}} u\right\|_{L_{I}^{q_{2}} L_{x}^{r_{2}}}^{p-2}\left\||\nabla|^{s_{c}} u\right\|_{L_{I}^{q_{1}} L_{x}^{r_{1}}} \\
& \leq c_{N, \gamma}\left\||\nabla|^{s_{c}} u\right\|_{L_{I}^{q_{2}} L_{x}^{r_{2}}}^{2(p-1)}\left\||\nabla|^{s_{c}} u\right\|_{L_{I}^{q_{1}} L_{x}^{r_{1}}} .
\end{aligned}
$$

Combining (2.46) and (2.47) along with (2.45), we get for $u \in B_{\text {in }}$,

$$
\begin{aligned}
\left\||\nabla|^{s_{c}} \Phi(u(t))\right\|_{L_{I}^{q_{1}} L_{x}^{r_{1}}} & +\left\||\nabla|^{s_{c}} \Phi(u(t))\right\|_{L_{I}^{q_{2}} L_{x}^{r_{2}}}+\|\Phi(u(t))\|_{L_{I}^{\infty} \dot{H}^{s_{c}}} \\
& \leq 3 c_{1}\left\|u_{0}\right\|_{\dot{H}_{x}^{s_{c}}}+6 c_{1} c_{N, \gamma} M_{i n}^{2 p-1} .
\end{aligned}
$$


Take $\left\|u_{0}\right\|_{\dot{H}_{x}^{s_{c}}}$ small enough so that

$$
2\left\|u_{0}\right\|_{\dot{H}_{x}^{s c}}^{2(p-1)} \leq \frac{1}{\left(12 c_{1}\right)^{2 p-1} c_{N, \gamma}}
$$

Set $M_{i n}=12 c_{1}\left\|u_{0}\right\|_{\dot{H}_{x}^{s_{c}}}$. Thus,

$$
M_{i n}^{2(p-1)} \leq \frac{1}{24 c_{1} c_{N, \gamma}}
$$

Take $T>0$ such that $\left\|e^{i t \Delta} u_{0}\right\|_{S\left(L^{2} ;[0, T]\right)} \leq \frac{M_{i n}}{4}$. Thus, for $u \in B_{\text {in }}$ with $T$ as above, (2.39) becomes

$$
\|\Phi(u(t))\|_{L_{I}^{q} L_{x}^{r}}+\|\Phi(u(t))\|_{L_{I}^{\infty} L_{x}^{2}} \leq \frac{M_{i n}}{4}+6 c_{1} c_{N, \gamma} \frac{M_{i n}}{24 c_{1} c_{N, \gamma}}<M_{i n}
$$

yielding $\Phi$ mapping $B_{i n}$ into itself. To see that $\Phi$ is a contraction, we carry similar calculations as above on the difference

$$
\begin{aligned}
d(\Phi(u(t)), \Phi(v(t))) \stackrel{\text { def }}{=} & \left\||\nabla|^{s_{c}}[\Phi(u(t))-\Phi(v(t))]\right\|_{L_{I}^{q_{1}} L_{x}^{r_{1}}} \\
& +\left\||\nabla|^{s_{c}}[\Phi(u(t))-\Phi(v(t))]\right\|_{L_{I}^{q_{2}} L_{x}^{r_{2}}}
\end{aligned}
$$

for $u, v \in B_{i n}$. We first apply Lemma 2.4.6 to get

$$
\begin{aligned}
d(\Phi(u(t)), \Phi(v(t))) \leq & 2 c_{1}\left\||\nabla|^{s_{c}}\left[\left(|x|^{-(N-\gamma)} *|u|^{p}\right)\left(|u|^{p-2} u-|v|^{p-2} v\right)\right]\right\|_{L_{I}^{q_{1}^{\prime}} L_{x}^{r_{1}^{\prime}}} \\
& +2 c_{1}\left\||\nabla|^{s_{c}}\left[\left(|x|^{-(N-\gamma)} *\left(|u|^{p}-|v|^{p}\right)\right)|v|^{p-2} v\right]\right\|_{L_{I}^{q_{1}^{\prime}} L_{x}^{r_{1}^{\prime}}} \\
\stackrel{\text { def }}{=} & 2 c_{1}\left(D_{1}+D_{2}\right) .
\end{aligned}
$$

We first use the product rule for $D_{1}$ and similar calculations as in (2.46) and (2.47) together with (2.18)

$$
\begin{aligned}
D_{1} \leq c_{N, \gamma}\left\||\nabla|^{s_{c}} u\right\|_{L_{I}^{q_{2}} L_{x}^{r_{2}}}^{p-1}\left\||\nabla|^{s_{c}} u\right\|_{L_{I}^{q_{1}} L_{x}^{r_{1}}}\left(\left\||\nabla|^{s_{c}} u\right\|_{L_{I}^{q_{2}} L_{x}^{r_{2}}}^{p-2}\right. & \text { (2.51) } \\
& \left.+\left\||\nabla|^{s_{c}} v\right\|_{L_{I}^{q_{2}} L_{x}^{r_{2}}}^{p-2}\right)\left\||\nabla|^{s_{c}}(u-v)\right\|_{L_{I}^{q_{2}} L_{x}^{r_{2}}} \\
& +c_{N, \gamma}\left\||\nabla|^{s_{c}} u\right\|_{L_{I}^{q_{2}} L_{x}^{r_{2}}}^{p}\left(\left\||\nabla|^{s_{c}} u\right\|_{L_{I}^{q_{2}} L_{x}^{r_{2}}}^{p-2}+\left\||\nabla|^{s_{c}} v\right\|_{L_{I}^{q_{2}} L_{x}^{r_{2}}}^{p-2}\right)\left\||\nabla|^{s_{c}}(u-v)\right\|_{L_{I}^{q_{1}} L_{x}^{r_{1}}} .
\end{aligned}
$$


Using again the product rule for $D_{2}$ and similar calculations as in (2.46) and (2.47) along with (2.19)

$$
\begin{gathered}
D_{2} \leq c_{N, \gamma}\left(\left\||\nabla|^{s_{c}} u\right\|_{L_{I}^{q_{2}} L_{x}^{r_{2}}}^{p-1}+\left\||\nabla|^{s_{c}} v\right\|_{L_{I}^{q_{2}} L_{x}^{r_{2}}}^{p-1}\right)\left\||\nabla|^{s_{c}}(u-v)\right\|_{L_{I}^{q_{1}} L_{x}^{r_{1}}}\left\||\nabla|^{s_{c}} v\right\|_{L_{I}^{q_{2}} L_{x}^{r_{2}}}^{p-1} \\
+c_{N, \gamma}\left\||\nabla|^{s_{c}} v\right\|_{L_{I}^{q_{2}} L_{x}^{r_{2}}}^{p-2}\left\||\nabla|^{s_{c}} v\right\|_{L_{I}^{q_{1}} L_{x}^{r_{1}}}\left(\left\||\nabla|^{s_{c}} u\right\|_{L_{I}^{q_{2}} L_{x}^{r_{2}}}^{p-1}\right. \\
\left.+\left\||\nabla|^{s_{c}} v\right\|_{L_{I}^{q_{2}} L_{x}^{r_{2}}}^{p-1}\right)\left\||\nabla|^{s_{c}}(u-v)\right\|_{L_{I}^{q_{2}} L_{x}^{r_{2}}}
\end{gathered}
$$

Combining the estimates for $D_{1}$ and $D_{2}$, we obtain for $u, v \in B_{\text {in }}$ that

$$
d(\Phi(u(t)), \Phi(v(t))) \leq 8 c_{1} c_{N, \gamma} M_{i n}^{2(p-1)} d(u, v)
$$

Taking $M_{i n}$ and $T$ as in (2.50) along with (2.49) implies that $\Phi$ is a contraction on $B_{\text {in }}$

(3.) $s_{c}=1$.

For $T>0$ and $M_{1}>0$ determined later, let

$$
u \in X_{1} \stackrel{\text { def }}{=} u \in C\left([0, T] ; \dot{H}^{1}\left(\mathbb{R}^{N}\right)\right) \cap L^{q}\left([0, T] ; \dot{W}^{1, r}\left(\mathbb{R}^{N}\right)\right) .
$$

Define

$$
B_{1}=\left\{u \in X_{1}: \max \left(\|\nabla u\|_{L_{t}^{q} L_{x}^{r}\left([0, T] \times \mathbb{R}^{N}\right)},\|u\|_{L_{t}^{\infty} \dot{H}_{x}^{1}\left([0, T] \times \mathbb{R}^{N}\right)}\right) \leq M_{1}\right\}
$$

We again prove that the operator defined in (2.23) is a contraction on the set $B_{1}$ for some $T>0$. Denoting by $I=[0, T]$ and using Lemma 2.4.6, we obtain

$$
\begin{aligned}
\|\nabla \Phi(u(t))\|_{L_{I}^{q} L_{x}^{r}}+ & \|\Phi(u(t))\|_{L_{I}^{\infty} \dot{H}_{x}^{1}} \\
& \leq 2 c_{1}\left\|u_{0}\right\|_{\dot{H}_{x}^{s_{c}}}+2 c_{1}\|\nabla F(u)\|_{L_{I}^{q^{\prime}} L_{x}^{r^{\prime}}}
\end{aligned}
$$

Using product rule, we have

$$
\begin{aligned}
\|\nabla F(u)\|_{L_{I}^{q^{\prime}} L_{x}^{r^{\prime}}} \leq & \left\|\left(|x|^{-(N-\gamma)} * \nabla\left(|u|^{p}\right)\right)|u|^{p-2} u\right\|_{L_{I}^{q^{\prime}} L_{x}^{r^{\prime}}} \\
& +\left\|\left(|x|^{-(N-\gamma)} *|u|^{p}\right) \nabla\left(|u|^{p-2} u\right)\right\|_{L_{I}^{q^{\prime}} L_{x}^{r^{\prime}}} \\
\stackrel{\text { def }}{=} & A_{1}+A_{2},
\end{aligned}
$$


where

$$
\begin{aligned}
A_{1} & \leq\left\||x|^{-(N-\gamma)} * \nabla\left(|u|^{p}\right)\right\|_{L_{I}^{2} L_{x}^{N-\gamma}} \frac{2 N}{N-\gamma} \|_{L_{I}^{\infty} L_{x}^{r}}^{p-1} \\
& \leq c_{N, \gamma}\left\||u|^{p-1} \nabla u\right\|_{L_{I}^{2} L_{x}^{N+\gamma}}^{\frac{2 N}{N+\gamma}}\|u\|_{L_{I}^{\infty} \dot{H}_{x}^{1}}^{p-1} \leq c_{N, \gamma}\|u\|_{L_{I}^{\infty} \dot{H}_{x}^{1}}^{2(p-1)}\|\nabla u\|_{L_{I}^{q} L_{x}^{r}}
\end{aligned}
$$

and

$$
\begin{aligned}
A_{2} & \leq\left\||x|^{-(N-\gamma)} *|u|^{p}\right\|_{L_{I}^{\infty} L_{x}^{N-\gamma}}^{\frac{2 N}{N-\gamma}}\|u\|_{L_{I}^{\infty} L_{x}^{r}}^{p-2}\|\nabla u\|_{L_{I}^{q} L_{x}^{r}} \\
& \leq c_{N, \gamma}\|u\|_{L_{I}^{\infty} L_{x}^{r}}^{p}\|u\|_{L_{I}^{\infty} \dot{H}^{1}}^{p-2}\|\nabla u\|_{L_{I}^{q} L_{x}^{r}} \leq c_{N, \gamma}\|u\|_{L_{I}^{\infty} \dot{H}_{x}^{1}}^{2(p-1)}\|\nabla u\|_{L_{I}^{q} L_{x}^{r}} .
\end{aligned}
$$

Combining (2.55) and (2.56) along with (2.54), we get for $u \in B_{1}$,

$$
\|\nabla \Phi(u(t))\|_{L_{I}^{q} L_{x}^{r}}+\|\Phi(u(t))\|_{L_{I}^{\infty} \dot{H}_{x}^{1}} \leq 2 c_{1}\left\|u_{0}\right\|_{\dot{H}_{x}^{1}}+4 c_{1} c_{N, \gamma} M_{1}^{2 p-1}
$$

Take $\left\|u_{0}\right\|_{\dot{H}_{x}^{s c}}$ small enough so that

$$
2\left\|u_{0}\right\|_{\dot{H}_{x}^{1}}^{2(p-1)} \leq \frac{1}{\left(8 c_{1}\right)^{2 p-1} c_{N, \gamma}}
$$

Set $M_{1}=8 c_{1}\left\|u_{0}\right\|_{\dot{H}_{x}^{1}}$. Thus,

$$
M_{1}^{2(p-1)} \leq \frac{1}{16 c_{1} c_{N, \gamma}}
$$

Take $T>0$ such that $\left\|e^{i t \Delta} \nabla u_{0}\right\|_{S\left(L^{2} ;[0, T]\right)} \leq \frac{M_{1}}{4}$. Thus, for $u \in B_{1}$ with $T$ as above, (2.39) becomes

$$
\|\nabla \Phi(u(t))\|_{L_{I}^{q} L_{x}^{r}}+\|\Phi(u(t))\|_{L_{I}^{\infty} \dot{H}_{x}^{1}} \leq \frac{M_{1}}{2}+4 c_{1} c_{N, \gamma} \frac{M_{1}}{16 c_{1} c_{N, \gamma}}<M_{1}
$$

yielding $\Phi$ mapping $B_{1}$ into itself. To see that $\Phi$ is a contraction, we carry similar calculations as above on the difference

$d(\Phi(u(t)), \Phi(v(t))) \stackrel{\text { def }}{=}\|\nabla[\Phi(u(t))-\Phi(v(t))]\|_{L_{I}^{q} L_{x}^{r}}+\|\Phi(u(t))-\Phi(v(t))\|_{L_{I}^{\infty} \dot{H}_{x}^{1}}$, 
for $u, v \in B_{1}$. We first apply Lemma 2.4.6 to get

$$
\begin{aligned}
d(\Phi(u(t)), \Phi(v(t))) \leq & 2 c_{1}\left\|\nabla\left[\left(|x|^{-(N-\gamma)} *|u|^{p}\right)\left(|u|^{p-2} u-|v|^{p-2} v\right)\right]\right\|_{L_{I}^{q^{\prime}} L_{x}^{r^{\prime}}} \\
& +2 c_{1}\left\|\nabla\left[\left(|x|^{-(N-\gamma)} *\left(|u|^{p}-|v|^{p}\right)\right)|v|^{p-2} v\right]\right\|_{L_{I}^{q^{\prime}} L_{x}^{r^{\prime}}} \\
\stackrel{\text { def }}{=} & 2 c_{1}\left(D_{1}+D_{2}\right) .
\end{aligned}
$$

We first use the product rule for $D_{1}$ and similar calculations as in (2.55) and (2.56) combined with (2.18)

$$
\begin{gathered}
D_{1} \leq c_{N, \gamma}\|u\|_{L_{t}^{\infty} \dot{H}_{x}^{1}}^{p-1}\|\nabla u\|_{L_{t}^{q} L_{x}^{r}}\left(\|u\|_{L_{t}^{\infty} H_{x}^{1}}^{p-2}+\|v\|_{L_{t}^{\infty} \dot{H}_{x}^{1}}^{p-2}\right)\|u-v\|_{L_{t}^{\infty} \dot{H}_{x}^{1}} \\
+\|u\|_{L_{t}^{\infty} \dot{H}_{x}^{1}}^{p}\left(\|u\|_{L_{t}^{\infty} \dot{H}_{x}^{1}}^{p-2}+\|v\|_{L_{t}^{\infty} \dot{H}_{x}^{1}}^{p-2}\right)\|\nabla(u-v)\|_{L_{t}^{q} L_{x}^{r}} .
\end{gathered}
$$

Using again the product rule for $D_{2}$ followed by similar calculations as in (2.55) and (2.56) with (2.19)

$$
\begin{aligned}
D_{2} \leq c_{N, \gamma} & \left(\|u\|_{L_{t}^{\infty} \dot{H}_{x}^{s_{c}}}^{p-1}+\|v\|_{L_{t}^{\infty} \dot{H}_{x}^{s_{c}}}^{p-1}\right)\left\||\nabla|^{s_{c}}(u-v)\right\|_{L_{t}^{q} L_{x}^{r}}\|v\|_{L_{t}^{\infty} \dot{H}_{x}^{s_{c}}}^{p-1} \\
& +\left(\|u\|_{L_{t}^{\infty} \dot{H}_{x}^{1}}^{p-1}+\|v\|_{L_{t}^{\infty} \dot{H}_{x}^{1}}^{p-1}\right)\|u-v\|_{L_{t}^{\infty} \dot{H}_{x}^{1}}\|v\|_{L_{t}^{\infty} \dot{H}_{x}^{1}}^{p-2}\left\||\nabla|^{s_{c}} v\right\|_{L_{t}^{q} L_{x}^{r}} .
\end{aligned}
$$

Combining the estimates for $D_{1}$ and $D_{2}$, we obtain for $u, v \in B_{1}$ that

$$
d(\Phi(u(t)), \Phi(v(t))) \leq 8 c_{1} c_{N, \gamma} M_{1}^{2(p-1)} d(u, v)
$$

Taking $M_{1}$ and $T$ as in (2.59) along with (2.58) implies that $\Phi$ is a contraction on $B_{1}$

(4.) $s_{c}>1 \Longrightarrow \gamma<N(p-1)-2 p$.

Case $p=2$ and $\gamma<N-4$, which requires $N>4$ since $\gamma>0$.

For $T>0$ and $M_{2}>0$ determined later, let

$$
X_{2}=C\left([0, T] ; \dot{H}^{s_{c}}\left(\mathbb{R}^{N}\right)\right) \cap L^{q}\left([0, T] ; \dot{W}^{s_{c}, r}\left(\mathbb{R}^{N}\right)\right),
$$


and define

$$
B_{2}=\left\{u \in X_{2}: \max \left(\left\||\nabla|^{s_{c}} u\right\|_{L_{t}^{q} L_{x}^{r}},\|\mid u\|_{L_{t}^{\infty} \dot{H}_{x}^{s_{c}}}\right) \leq M_{2}\right\}
$$

where $t \in[0, T]$ for some $T>0$ and $x \in \mathbb{R}^{N}$. Denoting by $I=[0, T]$ and applying Lemma 2.4.6 to the operator defined in (2.23), we obtain

$$
\left\||\nabla|^{s_{c}} \Phi(u(t))\right\|_{L_{I}^{q} L_{x}^{r}}+\|\Phi(u(t))\|_{L_{I}^{q} \dot{H}_{x}^{s_{c}}} \leq 2 c_{1}\left\|u_{0}\right\|_{\dot{H}^{s_{c}}}+2 c_{1}\left\||\nabla|^{s_{c}} F(u)\right\|_{L_{I}^{q^{\prime}} L_{x}^{r^{\prime}}},
$$

where $F(u)$ is defined in (2.24). Using the product rule and Hölder's inequality, we get

$$
\begin{aligned}
\left\||\nabla|^{s_{c}} F(u)\right\|_{L_{I}^{q^{\prime}} L_{x}^{r^{\prime}}} \leq & \left\|\left(|x|^{-(N-\gamma)} *|\nabla|^{s_{c}}\left(|u|^{2}\right)\right) u\right\|_{L_{t}^{q^{\prime}} L_{x}^{r^{\prime}}} \\
& +\left\|\left(|x|^{-(N-\gamma)} *|u|^{p}\right)|\nabla|^{s_{c}} u\right\|_{L_{t}^{q^{\prime}} L_{x}^{r^{\prime}}} \\
\stackrel{\text { def }}{=} & A_{1}+A_{2},
\end{aligned}
$$

where $A_{1}$ is estimated using Lemma 2.3.1 and Lemma 2.3.2 along with the formula for $s_{c}$

$$
\begin{aligned}
A_{1} & \leq\left\|\left(|x|^{-(N-\gamma)} *|\nabla|^{s_{c}}|u|^{2}\right)\right\|_{L_{I}^{\frac{3}{2}} \frac{L_{x}^{3 N-3 \gamma-2}}{3 N}}\|u\|_{L_{I}^{\infty} L_{x}^{\frac{2 N}{N-2 s_{c}}}} \\
& \leq c_{N, \gamma}\left\|u|\nabla|^{s_{c}} u\right\|_{L_{I}^{\frac{3}{2}} \frac{6 N}{L_{x}^{3 N+3 \gamma-2}}}\left\||\nabla|^{s_{c}} u\right\|_{L_{I}^{\infty} L_{x}^{2}} \\
& \leq c_{N, \gamma}\|u\|_{L_{I}^{q} L_{x}^{\frac{6 N}{3 \gamma+2}}}\left\||\nabla|^{s_{c}} u\right\|_{L_{I}^{q} L_{x}^{r}}\left\||\nabla|^{s_{c}} u\right\|_{L_{I}^{\infty} L_{x}^{2}} \\
& \leq c_{N, \gamma}\left\||\nabla|^{s_{c}} u\right\|_{L_{I}^{q} L_{x}^{r}}^{2}\left\||\nabla|^{s_{c}} u\right\|_{L_{I}^{\infty} L_{x}^{2}} .
\end{aligned}
$$

Here, we require $3 N-3 \gamma-2$ appearing in (2.62) to be positive, which is true if $\gamma<N-\frac{2}{3}$. Observe that since $s_{c}>1$ we have that $\gamma<N-4<N-\frac{2}{3}$. For $A_{2}$, we again use Hölder's inequality, Lemma 2.3.1 and Lemma 2.3.2 to obtain

$$
\begin{aligned}
A_{2} & \leq\left\||x|^{-(N-\gamma)} *|u|^{2}\right\|{ }_{L_{I}^{\frac{3}{2}} L_{x}^{\frac{3 N}{2}}}\left\||\nabla|^{s_{c}} u\right\|_{L_{I}^{\infty} L_{x}^{2}} \\
& \leq c_{N, \gamma}\|u\|_{L_{I}^{q} L_{x}^{\frac{6 N}{3 \gamma+2}}}^{2}\left\||\nabla|^{s_{c}} u\right\|_{L_{I}^{\infty} L_{x}^{2}} \leq c_{N, \gamma}\left\||\nabla|^{s_{c}} u\right\|_{L_{I}^{q} L_{x}^{r}}^{2}\left\||\nabla|^{s_{c}} u\right\|_{L_{I}^{\infty} L_{x}^{2}} .
\end{aligned}
$$


Thus, (2.61) yields

$$
\left\||\nabla|^{s_{c}} F(u)\right\|_{L_{I}^{q^{\prime}} L_{x}^{r^{\prime}}} \leq 2 c_{N, \gamma}\left\||\nabla|^{s_{c}} u\right\|_{L_{I}^{q} L_{x}^{r}}^{2}\left\||\nabla|^{s_{c}} u\right\|_{L_{I}^{\infty} L_{x}^{2}}
$$

Substituting (2.65) in (2.60), we get

$$
\begin{aligned}
& \left\||\nabla|^{s_{c}} \Phi(u(t))\right\|_{L_{I}^{q} L_{x}^{r}}+\|\Phi(u(t))\|_{L_{I}^{\infty} \dot{H}_{x}^{s_{c}}} \\
& \quad \leq 2 c_{1}\left\|u_{0}\right\|_{\dot{H}^{s_{c}}}+4 c_{1} c_{N, \gamma}\left\||\nabla|^{s_{c}} u\right\|_{L_{I}^{q} L_{x}^{r}}^{2}\left\||\nabla|^{s_{c}} u\right\|_{L_{I}^{\infty} L_{x}^{2}} .
\end{aligned}
$$

Take $\left\|u_{0}\right\|_{\dot{H}_{x}^{s c}}$ small enough so that

$$
2\left\|u_{0}\right\|_{\dot{H}_{x}^{s c}}^{2} \leq \frac{1}{\left(8 c_{1}\right)^{3} c_{N, \gamma}}
$$

Set $M_{2}=8 c_{1}\left\|u_{0}\right\|_{\dot{H}_{x}^{s c}}$. Thus,

$$
M_{2}^{2} \leq \frac{1}{16 c_{1} c_{N, \gamma}} .
$$

Take $T>0$ such that $\left\|e^{i t \Delta}\left(|\nabla|{ }^{s_{c}} u_{0}\right)\right\|_{S\left(L^{2} ;[0, T]\right)} \leq \frac{M_{2}}{4}$. Thus, for $u \in B_{2}$ with $T$ as above, we estimate (2.66) as follows

$$
\begin{aligned}
\|\Phi(u(t))\|_{L_{I}^{\infty} \dot{H}_{x}^{s c}} & +\left\||\nabla|^{s_{c}} \Phi(u(t))\right\|_{L_{I}^{q_{1}} L_{x}^{r}} \leq 2 c_{1}\left\|u_{0}\right\|_{\dot{H}_{x}^{s c}}+4 c_{1} c_{N, \gamma} M_{2}^{3} \\
& \leq \frac{M_{2}}{4}+4 c_{1} c_{N, \gamma} \frac{M_{2}}{16 c_{1} c_{N, \gamma}}<M_{2},
\end{aligned}
$$

yielding $\Phi$ mapping $B_{2}$ into itself. Now we need to show that the operator $\Phi$ is a contraction. This can be achieved by running the same argument as above on the difference

$$
\begin{aligned}
d(\Phi(u(t)), \Phi(v(t))) \stackrel{\text { def }}{=} & \left\||\nabla|^{s_{c}}[\Phi(u(t))-\Phi(v(t))]\right\|_{L_{I}^{q} L_{x}^{r}} \\
& +\left\||\nabla|^{s_{c}}[\Phi(u(t))-\Phi(v(t))]\right\|_{L_{I}^{\infty} L_{x}^{2}},
\end{aligned}
$$

for $u, v \in B_{T}$. We first apply Lemma 2.4.6 to get

$$
d(\Phi(u(t)), \Phi(v(t))) \leq 2 c_{1}\left\||\nabla|^{s_{c}}[\Phi(u(t))-\Phi(v(t))]\right\|_{L_{I}^{q^{\prime}} L_{x}^{r^{\prime}}},
$$


where

$$
\begin{aligned}
&\left\||\nabla|^{s_{c}}[\Phi(u(t))-\Phi(v(t))]\right\|_{L_{I}^{q^{\prime}} L_{x}^{r^{\prime}}} \leq\left\||\nabla|^{s_{c}}\left[\left(|x|^{-(N-\gamma)} *|u|^{2}\right)(u-v)\right]\right\|_{L_{I}^{q^{\prime}} L_{x}^{r^{\prime}}} \\
&+\left\||\nabla|^{s_{c}}\left[\left(|x|^{-(N-\gamma)} *\left(|u|^{2}-|v|^{2}\right)\right) v\right]\right\|_{L_{I}^{q^{\prime}} L_{x}^{r^{\prime}}} \\
& \stackrel{\text { def }}{=} D_{1}+D_{2} .
\end{aligned}
$$

For $D_{1}$ we first use the product rule

$$
\begin{aligned}
D_{1} \leq & \left\||x|^{-(N-\gamma)} *|\nabla|^{s_{c}}\left(|u|^{2}\right)\right\|_{L_{I}^{\frac{3}{2}} L_{x}^{\frac{6 N}{3 N-3 \gamma-2}}}\|u-v\|_{L_{I}^{\infty} L_{x}^{\frac{2 N}{N-2 s_{c}}}} \\
& +\left\||x|^{-(N-\gamma)} *|u|^{2}\right\|_{L_{I}^{\frac{3}{2}} L_{x}^{\frac{3 N}{2}}}\left\||\nabla|^{s_{c}}(u-v)\right\|_{L_{I}^{\infty} L_{x}^{2}},
\end{aligned}
$$

then using the similar calculations as in (2.63) and (2.64) yields

$$
D_{1} \leq 2 c_{N, \lambda}\left\||\nabla|^{s_{c}} u\right\|_{L_{I}^{q} L_{x}^{r}}^{2}\left\||\nabla|^{s_{c}}(u-v)\right\|_{L_{I}^{\infty} L_{x}^{2}}
$$

Again using the product rule, we have

$$
\begin{aligned}
D_{2} \leq & \left\||x|^{-(N-\gamma)} *|\nabla|^{s_{c}}\left(|u|^{2}-|v|^{2}\right)\right\|_{L_{I}^{\frac{3}{2}} \frac{6 N}{L_{x}^{3 N-3 \gamma-2}}}\|v\|_{L_{I}^{\infty} L_{x}^{\frac{2 N}{N-2 s_{c}}}} \\
& +\left\||x|^{-(N-\gamma)} *\left(|u|^{2}-|v|^{2}\right)\right\|_{L_{I}^{\frac{3}{2}} L_{x}^{\frac{3 N}{2}}}\left\||\nabla|^{s_{c}} v\right\|_{L_{I}^{\infty} L_{x}^{2} .}
\end{aligned}
$$

Using the similar calculations as above along with (2.19), we get

$$
D_{2} \leq 2 c_{N, \gamma}\left(\left\||\nabla|^{s_{c}} u\right\|_{L_{I}^{q} L_{x}^{r}}+\left\||\nabla|^{s_{c}} v\right\|_{L_{I}^{q} L_{x}^{r}}\right)\left\||\nabla|^{s_{c}}(u-v)\right\|_{L_{I}^{q} L_{x}^{r}}\left\||\nabla|^{s_{c}} v\right\|_{L_{I}^{\infty} L_{x}^{2}}
$$

Combining (2.69) and (2.70), we obtain for $u, v \in B_{2}$,

$$
d(\Phi(u(t)), \Phi(v(t))) \leq 8 c_{1} c_{N, \lambda} M_{2}^{2} d(u, v) .
$$

Taking $M_{2}$ as in (2.68) together with (2.67) implies that $\Phi$ is a contraction on $B_{2}$ for $p=2$ with $s_{c}>1$.

$\underline{\text { Case }} p>2$ and $\gamma<N(p-1)-2 p$. 
For $T>0$ and $M_{s}>0$ determined later, let

$$
u \in X_{s} \stackrel{\text { def }}{=} u \in C\left([0, T] ; \dot{H}^{s_{c}}\left(\mathbb{R}^{N}\right)\right) \cap L^{q}\left([0, T] ; \dot{W}^{s_{c}, r}\left(\mathbb{R}^{N}\right)\right)
$$

Define

$$
B_{s}=\left\{u \in X_{s}: \max \left(\left\||\nabla|^{s_{c}} u\right\|_{L_{t}^{q} L_{x}^{r}\left([0, T] \times \mathbb{R}^{N}\right)},\|u\|_{L_{t}^{\infty} \dot{H}_{x}^{s_{c}}\left([0, T] \times \mathbb{R}^{N}\right)}\right) \leq M_{s}\right\}
$$

We again prove that the operator defined in (2.23) is a contraction on the set $B_{1}$ for some $T>0$. Denoting by $I=[0, T]$ and using Lemma 2.4.6, we obtain

$$
\left\||\nabla|^{s_{c}} \Phi(u(t))\right\|_{L_{I}^{q} L_{x}^{r}}+\|\Phi(u(t))\|_{L_{I}^{\infty} \dot{H}_{x}^{s_{c}}} \leq 2 c_{1}\left\|u_{0}\right\|_{\dot{H}_{x}^{s_{c}}}+2 c_{1}\left\||\nabla|^{s_{c}} F(u)\right\|_{L_{I}^{q^{\prime}} L_{x}^{r^{\prime}}}
$$

Using product rule, we have

$$
\begin{aligned}
& \left\||\nabla|^{s_{c}} F(u)\right\|_{L_{I}^{q^{\prime}} L_{x}^{r^{\prime}}} \leq\left\|\left(|x|^{-(N-\gamma)} *|\nabla|^{s_{c}}\left(|u|^{p}\right)\right)|u|^{p-2} u\right\|_{L_{I}^{q^{\prime}} L_{x}^{r^{\prime}}} \\
& +\left\|\left(|x|^{-(N-\gamma)} *|u|^{p}\right)|\nabla|^{s_{c}}\left(|u|^{p-2} u\right)\right\|_{L_{I}^{q^{\prime}} L_{x}^{r^{\prime}}} \\
& \stackrel{\text { def }}{=} A_{1}+A_{2} \text {, }
\end{aligned}
$$

where $A_{1}$ is estimated using Hölder's inequality, Lemma 2.3.1 and Lemma 2.3.2 along with the formula for $s_{c}$

$$
\begin{aligned}
A_{1} & \leq\left\|\left(|x|^{-(N-\gamma)} *|\nabla|^{s_{c}}|u|^{p}\right)\right\|_{L_{I}^{q} L_{x}^{\frac{2 N}{N-\gamma}}}\|u\|_{L_{t}^{\infty} L_{x}^{\frac{2 N(p-1)}{\gamma+2}}}^{p-1} \\
& \leq c_{N, \gamma}\left\||u|^{p-1}|\nabla|^{s_{c}} u\right\|_{L_{I}^{q} L_{x}^{\frac{2 N}{N+\gamma}}}\left\||\nabla|^{s_{c}} u\right\|_{L_{I}^{\infty} L_{x}^{2}}^{p-1} \\
& \leq c_{N, \gamma}\|u\|^{p-1}{ }_{L_{t}^{\infty} L_{x}^{\frac{2 N(p-1)}{\gamma+2}}}\left\||\nabla|^{s_{c}} u\right\|_{L_{I}^{q} L_{x}^{r}}\left\||\nabla|^{s_{c}} u\right\|_{L_{I}^{\infty} L_{x}^{2}}^{p-1} \\
& \leq c_{N, \gamma}\left\||\nabla|^{s_{c}} u\right\|_{L_{I}^{q} L_{x}^{r}}\left\||\nabla|^{s_{c}} u\right\|_{L_{I}^{\infty} L_{x}^{2}}^{2(p-1)} .
\end{aligned}
$$


For $A_{2}$, we again use Hölder's inequality, Lemma 2.3.1 and Lemma 2.3.2 to obtain

$$
\begin{aligned}
A_{2} & \leq\left\||x|^{-(N-\gamma)} *|u|^{p}\right\|{ }_{L_{I}^{\infty} L_{x}^{\frac{2 N-\gamma(p-1)}{2 p-2)}}}\|u\|_{L_{t}^{\infty} L_{x}^{\frac{2 N(p-1)}{\gamma+2}}}^{p-2}\left\||\nabla|^{s_{c}} u\right\|_{L_{I}^{q} L_{x}^{r}} \\
& \leq c_{N, \gamma}\|u\|^{p}{ }_{L_{I}^{\infty} L_{x}^{\frac{2 N(p-1)}{\gamma+2}}}\left\||\nabla|^{s_{c}} u\right\|_{L_{I}^{\infty} L_{x}^{2}}^{p-2}\left\||\nabla|^{s_{c}} u\right\|_{L_{I}^{q} L_{x}^{r}} \\
& \leq c_{N, \gamma}\left\||\nabla|^{s_{c}} u\right\|_{L_{I}^{\infty} L_{x}^{2}}^{2(p-1)}\left\||\nabla|^{s_{c}} u\right\|_{L_{I}^{q} L_{x}^{r} .}
\end{aligned}
$$

Here, we require $2 p-\gamma(p-2)$ in (2.74) to be positive, which holds true if $\gamma<\frac{2 p}{p-2}$. This is the reason for an additional restriction (mentioned in the statement of the Proposition 2.6.3 (4)(b)) that $0<\gamma<\min \left(N, \frac{2 p}{p-2}\right)$. Thus, combining (2.73) and (2.75) with (2.72), (2.71) yields

$$
\begin{aligned}
\left\||\nabla|^{s_{c}} \Phi(u(t))\right\|_{L_{I}^{q} L_{x}^{r}} & +\|\Phi(u(t))\|_{L_{I}^{\infty} \dot{H}_{x}^{s_{c}}} \\
& \leq 2 c_{1}\left\|u_{0}\right\|_{\dot{H}_{x}^{s_{c}}}+4 c_{N, \gamma}\left\||\nabla|^{s_{c}} u\right\|_{L_{I}^{\infty} L_{x}^{2}}^{2(p-1)}\left\|\left.\nabla\right|^{s_{c}} u\right\|_{L_{I}^{q} L_{x}^{r}}
\end{aligned}
$$

Following a similar argument as in previous cases, we take $\left\|u_{0}\right\|_{\dot{H}_{x}^{s c}}$ small enough so that

$$
2\left\|u_{0}\right\|_{\dot{H}_{x}^{s c}}^{2(p-1)} \leq \frac{1}{\left(8 c_{1}\right)^{2 p-1} c_{N, \gamma}}
$$

and set $M_{s}=8 c_{1}\left\|u_{0}\right\|_{\dot{H}_{x}^{s_{c}}}$, which implies that

$$
M_{s}^{2(p-1)} \leq \frac{1}{8 c_{1} c_{N, \gamma}} .
$$

Taking $T>0$ such that $\left\|e^{i t \Delta}|\nabla|{ }^{s_{c}} u_{0}\right\|_{S\left(L^{2} ;[0, T]\right)} \leq \frac{M_{s}}{4}$, we get for $u \in B_{s}$,

$$
\left\||\nabla|^{s_{c}} \Phi(u(t))\right\|_{L_{I}^{q} L_{x}^{r}}+\|\Phi(u(t))\|_{L_{I}^{\infty} \dot{H}_{x}^{s_{c}}} \leq \frac{M_{s}}{4}+4 c_{1} c_{N, \gamma} \frac{M_{s}}{16 c_{1} c_{N, \gamma}}<M_{s},
$$

yielding $\Phi$ mapping $B_{s}$ into itself. Next, for $u, v \in B_{s}$, we again run similar calculations as above on the difference

$$
\begin{aligned}
d(\Phi(u(t)), \Phi(v(t))) \stackrel{\text { def }}{=} & \left\||\nabla|^{s_{c}}[\Phi(u(t))-\Phi(v(t))]\right\|_{L_{I}^{q} L_{x}^{r}} \\
& +\|\Phi(u(t))-\Phi(v(t))\|_{L_{I}^{\infty} \dot{H}_{x}^{s_{c}}}
\end{aligned}
$$


to show that $\Phi$ is a contraction. We first apply Lemma 2.4.6 to get

$$
\begin{aligned}
d(\Phi(u(t)), \Phi(v(t))) \leq & c_{1}\left\||\nabla|^{s_{c}}\left[\left(|x|^{-(N-\gamma)} *|u|^{p}\right)\left(|u|^{p-2} u-|v|^{p-2} v\right)\right]\right\|_{L_{I}^{q^{\prime}} L_{x}^{r^{\prime}}} \\
& +c_{1}\left\||\nabla|^{s_{c}}\left[\left(|x|^{-(N-\gamma)} *\left(|u|^{p}-|v|^{p}\right)\right)|v|^{p-2} v\right]\right\|_{L_{I}^{q^{\prime}} L_{x}^{r^{\prime}}} \\
\stackrel{\text { def }}{=} & c_{1}\left(D_{1}+D_{2}\right) .
\end{aligned}
$$

We then use the product rule for $D_{1}$ and similar calculations as in (2.73) and (2.75) along with (2.18)

$$
\begin{aligned}
& D_{1} \leq c_{N, \gamma}\|u\|_{L_{t}^{\infty} \dot{H}_{x}^{s_{c}}}^{p-1}\|\nabla u\|_{L_{t}^{q} L_{x}^{r}}\left(\|u\|_{L_{t}^{\infty} H_{x}^{s_{c}}}^{p-2}+\|v\|_{L_{t}^{\infty} \dot{H}_{x}^{s_{c}}}^{p-2}\right)\|u-v\|_{L_{t}^{\infty} \dot{H}_{x}^{s_{c}}} \\
&+\|u\|_{L_{t}^{\infty} \dot{H}_{x}^{s_{c}}}^{p}\left(\|u\|_{L_{t}^{\infty} \dot{H}_{x}^{s_{c}}}^{p-2}+\|v\|_{L_{t}^{\infty} \dot{H}_{x}^{s_{c}}}^{p-2}\right)\left\|\left.\nabla \nabla\right|^{s_{c}}(u-v)\right\|_{L_{t}^{q} L_{x}^{r}}
\end{aligned}
$$

Using again the product rule for $D_{2}$ and similar calculations as in (2.55) and (2.56)

$$
\begin{aligned}
D_{2} \leq c_{N, \gamma} & \left(\|u\|_{L_{t}^{\infty} \dot{H}_{x}^{s c}}^{p-1}+\|v\|_{L_{t}^{\infty} \dot{H}_{x}^{s_{c}}}^{p-1}\right)\|\nabla(u-v)\|_{L_{t}^{q} L_{x}^{r}}\|v\|_{L_{t}^{\infty} \dot{H}_{x}^{s_{c}}}^{p-1} \\
& +\left(\|u\|_{L_{t}^{\infty} \dot{H}_{x}^{s_{c}}}^{p-1}+\|v\|_{L_{t}^{\infty} \dot{H}_{x}^{s_{c}}}^{p-1}\right)\|u-v\|_{L_{t}^{\infty} \dot{H}_{x}^{s_{c}}}\|v\|_{L_{t}^{\infty} \dot{H}_{x}^{s_{c}}}^{p-2}\left\||\nabla|^{s_{c}} v\right\|_{L_{t}^{q} L_{x}^{r}} .
\end{aligned}
$$

Combining the estimates for $D_{1}$ and $D_{2}$, we obtain for $u, v \in B_{s}$ that

$$
d(\Phi(u(t)), \Phi(v(t))) \leq 8 c_{1} c_{N, \gamma} M_{s}^{2(p-1)} d(u, v)
$$

Taking $M_{s}$ and $T$ as in (2.78) along with (2.77) implies that $\Phi$ is a contraction on $B_{s}$.

Now continuous dependence with respect to $u_{0}$ is a direct consequence of the above analysis, we note that if $u$ and $v$ are the corresponding solutions of (2.23) with initial data $u_{0}$ and $v_{0}$, respectively, then

$$
u(t)-v(t)=e^{i t \Delta}\left(u_{0}-v_{0}\right)+i \int_{0}^{t} e^{i\left(t-t^{\prime}\right) \Delta}(F(u)-F(v))\left(t^{\prime}\right) d t^{\prime} .
$$

Thus, the argument used to estimate $D_{1}$ and $D_{2}$ in the above cases yield

$$
d(u(t), v(t)) \leq c_{1}\left\|u_{0}-v_{0}\right\|_{\dot{H}_{x}^{s_{c}}}+\frac{1}{2} d(u(t), v(t)) .
$$


This implies that if $\left\|u_{0}-v_{0}\right\|_{H_{x}^{s}}$ is small enough (see (2.40) for $s_{c}=0$, (2.49) for $0<$ $s_{c}<1,(2.58)$ for $s_{c}=1$ and (2.77) for $\left.s_{c}>1\right)$, we have that

$$
d(u(t), v(t)) \leq 2 c_{1}\left\|u_{0}-v_{0}\right\|_{\dot{H}_{x}^{s c}}
$$

and this completes the proof.

Note that the above result holds regardless of the focusing or defocusing cases; as a consequence, the same result holds in the inhomogeneous space $H^{s_{c}}$, which we prove next (see also [AR19]).

Proposition 2.6.4 Let $0<\gamma<N$ and $p \geq 2$ so that $s_{c} \geq 0$. Assume in addition that if $p$ is not an even integer, then $s_{c}<p-1$. Let $u_{0} \in H^{s_{c}}\left(\mathbb{R}^{N}\right)$. Then there exists a unique solution $u(x, t)$ of the equation (1.1) with data $u_{0}$ defined on $[0, T]$ for some $T>0$, and such that

(1.) for $s_{c}=0$ and $N \geq 1, u \in C\left([0, T] ; L_{x}^{2}\right) \cap L^{q}\left([0, T] ; L_{x}^{r}\right)$, where $(q, r)=$ $\left(2 p, \frac{2 N p}{N+\gamma}\right)$ is the $L^{2}$-admissible pair and $x \in \mathbb{R}^{N}$,

(2.) for $0<s_{c}<1$ and $N \geq 1, u \in C\left([0, T] ; H_{x}^{s_{c}}\right) \cap L^{q_{1}}\left([0, T] ; W_{x}^{s_{c}, r_{1}}\right) \cap L^{q_{2}}\left([0, T] ; W_{x}^{s_{c}, r_{2}}\right)$, where $\left(q_{1}, r_{1}\right)=\left(\frac{2 p}{1+s_{c}(p-1)}, \frac{2 N p}{N+\gamma}\right),\left(q_{2}, r_{2}\right)=\left(\frac{2 p}{1-s_{c}}, \frac{2 N p}{N+\gamma+2 s_{c} p}\right)$ are the $L^{2}$-admissible pairs and $x \in \mathbb{R}^{N}$,

(3.) for $s_{c}=1$ and $N \geq 3, u \in C\left([0, T] ; H_{x}^{1}\right) \cap L^{q}\left([0, T] ; W_{x}^{1, r}\right)$, where $(q, r)=$ $\left(2, \frac{2 N}{N-2}\right)$ is the $L^{2}$-admissible and $x \in \mathbb{R}^{N}$,

(4.) for $s_{c}>1, u \in C\left([0, T] ; H_{x}^{s_{c}}\right) \cap L^{q}\left([0, T] ; W_{x}^{s_{c}, r}\right)$, where $x \in \mathbb{R}^{N}$ and

- for $p=2($ thus, $N \geq 5),(q, r)=\left(3, \frac{6 N}{3 N-4}\right)$ is the $L^{2}$-admissible pair,

- for $p>2($ thus, $N \geq 3)$ and $0<\gamma<\min \left(N, \frac{2 p}{p-2}\right)$, the $L^{2}$-admissible pair is $(q, r)=\left(2, \frac{2 N}{N-2}\right)$. 
Moreover, for all $0<\widetilde{T}<T$, the continuous dependence upon the initial data holds.

Proof. The proof of (1.) stays the same as in Proposition 2.6.3. For other cases, we only need to include the estimate for the inhomogeneous part to prove that $\Phi$ defined in (2.23) is a contraction on the corresponding set for some $T>0$.

(2.) For $T>0$ and $M_{\text {in }}>0$ determined later, let

$u \in \mathcal{X}_{\text {in }} \stackrel{\text { def }}{=} C\left([0, T] ; H^{s_{c}}\left(\mathbb{R}^{N}\right)\right) \cap L^{q_{1}}\left([0, T] ; W^{s_{c}, r_{1}}\left(\mathbb{R}^{N}\right)\right) \cap L^{q_{2}}\left([0, T] ; W^{s_{c}, r_{2}}\left(\mathbb{R}^{N}\right)\right)$.

Define

$$
\begin{array}{r}
\mathcal{B}_{i n}=\left\{u \in \mathcal{X}_{i n}: \max \left(\left\||\nabla|^{s_{c}} u\right\|_{\left.L_{I}^{q_{1}} L_{x}^{r_{1}},\left\||\nabla|^{s_{c}} u\right\|_{L_{I}^{q_{2}} L_{x}^{r_{2}}}\right) \leq 2 M_{i n}}\right.\right. \\
\text { and } \left.\|u\|_{L_{I}^{q_{1}} L_{x}^{r_{1}}} \leq 6 c_{1}\left\|u_{0}\right\|_{L_{x}^{2}}\right\},
\end{array}
$$

for some $t \in I=[0, T]$. Estimating the inhomogeneous part using Lemma 2.4.6, we have

$$
\begin{aligned}
\|\Phi(u(t))\|_{L_{I}^{q_{1}} L_{x}^{r_{1}}} & +\|\Phi(u(t))\|_{L_{I}^{q_{2}} L_{x}^{r_{2}}}+\|\Phi(u(t))\|_{L_{I}^{\infty} L_{x}^{2}} \\
& \leq 3 c_{1}\left\|u_{0}\right\|_{L_{x}^{2}}+3 c_{1}\|F(u)\|_{L_{I}^{q_{1}^{\prime}} L_{x}^{r_{1}^{\prime}} .}
\end{aligned}
$$

Using Hölder's inequality, Lemma 2.3.1 and Lemma 2.3.2, we estimate

$$
\begin{aligned}
\|F(u)\|_{L_{I}^{q_{1}^{\prime}} L_{x}^{r_{1}^{\prime}}} & \leq\left\|\left(|x|^{-(N-\gamma)} *|u|^{p}\right)\right\|_{L_{I}^{2} L_{x}^{N-\gamma}}\|u\|_{L_{I}^{q_{2}} L_{x}^{r_{1}}}^{p-1} \leq c_{N, \gamma}\|u\|_{L_{I}^{2 p} \frac{2 N p}{L_{x}^{N+\gamma}}}^{p}\|u\|_{L_{I}^{q_{2}} L_{x}^{r_{1}}}^{p-1} \\
& \leq c_{N, \gamma}\|u\|_{L_{I}^{q_{2}} L_{x}^{r_{1}}}^{p-1}\|u\|_{L_{I}^{q_{1}} L_{x}^{r_{1}}}\left\||\nabla|^{s_{c}} u\right\|_{L_{I}^{q_{2}} L_{x}^{r_{2}}}^{r^{2}} \\
& \leq\left. c_{N, \gamma}\|\| \nabla\right|^{s_{c}} u\left\|_{L_{I}^{q_{2}} L_{x}^{r_{2}}}^{2(p-1)}\right\| u \|_{L_{I}^{q_{1}} L_{x}^{r_{1}}}
\end{aligned}
$$

Using (2.80), we write (2.79) as

$$
\begin{aligned}
\|\Phi(u(t))\|_{L_{I}^{q_{1}} L_{x}^{r_{1}}} & +\|\Phi(u(t))\|_{L_{I}^{q_{2}} L_{x}^{r_{2}}}+\|\Phi(u(t))\|_{L_{I}^{\infty} L_{x}^{2}} \\
& \leq 3 c_{1}\left\|u_{0}\right\|_{L_{x}^{2}}+3 c_{1} c_{N, \gamma}\left\|\left.\nabla\right|^{s_{c}} u\right\|_{L_{I}^{q_{2}} L_{x}^{r_{2}}}^{2(p-1)}\|u\|_{L_{I}^{q_{1}} L_{x}^{r_{1}}}
\end{aligned}
$$


Then for $u \in \mathcal{B}_{\text {in }}$, we have

$$
\begin{aligned}
\|\Phi(u(t))\|_{L_{I}^{q_{1}} L_{x}^{r_{1}}} & +\|\Phi(u(t))\|_{L_{I}^{q_{2}} L_{x}^{r_{2}}}+\|\Phi(u(t))\|_{L_{I}^{\infty} L_{x}^{2}} \\
& \leq 3 c_{1}\left\|u_{0}\right\|_{L_{x}^{2}}\left(1+2^{2 p-1} 3 c_{1} c_{N, \gamma} M_{i n}^{2(p-1)}\right) .
\end{aligned}
$$

Next, invoking (2.48) for $u \in \mathcal{B}_{i n}$, we have

$$
\begin{aligned}
\left\||\nabla|^{s_{c}} \Phi(u(t))\right\|_{L_{I}^{q_{1}} L_{x}^{r_{1}}} & +\left\||\nabla|^{s_{c}} \Phi(u(t))\right\|_{L_{I}^{q_{2}} L_{x}^{r_{2}}}+\|\Phi(u(t))\|_{L_{I}^{\infty} \dot{H}^{s_{c}}} \\
& \leq 3 c_{1}\left\|u_{0}\right\|_{\dot{H}_{x}^{s_{c}}}+3 c_{1} c_{N, \gamma} 2^{2 p} M_{i n}^{2 p-1} .
\end{aligned}
$$

Take $\left\|u_{0}\right\|_{\dot{H}_{x}^{s_{c}}}$ small enough so that

$$
\left\|u_{0}\right\|_{\dot{H}_{x}^{s c}}^{2(p-1)} \leq \frac{1}{2^{2(p+1)}\left(3 c_{1}\right)^{2 p-1} c_{N, \gamma}}
$$

Set $M_{i n}=3 c_{1}\left\|u_{0}\right\|_{\dot{H}_{x}^{s_{c}}}$. Thus,

$$
M_{i n}^{2(p-1)} \leq \frac{1}{2^{2(p+1)} 3 c_{1} c_{N, \gamma}} .
$$

Take $T>0$ such that $\left\|e^{i t \Delta}\left(|\nabla|{ }^{s_{c}} u_{0}\right)\right\|_{S\left(L^{2} ;[0, T]\right)} \leq M_{i n}$. Thus, using (2.84) and (2.85) on (2.82) and (2.83), we have

$$
\begin{aligned}
\|\Phi(u(t))\|_{L_{I}^{q_{1}} L_{x}^{r_{1}}}+\|\Phi(u(t))\|_{L_{I}^{q_{2}} L_{x}^{r_{2}}}+\|\Phi(u(t))\|_{L_{I}^{\infty} L_{x}^{2}} & \leq 3 c_{1}\left\|u_{0}\right\|_{L_{x}^{2}}\left(1+\frac{1}{8}\right) \\
& <6 c_{1}\left\|u_{0}\right\|_{L_{x}^{2}},
\end{aligned}
$$

and

$$
\begin{aligned}
\left\||\nabla|^{s_{c}} \Phi(u(t))\right\|_{L_{I}^{q_{1}} L_{x}^{r_{1}}}+\left\||\nabla|^{s_{c}} \Phi(u(t))\right\|_{L_{I}^{q_{2}} L_{x}^{r_{2}}} & +\|\Phi(u(t))\|_{L_{I}^{\infty} \dot{H}^{s_{c}}} \\
& \leq M_{i n}+\frac{M_{i n}}{4}<2 M_{i n} .
\end{aligned}
$$

Hence, (2.86) and (2.87) implies that $\Phi$ maps $\mathcal{B}_{\text {in }}$ into itself. The rest of the argument as in the proof of Proposition 2.6.3 part (2.). 
(3.) For $T>0$ and $M_{1}>0$ determined later, let

$$
u \in \mathcal{X}_{1} \stackrel{\text { def }}{=} u \in C\left([0, T] ; H^{1}\left(\mathbb{R}^{N}\right)\right) \cap L^{q}\left([0, T] ; W^{1, r}\left(\mathbb{R}^{N}\right)\right) .
$$

Define

$$
\begin{array}{r}
\mathcal{B}_{1}=\left\{u \in \mathcal{X}_{1}: \max \left(\|\nabla u\|_{L_{t}^{q} L_{x}^{r}\left([0, T] \times \mathbb{R}^{N}\right)},\|u\|_{L_{t}^{\infty} \dot{H}_{x}^{1}\left([0, T] \times \mathbb{R}^{N}\right)}\right) \leq M_{1}\right. \\
\text { and } \left.\|u\|_{L_{I}^{q} L_{x}^{r}} \leq 4 c_{1}\left\|u_{0}\right\|_{L_{x}^{2}}\right\} .
\end{array}
$$

Estimating the inhomogeneous part again, using Lemma 2.4.6, we have

$$
\|\Phi(u(t))\|_{L_{I}^{q} L_{x}^{r}}+\|\Phi(u(t))\|_{L_{I}^{\infty} L_{x}^{2}} \leq 2 c_{1}\left\|u_{0}\right\|_{L_{x}^{2}}+2 c_{1}\|F(u)\|_{L_{I}^{q^{\prime}} L_{x}^{r^{\prime}}}
$$

Using Hölder's inequality, Lemma 2.3.1 and Lemma 2.3.2, we estimate

$$
\begin{aligned}
\|F(u)\|_{L_{I}^{q^{\prime}} L_{x}^{r^{\prime}}} & \leq\left\|\left(|x|^{-(N-\gamma)} *|u|^{p}\right)\right\|_{L_{I}^{2} L_{x}^{N-\gamma}}\|u\|_{L_{I}^{\infty} L_{x}^{r}}^{p-1} \leq c_{N, \gamma}\|u\|_{L_{I}^{2 p} L_{x}^{r}}^{p}\|u\|_{L_{I}^{\infty} L_{x}^{r}}^{p-1} \\
& \leq c_{N, \gamma}\|u\|_{L_{I}^{\infty} L_{x}^{r}}^{p-1}\|u\|_{L_{I}^{q} L_{x}^{r}}\|u\|_{L_{I}^{\infty} \dot{H}_{x}^{1}}^{p-1} \\
& \leq c_{N, \gamma}\|u\|_{L_{I}^{\infty} \dot{H}_{x}^{1}}^{2(p-1)}\|u\|_{L_{I}^{q} L_{x}^{r}}
\end{aligned}
$$

Using (2.89), we write (2.88) as

$$
\|\Phi(u(t))\|_{L_{I}^{q} L_{x}^{r}}+\|\Phi(u(t))\|_{L_{I}^{\infty} L_{x}^{2}} \leq 2 c_{1}\left\|u_{0}\right\|_{L_{x}^{2}}+2 c_{1} c_{N, \gamma}\|u\|_{L_{I}^{\infty} \dot{H}_{x}^{1}}^{2(p-1)}\|u\|_{L_{I}^{q} L_{x}^{r}} .
$$

Then for $u \in \mathcal{B}_{1}$, we have

$$
\|\Phi(u(t))\|_{L_{I}^{q} L_{x}^{r}}+\|\Phi(u(t))\|_{L_{I}^{\infty} L_{x}^{2}} \leq 2 c_{1}\left\|u_{0}\right\|_{L_{x}^{2}}\left(1+2^{2 p} c_{1} c_{N, \gamma} M_{1}^{2(p-1)}\right) .
$$

Next, invoking (2.57) for $u \in \mathcal{B}_{1}$, we have

$$
\|\nabla \Phi(u(t))\|_{L_{I}^{q} L_{x}^{r}}+\|\Phi(u(t))\|_{L_{I}^{\infty} \dot{H}_{x}^{1}} \leq 2 c_{1}\left\|u_{0}\right\|_{\dot{H}_{x}^{1}}+2^{2 p+1} c_{1} c_{N, \gamma} M_{1}^{2 p-1} .
$$

Take $\left\|u_{0}\right\|_{\dot{H}_{x}^{s c}}$ small enough so that

$$
\left\|u_{0}\right\|_{\dot{H}_{x}^{s c}}^{2(p-1)} \leq \frac{1}{2^{4 p}\left(c_{1}\right)^{2 p-1} c_{N, \gamma}}
$$


Set $M_{1}=2 c_{1}\left\|u_{0}\right\|_{\dot{H}_{x}^{s_{c}}}$. Thus,

$$
M_{1}^{2(p-1)} \leq \frac{1}{2^{2(p+1)} c_{1} c_{N, \gamma}} .
$$

Take $T>0$ such that $\left\|e^{i t \Delta}\left(\nabla u_{0}\right)\right\|_{S\left(L^{2} ;[0, T]\right)} \leq M_{1}$. Thus, using (2.92) and (2.93) on (2.90) and (2.91), we have

$$
\|\Phi(u(t))\|_{L_{I}^{q} L_{x}^{r}}+\|\Phi(u(t))\|_{L_{I}^{\infty} L_{x}^{2}} \leq 2 c_{1}\left\|u_{0}\right\|_{L_{x}^{2}}\left(1+\frac{1}{4}\right)<4 c_{1}\left\|u_{0}\right\|_{L_{x}^{2}}
$$

and

$$
\|\nabla \Phi(u(t))\|_{L_{I}^{q} L_{x}^{r}}+\|\Phi(u(t))\|_{L_{I}^{\infty} \dot{H}^{1}} \leq M_{1}+\frac{M_{1}}{2}<2 M_{1}
$$

Hence, (2.94) and (2.95) implies that $\Phi$ maps $\mathcal{B}_{1}$ into itself. The rest of the argument as in the proof of Proposition 2.6.3 part (3.).

(4.) $s_{c}>1 \Longrightarrow \gamma<N(p-1)-2 p$.

Case $p=2$ and $\gamma<N-4$, which requires $N>4$ since $\gamma>0$.

For $T>0$ and $M_{2}>0$ determined later, let

$$
\mathcal{X}_{2}=C\left([0, T] ; H^{s_{c}}\left(\mathbb{R}^{N}\right)\right) \cap L^{q}\left([0, T] ; W^{s_{c}, r}\left(\mathbb{R}^{N}\right)\right)
$$

and define

$$
\begin{aligned}
\mathcal{B}_{2}=\left\{u \in X_{2}: \max \left(\left\||\nabla|^{s_{c}} u\right\|_{L_{I}^{q} L_{x}^{r}},\|u\|_{L_{I}^{\infty} \dot{H}_{x}^{s_{c}}}\right)\right. & \leq M_{2} \\
\text { and }\|u\|_{L_{I}^{q} L_{x}^{r}} & \left.\leq 4 c_{1}\left\|u_{0}\right\|_{L^{2}}\right\},
\end{aligned}
$$

where $t \in I=[0, T]$ for some $T>0$ and $x \in \mathbb{R}^{N}$. Estimating the inhomogeneous part again, using Lemma 2.4.6, we have

$$
\|\Phi(u(t))\|_{L_{I}^{q} L_{x}^{r}}+\|\Phi(u(t))\|_{L_{I}^{\infty} L_{x}^{2}} \leq 2 c_{1}\left\|u_{0}\right\|_{L_{x}^{2}}+2 c_{1}\|F(u)\|_{L_{I}^{q^{\prime}} L_{x}^{r^{\prime}}}
$$


Using Hölder's inequality, Lemma 2.3.1 and Lemma 2.3.2, we estimate

$$
\begin{aligned}
\|F(u)\|_{L_{I}^{q^{\prime}} L_{x}^{r^{\prime}}} & \leq\left\|\left(|x|^{-(N-\gamma)} *|u|^{2}\right)\right\|_{L_{I}^{\frac{3}{2}} L_{x}^{3 N-3 \gamma-2}}\|u\|_{L_{I}^{\infty} L_{x}^{\frac{2 N}{N-2 s_{c}}}} \\
& \leq c_{N, \gamma}\|u\|_{L_{I}^{q} L_{x}^{3 N+3 \gamma-2}}^{2}\|u\|_{L_{I}^{\infty} \dot{H}_{x}^{s_{c}}} \\
& \leq c_{N, \gamma}\|u\|_{L_{I}^{q} L_{x}^{\frac{6 N}{3 \gamma+2}}}\|u\|_{L_{I}^{q} L_{x}^{r}}\|u\|_{L_{I}^{\infty} \dot{H}_{x}^{s_{c}}} \\
& \leq c_{N, \gamma}\left\||\nabla|^{s_{c}} u\right\|_{L_{I}^{q} L_{x}^{r}}\|u\|_{L_{I}^{q} L_{x}^{r}}\|u\|_{L_{I}^{\infty} \dot{H}_{x}^{s_{c}}}
\end{aligned}
$$

Again, we require $3 N-3 \gamma-2$ appearing in (2.97) to be positive, which is true if $\gamma<N-\frac{2}{3}$, and since $s_{c}>1$, we have that $\gamma<N-4<N-\frac{2}{3}$. Using (2.98) and for $u \in \mathcal{B}_{1}$, we write $(2.96)$ as

$$
\|\Phi(u(t))\|_{L_{I}^{q} L_{x}^{r}}+\|\Phi(u(t))\|_{L_{I}^{\infty} L_{x}^{2}} \leq 2 c_{1}\left\|u_{0}\right\|_{L_{x}^{2}}\left(1+8 c_{1} c_{N, \gamma} M_{2}^{2}\right) .
$$

Next, invoking (2.66) for $u \in \mathcal{B}_{2}$, we have

$$
\left\||\nabla|^{s_{c}} \Phi(u(t))\right\|_{L_{I}^{q} L_{x}^{r}}+\|\Phi(u(t))\|_{L_{I}^{\infty} \dot{H}_{x}^{s_{c}}} \leq 2 c_{1}\left\|u_{0}\right\|_{\dot{H}_{x}^{s_{c}}}+32 c_{1} c_{N, \gamma} M_{2}^{3} .
$$

Take $\left\|u_{0}\right\|_{\dot{H}_{x}^{s c}}$ small enough so that

$$
\left\|u_{0}\right\|_{\dot{H}_{x}^{s_{c}}}^{2} \leq \frac{1}{2^{8}\left(c_{1}\right)^{3} c_{N, \gamma}} .
$$

Set $M_{2}=2 c_{1}\left\|u_{0}\right\|_{\dot{H}_{x}^{s_{c}}}$. Thus,

$$
M_{2}^{2} \leq \frac{1}{2^{6} c_{1} c_{N, \gamma}} .
$$

Take $T>0$ such that $\left\|e^{i t \Delta}\left(\nabla u_{0}\right)\right\|_{S\left(L^{2} ;[0, T]\right)} \leq M_{2}$. Thus, using (2.101) and (2.102) on (2.99) and (2.100), we have

$$
\|\Phi(u(t))\|_{L_{I}^{q} L_{x}^{r}}+\|\Phi(u(t))\|_{L_{I}^{\infty} L_{x}^{2}} \leq 2 c_{1}\left\|u_{0}\right\|_{L_{x}^{2}}\left(1+\frac{1}{8}\right)<4 c_{1}\left\|u_{0}\right\|_{L_{x}^{2}}
$$

and

$$
\|\nabla \Phi(u(t))\|_{L_{I}^{q} L_{x}^{r}}+\|\Phi(u(t))\|_{L_{I}^{\infty} \dot{H}^{1}} \leq M_{2}+\frac{M_{2}}{2}<2 M_{2}
$$


Hence, (2.103) and (2.104) implies that $\Phi$ maps $\mathcal{B}_{1}$ into itself. The rest of the argument as in the proof of Proposition 2.6.3 part (4.) for $p=2$.

Case $p>2$ and $\gamma<N(p-1)-2 p$.

For $T>0$ and $M_{s}>0$ determined later, let

$$
u \in \mathcal{X}_{s} \stackrel{\text { def }}{=} u \in C\left([0, T] ; H^{s_{c}}\left(\mathbb{R}^{N}\right)\right) \cap L^{q}\left([0, T] ; W^{s_{c}, r}\left(\mathbb{R}^{N}\right)\right)
$$

Define

$$
\begin{array}{r}
\mathcal{B}_{s}=\left\{u \in \mathcal{X}_{s}: \max \left(\left\||\nabla|^{s_{c}} u\right\|_{L_{t}^{q} L_{x}^{r}\left([0, T] \times \mathbb{R}^{N}\right)},\|u\|_{L_{t}^{\infty} \dot{H}_{x}^{s c}\left([0, T] \times \mathbb{R}^{N}\right)}\right) \leq M_{s}\right. \\
\text { and } \left.\|u\|_{L_{I}^{q} L_{x}^{r}} \leq 4 c_{1}\left\|u_{0}\right\|_{L^{2}}\right\}
\end{array}
$$

Estimating the inhomogeneous part again, using Lemma 2.4.6, we have

$$
\|\Phi(u(t))\|_{L_{I}^{q} L_{x}^{r}}+\|\Phi(u(t))\|_{L_{I}^{\infty} L_{x}^{2}} \leq 2 c_{1}\left\|u_{0}\right\|_{L_{x}^{2}}+2 c_{1}\|F(u)\|_{L_{I}^{q^{\prime}} L_{x}^{r^{\prime}}}
$$

Using Hölder's inequality, Lemma 2.3.1 and Lemma 2.3.2, we estimate

$$
\begin{aligned}
& \|F(u)\|_{L_{I}^{q^{\prime}} L_{x}^{r^{\prime}}} \leq\left\|\left(|x|^{-(N-\gamma)} *|u|^{p}\right)\right\|_{L_{I}^{q} L_{x}^{\frac{2 N}{N-\gamma}}}\|u\|_{L_{I}^{\infty} L_{x}^{\frac{2 N(p-1)}{\gamma+2}}}^{p} \\
& \leq c_{N, \gamma}\left\||u|^{p}\right\|_{L_{I}^{q} L_{x}^{N+\gamma}}^{\frac{2 N}{N+\gamma}}\|u\|_{L_{I}^{\infty} \dot{H}_{x}^{s_{c}}}^{p-1} \\
& \leq c_{N, \gamma}\|u\|_{L_{I}^{\infty} \frac{2 N(p-1)}{L_{x}}}^{p-1}\|u\|_{L_{I}^{q} L_{x}^{r}}\|u\|_{L_{I}^{\infty} \dot{H}_{x}^{s c}}^{p-1} \\
& \leq c_{N, \gamma}\|u\|_{L_{I}^{\infty} \dot{H}_{x}^{s_{c}}}^{2(p-1)}\|u\|_{L_{I}^{q} L_{x}^{r}} .
\end{aligned}
$$

Using (2.106) and for $u \in \mathcal{B}_{1}$, we write (2.105) as

$$
\|\Phi(u(t))\|_{L_{I}^{q} L_{x}^{r}}+\|\Phi(u(t))\|_{L_{I}^{\infty} L_{x}^{2}} \leq 2 c_{1}\left\|u_{0}\right\|_{L_{x}^{2}}\left(1+2^{2 p} c_{1} c_{N, \gamma} M_{s}^{2(p-1)}\right) .
$$

Next, invoking (2.76) for $u \in \mathcal{B}_{s}$, we have

$$
\left\||\nabla|^{s_{c}} \Phi(u(t))\right\|_{L_{I}^{q} L_{x}^{r}}+\|\Phi(u(t))\|_{L_{I}^{\infty} \dot{H}_{x}^{s_{c}}} \leq 2 c_{1}\left\|u_{0}\right\|_{\dot{H}_{x}^{s_{c}}}+2^{2 p+1} c_{1} c_{N, \gamma} M_{s}^{2 p-1}
$$


Note that (2.108) requires $2 p-\gamma(p-2)>0$, which holds true if $\gamma<\frac{2 p}{p-2}$ (see (2.74)). This is the reason for an additional restriction (mentioned in the statement of the Proposition 2.6.4 (4)(b)) that $0<\gamma<\min \left(N, \frac{2 p}{p-2}\right)$. Take $\left\|u_{0}\right\|_{\dot{H}_{x}^{s_{c}}}$ small enough so that

$$
\left\|u_{0}\right\|_{\dot{H}_{x}^{s c}}^{2(p-1)} \leq \frac{1}{2^{4 p}\left(c_{1}\right)^{2 p-1} c_{N, \gamma}}
$$

Set $M_{s}=2 c_{1}\left\|u_{0}\right\|_{\dot{H}_{x}^{s_{c}}}$. Thus,

$$
M_{s}^{2(p-1)} \leq \frac{1}{2^{2(p+1)} c_{1} c_{N, \gamma}}
$$

Take $T>0$ such that $\left\|e^{i t \Delta}\left(\nabla u_{0}\right)\right\|_{S\left(L^{2} ;[0, T]\right)} \leq M_{s}$. Thus, using (2.109) and (2.110) on (2.107) and (2.108), we have

$$
\|\Phi(u(t))\|_{L_{I}^{q} L_{x}^{r}}+\|\Phi(u(t))\|_{L_{I}^{\infty} L_{x}^{2}} \leq 2 c_{1}\left\|u_{0}\right\|_{L_{x}^{2}}\left(1+\frac{1}{4}\right)<4 c_{1}\left\|u_{0}\right\|_{L_{x}^{2}}
$$

and

$$
\left\||\nabla|^{s_{c}} \Phi(u(t))\right\|_{L_{I}^{q} L_{x}^{r}}+\|\Phi(u(t))\|_{L_{I}^{\infty} \dot{H}^{s_{c}}} \leq M_{s}+\frac{M_{s}}{2}<2 M_{s} .
$$

Hence, (2.111) and (2.112) implies that $\Phi$ maps $\mathcal{B}_{1}$ into itself. The rest of the argument as in the proof of Proposition 2.6.3 part (4.) for $p>2$.

The continuous dependence also follows from an exactly similar argument as in Proposition 2.6.3.

\subsection{Local well-posedness in $\dot{H}^{s}$ for $0<s<1$}

In this section we show the local well-posedness in $\dot{H}^{s}$ (sub-critical cases, $0 \leq s<1$ ) using Besov spaces for $s>s_{c}$, where the choice of $0<s<1$ depends on the value of $p \geq 2$ and $0<\gamma<N$. 
Proposition 2.7.1 Let $N \geq 1,0<\gamma<N, 2 \leq p<1+\frac{\gamma+2}{N-2 s}$ (i.e., $s>s_{c}$ ), in addition, we also assume that $0<s<(N-\gamma) / 2$. Let $u_{0} \in \dot{H}^{s}\left(\mathbb{R}^{N}\right)$. Then there exists a unique solution $u(x, t)$ of the equation (1.1) with data $u_{0}$ defined on $I=[0, T]$ for some $T>0$, and such that

$$
u \in \mathcal{Y} \stackrel{\text { def }}{=} C\left(I ; \dot{H}^{s}\left(\mathbb{R}^{N}\right)\right) \cap L^{q}\left(I ; \dot{B}_{r, 2}^{s}\left(\mathbb{R}^{N}\right)\right)
$$

where the pair $(q, r)$ is the $L^{2}$-admissible pair given by

$$
(q, r)=\left(\frac{4 p}{(p-1)(N-2 s)-\gamma}, \frac{2 N p}{N+\gamma+2 s(p-1)}\right) .
$$

Here, $(p-1)(N-2 s) \geq N-2 s>\gamma$, since $p \geq 2$ and $s<(N-\gamma) / 2$.

Proof. For $T>0$ and $M>0$ determined later, let

$$
S_{b}=\left\{u \in \mathcal{Y}:\|u\|_{L_{I}^{q} \dot{B}_{r, 2}^{s}} \leq M_{b}\right\}
$$

We again prove that the operator defined in (2.23) is a contraction on the set $S_{b}$ for some $T>0$. Denoting by $I=[0, T]$, using Lemma 2.4.9 and (2.14), we obtain

$$
\begin{aligned}
\|\Phi(u(t))\|_{L_{I}^{q} \dot{B}_{r, 2}^{s}} & \leq c_{1}\left\|u_{0}\right\|_{\dot{H}_{x}^{s}}+c_{1}\|F(u)\|_{L_{t}^{q^{\prime} \dot{B}_{r^{\prime}, 2}^{s}}} \\
& \leq c_{1}\left\|u_{0}\right\|_{\dot{H}_{x}^{s}}+c_{1}\left\||\nabla|^{s} F(u)\right\|_{L_{I}^{q^{\prime}} L_{x}^{r^{\prime}}}
\end{aligned}
$$

Using Hölder's in time for the second term on the right-hand side of (2.114), we have

$$
\left\||\nabla|^{s} F(u)\right\|_{L_{I}^{q^{\prime}} L_{x}^{r^{\prime}}} \lesssim T^{\delta}\left\||\nabla|^{s} F(u)\right\|_{L_{I}^{\frac{q}{2 p^{2-1}} L_{x}^{r^{\prime}}}}
$$

where $\delta=\frac{1}{q^{\prime}}-\frac{2 p-1}{q}>0$. Using product rule along with Hölder's inequality, we get

$$
\begin{aligned}
\left\||\nabla|^{s} F(u)\right\|_{L_{I}^{q} L_{x}^{r^{\prime}}} \leq & \left\|\left(|x|^{-(N-\gamma)} *|\nabla|^{s}\left(|u|^{p}\right)\right)|u|^{p-2} u\right\|_{L_{I}^{2 p-1} L_{x}^{r^{\prime}}} \\
& +\left\|\left(|x|^{-(N-\gamma)} *|u|^{p}\right)|\nabla|^{s}\left(|u|^{p-2} u\right)\right\|_{L_{I}^{\frac{q}{2 p-1}} L_{x}^{r^{\prime}}} \\
\stackrel{\text { def }}{=} & A_{1}+A_{2},
\end{aligned}
$$


where for $A_{1}$, Hölder's inequality, Lemmas 2.3.1 and 2.3.2 give

$$
\begin{aligned}
A_{1} & \leq\left\||x|^{-(N-\gamma)} *|\nabla|^{s}\left(|u|^{p}\right)\right\|_{L_{I}^{q / p} L_{x}^{\frac{2 N}{N-\gamma}}}\|u\|_{L_{I}^{q} L_{x}^{\frac{2 N p}{N-2 s+\gamma}}}^{p-1} \\
& \leq c_{N, \gamma}\left\||u|^{p-1}|\nabla|^{s} u\right\|_{L_{I}^{q / p} L_{x}^{N+\gamma}}\left\||\nabla|^{s} u\right\|_{L_{I}^{q} L_{x}^{r}}^{p-1} \leq c_{N, \gamma}\left\||\nabla|^{s} u\right\|_{L_{I}^{q} L_{x}^{r}}^{2 p-1},
\end{aligned}
$$

and estimating $A_{2}$ in a similar fashion, we obtain

$$
\begin{gathered}
A_{2} \leq\left\||x|^{-(N-\gamma)} *|u|^{p}\right\|_{L_{I}^{q / p} L_{x}^{N-2 s-\gamma}}\|u\|_{L_{I}^{q} L_{x}^{\frac{2 N}{N-2 s+\gamma}}}^{p-2}\left\||\nabla|^{s} u\right\|_{L_{I}^{q} L_{x}^{r}} \\
\leq c_{N, \gamma}\|u\|_{L_{I}^{q} L_{x}^{\frac{2 N}{N-2 s+\gamma}}}^{p}\left\||\nabla|^{s} u\right\|_{L_{I}^{q} L_{x}^{r}}^{p-1} \leq c_{N, \gamma}\left\||\nabla|^{s} u\right\|_{L_{I}^{q} L_{x}^{r}}^{2 p-1} .
\end{gathered}
$$

Using (2.13), we have

$$
A_{1} \leq c_{N, \gamma}\|u\|_{L_{I}^{q} \dot{B}_{r, 2}^{s}}^{2 p-1} \quad \text { and } \quad A_{2} \leq c_{N, \gamma}\|u\|_{L_{I}^{q} \dot{B}_{r, 2}^{s}}^{2 p-1}
$$

Combining (2.118) and (2.115) along with (2.114), we get for $u \in S_{b}$,

$$
\|\Phi(u(t))\|_{L_{I}^{q} \dot{B}_{r, 2}^{s}} \leq c_{1}\left\|u_{0}\right\|_{\dot{H}_{x}^{s}}+2 c_{1} c_{N, \gamma} T^{\delta} M_{b}^{2 p-1}
$$

Similarly, we have

$$
\|\Phi(u(t))\|_{L_{I}^{\infty} \dot{H}_{x}^{s}} \leq c_{1}\left\|u_{0}\right\|_{\dot{H}_{x}^{s}}+2 c_{1} c_{N, \gamma} T^{\delta} M_{b}^{2 p-1}
$$

Adding (2.119) and (2.120), we get

$$
\|\Phi(u(t))\|_{L_{I}^{q} \dot{B}_{r, 2}^{s}}+\|\Phi(u(t))\|_{L_{I}^{\infty} \dot{H}_{x}^{s}} \leq 2 c_{1}\left\|u_{0}\right\|_{\dot{H}_{x}^{s}}+4 c_{1} c_{N, \gamma} T^{\delta} M_{b}^{2 p-1}
$$

Set $M_{b}=4 c_{1}\left\|u_{0}\right\|_{\dot{H}_{x}^{s}}$ and take $T$ such that

$$
4 c_{1} c_{N, \gamma} T^{\delta} M_{b}^{2(p-1)}<\frac{1}{4}
$$

which implies that the right-hand side of (2.121) is bounded by $M_{b}$. Therefore, $T$ given by (2.122) gives that $\Phi$ maps $S_{b}$ to itself. Next, we show that $\Phi$ is a contraction by running 
similar calculations as above on the difference

$$
\begin{aligned}
d(\Phi(u(t)), \Phi(v(t))) & \stackrel{\text { def }}{=}\|\Phi(u(t))-\Phi(v(t))\|_{L_{I}^{q} \dot{B}_{r, 2}^{s}} \\
& \leq c_{1} T^{\delta}\left\||\nabla|^{s}[\Phi(u(t))-\Phi(v(t))]\right\|_{L_{I}^{\frac{q}{2 p-1}} L_{x}^{r^{\prime}}} \\
& \leq c_{1} T^{\delta}\left\||\nabla|^{s}\left[\left(|x|^{-(N-\gamma)} *|u|^{p}\right)\left(|u|^{p-2} u-|v|^{p-2} v\right)\right]\right\|_{L_{I}^{\frac{q}{2 p-1}} L_{x}^{r^{\prime}}} \\
& +c_{1} T^{\delta}\left\||\nabla|^{s}\left[\left(|x|^{-(N-\gamma)} *\left(|u|^{p}-|v|^{p}\right)\right)|v|^{p-2} v\right]\right\|_{L_{I}^{\frac{q}{2 p-1}} L_{x}^{r^{\prime}}} \\
& \stackrel{\text { def }}{=} c_{1} T^{\delta}\left(D_{1}+D_{2}\right),
\end{aligned}
$$

where we have used Lemma 2.4.9 along with (2.14) and Hölder's inequality in time.

We first use product rule for $D_{1}$ and similar calculations as in (2.116) and (2.117) together with (2.18) to obtain

$$
D_{1} \leq 2 c_{N, \gamma}\left\||\nabla|^{s} u\right\|_{L_{I}^{q} L_{x}^{r}}^{p}\left(\left\||\nabla|^{s} u\right\|_{L_{I}^{q} L_{x}^{r}}^{p-2}+\left\||\nabla|^{s} v\right\|_{L_{I}^{q} L_{x}^{r}}^{p-2}\right)\left\||\nabla|^{s}(u-v)\right\|_{L_{I}^{q} L_{x}^{r}} .
$$

Again, first using product rule for $D_{2}$ and similar calculations as in (2.116) and (2.117) along with (2.19), we have

$$
D_{2} \leq 2 c_{N, \gamma}\left(\left\||\nabla|^{s} u\right\|_{L_{I}^{q} L_{x}^{r}}^{p-1}+\left\||\nabla|^{s} v\right\|_{L_{I}^{q} L_{x}^{r}}^{p-1}\right)\left\||\nabla|^{s}(u-v)\right\|_{L_{I}^{q} L_{x}^{r}}\left\||\nabla|^{s} v\right\|_{L_{I}^{q} L_{x}^{r}}^{p-1} .
$$

Combining the estimates for $D_{1}$ and $D_{2}$ along with (2.13), for $u, v \in S_{b}$, we get

$$
d(\Phi(u(t)), \Phi(v(t))) \leq 8 c_{1} c_{N, \gamma} T^{\delta} M_{b}^{2(p-1)} d(u, v) .
$$

This together with (2.122) implies that $\Phi$ is a contraction on $S_{b}$.

\subsection{Ground state solutions}

The equation (1.1) admits solitary waves solutions of the form $u(x, t)=e^{i t} Q(x)$, where $Q$ solves the nonlinear nonlocal elliptic equation

$$
-Q+\Delta Q+\left(|x|^{-(N-\gamma)} *|Q|^{p}\right)|Q|^{p-2} Q=0 .
$$


The equation (2.123) is known as the nonlinear Choquard or Choquard-Pekar equation. A special case of (2.123) when $N=3, p=2$, and $\gamma=2$,

$$
\Delta Q-Q+\left(|x|^{-1} *|Q|^{2}\right) Q=0
$$

appeared back in 1954 in the work of S. I. Pekar [Pek54] describing the quantum mechanics of a polaron at rest. Lieb in [Lie 77] mentions it in the context of the Hartree-Fock theory of plasma, pointing out that $\mathrm{P}$. Choquard proposed investigating minimization of the corresponding functional in 1976. In $1996 \mathrm{R}$. Penrose proposed equation (2.124) as a model of self-gravitating matter, in which quantum state reduction is understood as a gravitational phenomenon, see [MPT98].

The existence of positive solutions to (2.124) was first proved by Lieb [Lie77], see also Lions [Lio80], [Lio84a]. The general existence result of positive solutions along with the regularity and radial symmetry of solutions to (2.123) for $\frac{N+\gamma}{N}<p<\frac{N+\gamma}{N-2}$ with $0<\gamma<N$ was shown by Moroz and Van Schaftingen [MVS13] (see also a review by Moroz and Van Schaftingen [MVS17] and references therein).

The uniqueness proof ${ }^{1}$ for $p=2$ with $\gamma=2$ in dimension $N=3$ dates back to 1976-77 work of Lieb [Lie77] and later in 2009 was extended to the dimension $N=4$ by Krieger, Lenzmann and Raphaël in [KLR09]; the uniqueness in the pseudo-relativistic three dimensional version of (2.124) was established by Lenzmann [Len09]. We review the proof of uniqueness for any (reasonable) $N$ (and $p=2, \gamma=2$ ). For other cases of $\gamma$ and $p$, it is an intricate issue, and while several authors made attempts to obtain uniqueness, it is still an open question. A recent work [Xia16] shows uniqueness and nondegeneracy of the ground state for $p=2+\epsilon$, i.e., when $p$ is sufficiently close to 2

\footnotetext{
${ }^{1}$ In certain existing literature there seem to be a misconception about the uniqueness of the ground state even in the standard $(p=2)$ Hartree equation: statements such as "take the positive unique ground state solution $Q$ of the equation $\Delta Q-Q+\left(|x|^{-b} *|Q|^{2}\right) Q=0$ " are not justified for any $0<b<N$ as the uniqueness of the ground state is only proved when $b=N-2$, $2<N<6$, see Section 2.8.2.
} 
in dimension $N=3$ and $\gamma=2$ via perturbation methods. We note that the proof of uniqueness for the nonlinear elliptic equation with convolution (2.123) differs from the corresponding results for the NLS-type equations (e.g., with $|u|^{p-1} u$ type nonlinearity), for which it is given, for example, by Kwong [Kwo89] and Berestycki and Lions [BL83a][BL83b]. The proof in the Hartree case uses Newton's theorem for the convolution in (2.124) and linearity in $Q$ outside of the convolution $(p=2)$, see more on this in Section 2.8.2. In Chapters 3 and 4, we do not need the uniqueness, it suffices to use minimizing properties of the Weinstein-type functional and the value of the sharp constant in the Gagliardo-Nirenberg convolution type inequality via ground state solutions as that value will be unique. Thus, we denote by $Q$ any ground state solution of (2.123) and use such quantities as $M[Q],\|\nabla Q\|_{L^{2}}$ and $E[Q]$, which are uniquely obtained from the sharp constant. In Chapter 5, specifically Section 5.3 to prove the Theorem 5.3.2, we take a ground state solution $Q$.

\subsubsection{Properties of the ground state}

We would like to study how large the initial data can be taken to have the property of global existence. As in most focusing dispersive equations, there is typically a (sharp) threshold, which can be identified via the so-called ground state. However, one would need to know that such ground state solutions exist, whether they are unique (perhaps up to certain symmetries), and if ground state solutions can be obtained as minimizers of a certain functional (as it was originally done by Weinstein for the NLS equation in [Wei83]). Minimization will identify the value of the threshold via some sharp constants of inequalities, from which the functional is derived. We proceed along this route: we consider an appropriate interpolation inequality, set up a functional, minimize it and iden-

tify the sharp constant. One property that we do not know is if the minimizer is unique. 
Nevertheless, for the purposes of Chapters 3 and 4, it is sufficient to use the value of the sharp constant (for Chapter 5, Section 5.3, we choose a ground state solution $Q$ ).

We start with the Gagliardo-Nirenberg type inequality of convolution type. For brevity we denote

$$
Z(u)=\int_{\mathbb{R}^{N}}\left(|x|^{-(N-\gamma)} *|u|^{p}\right)|u|^{p} d x .
$$

Lemma 2.8.1 Suppose $p \geq 2$ and $0<\gamma<N$, so that $0 \leq s_{c}<1$. Then

$$
Z(u) \leq C_{G N}\|\nabla u\|_{L^{2}}^{N p-(N+\gamma)}\|u\|_{L^{2}}^{N+\gamma-(N-2) p}
$$

Moreover, the equality is attained on ground state solutions $Q$, which solve ${ }^{2}$

$$
-\left(\frac{N+\gamma}{2 p}-\frac{N-2}{2}\right) Q+\left(\frac{N}{2}-\frac{N+\gamma}{2 p}\right) \Delta Q+\left(|x|^{-(N-\gamma)} *|Q|^{p}\right)|Q|^{p-2} Q=0
$$

and the sharp constant for (2.126) is attained at (any ground state) $Q$, which may be expressed as $C_{G N}=\|Q\|_{L^{2}\left(\mathbb{R}^{N}\right)}^{-2(p-1)}$.

Remark 2.8.2 We note that ground state solutions $Q$ are positive, vanishing at infinity solutions, which are radial (modulo translations). These and other properties are investigated in [MVS13], see also early works on the Hartree case in $\mathbb{R}^{3}$ in [Lie77], [Lie83], [Lio80], [Lio84a], [Lio84b]. As we mentioned in the beginning of Section 2.8 the uniqueness is only known in the case $p=2, \gamma=2$ and $N \geq 3$ (also for $p=2+\epsilon, \gamma=2$ in dimension $N=3$ ).

Proof. We consider the Weinstein-type functional for functions $u \in H^{1}\left(\mathbb{R}^{N}\right) \backslash\{0\}$

$$
J(u)=\frac{\|u\|_{L^{2}}^{(N+\gamma)-(N-2) p}\|\nabla u\|_{L^{2}}^{N p-(N+\gamma)}}{Z(u)} .
$$

\footnotetext{
${ }^{2}$ In this equation we use the normalization for $Q$ as in Weinstein [Wei83] when $\|Q\|_{L^{2}}=$ $\|\nabla Q\|_{L^{2}}=Z(Q)$. Below we rescale $Q$ to have the elliptic equation with unit coefficients.
} 
We mention that since we are interested in minimizing the value of $J$, replacing $u$ with its symmetric decreasing rearrangement will decrease both the $L^{2}$ norm and the $H^{1}$ norm (by Pólya-Szegö inequalities, see [LL01, Section 7.17]). On the other hand, the symmetric decreasing rearrangement will increase the value of $Z(u)$ by Riesz's inequality (see, [LL01, Section 3.7]), and thus, also will decrease the value of $J$. Hence, we can consider only radially symmetric functions $u=u(r)$, which are radially non-increasing (this is up to translations).

We proceed as in Weinstein [Wei83] by defining

$$
\eta=\inf \left\{J(u): u \in H_{\text {rad }}^{1} \backslash\{0\}\right\}
$$

Since $J(u)>0$, there exists a minimizing sequence $\left\{u_{k}\right\}$ such that $\eta=\lim _{k \rightarrow \infty} J\left(u_{k}\right)<\infty$. Note that if we set $u_{\lambda, \mu}=\mu u(\lambda x)$, then $\left\|u_{\lambda, \mu}\right\|_{L^{2}}^{2}=\lambda^{-N} \mu^{2}\|u\|_{L^{2}}^{2} \quad$ and $\quad\left\|\nabla u_{\lambda, \mu}\right\|_{L^{2}}^{2}=$ $\lambda^{2-N} \mu^{2}\|\nabla u\|_{L^{2}}^{2}$. By choosing $\lambda_{k}=\left\|u_{k}\right\|_{L^{2}} /\left\|\nabla u_{k}\right\|_{L^{2}}$ and $\mu_{k}=\left\|u_{k}\right\|_{L^{2}}^{\frac{N}{2}-1} /\left\|\nabla u_{k}\right\|_{L^{2}}^{\frac{N}{2}}$, we obtain the sequence $\left\{u_{\lambda_{k}, \mu_{k}}\right\}$, denoting it also by $\left\{u_{k}\right\}$, with $\left\|\nabla u_{k}\right\|_{L^{2}}=\left\|u_{k}\right\|_{L^{2}}=1$. Thus, $\left\{u_{k}\right\}$ is a bounded non-negative sequence in $H^{1}$. Hence, there exists $u^{*} \in H^{1} \backslash$ $\{0\}$, radial, nonnegative and non-increasing, such that a subsequence of $\left\{u_{k}\right\}$ converges weakly in $H^{1}$ to $u^{*}$ with $\left\|u^{*}\right\|_{L^{2}} \leq 1$ and $\left\|\nabla u^{*}\right\|_{L^{2}} \leq 1$.

We next claim that $Z\left(u^{*}\right)=\lim _{k \rightarrow \infty} Z\left(u_{k}\right)$, which is justified as follows: since $\left\{u_{k}\right\}$ is uniformly bounded in $\dot{H}_{r a d}^{1}$, we have $u_{k} \rightarrow u^{*}$ in $L^{\frac{2 N p}{N+\gamma}}$ (note that $2<\frac{2 N p}{N+\gamma}<\frac{2 N}{N-2}$ ). Now evaluating the difference, we obtain

$$
\begin{aligned}
Z\left(u_{k}\right)-Z\left(u^{*}\right)= & \int_{\mathbb{R}^{N}}\left(|\cdot|^{-(N-\gamma)} *\left|u_{k}\right|^{p}\right)\left(\left|u_{k}\right|^{p}-\left|u^{*}\right|^{p}\right) d x \\
& +\int_{\mathbb{R}^{N}}\left(|\cdot|^{-(N-\gamma)} *\left(\left|u_{k}\right|^{p}-\left|u^{*}\right|^{p}\right)\right)\left|u^{*}\right|^{p} d x \\
\lesssim & \left\|u_{k}\right\|_{L^{\frac{2 N p}{N+\gamma}}}^{p}\left\|\left|u_{k}\right|^{p}-\left|u^{*}\right|^{p}\right\|_{L^{\frac{2 N}{N+\gamma}}}+\left\|\left|u_{k}\right|^{p}-\left|u^{*}\right|^{p}\right\|_{L^{\frac{2 N}{N+\gamma}}}\left\|u^{*}\right\|_{\frac{2 N p}{N+\gamma}}^{p} \underset{k \rightarrow \infty}{\longrightarrow} 0 .
\end{aligned}
$$

We can now conclude

$$
\eta \leq J\left(u^{*}\right) \leq \frac{1}{Z\left(u^{*}\right)}=\lim _{k \rightarrow \infty} J\left(u_{k}\right)=\eta
$$


This implies that $\left\|u^{*}\right\|_{L^{2}}=\left\|\nabla u^{*}\right\|_{L^{2}}=1$, and also $u_{k} \rightarrow u^{*}$ strongly in $H^{1}$. Therefore, $u^{*}$ is indeed a minimizer of $J$.

Next we note that a minimizer $u^{*}$ satisfies the Euler - Lagrange equation

$$
\left.\frac{d}{d \epsilon}\right|_{\epsilon=0} J\left(u^{*}+\epsilon h\right)=0 \quad \text { for all } h \in C_{0}^{\infty}
$$

which, with $\left\|u^{*}\right\|_{L}^{2}=1$ and $\left\|\nabla u^{*}\right\|_{L^{2}}=1$, can be written as

$$
-\left(\frac{N+\gamma}{2 p}-\frac{N-2}{2}\right) u^{*}+\left(\frac{N}{2}-\frac{N+\gamma}{2 p}\right) \Delta u^{*}+\eta\left(|x|^{-(N-\gamma)} *\left|u^{*}\right|^{p}\right)\left|u^{*}\right|^{p-1}=0
$$

With equality in (2.129), we have $C_{G N}=\frac{1}{\eta}=Z\left(u^{*}\right)$. Recall that $u^{*}$ is a positive, vanishing at infinity function, satisfying the above equation, thus, it is a ground state solution of (2.130) with the normalization $\left\|u^{*}\right\|_{L^{2}}=\left\|\nabla u^{*}\right\|_{L^{2}}=1$.

Setting $Q=\eta^{\frac{1}{2(p-1)}} u^{*}$, we obtain that $Q$ satisfies (2.127). With this rescaling, we have $\|Q\|_{L^{2}}^{2}=\|\nabla Q\|_{L^{2}}^{2}=Z(Q)=\eta^{\frac{1}{2(p-1)}}$, and the sharp constant $C_{G N}=\frac{1}{\eta} \equiv 1 /\|Q\|_{L^{2}}^{2(p-1)}$.

Note that $\eta$ is the infimum, it uniquely determines $C_{G N}$ or such a quantity as $\|Q\|_{L^{2}}$.

One can also use another approach to find $C_{G N}$ and compute Pohozhaev identities for the equation (2.127): first, multiplying (2.127) by $Q$ and integrating to obtain

$$
\left(\frac{N+\gamma}{2 p}-\frac{N-2}{2}\right)\|Q\|_{L^{2}}^{2}+\left(\frac{N}{2}-\frac{N+\gamma}{2 p}\right)\|\nabla Q\|_{L^{2}}^{2}=Z(Q)
$$

Secondly, multiplying (2.127) by $x \cdot \nabla Q$ and integrating, yields

$$
\frac{N}{2}\left(\frac{N+\gamma}{2 p}-\frac{N-2}{2}\right)\|Q\|_{L^{2}}^{2}+\frac{N-2}{2}\left(\frac{N}{2}-\frac{N+\gamma}{2 p}\right)\|\nabla Q\|_{L^{2}}^{2}=\frac{N+\gamma}{2 p} Z(Q)
$$

which also gives

$$
Z(Q)=\|Q\|_{L^{2}}^{2}=\|\nabla Q\|_{L^{2}}^{2}
$$

and substituting these values into (2.126), we obtain $\eta \equiv C_{G N, s h a r p}=\|Q\|_{L^{2}}^{-2(p-1)}$. 
Remark 2.8.3 As in [HRO7] and [HR08] for the nonlinear Schrödinger equation, we observe that the quantities

$$
\left\|u_{0}\right\|_{L^{2}\left(\mathbb{R}^{N}\right)}^{1-s_{c}}\left\|\nabla u_{0}\right\|_{L^{2}\left(\mathbb{R}^{N}\right)}^{s_{c}} \quad \text { and } \quad M\left[u_{0}\right]^{1-s_{c}} E\left[u_{0}\right]^{s_{c}}
$$

are also scale-invariant, recall $s_{c}=\frac{N}{2}-\frac{\gamma+2}{2(p-1)}$, in the generalized Hartree equation, and for $s_{c}>0$ with $\theta=\frac{1-s_{c}}{s_{c}}$ we define

- renormalized mass-energy: $\mathcal{M E}[u]=\frac{M[u]^{\theta} E[u]}{M[Q]^{\theta} E[Q]}$,

- renormalized gradient (dependent on $t): \mathcal{G}[u(t)]=\frac{\|u\|_{L^{2}\left(\mathbb{R}^{N}\right)}^{\theta}\|\nabla u(t)\|_{L^{2}\left(\mathbb{R}^{N}\right)}}{\|Q\|_{L^{2}\left(\mathbb{R}^{N}\right)}^{\theta}\|\nabla Q\|_{L^{2}\left(\mathbb{R}^{N}\right)}}, \quad$ and - renormalized momentum: $\mathcal{P}[u]=\frac{\|u\|_{L^{2}\left(\mathbb{R}^{N}\right)}^{\theta-1} P[u]}{\|Q\|_{L^{2}\left(\mathbb{R}^{N}\right)}^{\theta}\|\nabla Q\|_{L^{2}\left(\mathbb{R}^{N}\right)}}$.

Remark 2.8.4 It is convenient to rescale $Q$ as $Q(x)=\beta^{\frac{1}{2(p-1)}} \widetilde{Q}\left(\frac{\sqrt{\beta}}{\alpha} x\right)$, which gives the equation (2.123) (with all unit coefficients) for $Q$ instead of (2.127) for $\tilde{Q}$. Here, $\alpha^{2}=\frac{N(p-1)-\gamma}{2 p}$ and $\beta=\frac{N+\gamma-(N-2) p}{2 p}$. From now on we only use $\widetilde{Q}$ (denoting it again by Q), solving (2.123) and the sharp constant

$$
C_{G N}=\frac{2 p}{N(p-1)-\gamma}\left(\frac{N+\gamma-(N-2) p}{N(p-1)-\gamma}\right)^{\frac{N(p-1)-\gamma}{2}-1} \frac{1}{\|Q\|_{L^{2}}^{2(p-1)}} .
$$

For future reference we also compute,

$$
M[Q]^{\theta} E[Q]=\frac{s_{c}(p-1)}{2 s_{c}(p-1)+2}\|Q\|_{L^{2}}^{2 \theta}\|\nabla Q\|_{L^{2}}^{2}
$$

and

$$
\|Q\|_{L^{2}}^{1-s_{c}}\|\nabla Q\|_{L^{2}}^{s_{c}}=\left(\frac{p\left(C_{G N}\right)^{-1}}{s_{c}(p-1)+1}\right)^{\frac{1}{2(p-1)}}
$$

\subsubsection{Uniqueness of the ground state for $p=2, \gamma=2$}

Here, for completeness we review the uniqueness of the ground state argument to the nonlocal elliptic (Choquard) equation

$$
-Q+\Delta Q+\left(|x|^{-(N-2)} *|Q|^{2}\right) Q=0
$$


since the argument is different from that for a local (such as in semi-linear NLS) nonlinearity. As it was mentioned in the introduction, for $N=3$ the uniqueness was proved by Lieb [Lie83], a slightly different proof using the comparison argument is given in Lenzmann [Len09]; for $N=4$ it is proved in Krieger, Lenzmann and Raphaël [KLR09] via a combination of the above. We also follow the above arguments in $3 \mathrm{~d}$ and generalize it for $2<N<6$. The stationary equation (2.137) appears in the context of the Hartree equation only in dimensions $2<N<6$ : in dimension $N=6$ the Hartree equation is energy-critical, and thus, the corresponding elliptic equation will be different (lacking the linear term). While most of the arguments below work for dimensions 6 and higher, the equation (2.137) is only needed for $N<6$.

Theorem 2.8.5 Let $2<N<6$. The equation (2.137) has the unique positive, radial solution $Q$ in $H^{1}\left(\mathbb{R}^{N}\right)$.

The proof uses the following representation of the Newton's potential, which can be found in the textbook [LL01, Theorem 9.7].

Lemma 2.8.6 If $f$ is a radial $C^{\infty}$ function on $\mathbb{R}^{N}$, then

$$
-\left(\frac{1}{|x|^{N-2}} * f\right)(r)=\int_{0}^{r} K(r, s) f(s) d s-\left|\mathbb{S}^{N-1}\right| \int_{0}^{\infty} f(s) s d s
$$

where

$$
K(r, s)=\left|\mathbb{S}^{N-1}\right|\left(1-\left(\frac{s}{r}\right)^{N-2}\right) s \geq 0 \text { for } r \geq s
$$

Proof. (of Theorem 2.8.5) Using Lemma 2.8.6 for a radial $Q \in H^{1}\left(\mathbb{R}^{N}\right)$, we rewrite (2.137) as

$$
-Q^{\prime \prime}-\frac{N-1}{r} Q^{\prime}+\left(\int_{0}^{r} K(r, s) Q(s)^{2} d s\right) Q=a Q
$$


where $a=-1+\left|\mathbb{S}^{N-1}\right|\left(\int_{0}^{\infty} Q(s)^{2} s d s\right)>0$. Using the rescaling $Q(r) \mapsto a^{-1} Q\left(a^{-1 / 2} r\right)$, we obtain the version of (2.140) with $a=1$, namely,

$$
\left(-\frac{d^{2}}{d r^{2}}-\frac{N-1}{r} \frac{d}{d r}+U_{Q}(r)\right) Q(r)=Q(r)
$$

where

$$
U_{Q}(r)=\left(\int_{0}^{r} K(r, s) Q(s)^{2} d s\right) .
$$

Suppose $Q_{1}(r)$ and $Q_{2}(r)$ are two positive radial solutions of (2.142) in $H^{1}\left(\mathbb{R}^{N}\right)$ such that $Q_{1} \neq Q_{2}$ that solve the IVP

$$
\left\{\begin{array}{l}
Q^{\prime \prime}(r)+\frac{N-1}{r} Q^{\prime}(r)+Q(r)-U_{Q}(r) Q(r)=0 \\
Q(0)=Q_{0}, \quad Q^{\prime}(0)=0
\end{array}\right.
$$

The Volterra integral theory (for example, see [YRZ19, Lemmas 2.4-2.6 and Theorem 2.1]) guarantees existence and uniqueness of a local $C^{2}$ solution to the above initial-value problem for a given $Q(0)$ (note that $U_{Q}(r)$ is bounded, see details below). Therefore, if $Q_{1} \neq Q_{2}$, then $Q_{1}(0) \neq Q_{2}(0)$. Without loss of generality, assume that $Q_{1}(0)>Q_{2}(0)$, and by continuity we have $Q_{1}(r)>Q_{2}(r)$ on some interval $r>0$. We now prove that $Q_{1}(r)>Q_{2}(r)$ for all $r \geq 0$. Multiplying the equation (2.143) written for $Q_{1}$ with $Q_{2}$ and subtracting the same with indices reversed, we get

$$
Q_{1}^{\prime \prime} Q_{2}-Q_{1} Q_{2}^{\prime \prime}=-\frac{N-1}{r}\left(Q_{1}^{\prime} Q_{2}-Q_{1} Q_{2}^{\prime}\right)+\left(U_{Q_{1}}-U_{Q_{2}}\right) Q_{1} Q_{2}
$$

or, equivalently (multiplying by $r^{N-1}$ ),

$$
\frac{d}{d r}\left(r^{N-1}\left(Q_{1}^{\prime} Q_{2}-Q_{1} Q_{2}^{\prime}\right)\right)=r^{N-1}\left(U_{Q_{1}}-U_{Q_{2}}\right) Q_{1} Q_{2}
$$

Integrating (2.144), we obtain

$$
r^{N-1}\left(Q_{1}^{\prime}(r) Q_{2}(r)-Q_{1}(r) Q_{2}^{\prime}(r)\right)=\int_{0}^{r} s^{N-1}\left(U_{Q_{1}}(s)-U_{Q_{2}}(s)\right) Q_{1}(s) Q_{2}(s) d s .
$$


Suppose that $Q_{1}(r)$ intersects $Q_{2}(r)$ at $r_{1}>0$ for the first time. Then, the left-hand side of (2.145) at $r_{1}$ is non-positive because of monotonicity and decay of both $Q_{1}$ and $Q_{2}$ :

$$
r_{1}^{N-1} Q_{1}\left(r_{1}\right)\left(Q_{1}^{\prime}\left(r_{1}\right)-Q_{2}^{\prime}\left(r_{1}\right)\right) \leq 0
$$

however, the right-hand side of (2.145) satisfies

$$
\int_{0}^{r_{1}} s^{N-1} Q_{1}(s) Q_{2}(s)\left(U_{Q_{1}}(s)-U_{Q_{2}}(s)\right) d s>0,
$$

since both $Q_{1}(r), Q_{2}(r)>0$ along with $U_{Q_{1}}(r)>U_{Q_{2}}(r)$ for $0<r<r_{1}$. This leads to a contradiction, thus, $Q_{1}(r)$ and $Q_{2}(r)$ do not intersect, which implies that $Q_{1}(r)>$ $Q_{2}(r)$ must hold for all $r \geq 0$. Now we show that this fact also leads to a contradiction. Consider the two Schrödinger operators $H_{i}=-\Delta+U_{Q_{i}}, i=1,2$, with $U_{Q_{i}}(r)=$ $\int_{0}^{r}\left(1-\left(\frac{s}{r}\right)^{N-2}\right) s Q_{i}^{2}(s) d s$. Recalling that a ground state $Q_{i}(r)$ asymptotically behaves as $r^{-\frac{N-1}{2}+\varepsilon} e^{-|x|}$ (in the case $p=2$ ), it is easy to observe that $U_{Q_{i}}$ is not only bounded, but increases to a horizontal asymptote $y=c_{N}=$ const. Hence, we can apply the classical Schrödinger operator theory (for example, [RS78, Chapter 13]) to show that both equations $H_{i} Q=Q, i=1,2$, have the unique positive ground state solution, respectively denoted by $Q_{i}$ (with the eigenvalue 1 as we rescaled the equation in (2.141)). This implies that $\left\langle H_{i} f, f\right\rangle \geq\|f\|_{L^{2}}$ for any $H^{1}$ function $f$ with equality holding on a multiple of $Q_{i}$, that is, when $f=c_{i} Q_{i}, i=1,2$, respectively. Now, since $H_{2}=H_{1}-\left(U_{Q_{1}}-U_{Q_{2}}\right)$, we obtain

$$
\left\|Q_{1}\right\|_{L^{2}}^{2} \leq\left\langle H_{2} Q_{1}, Q_{1}\right\rangle=\left\langle H_{1} Q_{1}, Q_{1}\right\rangle-\left\langle\left(U_{Q_{1}}-U_{Q_{2}}\right) Q_{1}, Q_{1}\right\rangle=\left\|Q_{1}\right\|_{L^{2}}^{2}-\delta,
$$

since $U_{Q_{1}}>U_{Q_{2}}$, yielding a contradiction. This implies that (2.140) (and hence (2.137)) can not have two distinct radial positive $H^{1}$ solutions. 


\section{CHAPTER 3}

\section{GLOBAL BEHAVIOR OF SOLUTIONS TO THE FOCUSING GENERALIZED HARTREE EQUATION}

The journey of thousand miles begins with one step. (Lao Tzu)

\subsection{Background and statement of main result}

A global solution $u(t)$ to (1.1) is said to scatter in $H^{s}\left(\mathbb{R}^{N}\right)$ as $t \rightarrow+\infty$, if there exists $u^{+} \in H^{s}\left(\mathbb{R}^{N}\right)$ such that

$$
\lim _{t \rightarrow+\infty}\left\|u(t)-e^{i t \Delta} u^{+}\right\|_{H^{s}\left(\mathbb{R}^{N}\right)}=0 .
$$

There are a number of early works on global existence, asymptotic behavior of solutions and scattering theory for the standard Hartree equation (1.2). Studies trace back to Ginibre and Velo [GV80], where the local well-posedness is established and the authors also prove asymptotic completeness for a repulsive potential. Hayashi and Tsutsumi [HT87] continued to develop the scattering theory and obtain the asymptotic completeness of wave operators in $H^{m} \cap L^{p}\left(|x|^{\beta} d x\right)$. We refer the reader to Ginibre and Ozawa [GO93] for results in the case of the convolution with $|x|^{-1}$, or $N-\gamma=1$, for $N \geq 2$; to Ginibre and Velo [GV00c] for $2<N-\gamma<\min (4, N)$ when $N \geq 3$. In a sequence of papers [GV00a]-[GV01] Ginibre and Velo considered the time-dependent potential $\pm t^{\mu-\gamma}|x|^{-\mu}$ and studied the asymptotic dynamics and scattering (for any data in the repulsive case or small data otherwise) first when the convolution power is $\frac{1}{2}<N-\gamma<1$ in [GV00a], and then in the whole range $0<N-\gamma \leq 1$ in [GV00b]. These two papers are written in the framework of Sobolev spaces with the assumption $\mu \leq N-2(N \geq 3)$. In [GV01] the Hartree was treated in Gevrey spaces, which made it possible to cover the whole range $0<\mu \leq N$ with an arbitrary space dimensions $N \geq 1$. Hayashi, Naumkin and Ozawa 
studied the Hartree equation with $N-\gamma=1(N \geq 2)$ and initial data in a weighted Sobolev space $H^{0, \alpha} \cap H^{\alpha, 0}$ with $\frac{1}{2}<\alpha<\frac{N}{2}$ in [HNO98].

Our aim is to understand global behavior and dynamics of solutions to the generalized Hartree (1.1), in particular, how the nonlocal potential with the flexibility of different powers in nonlinearity may influence the global behavior and dynamics of solutions either with infinite or finite time of existence. We are also curious whether solutions behave in a manner similar to local potentials as, for example, in the standard semilinear Schrödinger equation with $|u|^{p-1} u$ nonlinearity, or if nonlocality creates significant differences in solutions behavior. In addition, we want to develop methods needed to study such solutions.

Here we describe the global behavior of solutions to (1.1) with $H^{1}$ initial data in the inter-critical regime $\left(0<s_{c}<1\right)$, provided that $p \geq 2$, that is,

$$
1+\frac{\gamma+2}{N}<p<1+\frac{\gamma+2}{N-2}, 0<\gamma<N \text { and } p \geq 2
$$

with the appropriate modification of the right-hand side for $N=1,2(p<\infty)$. (As a byproduct, we also obtain small data theory in the energy-subcritical setting, $s_{c}<1$.) Since we also have the local well-posedness at the critical regularity $\dot{H}^{s_{c}}, s_{c} \geq 0$, which is not necessarily conserved (or even bounded in the focusing case), see Chapter 2, Section 2.6, Propositions 2.6.3 and 2.6.4. Thus, similar to $H^{1}$ case we extend the local existence to the global existence for small $\dot{H}^{s_{c}}$ data. We then establish a dichotomy for global vs. finite time solutions (with $H^{1}$ initial data) under the mass-energy threshold and show $H^{1}$ scattering for the global solutions, following the concentration-compactness approach of Kenig and Merle [KM06], and divergence along a time sequence for nonradial infinite variance data (also via concentration-compactness method). This is in the spirit of [HR08], [DHR08], [HR10] and further generalizations [FXC11], [AN13], [Gue14], for the focusing nonlinear Schrödinger equation. 
We now state the main result of the present chapter about solutions behavior under the mass-energy threshold. We consider (1.1) with given $N, \gamma$, and $p \geq 2$ satisfying (3.1) so that $s_{c}$ defined by $(2.1)$ is $0<s_{c}<1$. Also, recall the definition of $\mathcal{M E}, \mathcal{G}, \mathcal{P}$ from Remark 2.8.3. We then first consider solutions with zero momentum.

Theorem 3.1.1 (Zero momentum) Let $u_{0} \in H^{1}\left(\mathbb{R}^{N}\right)$ with $P\left[u_{0}\right]=0$ and let $u(t)$ be the corresponding solution to (1.1) with the maximal time interval of existence $\left(T_{*}, T^{*}\right)$. Suppose that $\mathcal{M E}\left[u_{0}\right]<1$.

1. If $\mathcal{G}\left[u_{0}\right]<1$, then

(a) the solution exists globally in time with $\mathcal{G}[u(t)]<1$ for all $t \in \mathbb{R}$, and

(b) $u(t)$ scatters in $H^{1}$, in other words, there exists $u_{ \pm} \in H^{1}$ such that

$$
\lim _{t \rightarrow \pm \infty}\left\|u(t)-e^{i t \Delta} u_{ \pm}\right\|_{H^{1}\left(\mathbb{R}^{N}\right)}=0
$$

2. If $\mathcal{G}\left[u_{0}\right]>1$, then $\mathcal{G}[u(t)]>1$ for all $t \in\left(T_{*}, T^{*}\right)$. Moreover, if

(a) $|x| u_{0} \in L^{2}\left(\mathbb{R}^{N}\right)$ (finite variance) or $u_{0}$ is radial, then the solution blows up in finite time,

(b) $u_{0}$ is of infinite variance and nonradial, then either the solution blows up in finite time or there exits a sequence of times $t_{n} \rightarrow+\infty$ (or $\left.t_{n} \rightarrow-\infty\right)$ such that $\left\|\nabla u\left(t_{n}\right)\right\|_{L^{2}\left(\mathbb{R}^{N}\right)} \rightarrow \infty$.

The general case when $P\left[u_{0}\right] \neq 0$ is given by the following

Theorem 3.1.2 Let $u_{0} \in H^{1}\left(\mathbb{R}^{N}\right)$ and $u(t)$ be the corresponding solution to (1.1) with the maximal time interval of existence $\left(T_{*}, T^{*}\right)$. Assume that

$$
\mathcal{M E}\left[u_{0}\right]-\frac{N(p-1)-\gamma}{N(p-1)-\gamma-2} \mathcal{P}\left[u_{0}\right]^{2}<1
$$


1. If

$$
\mathcal{G}\left[u_{0}\right]^{2}-\mathcal{P}\left[u_{0}\right]^{2}<1
$$

then

(a) the solution exists globally in time with $\mathcal{G}[u(t)]^{2}-\mathcal{P}\left[u_{0}\right]^{2}<1$ for all $t \in \mathbb{R}$, and

(b) $u(t)$ scatters in $H^{1}$, i.e., there exists $u_{ \pm} \in H^{1}$ such that

$$
\lim _{t \rightarrow \pm \infty}\left\|u(t)-e^{i t \Delta} u_{ \pm}\right\|_{H^{1}\left(\mathbb{R}^{N}\right)}=0
$$

2. If

$$
\mathcal{G}\left[u_{0}\right]^{2}-\mathcal{P}\left[u_{0}\right]^{2}>1
$$

then $\mathcal{G}[u(t)]^{2}-\mathcal{P}\left[u_{0}\right]^{2}>1$ for all $t \in\left(T_{*}, T^{*}\right)$. Moreover, if

(a) $|x| u_{0} \in L^{2}\left(\mathbb{R}^{N}\right)$ or $u_{0}$ is radial, then the solution blows up in finite time,

(b) $u_{0}$ is of infinite variance and nonradial, then either the solution blows up in finite time or there exits a sequence of times $t_{n} \rightarrow+\infty\left(\right.$ or $\left.t_{n} \rightarrow-\infty\right)$ such that $\left\|\nabla u\left(t_{n}\right)\right\|_{L^{2}\left(\mathbb{R}^{N}\right)} \rightarrow \infty$.

While we follow the strategy of [HR08], [Gue14], [DHR08] and [HR10], the fundamental difference is in the nonlocal potential, and control of convolution terms arising in various steps of our work. For example, to obtain local well-posedness and small data theory in $H^{1}$ we do not get the contraction automatically as the difference produces extra terms that result from convolution. We use Lemma 2.3.1 to estimate the inhomogeneous term in Duhamel's formula via Strichartz estimate in Proposition 3.2.1, Proposition 3.2.2, Theorem 3.2.4, Theorem 3.2.5 and in Theorem 3.4.3 (Claim 3.4.5). Also note that to control the potential energy in Proposition 3.4.2 and in Lemma 2.8.1, we rely on $L_{x}^{\frac{2 N p}{N+\gamma}}$ 
norm (using the assumption that $s_{c}<1$ ) along with the Lemma 2.3.1. Moreover, the local virial identity (3.100), (3.101), (3.102) in Theorem 3.3.1 and Theorem 3.5.1 has some extra terms involving convolution which demands a careful study and application of convolution properties, Lemma 2.3.1 and Lemma 2.3.3.

\subsection{Small data theory}

We investigate the global existence of small data in $H^{1}$ and $\dot{H}^{s_{c}}$ (as we now have the local well-posedness in both cases) and $H^{1}$ scattering of global solutions with initial data in $H^{1}$. At the end of section 3.2 we also include the long-time perturbation argument. This may appear to be standard, however, we give a careful and detailed proof demonstrating how we tackle the nonlocal potential term. In this section we consider the integral equation (2.16) with $u_{0} \in H^{1}\left(\mathbb{R}^{N}\right)$ and $0<\gamma<N$ with $p \geq 2$ satisfying

$$
\begin{cases}1+\frac{\gamma+2}{N} \leq p<1+\frac{\gamma+2}{N-2}, & \text { if } N \geq 3 \\ 1+\frac{\gamma+2}{N} \leq p<\infty, & \text { if } N=1,2 .\end{cases}
$$

In the energy-subcritical case $\left(s_{c}<1\right)$ it is possible to obtain $\dot{H}^{s_{c}}$ small data theory, replacing the right-hand side bound below in (3.12) with the $\dot{H}^{s_{c}}$ norm (instead of $H^{1}$ norm) as done in [HR07], [Gue14]. This requires fractional derivatives, introduction of different Strichartz pairs and considering different cases of smoothness, depending on $p$ and $s_{c}$; see Proposition 3.2.2. We first take $H^{1}$ small data, and thus, we consider the bound on the right-hand side of (3.12) by the full $H^{1}$ norm. Also note that while the norm on the left-hand side of (3.12) is at the $H^{s_{c}}$ level, it can be replaced with the norms at the $H^{1}$ level, that is, by $\|u\|_{S\left(L^{2}\right)}+\|\nabla u\|_{S\left(L^{2}\right)}$ (by the interpolation and then separating it into the sum by Peter-Paul), which we will do in the proof. For brevity, we chose to state (3.12) at the $H^{s_{c}}$ level. Furthermore, we note that Proposition 3.2.1 also holds true for the 
$L^{2}$-critical equations $\left(s_{c}=0\right)$ with $u_{0} \in H^{1}\left(\mathbb{R}^{N}\right)$ and (3.12) reduces just to one condition (3.11).

In the rest of the Chapter 3, for given $N, p, \gamma$, and hence, a fixed $0<s_{c}<1$, we use the following $L^{2}$-admissible pairs :

$$
\left(q_{1}, r_{1}\right)=\left(\frac{2 p}{1+s_{c}(p-1)}, \frac{2 N p}{N+\gamma}\right)
$$

and

$$
\left(q_{2}, r_{2}\right)=\left(\frac{2 p}{1-s_{c}}, \frac{2 N p}{N+\gamma+2 p s_{c}}\right) .
$$

Observe that $s_{c}<1$ implies $\frac{2 p}{1+s_{c}(p-1)}>2$. As an $L^{2}$-dual admissible pair we take

$$
\left(q_{1}^{\prime}, r_{1}^{\prime}\right)=\left(\frac{2 p}{2 p-1-s_{c}(p-1)}, \frac{2 N p}{2 N p-N-\gamma}\right)
$$

The specific $\dot{H}^{s_{c}}$-admissible pair we use is

$$
\left(q_{2}, r_{1}\right)=\left(\frac{2 p}{1-s_{c}}, \frac{2 N p}{N+\gamma}\right)
$$

and the $\dot{H}^{-s_{c}}$ dual admissible pair is given by

$$
\left(q_{3}^{\prime}, r_{1}^{\prime}\right)=\left(\frac{2 p}{(2 p-1)\left(1-s_{c}\right)}, \frac{2 N p}{2 N p-N-\gamma}\right) .
$$

Note that $\left(q_{3}, r_{1}\right)=\left(\frac{2 p}{1+s_{c}(2 p-1)}, \frac{2 N p}{N+\gamma}\right)$ is an $\dot{H}^{-s_{c}}$ admissible pair. One can verify that $s_{c}<1$ imply both $\frac{2 p}{1+s_{c}(2 p-1)}>\frac{2}{1+s_{c}}$ and $\frac{2 p}{1+s_{c}(2 p-1)}<\frac{1}{s_{c}}$, thus, confirming to be in the range of (2.11). We point out that all the pairs defined above satisfy both Lemma 2.4.6 along with the respective ranges defined in (2.10) and (2.11). In what follows (at least for $0<s_{c}<1$ ), we will work with the pairs defined above. However, one can take any pair $(q, r)$ satisfying (2.10) and (2.11), and thus, we utilize the notation introduced in Section 2.4 (after Lemma 2.4.6) to indicate that it is possible to cover the entire range by taking an appropriate pair from (2.10) and (2.11). 
Proposition 3.2.1 (Small data theory in $H^{1}$ ) Let $p \geq 2$ satisfy (3.5) with $0<\gamma<N$ and $u_{0} \in H^{1}\left(\mathbb{R}^{N}\right)$. Suppose $\left\|u_{0}\right\|_{H^{1}} \leq A$. There exists $\delta=\delta(A)>0$ such that if $\left\|e^{i t \Delta} u_{0}\right\|_{S\left(\dot{H}^{\left.s_{c}\right)}\right.} \leq \delta$, then there exists a unique global solution $u$ of $(1.1)$ in $H^{1}\left(\mathbb{R}^{N}\right)$ such that

$$
\|u\|_{S\left(\dot{H}^{s_{c}}\right)} \leq 2\left\|e^{i t \Delta} u_{0}\right\|_{S\left(\dot{H}^{s_{c}}\right)}
$$

and

$$
\left\||\nabla|^{s_{c}} u\right\|_{S\left(L^{2}\right)} \leq 2 c\left\|u_{0}\right\|_{H^{1}}
$$

where c depends on constants from the Gagliardo-Nirenberg interpolation estimate and the Strichartz inequality.

Proof. First, note that by Lemma 2.4.7 and Sobolev estimates, we can track the dependence of $\delta$ on $A$ (if needed, splitting the time interval). Next, denote

$$
B=\left\{u:\|u\|_{S\left(\dot{H}^{s_{c}}\right)} \leq 2\left\|e^{i t \Delta} u_{0}\right\|_{S\left(\dot{H}^{s^{c}}\right)} \text { and }\left.\|\| \nabla\right|^{s_{c}} u\left\|_{S\left(L^{2}\right)} \leq 2 c\right\| u_{0} \|_{H^{1}}\right\}
$$

and define

$$
\Phi_{u_{0}}(u)=e^{i t \Delta} u_{0}+i \int_{0}^{t} e^{i\left(t-t^{\prime}\right) \Delta} F\left(u\left(t^{\prime}\right)\right) d t^{\prime},
$$

where $F(u)=\left(|x|^{-(N-\gamma)} *|u|^{p}\right)|u|^{p-2} u$. Applying the triangle inequality and Lemmas 2.4.6 to (3.13), we obtain

$$
\left\|\Phi_{u_{0}}(u)\right\|_{S\left(\dot{H}^{s_{c}}\right)} \leq\left\|e^{i t \Delta} u_{0}\right\|_{S\left(\dot{H}^{s_{c}}\right)}+c\|F(u)\|_{S^{\prime}\left(\dot{H}^{-s_{c}}\right)}
$$

We will use the pair $\left(q_{3}^{\prime}, r_{1}^{\prime}\right)$ (defined in (3.10)) on the right-hand side of the above inequality. Using the Hölder's inequality yields

$$
\|F(u)\|_{L_{t}^{q_{3}^{\prime}} L_{x}^{r_{1}^{\prime}}} \leq\left\||x|^{-(N-\gamma)} *|u|^{p}\right\|_{L_{t}^{1-s_{c}}} L_{x}^{\frac{2 N}{N-\gamma}}\|u\|_{L_{t}^{q_{2}} L_{x}^{r_{1}}}^{p-1}
$$

Applying Lemma 2.3.1 for $N>\gamma$, we estimate

$$
\left\||x|^{-(N-\gamma)} *|u|^{p}\right\|_{L_{t}^{\frac{2}{1-s_{c}}}} \frac{2 N}{L_{x}^{N-\gamma}} \leq c_{N, p, \gamma}\|u\|_{L_{t}^{q_{2}} L_{x}^{r_{1}}}^{p}
$$


Using (3.14), we can write the estimate (3.36) as

$$
\|F(u)\|_{S^{\prime}\left(\dot{H}^{-s_{c}}\right)} \leq c_{N, p, \gamma}\|u\|_{S\left(\dot{H}^{s_{c}}\right)}^{p}\|u\|_{S\left(\dot{H}^{s_{c}}\right)}^{p-1} .
$$

Thus, for $u \in B$, (3.15) gives

$$
\|F(u)\|_{S^{\prime}\left(\dot{H}^{-s_{c}}\right)} \leq c_{N, p, \gamma} 2^{2 p-1}\left\|e^{i t \Delta} u_{0}\right\|_{S\left(\dot{H}^{s_{c}}\right)}^{2 p-1} .
$$

Inserting (3.16) into (3.35) and redefining the constant $c_{N, p, \gamma} c=: c_{1}$, we have

$$
\left\|\Phi_{u_{0}}(u)\right\|_{S\left(\dot{H}^{s_{c}}\right)} \leq\left\|e^{i t \Delta} u_{0}\right\|_{S\left(\dot{H}^{s_{c}}\right)}\left(1+c_{1} 2^{2 p-1}\left\|e^{i t \Delta} u_{0}\right\|_{S\left(\dot{H}^{s_{c}}\right)}^{2(p-1)}\right)
$$

and thus, we need

$$
c_{1} 2^{2 p-1}\left\|e^{i t \Delta} u_{0}\right\|_{S\left(\dot{H}^{s_{c}}\right)}^{2(p-1)} \leq 1
$$

To estimate $\left\||\nabla|^{s_{c}} \Phi_{u_{0}}(u)\right\|_{S\left(L^{2}\right)}$, we recall the Gagliardo-Nirenberg interpolation inequality

$$
\left\||\nabla|^{s_{c}} v\right\|_{L^{2}} \leq c_{G N}\|\nabla v\|_{L^{2}}^{s_{c}}\|v\|_{L^{2}}^{1-s_{c}}
$$

and taking $v=\Phi_{u_{0}}(u)$, we bound the $L^{2}$ and $\dot{H}^{1}$ norms as

$$
\left\|\Phi_{u_{0}}(u)\right\|_{S\left(L^{2}\right)} \leq c\left\|u_{0}\right\|_{L^{2}}+c\|F(u)\|_{S^{\prime}\left(L^{2}\right)} .
$$

From Hölder's inequality, we get

$$
\|F(u)\|_{L_{t}^{q_{1}^{\prime}} L_{x}^{r_{1}^{\prime}}} \leq\left\||x|^{-(N-\gamma)} *|u|^{p}\right\|_{L_{t}^{2} L_{x}^{N-\gamma}}\|u\|_{L_{t}^{q_{2}} L_{x}^{r_{1}}}^{p} .
$$

We estimate the convolution term in (3.18) again by Lemma 2.3.1 for $N>\gamma$ and then use Hölder's to obtain

$$
\begin{aligned}
\|F(u)\|_{S^{\prime}\left(L^{2}\right)} & \leq c_{N, p, \gamma}\left\||u|^{p}\right\|_{L_{t}^{2} L_{x}^{\frac{2 N}{N+\gamma}}}\|u\|_{L_{t}^{q_{2}} L_{x}^{r_{1}}}^{p-1} \\
& \leq c_{N, p, \gamma}\|u\|_{L_{t}^{q_{2}} L_{x}^{r_{1}}}^{p-1}\|u\|_{L_{t}^{q_{1}} L_{x}^{r_{1}}}\|u\|_{L_{t}^{q_{2}} L_{x}^{r_{1}}}^{p-1} \\
& \leq c_{N, p, \gamma}\|u\|_{S\left(\dot{H}^{s_{c}}\right)}^{2(p-1)}\|u\|_{S\left(L^{2}\right)} .
\end{aligned}
$$


Using (2.12) (and triangle inequality) in (3.13), we get

$$
\left\|\nabla \Phi_{u_{0}}(u)\right\|_{S\left(L^{2}\right)} \leq c\left\|\nabla u_{0}\right\|_{L^{2}}+c\|\nabla F(u)\|_{S^{\prime}\left(L^{2}\right)},
$$

where the nonlinear term is estimated as

$$
\begin{aligned}
& \|\nabla F(u)\|_{L_{t}^{q_{1}^{\prime}} L_{x}^{r_{1}^{\prime}}} \leq\left\||x|^{-(N-\gamma)} *|u|^{p}\right\|_{L_{t}^{1-s_{c}}} \frac{2 N}{L_{x}^{N-\gamma}}\left\|\nabla\left(|u|^{p-2} u\right)\right\|_{L_{t}^{\frac{2 p}{p-\left(1-s_{c}\right)}} L_{x}^{\frac{2 N p}{(N+\gamma)(p-1)}}} \\
& +\left\||x|^{-(N-\gamma)} * \nabla\left(|u|^{p}\right)\right\|_{L_{t}^{2} L_{x}^{2 N}-\gamma}\|u\|_{L_{t}^{q_{2}} L_{x}^{r_{1}}}^{p-1} \\
& \leq c_{N, p, \gamma}\|u\|_{L_{t}^{q_{2}} L_{x}^{r_{1}}}^{2(p-1)}\|\nabla u\|_{L_{t}^{q_{1}} L_{x}^{r_{1}}}+c_{N, p, \gamma}\left\|\nabla\left(|u|^{p}\right)\right\|_{L_{t}^{2} L_{x}^{\frac{2 N}{N+\gamma}}}\|u\|_{L_{t}^{q_{2}} L_{x}^{r_{1}}}^{p-1} \\
& \leq c_{N, p, \gamma}\|u\|_{L_{t}^{q_{2}} L_{x}^{r_{1}}}^{2(p-1)}\|\nabla u\|_{L_{t}^{q_{1}} L_{x}^{r_{1}}}+c_{N, p, \gamma}\|\nabla u\|_{L_{t}^{q_{1}} L_{x}^{r_{1}}\|u\|_{L_{t}^{q_{2}} L_{x}^{r_{1}}}^{2(p-1)}} \\
& \leq 2 c_{N, p, \gamma}\|u\|_{S\left(\dot{H}^{s^{c}}\right)}^{2(p-1)}\|\nabla u\|_{S\left(L^{2}\right)} \text {. }
\end{aligned}
$$

Combining (3.17) and (3.20), and applying (3.19) and (3.21), we obtain

$$
\begin{aligned}
\left\|\Phi_{u_{0}}(u)\right\|_{S\left(L^{2}\right)}+\left\|\nabla \Phi_{u_{0}}(u)\right\|_{S\left(L^{2}\right)} \leq & c\left(\left\|u_{0}\right\|_{L^{2}}+\left\|\nabla u_{0}\right\|_{L^{2}}\right) \\
& +c_{1}\|u\|_{S\left(\dot{H}^{\left.s_{c}\right)}\right.}^{2(p-1)}\left(\|u\|_{S\left(L^{2}\right)}+\|\nabla u\|_{S\left(L^{2}\right)}\right) \\
\leq & c\left\|u_{0}\right\|_{H^{1}}+2^{2 p-1} c_{1} c\left\|e^{i t \Delta} u_{0}\right\|_{S\left(\dot{H}^{\left.s_{c}\right)}\right.}^{2(p-1)}\left\|u_{0}\right\|_{H^{1}} \\
\leq & c\left\|u_{0}\right\|_{H^{1}}\left(1+2^{2 p-1} c_{1}\left\|e^{i t \Delta} u_{0}\right\|_{S\left(\dot{H}^{s_{c}}\right)}^{2(p-1)}\right),
\end{aligned}
$$

where $c_{N, p, \gamma} c=: c_{1}$. Now, if we take

$$
2^{2 p-1} c_{1}\left\|e^{i t \Delta} u_{0}\right\|_{S\left(\dot{H}^{s_{c}}\right)}^{2(p-1)} \leq 1
$$

and recalling that $\left\|e^{i t \Delta} u_{0}\right\|_{S\left(\dot{H}^{s_{c}}\right)}<\delta$, then (3.22) would give the required bound for the space $B: 2 c\left\|u_{0}\right\|_{H^{1}}$. Hence, choosing $\delta<\delta_{0}=\frac{1}{2} \sqrt[2(p-1)]{\frac{1}{2 c_{1}}}$ implies that $\Phi_{u_{0}} \in B$. Now we show that $\Phi_{u_{0}}(u)$ is a contraction on $B$ with the metric

$$
d(u, v)=\|u-v\|_{S\left(L^{2}\right)}+\|\nabla(u-v)\|_{S\left(L^{2}\right)}+\|u-v\|_{S\left(\dot{H}^{s_{c}}\right)} .
$$


(The last norm is included for convenience.) For $u, v \in B$, by Lemma 2.4.6, we obtain

$$
\left\|\Phi_{u_{0}}(u)-\Phi_{u_{0}}(v)\right\|_{S\left(\dot{H}^{\left.s_{c}\right)}\right.} \leq c\|F(u)-F(v)\|_{S^{\prime}\left(\dot{H}^{\left.-s_{c}\right)}\right.},
$$

and

$$
\left\|(1+\nabla)\left(\Phi_{u_{0}}(u)-\Phi_{u_{0}}(v)\right)\right\|_{S\left(L^{2}\right)} \leq c\|(1+\nabla)(F(u)-F(v))\|_{S^{\prime}\left(L^{2}\right)} .
$$

The triangle inequality applied to the right-hand side of (3.24) yields

$$
\begin{aligned}
\|F(u)-F(v)\|_{S^{\prime}\left(\dot{H}^{\left.-s_{c}\right)}\right.} \leq & \left\|\left(|x|^{-(N-\gamma)} *|u|^{p}\right)\left(|u|^{p-2} u-|v|^{p-2} v\right)\right\|_{{S^{\prime}\left(\dot{H}^{-s_{c}}\right)}} \\
& +\left\|\left(|x|^{-(N-\gamma)} *\left(|u|^{p}-|v|^{p}\right)\right)|v|^{p-2} v\right\|_{S^{\prime}\left(\dot{H}^{-s_{c}}\right)}
\end{aligned}
$$

where we have added and subtracted the term $\left(|x|^{-(N-\gamma)} *|u|^{p}\right)|v|^{p-2} v$ to the difference. Using the pair $\left(q_{3}^{\prime}, r_{1}^{\prime}\right)$ (defined in (3.10)) along with (2.18), (2.19) and calculations in (3.36), we obtain

$$
\begin{aligned}
& \|F(u)-F(v)\|_{S^{\prime}\left(\dot{H}^{-s_{c}}\right)} \leq c_{N, p, \gamma}\left\||x|^{-(N-\gamma)} *|u|^{p}\right\|_{L_{t}^{\frac{q_{2}}{p}} \frac{2 N}{L_{x}^{N-\gamma}}}\left\||u|^{p-2} u-|v|^{p-2} v\right\|_{L_{t}^{\frac{q_{2}}{p-1}}} \frac{r_{1}}{L_{x}^{p-1}} \\
& +c_{N, p, \gamma}\left\||x|^{-(N-\gamma)} *\left(|u|^{p}-|v|^{p}\right)\right\|_{L_{t}^{\frac{q_{2}}{p}} L_{x}^{\frac{2 N}{N-\gamma}}}\|v\|_{S\left(\dot{H}^{s_{c}}\right)}^{p-1} \\
& \leq c_{N, p, \gamma}\|u\|_{L_{t}^{q_{2}} L_{x}^{r_{1}}}^{p}\left(\|u\|_{L_{t}^{q_{2}} L_{x}^{r_{1}}}^{p-2}+\|v\|_{L_{t}^{q_{2}} L_{x}^{r_{1}}}\right)\|u-v\|_{L_{t}^{q_{2}} L_{x}^{r_{1}}} \\
& +c_{N, p, \gamma}\left\||u|^{p}-|v|^{p}\right\|_{L_{t}^{\frac{q_{2}}{p}} L_{x}^{\frac{r_{1}}{p}}\|v\|_{S\left(\dot{H}^{s c}\right)}^{p-1}} \\
& \leq c_{N, p, \gamma}\|u\|_{S\left(\dot{H}^{s_{c}}\right)}^{p}\left(\|u\|_{S\left(\dot{H}^{s_{c}}\right)}^{p-2}+\|v\|_{S\left(\dot{H}^{s^{c}}\right)}^{p-2}\right)\|u-v\|_{S\left(\dot{H}^{s_{c}}\right)} \\
& +c_{N, p, \gamma}\left(\|u\|_{S\left(\dot{H}^{s c}\right)}^{p-1}+\|v\|_{S\left(\dot{H}^{\left.s_{c}\right)}\right.}^{p-1}\right)\|u-v\|_{S\left(\dot{H}^{s_{c}}\right)}\|v\|_{S\left(\dot{H}^{s_{c}}\right)}^{p-1} .
\end{aligned}
$$

For $u, v \in B$, we have that

$$
\|F(u)-F(v)\|_{S^{\prime}\left(\dot{H}^{\left.-s_{c}\right)}\right.} \leq 2^{2 p} c_{N, p, \gamma}\left\|e^{i t \Delta} u_{0}\right\|_{S\left(\dot{H}^{s c}\right)}^{2(p-1)}\|u-v\|_{S\left(\dot{H}^{s}\right)} .
$$

Combining (3.24) with (3.26), we obtain

$$
\left\|\Phi_{u_{0}}(u)-\Phi_{u_{0}}(v)\right\|_{S\left(\dot{H}^{s^{c}}\right)} \leq 2^{2 p} c_{1}\left\|e^{i t \Delta} u_{0}\right\|_{S\left(\dot{H}^{s c}\right)}^{2(p-1)}\|u-v\|_{S\left(\dot{H}^{s c}\right)} .
$$


Next, we estimate the difference from (3.25) using again the triangle inequality and Hölder's along with the pair $\left(q_{1}^{\prime}, r_{1}^{\prime}\right)$ (defined in (3.8))

$$
\begin{aligned}
& \|F(u)-F(v)\|_{S^{\prime}\left(L^{2}\right)} \leq\left\|\left(|x|^{-(N-\gamma)} *|u|^{p}\right)\left(|u|^{p-2} u-|v|^{p-2} v\right)\right\|_{S^{\prime}\left(L^{2}\right)} \\
& +\left\|\left(|x|^{-(N-\gamma)} *\left(|u|^{p}-|v|^{p}\right)\right)|v|^{p-2} v\right\|_{S^{\prime}\left(L^{2}\right)} \\
& \leq c_{N, p, \gamma}\left\||x|^{-(N-\gamma)} *|u|^{p}\right\|_{L_{t}^{\frac{q_{2}}{p}} L_{x}^{\frac{2 N}{N-\gamma}}}\left\||u|^{p-2} u-|v|^{p-2} v\right\|_{L_{t}^{\frac{2 p}{p-1+s_{c}}} \frac{r_{x}^{p-1}}{L^{p-1}}} \\
& +c_{N, p, \gamma}\left\||x|^{-(N-\gamma)} *\left(|u|^{p}-|v|^{p}\right)\right\|_{L_{t}^{2} L_{x}^{\frac{2 N}{N-\gamma}}}\|v\|_{L_{t}^{q_{2}} L_{x}^{r_{1}}}^{p-1}
\end{aligned}
$$

Apply (2.18), (2.19) and calculations in (3.18), (3.19) on the right-hand side of above estimate to obtain

$$
\begin{aligned}
\|F(u)-F(v)\|_{S^{\prime}\left(L^{2}\right) \leq} & c_{N, p, \gamma}\|u\|_{L_{t}^{q_{2} L_{x}^{r_{1}}}}^{p}\left(\|u\|_{L_{t}^{q_{2}} L_{x}^{r_{1}}}^{p-2}+\|v\|_{L_{t}^{q_{2} L_{x}^{r_{1}}}}^{p-2}\right)\|u-v\|_{L_{t}^{q_{1}} L_{x}^{r_{1}}} \\
& +\left.c_{N, p, \gamma}\|\| u\right|^{p}-|v|^{p}\left\|_{L_{t}^{2} L_{x}^{\frac{r_{1}}{p}}\|v\|_{L_{t}^{q_{2}} L_{x}^{r_{1}}}^{p-1}} \leq c_{N, p, \gamma}\right\| u\left\|_{S\left(\dot{H}^{s_{c}}\right)}^{p}\left(\|u\|_{S\left(\dot{H}^{\left.s_{c}\right)}\right.}^{p-2}+\|v\|_{S\left(\dot{H}^{s_{c}}\right)}^{p-2}\right)\right\| u-v \|_{S\left(L^{2}\right)} \\
& +c_{N, p, \gamma}\left(\|u\|_{S\left(\dot{H}^{s^{c}}\right)}^{p-1}+\|v\|_{S\left(\dot{H}^{s_{c}}\right)}^{p-1}\right)\|u-v\|_{S\left(L^{2}\right)}\|v\|_{S\left(\dot{H}^{s_{c}}\right)}^{p-1}
\end{aligned}
$$

For $u, v \in B$, we have

$$
\|F(u)-F(v)\|_{S^{\prime}\left(L^{2}\right)} \leq 2^{2 p} c_{N, p, \gamma}\left\|e^{i t \Delta} u_{0}\right\|_{S\left(\dot{H}^{s}\right)}^{2(p-1)}\|u-v\|_{S\left(L^{2}\right)}
$$

Combining (3.25) with (3.28), we obtain

$$
\left\|\Phi_{u_{0}}(u)-\Phi_{u_{0}}(v)\right\|_{S\left(L^{2}\right)} \leq 2^{2 p} c_{1}\left\|e^{i t \Delta} u_{0}\right\|_{S\left(\dot{H}^{s c}\right)}^{2(p-1)}\|u-v\|_{S\left(L^{2}\right)} .
$$

Finally, estimating the difference in (3.25) with the gradient, we obtain

$$
\begin{aligned}
\|\nabla(F(u)-F(v))\|_{S^{\prime}\left(L^{2}\right)} \leq & \left\|\nabla\left[\left(|x|^{-(N-\gamma)} *|u|^{p}\right)\left(|u|^{p-2} u-|v|^{p-2} v\right)\right]\right\|_{S^{\prime}\left(L^{2}\right)} \\
& +\left\|\nabla\left[\left(|x|^{-(N-\gamma)} *\left(|u|^{p}-|v|^{p}\right)\right)|v|^{p-2} v\right]\right\|_{S^{\prime}\left(L^{2}\right)}
\end{aligned}
$$


Using again the pair $\left(q_{1}^{\prime}, r_{1}^{\prime}\right)$ (defined in (3.8)) and (3.35) along with the calculations for (3.22), we get

$$
\begin{aligned}
(3.30) & \leq c_{N, p, \gamma}\|u\|_{S\left(\dot{H}^{s_{c}}\right)}^{p}\left(\|u\|_{S\left(\dot{H}^{s_{c}}\right)}^{p-2}+\|v\|_{S\left(\dot{H}^{s_{c}}\right)}^{p-2}\right)\|\nabla(u-v)\|_{S\left(L^{2}\right)} \\
& +c_{N, p, \gamma}\|u\|_{S\left(\dot{H}^{s^{c}}\right)}^{p-1}\|\nabla u\|_{S\left(L^{2}\right)}\left(\|u\|_{S\left(\dot{H}^{s}\right)}^{p-2}+\|v\|_{S\left(\dot{H}^{s^{c}}\right)}^{p-2}\right)\|u-v\|_{S\left(\dot{H}^{s_{c}}\right)} .
\end{aligned}
$$

Similarly, we obtain

$$
\begin{aligned}
(3.31) & \leq c_{N, p, \gamma}\left(\|u\|_{S\left(\dot{H}^{s}\right)}^{p-1}+\|v\|_{S\left(\dot{H}^{s_{c}}\right)}^{p-1}\right)\|\nabla(u-v)\|_{S\left(L^{2}\right)}\|v\|_{S\left(\dot{H}^{s^{c}}\right)}^{p-1} \\
& +c_{N, p, \gamma}\left(\|u\|_{S\left(\dot{H}^{s}\right)}^{p-1}+\|v\|_{S\left(\dot{H}^{s^{c}}\right)}^{p-1}\right)\|u-v\|_{S\left(\dot{H}^{s_{c}}\right)}\|\nabla v\|_{S\left(L^{2}\right)}\|v\|_{S\left(\dot{H}^{s_{c}}\right)}^{p-2}
\end{aligned}
$$

Then for $u, v \in B$, we have

$$
\begin{aligned}
& \left\|\nabla\left(\Phi_{u_{0}}(u)-\Phi_{u_{0}}(v)\right)\right\|_{S\left(L^{2}\right)} \leq c\|\nabla(F(u)-F(v))\|_{S^{\prime}\left(L^{2}\right)} \\
& \quad \leq 2^{2 p} c_{1}\left\|e^{i t \Delta} u_{0}\right\|_{S\left(\dot{H}^{s^{c}}\right)}^{2(p-1)}\|\nabla(u-v)\|_{S\left(L^{2}\right)}+2^{2 p} c c_{1}\left\|u_{0}\right\|_{H^{1}}\left\|e^{i t \Delta} u_{0}\right\|_{S\left(\dot{H}^{s c}\right)}^{2 p-3}\|u-v\|_{S\left(\dot{H}^{s}\right)}
\end{aligned}
$$

Putting together the estimates (3.27), (3.28) and (3.32) along with (3.23) (definition of metric $d$ ), we get

$$
d\left(\Phi_{u_{0}}(u), \Phi_{u_{0}}(v)\right) \leq 2^{2 p} \tilde{c}_{1}\left\|u_{0}\right\|_{H^{1}}\left\|e^{i t \Delta} u_{0}\right\|_{S\left(\dot{H}^{s_{c}}\right)}^{2 p-3} d(u, v) \leq \frac{1}{2} d(u, v),
$$

for $\delta_{1} \leq \sqrt[2 p-3]{\frac{1}{2^{2 p+1} \tilde{c}_{1} A}}$ and $\tilde{c}_{1}=\max \left(c_{1}, c c_{1}\right)$. Finally, taking $\delta \leq \min \left(\delta_{0}, \delta_{1}\right)$ concludes that $\Phi_{u_{0}}$ is a contraction.

Now we give a similar result in $\dot{H}^{s_{c}}$ (see also [AR19]), which makes it possible to extend the local existence to the larger time intervals. First, we set

$$
\|u\|_{W^{s_{c}}} \stackrel{\text { def }}{=} \begin{cases}\|u\|_{L_{t}^{\frac{2 p}{1-s_{c}}} L_{x}^{\frac{2 N p}{N+\gamma}},} & \text { for } 0<s_{c}<1, \\ \max _{L_{t}^{\infty} L_{x}^{\frac{2 N}{N-2}},}\left(\|u\|_{L_{t}^{3} L_{x}^{\frac{6 N}{3 \gamma+2}},\|u\|_{\left.L_{t}^{\infty} L_{x}^{\frac{2 N}{\gamma+2}}\right),}}\right. & \text { for } s_{c}=1, \\ \|u\|_{L_{t}^{\infty} L_{L^{2 N(p-1)}}^{\gamma+2}}, & \text { for } s_{c}>1 \text { and } p>2,\end{cases}
$$


where all the above pairs corresponding to $W^{s_{c}}$ are $\dot{H}^{s_{c}}$-admissible pairs and

$$
\|u\|_{S^{0}} \stackrel{\text { def }}{=} \begin{cases}\|u\|_{L_{t}^{\frac{2 p}{1+s_{c}(p-1)}} \frac{2 N p}{L_{x}^{N+\gamma}},} & \text { for } 0<s_{c}<1 \\ L_{L_{t}^{2} L_{x}^{\frac{2 N}{N-2}},} & \text { for } s_{c}=1, \\ \max \left(\|u\|_{\left.L_{t}^{3} L_{x}^{\frac{6 N}{3 N-4}},\|u\|_{L_{t}^{\infty} L_{x}^{2}}\right),}\right. & \text { for } s_{c}>1 \text { and } p=2, \\ \|u\|_{L_{t}^{2} L_{x}^{\frac{2 N}{N-2}},} & \text { for } s_{c}>1 \text { and } p>2\end{cases}
$$

where all the pairs corresponding to $S^{0}$ are $L^{2}$-admissible pairs.

Proposition 3.2.2 Let $\gamma, N$ and $p$ be as in Proposition 2.6.3 so that $s_{c} \geq 0$. Assume in addition that if $p$ is not an even integer, then $s_{c}<p-1$. Let $u_{0} \in \dot{H}^{s_{c}}\left(\mathbb{R}^{N}\right)$ with $\left\|u_{0}\right\|_{\dot{H}^{s_{c}}} \leq A$. Then there exists $\delta=\delta(A)>0$ such that if $\left\|e^{i t \Delta} u_{0}\right\|_{W^{s_{c}}} \leq \delta$, then there exists a unique global solution $u$ of (1.1) in $\dot{H}^{s_{c}}\left(\mathbb{R}^{N}\right)$ such that

$$
\|u\|_{W^{s_{c}}} \leq 2\left\|e^{i t \Delta} u_{0}\right\|_{W^{s_{c}}}
$$

and

$$
\left\||\nabla|^{s_{c}} u\right\|_{S^{0}} \leq 2 c_{1}\left\|u_{0}\right\|_{\dot{H}^{s_{c}}}
$$

Proof. Denote

$$
B_{s d}=\left\{u:\|u\|_{W^{s_{c}}} \leq 2\left\|e^{i t \Delta} u_{0}\right\|_{W^{s_{c}}} \quad \text { and } \quad\left\||\nabla|^{s_{c}} u\right\|_{S^{0}} \leq 2 c_{1}\left\|u_{0}\right\|_{\dot{H}^{s_{c}}}\right\} .
$$

We divide the analysis into several cases:

1. $0<s_{c}<1$. Applying the triangle inequality and Lemma 2.4.7 to (3.13), we obtain

$$
\begin{aligned}
\left\|\Phi_{u_{0}}(u)\right\|_{W^{s_{c}}} & =\left\|\Phi_{u_{0}}(u)\right\|_{L_{t}^{q_{2}} L_{x}^{r_{1}}} \\
& \leq\left\|e^{i t \Delta} u_{0}\right\|_{L_{t}^{q_{2}} L_{x}^{r_{1}}}+c\left\||\nabla|^{s_{c}} \int_{0}^{t} e^{i\left(t-t^{\prime}\right) \Delta} N\left(u\left(t^{\prime}\right)\right) d t^{\prime}\right\|_{L_{t}^{q_{2}} L_{x}^{r_{2}}} \\
& \leq\left\|e^{i t \Delta} u_{0}\right\|_{L_{t}^{q_{2}} L_{x}^{r_{1}}}+c c_{1}\left\||\nabla|^{s_{c}} N(u)\right\|_{L_{t}^{q_{1}^{\prime}} L_{x}^{r_{1}^{\prime}}}
\end{aligned}
$$


where $r_{1}=\frac{2 N p}{N+\gamma}, r_{2}=\frac{2 N p}{N+\gamma+2 s_{c} p}$ and $q_{1}=\frac{2 p}{1+s_{c}(p-1)}, q_{2}=\frac{2 p}{1-s_{c}}$ satisfy the conditions (2.5) and (2.4), respectively. The triangle inequality along with Lemma 2.4.6 to $(3.13)$ gives

$$
\begin{aligned}
\left\|\left.|\nabla|\right|^{s_{c}} \Phi_{u_{0}}(u)\right\|_{S^{0}} & =\left\||\nabla|^{s_{c}} \Phi_{u_{0}}(u)\right\|_{L_{t}^{q_{1}} L_{x}^{r_{1}}} \\
& \leq c_{1}\left\|u_{0}\right\|_{\dot{H}^{s_{c}}}+c_{1}\left\||\nabla|^{s_{c}} N(u)\right\|_{L_{t}^{q_{1}^{\prime}} L_{x}^{r_{1}^{\prime}}}
\end{aligned}
$$

Using (2.45) along with Lemma 2.3.2, (2.46) and (2.47), we get

$$
\left\||\nabla|^{s_{c}} N(u)\right\|_{L_{t}^{q_{1}^{\prime}} L_{x}^{r_{1}^{\prime}}} \leq 2 c_{N, \gamma, p}\|u\|_{L_{t}^{q_{2}} L_{x}^{r_{1}}}^{2(p-1}\left\||\nabla|^{s_{c}} u\right\|_{L_{t}^{q_{1}} L_{x}^{r_{1}}}
$$

Therefore, (3.35) gives

$$
\left\|\Phi_{u_{0}}(u)\right\|_{W^{s_{c}}} \leq\left\|e^{i t \Delta} u_{0}\right\|_{W^{s_{c}}}+2 c c_{1} c_{N, \gamma, p}\|u\|_{W^{s_{c}}}^{2(p-1)}\left\||\nabla|^{s_{c}} u\right\|_{S^{0}}
$$

and (3.36) gives

$$
\left\||\nabla|^{s_{c}} \Phi_{u_{0}}(u)\right\|_{S^{0}} \leq c_{1}\left\|u_{0}\right\|_{\dot{H}^{s_{c}}}+2 c_{1} c_{N, \gamma, p}\|u\|_{W^{s_{c}}}^{2(p-1)}\left\||\nabla|^{s_{c}} u\right\|_{S^{0}} .
$$

Thus, from (3.37) for $u \in B_{s d}$, we obtain

$$
\left\|\Phi_{u_{0}}(u)\right\|_{W^{s_{c}}} \leq\left\|e^{i t \Delta} u_{0}\right\|_{W^{s_{c}}}\left(1+2^{2 p} c c_{1}^{2} c_{N, \gamma, p}\left\|e^{i t \Delta} u_{0}\right\|_{W^{s_{c}}}^{2 p-3} A\right)
$$

which implies that we need

$$
2^{2 p} c c_{1}^{2} c_{N, \gamma, p}\left\|e^{i t \Delta} u_{0}\right\|_{W^{s_{c}}}^{2 p-3} A \leq 1
$$

Similarly, from (3.38) for $u \in B_{s d}$, we obtain

$$
\left\||\nabla|{ }^{s_{c}} \Phi_{u_{0}}(u)\right\|_{S^{0}} \leq c_{1}\left\|u_{0}\right\|_{\dot{H}^{s_{c}}}\left(1+2^{2 p} c_{1} c_{N, \gamma, p}\left\|e^{i t \Delta} u_{0}\right\|_{W^{s_{c}}}^{2(p-1)}\right),
$$

which implies that we require

$$
2^{2 p} c_{1} c_{N, \gamma, p}\left\|e^{i t \Delta} u_{0}\right\|_{W^{s_{c}}}^{2(p-1)} \leq 1 .
$$


Therefore, from (3.39) and (3.40), choosing

$$
\delta<\delta_{0} \leq \min \left(\frac{1}{\sqrt[2 p-3]{2^{2 p} c c_{1}^{2} c_{N, \gamma, p} A}}, \frac{1}{\sqrt[2(p-1)]{2^{2 p} c_{1} c_{N, \gamma, p}}}\right)
$$

implies that $\Phi_{u_{0}} \in B_{s d}$. Now we show that $\Phi_{u_{0}}(u)$ is a contraction on $B_{s d}$ with the metric

$$
d(u, v)=\left\||\nabla|^{s_{c}}(u-v)\right\|_{S^{0}}+\|u-v\|_{W^{s_{c}}} .
$$

For $u, v \in B$, by Lemmas 2.4.6 and 2.4.7, we obtain

$$
d\left(\Phi_{u_{0}}(u), \Phi_{u_{0}}(v)\right) \leq 2 \tilde{c}_{1}\left\||\nabla|^{s_{c}}[N(u)-N(v)]\right\|_{L_{t}^{q_{1}^{\prime}} L_{x}^{r_{1}^{\prime}}}
$$

where $\tilde{c}_{1}=\max \left(c c_{1}, c_{1}\right)$. The triangle inequality applied to the term on the righthand side of (3.41) yields

$$
\begin{aligned}
\||\nabla|^{s_{c}}(N(u) & -N(v)) \|_{L_{t}^{q_{1}^{\prime}} L_{x}^{r_{1}^{\prime}}} \\
\leq & \left\||\nabla|^{s_{c}}\left[\left(|x|^{-(N-\gamma)} *|u|^{p}\right)\left(|u|^{p-2} u-|v|^{p-2} v\right)\right]\right\|_{L_{t}^{q_{1}^{\prime}} L_{x}^{r_{1}^{\prime}}} \\
& +\left\||\nabla|^{s_{c}}\left[\left(|x|^{-(N-\gamma)} *\left(|u|^{p}-|v|^{p}\right)\right)|v|^{p-2} v\right]\right\|_{L_{t}^{q_{1}^{\prime}} L_{x}^{r_{1}^{\prime}}}
\end{aligned}
$$

Using (2.18), Hölder's inequality and Lemma 2.3.1, we obtain

$$
\begin{aligned}
& (3.42) \leq\left\||x|^{-(N-\gamma)} *|u|^{p}\right\|_{L_{t}^{\frac{q_{2}}{p}} \frac{2 N}{L_{x}^{N-\gamma}}}\left\||\nabla|^{s_{c}}\left(|u|^{p-2} u-|v|^{p-2} v\right)\right\|_{L_{t}^{\frac{2 p}{p-1+s_{c}}} \frac{r_{x}^{p-1}}{L_{x}}} \\
& +|||x|^{-(N-\gamma)} *|\nabla|^{s_{c}}\left(|u|^{p}\right)\left\|_{L_{t}^{2} L_{x}^{\frac{2 N}{N-\gamma}}}\right\||u|^{p-2} u-|v|^{p-2} v \|_{L_{t}^{\frac{q_{2}}{p-1}} \frac{r_{1}}{L_{x}^{p-1}}} \\
& \leq c_{N, \gamma, p}\|u\|_{L_{t}^{q_{2}} L_{x}^{r_{1}}}^{p}\left(\|u\|_{L_{t}^{q_{2}} L_{x}^{r_{1}}}^{p-2}+\|v\|_{L_{t}^{q_{2}} L_{x}^{r_{1}}}^{p-2}\right)\left\||\nabla|^{s_{c}}(u-v)\right\|_{L_{t}^{q_{1}} L_{x}^{r_{1}}} \\
& +c_{N, \gamma, p}\|u\|_{L_{t}^{q_{2}} L_{x}^{r_{1}}}^{p-1}\left\||\nabla|^{s_{c}} u\right\|_{L_{t}^{q_{1}} L_{x}^{r_{1}}}\left(\|u\|_{L_{t}^{q_{2}} L_{x}^{r_{1}}}^{p-2}+\|v\|_{L_{t}^{q_{2}} L_{x}^{r_{1}}}^{p-2}\right)\|u-v\|_{L_{t}^{q_{2}} L_{x}^{r_{1}}} .
\end{aligned}
$$


Similarly, using (2.19), Hölder's inequality and Lemma 2.3.1, we also obtain

$$
\begin{aligned}
& \text { (3.43) } \leq\left\||x|^{-(N-\gamma)} *\left(|u|^{p}-|v|^{p}\right)\right\|_{L_{t}^{\frac{q_{2}}{p}} L_{x}^{\frac{2 N}{N-\gamma}}}\left\||\nabla|^{s_{c}}\left(|v|^{p-2} v\right)\right\|_{L_{t}^{\frac{2 p}{p-1+s_{c}}} L_{x}^{\frac{r_{1}}{p-1}}} \\
& +\left\||x|^{-(N-\gamma)} *|\nabla|^{s_{c}}\left(|u|^{p}-|v|^{p}\right)\right\|_{L_{t}^{2} L_{x}^{\frac{2 N}{N-\gamma}}}\|v\|_{L_{t}^{q_{2}} L_{x}^{r_{1}}}^{p-1} \\
& \leq c_{N, \gamma, p}\left(\|u\|_{L_{t}^{q_{2}} L_{x}^{r_{1}}}^{p-1}+\|v\|_{L_{t}^{q_{2}} L_{x}^{r_{1}}}^{p-1}\right)\|u-v\|_{L_{t}^{q_{2}} L_{x}^{r_{1}}}\|v\|_{L_{t}^{q_{2}} L_{x}^{r_{1}}}^{p-2}\left\||\nabla|^{s_{c}} v\right\|_{L_{t}^{q_{1}} L_{x}^{r_{1}}}
\end{aligned}
$$

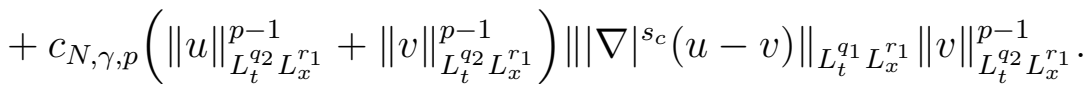

Thus, for $u, v \in B_{s d}$, we have

$$
\left\||\nabla|^{s_{c}}[N(u)-N(v)]\right\|_{L_{t}^{q_{1}^{\prime}} L_{x}^{r_{1}^{\prime}}} \leq 2^{2 p+1} c_{1} c_{N, p, \gamma} A\left\|e^{i t \Delta} u_{0}\right\|_{W^{s_{c}}}^{2 p-3} d(u, v) .
$$

Combining (3.44) with (3.41), we get

$$
d\left(\Phi_{u_{0}}(u), \Phi_{u_{0}}(v)\right) \leq 2^{2(p+1)} \tilde{c}_{1}^{2} c_{N, p, \gamma} A\left\|e^{i t \Delta} u_{0}\right\|_{W^{s c}}^{2 p-3} d(u, v) \leq \frac{1}{2} d(u, v)
$$

for $\delta_{1} \leq \sqrt[2 p-3]{\frac{1}{2^{2 p+3} \tilde{c}_{1}^{2} c_{N, p, \gamma} A}}$. Finally, taking $\delta \leq \min \left(\delta_{0}, \delta_{1}\right)$ concludes that $\Phi_{u_{0}}$ is a contraction.

2. $s_{c}=1$. Applying the triangle inequality and Lemma 2.4 .7 to (3.13), we obtain

$$
\left\|\Phi_{u_{0}}(u)\right\|_{W^{1}}=\left\|\Phi_{u_{0}}(u)\right\|_{L_{t}^{\infty} L_{x}^{r}} \leq\left\|e^{i t \Delta} u_{0}\right\|_{L_{t}^{\infty} L_{x}^{r}}+c c_{1}\|\nabla N(u)\|_{L_{t}^{2} L_{x}^{r^{\prime}}}
$$

where $(\infty, 2)$ and $\left(2, \frac{2 N}{N-2}\right)$ satisfy conditions (2.4) and (2.5). The triangle inequality along with Lemma 2.4 .6 yields

$$
\left\|\nabla \Phi_{u_{0}}(u)\right\|_{S^{0}}=\left\|\nabla \Phi_{u_{0}}(u)\right\|_{L_{t}^{2} L_{x}^{r}} \leq c_{1}\left\|u_{0}\right\|_{\dot{H}^{1}}+c_{1}\|\nabla N(u)\|_{L_{t}^{2} L_{x}^{r^{\prime}}}
$$

Using (2.54) along with (2.55) and (2.56), we get

$$
\|\nabla N(u)\|_{L_{t}^{2} L_{x}^{r^{\prime}}} \leq 2 c_{N, \gamma, p}\|u\|_{L_{t}^{\infty} L_{x}^{r}}^{2(p-1)}\|\nabla u\|_{L_{t}^{2} L_{x}^{r}}
$$

Therefore, (3.35) gives

$$
\left\|\Phi_{u_{0}}(u)\right\|_{W^{1}} \leq\left\|e^{i t \Delta} u_{0}\right\|_{W^{1}}+2 c c_{1} c_{N, \gamma, p}\|u\|_{W^{1}}^{2(p-1)}\|\nabla u\|_{S^{0}},
$$


and (3.36) gives

$$
\left\|\nabla \Phi_{u_{0}}(u)\right\|_{S^{0}} \leq c_{1}\left\|u_{0}\right\|_{\dot{H}^{1}}+2 c_{1} c_{N, \gamma, p}\|u\|_{W^{1}}^{2(p-1)}\|\nabla u\|_{S^{0}} .
$$

For contraction, we define the metric

$$
d(u, v)=\|\nabla(u-v)\|_{S^{0}}+\|u-v\|_{W^{1}},
$$

and use Sobolev embedding and Strichartz estimates along with triangle inequality, to obtain

$$
\begin{aligned}
d\left(\phi_{u_{0}}(u), \phi_{u_{0}}(v)\right) & \leq 2 \tilde{c}_{1}\|\nabla[N(u)-N(v)]\|_{L_{t}^{2} L_{x}^{r^{\prime}}} \\
& \leq 2 \tilde{c}_{1}\left\|\nabla\left[\left(|x|^{-(N-\gamma)} *|u|^{p}\right)\left(|u|^{p-2} u-|v|^{p-2} v\right)\right]\right\|_{L_{t}^{2} L_{x}^{r^{\prime}}} \\
& +2 \tilde{c}_{1}\left\|\nabla\left[\left(|x|^{-(N-\gamma)} *\left(|u|^{p}-|v|^{p}\right)\right)|v|^{p-2} v\right]\right\|_{L_{t}^{2} L_{x}^{r^{\prime}}}
\end{aligned}
$$

Using (2.18), Hölder's inequality and Lemma 2.3.1, we obtain

$$
\begin{aligned}
(3.47) \leq & \left\||x|^{-(N-\gamma)} *|u|^{p}\right\|_{L_{t}^{\infty} L_{x}^{\frac{2 N}{N-\gamma}}}\left\|\nabla\left(|u|^{p-2} u-|v|^{p-2} v\right)\right\|_{L_{t}^{2} L_{x}^{\frac{r}{p-1}}} \\
& +\left\||x|^{-(N-\gamma)} * \nabla\left(|u|^{p}\right)\right\|_{L_{t}^{2} L_{x}^{\frac{2 N}{N-\gamma}}}\left\||u|^{p-2} u-|v|^{p-2} v\right\|_{L_{t}^{\infty} L_{x}^{\frac{r}{p-1}}} \\
\leq & c_{N, \gamma, p}\|u\|_{L_{t}^{\infty} L_{x}^{r}}^{p}\left(\|u\|_{L_{t}^{\infty} L_{x}^{r}}^{p-2}+\|v\|_{L_{t}^{\infty} L_{x}^{r}}^{p-2}\right)\|\nabla(u-v)\|_{L_{t}^{2} L_{x}^{r}} \\
& +c_{N, \gamma, p}\|u\|_{L_{t}^{\infty} L_{x}^{r}}^{p-1}\|\nabla u\|_{L_{t}^{2} L_{x}^{r}}\left(\|u\|_{L_{t}^{\infty} L_{x}^{r}}^{p-2}+\|v\|_{L_{t}^{\infty} L_{x}^{r}}^{p-2}\|u-v\|_{L_{t}^{\infty} L_{x}^{r}} .\right.
\end{aligned}
$$

Similarly, using (2.19), Hölder's inequality and Lemma 2.3.1, we also obtain

$$
\begin{aligned}
(3.48) \leq & \left\||x|^{-(N-\gamma)} *\left(|u|^{p}-|v|^{p}\right)\right\|_{L_{t}^{\infty} L_{x}^{N-\gamma}}\|v\|_{L_{t}^{\infty} L_{x}^{r}}^{p-2}\|\nabla v\|_{L_{t}^{2} L_{x}^{r}} \\
& +\left\||x|^{-(N-\gamma)} * \nabla\left(|u|^{p}-|v|^{p}\right)\right\|_{L_{t}^{2} L_{x}^{N-\gamma}}\|v\|_{L_{t}^{\infty} L_{x}^{r}}^{p-1} \\
\leq & c_{N, \gamma, p}\left(\|u\|_{L_{t}^{\infty} L_{x}^{r}}^{p-1}+\|v\|_{L_{t}^{\infty} L_{x}^{r}}^{p-1}\right)\|u-v\|_{L_{t}^{\infty} L_{x}^{r}}\|v\|_{L_{t}^{\infty} L_{x}^{r}}^{p-2}\|\nabla v\|_{L_{t}^{2} L_{x}^{r}} \\
& +c_{N, \gamma, p}\left(\|u\|_{L_{t}^{\infty} L_{x}^{r}}^{p-1}+\|v\|_{L_{t}^{\infty} L_{x}^{r}}^{p-1}\right)\|\nabla(u-v)\|_{L_{t}^{2} L_{x}^{r}}\|v\|_{L_{t}^{\infty} L_{x}^{r}}^{p-1}
\end{aligned}
$$

Rest of the details are similar to the inter-critical case $\left(0<s_{c}<1\right)$. 
3. $s_{c}>1$.

Case $p=2$. Using triangle inequality and Lemma 2.4.7, we get

$$
\begin{aligned}
\left\|\Phi_{u_{0}}(u)\right\|_{L_{t}^{q} L_{x}^{r_{1}}} & +\left\|\Phi_{u_{0}}(u)\right\|_{L_{t}^{\infty} L_{x}^{r_{2}}} \\
\leq & \left\|e^{i t \Delta} u_{0}\right\|_{L_{t}^{q} L_{x}^{r_{1}}}+c\left\||\nabla|^{s_{c}} \int_{0}^{t} e^{i\left(t-t^{\prime}\right) \Delta} N\left(u\left(t^{\prime}\right)\right) d t^{\prime}\right\|_{L_{t}^{q} L_{x}^{r}} \\
& +\left\|e^{i t \Delta} u_{0}\right\|_{L_{t}^{\infty} L_{x}^{r_{2}}}+c\left\||\nabla|^{s_{c}} \int_{0}^{t} e^{i\left(t-t^{\prime}\right) \Delta} N\left(u\left(t^{\prime}\right)\right) d t^{\prime}\right\|_{L_{t}^{\infty} L_{x}^{2}} \\
\leq & \left\|e^{i t \Delta} u_{0}\right\|_{L_{t}^{q} L_{x}^{r_{1}}}+\left\|e^{i t \Delta} u_{0}\right\|_{L_{t}^{\infty} L_{x}^{r_{2}}}+2 c c_{1}\left\||\nabla|^{s_{c}} N(u)\right\|_{L_{t}^{q^{\prime}} L_{x}^{r^{\prime}}},
\end{aligned}
$$

where the pairs $\left(3, \frac{6 N}{3 N-4}\right),(\infty, 2)$ satisfy the conditions (2.4) and (2.5) with $(\tilde{q}, \tilde{r})$ $=\left(3, \frac{6 N}{3 N-4}\right)$. Using the fractional product rule, we have

$$
\begin{aligned}
\left\||\nabla|^{s_{c}} N(u)\right\|_{L_{I}^{q^{\prime}} L_{x}^{r^{\prime}}} \leq & \left\|\left(|x|^{-(N-\gamma)} *|\nabla|^{s_{c}}\left(|u|^{2}\right)\right) u\right\|_{L_{t}^{q^{\prime}} L_{x}^{r^{\prime}}} \\
& +\left\|\left(|x|^{-(N-\gamma)} *|u|^{2}\right)|\nabla|^{s_{c}} u\right\|_{L_{t}^{q^{\prime}} L_{x}^{r^{\prime}}}
\end{aligned}
$$

where (3.50) is estimated using Lemma 2.3.1 and Hölder's inequality along with the formula for $s_{c}$

$$
\begin{aligned}
& (3.50) \leq\left\|\left(|x|^{-(N-\gamma)} *|\nabla|^{s_{c}}|u|^{2}\right)\right\|_{L_{t}^{\frac{3}{2}} L_{x}^{\frac{6 N}{3 N-3 \gamma-2}}}\|u\|_{L_{t}^{\infty} L_{x}^{r_{2}}} \\
& \leq c_{N, \gamma}\left\|u|\nabla|^{s_{c}} u\right\|_{L_{t}^{\frac{3}{2}} L_{x}^{\frac{6 N}{3 N+3 \gamma-2}}}\|u\|_{L_{t}^{\infty} L_{x}^{r_{2}}} \\
& \leq c_{N, \gamma}\|u\|_{L_{t}^{q} L_{x}^{r_{1}}}\left\||\nabla|^{s_{c}} u\right\|_{L_{t}^{q} L_{x}^{r}}\|u\|_{L_{t}^{\infty} L_{x}^{r_{2}}}
\end{aligned}
$$

Here, we again require $3 N-3 \gamma-2>0$ (as in (2.62)) and since $s_{c}>1$ we have that $\gamma<N-4<N-\frac{2}{3}$. For (3.51), we again use Hölder's inequality and Lemma 2.3.1 to obtain

$$
\begin{aligned}
(3.51) & \leq\left\||x|^{-(N-\gamma)} *|u|^{2}\right\|_{L_{t}^{\frac{3}{2}} L_{x}^{\frac{3 N}{2}}}\left\||\nabla|^{s_{c}} u\right\|_{L_{t}^{\infty} L_{x}^{2}} \\
& \leq c_{N, \gamma}\|u\|_{L_{t}^{q} L_{x}^{r_{1}}}^{2}\left\||\nabla|^{s_{c}} u\right\|_{L_{t}^{\infty} L_{x}^{2}}
\end{aligned}
$$


Putting (3.49) together with (3.52) and (3.53), we get

$$
\left\|\Phi_{u_{0}}(u)\right\|_{W^{s_{c}}} \leq\left\|e^{i t \Delta} u_{0}\right\|_{W^{s_{c}}}+2 c c_{1} c_{N, \gamma}\|u\|_{W^{s_{c}}}^{2}\left\||\nabla|^{s_{c}} u\right\|_{S^{0}} .
$$

Similarly, we obtain

$$
\left\||\nabla|^{s_{c}} \Phi_{u_{0}}(u)\right\|_{S^{0}} \leq c_{1}\left\|u_{0}\right\|_{\dot{H}^{s_{c}}}+2 c_{1} c_{N, \gamma}\|u\|_{W^{s_{c}}}^{2}\left\||\nabla|^{s_{c}} u\right\|_{S^{0}} .
$$

For contraction, we again define same metric

$$
d(u, v)=\left\||\nabla|^{s_{c}}(u-v)\right\|_{S^{0}}+\|u-v\|_{W^{s_{c}}},
$$

and use Lemmas 2.4.6 and 2.4.7 along with triangle inequality, to obtain

$$
d\left(\Phi_{u_{0}}(u)-\Phi_{u_{0}}(v)\right) \leq 2 \tilde{c}_{1}\left\||\nabla|^{s_{c}}[N(u)-N(v)]\right\|_{L_{t}^{q^{\prime}} L_{x}^{r^{\prime}}}
$$

where

$$
\begin{aligned}
\||\nabla|^{s_{c}}[N(u) & -N(v)] \|_{L_{t}^{q^{\prime}} L_{x}^{r^{\prime}}} \\
& \leq\left\||\nabla|^{s_{c}}\left[\left(|x|^{-(N-\gamma)} *|u|^{2}\right)(u-v)\right]\right\|_{L_{t}^{q^{\prime}} L_{x}^{r^{\prime}}} \\
& +\left\||\nabla|^{s_{c}}\left[\left(|x|^{-(N-\gamma)} *\left(|u|^{2}-|v|^{2}\right)\right) v\right]\right\|_{L_{t}^{q^{\prime}} L_{x}^{r^{\prime}}}
\end{aligned}
$$

Using (2.18), Hölder's inequality and Lemma 2.3.1, we obtain

$$
\begin{aligned}
\text { (3.54) } \leq & \left\||x|^{-(N-\gamma)} *|u|^{2}\right\|_{L_{t}^{3 / 2} L_{x}^{\frac{3 N}{2}}}\left\||\nabla|^{s_{c}}(u-v)\right\|_{L_{t}^{\infty} L_{x}^{2}} \\
& +\left\||x|^{-(N-\gamma)} *|\nabla|^{s_{c}}\left(|u|^{2}\right)\right\|_{L_{t}^{3 / 2} L_{x}^{\frac{6 N}{3-3 \gamma-2}}}\|u-v\|_{L_{t}^{\infty} L_{x}^{r_{2}}} \\
\leq & c_{N, \gamma}\|u\|_{L_{t}^{q} L_{x}^{r_{1}}}^{2}\left\||\nabla|^{s_{c}}(u-v)\right\|_{L_{t}^{\infty} L_{x}^{2}} \\
& +c_{N, \gamma}\|u\|_{L_{t}^{q} L_{x}^{r_{1}}}\left\||\nabla|^{s_{c}} u\right\|_{L_{t}^{q} L_{x}^{r}}\|u-v\|_{L_{t}^{\infty} L_{x}^{r_{2}}} \\
\leq & c_{N, \gamma}\|u\|_{W^{s_{c}}}^{2}\left\||\nabla|^{s_{c}}(u-v)\right\|_{S^{0}}+c_{N, \gamma}\|u\|_{W^{s_{c}}}\left\||\nabla|^{s_{c}} u\right\|_{S^{0}}\|u-v\|_{W^{s_{c}}} .
\end{aligned}
$$


Similarly, using (2.19), Hölder's inequality and Lemma 2.3.1, we also obtain

$$
\begin{aligned}
(3.55) \leq & \left\||x|^{-(N-\gamma)} *\left(|u|^{2}-|v|^{2}\right)\right\|_{L_{t}^{3 / 2} L_{x}^{\frac{3 N}{2}}}\left\||\nabla|^{s_{c}} v\right\|_{L_{t}^{\infty} L_{x}^{2}} \\
& +\left\||x|^{-(N-\gamma)} *|\nabla|^{s_{c}}\left(|u|^{2}-|v|^{2}\right)\right\|_{L_{t}^{3 / 2} L_{x}^{\frac{6 N}{3 N-3 \gamma-2}}}\|v\|_{L_{t}^{\infty} L_{x}^{r_{2}}} \\
\leq & c_{N, \gamma}\left(\|u\|_{W^{s_{c}}}+\|v\|_{W^{s_{c}}}\right)\|u-v\|_{W^{s_{c}}}\left\||\nabla|^{s_{c}} v\right\|_{S^{0}} \\
& +c_{N, \gamma}\left(\|u\|_{W^{s_{c}}}+\|v\|_{W^{s_{c}}}\right)\left\||\nabla|^{s_{c}}(u-v)\right\|_{S^{0}}\|v\|_{W^{s_{c}}} .
\end{aligned}
$$

The rest of details are similar to the inter-critical case $\left(0<s_{c}<1\right)$.

Case $p>2$. Again, using triangle inequality and Lemma 2.4.7, we have

$$
\begin{aligned}
\left\|\phi_{u_{0}}(u)\right\|_{W^{s_{c}}} & =\left\|\phi_{u_{0}}(u)\right\|_{L_{t}^{\infty} L_{x}^{r_{1}}} \\
& \leq\left\|e^{i t \Delta} u_{0}\right\|_{L_{t}^{\infty} L_{x}^{r_{1}}}+c\left\||\nabla|^{s_{c}} \int_{0}^{t} e^{i\left(t-t^{\prime}\right) \Delta} N\left(u\left(t^{\prime}\right)\right) d t^{\prime}\right\|_{L_{t}^{\infty} L_{x}^{2}} \\
& \leq\left\|e^{i t \Delta} u_{0}\right\|_{L_{t}^{\infty} L_{x}^{r_{1}}}+c c_{1}\left\||\nabla|^{s_{c}} N(u)\right\|_{L_{t}^{q^{\prime}} L_{x}^{r^{\prime}}},
\end{aligned}
$$

where $(\infty, 2)$ and $\left(2, \frac{2 N}{N-2}\right)$ satisfy the conditions (2.4) and (2.5). Using the fractional product rule, we get

$$
\begin{aligned}
\left\||\nabla|^{s_{c}} N(u)\right\|_{L_{I}^{q^{\prime}} L_{x}^{r^{\prime}}} \leq & \left\|\left(|x|^{-(N-\gamma)} *|\nabla|^{s_{c}}\left(|u|^{p}\right)\right)|u|^{p-2} u\right\|_{L_{t}^{q^{\prime}} L_{x}^{r^{\prime}}} \\
& +\left\|\left(|x|^{-(N-\gamma)} *|u|^{p}\right)|\nabla|^{s_{c}}\left(|u|^{p-2} u\right)\right\|_{L_{t}^{q^{\prime}} L_{x}^{r^{\prime}}},
\end{aligned}
$$

where (3.57) is estimated using Hölder's inequality and Lemma 2.3.1 along with the formula for $s_{c}$

$$
\begin{aligned}
\text { (3.57) } & \leq\left\|\left(|x|^{-(N-\gamma)} *|\nabla|^{s_{c}}|u|^{p}\right)\right\|_{L_{t}^{q} L_{x}^{\frac{2 N}{N-\gamma}}}\|u\|_{L_{t}^{\infty} L_{x}^{r_{1}}}^{p-1} \\
& \leq c_{N, \gamma, p}\left\||u|^{p-1}|\nabla|^{s_{c}} u\right\|_{L_{t}^{q} L_{x}^{\frac{2 N}{N+\gamma}}}\|u\|_{L_{t}^{\infty} L_{x}^{r_{1}}}^{p-1} \\
& \leq c_{N, \gamma, p}\|u\|_{L_{t}^{\infty} L_{x}^{r_{1}^{1}}}^{2(p-1)}\left\|\left.\nabla\right|^{s_{c}} u\right\|_{L_{t}^{q} L_{x}^{r} .}
\end{aligned}
$$


For (3.58), we again use Hölder's inequality and Lemma 2.3.1 to obtain

$$
\begin{aligned}
(3.58) & \leq\left\||x|^{-(N-\gamma)} *|u|^{p}\right\|_{L_{I}^{\infty} \frac{2 N(p-1)}{2 p-\gamma(p-2)}}\|u\|_{L_{t}^{\infty} L_{x}^{r_{1}}}^{p-2}\left\||\nabla|^{s_{c}} u\right\|_{L_{I}^{q} L_{x}^{r}} \\
& \leq c_{N, \gamma, p}\|u\|_{L_{I}^{\infty} L_{x}^{r_{1}}}^{2(p-1)}\left\||\nabla|^{s_{c}} u\right\|_{L_{I}^{q} L_{x}^{r} .}
\end{aligned}
$$

Here, we again require $2 p-\gamma(p-2)>0$ as in (2.74), and therefore, $0<\gamma<$ $\min \left(N, \frac{2 p}{p-2}\right)$, see the statement of the Theorem 2.6.2 (4)(b) or Theorem 2.6.4 (4)(b). Combining (3.56) with (3.59) and (3.60), we get

$$
\left\|\phi_{u_{0}}(u)\right\|_{W^{s_{c}}} \leq\left\|e^{i t \Delta} u_{0}\right\|_{W^{s_{c}}}+2 c c_{1} c_{N, \gamma, p}\|u\|_{W^{s_{c}}}^{2(p-1)}\left\||\nabla|^{s_{c}} u\right\|_{S^{0}} .
$$

Similarly, we obtain

$$
\left\||\nabla|^{s_{c}} \Phi_{u_{0}}(u)\right\|_{S^{0}} \leq c_{1}\left\|u_{0}\right\|_{\dot{H}^{s_{c}}}+2 c_{1} c_{N, \gamma}\|u\|_{W^{s_{c}}}^{2(p-1)}\left\||\nabla|^{s_{c}} u\right\|_{S^{0}} .
$$

For contraction, we again define the same metric as before,

$$
d(u, v)=\left\||\nabla|^{s_{c}}(u-v)\right\|_{S^{0}}+\|u-v\|_{W^{s_{c}}},
$$

and use Lemmas 2.4.6 and 2.4.7 along with triangle inequality

$$
\begin{aligned}
\||\nabla|^{s_{c}}\left[\Phi_{u_{0}}(u)\right. & \left.-\Phi_{u_{0}}(v)\right] \|_{S\left(L^{2}\right)} \\
\leq & \left\||\nabla|^{s_{c}}\left[\left(|x|^{-(N-\gamma)} *|u|^{p}\right)\left(|u|^{p-2} u-|v|^{p-2} v\right)\right]\right\|_{L_{t}^{q^{\prime}} L_{x}^{r^{\prime}}} \\
& +\left\||\nabla|^{s_{c}}\left[\left(|x|^{-(N-\gamma)} *\left(|u|^{p}-|v|^{p}\right)\right)|v|^{p-2} v\right]\right\|_{L_{t}^{q^{\prime}} L_{x}^{r^{\prime}}}
\end{aligned}
$$

Using (2.18), Hölder's inequality and Lemma 2.3.1, we obtain

$$
\begin{gathered}
(3.61) \leq\left\||x|^{-(N-\gamma)} *|u|^{p}\right\|_{L_{t}^{\infty} L_{x}^{2 p-\gamma(p-2)}} \frac{2 N(p-1)}{2 p-\gamma}\left(\|u\|_{L_{t}^{\infty} L_{x}^{r_{1}}}^{p-2}\right. \\
+\left.\|v\|_{L_{t}^{\infty} L_{x}^{r_{1}}}^{p-2}\|\| \nabla\right|^{s_{c}}(u-v) \|_{L_{t}^{q} L_{x}^{r}} \\
+\left\||x|^{-(N-\gamma)} *|\nabla|^{s_{c}}\left(|u|^{p}\right)\right\|_{L_{t}^{q} L_{x}^{N-\gamma}} \frac{2 N}{N-\gamma}\left(\|u\|_{L_{t}^{\infty} L_{x}^{r_{1}}}^{p-2}+\|v\|_{L_{t}^{\infty} L_{x}^{r_{1}}}^{p-2}\right)\|u-v\|_{L_{t}^{\infty} L_{x}^{r_{1}}} \\
\leq c_{N, \gamma, p}\|u\|_{W^{s_{c}}}^{p}\left(\|u\|_{W^{s_{c}}}^{p-2}+\|v\|_{W^{s_{c}}}^{p-2}\right)\left\||\nabla|^{s_{c}}(u-v)\right\|_{S^{0}} \\
+c_{N, \gamma, p}\|u\|_{W^{s_{c}}}^{p-1}\left\|\left.\nabla\right|^{s_{c}} u\right\|_{S^{0}}\left(\|u\|_{W^{s_{c}}}^{p-2}+\|v\|_{W^{s_{c}}}^{p-2}\right)\|u-v\|_{W^{s_{c}}} .
\end{gathered}
$$


Similarly, using (2.19), Hölder's inequality and Lemma 2.3.1, we also obtain

$$
\begin{aligned}
(3.62) \leq & \left\||x|^{-(N-\gamma)} *\left(|u|^{p}-|v|^{p}\right)\right\|{ }_{L_{t}^{\infty} \frac{2 N(p-1)}{L_{x}^{2 p-\gamma(p-2)}}}\|v\|_{L_{t}^{\infty} L_{x}^{r_{1}}}^{p-2}\left\||\nabla|^{s_{c}} v\right\|_{L_{t}^{q} L_{x}^{r}} \\
& +\left\||x|^{-(N-\gamma)} *|\nabla|^{s_{c}}\left(|u|^{p}-|v|^{p}\right)\right\|_{L_{t}^{q} L_{x}^{\frac{2 N}{N-\gamma}}}\|v\|_{L_{t}^{\infty} L_{x}^{r_{1}}}^{p-1} \\
\leq & c_{N, \gamma, p}\left(\|u\|_{W^{s_{c}}}^{p-1}+\|v\|_{W^{s_{c}}}^{p-1}\right)\|u-v\|_{W^{s_{c}}}\|v\|_{W^{s_{c}}}^{p-1}\left\|\left.\nabla\right|^{s_{c}} v\right\|_{S^{0}} \\
& +c_{N, \gamma, p}\left(\|u\|_{W^{s_{c}}}^{p-1}+\|v\|_{W^{s_{c}}}^{p-1}\right)\left\||\nabla|^{s_{c}}(u-v)\right\|_{S^{0}}\|v\|_{W^{s_{c}}}^{p-1} .
\end{aligned}
$$

Rest of the details are similar to the inter-critical case $\left(0<s_{c}<1\right)$.

This concludes the proof of Proposition 3.2.2.

We also have the local well-posedness in $\dot{H}^{s}$ (for $s>s_{c}$ ) (Proposition 2.7.1), as a result one can pose a question: whether it is possible to obtain the global existence for small data in $\dot{H}^{s}$ for $s>s_{c}$ ? The answer is yes, which we investigate next. The following Proposition is proved using Besov spaces (it is also possible to prove it without Besov spaces, for this work we show one possible approach). We define

$$
\|u\|_{\dot{B}_{S\left(\dot{H}^{s}\right)}^{0}} \stackrel{\text { def }}{=}\|u\|_{L_{t}^{q_{2}} \dot{B}_{r_{1}, 2}^{s_{c}}} \text { and }\|u\|_{\dot{B}_{S\left(L^{2}\right)}^{s}} \stackrel{\text { def }}{=}\|u\|_{L_{t}^{q_{1}} \dot{B}_{r_{1}, 2}^{s_{c}}},
$$

where $\left(q_{2}, r_{1}\right)$ and $\left(q_{1}, r_{1}\right)$ are defined in (3.9) and (3.6), respectively. We recall that with these pairs we can consider the whole range defined by (2.10).

Proposition 3.2.3 (Small data theory in $\dot{H}^{s}$ ) Let $p \geq 2, N \geq 1$ and $0<\gamma<N$ so that $0<s_{c}<1$. Suppose $s>s_{c}$ and assume that $u_{0} \in \dot{H}^{s}\left(\mathbb{R}^{N}\right)$. Also, assume that $\left\|u_{0}\right\|_{\dot{H}^{s}} \leq A$. There exists $\delta=\delta(A)>0$ such that if $\left\|e^{i t \Delta} u_{0}\right\|_{\dot{B}_{S\left(\dot{H}^{s}\right)}^{0}} \leq \delta$, then the solution $u$ of (1.1) exists globally in $\dot{H}^{s}\left(\mathbb{R}^{N}\right)$ such that

$$
\|u\|_{\dot{B}_{S\left(\dot{H}^{s c}\right)}^{0}} \leq 2\left\|e^{i t \Delta} u_{0}\right\|_{\dot{B}_{S\left(\dot{H}^{s c}\right)}^{0}}
$$

and

$$
\|u\|_{\dot{B}_{S\left(L^{2}\right)}^{s}} \leq 2 c\left\|u_{0}\right\|_{\dot{H}^{s}}
$$




\section{Proof. Denote}

$$
\mathcal{X}_{s d}=\left\{u:\|u\|_{\dot{B}_{S\left(\dot{H}^{s}\right)}^{0}} \leq 2\left\|e^{i t \Delta} u_{0}\right\|_{\dot{B}_{S\left(\dot{H}^{s}\right)}^{0}},\|u\|_{\dot{B}_{S\left(L^{2}\right)}^{s}} \leq 2 c\left\|u_{0}\right\|_{\dot{H}^{s}}\right\}
$$

and consider $\Phi_{u_{0}}$ as defined in (3.13). Combining triangle inequality and Lemma 2.4.9 along with (2.15), we obtain

$$
\left\|\Phi_{u_{0}}(u)\right\|_{\dot{B}_{S\left(\dot{H}^{\left.s_{c}\right)}\right.}^{0}} \leq\left\|e^{i t \Delta} u_{0}\right\|_{\dot{B}_{S\left(\dot{H}^{\left.s_{c}\right)}\right.}^{0}}+c\left\||\nabla|^{s_{c}} F(u)\right\|_{S^{\prime}\left(L^{2}\right)},
$$

where $F(u)$ is defined in (2.24). We use the estimates (2.46) and (2.47) from Proposition 2.6.3 along with (2.13) to rewrite (3.65) as

$$
\left\|\Phi_{u_{0}}(u)\right\|_{\dot{B}_{S\left(\dot{H}^{\left.s_{c}\right)}\right.}^{0}} \leq\left\|e^{i t \Delta} u_{0}\right\|_{\dot{B}_{S\left(\dot{H}^{s} s^{\prime}\right)}^{0}}+2 c_{1}\|u\|_{\dot{B}_{S\left(\dot{H}^{s}\right)}^{0}}^{2(p-1)}\|u\|_{\dot{B}_{S\left(L^{2}\right)}^{s}},
$$

where $c_{1}:=c c_{p, \gamma, N}$. Thus, for $u \in \mathcal{X}_{s d}$, (3.66) gives

$$
\left\|\Phi_{u_{0}}(u)\right\|_{\dot{B}_{S\left(\dot{H}^{s_{c}}\right)}^{0}} \leq\left\|e^{i t \Delta} u_{0}\right\|_{\dot{B}_{S\left(\dot{H}^{s_{c}}\right)}^{0}}\left(1+2^{2 p} c_{1} c\left\|e^{i t \Delta} u_{0}\right\|_{\dot{B}_{S\left(\dot{H}^{s}\right)}^{0}}^{2 p-3} A\right),
$$

thus, we require

$$
2^{2 p} c_{1} c\left\|e^{i t \Delta} u_{0}\right\|_{\dot{B}_{S\left(H^{s_{c}}\right)}^{0}}^{2 p-3} A \leq 1
$$

Using Lemma 2.4.9 along with (2.14), we have

$$
\left\|\Phi_{u_{0}}(u)\right\|_{\dot{B}_{S\left(L^{2}\right)}^{s}} \leq c\left\|u_{0}\right\|_{\dot{H}^{s}}+\left.c\|\| \nabla\right|^{s} F(u) \|_{S^{\prime}\left(L^{2}\right)} .
$$

Again, using (2.46) and (2.47) along with (2.13), we write (3.69) for $u \in \mathcal{X}_{s d}$ as

$$
\left\|\Phi_{u_{0}}(u)\right\|_{\dot{B}_{S\left(L^{2}\right)}^{s}} \leq c\left\|u_{0}\right\|_{\dot{H}^{s}}\left(1+2^{2 p} c_{1}\left\|e^{i t \Delta} u_{0}\right\|_{\left.\dot{B}_{S\left(\dot{H}^{s}\right)}^{0}-1\right)}^{2(p-1)}\right),
$$

thus, we require

$$
2^{2 p} c_{1}\left\|e^{i t \Delta} u_{0}\right\|_{\dot{B}_{S\left(\dot{H}^{s}\right)}^{0}}^{2(p-1)} \leq 1
$$

Recalling that $\left\|e^{i t \Delta} u_{0}\right\|_{\dot{B}_{S\left(\dot{H}^{s}\right)}^{0}}<\delta$, then from (3.68) and (3.70), choosing

$$
\delta<\delta_{0} \leq \min \left(\frac{1}{\sqrt[2 p-3]{2^{2 p+1} c_{1} c A}}, \frac{1}{\sqrt[2(p-1)]{2^{2 p+1} c_{1}}}\right)
$$


implies that $\Phi_{u_{0}} \in \mathcal{X}_{s d}$. Now we show that $\Phi_{u_{0}}(u)$ is a contraction on $\mathcal{X}_{s d}$ with the metric

$$
d(u, v)=\|u-v\|_{\dot{B}_{S\left(\dot{H}^{\left.s_{c}\right)}\right.}^{0}}+\|u-v\|_{\dot{B}_{S\left(L^{2}\right)}^{s}} .
$$

For $u, v \in \mathcal{X}_{s d}$, by Lemma 2.4.9 together with (2.15), we obtain

$$
\left\|\Phi_{u_{0}}(u)-\Phi_{u_{0}}(v)\right\|_{\dot{B}_{S\left(\dot{H}^{s}\right)}^{0}} \leq c\|F(u)-F(v)\|_{S^{\prime}\left(\dot{H}^{-s_{c}}\right)} .
$$

Similarly, from Lemma 2.4.9 and (2.14), we get

$$
\left\|\Phi_{u_{0}}(u)-\Phi_{u_{0}}(v)\right\|_{\dot{B}_{S\left(L^{2}\right)}^{s}} \leq c\left\||\nabla|^{s}(F(u)-F(v))\right\|_{S^{\prime}\left(L^{2}\right)}
$$

Using the estimate (3.26) for (3.71) along with (2.13), we have

$$
\left\|\Phi_{u_{0}}(u)-\Phi_{u_{0}}(v)\right\|_{\dot{B}_{S\left(\dot{H}^{s}\right)}^{0}} \leq 2^{2 p+1} c_{1} c\left\|e^{i t \Delta} u_{0}\right\|_{\dot{B}_{S\left(\dot{H}^{s}\right)}^{0}}^{2(p-1)}\|u-v\|_{\dot{B}_{S\left(\dot{H}^{s}\right)}^{0}}
$$

and for (3.72), we use the estimates (2.46) and (2.47) (to perform similar calculations as in (2.51) and (2.52)) along with (2.13) to get

$$
\left\|\Phi_{u_{0}}(u)-\Phi_{u_{0}}(v)\right\|_{\dot{B}_{S\left(L^{2}\right)}^{s}} \leq 2^{2 p+1} c_{1} c\left\|e^{i t \Delta} u_{0}\right\|_{\dot{B}_{S\left(H^{s} c\right)}^{0}}^{2(p-1)}\|u-v\|_{\dot{B}_{S\left(L^{2}\right)}^{s}}
$$

Combining (3.73) and (3.74), we obtain

$$
d\left(\Phi_{u_{0}}(u), \Phi_{u_{0}}(v)\right) \leq 2^{2(p+1)} c c_{1}\left\|e^{i t \Delta} u_{0}\right\|_{\dot{B}_{S\left(\dot{H}^{s c}\right)}^{0}}^{2(p-1)} d(u, v) \leq \frac{1}{2} d(u, v)
$$

for $\delta_{1}<\sqrt[2(p-1)]{\left(2^{2 p+3} c c_{1}\right)^{-1}}$. Taking $\delta \leq \min \left(\delta_{0}, \delta_{1}\right)$ implies that $\Phi_{u_{0}}$ is a contraction.

Next we establish the scattering in $H^{1}\left(\mathbb{R}^{N}\right)$.

Theorem 3.2.4 ( $H^{1}$ scattering) Let $u(t)$ be a global solution to (1.1) with initial data $u_{0} \in H^{1}\left(\mathbb{R}^{N}\right)$. If u is globally finite in the $\dot{H}^{s_{c}}$-admissible Strichartz norm, i.e., $\|u\|_{S\left(\dot{H}^{\left.s_{c}\right)}\right.}<$ $+\infty$ and uniformly bounded in $H^{1}\left(\mathbb{R}^{N}\right)$ norm, i.e., $\sup _{t \in \mathbb{R}^{+}}\|u(t)\|_{H^{1}} \leq B$, then $u(t)$ scatters in $H^{1}\left(\mathbb{R}^{N}\right)$ as $t \rightarrow+\infty$, i.e., there exists $u^{+} \in H^{1}\left(\mathbb{R}^{N}\right)$ such that

$$
\lim _{t \rightarrow+\infty}\left\|u(t)-e^{i t \Delta} u^{+}\right\|_{H^{1}}=0
$$


Proof. The assumption $\|u\|_{S\left(\dot{H}^{s_{c}}\right)}<+\infty$ implies that there exists $M$ such that

$$
M=\|u\|_{L_{t}^{1-s_{c}}} \frac{2 p p}{L_{x}^{N+\gamma}}<+\infty .
$$

Recall that $\left(\frac{2 p}{1-s_{c}}, \frac{2 N p}{N+\gamma}\right)$ is an $\dot{H}^{s_{c}}$-admissible pair. Let $\widetilde{M}=M^{\frac{2 p}{1-s_{c}}}$. Given $\delta>0$ we can decompose $[0,+\infty)=\cup_{j=1}^{\widetilde{M}} I_{j}$, where $I_{j}=\left[t_{j}, t_{j+1}\right)$ such that for each $j$, we have

$$
\|u\|_{L_{I_{j}}^{\frac{2 p}{1-s_{c}}} L_{x}^{\frac{2 N p}{N+\gamma}}}<\delta
$$

Hence, by the triangle inequality and Lemma (2.4.6) applied to the integral equation (2.16) on $I_{j}$, we have

$$
\|u\|_{S\left(L^{2} ; I_{j}\right)} \leq c\left\|u\left(t_{j}\right)\right\|_{L^{2}}+c\left\|\left(|x|^{-(N-\gamma)} *|u|^{p}\right)|u|^{p-2} u\right\|_{S^{\prime}\left(L^{2} ; I_{j}\right)}
$$

From (3.19), we have

$$
\left\|\left(|x|^{-(N-\gamma)} *|u|^{p}\right)|u|^{p-2} u\right\|_{S^{\prime}\left(L^{2} ; I_{j}\right)} \leq c_{N, p, \gamma}\|u\|_{S\left(\dot{H}^{s^{c}} ; I_{j}\right)}^{2(p-1)}\|u\|_{S\left(L^{2} ; I_{j}\right)} .
$$

Thus, (3.75) combined with (3.76) and the assumption $\sup _{t \in \mathbb{R}^{+}}\|u(t)\|_{H^{1}} \leq B$ implies

$$
\|u\|_{S\left(L^{2} ; I_{j}\right)} \leq c B+c_{1} \delta^{2(p-1)}\|u\|_{S\left(L^{2} ; I_{j}\right)}
$$

Similarly, using Lemma 2.4.7 for $s=1$ along with (3.21) yields

$$
\begin{aligned}
\|\nabla u\|_{S\left(L^{2} ; I_{j}\right)} & \leq c\left\|\nabla u\left(t_{j}\right)\right\|_{L}^{2}+c\left\|\nabla\left(\left(|x|^{-(N-\gamma)} *|u|^{p}\right)|u|^{p-2} u\right)\right\|_{S^{\prime}\left(L^{2} ; I_{j}\right)} \\
& \lesssim c B+2 c_{1}\|u\|_{S\left(\dot{H}^{s_{c}} ; I_{j}\right)}^{2(p-1)}\|\nabla u\|_{S\left(L^{2} ; I_{j}\right)} \\
& \lesssim c B+2 c_{1} \delta^{2(p-1)}\|\nabla u\|_{S\left(L^{2} ; I_{j}\right)} .
\end{aligned}
$$

Combining (3.77) and (3.78), we get

$$
\|u\|_{S\left(L^{2} ; I_{j}\right)}+\|\nabla u\|_{S\left(L^{2} ; I_{j}\right)} \leq 2 c B+2 c_{1} \delta^{2(p-1)}\left(\|u\|_{S\left(L^{2} ; I_{j}\right)}+\|\nabla u\|_{S\left(L^{2} ; I_{j}\right)}\right) .
$$

Performing the summation over $I_{j}$, we obtain

$$
\|u\|_{S\left(L^{2}\right)}+\|\nabla u\|_{S\left(L^{2}\right)} \leq 2 c B M^{\frac{2 p}{1-s_{c}}}+2 c_{1} \delta^{2(p-1)}\left(\|u\|_{S\left(L^{2}\right)}+\|\nabla u\|_{S\left(L^{2}\right)}\right)
$$


which implies that

$$
\left(1-2 c_{1} \delta^{2(p-1)}\right)\left(\|u\|_{S\left(L^{2}\right)}+\|\nabla u\|_{S\left(L^{2}\right)}\right) \lesssim 2 c B M^{\frac{2 p}{1-s_{c}}} .
$$

Thus, for small $\delta$, we require that $1-2 \delta^{2(p-1)} \leq \frac{1}{2}$, so that

$$
\|u\|_{S\left(L^{2}\right)}+\|\nabla u\|_{S\left(L^{2}\right)} \leq 4 c B M^{\frac{2 p}{1-s_{c}}}
$$

Now, we define the wave operator

$$
u^{+}=u_{0}+i \int_{0}^{+\infty} e^{-i t^{\prime} \Delta}\left(|x|^{-(N-\gamma)} *|u|^{p}\right)|u|^{p-2} u\left(t^{\prime}\right) d t^{\prime} .
$$

By the same arguments as before, we have

$$
\left\|u^{+}\right\|_{L^{2}} \leq c\left\|u_{0}\right\|_{L^{2}}+c_{1}\|u\|_{S\left(\dot{H}^{s_{c}}\right)}^{2(p-1)}\|u\|_{S\left(L^{2}\right)},
$$

and

$$
\left\|\nabla u^{+}\right\|_{L^{2}} \leq c\left\|\nabla u_{0}\right\|_{L^{2}}+2 c_{1}\|u\|_{S\left(\dot{H}^{s_{c}}\right)}^{2(p-1)}\|\nabla u\|_{S\left(L^{2}\right)} .
$$

Finally, by initial assumptions, we get

$$
\left\|u^{+}\right\|_{L^{2}}+\left\|\nabla u^{+}\right\|_{L^{2}} \leq c B+2 c_{1} M^{2(p-1)}\left(\|u\|_{S\left(L^{2}\right)}+\|\nabla u\|_{S\left(L^{2}\right)}\right) .
$$

Using (3.79), we obtain that $\left\|u^{+}\right\|_{H^{1}} \leq$ constant. This implies that $u^{+} \in H^{1}\left(\mathbb{R}^{N}\right)$. From (3.80) and the integral equation (2.16), we have

$$
u(t)-e^{i t \Delta} u^{+}=-i \int_{t}^{+\infty} e^{i\left(t-t^{\prime}\right) \Delta}\left(|x|^{-(N-\gamma)} *|u|^{p}\right)|u|^{p-2} u\left(t^{\prime}\right) d t^{\prime}
$$

Again using the similar computation, we obtain

$$
\left\|u(t)-e^{i t \Delta} u^{+}\right\|_{L^{2}} \leq c_{1}\|u\|_{\left.S\left(\dot{H}^{s_{c} ;} ; t,+\infty\right)\right)}^{2(p-1)}\|u\|_{S\left(L^{2} ;[t,+\infty)\right)},
$$

and

$$
\left\|\nabla\left(u(t)-e^{i t \Delta} u^{+}\right)\right\|_{L^{2}} \leq c_{1}\|u\|_{\left.S\left(\dot{H}^{s_{c} ;} ; t,+\infty\right)\right)}^{2(p-1)}\|\nabla u\|_{S\left(L^{2} ;[t,+\infty)\right)} .
$$


While obtaining (3.79), we have observed that the Strichartz norm on $[0,+\infty)$ for the above expression is bounded, therefore, the tail has to vanish as $t \rightarrow+\infty$, and thus, $\|u\|_{S\left(\dot{H}^{\left.s^{c} ;[t,+\infty)\right)}\right.} \rightarrow 0$ as $t \rightarrow+\infty$. Hence,

$$
\lim _{t \rightarrow+\infty}\left\|u(t)-e^{i t \Delta} u^{+}\right\|_{\dot{H}^{1}}=0
$$

We note that Theorem 3.2.4 with initial data $u_{0} \in H^{1}\left(\mathbb{R}^{N}\right)$ also holds in the $L^{2}$-critical case $\left(s_{c}=0\right.$ or $\left.p=1+\frac{\gamma+2}{N} \geq 2\right)$. One can also obtain a similar result for the energycritical case $\left(s_{c}=1\right)$ but with a different selection of Strichartz pairs.

We now prove the long time perturbation result in the spirit of [HR08], which is one of the necessary ingredients in the subsequent analysis, specifically, used in Theorem 3.4.3.

Theorem 3.2.5 (Long time perturbation) For each $A \gg 1$, there exists $\epsilon_{0}=\epsilon_{0}(A) \ll$ 1 and $c=c(A) \gg 1$ such that the following holds. Let $u=u(x, t) \in H^{1}\left(\mathbb{R}^{N}\right)$ for all time t and solve (1.1). Let $\widetilde{u}=\widetilde{u}(x, t) \in H^{1}\left(\mathbb{R}^{N}\right)$ for all $t$ and define e to be

$$
e \stackrel{\text { def }}{=} i \widetilde{u}_{t}+\Delta \widetilde{u}+\left(|x|^{-(N-\gamma)} *|\widetilde{u}|^{p}\right)|\widetilde{u}|^{p-2} \widetilde{u}
$$

Suppose that

$$
\|\widetilde{u}\|_{S\left(\dot{H}^{s_{c}}\right)} \leq A, \quad\|e\|_{S^{\prime}\left(\dot{H}^{-s_{c}}\right)} \leq \epsilon_{0}
$$

and

$$
\left\|e^{i\left(t-t_{0}\right) \Delta}\left(u\left(t_{0}\right)-\widetilde{u}_{0}\left(t_{0}\right)\right)\right\|_{S\left(\dot{H}^{s_{c}}\right)} \leq \epsilon_{0} .
$$

Then

$$
\|u\|_{S\left(\dot{H}^{s_{c}}\right)} \leq c=c(A)<+\infty .
$$


Proof. Denote by $w$ the perturbation of $u$ : $w=u-\widetilde{u}$. Set $W(\widetilde{u}, w)=F(u)-F(\widetilde{u})=$ $F(\widetilde{u}+w)-F(\widetilde{u})$, for $F(u)=\left(|x|^{-(N-\gamma)} *|u|^{p}\right)|u|^{p-2} u$. Then, $w$ solves

$$
i w_{t}+\Delta w+W(\widetilde{u}, w)-e=0
$$

Since $\|\widetilde{u}\|_{S\left(\dot{H}^{s}\right)} \leq A$, we can partition the interval $\left[t_{0},+\infty\right)$ into $K=K(A)$ intervals $I_{j}=$ $\left[t_{j}, t_{j+1}\right]$ such that for each $j,\|\widetilde{u}\|_{S\left(\dot{H}^{\left.s_{c} ; I_{j}\right)}\right.} \leq \delta$. Note that the number of intervals depends only on $A$, however, the intervals themselves depend upon $\widetilde{u}$. The integral equation of $w$ at time $t_{j}$ is given by

$$
w(t)=e^{i\left(t-t_{j}\right) \Delta} w\left(t_{j}\right)+i \int_{t_{j}}^{t} e^{i\left(t-t^{\prime}\right) \Delta}(W-e)\left(t^{\prime}\right) d t^{\prime} .
$$

Applying Lemma 2.4.6 to (3.84) for each $I_{j}$, we obtain

$$
\begin{aligned}
\|w\|_{S\left(\dot{H}^{\left.s_{c} ; I_{j}\right)}\right.} & \leq\left\|e^{i\left(t-t_{j}\right) \Delta} w\left(t_{j}\right)\right\|_{S\left(\dot{H}^{s_{c} ; I_{j}}\right)}+c\|W(\widetilde{u}, w)\|_{S^{\prime}\left(\dot{H}^{\left.-s_{c} ; I_{j}\right)}\right.}+c\|e\|_{S^{\prime}\left(\dot{H}^{-s_{c} ; I_{j}}\right)} \\
& \leq\left\|e^{i\left(t-t_{j}\right) \Delta} w\left(t_{j}\right)\right\|_{S\left(\dot{H}^{s_{c} ; I_{j}}\right)}+c\|W(\widetilde{u}, w)\|_{S^{\prime}\left(\dot{H}^{-s_{c} ; I_{j}}\right)}+c \epsilon_{0} .
\end{aligned}
$$

Next we estimate

$$
\|W(\widetilde{u}, w)\|_{S^{\prime}\left(\dot{H}^{-s_{c} ; I_{j}}\right)} \lesssim\|F(\widetilde{u}+w)-F(\widetilde{u})\|_{L_{I_{j}}^{q_{3}^{\prime}} L_{x}^{r_{1}^{\prime}}}
$$

Adding and subtracting $\left(|x|^{-(N-\gamma)} *|\widetilde{u}+w|^{p}\right)|\widetilde{u}|^{p-2} \widetilde{u}$, we obtain

$$
\begin{aligned}
\|W(\widetilde{u}, w)\|_{S^{\prime}\left(\dot{H}^{-s_{c}} ; I_{j}\right)} \lesssim & \left\|\left(|x|^{-(N-\gamma)} *|\widetilde{u}+w|^{p}\right)\left(|\widetilde{u}+w|^{p-2}(\widetilde{u}+w)-|\widetilde{u}|^{p-2} \widetilde{u}\right)\right\|_{L_{I_{j}}^{q_{3}^{\prime}} L_{x}^{r_{1}^{\prime}}} \\
& +\left\|\left(|x|^{-(N-\gamma)} *\left(|\widetilde{u}+w|^{p}-|\widetilde{u}|^{p}\right)\right)|\widetilde{u}|^{p-2} \widetilde{u}\right\|_{L_{I_{j}}^{q_{3}^{\prime}} L_{x}^{r_{1}^{\prime}}}
\end{aligned}
$$

Using the calculations similar to (3.36), we get

$$
\begin{aligned}
\|W(\widetilde{u}, w)\|_{S^{\prime}\left(\dot{H}^{-s_{c}} ; I_{j}\right)} \leq & c_{N, \gamma}\|\widetilde{u}+w\|_{L_{I_{j}}^{q_{2}} L_{x}^{r_{1}}}^{p}\left\|\widetilde{u}+\left.w\right|^{p-2}(\widetilde{u}+w)-|\widetilde{u}|^{p-2} \widetilde{u}\right\|_{L_{I_{j}}^{\frac{q_{2}}{p-1}} \frac{r_{1}}{L_{x}^{p-1}}} \\
& +c_{N, \gamma}\|\| \widetilde{u}+\left.w\right|^{p}-|\widetilde{u}|^{p} \|_{L_{I_{j}}^{\frac{q_{2}}{p}} L_{x}^{\frac{r_{1}}{p}}\|\widetilde{u}\|_{L_{I_{j}}^{q_{2}} L_{x}^{r_{1}}}^{p-1}}
\end{aligned}
$$


Using (2.18) and (2.19) yields

$$
\begin{aligned}
\|W(\widetilde{u}, w)\|_{S^{\prime}\left(\dot{H}^{-s_{c}} ; I_{j}\right)} \leq & c_{N, \gamma}\|\widetilde{u}+w\|_{L_{I_{j}}^{q_{2}} L_{x}^{r_{1}}}^{p}\|w\|_{L_{I_{j}}^{q_{2}} L_{x}^{r_{1}}}\left(\|\widetilde{u}+w\|_{L_{I_{j}}^{q_{2}} L_{x}^{r_{1}}}^{p-2}+\|\widetilde{u}\|_{L_{I_{j}}^{q_{2}} L_{x}^{r_{1}}}^{p-2}\right) \\
& +c_{N, \gamma}\|w\|_{L_{I_{j}}^{q_{2}} L_{x}^{r_{1}}}\left(\|\widetilde{u}+w\|_{L_{I_{j}}^{q_{2}} L_{x}^{r_{1}}}^{p-1}+\|\widetilde{u}\|_{L_{I_{j}}^{q_{2}} L_{x}^{r_{1}}}^{p-1}\right)\|\widetilde{u}\|_{L_{I_{j}}^{q_{2}} L_{x}^{r_{1}}}^{p-1}
\end{aligned}
$$

We use the fact that $(a+b)^{p} \lesssim_{p} a^{p}+b^{p}$ for the $\|\widetilde{u}+w\|_{L_{I_{j}}^{q_{2}} L_{x}^{r_{1}}}$ terms in (3.86) and (3.87) to obtain

$$
\begin{aligned}
\|W(\widetilde{u}, w)\|_{S^{\prime}\left(\dot{H}-s_{c} ; I_{j}\right)} \lesssim & c_{N, \gamma}\left(\|\widetilde{u}\|_{L_{I_{j}}^{q_{2}} L_{x}^{r_{1}}}^{p}+\|w\|_{L_{I_{j}}^{q_{2}} L_{x}^{r_{1}}}^{p}\right)\|w\|_{L_{I_{j}}^{q_{2}} L_{x}^{r_{1}}}\left(\|w\|_{L_{I_{j}}^{q_{2}} L_{x}^{r_{1}}}^{p-2}+\|\widetilde{u}\|_{L_{I_{j}}^{q_{2}} L_{x}^{r_{1}}}^{p-2}\right) \\
& +c_{N, \gamma}\|w\|_{L_{I_{j}}^{q_{2}} L_{x}^{r_{1}}}\left(\|\widetilde{u}\|_{L_{I_{j}}^{q_{2}} L_{x}^{r_{1}}}^{p-1}+\|w\|_{L_{I_{j}}^{q_{2}} L_{x}^{r_{1}}}^{p-1}\right)\|\widetilde{u}\|_{L_{I_{j}}^{q_{2}} L_{x}^{r_{1}}}^{p-1} .
\end{aligned}
$$

Since $\left(q_{2}, r_{1}\right)$ is a $\dot{H}^{s_{c}}$ admissible pair by our assumption $\|\widetilde{u}\|_{S\left(\dot{H}^{s_{c}} ; I_{j}\right)} \leq \delta$, we obtain

$$
\begin{aligned}
\|W(\widetilde{u}, w)\|_{S^{\prime}\left(\dot{H}^{-s_{c} ; I_{j}}\right)} \lesssim & c_{N, \gamma}\left(\delta^{p}+\|w\|_{L_{I_{j}}^{q_{2}} L_{x}^{r_{1}}}^{p}\right)\|w\|_{L_{I_{j}}^{q_{2}} L_{x}^{r_{1}}}\left(\|w\|_{L_{I_{j}}^{q_{2}} L_{x}^{r_{1}}}^{p-2}+\delta^{p-2}\right) \\
& +c_{N, \gamma}\|w\|_{L_{I_{j}}^{q_{2}} L_{x}^{r_{1}}}\left(\delta^{p-1}+\|w\|_{L_{I_{j}}^{q_{2}} L_{x}^{r_{1}}}^{p-1}\right) \delta^{p-1} .
\end{aligned}
$$

Substituting the above estimate in (3.85),

$$
\begin{aligned}
\|w\|_{S\left(\dot{H}^{s_{c} ; I_{j}}\right)} \lesssim & \left\|e^{i\left(t-t_{j}\right) \Delta} w\left(t_{j}\right)\right\|_{S\left(\dot{H}^{s} ; I_{j}\right)}+c_{1} \delta^{p}\|w\|_{S\left(\dot{H}^{s} ; I_{j}\right)}^{p-1}+2 c_{1} \delta^{2(p-1)}\|w\|_{S\left(\dot{H}^{s_{c} ; I_{j}}\right)} \\
& +c_{1} \delta^{p-2}\|w\|_{S\left(\dot{H}^{s} ; I_{j}\right)}^{p+1}+c_{1} \delta^{p-1}\|w\|_{S\left(\dot{H}^{s} ; I_{j}\right)}^{p}+c_{1}\|w\|_{S\left(\dot{H}^{\left.s_{c} ; I_{j}\right)}\right.}^{2 p-1}+c \epsilon_{0} .
\end{aligned}
$$

Let $\|w\|_{S\left(\dot{H}^{\left.s_{c} ; I_{j}\right)}\right.} \leq \widetilde{c} \delta$. If $c_{1} \widetilde{c} \delta^{2(p-1)} \leq \frac{1}{12}$, by choosing $\delta \leq \min \left(1, \delta_{1}\right)$, where $\delta_{1}=$ $\sqrt[2(p-1)]{\frac{1}{12 c_{1} \widetilde{c}}}$ together with (3.82), we can make sure that at time $t_{j},\left\|e^{i\left(t-t_{j}\right) \Delta} w\left(t_{j}\right)\right\|_{S\left(\dot{H}^{s_{c}}\right)} \leq$ $\epsilon_{1}$, where $\epsilon_{1}$ depends on $\epsilon_{0}$, thus, we take

$$
\left\|e^{i\left(t-t_{j}\right) \Delta} w\left(t_{j}\right)\right\|_{S\left(\dot{H}^{\left.s^{c} ; I_{j}\right)}\right.}+c \epsilon_{0} \leq \min \left(1, \frac{\delta_{1}}{2}\right) .
$$

Therefore, (3.88) ensures that,

$$
\|w\|_{S\left(\dot{H}^{s_{c} ; I_{j}}\right)} \leq 2\left\|e^{i\left(t-t_{j}\right) \Delta} w\left(t_{j}\right)\right\|_{S\left(\dot{H}^{s_{c}} ; I_{j}\right)}+2 c \epsilon_{0} .
$$


Taking $t=t_{j+1}$ in (3.84), applying $e^{i\left(t-t_{j+1}\right) \Delta}$ to both sides and repeating the similar argument used for (3.89) (since the Duhamel integral is confined to $I_{j}=\left[t_{j}, t_{j+1}\right]$ ), we obtain

$$
\left\|e^{i\left(t-t_{j+1}\right) \Delta} w\left(t_{j+1}\right)\right\|_{S\left(\dot{H}^{s_{c}}\right)} \leq 2\left\|e^{i\left(t-t_{j}\right) \Delta} w\left(t_{j}\right)\right\|_{S\left(\dot{H}^{s_{c}}\right)}+2 c \epsilon_{0}
$$

Iterating down to $j=0$ and using (3.82), we get

$$
\left\|e^{i\left(t-t_{j}\right) \Delta} w\left(t_{j}\right)\right\|_{S\left(\dot{H}^{s_{c}}\right)} \leq 2^{j}\left\|e^{i\left(t-t_{0}\right) \Delta} w\left(t_{0}\right)\right\|_{S\left(\dot{H}^{s_{c}}\right)}+\left(2^{j}-1\right) 2 c \epsilon_{0} \leq 2^{j+2} c \epsilon_{0} .
$$

Now to satisfy the assumption (3.88) for all intervals $I_{j}, 0 \leq j \leq n-1$, we require that

$$
2^{n+2} c \epsilon_{0} \leq \min \left(1, \frac{\delta_{1}}{2}\right) .
$$

This quantifies $\epsilon_{0}$ in terms of $n$ (number of time subintervals), which is determined by $A$ (given). Hence, substituting $w=u-\tilde{u}$ on the left-hand side of (3.84) and applying Lemma 2.4.6, we obtain

$$
\|u\|_{S\left(\dot{H}^{s c}\right)} \leq\left\|e^{i\left(t-t_{j}\right) \Delta} w\left(t_{j}\right)\right\|_{S\left(\dot{H}^{s_{c}}\right)}+c\|W(\widetilde{u}, w)\|_{S^{\prime}\left(\dot{H}^{\left.-s_{c} ; I_{j}\right)}\right.}+c \epsilon_{0}+\|\widetilde{u}\|_{S\left(\dot{H}^{s^{c}}\right)}
$$

Thus, by repeating the argument used to deduce (3.89) and using (3.90) (3.81) and (3.91), we can conclude that

$$
\|u\|_{S\left(\dot{H}^{s_{c}}\right)} \leq c(A)
$$

\subsection{Dichotomy: Global vs blow up solutions}

In Section 3.3 we obtain the proof of Theorem 3.1.1 part (1)(a) and part (2). We show that the condition in Theorem 3.1.1 is sharp.

Theorem 3.3.1 Consider (1.1) with $u_{0} \in H^{1}\left(\mathbb{R}^{N}\right)$ and $0<s_{c}<1$. Assume that

$$
\mathcal{M E}\left[u_{0}\right]<1
$$




$$
\mathcal{G}\left[u_{0}\right]<1
$$

then the solution $u(t)$ exists for all $t \in \mathbb{R}$ (i.e., $I=\mathbb{R})$, and

$$
\mathcal{G}[u(t)]<1
$$

If

$$
\mathcal{G}\left[u_{0}\right]>1
$$

then for $t \in I=(-T, T)$

$$
\mathcal{G}[u(t)]>1
$$

Moreover, if either $x\left|u_{0}\right| \in L^{2}\left(\mathbb{R}^{N}\right)$ or $u_{0}$ is radial, then I is finite, and thus, the solution blows up in finite time.

The proof of this theorem goes along the established convexity arguments and the relevant Gagliardo-Nirenberg inequality with its sharp constant, the constants and coefficients are specific for the generalized Hartree case. The localized virial part deals with the convolution term, and thus, is new.

Proof. Using the energy conservation and (2.126), we have

$$
\begin{aligned}
& \mathcal{M E}[u]=\left(\frac{1}{2}\|\nabla u\|_{L^{2}\left(\mathbb{R}^{N}\right)}^{2}\left\|u_{0}\right\|_{L^{2}\left(\mathbb{R}^{N}\right)}^{2 \theta}-\frac{1}{2 p} Z(u)\left\|u_{0}\right\|_{L^{2}\left(\mathbb{R}^{N}\right)}^{2 \theta}\right) \frac{1}{M[Q]^{\theta} E[Q]} \\
& \geq\left(\frac{1}{2}\|\nabla u\|_{L^{2}\left(\mathbb{R}^{N}\right)}^{2}\left\|u_{0}\right\|_{L^{2}\left(\mathbb{R}^{N}\right)}^{2 \theta}-\frac{C_{G N}}{2 p}\left(\|\nabla u\|_{L^{2}}\left\|u_{0}\right\|_{L^{2}}^{\theta}\right)^{2 s_{c}(p-1)+2}\right) \frac{1}{M[Q]^{\theta} E[Q]} .
\end{aligned}
$$

Using (2.135) and (2.136) and the value of $C_{G N}$, we get

$$
\mathcal{M E}[u] \geq \frac{s_{c}(p-1)+1}{s_{c}(p-1)} \mathcal{G}[u(t)]^{2}-\frac{1}{s_{c}(p-1)}(\mathcal{G}[u(t)])^{2 s_{c}(p-1)+2} .
$$


For $x>0$ define $f(x)=\frac{s_{c}(p-1)+1}{s_{c}(p-1)} x^{2}-\frac{1}{s_{c}(p-1)} x^{2 s_{c}(p-1)+2}$. Since $s_{c}>0$ and $p \geq 2$, $\operatorname{deg}(f)>2$. Therefore,

$$
f^{\prime}(x)=\frac{2 s_{c}(p-1)+2}{s_{c}(p-1)}\left(1-x^{2 s_{c}(p-1)}\right) x,
$$

which implies that $f^{\prime}(x)=0$ when $x_{0}=0$ and $x_{1}=1$. Observe that $f\left(x_{0}\right)=0$ and $f\left(x_{1}\right)=1$. Since $f(x)>0$ for $0<x<1$, we conclude that the graph of $f(x)$ has a local minimum at the left end point $x_{0}$ and a local maximum at $x_{1}$. Furthermore, this along with the assumption (3.92) implies that there exists a $\delta_{1}>0$ such that $\mathcal{M E}[u]<1-\delta_{1}$. Combining this with (3.97), we obtain

$$
f(x) \leq 1-\delta_{1}<1=f\left(x_{1}\right)
$$

If initially we have (3.93), i.e., $\mathcal{G}\left[u_{0}\right]<x_{1}=1$, then by (3.98) and the continuity of $\|\nabla u(t)\|_{L^{2}\left(\mathbb{R}^{N}\right)}$ in $t$, we conclude that $\mathcal{G}[u(t)]<1$ for all time $t \in I$, which yields (3.94). This implies that $\dot{H}^{1}$ norm is bounded for all $t$ and we have global existence. Similarly, if initially we have (3.95), i.e., $\mathcal{G}\left[u_{0}\right]>x_{1}=1$, then by (3.98) and the continuity of $\|\nabla u(t)\|_{L^{2}\left(\mathbb{R}^{N}\right)}$ in $t$, we conclude that $\mathcal{G}[u(t)]>1$ for all time $t \in I$, which yields (3.96).

From (3.98) we have that $y=\mathcal{M E}[u]$ intersects the graph of $f(x)$ at two points. Then from the assumptions $\mathcal{M E}[u]<1$ and $\mathcal{G}\left[u_{0}\right]>1$, we deduce that there exists $\delta_{1}>0$ and $\delta_{2}=\delta_{2}\left(\delta_{1}\right)$ such that for all $t \in I \mathcal{M E}[u]<1-\delta_{1}$ and $\mathcal{G}[u(t)]^{2}>1+\delta_{2}$, respectively.

Next if, $x u_{0} \in L^{2}\left(\mathbb{R}^{N}\right)$, we write the virial identity as

$$
V_{t t}=16\left(s_{c}(p-1)+1\right) E\left[u_{0}\right]-8 s_{c}(p-1)\|\nabla u\|_{L^{2}\left(\mathbb{R}^{N}\right)}^{2}
$$

Multiplying the virial identity by $M\left[u_{0}\right]^{\theta}$ and proceeding as in [HR08], [Gue14], we get

$$
M\left[u_{0}\right]^{\theta} V_{t t}<-8 s_{c}(p-1) \delta M[Q]^{\theta}\|\nabla Q\|_{L^{2}}^{2}<0,
$$

which by the convexity argument implies that the time interval $I$ must be finite, thus, having blow-up in finite time. 
If $u_{0}$ is radial, define $\phi \in C^{\infty}(\mathbb{R})$,

$$
\phi(|x|)=\left\{\begin{array}{lc}
\frac{|x|^{2}}{2} & 0 \leq|x| \leq 2 \\
1 & r \geq 3
\end{array}\right.
$$

such that $\phi$ is smooth for $2<r<3$ and $\partial_{r}^{2} \phi(r) \leq 1$ for all $r \geq 0$. Now, for $R>0$ large, let $\phi_{R}=R^{2} \phi\left(\frac{|x|}{R}\right)$. Define the localized variance

$$
V_{l o c}(t)=\int \phi_{R}(x)|u(x, t)|^{2} d x
$$

and compute the second derivative to obtain

$$
\begin{aligned}
\partial_{t}^{2} V_{l o c}(t)= & 4 \int_{\mathbb{R}^{N}} \phi_{R}^{\prime \prime}|\nabla u|^{2} d x-\int_{\mathbb{R}^{N}} \Delta^{2} \phi_{R}|u|^{2} d x \\
& -\frac{2(p-2)}{p} \int_{\mathbb{R}^{N}} \Delta \phi_{R} \frac{|u(x)|^{p}|u(y)|^{p}}{|x-y|^{N-\gamma}} d x d y \\
& -\frac{4(N-\gamma)}{p} \int_{\mathbb{R}^{N}} \int_{\mathbb{R}^{N}} \nabla \phi_{R} \frac{(x-y)|u(x)|^{p}|u(y)|^{p}}{|x-y|^{N-\gamma+2}} d x d y .
\end{aligned}
$$

We bound the two terms in (3.100) using $\Delta \phi_{R}=N$ and $\Delta^{2} \phi_{R}=0$ for $|x| \leq 2 R$ as follows

$$
\begin{aligned}
& 4 \int \phi_{R}^{\prime \prime}|\nabla u|^{2} d x \leq 4 \int_{\mathbb{R}^{N}}|\nabla u|^{2} d x, \\
- & \int \Delta^{2} \phi_{R}|u|^{2} d x \leq \frac{c}{R^{2}} \int_{2 R<|x|<3 R}|u|^{2} d x .
\end{aligned}
$$

Estimate (3.101) using again the fact that $\Delta \phi_{R}(r)=N$

$$
\begin{aligned}
& -\frac{2(p-2)}{p} \int_{\mathbb{R}^{N}} \Delta \phi_{R}\left(|x|^{-(N-\gamma)} *|u|^{p}\right)|u|^{p} d x \\
\leq & -\frac{2(p-2)}{p}\left(N \int_{|x| \leq 2 R}\left(|x|^{-(N-\gamma)} *|u|^{p}\right)|u|^{p} d x+c \int_{2 R<|x|^{<3 R}}\left(|x|^{-(N-\gamma)} *|u|^{p}\right)|u|^{p} d x\right) \\
\leq & -\frac{2 N(p-2)}{p} \int_{\mathbb{R}^{N}}\left(|x|^{-(N-\gamma)} *|u|^{p}\right)|u|^{p} d x+c_{1} \int_{|x|>2 R}\left(|x|^{-(N-\gamma)} *|u|^{p}\right)|u|^{p} d x .
\end{aligned}
$$


Next we turn our attention to the term in (3.102), which can be rewritten as

$$
\begin{aligned}
(3.102)=- & \frac{4(N-\gamma)}{p} \int_{\mathbb{R}^{N}} \int_{\mathbb{R}^{N}} \frac{R}{|x|} \phi^{\prime}\left(\frac{|x|}{R}\right) \frac{x(x-y)|u(x)|^{p}|u(y)|^{p}}{|x-y|^{N-\gamma+2}} d x d y \\
=- & \frac{4(N-\gamma)}{p} \int_{\mathbb{R}^{N}} \int_{\mathbb{R}^{N}} \frac{x(x-y)|u(x)|^{p}|u(y)|^{p}}{|x-y|^{N-\gamma+2}} d x d y \\
& +\frac{4(N-\gamma)}{p} \int_{\mathbb{R}^{N}} \int_{\mathbb{R}^{N}}\left(1-\frac{R}{|x|} \phi^{\prime}\left(\frac{|x|}{R}\right)\right) \frac{x(x-y)|u(x)|^{p}|u(y)|^{p}}{|x-y|^{N-\gamma+2}} d x d y \\
= & -\frac{2(N-\gamma)}{p} \int_{\mathbb{R}^{N}} \int_{\mathbb{R}^{N}} \frac{|u(x)|^{p}|u(y)|^{p}}{|x-y|^{N-\gamma}} d x d y \\
& +\frac{4(N-\gamma)}{p} \int_{\mathbb{R}^{N}} \int_{\mathbb{R}^{N}}\left(1-\frac{R}{|x|} \phi^{\prime}\left(\frac{|x|}{R}\right)\right) \frac{x(x-y)|u(x)|^{p}|u(y)|^{p}}{|x-y|^{N-\gamma+2}} d x d y
\end{aligned}
$$

Combining the above expression with (3.103), (3.104) and (3.105), we write

$$
\begin{aligned}
\partial_{t}^{2} V_{l o c}(t) \leq & 4 \int_{\mathbb{R}^{N}}|\nabla u|^{2}+\frac{c}{R^{2}} \int_{2 R<|x|<3 R}|u|^{2}+c_{1} \int_{|x|>2 R}\left(|x|^{-(N-\gamma)} *|u|^{p}\right)|u|^{p} d x \\
& -\left(\frac{2 N(p-2)}{p}+\frac{2(N-\gamma)}{p}\right) \int_{\mathbb{R}^{N}}\left(|x|^{-(N-\gamma)} *|u|^{p}\right)|u|^{p} d x \\
& +\frac{4(N-\gamma)}{p} \int_{\mathbb{R}^{N}} \int_{\mathbb{R}^{N}}\left(1-\frac{R}{|x|} \phi^{\prime}\left(\frac{|x|}{R}\right)\right) \frac{x(x-y)|u(x)|^{p}|u(y)|^{p}}{|x-y|^{N-\gamma+2}} d x d y .
\end{aligned}
$$

Writing the above inequality in terms of energy and gradient, we get

$$
\begin{aligned}
\partial_{t}^{2} V_{l o c}(t) \leq & 4(N(p-1)-\gamma) E\left[u_{0}\right]-(2(N(p-1)-\gamma)-4) \int_{\mathbb{R}^{N}}|\nabla u|^{2} d x \\
& +\frac{c}{R^{2}} \int_{2 R<|x|<3 R}|u|^{2} d x+c_{1} \int_{|x|>2 R}\left(|x|^{-(N-\gamma)} *|u|^{p}\right)|u|^{p} d x \\
& +\frac{4(N-\gamma)}{p} \int_{\mathbb{R}^{N}} \int_{\mathbb{R}^{N}}\left(1-\frac{R}{|x|} \phi^{\prime}\left(\frac{|x|}{R}\right)\right) \frac{x(x-y)|u(x)|^{p}|u(y)|^{p}}{|x-y|^{N-\gamma+2}} d x d y .
\end{aligned}
$$

The second term in the expression (3.107) can be estimated as

$$
\begin{aligned}
& \int_{|x|>2 R}\left(|x|^{-(N-\gamma)} *|u|^{p}\right)|u|^{p} d x \lesssim\left\||x|^{-(N-\gamma)} *|u|^{p}\right\|_{L_{|x|>2 R}^{\frac{2 N}{N-\gamma}}\|u\|^{p}}^{p} \sum_{L_{|x|>2 R}^{N+\gamma}}^{\frac{2 N p}{N+\gamma}} \text { (Hölder's) } \\
& \lesssim\|u\|_{L_{|x|>2 R}^{2 p}}^{\frac{2 N p}{N+\gamma}} \quad(\text { Lemma 2.3.1) } \\
& \lesssim \frac{1}{R^{\frac{(N-1)(N(p-1)-\gamma)}{N}}}\|\nabla u\|_{L^{2}}^{\frac{N(p-1)-\gamma}{N}}\|u\|_{L^{2}}^{\frac{N(p+1)+\gamma}{N}} \text { (radial Sobolev). }
\end{aligned}
$$


We rewrite the integral in (3.108), using symmetry, as follows

$\frac{1}{2} \int_{\mathbb{R}^{N}} \int_{\mathbb{R}^{N}}\left(\left(1-\frac{R}{|x|} \phi^{\prime}\left(\frac{|x|}{R}\right)\right) x-\left(1-\frac{R}{|y|} \phi^{\prime}\left(\frac{|y|}{R}\right)\right) y\right) \frac{(x-y)|u(x)|^{p}|u(y)|^{p}}{|x-y|^{N-\gamma+2}} d x d y$,

which can be broken down into the following regions (observe that the integral vanishes in the region $|x| \leq 2 R$ );

- Region I: $|x| \approx|y|$. In this region we have

$$
|x|>2 R,|y|>2 R \text {. }
$$

Observe that

$$
\left|\left(1-\frac{R}{|x|} \phi^{\prime}\left(\frac{|x|}{R}\right)\right) x-\left(1-\frac{R}{|y|} \phi^{\prime}\left(\frac{|y|}{R}\right)\right) y\right| \lesssim|x-y| .
$$

We estimate (3.110) in a similar fashion as (3.109) to obtain

$$
\left.\iint \frac{\chi_{|y|>2 R}|u(y)|^{p}}{|x-y|^{N-\gamma}} \chi_{|x|>2 R}|u(x)|^{p} d x d y \lesssim \frac{1}{R^{\frac{(N-1)(N(p-1)-\gamma)}{N}}}|| \nabla u\right|_{L^{2}} ^{\frac{N(p-1)-\gamma}{N}}\|u\|_{L^{2}}^{\frac{N(p+1)+\gamma}{N}} .
$$

- Region II: $\max \{|x|,|y|\} \gg \min \{|x|,|y|\}$ and $\max \{|x|,|y|\}>2 R$. We consider two cases:

- Case (a): $|x| \ll|y| \approx|x-y|, \quad|y|>2 R$ and $|x|<2 R$. In this case (3.110) becomes

$$
\iint \frac{1}{|x-y|^{N-\gamma}} \chi_{|y|>2 R}|u(y)|^{p}|u(x)|^{p} d x d y,
$$

since using the triangle inequality and the definition of $\phi$, we have

$$
\begin{aligned}
& \left|\left(1-\frac{R}{|x|} \phi^{\prime}\left(\frac{|x|}{R}\right)\right) x-\left(1-\frac{R}{|y|} \phi^{\prime}\left(\frac{|y|}{R}\right)\right) y\right| \\
& \leq|x|\left(1-\frac{R}{|x|} \phi^{\prime}\left(\frac{|x|}{R}\right)\right)+|y|\left(1-\frac{R}{|y|} \phi^{\prime}\left(\frac{|y|}{R}\right)\right) \\
& \lesssim|y| \approx|x-y|
\end{aligned}
$$


since $1-\frac{R}{|x|} \phi^{\prime}\left(\frac{|x|}{R}\right)<1$ and $1-\frac{R}{|y|} \phi^{\prime}\left(\frac{|y|}{R}\right)>\frac{1}{2}$. Again using Hölder's inequality, Lemma 2.3.1 and radial Sobolev as in (3.109), we bound the above integral by

$$
\frac{1}{R^{\frac{(N-1)(N(p-1)-\gamma)}{N}}}\|\nabla u\|_{L^{2}}^{\frac{N(p-1)-\gamma}{N}}\|u\|_{L^{2}}^{\frac{N(p+1)+\gamma}{N}} .
$$

- Case (b): $|y| \ll|x| \approx|x-y|, \quad|x|>2 R$ and $|y|<2 R$. This case is symmetric and treated with a similar argument as in Case (a).

Combining (3.109), (3.111) and (3.112), we get

$$
\begin{aligned}
\partial_{t}^{2} V_{l o c}(t) \leq & 8\left(s_{c}(p-1)+1\right) E\left[u_{0}\right]-4 s_{c}(p-1) \int_{\mathbb{R}^{N}}|\nabla u|^{2}+\frac{c}{R^{2}} \int_{2 R<|x|<3 R}|u|^{2} \\
& +\frac{\tilde{c}}{R^{\frac{(N-1)(N(p-1)-\gamma)}{N}}}\|\nabla u\|_{L^{2}}^{\frac{N(p-1)-\gamma}{N}}\|u\|_{L^{2}}^{\frac{N(p+1)+\gamma}{N}} .
\end{aligned}
$$

Using Young's inequality to separate the $L^{2}$ norm and gradient term in the last term, we obtain

$$
\begin{aligned}
\partial_{t}^{2} V_{l o c}(t) \leq & 8\left(s_{c}(p-1)+1\right) E\left[u_{0}\right]-4 s_{c}(p-1) \int_{\mathbb{R}^{N}}|\nabla u|^{2}+\frac{c}{R^{2}} \int_{2 R<|x|<3 R}|u|^{2} \\
& +\epsilon\|\nabla u\|_{L^{2}}^{2}+\frac{c(\epsilon, N)}{R^{\frac{2(N-1)(N(p-1)-\gamma)}{N(3-p)+\gamma}}\|u\|_{L^{2}}^{\frac{2(N(p+1)+\gamma)}{N(3-p)+\gamma}} .}
\end{aligned}
$$

Multiplying the above expression by $M\left[u_{0}\right]^{\theta}$ and using the similar argument as in the case of finite variance, we get

$$
\begin{aligned}
M\left[u_{0}\right]^{\theta} \partial_{t}^{2} V_{l o c}(t) \leq & 8\left(s_{c}(p-1)+1\right) M\left[u_{0}\right]^{\theta} E\left[u_{0}\right]-\left(4 s_{c}(p-1)-\epsilon\right)\|u\|_{L^{2}}^{2 \theta}\|\nabla u\|_{L^{2}}^{2} \\
& +\frac{c}{R^{2}}\|u\|_{L^{2}}^{2+2 \theta}+\frac{c(\epsilon, N)}{R^{\frac{2(N-1)(N(p-1)-\gamma)}{N(3-p)+\gamma}}}\|u\|_{L^{2}}^{\frac{2(N(p-p)+\gamma}{2(3-\gamma)+2 \theta}}
\end{aligned}
$$

which can be re-written as

$$
\begin{aligned}
& M\left[u_{0}\right]^{\theta} \partial_{t}^{2} V_{l o c}(t) \leq 4 s_{c}(p-1)\left(1-\delta_{1}\right) M[Q]^{\theta}\|\nabla Q\|_{L^{2}}^{2}+\frac{c}{R^{2}}\|u\|_{L^{2}}^{2+2 \theta} \\
& \quad-\left(4 s_{c}(p-1)-\epsilon\right)\left(1+\delta_{2}\right) M[Q]^{\theta}\|\nabla Q\|_{L^{2}}^{2}+\frac{c(\epsilon, N)}{R^{\frac{2(N-1)(N(p-1)-\gamma)}{N(3-p)+\gamma}}\|u\|_{L^{2}}^{\frac{2(N(p+1)+\gamma)}{N(3-\gamma) \gamma}}} .
\end{aligned}
$$


Choose

$$
0<\epsilon<\frac{4 s_{c}(p-1)\left(\delta_{1}+\delta_{2}\right)}{1+\delta_{2}}
$$

and $R=R\left(\epsilon, \delta_{1}, N, p, \gamma, M\left[u_{0}\right]\right)$ large enough to obtain

$$
M\left[u_{0}\right]^{\theta} \partial_{t}^{2} V_{l o c}(t) \leq-c(\epsilon, N, p, \gamma)
$$

where $c(\epsilon, N, p, \gamma)>0$, implying that the maximum interval of existence $I$ is finite.

The following lemmas provide some additional estimates that will be needed for the compactness and rigidity results in Section 3.4-3.5.

Lemma 3.3.2 (Comparison of Energy and Gradient) Let $u_{0} \in H^{1}\left(\mathbb{R}^{N}\right)$ satisfy (3.92) and (3.93). Then

$$
\frac{s_{c}(p-1)}{2 s_{c}(p-1)+2}\|\nabla u\|_{L^{2}\left(\mathbb{R}^{N}\right)}^{2} \leq E[u] \leq \frac{1}{2}\|\nabla u\|_{L^{2}\left(\mathbb{R}^{N}\right)}^{2}
$$

Proof. The second inequality immediately follows from the definition of energy. The first inequality is obtained by using Lemma 2.8.1, (3.94) and (2.136)

$$
\begin{aligned}
E[u] & \geq \frac{1}{2}\|\nabla u\|_{L^{2}\left(\mathbb{R}^{N}\right)}^{2}\left(1-\frac{C_{G N}}{p}\left(\|\nabla u\|_{L^{2}\left(\mathbb{R}^{N}\right)}^{s_{c}}\|u\|_{L^{2}\left(\mathbb{R}^{N}\right)}^{1-s_{c}}\right)^{2(p-1)}\right) \\
& >\frac{1}{2}\|\nabla u\|_{L^{2}\left(\mathbb{R}^{N}\right)}^{2}\left(1-\frac{C_{G N}}{p}\left(\|\nabla Q\|_{L^{2}\left(\mathbb{R}^{N}\right)}^{s}\|Q\|_{L^{2}\left(\mathbb{R}^{N}\right)}^{1-s}\right)^{2(p-1)}\right) \\
& =\frac{s(p-1)}{2(s(p-1)+1)}\|\nabla u\|_{L^{2}\left(\mathbb{R}^{N}\right)}^{2},
\end{aligned}
$$

as desired.

Lemma 3.3.3 (Lower bound on the convexity of variance) Let $u_{0} \in H^{1}\left(\mathbb{R}^{N}\right)$ satisfy (3.92) and (3.93). Then for all $t \in \mathbb{R}$

$$
16 E[u]\left(1-(\mathcal{M E}[u])^{s_{c}(p-1)}\right) \leq 8\left(\|\nabla u\|_{L^{2}}^{2}-\frac{s_{c}(p-1)+1}{p} Z(u)\right) .
$$


Proof. Multiplying the first inequality in (3.113) with $M[u]^{\theta}$, where $\theta=\frac{1-s_{c}}{s_{c}}$, dividing it by $\|\nabla Q\|_{L^{2}\left(\mathbb{R}^{N}\right)}^{2}\|Q\|_{L^{2}\left(\mathbb{R}^{N}\right)}^{2 \theta}$ and using (2.135), we get

$$
|\mathcal{G}[u(t)]|^{2}=\frac{\|u(t)\|_{L^{2}\left(\mathbb{R}^{N}\right)}^{2 \theta}\|\nabla u(t)\|_{L^{2}\left(\mathbb{R}^{N}\right)}^{2}}{\|Q\|_{L^{2}\left(\mathbb{R}^{N}\right)}^{2 \theta}\|\nabla Q\|_{L^{2}\left(\mathbb{R}^{N}\right)}^{2}} \leq \frac{M[u]^{\theta} E[u]}{M[Q]^{\theta} E[Q]}=\mathcal{M E}[u],
$$

which implies that $\mathcal{G}[u(t)] \leq \sqrt{\mathcal{M E}}$. Applying (2.126) to the virial identity and using (2.136), we obtain

$$
\begin{aligned}
8\left(\|\nabla u\|_{L^{2}\left(\mathbb{R}^{N}\right)}^{2}-\frac{s_{c}(p-1)+1}{p} Z(u)\right) & \geq 8\|\nabla u\|_{L^{2}\left(\mathbb{R}^{N}\right)}^{2}\left(1-|\mathcal{G}[u(t)]|^{2 s_{c}(p-1)}\right) \\
& \geq 8\|\nabla u\|_{L^{2}\left(\mathbb{R}^{N}\right)}^{2}\left(1-(\mathcal{M E})^{s_{c}(p-1)}\right) \\
& \geq 16 E[u]\left(1-(\mathcal{M E}[u])^{s_{c}(p-1)}\right)
\end{aligned}
$$

where the last inequality follows from the second inequality in (3.113).

Lemma 3.3.4 (Existence of wave operators) Suppose $\psi^{+} \in H^{1}\left(\mathbb{R}^{N}\right)$ and

$$
\left\|\psi^{+}\right\|_{L^{2}}^{2 \theta}\left\|\nabla \psi^{+}\right\|_{L^{2}}^{2} \leq \mu^{2}\left(\frac{2 s_{c}(p-1)+2}{s_{c}(p-1)}\right) M[Q]^{\theta} E[Q]
$$

for some $0<\mu \leq\left(\frac{s_{c}(p-1)}{2 s_{c}(p-1)+2}\right)^{\frac{1}{2}}<1$. Then there exists $v_{0} \in H^{1}\left(\mathbb{R}^{N}\right)$ such that $v(t)$, solving (1.1) with initial data $v_{0}$, is global in $H^{1}\left(\mathbb{R}^{N}\right)$ with

$$
\left\|v_{0}\right\|_{L^{2}}^{\theta}\|\nabla v(t)\|_{L^{2}} \leq\|Q\|_{L^{2}}^{\theta}\|\nabla Q\|_{L^{2}}, \quad M[v]=\left\|\psi^{+}\right\|_{L^{2}}^{2}, \quad E[v]=\frac{1}{2}\left\|\nabla \psi^{+}\right\|_{L^{2}}^{2}
$$

and

$$
\left\|v(t)-e^{i t \Delta} \psi^{+}\right\|_{H^{1}} \rightarrow 0 \quad \text { as } \quad t \rightarrow \infty .
$$

Moreover, if $\left\|e^{i t \Delta} \psi^{+}\right\|_{S\left(\dot{H}^{s c}\right)} \leq \delta$, then

$$
\left\|v_{0}\right\|_{\dot{H}^{s_{c}}} \leq 2\left\|\psi^{+}\right\|_{\dot{H}^{s_{c}}} \quad \text { and } \quad\|v\|_{S\left(\dot{H}^{s_{c}}\right)} \leq 2\left\|e^{i t \Delta} \psi^{+}\right\|_{S\left(\dot{H}^{s_{c}}\right)} .
$$

Proof. We consider the integral equation

$$
v(t)=e^{i t \Delta} \psi^{+}-i \int_{t}^{\infty} e^{i\left(t-t^{\prime}\right) \Delta}\left(\left(|x|^{-(N-\gamma)} *|u|^{p}\right)|u|^{p-2} u\right)\left(t^{\prime}\right) d t^{\prime}
$$


which we would like to solve for all $t$. Note that for $T>0$ by Theorem 3.2.1 (small data theory) there exists $\delta>0$ such that $\left\|e^{i t \Delta} \psi^{+}\right\|_{S\left(\dot{H}^{s_{c}} ;[T, \infty)\right)} \leq \delta$. Thus, we solve the equation (3.116) in $H^{1}$ for $t \geq T$ with $T$ large. Estimating (3.116) in $S\left(L^{2}\right)$ for $t \geq T$, we obtain

$$
\begin{aligned}
\|\nabla v\|_{S\left(L^{2} ;[T, \infty)\right)} & \lesssim\left\|e^{i t \Delta} \nabla \psi^{+}\right\|_{S\left(L^{2} ;[T, \infty)\right)}+\left\|\nabla\left[\left(|\cdot|^{-(N-\gamma)} *|v|^{p}\right)|v|^{p-2} v\right]\right\|_{S^{\prime}\left(L^{2} ;[T, \infty)\right)} \\
& \lesssim\left\|\psi^{+}\right\|_{\dot{H}^{1}}+\|v\|_{\left.S\left(\dot{H}^{s c} ; T, \infty\right)\right)}^{2(p-1)}\|\nabla v\|_{S\left(L^{2} ;[T, \infty)\right)} .
\end{aligned}
$$

Taking $T$ sufficiently large so that $\|v\|_{S\left(\dot{H}^{s^{c} ;[T, \infty)}\right)}^{2(p-1)} \leq \frac{1}{2}$, we get $\|\nabla v\|_{S\left(L^{2} ;[T, \infty)\right)} \lesssim 2\left\|\psi^{+}\right\|_{\dot{H}^{1}}$. Using the above inequality, we obtain in a similar fashion,

$$
\begin{aligned}
\left\|\nabla\left(v-e^{i t \Delta} \psi^{+}\right)\right\|_{S\left(L^{2} ;[T, \infty)\right)} & \leq\left\|\nabla\left[\left(|\cdot|^{-(N-\gamma)} *|v|^{p}\right)|v|^{p-2} v\right]\right\|_{S^{\prime}\left(L^{2} ;[T, \infty)\right)} \\
& \leq\|v\|_{S\left(\dot{H}^{s_{c}} ;[T, \infty)\right)}^{2(p-1)}\|\nabla v\|_{S\left(L^{2} ;[T, \infty)\right)} \\
& \leq c\left\|\psi^{+}\right\|_{\dot{H}^{1}},
\end{aligned}
$$

hence, $\left\|\nabla\left(v-e^{i t \Delta} \psi^{+}\right)\right\|_{S\left(L^{2} ;[T, \infty)\right)} \rightarrow 0$ as $T \rightarrow \infty$. Since, by Theorem 3.2.4 $\left(H^{1}\right.$ scattering), we have $v-e^{i t \Delta} \psi^{+} \rightarrow 0$ in $H^{1}$ as $t \rightarrow \infty$ and the decay estimate together with the embedding $H^{1}\left(\mathbb{R}^{N}\right) \hookrightarrow L^{q}\left(\mathbb{R}^{N}\right)$ with $q \leq \frac{2 N}{N-2}$ for $N \geq 3, q<\infty$ for $N=2$ and $q \leq \infty$ for $N=1$ implies

$$
Z\left(e^{i t \Delta} \psi^{+}\right) \lesssim\left\|e^{i t \Delta} \psi^{+}\right\|_{L^{\frac{2 N p}{N+\gamma}}} \leq|t|^{-\frac{N p-N-\gamma}{2 p}}\left\|\psi^{+}\right\|_{H^{1}}
$$

thus, $Z\left(e^{i t \Delta} \psi^{+}\right) \rightarrow 0$ in $L^{\frac{2 N p}{N+\gamma}}$ as $t \rightarrow \infty$. Since $\lim _{t \rightarrow+\infty}\|v(t)\|_{H^{1}}=\left\|\nabla \psi^{+}\right\|_{H^{1}}$, we have

$$
\begin{aligned}
E[v] & =\frac{1}{2}\|\nabla v\|_{L^{2}}^{2}-\frac{1}{2 p} \int\left(|x|^{-(N-\gamma)} *|v|^{p}\right)|v|^{p} d x \\
& =\lim _{t \rightarrow \infty}\left(\frac{1}{2}\left\|\nabla e^{i t \Delta} \psi^{+}\right\|_{L^{2}}^{2}-\frac{1}{2 p} \int\left(|x|^{-(N-\gamma)} *\left|e^{i t \Delta} \psi^{+}\right|^{p}\right)\left|e^{i t \Delta} \psi^{+}\right|^{p}\right) \\
& =\frac{1}{2}\left\|\nabla \psi^{+}\right\|_{L^{2}}^{2}
\end{aligned}
$$


and $M[v]=\lim _{t \rightarrow \infty}\left\|e^{i t \Delta} \psi^{+}\right\|_{L^{2}}^{2}=\left\|\psi^{+}\right\|_{L^{2}}^{2}$. Note that by (3.115) we now have

$$
M[v]^{\theta} E[v]=\frac{1}{2}\left\|\psi^{+}\right\|_{L^{2}}^{2 \theta}\left\|\nabla \psi^{+}\right\|_{L^{2}}^{2} \leq \mu^{2}\left(\frac{2 s_{c}(p-1)+2}{s_{c}(p-1)}\right) M[Q]^{\theta} E[Q]
$$

and by our choice of $\mu$ we conclude that $M[v]^{\theta} E[v]<M[Q]^{\theta} E[Q]$. Moreover,

$$
\begin{aligned}
\lim _{t \rightarrow \infty}\|v(t)\|_{L^{2}}^{2 \theta}\|\nabla v(t)\|_{L^{2}}^{2} & =\left\|\psi^{+}\right\|_{L^{2}}^{2 \theta}\left\|\nabla \psi^{+}\right\|_{L^{2}}^{2} \\
& \leq \mu^{2}\left(\frac{2 s_{c}(p-1)+2}{s_{c}(p-1)}\right) M[Q]^{\theta} E[Q] \\
& =\mu^{2}\|Q\|_{L^{2}}^{2 \theta}\|\nabla Q\|_{L^{2}}^{2},
\end{aligned}
$$

where the inequality is due to (3.115) and last equality is from (2.136). We can take $T>0$ sufficiently large so that $\|v(T)\|_{L^{2}}^{\theta}\|\nabla v(T)\|_{L^{2}}<\mu\|Q\|_{L^{2}}^{\theta}\|\nabla Q\|_{L^{2}}$. Since $\mu<1$, by Theorem 3.3.1 (global existence of solutions), we evolve $v(T)$ from time $T$ back to time 0 and obtain $v$ with initial data $v_{0} \in H^{1}$ for all time $t \in[0, \infty)$ with the desired properties.

\subsection{Compactness}

\subsubsection{Roadmap}

To characterize the behavior of global solutions to (1.1), we must show that if $\mathcal{M E}[u]<1$ and $\mathcal{G}\left[u_{0}\right]<1$, then the global-in-time $\dot{H}^{s_{c}}$ Strichartz norm is finite, i.e., $\|u\|_{S_{\left(\dot{H}^{s_{c}}\right)}}<$ $\infty$. This would imply that $\|\nabla u(t)\|_{L^{2}} \leq C$, and thus, $I=(-\infty, \infty)$. For completeness we provide the blueprint below, which is derived from the works of Holmer and Roudenko [HR08], Duyckaerts, Holmer and Roudenko [DHR08] for the 3d cubic nonlinear Schrödinger equation and Kenig and Merle [KM06] for the energy-critical nonlinear Schrödinger equation. 


\section{First Stage: Small data theory}

Using Lemma 3.3.2, we have

$$
\left\|u_{0}\right\|_{\dot{H}^{s_{c}}}^{2(p-1)} \leq\left(\left\|u_{0}\right\|_{L^{2}}^{\theta}\left\|\nabla u_{0}\right\|_{L^{2}}\right)^{2 s_{c}(p-1)}<\left(\frac{2 p}{p-1}\right)^{s_{c}(p-1)}\left(M[u]^{\theta} E[u]\right)^{s_{c}(p-1)} .
$$

If $\mathcal{G}\left[u_{0}\right]<1$ and $\mathcal{M E}[u]<\left(\frac{p-1}{2 p}\right) \frac{\delta_{s d}^{2 / s_{c}}}{M[Q]^{\theta} E[Q]}$, then from the above inequality, we obtain $\left\|u_{0}\right\|_{\dot{H}^{s_{c}}} \leq \delta_{s d}$, which by Strichartz estimates gives $\left\|e^{i t \Delta} u_{0}\right\|_{S\left(\dot{H}^{s^{c}}\right)} \leq c \delta_{s d}$. Therefore, Theorem 3.2.1 (small data theory) implies that there exists a $\delta>0$ such that if $\mathcal{G}\left[u_{0}\right]<1$ and $\mathcal{M E}[u]<\delta$, then $T^{*}=+\infty$ and $\left\|u_{0}\right\|_{\dot{H}^{s_{c}}}<\infty$. This gives us the basis for induction.

Second stage: Construction of critical solution (via induction on scattering threshold)

Let $(\mathcal{M E})_{c}$ be the supremum over all $\delta>0$ for which the following is true:

"If $u_{0} \in H^{1}\left(\mathbb{R}^{N}\right)$ with $\mathcal{G}\left[u_{0}\right]<1$ and $\mathcal{M E}[u]<\delta$ such that $\delta=\delta\left(M[Q]^{1-s} E[Q]^{s}\right)$, then $T^{*}=+\infty$ and $\left\|u_{0}\right\|_{\dot{H}^{s_{c}}}<\infty$."

If $(\mathcal{M E})_{c}=1$, then we are done, since $Q$ (soliton) does not scatter. So, we assume that $(\mathcal{M E})_{c}<1$. This implies (by definition of $(\mathcal{M E})_{c}$ ) that there exists a sequence of solutions $\left\{u_{n}\right\}$ to (1.1) with initial data $u_{n, 0} \in H^{1}\left(\mathbb{R}^{N}\right)$ that approach the threshold $(\mathcal{M E})_{c}$ from above but do not scatter, i.e., there exists a sequence $u_{n, 0} \in H^{1}\left(\mathbb{R}^{N}\right)$ such that

$$
\mathcal{G}\left[u_{n, 0}\right]<1 \text { and } \mathcal{M E}\left[u_{n, 0}\right] \searrow(\mathcal{M E})_{c} \text { as } n \rightarrow \infty
$$

for which $\left\|u_{n}\right\|_{S\left(\dot{H}^{s}\right)}=+\infty$. Using the profile decomposition (Theorem 3.4.1) on the sequence of initial data $\left\{u_{n, 0}\right\}$, we prove the existence of an $H^{1}$ solution $u_{c}$ to (1.1) with initial data $u_{c, 0}$ such that

$$
\mathcal{G}\left[u_{c, 0}\right]<1 \text { and } \mathcal{M E}\left[u_{c}\right]=(\mathcal{M E})_{c}
$$

i.e., it lies exactly at the threshold $(\mathcal{M E})_{c}$, but $u_{c}$ does not scatter (Theorem 3.4.3). 
Third stage: Localization of critical solution (setting the premise for rigidity theorem)

The critical solution $u_{c}(t)$, constructed in the second stage, will have the property that $K=\left\{u_{c}(t) \mid t \in[0, \infty)\right\}$ is precompact in $H^{1}\left(\mathbb{R}^{N}\right)$ (Proposition 3.4.6). This will allow us to show that for a given $\epsilon>0$, there is an $R>0$ such that

$$
\int_{|x+x(t)|>R}\left|\nabla u\left(x, t_{n}\right)\right|^{2} d x \leq \epsilon
$$

uniformly in $t$ (Lemma 3.4.7). Together with the zero momentum hypothesis (Lemma 3.4.8), this controls the growth of path $x(t)$ (Lemma 3.4.9).

Final Stage: Rigidity theorem (Theorem 3.5.1)

Appealing to this uniform localization and control of $x(t)$, we invoke the Rigidity theorem, which leads to contradiction that such compact solution in $H^{1}$ exists unless it is a trivial solution, which scatters. Therefore, the assumption $(\mathcal{M E})_{c}<1$ is not valid, concluding the proof.

We now fill in the necessary details.

\subsubsection{Profile decomposition}

Theorem 3.4.1 (Linear Profile decomposition) Let $\phi_{n}(x)$ be a uniformly bounded sequence in $H^{1}\left(\mathbb{R}^{N}\right)$. Then for each $M \in \mathbb{N}$ there exists a subsequence of $\phi_{n}(x)$ (also denoted $\left.\phi_{n}(x)\right)$, such that, for each $1 \leq j \leq M$,

1. there exist, fixed in $n$, a profile $\psi^{j} \in H^{1}\left(\mathbb{R}^{N}\right)$,

2. there exists a sequence (in $n$ ) $t_{n}^{j}$ of time shifts,

3. there exists a sequence (in $n$ ) $x_{n}^{j}$ of space shifts,

4. there exists a sequence (in $n) W_{n}^{M}(x)$ of remainders in $H^{1}\left(\mathbb{R}^{N}\right)$, such that

$$
\phi_{n}(x)=\sum_{j=1}^{M} e^{-i t_{n}^{j} \Delta} \psi^{j}\left(x-x_{n}^{j}\right)+W_{n}^{M}(x)
$$


with the properties:

- Pairwise divergence for the time and space sequences. For $1 \leq k \neq j \leq M$,

$$
\lim _{n \rightarrow \infty}\left|t_{n}^{j}-t_{n}^{k}\right|+\left|x_{n}^{j}-x_{n}^{k}\right|=+\infty
$$

- Asymptotic smallness for the remainder sequence

$$
\lim _{M \rightarrow \infty}\left(\lim _{n \rightarrow \infty}\left\|e^{i t \Delta} W_{n}^{M}\right\|_{S\left(\dot{H}^{s_{c}}\right)}\right)=0
$$

- Asymptotic Pythagorean expansion. For fixed $M \in \mathbb{N}$ and for any $0 \leq s \leq 1$, we have

$$
\left\|\phi_{n}\right\|_{\dot{H}^{s}}=\sum_{j=1}^{M}\left\|\psi^{j}\right\|_{\dot{H}^{s}}^{2}+\left\|W_{n}^{M}\right\|_{\dot{H}^{s}}^{2}+o_{n}(1) .
$$

Proof. Since $\phi_{n}$ is assumed to be uniformly bounded in $H^{1}$, then $\left\|\phi_{n}\right\|_{H^{1}\left(\mathbb{R}^{N}\right)} \leq C_{1}$, for some positive constant $C_{1}$. Note the interpolation inequality

$$
\|v\|_{L_{t}^{q} L_{x}^{r}} \leq\|v\|_{L_{t}^{\tilde{q}} L_{x}^{\tilde{r}}}^{1-\theta}\|v\|_{L_{t}^{\infty} L_{x}^{\frac{2 N}{N-2 s_{c}}}}^{\theta},
$$

where $(q, r)$ is any $\dot{H}^{s_{c}}$ admissible pair. Let $\tilde{r}=k r$ for $k>1, \tilde{q}=\frac{4 r k}{r k\left(N-2 s_{c}\right)-2 N}$ and $\theta=\frac{(k-1) 2 d}{r k\left(N-2 s_{c}\right)-2 N}$ so that $(\tilde{q}, \tilde{r})$ is also an $\dot{H}^{s_{c}}$ admissible pair for $0<s_{c}<1$ with $0<\theta<1$. By this inequality and Strichartz estimates we get

$$
\left\|e^{i t \Delta} W_{n}^{M}\right\|_{L_{t}^{q} L_{x}^{r}} \leq c\left\|W_{n}^{M}\right\|_{\dot{H}^{s c}\left(\mathbb{R}^{N}\right)}^{1-\theta}\left\|e^{i t \Delta} W_{n}^{M}\right\|_{L_{t}^{\infty} L_{x}^{\frac{2 N}{N-2 s_{c}}}}^{\theta} .
$$

Our aim is to write the profile as $\phi_{n}(x)=\sum_{j=1}^{M} e^{-i t_{n}^{j} \Delta} \psi^{j}\left(x-x_{n}^{j}\right)+W_{n}^{M}(x)$. Since $\left\|W_{n}^{M}\right\|_{\dot{H}^{s_{c}\left(\mathbb{R}^{N}\right)}} \leq C_{1}$, thus, by (3.121), it is sufficient to show that

$$
\lim _{M \rightarrow \infty}\left(\limsup _{n \rightarrow \infty}\left\|e^{i t \Delta} W_{n}^{M}\right\|_{L_{t}^{\infty} L_{x}^{\frac{2 N}{N-2 s_{c}}}}\right)=0 .
$$

We start with the construction of $\psi_{n}^{1}$. Let

$$
A_{1}=\limsup _{n \rightarrow+\infty}\left\|e^{i t \Delta} \phi_{n}\right\|_{L_{t}^{\infty} L_{x}^{\frac{2 N}{N-2 s_{c}}}} .
$$


If $A_{1}=0$, taking $\psi^{j}=0$ for all $1 \leq j \leq M$ completes the construction. Indeed, for an arbitrary $\dot{H}^{s_{c}}$ admissible pair $(q, r)$ we have

$$
\left\|e^{i t \Delta} \phi_{n}\right\|_{L_{t}^{q} L_{x}^{r}} \leq c\left\|\phi_{n}\right\|_{\dot{H}^{s_{c}}\left(\mathbb{R}^{N}\right)}^{1-\theta}\left\|e^{i t \Delta} \phi_{n}\right\|_{L_{t}^{\infty} L_{x}^{N-2 s_{c}}}^{\theta}
$$

since $A_{1}=\lim \sup _{n}\left\|e^{i t \Delta} \phi_{n}\right\|_{L_{t}^{\infty} \frac{2 N}{L_{x}^{N-2 s_{c}}}}=0$, we have $\lim \sup _{n}\left\|e^{i t \Delta} \phi_{n}\right\|_{S\left(\dot{H}^{s_{c}}\right)}=0$ and we can take $\psi^{j}=0$ for all $j$. Suppose now that $A_{1}>0$ and $C_{1}=\lim \sup _{n}\left\|\phi_{n}\right\|_{H^{1}\left(\mathbb{R}^{N}\right)}<$ $\infty$. Extracting a subsequence from $\phi_{n}$, we show that there exist sequences $t_{n}^{1}, x_{n}^{1}$ and $\psi^{1} \in H^{1}\left(\mathbb{R}^{N}\right)$ such that $e^{i t_{n}^{1} \Delta} \phi_{n}\left(\cdot+x_{n}^{1}\right) \rightarrow \psi^{1}$ weakly in $H^{1}\left(\mathbb{R}^{N}\right)$ and

$$
K\left\|\psi^{1}\right\|_{\dot{H}^{s_{c}}} \geq \frac{A_{1}^{\frac{N-2 s_{c}^{2}}{2 s_{c}\left(1-s_{c}\right)}}}{C_{1}^{\frac{N-2 s_{c}}{2 s_{c}\left(1-s_{c}\right)}}}
$$

where $K>0$ is a constant independent of all parameters. Let $\chi$ be a radially symmetric and real-valued function such that $0 \leq \chi \leq 1, \chi(\xi)=1$ for $|\xi| \leq 1$ and $\chi(\xi)=0$ for $|\xi| \geq 2$. Given $r>0$, define $\chi_{r}$ by $\widehat{\chi}_{r}(\xi)=\chi(\xi / r)$. From Lemma 2.3.2 and the fact that $e^{i t \Delta}$ is an isometry in $H^{s_{c}}$, we have

$$
\begin{aligned}
\left\|e^{i t \Delta} \phi_{n}-\chi_{r} * e^{i t \Delta} \phi_{n}\right\|_{L_{t}^{\infty} L_{x}^{N-2 s_{c}}}^{2} & \leq C\left\|e^{i t \Delta} \phi_{n}-\chi_{r} * e^{i t \Delta} \phi_{n}\right\|_{L_{t}^{\infty} H_{x}^{s_{c}}}^{2} \\
& \leq C \int|\xi|^{2 s_{c}}\left(1-\widehat{\chi_{r}}(\xi)\right)^{2}\left|\widehat{\phi_{n}}(\xi)\right|^{2} d \xi \\
& \leq C \int_{|\xi|>r}|\xi|^{-2\left(1-s_{c}\right)}|\xi|^{2}\left|\hat{\phi}_{n}(\xi)\right|^{2} d \xi \\
& \leq \frac{C}{r^{2\left(1-s_{c}\right)}}\left\|\phi_{n}\right\|_{\dot{H}^{1}}^{2} \leq \frac{C C_{1}^{2}}{r^{2\left(1-s_{c}\right)}}
\end{aligned}
$$

Taking

$$
r=\left(\frac{4 \sqrt{C} C_{1}}{A_{1}}\right)^{\frac{1}{1-s_{c}}}
$$

and using the definition of $A_{1}$ along with the triangle inequality, for large $n$, we have

$$
\left\|\chi_{r} * e^{i t \Delta} \phi_{n}\right\|_{L_{t}^{\infty} L_{x}^{\frac{2 N}{N-2 s}}} \geq \frac{A_{1}}{2} .
$$


Performing an interpolation gives

$$
\begin{aligned}
\left\|\chi_{r} * e^{i t \Delta} \phi_{n}\right\|_{L_{t}^{\infty} L_{x}^{N-2 s_{c}}}^{N} & \leq\left\|\chi_{r} * e^{i t \Delta} \phi_{n}\right\|_{L_{t}^{\infty} L_{x}^{2}}^{N-2 s_{c}}\left\|\chi_{r} * e^{i t \Delta} \phi_{n}\right\|_{L_{t}^{\infty} L_{x}^{\infty}}^{2 s_{c}} \\
& \leq C_{1}^{N-2 s}\left\|\chi_{r} * e^{i t \Delta} \phi_{n}\right\|_{L_{t}^{\infty} L_{x}^{\infty}}^{2 s_{c}}
\end{aligned}
$$

where the second inequality follows from the fact that $\left|\hat{\chi}_{r}\right| \leq 1, L^{2}$ isometry property of the operator $e^{i t \Delta}$ and the fact that $C_{1}=\lim \sup _{n}\left\|\phi_{n}\right\|_{H^{1}\left(\mathbb{R}^{N}\right)}<\infty$. Therefore, combining (3.125) and (3.126), we get

$$
\left\|\chi_{r} * e^{i t \Delta} \phi_{n}\right\|_{L_{t}^{\infty} L_{x}^{\infty}} \geq\left(\frac{A_{1}}{2 C_{1}^{\frac{N-2 s_{c}}{N}}}\right)^{\frac{N}{2 s_{c}}}
$$

Let there exists sequences $t_{n}^{1}, x_{n}^{1}$ such that for each $n \in \mathbb{N}$

$$
\left|\chi_{r} * e^{i t_{n}^{1} \Delta} \phi_{n}\left(x_{n}^{1}\right)\right| \geq \frac{1}{2}\left(\frac{A_{1}}{2 C_{1}^{\frac{N-2 s_{c}}{N}}}\right)^{\frac{N}{2 s_{c}}}
$$

or, equivalently

$$
\frac{1}{2}\left(\frac{A_{1}}{2 C_{1}^{\frac{N-2 s_{c}}{N}}}\right)^{\frac{N}{2 s_{c}}} \leq\left|\int_{\mathbb{R}^{N}} \chi_{r}\left(x_{n}^{1}-y\right) e^{i t_{n}^{1} \Delta} \phi_{n}(y) d y\right|
$$

Since $e^{i t \Delta}$ is translation invariant, i.e.,

$$
\left|\left(e^{i t \Delta} f(x+h)\right)^{\wedge}\right|=\left|e^{i \xi h}\left(e^{i t \Delta} f(x)\right)^{\wedge}\right|=\left|\left(e^{i t \Delta} f(x)\right)^{\wedge}\right|
$$

and an $H^{1}$ isometry, (i.e., $\left\|e^{i t_{n}^{1} \Delta} \phi_{n}\left(x_{n}^{1}\right)\right\|_{H^{1}}=\left\|\phi_{n}\right\|_{H^{1}} \leq C_{1}$ ), we consider the sequence $e^{i t_{n}^{1} \Delta} \phi_{n}\left(\cdot+x_{n}^{1}\right)$, which is uniformly bounded in $H^{1}$ (with the same constant as $\phi_{n}$ ) and passing to a subsequence it follows that $e^{i t_{n}^{1} \Delta} \phi_{n}\left(\cdot+x_{n}^{1}\right) \rightarrow \psi^{1}$ weakly in $H^{1}$ with $\psi^{1} \in H^{1}$ and $\left\|\psi^{1}\right\|_{H^{1}} \leq \lim \sup _{n}\left\|\phi_{n}\right\|_{H^{1}} \leq C_{1}$. Therefore, from (3.127), we have

$$
\frac{1}{4}\left(\frac{A_{1}}{2 C_{1}^{\frac{N-2 s_{c}}{N}}}\right)^{\frac{N}{2 s_{c}}} \leq\left|\int_{\mathbb{R}^{N}} \chi_{r}\left(x_{n}^{1}-y\right) \psi^{1}(y) d y\right|
$$

By Plancherel formula applied to the right-hand side of the above inequality, we obtain $\frac{1}{4}\left(\frac{A_{1}}{2 C_{1}^{\frac{N-2 s_{c}}{N}}}\right)^{\frac{N}{2 s_{c}}} \leq\left|\int_{\mathbb{R}^{N}} \chi_{r}\left(x_{n}^{1}-y\right) \psi^{1}(y) d y\right|=\left.\left|\int_{\mathbb{R}^{N}}\right| \xi\right|^{-2 s_{c}} \widehat{\chi}_{r}\left(x_{n}^{1}-y\right)|\xi|^{2 s_{c}} \widehat{\psi^{1}}(y) d y \mid$ 
and Cauchy-Schwarz to the rightmost term in the above inequality gives

$$
\frac{1}{4}\left(\frac{A_{1}}{2 C_{1}^{\frac{N-2 s_{c}}{N}}}\right)^{\frac{N}{2 s_{c}}} \leq\left\|\chi_{r}\right\|_{\dot{H}^{-s_{c}}}\left\|\psi^{1}\right\|_{\dot{H}^{s}} .
$$

Since $\left\|\chi_{r}\right\|_{\dot{H}^{-s_{C}}}^{2} \leq C \int_{0}^{2 r} \rho^{-2 s_{c}} \rho^{N-1} d \rho \leq C r^{\frac{N-2 s_{c}}{2}}$, here we have used the same radius chosen in (3.124), which implies

$$
\left\|\psi^{1}\right\|_{\dot{H}^{s_{c}}} \geq \frac{1}{4}\left(\frac{A_{1}}{2 C_{1}^{\frac{N-2 s_{c}}{N}}}\right)^{\frac{N}{2 s_{c}}} \frac{1}{C r^{\frac{N-2 s_{c}}{2}}} .
$$

Therefore, injecting (3.124) into the above estimate yields (3.123).

Now, define $W_{n}^{1}(x)=\phi_{n}(x)-e^{-i t_{n}^{1} \Delta} \psi^{1}\left(x-x_{n}^{1}\right)$. Since $e^{i t_{n}^{1} \Delta} \phi_{n}\left(\cdot+x_{n}^{1}\right) \rightarrow \psi^{1}$ weakly in $H^{1}$, it follows that $e^{i t_{n}^{1} \Delta} W_{n}^{1} \rightarrow 0$ and $\left\langle\phi_{n}, e^{-i t_{n}^{1} \Delta} \psi^{1}\right\rangle_{\dot{H}^{s}}=\left\langle e^{i t_{n}^{1} \Delta} \phi_{n}, \psi^{1}\right\rangle_{\dot{H}^{s}}=\left\|\psi^{1}\right\|_{\dot{H}^{s}}^{2}$. Moreover, $\left\|W_{n}^{1}\right\|_{\dot{H}^{s}}^{2}=\left\|\phi_{n}\right\|_{\dot{H}^{s}}^{2}-\left\|\psi^{1}\right\|_{\dot{H}^{s}}^{2}$ as $n \rightarrow \infty$, which with $s=0$ and $s=1$ implies $\left\|W_{n}^{1}\right\|_{H^{1}} \leq C_{1}$.

Next, we construct $\psi_{n}^{j}$ for $j \geq 2$. We construct the functions $\phi^{j}$ inductively by assuming that $\psi^{j-1}$ 's are known. Let $M \geq 2$. Assuming that $\psi^{j}, x_{n}^{j}, t_{n}^{j}$ and $W_{n}^{j}$ are known for $1 \leq j \leq M-1$, we consider

$$
A_{M}=\limsup _{n}\left\|e^{i t \Delta} W_{n}^{M-1}\right\|_{L_{t}^{\infty} L_{x}^{\frac{2 N}{N-2 s_{c}}}} .
$$

If $A_{M}=0$, then we take $\psi^{j}=0$ for $j \geq M$ as in previous step. Assume $A_{M}>$ 0. Applying the previous step (construction for $\psi^{1}$ ) to the sequence $W_{n}^{M-1}$, we obtain, sequences $x_{n}^{M}, t_{n}^{M}$ and a function $\psi^{M} \in H^{1}$ such that $e^{i t_{n}^{M} \Delta} W_{n}^{M-1}\left(\cdot+x_{n}^{M}\right) \rightarrow \psi^{M}$ in $H^{1}$ and

$$
K\left\|\psi^{M}\right\|_{\dot{H}^{s_{c}}} \geq \frac{A_{M}^{\frac{N-2 s_{c}^{2}}{2 s_{c}\left(1-s_{c}\right)}}}{C_{M}^{\frac{N-2 s_{c}}{2 s_{c}\left(1-s_{c}\right)}}}, \quad \text { where } C_{M}=\limsup _{n}\left\|e^{i t \Delta} W_{n}^{M-1}\right\|_{H^{1}}
$$

We define $W_{n}^{M}(x)=W_{n}^{M-1}(x)-e^{-i t_{n}^{M} \Delta} \psi^{M}\left(x-x_{n}^{M}\right)$. We show (3.118) and (3.120) holds by induction. Assume (3.120) holds for $M-1$. Expanding

$$
\left\|W_{n}^{M}\right\|_{\dot{H}^{s}}^{2}=\left\|e^{i t_{n}^{M} \Delta} W_{n}^{M}\left(\cdot+x_{n}^{M}\right)\right\|_{\dot{H}^{s}}^{2}=\left\|e^{i t_{n}^{M} \Delta} W_{n}^{M-1}\left(\cdot+x_{n}^{M}\right)-\psi^{M}(x)\right\|_{\dot{H}^{s}}^{2}
$$


and using the weak convergence, we obtain (3.120) at $M$. Assume now that (3.118) holds for $j, k \in\{1, \ldots, M-1\}$ with $j \neq k$. Let $1 \leq j \leq M-1$. We will show inductively that $\left|t_{n}^{M}-t_{n}^{j}\right|+\left|x_{n}^{M}-x_{n}^{j}\right| \rightarrow+\infty$ by assuming that

$$
\left|t_{n}^{M}-t_{n}^{j+1}\right|+\left|x_{n}^{M}-x_{n}^{j+1}\right| \rightarrow+\infty, \ldots,\left|t_{n}^{M}-t_{n}^{M-1}\right|+\left|x_{n}^{M}-x_{n}^{M-1}\right| \rightarrow+\infty .
$$

Suppose, passing to a subsequence that $t_{n}^{M}-t_{n}^{j} \rightarrow t^{M_{j}}$ and $x_{n}^{M}-x_{n}^{j} \rightarrow x^{M_{j}}$ finite. Note that

$$
\begin{aligned}
e^{i t_{n}^{M} \Delta} W_{n}^{M-1}\left(x+x_{n}^{M}\right)= & e^{i\left(t_{n}^{M}-t_{n}^{j}\right) \Delta}\left(e^{i t_{n}^{j} \Delta} W_{n}^{j-1}\left(x+x_{n}^{j}\right)-\psi^{j}\left(x+x_{n}^{j}\right)\right) \\
& -\sum_{k=j+1}^{M-1} e^{i\left(t_{n}^{j}-t_{n}^{k}\right) \Delta} \psi_{n}^{k}\left(x+x_{n}^{j}-x_{n}^{k}\right) .
\end{aligned}
$$

By (3.118), the above summation term converges to 0 weakly in $H^{1}$ as $n$ tends to infinity and by the definition of $W_{n}^{j-1}$, we have $e^{i t_{n}^{j} \Delta} W_{n}^{j-1}\left(\cdot+x_{n}^{j}\right) \rightarrow \psi^{j}$ in $H^{1}$, which implies that the other term on the right-hand side of the previous expression goes to 0 weakly in $H^{1}$ as well as $n \rightarrow+\infty$, while the left side converges weakly to a nonzero $\psi^{M}$ by the definition of $W_{n}^{M-1}$, which leads to a contradiction. Thus, we deduce that $\left|t_{n}^{M}-t_{n}^{j}\right|+\left|x_{n}^{M}-x_{n}^{j}\right| \rightarrow$ $+\infty$ for $k=M$, which shows that (3.118) must hold for all $M$. Finally, we show (3.119). If for all $M, A_{M}>0$, then for a fixed $s_{c}$ by (3.128)

$$
\sum_{M \geq 1} \frac{A_{M}^{\frac{N-2 s_{c}^{2}}{2 s_{c}\left(1-s_{c}\right)}}}{K C_{M}^{\frac{N-2 s_{c}}{2 s_{c}\left(1-s_{c}\right)}}} \leq \sum_{n \geq 1}\left\|\psi^{M}\right\|_{\dot{H}^{s_{c}}}^{2} \leq \lim _{n \rightarrow+\infty}\left\|\phi_{n}\right\|_{\dot{H}^{s_{c}}}^{2}<\infty,
$$

where the second inequality follows from (3.120). Therefore, $A_{M} \rightarrow 0$ as $M \rightarrow \infty$, i.e., (3.122) holds. Combining (3.122) with (3.121) implies (3.119), thus, completing the proof.

Proposition 3.4.2 (Energy Pythagorean expansion) Under the assumptions of Theorem 3.4.1, we have

$$
E\left[\phi_{n}\right]=\sum_{j=1}^{M} E\left[e^{-i t_{n}^{j} \Delta} \psi^{j}\right]+E\left[W_{n}^{M}\right]+o_{n}(1)
$$


Proof. By the definition of energy, $E[u]$, and (3.120) for $s=1$, it is sufficient to establish for all $M \geq 1$,

$$
Z\left(\phi_{n}\right)=\sum_{j=1}^{M} Z\left(e^{-i t_{n}^{j} \Delta} \psi^{j}\right)+Z\left(W_{n}^{M}\right)+o_{n}(1),
$$

where $Z(u)=\int_{\mathbb{R}^{N}}\left(|x|^{-(N-\gamma)} *|u|^{p}\right)|u|^{p}$.

Step 1. Pythagorean expansion of a sum of orthogonal profiles. We show that for $M \geq 1$ fixed, the orthogonality condition (3.118) implies

$$
Z\left(\sum_{j=1}^{M} e^{-i t_{n}^{j} \Delta} \psi^{j}\left(\cdot-x_{n}^{j}\right)\right)=\sum_{j=1}^{M} Z\left(e^{-i t_{n}^{j} \Delta} \psi^{j}\right)+o_{n}(1)
$$

By rearranging and reindexing, we can find $M_{0} \leq M$ such that

- For $1 \leq j \leq M_{0}$, we have that $t_{n}^{j}$ is bounded in $n$.

- For $M_{0}+1 \leq j \leq M$, we have that $\left|t_{n}^{j}\right| \rightarrow \infty$ as $n \rightarrow \infty$.

Passing to a subsequence, we may assume that for each $1 \leq j \leq M_{0}, t_{n}^{j}$ converges (in $n$ ), and by adjusting the profiles $\psi^{j}$ 's we can take $t_{n}^{j}=0$. Note that either for $1 \leq k \leq M_{0}$ we have $t_{n}^{k} \rightarrow 0$ or for $M_{0}+1 \leq k \leq M$ we have $\left|t_{n}^{k}\right| \rightarrow \infty$ as $n \rightarrow \infty$. So if $t_{n}^{k} \rightarrow 0$, then from (3.120) we have $\left|x_{n}^{j}-x_{n}^{k}\right| \rightarrow \infty$ as $n \rightarrow \infty$, which implies

$$
Z\left(\sum_{j=1}^{M_{0}} \psi^{j}\left(\cdot-x_{n}^{j}\right)\right)=\sum_{j=1}^{M_{0}} Z\left(\psi^{j}\right)+o_{n}(1) .
$$

Now if $\left|t_{n}^{k}\right| \rightarrow \infty$ as $n \rightarrow \infty$, for a function $\tilde{\psi} \in \dot{H}^{\frac{N(p-1)-\gamma}{2 p}} \cap L^{\frac{p+1}{p}}$, by Hardy-LittlewoodSobolev, Sobolev embedding and $L^{p}$ space-time decay estimate, we obtain

$$
Z\left(e^{-i t_{n}^{k} \Delta} \psi^{k}\right) \lesssim\left\|e^{-i t_{n}^{k} \Delta} \psi^{k}\right\|_{L^{\frac{2 N p}{N+\gamma}}}^{2 p} \lesssim\left\|\psi^{k}-\tilde{\psi}\right\|_{\dot{H}^{\frac{N(p-1)-\gamma}{2 p}}}+\left|t_{n}^{k}\right|^{-\frac{N(p-1)}{2(p+1)}}\|\tilde{\psi}\|_{L^{\frac{p+1}{p}}}
$$

Approximating $\psi^{k}$ by $\tilde{\psi} \in C_{0}^{\infty}$ in $\dot{H}^{\frac{N(p-1)-\gamma}{2 p}}$ and sending $n \rightarrow \infty$, we obtain

$$
\lim _{n \rightarrow+\infty} Z\left(e^{-i t_{n}^{k} \Delta} \psi^{k}\right) \lesssim \lim _{n \rightarrow+\infty}\left\|e^{-i t_{n}^{k} \Delta} \psi^{k}\right\|_{L^{\frac{2 N p}{N+\gamma}}}^{2 p}=0
$$


Thus, combining (3.132) and (3.133) together yields,

$$
\begin{aligned}
& Z\left(\sum_{j=1}^{M} e^{-i t_{n}^{j} \Delta} \psi^{j}\right)=Z\left(\sum_{j=1}^{M_{0}} \psi^{j}+\sum_{j=M_{0}+1}^{M} e^{-i t_{n}^{j} \Delta} \psi^{j}\right) \\
& \quad=Z\left(\sum_{j=1}^{M_{0}} \psi^{j}\right)+\sum_{j=M_{0}+1}^{M} Z\left(e^{-i t_{n}^{j} \Delta} \psi^{j}\right)+o_{n}(1)=\sum_{j=1}^{M} Z\left(e^{-i t_{n}^{j} \Delta} \psi^{j}\right)+o_{n}(1),
\end{aligned}
$$

which is the right-hand side of the expansion (3.131).

Step 2. Ending the proof. Note that

$$
\begin{aligned}
\left\|W_{n}^{M}\right\|_{L^{\frac{2 N p}{N+\gamma}}}^{2 p} & \leq\left\|e^{i t \Delta} W_{n}^{M}\right\|_{L_{t}^{\infty} L_{x}^{\frac{2 N p}{N+\gamma}}}^{2 p} \leq\left\|e_{n}^{i t \Delta} W_{L_{t}^{\infty} L_{x}^{\frac{2 N}{N-2 s_{c}}}}^{M}\right\|_{n}^{p}\left\|e_{L_{t}^{\infty} L_{x}^{\frac{2 N p}{N+\gamma-2\left(1-s_{c}\right)}}}^{M}\right\|^{p} \\
& \leq\left\|e^{i t \Delta} W_{n}^{M}\right\|_{L_{t}^{\infty} L_{x}^{\frac{2 N}{N-2 s_{c}}}}^{p}\left\|e^{i t \Delta} W_{n}^{M}\right\|_{L_{t}^{\infty} \dot{H}_{x}^{1}}^{p} \leq\left\|e^{i t \Delta} W_{n}^{M}\right\|_{L_{t}^{\infty} L_{x}^{\frac{2 N}{N-2 s_{c}}}}^{p} \sup _{n}\left\|\phi_{n}\right\|_{H^{1}}^{p} .
\end{aligned}
$$

Since $\dot{H}^{s_{c}} \hookrightarrow L^{\frac{2 N}{N-2 s_{c}}}$, i.e., $\left(\infty, \frac{2 N}{N-2 s_{c}}\right)$ is an $\dot{H}^{s_{c}}$ admissible pair, by (3.119), we get

$$
\lim _{M \rightarrow+\infty}\left(\lim _{n \rightarrow+\infty}\left\|W_{n}^{M}\right\|_{\frac{2 N p}{N+\gamma}}^{2 p}\right)=0 .
$$

Let $M \geq 1$ and $\varepsilon>0$. Note that $\left\{\phi_{n}\right\}_{n}$ is uniformly bounded in $L^{\frac{2 N p}{N+\gamma}}$, as it is uniformly bounded in $H^{1}$ by the hypothesis. Hence, by (3.134) $\left\{W_{n}^{M}\right\}_{n}$ is also uniformly bounded in $L^{\frac{2 N p}{N+\gamma}}$. Hence, we can choose $M_{1}>M$ and $n_{1}$ such that for $n>n_{1}$, we have

$$
\begin{gathered}
\left|Z\left(\phi_{n}\right)-Z\left(\phi_{n}-W_{n}^{M_{1}}\right)\right|+\left|Z\left(W_{n}^{M}-W_{n}^{M_{1}}\right)-Z\left(W_{n}^{M}\right)\right| \\
\leq C\left[\left\|W_{n}^{M_{1}}\right\|_{L^{\frac{2 N p}{N+\gamma}}}\left(\sup _{n}\left\|\phi_{n}\right\|_{L^{\frac{2 N p}{N+\gamma}}}^{2 p-1}+\sup _{n}\left\|W_{n}^{M}\right\|_{L^{\frac{2 N p}{N+\gamma}}}^{2 p-1}\right)\right]+C\left\|W_{n}^{M_{1}}\right\|_{L^{\frac{2 N p}{N+\gamma}}}^{2 p} \leq \varepsilon,
\end{gathered}
$$

where we have used the triangle inequality to estimate

$$
\left|\left\|W_{n}^{M}-\left.W_{n}^{M_{1}}\right|_{L^{\frac{2 N p}{N+\gamma}}} ^{2 p}-\right\| W_{n}^{M}\right|_{L^{\frac{2 N p}{N+\gamma}}}^{2 p}\left|\lesssim \| W_{n}^{M_{1}}\right|_{L^{\frac{2 N p}{N+\gamma}}}^{2 p},
$$

and by observing that $a^{2 p}>a(a-b)^{2 p-1}$ together with the triangle inequality, we estimate

$$
\begin{aligned}
\left|\left\|\phi_{n}-W_{n}^{M_{1}}\right\|_{L^{\frac{2 N p}{N+\gamma}}}^{2 p}-\left\|\phi_{n}\right\|_{L^{\frac{2 N p}{N+\gamma}}}^{2 p}\right| & \lesssim\left(\left\|\phi_{n}-W_{n}^{M_{1}}\right\|_{L^{\frac{2 N p}{N+\gamma}}}-\left\|\phi_{n}\right\|_{L^{\frac{2 N p}{N+\gamma}}}\right)\left\|\phi_{n}-W_{n}^{M}\right\|_{L^{\frac{2 N p}{N+\gamma}}}^{2 p-1} \\
& \lesssim\left\|W_{n}^{M_{1}}\right\|_{L^{\frac{2 N p}{N+\gamma}}}\left(\sup _{n}\left\|\phi_{n}\right\|_{L^{\frac{2 N p}{N+\gamma}}}^{2 p-1} \sup _{n}\left\|W_{n}^{M}\right\|_{L^{\frac{2 N p}{N+\gamma}}}^{2 p-1}\right) .
\end{aligned}
$$


Choose $n_{2} \geq n_{1}$ such that for $n \geq n_{2}$, by (3.131), we get

$$
\left|Z\left(\phi_{n}-W_{n}^{M_{1}}\right)-\sum_{j=1}^{M_{1}} Z\left(e^{-i t_{n}^{j} \Delta} \psi^{j}\right)\right| \leq \varepsilon .
$$

Using the definition of $W_{n}^{j}$, we expand $W_{n}^{M}-W_{n}^{M_{1}}$, to obtain

$$
W_{n}^{M}-W_{n}^{M_{1}}=\sum_{j=M+1}^{M_{1}} e^{-i t_{n}^{j} \Delta} \psi^{j}\left(\cdot-x_{j}\right) .
$$

By (3.131) there exists $n_{3} \geq n_{2}$ such that for $n \geq n_{3}$,

$$
\left|Z\left(W_{n}^{M}-W_{n}^{M_{1}}\right)-\sum_{j=M+1}^{M_{1}} Z\left(e^{-i t_{n}^{j} \Delta} \psi^{j}\right)\right| \leq \varepsilon .
$$

Thus, for $n \geq n_{3}$, by (3.135), (3.136) and (3.137), we obtain

$$
\begin{aligned}
Z\left(\phi_{n}\right) & -\sum_{j=1}^{M} Z\left(e^{-i t_{n}^{j} \Delta} \psi^{j}\right)-Z\left(W_{n}^{M}\right) \\
= & \mid Z\left(\phi_{n}\right)-Z\left(\phi_{n}-W_{n}^{M_{1}}\right)+Z\left(\phi_{n}-W_{n}^{M_{1}}\right)-\sum_{j=1}^{M_{1}} Z\left(e^{-i t_{n}^{j} \Delta} \psi^{j}\right)+Z\left(W_{n}^{M}-W_{n}^{M_{1}}\right) \\
& -Z\left(W_{n}^{M}\right)+\sum_{j=M+1}^{M_{1}} Z\left(e^{-i t_{n}^{j} \Delta} \psi^{j}\right)-Z\left(W_{n}^{M}-W_{n}^{M_{1}}\right) \mid \leq 3 \varepsilon
\end{aligned}
$$

which implies (3.130).

\subsubsection{Critical solution}

In subsection 3.4.3, we study a critical solution of (1.1), denoted by $u_{c}(t)$. The main ingredients are Theorem 3.4.1 and Proposition 3.4.2 (proved in previous subsection) along with Theorem 3.2.5 (long time perturbation theory).

Theorem 3.4.3 (Existence of critical solution) Let $0<s_{c}<1$. There exists a global solution $u_{c}(t) \in H^{1}\left(\mathbb{R}^{N}\right)$ of (1.1) with initial data $u_{c, 0} \in H^{1}\left(\mathbb{R}^{N}\right)$ such that $\left\|u_{c, 0}\right\|_{L^{2}}=1$, $(\mathcal{M E})_{c}<1$, where $(\mathcal{M E})_{c}=\frac{E\left[u_{c}\right]}{M[Q]^{\theta} E[Q]}, \mathcal{G}\left[u_{c}(t)\right]<1$ for all $0 \leq t \leq+\infty$, and

$$
\left\|u_{c}\right\|_{S\left(\dot{H}^{s_{c}}\right)}=+\infty
$$


Proof. We consider a sequence of solutions $u_{n}(t)$ with corresponding initial data $u_{n, 0}$ such that $\mathcal{G}_{u_{n}}(0)<1$ and $\mathcal{M E}\left[u_{n}\right] \searrow(\mathcal{M E})_{c}$ as $n \rightarrow+\infty$. Each $u_{n}$ is global and nonscattering i.e. $\left\|u_{n}\right\|_{S(\dot{H})^{s_{c}}}=+\infty$. Without lost of generality, rescale the solutions so that $\left\|u_{n, 0}\right\|_{L^{2}}=1$, thus, $\left\|\nabla u_{n, 0}\right\|_{L^{2}}<\|Q\|_{L^{2}}^{\theta}\|\nabla Q\|_{L^{2}}$ and $\mathcal{M E}\left[u_{n}\right] \searrow(\mathcal{M E})_{c}$. Applying the linear profile expansion Theorem 3.4.1 to $u_{n, 0}$ (which is now uniformly bounded in $H^{1}$ ), we have

$$
u_{n, 0}(x)=\sum_{j=1}^{M} e^{-i t_{n}^{j} \Delta} \psi^{j}\left(x-x_{n}^{j}\right)+W_{n}^{M}(x) .
$$

By the energy Pythagorean expansion (Proposition 3.4.2), we have

$$
\begin{aligned}
\sum_{j=1}^{M} \lim _{n \rightarrow+\infty} \frac{E\left[e^{-i t_{n}^{j} \Delta} \psi^{j}\right]}{M[Q]^{1-s_{c}} E[Q]^{s_{c}}} & +\lim _{n \rightarrow+\infty} \frac{E\left[W_{n}^{M}\right]}{M[Q]^{1-s_{c}} E[Q]^{s_{c}}} \\
& =\lim _{n \rightarrow+\infty} \frac{E\left[u_{n, 0}\right]}{M[Q]^{1-s_{c}} E[Q]^{s_{c}}}=(\mathcal{M E})_{c}
\end{aligned}
$$

and since by Lemma 3.113 (comparison of gradient and energy) each energy is $\geq 0$,

$$
E\left[e^{-i t_{n}^{j} \Delta} \psi^{j}\right] \geq c(N, p, \gamma)\left\|\nabla \psi^{j}\right\|_{L^{2}} \geq 0
$$

we have that

$$
\lim _{n \rightarrow+\infty} \frac{E\left[e^{-i t_{n}^{j} \Delta} \psi^{j}\right]}{M[Q]^{1-s_{c}} E[Q]^{s_{c}}} \leq(\mathcal{M E})_{c} \forall j .
$$

Also, for $s=0$ in (3.120), we have

$$
\sum_{j=1}^{M}\left\|\psi^{j}\right\|_{L^{2}}^{2}+\lim _{n \rightarrow+\infty}\left\|W_{n}^{M}\right\|_{L^{2}}^{2} \leq \lim _{n \rightarrow+\infty}\left\|u_{n, 0}\right\|_{L^{2}}^{2}=1
$$

This implies that

$$
\sum_{j=1}^{M}\left\|\psi^{j}\right\|_{L^{2}}^{2} \leq 1
$$

Again, Pythagorean expansion (3.120) for $s=1$ yields

$$
\sum_{j=1}^{M}\left\|\nabla \psi^{j}\right\|_{L^{2}}^{2}+\lim _{n \rightarrow+\infty}\left\|\nabla W_{n}^{M}\right\|_{L^{2}}^{2} \leq \lim _{n \rightarrow+\infty}\left\|\nabla u_{n, 0}\right\|_{L^{2}}^{2} \leq\|Q\|_{L^{2}}^{2 \theta}\|\nabla Q\|_{L^{2}}^{2}
$$


thus, we have

$$
\left\|\nabla \psi^{j}\right\|_{L^{2}} \leq\|Q\|_{L^{2}}^{\theta}\|\nabla Q\|_{L^{2}}
$$

Similarly, for all $M \in \mathbb{N}$ we get

$$
\lim _{n \rightarrow+\infty}\left\|W_{n}^{M}\right\|_{L^{2}}^{2} \leq 1 \quad \text { and } \quad \lim _{n \rightarrow+\infty}\left\|\nabla W_{n}^{M}\right\|_{L^{2}} \leq\|Q\|_{L^{2}}^{\theta}\|\nabla Q\|_{L^{2}}
$$

and for large $n$

$$
E\left[W_{n}^{M}\right] \geq 0
$$

Consider the sequence $\left\{t_{n}^{j}\right\}$ given by Theorem 3.4.1. Combining (3.143) and (3.145), we get

$$
\left\|e^{-i t_{n}^{j} \Delta} \psi^{j}\right\|_{L^{2}}\left\|\nabla e^{-i t_{n}^{j} \Delta} \psi^{j}\right\|_{L^{2}} \leq\|Q\|_{L^{2}}^{\theta}\|\nabla Q\|_{L^{2}}
$$

Now for each $\psi^{j} \in H^{1}$ by applying Lemma 3.3.4 (existence of wave operator), we obtain a function $\widetilde{\psi}^{j} \in H^{1}$, which we say is the nonlinear profile (corresponding to the linear profile $\left.\psi^{j}\right)$. Set $\widetilde{\psi}^{j}=\operatorname{NLF}\left(t_{n}^{j}\right)\left(e^{-i t_{n}^{j} \Delta} \psi^{j}\right)$ such that $\operatorname{NLF}\left(-t_{n}^{j}\right) \widetilde{\psi}^{j}=e^{-i t_{n}^{j} \Delta} \psi^{j}$. We consider two cases:

Case 1: $\left|t_{n}^{j}\right| \rightarrow \pm \infty$. If $\left|t_{n}^{j}\right| \rightarrow+\infty$, we have $\left\|e^{-i t_{n}^{k} \Delta} \psi^{k}\right\|_{L^{\frac{2 N p}{N+\gamma}}}^{2 p} \rightarrow 0$ as discussed in Proposition 3.4.2, and thus, using (3.141)

$$
\frac{1}{2} \frac{\left\|\psi^{j}\right\|_{L^{2}}^{\theta}\left\|\nabla \psi^{j}\right\|_{L^{2}}}{M[Q]^{\theta} E[Q]}<(\mathcal{M E})_{c} .
$$

By Lemma 3.3.4 (existence of wave operator) with $\omega=\left(\frac{s_{c}(p-1)+1}{s_{c}(p-1)}\right)^{s_{c} / 2}<1$, there exists $\widetilde{\psi^{j}} \in H^{1}\left(\mathbb{R}^{N}\right)$ such that

$$
\begin{array}{r}
M[\widetilde{\psi}]=M\left[\psi^{j}\right] \text { and } E[\widetilde{\psi}]=\frac{1}{2}\left\|\nabla \psi^{j}\right\|_{L^{2}}^{2}, \\
\left\|\nabla \operatorname{NLF}(t) \widetilde{\psi^{j}}\right\|_{L^{2}}^{s_{c}}\left\|\widetilde{\psi}^{j}\right\|_{L^{2}}^{1-s_{c}}<\|\nabla Q\|_{L^{2}}^{s_{c}}\|Q\|_{L^{2}}^{1-s_{c}},
\end{array}
$$


and

$$
\left\|\operatorname{NLF}\left(-t_{n}^{j}\right) \widetilde{\psi}^{j}-e^{-i t_{n}^{j} \Delta} \psi^{j}\right\|_{H^{1}} \rightarrow 0
$$

Since $M\left[\psi^{j}\right]<1$, using (3.148) and (3.149), we obtain $\mathcal{M E}\left[\widetilde{\psi}^{j}\right]<(\mathcal{M E})_{c}$. Therefore, the definition of $(\mathcal{M E})_{c}$ together with (3.150) implies that

$$
\left\|\operatorname{NLF}(-t) \widetilde{\psi}^{j}\right\|_{S\left(\dot{H}^{s_{c}}\right.}<+\infty
$$

Similarly, if $\left|t_{n}^{j}\right| \rightarrow-\infty$, Lemma 3.3.4 (existence of wave operator) yields

$$
\left\|\operatorname{NLF}\left(t_{n}^{j}\right) \widetilde{\psi}^{j}-e^{i t_{n}^{j} \Delta} \psi^{j}\right\|_{H^{1}} \rightarrow 0 \quad \text { and } \quad\left\|\operatorname{NLF}(t) \widetilde{\psi}^{j}\right\|_{S\left(\dot{H}^{s_{c}}\right.}<+\infty
$$

Case 2: $t_{n}^{j}$ is bounded (as $n \rightarrow \infty$ ). Adjusting the profiles $\psi^{j}$ we restrict it to the case $t_{n}^{j}=0$. Thus, (3.118) reduces to $\left|x_{n}^{j}-x_{n}^{k}\right| \rightarrow+\infty$ as $n \rightarrow \infty$ and continuity of linear flow in $H^{1}$ leads to $e^{-i t_{n}^{j} \Delta} \psi^{j} \rightarrow \psi^{j}$ strongly in $H^{1}$ as $n \rightarrow \infty$. In this scenario, we can simply let

$$
\widetilde{\psi}^{j}=\operatorname{NLF}(0) e^{-i 0 \Delta} \psi^{j}=\psi^{j} .
$$

Therefore, in both the cases, we have a new nonlinear profile $\widetilde{\psi}^{j}$ associated to each original linear profile $\psi^{j}$ satisfying (3.151) and (3.152). The idea now is to apply a nonlinear flow to $\phi_{n}(x)$ and approximate it by a combination of "nonlinear bumps", i.e.,

$$
\operatorname{NLF}(t) \phi_{n}(x) \approx \sum_{j=1}^{M} \operatorname{NLF}\left(t-t_{n}^{j}\right) \widetilde{\psi}^{j}\left(x-x_{n}^{j}\right) .
$$

To carry out the argument, we introduce the nonlinear evolution of each separate initial condition $u_{n, 0}=\phi_{n}$ :

$$
u_{n}(t)=\operatorname{NLF}(t) \phi_{n}(x)=\operatorname{NLF}(t) u_{n, 0},
$$

the nonlinear evolution of each separate nonlinear profile ("bump"):

$$
v^{j}(t)=\operatorname{NLF}(t) \widetilde{\psi}^{j}
$$


and a linear sum of nonlinear evolutions of those "bumps":

$$
\widetilde{u}_{n}(t, x)=\sum_{j=1}^{M} v^{j}\left(t-t_{n}^{j}, x-x_{n}^{j}\right) .
$$

We now think of $u_{n, 0}=\phi_{n}$ as a sum of nonlinear bumps $\widetilde{\psi}^{j}$ and $u_{n}(t)$ is a nonlinear evolution of their entire sum. On the other hand, $\widetilde{u}_{n}(t)$ is a sum of nonlinear evolutions of each bump and we want to compare $u_{n}(t)$ with $\widetilde{u}_{n}(t)$. Also, note that if we just had the linear evolutions, then both $u_{n}(t)$ and $\widetilde{u}_{n}(t)$ would be the same. Thus, $u_{n}(t)$ satisfies

$$
i\left(u_{n}\right)_{t}+\Delta u_{n}+\left(|x|^{-(N-\gamma)} *\left|u_{n}\right|^{p}\right)\left|u_{n}\right|^{p-2} u_{n}=0,
$$

and $\widetilde{u}_{n}(t)$ satisfies

$$
i\left(\widetilde{u}_{n}\right)_{t}+\Delta \widetilde{u}_{n}+\left(|x|^{-(N-\gamma)} *\left|\widetilde{u}_{n}\right|^{p}\right)\left|\widetilde{u}_{n}\right|^{p-2} \widetilde{u}_{n}=\widetilde{e}_{n}^{M},
$$

where

$$
\widetilde{e}_{n}^{M}=\left(|x|^{-(N-\gamma)} *\left|\widetilde{u}_{n}\right|^{p}\right)\left|\widetilde{u}_{n}\right|^{p-2} \widetilde{u}_{n}-\sum_{j=1}^{M}\left(|x|^{-(N-\gamma)} *\left|v^{j}\left(t-t_{n}^{j}, \cdot-x_{n}^{j}\right)\right|^{p}\right)\left|v^{j}\right|^{p-2} v^{j} .
$$

We also define

$$
\widetilde{W}_{n}^{M}=W_{n}^{M}+\sum_{j=1}^{M}\left(e^{-i t_{n}^{j} \Delta} \psi^{j}\left(x-x_{n}^{j}\right)-\operatorname{NLF}\left(-t_{n}^{j}\right) \widetilde{\psi}^{j}\left(x-x_{n}^{j}\right)\right),
$$

and using (3.117) we write

$$
u_{n, 0}=\sum_{j=1}^{M} \operatorname{NLF}\left(-t_{n}^{j}\right) \widetilde{\psi}^{j}\left(x-x_{n}^{j}\right)+\widetilde{W}_{n}^{M},
$$

such that $u_{n, 0}-\widetilde{u}(0)=\widetilde{W_{n}^{M}}$. Applying triangle inequality together with (2.3), we estimate

$$
\left\|e^{i t \Delta} \widetilde{W}_{n}^{M}\right\|_{S\left(\dot{H}^{\left.s_{c}\right)}\right.} \lesssim\left\|e^{i t \Delta} W_{n}^{M}\right\|_{S\left(\dot{H}^{s_{c}}\right)}+\sum_{j=1}^{M}\left\|e^{-i t_{n}^{j} \Delta} \psi^{j}-N L F\left(-t_{n}^{j}\right) \widetilde{\psi}^{j}\right\|_{H^{1}} .
$$

By (3.151) and (3.119) we have that

$$
\lim _{M \rightarrow \infty}\left(\lim _{n \rightarrow \infty}\left\|e^{i t \Delta} \widetilde{W}_{n}^{M}\right\|_{S\left(\dot{H}^{s_{c}}\right)}\right)=0 .
$$


We now approximate $u_{n}$ by $\widetilde{u}_{n}$. Then from the Theorem 3.2.5 (long time perturbation theory) and (3.152) it follows that for $n$ large enough, $\left\|u_{n}\right\|_{S\left(\dot{H}^{s_{c}}\right)}<+\infty$, which is a contradiction, since $u_{n}$ is non-scattering. We assume the following two claims, which we prove later.

Claim 3.4.4 There exists a constant $A$ independent of $M$, and for every $M$, there exists $n_{0}=n_{0}(M)$ such that if $n>n_{0}$, then $\left\|\widetilde{u}_{n}\right\|_{S\left(\dot{H}^{s_{c}}\right)} \leq A$.

Claim 3.4.5 For each $M$ and $\epsilon>0$, there exists $n_{1}=n_{1}(M ; \epsilon)$ such that if $n>n_{1}$, then $\left\|\widetilde{e}_{n}^{M}\right\|_{S^{\prime}\left(\dot{H}^{-s_{c}}\right)} \leq \epsilon$.

By (3.155), for any $\epsilon>0$ there exists $M_{1}=M_{1}(\epsilon)$ sufficiently large such that for each $M>M_{1}$ there exists $n_{2}=n_{2}(M)$ such that $n>n_{2}$ implies

$$
\left\|e^{i t \Delta}\left(\tilde{u}_{n}(0)-u_{n}(0)\right)\right\|_{S\left(\dot{H}^{s}\right)} \leq \epsilon .
$$

Thus, if the Claim 3.4.4 and Claim 3.4.5 hold true, using Theorem 3.2.5 for sufficiently large $M$ and $n=\max \left(n_{0}, n_{1}, n_{2}\right)$, we obtain $\left\|u_{n}\right\|_{S\left(\dot{H}^{s_{c}}\right)}<\infty$, a contradiction, since $u_{n}$ is non-scattering. Now there are two possible scenarios in the profile decomposition (3.154):

Scenario 1: More that one $\widetilde{\psi}^{j} \neq 0$. By (3.142), we must have $M\left[e^{-i t_{n}^{j} \Delta} \widetilde{\psi}^{j}\right]<1$ for each $j$, which by energy decomposition, for large enough $n$ yields

$$
\frac{M\left[\operatorname{NLF}(t) \widetilde{\psi}^{j}\right]^{1-s_{c}} E\left[\operatorname{NLF}(t) \widetilde{\psi}^{j}\right]^{s_{c}}}{M[Q]^{1-s_{c}} E[Q]^{s_{c}}}=\frac{M\left[\widetilde{\psi}^{j}\right]^{1-s_{c}} E\left[\widetilde{\psi}^{j}\right]^{s_{c}}}{M[Q]^{1-s_{c}} E[Q]^{s_{c}}}=\mathcal{M E}\left[\widetilde{\psi}^{j}\right]<(M E)_{c} .
$$

Now, since $\left\|\operatorname{NLF}(t) \widetilde{\psi}^{j}\left(\cdot-x_{n}^{j}\right)\right\|_{S\left(\dot{H}^{s_{c}}\right)}<+\infty$, the right-hand side of (3.154) is bounded in $S\left(\dot{H}^{s_{c}}\right)$. By (3.155), we conclude that $\left\|\operatorname{NLF}(t) u_{n, 0}\right\|_{S\left(\dot{H}^{s_{c}}\right)}<+\infty$, which is a contradiction.

Scenario 2: Suppose $\widetilde{\psi}^{1} \neq 0$ and $\widetilde{\psi}^{j}=0$ for all $j \geq 2$. Hence, we have

$$
u_{n, 0}=\operatorname{NLF}\left(-t_{n}^{1}\right) \widetilde{\psi}^{1}\left(x-x_{n}^{1}\right)+\widetilde{W}_{n}^{1}
$$


with

$$
M\left[\widetilde{\psi}^{1}\right]<1, \quad \mathcal{M E}\left[\widetilde{\psi}^{1}\right] \leq(\mathcal{M E})_{c}, \text { and } \lim _{n \rightarrow+\infty}\left\|e^{i t \Delta}(t) \widetilde{W}_{n}^{1}\right\|_{S\left(\dot{H}^{s_{c}}\right)}=0
$$

Let $u_{c}$ be the global solution to (1.1) with initial data $u_{c, 0}=\widetilde{\psi}^{1}$ i.e., $u_{c}(t)=\operatorname{NLF}(t) \widetilde{\psi}^{1}$. Assume by contradiction that $\left\|u_{c}\right\|_{S\left(\dot{H}^{s^{c}}\right)}<+\infty$. Let $\widetilde{u}_{n}(t)=\operatorname{NLF}\left(t-t_{n}^{1}\right) \widetilde{\psi}^{1}$, then

$$
\left\|\widetilde{u}_{n}(t)\right\|_{S\left(\dot{H}^{s_{c}}\right)}=\left\|\mathrm{NLF}\left(t-t_{n}^{1}\right) \widetilde{\psi}^{1}\right\|_{S\left(\dot{H}^{s_{c}}\right)}=\left\|u_{c}\right\|_{S\left(\dot{H}^{s_{c}}\right)}<+\infty .
$$

Therefore, using the long time perturbation theory with $e=0$, we deduce that $\left\|u_{n}\right\|_{S\left(\dot{H}^{s} c\right)}<$ $+\infty$, which is a contradiction, since by construction $u_{n}$ is non-scattering. It only remains to establish Claims 3.4.4 and 3.4.5.

Proof of Claim 3.4.4: Let $M_{0}$ be large enough so that

$$
\left\|e^{i t \Delta} \widetilde{W}_{n}^{M_{0}}\right\|_{S\left(\dot{H}^{s c}\right)} \leq \delta_{s d}
$$

Then by (3.151), (3.153) and (3.119) for each $j>M_{0}$, we have $\left\|e^{i t \Delta} \psi^{j}\right\|_{S\left(\dot{H}^{s_{c}}\right)} \leq \delta_{s d}$, and by Lemma 3.3.4 (existence of wave operator), we obtain

$$
\left\|v^{j}\right\|_{S\left(\dot{H}^{s_{c}}\right)} \leq 2\left\|e^{i t \Delta} \psi^{j}\right\|_{S\left(\dot{H}^{s_{c}}\right)} \text { for } j>M_{0} .
$$

Note that the pair $\left(\frac{2(N+2)}{N-2 s_{c}}, \frac{2(N+2)}{N-2 s_{c}}\right)$ is $\dot{H}^{s_{c}}$ admissible. Hence, we have

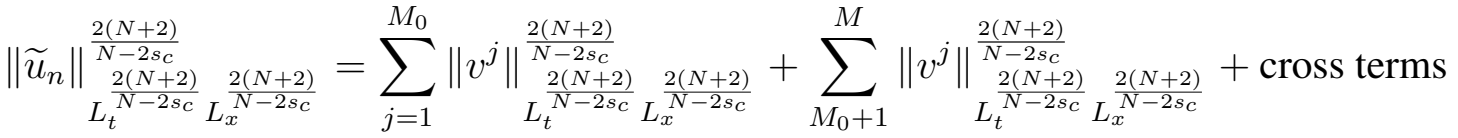

$$
\begin{aligned}
& \leq \sum_{j=1}^{M_{0}}\left\|v^{j}\right\|_{L_{t}^{\frac{2\left(N-2 s_{c}\right.}{N-2 s_{c}}}}^{\frac{2(N+2)}{L_{x}^{N-2 s_{c}}}}+2^{\frac{2(N+2)}{N-2 s_{c}}} \sum_{M_{0}+1}^{M}\left\|e^{i t \Delta} \psi^{j}\right\|_{L_{t}^{\frac{2\left(N+2 s_{c}\right.}{N-2 s_{c}}}}^{\frac{2(N+2)}{L_{x}^{N-2 s_{c}}}}+\text { cross terms. }
\end{aligned}
$$

On the other hand, by (3.117) we have

$$
\begin{aligned}
& \left\|e^{i t \Delta} \phi_{n}\right\|^{\frac{2(N+2)}{N-2 s_{c}}} \frac{2(N+2)}{L_{t}^{N-2 s_{c}}} L_{x}^{\frac{2(N+2)}{N-2 s_{c}}}
\end{aligned}
$$

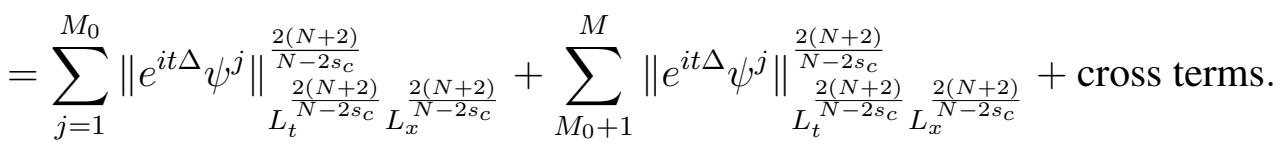


By (3.118) and taking $n_{0}=n_{0}(M)$ sufficiently large, we make the "cross terms" $\leq 1$. Since $\left\|e^{i t \Delta} \phi_{n}\right\|_{L_{t}^{\frac{2(N+2)}{N-2 s_{c}}} L_{x}^{\frac{2(N+2)}{N-2 s_{c}}}} \leq c\left\|\phi_{n}\right\|_{\dot{H}^{s_{c}}} \leq c_{1}$, (3.157) implies that the second term on the right-hand side of the above expression is bounded independent of $M$ provided $n>n_{0}$. And, thus (3.156) gives that $\left\|\tilde{u}_{n}\right\|_{L_{t}^{\frac{2(N+2)}{N-2 s}}} \frac{2(N+2)}{L_{x}^{N-2 s}}$ is also bounded independent of $M$ provided $n>n_{0}$.

Similar argument proves that $\left\|\widetilde{u}_{n}\right\|_{L_{t}^{\infty} L_{x}^{\frac{2 N}{N-2 s_{c}}}}$ is bounded independent of $M$ provided $n>n_{0}$. Interpolation between these exponents gives that $\left\|\widetilde{u}_{n}\right\|_{L_{t}^{1-s_{c}}} L_{x}^{\frac{2 N p}{N+\gamma}}$ is bounded independent of $M$ provided $n>n_{0}$. Finally, by applying Lemma 2.4.6 to the integral equation of $i\left(\widetilde{u}_{n}\right)_{t}+\Delta \widetilde{u}_{n}+\left(|x|^{-(N-\gamma)} *\left|\widetilde{u}_{n}\right|^{p}\right)\left|\widetilde{u}_{n}\right|^{p-2} \widetilde{u}_{n}=\widetilde{e}_{n}^{M}$ and using $\left\|\widetilde{e}_{n}^{M}\right\|_{S^{\prime}\left(\dot{H}^{-s_{c}}\right)} \leq \epsilon$ (see Claim 3.4.5), we obtain that $\left\|\widetilde{u}_{n}\right\|_{S\left(\dot{H}^{s_{c}}\right)}$ is bounded independent of $M$ for $n>n_{0}$.

Proof of Claim 3.4.5: Recall that $\left(\frac{2 p}{s_{c}(2 p-1)+1}, \frac{2 N p}{N+\gamma}\right)$ is an $\dot{H}^{-s_{c}}$ admissible pair. Then

$$
\left\|\widetilde{e}_{n}^{M}\right\|_{S^{\prime}\left(\dot{H}^{-s_{c}}\right)} \leq\left\|\widetilde{e}_{n}^{M}\right\|_{L_{t}^{\left(1-s_{c}\right)(2 p-1)}} \frac{2 p}{L_{x}^{2 N p-N-\gamma}} .
$$

Observe that expansion of $\widetilde{e}_{n}^{M}$ consists of cross terms of the form

$$
\sum_{j=1}^{M} \sum_{k=1}^{M} \sum_{\substack{l=1 \\ k \neq l}}^{M}\left(|x|^{-(N-\gamma)} *\left|v^{j}\left(t-t_{n}^{j}\right)\right|^{p}\right)\left|v^{k}\left(t-t_{n}^{k}\right)\right|^{p-2} v^{l}\left(t-t_{n}^{l}\right)
$$

where all of $j, k$ and $l$ are not same. Assume, without loss of generality, that $k \neq l$, and thus, $\left|t_{n}^{k}-t_{n}^{l}\right| \rightarrow \infty$ as $n \rightarrow+\infty$. So, we estimate

$$
\begin{aligned}
\|\left(|x|^{-(N-\gamma)}\right. & \left.*\left|v^{j}\left(t-t_{n}^{j}\right)\right|^{p}\right)\left|v^{k}\left(t-t_{n}^{k}\right)\right|^{p-2} v^{l}\left(t-t_{n}^{l}\right) \|_{L_{t}^{\frac{2 p}{\left(1-s_{c}\right)(2 p-1)}} L_{x}^{\frac{2 N p}{2 N p-N-\gamma}}} \\
& \leq\left\|v^{j}\right\| \frac{2 p}{L_{t}^{1-s_{c}}} \frac{2 N p}{L_{x}^{N+\gamma}}\left\|\left|v^{k}\left(t-t_{n}^{k}\right)\right|^{p-2} v^{l}\left(t-t_{n}^{l}\right)\right\|_{L_{t}^{\frac{2 p}{\left(1-s_{c}\right)(p-1)}}} \frac{2 N p}{L_{x}^{(N+\gamma)(p-1)}}
\end{aligned}
$$

Since both $v^{k}$ and $v^{l}$ belong to $L_{t}^{\frac{2 p}{1-s_{c}}} L_{x}^{\frac{2 N p}{N+\gamma}}$, then

$$
\left\|\left|v^{k}\left(t-\left(t_{n}^{k}-t_{n}^{l}\right)\right)\right|^{p-2} v^{l}(t)\right\|_{L_{t}^{\left(1-s_{c}\right)(p-1)}} \frac{2 N p}{L_{x}^{(N+\gamma)(p-1)}} \rightarrow 0
$$

This gives Claim 3.4.5, which completes the proof of Theorem 3.4.3. 
We now prove the precompactness of the flow associated to the critical solution $u_{c}$.

Proposition 3.4.6 (Precompactness of the flow of the critical solution) Assume $u_{c}$ as in Theorem 3.4.3. Then there exists a continuous path $x(t)$ in $\mathbb{R}^{N}$ such that

$$
K=\left\{u_{c}(\cdot-x(t), t) \mid t \in[0, \infty)\right\}
$$

is precompact in $H^{1}$ (i.e., $\bar{K}$ is compact in $H^{1}$ ).

Proof. Take a sequence $t_{n} \rightarrow+\infty$; we want to show that $u_{c}\left(t_{n}\right)$ has a converging subsequence in $H^{1}\left(\mathbb{R}^{N}\right)$. Note that if $\left\{t_{n}\right\}$ is bounded, we may assume $t_{n}$ converges to some finite $t^{*}$ and by the continuity of the solution in $H^{1}\left(\mathbb{R}^{N}\right)$, we get the desired result. Let $\phi_{n}=u_{c}\left(t_{n}\right)$ be a uniformly bounded sequence in $H^{1}\left(\mathbb{R}^{N}\right)$ in linear profile decomposition (Theorem 3.4.1). Thus, by Theorem 3.4.3, there exists profiles $\widetilde{\psi}^{j}$, time sequences $t_{n}^{j}$, space sequences $x_{n}^{j}$ and an error $\widetilde{W}_{n}^{M}$ such that

$$
u_{c}\left(t_{n}\right)=\sum_{j=1}^{M} N L F\left(-t_{n}^{j}\right) \widetilde{\psi}^{j}\left(x-x_{n}^{j}\right)+\widetilde{W}_{n}^{M}(x)
$$

with $\left|t_{n}^{j}-t_{n}^{k}\right|+\left|x_{n}^{j}-x_{n}^{k}\right| \rightarrow+\infty$ as $n \rightarrow+\infty$ for fixed $j \neq k$. By energy Pythagorean expansion (Proposition 3.4.2)

$$
\sum_{j=1}^{M} E\left[\widetilde{\psi}^{j}\right]+\lim _{n \rightarrow+\infty} E\left[\widetilde{W}_{n}^{M}\right]=E\left[u_{c}\right]=(\mathcal{M E})_{c} M[Q]^{1-s} E[Q]^{s},
$$

since each energy is $\geq 0$ by Lemma 3.3.2 (comparison of gradient and energy), we have

$$
\lim _{n \rightarrow+\infty} E\left[N L F\left(-t_{n}^{j}\right) \widetilde{\psi}^{j}\left(x-x_{n}^{j}\right)\right] \leq(\mathcal{M E})_{c} M[Q]^{1-s} E[Q]^{s} \quad \forall j
$$

Also, for $s=0$ in (3.120), we get

$$
\sum_{j=1}^{M} M\left[\widetilde{\psi}^{j}\left(x-x_{n}^{j}\right)\right]+\lim _{n \rightarrow+\infty} M\left[\widetilde{W}_{n}^{M}\right]=\lim _{n \rightarrow+\infty} M\left[u_{c}\right]=1
$$


We follow a similar argument as in Theorem 3.4.3 to show that only Scenario 2 holds, i.e., only $\psi^{1} \neq 0$ and $\psi^{j}=0$ for all $2 \leq j \leq M$, so that

$$
u_{c}\left(t_{n}\right)=N L F\left(-t_{n}^{1}\right) \widetilde{\psi}^{1}\left(x-x_{n}^{1}\right)+\widetilde{W}_{n}^{M}
$$

such that

$$
M\left[\widetilde{\psi}^{1}\right]=M\left[u_{c}\right]=1 \quad \lim _{n \rightarrow+\infty} \mathcal{M E}\left[N L F\left(-t_{n}^{1}\right) \widetilde{\psi}^{1}\left(x-x_{n}^{1}\right)\right]=(\mathcal{M E})_{c}
$$

Using (3.159) and (3.160) along with (3.162), we get

$$
\lim _{n \rightarrow+\infty} M\left[\widetilde{W}_{n}^{M}\right]=0 \quad \text { and } \quad \lim _{n \rightarrow+\infty} E\left[\widetilde{W}_{n}^{M}\right]=0
$$

Therefore, Lemma 3.3.2 (comparison of energy and gradient) gives

$$
\lim _{n \rightarrow+\infty}\left\|\widetilde{W_{n}^{M}}\right\|_{H^{1}}=0
$$

Now, we show that $t_{n}^{1}$ converges to some finite $t^{*}$. Since then $e^{-i t_{n}^{1} \Delta} \widetilde{\psi}^{1} \rightarrow e^{-i t^{*} \Delta} \widetilde{\psi}^{1}$, combining this with (3.163) and (3.161) implies that $u_{c}\left(t_{n}\right)$ converges in $H^{1}\left(\mathbb{R}^{N}\right)$. Thus, for each $n$, select $x\left(t_{n}^{1}\right)=: x_{n}^{1} \in \mathbb{R}^{N}$ such that $u_{c}\left(\cdot-x\left(t_{n}^{1}\right), t_{n}^{1}\right) \in K$. Define $x(t)$ be the continuous function that connects $x\left(t_{n}^{1}\right)$ to $x\left(t_{n+1}^{1}\right)$ by a straight line in $\mathbb{R}^{N}$. Suppose the opposite happens, i.e., $\left|t_{n}^{1}\right| \rightarrow+\infty$, we have two cases. First, consider $t_{n}^{1} \rightarrow-\infty$. Then applying nonlinear flow to (3.161), we obtain

$$
\begin{aligned}
\left\|N L F(t) u_{c}\left(t_{n}\right)\right\|_{S\left(\dot{H}^{s} ;[0,+\infty)\right)} \leq & \left\|\operatorname{LLF}\left(t-t_{n}^{1}\right) \widetilde{\psi}^{1}\left(x-x_{n}^{1}\right)\right\|_{S\left(\dot{H}^{s_{c}} ;[0,+\infty)\right)} \\
& +\left\|N L F(t) \widetilde{W_{n}^{M}}\right\|_{S\left(\dot{H}^{s_{c}} ;[0,+\infty)\right)}
\end{aligned}
$$

Since $t_{n}^{1} \rightarrow-\infty$, we get

$$
\left\|N L F\left(t-t_{n}^{1}\right) \widetilde{\psi}^{1}\left(x-x_{n}^{1}\right)\right\|_{S\left(\dot{H}^{s^{c}} ;[0,+\infty)\right)}=\left\|N L F(t) \widetilde{\psi}^{1}\left(x-x_{n}^{1}\right)\right\|_{S\left(\dot{H}^{s_{c}} ;\left[-t_{n}^{1},+\infty\right)\right)} \leq \frac{\delta}{2},
$$

and by (2.3) along with (3.163), we get

$$
\left\|N L F(t) \widetilde{W}_{n}^{M}\right\|_{S\left(\dot{H}^{s}\right)} \leq \frac{\delta}{2}
$$


for $\delta>0$ (given) for sufficiently large $n, M$. Thus,

$$
\left\|N L F(t) u_{c}\left(t_{n}\right)\right\|_{S\left(\dot{H}^{s} ;[0,+\infty)\right)} \leq \delta
$$

Choosing $\delta>0$ sufficiently small, we obtain $\left\|u_{c}\right\|_{S\left(\dot{H}^{s_{c}}\right)} \leq 2 \delta$ (by the small data scattering theory Theorem 3.2.1), which is in contradiction with (3.138). With the similar argument, for $n$ large, assuming $t_{n}^{1} \rightarrow+\infty$, we obtain

$$
\left\|N L F(t) u_{c}\left(t_{n}\right)\right\|_{S\left(\dot{H}^{s^{c}} ;(-\infty, 0]\right)} \leq \delta
$$

and thus, Theorem 3.2.1 implies that $\left\|u_{c}\left(t_{n}\right)\right\|_{S\left(\dot{H}^{s} ;\left(-\infty, t_{n}\right]\right)} \leq 2 \delta$. Sending $n \rightarrow+\infty$ in the above expression, we have $t_{n} \rightarrow+\infty$, thus, $\left\|u_{c}\left(t_{n}\right)\right\|_{S\left(\dot{H}^{s_{c}}\right)} \leq 2 \delta$, again a contradiction. Hence, $t_{n}^{1}$ must converge to some finite $t^{*}$.

Lemma 3.4.7 (Precompactness of the flow implies uniform localization) Let u be a solution to (1.1) such that

$$
K=\{u(\cdot-x(t), t) \mid t \in[0, \infty)\}
$$

is precompact in $H^{1}$. Then for each $\epsilon>0$, there exists $R>0$ so that

$$
\int_{|x+x(t)|>R}|\nabla u(x, t)|^{2}+|u(x, t)|^{2} d x<\epsilon
$$

for all $0 \leq t<\infty$.

Proof. Suppose not, then there exists $\epsilon>0$ and a sequence of times $t_{n}$ such that

$$
\int_{\left|x+x\left(t_{n}\right)\right|>R_{n}}\left|\nabla u\left(x, t_{n}\right)\right|^{2}+\left|u\left(x, t_{n}\right)\right|^{2} d x \geq \epsilon .
$$

By a change of variables, we get

$$
\int_{|x|>R_{n}}\left|\nabla u\left(x-x\left(t_{n}\right), t_{n}\right)\right|^{2}+\left|u\left(x-x\left(t_{n}\right), t_{n}\right)\right|^{2} d x \geq \epsilon
$$


Since $K$ is precompact, there exists $\phi \in H^{1}$ such that, passing to a subsequence of $t_{n}$, we have $u\left(\cdot-x\left(t_{n}\right), t_{n}\right) \rightarrow \phi$ in $H^{1}$. Taking $R=\max _{n} R_{n}$, (3.165) implies

$$
\int_{|x|>R}|\nabla \phi(x)|^{2}+|\phi(x)|^{2} d x \geq \epsilon
$$

which is a contradiction to the fact $\phi \in H^{1}$. Thus, (3.164) holds.

Lemma 3.4.8 (Zero momentum) Let $u_{c}$ be the critical solution constructed in Theorem 3.4.3 and assume $(\mathcal{M E})_{c}<1$. Then $P\left[u_{c}\right]=\operatorname{Im} \int \bar{u}_{c} \nabla u_{c} d x=0$.

Proof. Assume $P\left[u_{c}\right] \neq 0$, then for some $\xi_{0} \in \mathbb{R}^{N}$ consider the transformed solution

$$
\widetilde{u}_{c}(x, t)=e^{i x \cdot \xi_{0}} e^{\left.-t\left|\xi_{0}\right|^{2}\right)} u_{c}\left(x-\xi_{0} t, t\right)
$$

We compute

$$
\left\|\nabla \widetilde{u}_{c}\right\|_{L^{2}}^{2}=\left|\xi_{0}\right|^{2} M\left[u_{c}\right]+2 \xi_{0} \cdot P\left[u_{c}\right]+\left\|\nabla u_{c}\right\|_{L^{2}}^{2}
$$

Observe that $M\left[\widetilde{u}_{c}\right]=M\left[u_{c}\right], P\left[\widetilde{u}_{c}\right]=\xi_{0} M\left[u_{c}\right]+P\left[u_{c}\right]$ and

$$
E\left[\widetilde{u}_{c}\right]=\frac{1}{2}\left|\xi_{0}\right|^{2} M\left[u_{c}\right]+\xi_{0} \cdot P\left[u_{c}\right]+E\left[u_{c}\right]
$$

Choose $\xi_{0}=-\frac{P\left[u_{c}\right]}{M\left[u_{c}\right]}$, then $P\left[\widetilde{u}_{c}\right]=0$ and

$$
\left\|\nabla \widetilde{u}_{c}\right\|_{L^{2}}^{2}=\left\|\nabla u_{c}\right\|_{L^{2}}^{2}-\frac{P\left[u_{c}\right]^{2}}{M\left[u_{c}\right]}, \quad E\left[\widetilde{u}_{c}\right]=E\left[u_{c}\right]-\frac{1}{2} \frac{P\left[u_{c}\right]^{2}}{M\left[u_{c}\right]}, \quad M\left[\widetilde{u}_{c}\right]=M\left[u_{c}\right] .
$$

Thus,

$$
\mathcal{M}^{\theta} \mathcal{E}\left[\widetilde{u}_{c}\right]=\mathcal{M}^{\theta} E\left[u_{c}\right]-\frac{s_{c}(p-1)+1}{s_{c}(p-1)}\left(\mathcal{P}\left[u_{c}\right]\right)^{2 / s_{c}}<1
$$

and

$$
\mathcal{G}\left[\widetilde{u}_{c, 0}\right]^{2 / s_{c}}=\mathcal{G}\left[u_{c, 0}\right]^{2 / s_{c}}-\left(\mathcal{P}\left[u_{c}\right]\right)^{2 / s_{c}}<1
$$

By Theorem 3.4.3, $\left\|u_{c}\right\|_{S\left(\dot{H}^{s_{c}}\right)}=+\infty$, and hence, $\left\|\widetilde{u}_{c}\right\|_{S\left(\dot{H}^{s_{c}}\right)}=+\infty$, which contradicts the definition of $u_{c}$. Hence, $P\left[u_{c}\right]$ must be zero. 
Since now $P\left[u_{c}\right]=0$ (see Lemma 3.4.8), this implies that $\int x\left|u_{c}(x, t)\right|^{2} d x=$ constant, provided it is finite. Also, note that

$$
\frac{\partial}{\partial t} \int x|u(x, t)|^{2} d x=2 N P[u] .
$$

We will adapt this with a localized version for a suitably large radius $R>0$. To envelope the entire path $x(t)$ over $\left[T, T_{1}\right]$ the localization $R$ should be taken large enough over the same interval $\left[T, T_{1}\right]$. We can use the precompactness of the translated flow $u_{c}(\cdot-x(t), t)$ and the zero momentum to prove that the localized center of mass is nearly conserved. By the localization of $u_{c}$ in $H^{1}$ around $x(t)$ and the near conservation of localized center of mass we constrain parameter $x(t)$ from going too quickly to $+\infty$.

Lemma 3.4.9 (Control over $x(t)$ ) Let $u$ be a solution of (1.1) defined on $[0,+\infty)$ such that $P[u]=0$ and $K=\{u(\cdot-x(t), t) \mid t \in[0, \infty)\}$ is precompact in $H^{1}$, for some continuous function $x(\cdot)$. Then

$$
\frac{x(t)}{t} \rightarrow 0 \quad \text { as } t \rightarrow+\infty
$$

Proof. We argue by contradiction, i.e., suppose (3.166) does not hold. Then there exists a sequence $t_{n} \rightarrow+\infty$ such that $\left|x\left(t_{n}\right)\right| / t_{n} \geq \epsilon_{0}$ for some $\epsilon_{0}>0$. Without loss of generality we may assume $x(0)=0$. For $R>0$, let

$$
t_{0}(R)=\inf \{t \geq 0:|x(t)| \geq R\}
$$

i.e., $t_{0}(R)$ is the first time when $x(t)$ reaches the boundary of the ball of radius $R$. By continuity of $x(t)$, the value $t_{0}(R)$ is well-defined. Moreover, the following properties hold: (1) $t_{0}(R)>0$; (2) $|x(t)|<R$ for $0 \leq t<t_{0}(R)$; and (3) $\left|x\left(t_{0}(R)\right)\right|=R$.

Define $R_{n}=\left|x\left(t_{n}\right)\right|$ and $\tilde{t}_{n}=t_{0}\left(R_{n}\right)$. Note that $t_{n} \geq \tilde{t}_{n}$, which combined with $\left|x\left(t_{n}\right)\right| / t_{n} \geq \epsilon_{0}$ gives $R_{n} / \tilde{t}_{n} \geq \epsilon_{0}$. Since $t_{n} \rightarrow+\infty$ and $\left|x\left(t_{n}\right)\right| / t_{n} \geq \epsilon_{0}$, we have $R_{n}=\left|x\left(t_{n}\right)\right| \rightarrow+\infty$. Thus, $\tilde{t}_{n}=t_{0}\left(R_{n}\right) \rightarrow+\infty$. This allows us to forget about $t_{n}$ and work on the time interval $\left[0, \tilde{t}_{n}\right]$ with following properties: 
1. $|x(t)|<R_{n}$ for $0 \leq t<\tilde{t}_{n}$;

2. $\left|x\left(\tilde{t}_{n}\right)\right|=R_{n}$;

3. $\frac{R_{n}}{\tilde{t}_{n}} \geq \epsilon_{0}$ and $\tilde{t}_{n} \rightarrow+\infty$.

By the precompactness of $K$ and Lemma 3.4.7, it follows that for any $\epsilon>0$ there exists $R_{0}(\epsilon) \geq 0$ such that for any $t \geq 0$,

$$
\int_{|x+x(t)|>R_{0}(\epsilon)}|\nabla u|^{2}+|u|^{2} d x<\epsilon .
$$

For $x \in \mathbb{R}$, let $\theta(x) \in C_{\text {comp }}^{\infty}(\mathbb{R})$ be such that $\theta(x)=x$, for $-1 \leq x \leq 1, \theta(x)=0$ for $|x| \geq 2^{1 / N},|\theta(x)| \leq|x|,\left\|\theta^{\prime}\right\|_{L^{\infty}} \leq 4$, and $\|\theta\|_{L^{\infty}} \leq 2$.

Set $\phi(x)=\left(\theta\left(x_{1}\right), \theta\left(x_{2}\right), \ldots, \theta\left(x_{N}\right)\right)$, where $x=\left(x_{1}, x_{2}, \ldots, x_{N}\right) \in \mathbb{R}^{N}$. Then $\phi(x)=x$ for $|x| \leq 1$ and $\|\phi\|_{L^{\infty}} \leq 2$. For $R>0$, let $\phi_{R}(x)=R \phi(x / R)$. Assume $z_{R}: \mathbb{R} \rightarrow \mathbb{R}^{N}$ to be the truncated center of mass given by

$$
z_{R}(t)=\int \phi_{R}(x)|u(x, t)|^{2} d x
$$

Then

$$
\left[z_{R}^{\prime}(t)\right]_{j}=2 \operatorname{Im} \int \theta^{\prime}\left(x_{j} / R\right) \partial_{j} u \bar{u} d x
$$

with $z_{R}^{\prime}(t)=\left(\left[z_{R}^{\prime}(t)\right]_{1},\left[z_{R}^{\prime}(t)\right]_{2}, \ldots,\left[z_{R}^{\prime}(t)\right]_{N}\right)$. Since $\theta^{\prime}\left(x_{j} / R\right)=1$ for $\left|x_{j}\right| \leq 1$, the zero momentum condition implies

$$
\operatorname{Im} \int_{\left|x_{j}\right| \leq R} \partial_{j} u \bar{u} d x=-\operatorname{Im} \int_{\left|x_{j}\right|>R} \partial_{j} u \bar{u} d x
$$

and therefore,

$$
\left[z_{R}^{\prime}(t)\right]_{j}=-2 \operatorname{Im} \int_{\left|x_{j}\right| \geq R} \partial_{j} u \bar{u} d x+2 \operatorname{Im} \int_{\left|x_{j}\right| \geq R} \theta^{\prime}\left(x_{j} / R\right) \partial_{j} u \bar{u} d x
$$

from which we obtain

$$
\left|z_{R}^{\prime}(t)\right| \leq 5 \int_{|x| \geq R}|\nabla u|^{2}+|u|^{2} d x
$$


Set $\tilde{R}_{n}=R_{n}+R_{0}(\epsilon)$. Note that for $0 \leq t \leq \tilde{t}_{n}$ and $|x|>\tilde{R}_{n}$, we have $|x+x(t)| \geq$ $\tilde{R}_{n}-R_{n}=R_{0}(\epsilon)$, and thus, by (3.167) and (3.168), we obtain

$$
\left|z_{\tilde{R}_{n}}^{\prime}(t)\right| \leq 5 \epsilon
$$

Now we obtain an upper bound for $z_{\tilde{R}_{n}}(0)$ and a lower bound for $z_{\tilde{R}_{n}}(t)$.

$$
z_{\tilde{R}_{n}}(0)=\int_{|x|<R_{0}(\epsilon)} \phi_{\tilde{R}_{n}}(x)\left|u_{0}(x)\right|^{2} d x+\int_{|x+x(0)| \geq R_{0}(\epsilon)} \phi_{\tilde{R}_{n}}(x)\left|u_{0}(x)\right|^{2} d x,
$$

and thus, by (3.167), we have

$$
\left|z_{\tilde{R}_{n}}(0)\right| \leq R_{0}(\epsilon) M[u]+2 \tilde{R}_{n} \epsilon
$$

For $0 \leq t \leq \tilde{t}_{n}$, we write

$$
z_{\tilde{R}_{n}}(t)=\int_{|x+x(t)|<R_{0}(\epsilon)} \phi_{\tilde{R}_{n}}(x)|u(x, t)|^{2} d x+\int_{|x+x(t)| \geq R_{0}(\epsilon)} \phi_{\tilde{R}_{n}}(x)|u(x, t)|^{2} d x .
$$

To estimate the second term, we observe that $\left|\phi_{\tilde{R}_{n}}(x)\right| \leq 2 \tilde{R}_{n}$, combining it with (3.167), we obtain

$$
\left.\left|\int_{|x+x(t)| \geq R_{0}(\epsilon)} \phi_{\tilde{R}_{n}}(x)\right| u(x, t)\right|^{2} d x \mid \leq 2 \tilde{R}_{n} \epsilon .
$$

To estimate the first term, we first note that $|x| \leq|x+x(t)|+|x(t)| \leq R_{0}(\epsilon)+R_{n}=\tilde{R}_{n}$, and thus, $\phi_{\tilde{R}_{n}}(x)=x$. So, we rewrite the first term as

$$
\begin{aligned}
& \int_{|x+x(t)|<R_{0}(\epsilon)}(x+x(t))|u(x, t)|^{2} d x-x(t) \int_{|x+x(t)|<R_{0}(\epsilon)}|u(x, t)|^{2} d x \\
= & \int_{|x+x(t)|<R_{0}(\epsilon)}(x+x(t))|u(x, t)|^{2} d x-x(t) M[u]+x(t) \int_{|x+x(t)| \geq R_{0}(\epsilon)}|u(x, t)|^{2} d x \\
= & I_{1}-x(t) M[u]+I_{2} .
\end{aligned}
$$

Observe that $\left|I_{1}\right| \leq R_{0}(\epsilon) M[u]$ and by (3.167), $\left|I_{2}\right| \leq|x(t)| \epsilon \leq \tilde{R}_{n} \epsilon$. Thus,

$$
\left|z_{\tilde{R}_{n}}(t)\right| \geq|x(t)| M[u]-R_{0}(\epsilon) M[u]-3 \tilde{R}_{n} \epsilon
$$


Taking $t=\tilde{t}_{n}$, we get

$$
\left|z_{\tilde{R}_{n}}\left(\tilde{t}_{n}\right)\right| \geq \tilde{R}_{n}(M[u]-3 \epsilon)-R_{0}(\epsilon) M[u]
$$

Combining (3.169), (3.170) and (3.171), we have

$$
\begin{aligned}
5 \epsilon \tilde{t}_{n} & \geq \int_{0}^{\tilde{t}_{n}}\left|z_{\tilde{R}_{n}}^{\prime}(t)\right| d t \geq\left|\int_{0}^{\tilde{t}_{n}} z_{\tilde{R}_{n}}^{\prime}(t) d t\right| \geq\left|z_{\tilde{R}_{n}}\left(\tilde{t}_{n}\right)-z_{\tilde{R}_{n}}(0)\right| \\
& \geq \tilde{R}_{n}(M[u]-5 \epsilon)-2 R_{0}(\epsilon) M[u] .
\end{aligned}
$$

Dividing by $\tilde{t}_{n}$ and using that $\tilde{R}_{n} \geq R_{n}$ (assume $\epsilon \leq \frac{1}{5} M[u]$ ), we obtain

$$
5 \epsilon \geq \frac{R_{n}}{\tilde{t}_{n}}(M[u]-5 \epsilon)-\frac{2 R_{0}(\epsilon) M[u]}{\tilde{t}_{n}} .
$$

Since $R_{n} / \tilde{t}_{n} \geq \epsilon_{0}$, we have

$$
5 \epsilon \geq \epsilon_{0}(M[u]-5 \epsilon)-\frac{2 R_{0}(\epsilon) M[u]}{\tilde{t}_{n}} .
$$

Take $\epsilon=M[u] \epsilon_{0} / 16$ (assume $\epsilon_{0} \leq 1$ ), and then send $n \rightarrow+\infty$. Since $\tilde{t}_{n} \rightarrow+\infty$, we obtain a contradiction.

\subsection{Rigidity Theorem}

Theorem 3.5.1 (Rigidity) Let $u$ be the global solution of (1.1) with initial data $u_{0} \in$ $H^{1}\left(\mathbb{R}^{N}\right)$ satisfying $P\left[u_{0}\right]=0, \mathcal{M E}\left[u_{0}\right]<1$ and $\mathcal{G}\left[u_{0}\right]<1$. Suppose

$$
K=\{u(\cdot-x(t), t) \mid t \in[0, \infty)\}
$$

is precompact in $H^{1}$. Then $u_{0} \equiv 0$.

Proof. Let $\phi \in C_{0}^{\infty}$ be radial, with $\phi(x)=|x|^{2}$ for $|x| \leq 1$ and 0 for $|x| \geq 2$. For $R>0$, let $\phi_{R}(x)=R^{2} \phi(x / R)$. Define

$$
V_{l o c}(t)=\int \phi_{R}(x)|u(x, t)|^{2} d x \Longrightarrow V_{l o c}^{\prime}(t)=2 R \operatorname{Im} \int \bar{u}(t) \cdot \nabla u(t)(\nabla \phi)\left(\frac{x}{R}\right) d x .
$$


Using Hölder's inequality, we get

$$
\left|V_{l o c}^{\prime}(t)\right| \leq C R \int_{|x| \leq 2 R}|u(t)||\nabla u(t)| d x \leq C R\|u(t)\|_{L^{2}}^{2\left(1-s_{c}\right)}\|\nabla u(t)\|_{L^{2}}^{2 s_{c}} .
$$

The second derivative, using the definition of $\phi$ and symmetrization, yields

$$
\begin{aligned}
V_{l o c}^{\prime \prime}(t) \geq & 8 \int_{|x| \leq R}|\nabla u|^{2}-\frac{4(N(p-1)-\gamma)}{p} \int_{|x| \leq R}\left(|x|^{-(N-\gamma)} *|u|^{p}\right)|u|^{p} \\
& -\frac{c}{R^{2}} \int_{R<|x|<2 R}|u|^{2}+4 \int_{R<|x|<2 R} \phi^{\prime \prime}\left(\frac{|x|}{R}\right)|\nabla u|^{2} \\
& -\left(4 c\left(\frac{1}{2}-\frac{1}{p}\right)+\frac{2(N-\gamma)}{p}\right) \int_{R<|x|<2 R}\left(|x|^{-(N-\gamma)} *|u|^{p}\right)|u|^{p} \\
& +\frac{2(N-\gamma)}{p} \iint_{\Omega}\left(1-\frac{R}{|x|} \phi^{\prime}\left(\frac{|x|}{R}\right)\right) \frac{x(x-y)|u(x)|^{p}|u(y)|^{p}}{|x-y|^{N-\gamma+2}} d x d y \\
& -\frac{2(N-\gamma)}{p} \iint_{\Omega}\left(1-\frac{R}{|y|} \phi^{\prime}\left(\frac{|y|}{R}\right)\right) \frac{y(x-y)|u(x)|^{p}|u(y)|^{p}}{|x-y|^{N-\gamma+2}} d x d y
\end{aligned}
$$

We re-write the above estimate as follows

$$
\begin{aligned}
V_{l o c}^{\prime \prime}(t) \geq & \left(8 \int_{|x| \leq R}|\nabla u|^{2}-\frac{4(N(p-1)-\gamma)}{p} \int_{|x| \leq R}\left(|x|^{-(N-\gamma)} *|u|^{p}\right)|u|^{p}\right) \\
& -c_{1}\left(\int_{R<|x|<2 R}|\nabla u|^{2}+\frac{|u|^{2}}{R^{2}}+\left(|x|^{-(N-\gamma)} *|u|^{p}\right)|u|^{p}\right) \\
& +\frac{2(N-\gamma)}{p} \iint_{\Omega}\left(1-\frac{R}{|x|} \phi^{\prime}\left(\frac{|x|}{R}\right)\right) \frac{x(x-y)|u(x)|^{p}|u(y)|^{p}}{|x-y|^{N-\gamma+2}} d x d y \\
& -\frac{2(N-\gamma)}{p} \iint_{\Omega}\left(1-\frac{R}{|y|} \phi^{\prime}\left(\frac{|y|}{R}\right)\right) \frac{y(x-y)|u(x)|^{p}|u(y)|^{p}}{|x-y|^{N-\gamma+2}} d x d y
\end{aligned}
$$

where

$$
\Omega=\left\{(x, y) \in \mathbb{R}^{N} \times \mathbb{R}^{N}:|x|>R\right\} \cup\left\{(x, y) \in \mathbb{R}^{N} \times \mathbb{R}^{N}:|y|>R\right\} .
$$

Since $\{u(\cdot-x(t), t) \mid t \in[0, \infty)\}$ is precompact in $H^{1}\left(\mathbb{R}^{N}\right)$, by Lemma 3.4.7 there exists $R_{0} \geq 0$ such that taking $R \geq R_{0}+\sup _{t \in\left[T, T_{1}\right]}|x(t)|$, we obtain for all $t \in\left[T, T_{1}\right]$

$$
\int_{|x|>R_{0}}|\nabla u(x, t)|^{2}+|u(x, t)|^{2} d x<\frac{\epsilon}{8} .
$$


Using Hölder's inequality, Hardy-Littlewood-Sobolev inequality, and radial Sobolev inequality yields the existence of $R_{1}>0$ such that

$$
\begin{aligned}
& \int_{|x|>R_{1}}\left(|x|^{-(N-\gamma)} *|u|^{p}\right)|u|^{p} d x \\
& \leq c_{2}\left\||\cdot|^{-(N-\gamma)} *|u|^{p}\right\|_{\substack{\frac{2 N}{\mid x-\gamma} \\
L_{|x|>R_{1}}}}\|u\|_{\substack{2 N p \\
L_{|x|>R_{1}}^{N+\gamma}}}^{p} \text { (Hölder's inequality) } \\
& \leq c_{3}\|u\|_{\substack { L_{|x|>R_{1}} \\
\begin{subarray}{c}{N+\gamma \\
c_{4}{ L _ { | x | > R _ { 1 } } \\
\begin{subarray} { c } { N + \gamma \\
c _ { 4 } } }\end{subarray}}^{2 p} \text { (HLS inequality; Lemma 2.3.1) } \\
& \leq \frac{c_{4}}{R_{1}^{\frac{(N-1)(N(p-1)-\gamma)}{N}}}\|u\|_{\dot{H}^{1}}^{\frac{N(p-1)-\gamma}{N}}\|u\|_{L^{2}}^{\frac{N(p+1)+\gamma}{N}}<\frac{\epsilon}{8},
\end{aligned}
$$

where the second to last inequality follows from the radial Sobolev inequality and the last one follows from taking $R_{1}^{\frac{(N-1)(N(p-1)-\gamma)}{N}}>8 c_{4}\|u\|_{\dot{H}^{1}}^{\frac{N(p-1)-\gamma}{N}}\|u\|_{L^{2}}^{\frac{N(p+1)+\gamma}{N}}$.

Let $\epsilon=16 E[u]\left(1-(\mathcal{M E}[u])^{s_{c}(p-1)}\right) c_{1}^{-1}$ and take $R=\max \left\{R_{0}, R_{1}\right\}$, combine (3.177) and (3.178) to obtain

$$
c_{1}\left(\int_{|x|>R}|\nabla u|^{2}+\frac{|u|^{2}}{R^{2}}+\left(|x|^{-(N-\gamma)} *|u|^{p}\right)|u|^{p}\right) \leq 4 E[u]\left(1-(\mathcal{M E}[u])^{s_{c}(p-1)}\right) .
$$

Now we invoke Lemma 3.3.3 by splitting the integrals on the right side of the expression (3.114) into the regions $\{|x|>R\}$ and $\{|x|<R\}$ and use (3.179) to obtain the following bound, (3.174) $\geq 12 E[u]\left(1-(\mathcal{M E}[u])^{s_{c}(p-1)}\right)$. Next, putting together the terms $(3.175)$ and (3.176)

$$
\iint_{\Omega}\left(\left(1-\frac{R}{|x|} \phi^{\prime}\left(\frac{|x|}{R}\right)\right) x-\left(1-\frac{R}{|x|} \phi^{\prime}\left(\frac{|x|}{R}\right)\right) y\right) \frac{(x-y)|u(x)|^{p}|u(y)|^{p}}{|x-y|^{N-\gamma+2}} d x d y
$$

we upper bound (3.180), by following a similar argument from Theorem 3.3.1, to obtain

$$
(3.180) \leq \frac{c_{5}}{R^{\frac{(N-1)(N(p-1)-\gamma)}{N}}}\left\|\chi_{|x|>R} u\right\|_{\dot{H}^{1}}^{\frac{N(p-1)-\gamma}{N}}<\frac{\epsilon}{4}<4 E[u]\left(1-(\mathcal{M E}[u])^{s_{c}(p-1)}\right)
$$

with $R^{\frac{(N-1)(N(p-1)-\gamma)}{N}}>4 c_{5}\left\|\chi_{|x|>R} u\right\|_{\dot{H}^{1}}^{\frac{N(p-1)-\gamma}{N}}$. Putting everything together, we obtain

$$
V_{l o c}^{\prime \prime}(t) \geq 8 E[u]\left(1-(\mathcal{M E}[u])^{s_{c}(p-1)}\right)-\left|I_{R}\right| \geq 4 E[u]\left(1-(\mathcal{M E}[u])^{s_{c}(p-1)}\right) .
$$


By Lemma 3.4.9, there exists $T \geq 0$ such that for all $t \geq T$, we have $|x(t)| \leq \delta t$, with $\delta>0$ to be chosen later. Taking $R=R_{0}+\delta T_{1}$, we have that (3.175) holds for all $t \in\left[T, T_{1}\right]$, then integrating from $T$ to $T_{1}$, we obtain

$$
\left|V_{l o c}^{\prime}\left(T_{1}\right)-V_{l o c}^{\prime}(T)\right| \geq 4 E[u]\left(1-(\mathcal{M E}[u])^{s_{c}(p-1)}\right)\left(T_{1}-T\right) .
$$

On the other hand, from (3.173) and (3.93), we have that

$$
\left|V_{l o c}^{\prime}(t)\right| \leq C R\|u(t)\|_{L^{2}}^{2\left(1-s_{c}\right)}\|\nabla u(t)\|_{L^{2}}^{2 s_{c}} \leq C\left(R_{0}+\delta T_{1}\right)\|Q\|_{L^{2}}^{2\left(1-s_{c}\right)}\|\nabla Q\|_{L^{2}}^{2 s_{c}}
$$

Combining (3.182) and (3.183), we get

$$
4 E[u]\left(1-(\mathcal{M E}[u])^{s_{c}(p-1)}\right)\left(T_{1}-T\right) \leq C\left(R_{0}+\delta T_{1}\right)\|Q\|_{L^{2}}^{2\left(1-s_{c}\right)}\|\nabla Q\|_{L^{2}}^{2 s_{c}}
$$

Let $\delta=\frac{E[u]\left(1-(\mathcal{M E}[u])^{s_{c}(p-1)}\right)}{C\|Q\|_{L^{2}}^{2\left(1-s_{c}\right)}\|\nabla Q\|_{L^{2}}^{2 s_{c}}}$, then the above expression can be re-written as

$$
\begin{aligned}
3 E[u]\left(1-(\mathcal{M E}[u])^{s_{c}(p-1)}\right) T_{1} \leq & C R_{0}\|Q\|_{L^{2}}^{2\left(1-s_{c}\right)}\|\nabla Q\|_{L^{2}}^{2 s_{c}} \\
& +4 E[u]\left(1-(\mathcal{M E}[u])^{s_{c}(p-1)}\right) T
\end{aligned}
$$

taking $T_{1} \rightarrow+\infty$ implies that the left-hand side of the above expression goes to $\infty$ and we derive a contradiction (right-hand side is bounded), which can be resolved only if $E[u]=0$, implying that $u \equiv 0$.

\subsection{Divergence to infinity (Theorem 3.1.1 (2) part (b))}

The argument for part (2)b follows [HR10] and [Gue14] proof verbatim. We give a brief overview here for completeness.

Assume that there is no finite time blowup for a nonradial and infinite variance solution (from Theorem 3.1.1 part (2)b), thus, the solutions exist for all time (i.e., $T^{*}=+\infty$ ). Under the assumption of global existence, we study the behavior of $\mathcal{G}[u(t)]$ as $t \rightarrow+\infty$, 
and use a concentration compactness type argument to establish the divergence of $\mathcal{G}[u(t)]$ in $H^{1}$ as it was developed in [HR10], note that the concentration compactness and the rigidity arguments are used to prove a blowup property.

We first restate (in the spirit of [HR10]) the characterization of $Q$ from Lions [Lio84a], Theorem II.1, which can be considered for any minimizer $Q$.

Proposition 3.6.1 There exists a function $\epsilon(\rho)$, defined for small $\rho>0$ with $\lim _{\rho \rightarrow 0} \epsilon(\rho)=0$, such that for all $u \in H^{1}\left(\mathbb{R}^{N}\right)$ with

$$
|Z(u)-Z(Q)|+\left|\|u\|_{L^{2}}-\|Q\|_{L^{2}}\right|+\left|\|\nabla u\|_{L^{2}}-\|\nabla Q\|_{L^{2}}\right| \leq \rho,
$$

there is $\theta_{0} \in \mathbb{R}$ and $x_{0} \in \mathbb{R}^{N}$ such that

$$
\left\|u-e^{i \theta_{0}} Q\left(\cdot-x_{0}\right)\right\|_{H^{1}} \leq \epsilon(\rho) .
$$

This is equivalent to

Proposition 3.6.2 There exists a function $\epsilon(\rho)$ such that $\epsilon(\rho) \rightarrow 0$ as $\rho \rightarrow 0$ satisfying the following: Suppose there exists $\lambda>0$ such that

$$
\left|\mathcal{M E}[u]-\frac{s_{c}(p-1)+1}{s_{c}(p-1)}\left(1-\frac{\lambda^{2 s_{c}(p-1)}}{s_{c}(p-1)+1}\right) \lambda^{2}\right| \leq \rho \lambda^{2 s_{c}(p-1)+2}
$$

and

$$
|\mathcal{G}[u(t)]-\lambda| \leq \rho \begin{cases}\lambda^{2 s_{c}(p-1)+1} & \text { if } \lambda \leq 1 \\ \lambda & \text { if } \lambda \geq 1\end{cases}
$$

Then there exists $\theta_{0} \in \mathbb{R}$ and $x_{0} \in \mathbb{R}^{N}$ such that

$$
\left\|u-e^{i \theta_{0}} \lambda^{N / 2} \beta^{-\frac{s_{c}}{1-s_{c}}} Q\left(\lambda\left(\beta^{-\frac{3 s_{c}}{\left(1-s_{c}\right) N}} x-x_{0}\right)\right)\right\|_{L^{2}} \leq \beta^{\frac{s_{c}}{2\left(1-s_{c}\right)}} \epsilon(\rho)
$$

and

$$
\left\|\nabla\left[u-e^{i \theta_{0}} \lambda^{N / 2} \beta^{-\frac{s_{c}}{1-s_{c}}} Q\left(\lambda\left(\beta^{-\frac{3 s_{c}}{\left(1-s_{c}\right) N}} x-x_{0}\right)\right)\right]\right\|_{L^{2}} \leq \lambda \beta^{-\frac{s_{c}}{2\left(1-s_{c}\right)}} \epsilon(\rho),
$$

where $\beta=\left(\frac{M[u]}{M[Q]}\right)^{\theta}$. 
Suppose that $0 \leq \mathcal{M E}[u]<1$ and let $\mathcal{G}[u(t)]=\lambda>0$ be given. The "mass-energy" horizontal line for this $\lambda$ intersects the graph of parabola, $y=\frac{s_{c}(p-1)+1}{s_{c}(p-1)}\left(1-\frac{\lambda^{2 s_{c}(p-1)}}{s_{c}(p-1)+1}\right) \lambda^{2}$ at two places, i.e., there exists two solutions $0 \leq \lambda_{1}<1<\lambda_{2}$. The first case produces a solution that is global and scattering (Theorem 3.1.1 (1)) and the second case produces a solution, which either blows up in finite time (Theorem 3.1.1 (2)(a)) or diverges in infinite time (Theorem 3.1.1 (2)(b)) as shown in Section 3.6.

It is possible that $\mathcal{G}[u(t)]$ is much larger than 1 or $\lambda_{2}$. The following Proposition shows that it can not.

Proposition 3.6.3 Let $\mathcal{G}\left[u_{0}\right]=\lambda_{0}>1$. Then there exists $\rho_{0}=\rho_{0}\left(\lambda_{0}\right)>0$ (with the property that $\rho_{0} \rightarrow 0$ as $\lambda_{0} \searrow 1$ ) such that for any $\lambda \geq \lambda_{0}$, the following holds: There does NOT exist a solution $u(t)$ of (1.1) with $P[u]=0$ satisfying $\|u\|_{L^{2}}=\|Q\|_{L^{2}}$ and

$$
\frac{E[u]}{E[Q]}=\frac{s_{c}(p-1)+1}{s_{c}(p-1)}\left(1-\frac{\lambda^{2 s_{c}(p-1)}}{s_{c}(p-1)+1}\right) \lambda^{2}
$$

with

$$
\lambda \leq \frac{\|\nabla u(t)\|_{L^{2}}}{\|\nabla Q\|_{L^{2}}} \leq \lambda\left(1+\rho_{0}\right) \text { for all } t \geq 0
$$

Proof. The proof relies on Proposition 3.6.2 and is easy to adapt as done in [HR10] and [Gue14] following the same argument as in Theorem 3.5.1 (Section 3.5) in the present Chapter.

This proves that there is NO solution at the "mass-energy" line for $\lambda$ satisfying (3.191). We want to show that $\mathcal{G}[u(t)]$ on any "mass-energy" line with $\mathcal{M E}\left[u_{0}\right]<1$ and $\mathcal{G}[u(t)]>$ 1 will diverge to infinity. By contradiction, we assume that such solutions have bounded renormalized gradient $\mathcal{G}[u(t)]$ for all $t>0$.

We say the solution has a globally bounded gradient if there exists a solution at the "mass-energy" line for $\lambda$ such that $\lambda \leq \mathcal{G}[u(t)] \leq \sigma$ for all $t>0$. Observe that if the 
solution does not have a globally bounded gradient for some $\lambda$ and $\sigma$, then for any $\sigma^{\prime}<\sigma$ the solution still does not have globally bounded gradient. We are now in a position to define the threshold.

Definition 3.6.4 Fix $\lambda_{0}>1$. Let $\sigma_{c}=\sigma_{c}\left(\lambda_{0}\right)$ be the supremum of all $\sigma>\lambda_{0}$ such that the solution of (1.1) does NOT have a globally bounded gradient for all $\lambda$ such that $\lambda_{0} \leq \lambda \leq \sigma$

By Proposition 3.6.3, we have that $\lambda \leq \mathcal{G}[u(t)] \leq \lambda\left(1+\rho_{0}\right)$ does not hold for all $\lambda \geq \lambda_{0}$. We want to prove that $\sigma_{c}\left(\lambda_{0}\right)=+\infty$. By contradiction, assume that $\sigma_{c}\left(\lambda_{0}\right)$ is finite. Let $u(t)$ be a solution to (1.1) with initial data $u_{n, 0}$ at the "mass-energy" line for $\lambda>\lambda_{0}$, satisfying the hypothesis of Proposition 3.6.3. Moreover, we want to prove that $\mathcal{G}[u(t)] \rightarrow \infty$ over a sequence of times $\left\{t_{n}\right\} \rightarrow \infty$. Assume that such a sequence of times does not exist. This implies that there is a finite $\sigma$ satisfying $\lambda \leq \mathcal{G}[u(t)] \leq \sigma$ for all $t>0$. Invoking the nonlinear profile decomposition on the sequence $\left\{u_{n, 0}\right\}$ as done in Theorem 3.4.3 enables us to construct a "critical threshold solution" $u(t)=u_{c}(t)$ at the "massenergy" line for $\lambda_{c}$ with $\lambda_{0}<\lambda_{c}<\sigma_{c}\left(\lambda_{0}\right)$ and $\lambda_{c}<\mathcal{G}\left[u_{c}(t)\right]<\sigma_{c}\left(\lambda_{0}\right)$ for all $t>0$. At this point we note that the nonlinear profile decomposition gives the $\dot{H}^{1}$ asymptotic orthogonality at $t=0$, but we would need to extend this for $0 \leq t \leq T$. This can be done following the argument described in [HR10] (Lemma 6.3) and [Gue14] (Lemma 3.9). This critical threshold solution $u_{c}(t)$ will satisfy Proposition 3.4.6 (precompactness of the flow) and Lemma 3.4.7 (uniform localization). This localization property of $u_{c}(t)$ implies that $u_{c}(t)$ blows-up in finite time. The arguments from [HR10] (Proposition 3.2) and [Gue14] (Lemma 4.10) prove exactly that, which contradicts the boundedness of $u_{c}(t)$ in $H^{1}$, and hence, $u_{c}(t)$ can not exist, which means that our initial assumption that $\sigma_{c}\left(\lambda_{0}\right)<\infty$ is false. The proof of Theorem 3.1.1 is now complete. 


\section{CHAPTER 4}

\section{SCATTERING IN THE NLS AND GHARTREE EQUATIONS: AN ALTERNATIVE APPROACH}

To know the road ahead, ask those coming back. (attributed to Confucius)

\subsection{Setting up the problem}

Consider the two Cauchy problems: the focusing nonlinear Schrödinger (NLS) equation

$$
(\mathrm{NLS}) \quad\left\{\begin{array}{l}
i u_{t}+\Delta u+|u|^{p-1} u=0, \quad p>1, t \in \mathbb{R}, x \in \mathbb{R}^{N}, \\
u(x, 0)=u_{0}(x) \in H^{1}\left(\mathbb{R}^{N}\right)
\end{array}\right.
$$

and the focusing generalized Hartree equation

$$
(\mathrm{gH}) \quad\left\{\begin{array}{l}
i v_{t}+\Delta v+\left(|x|^{-(N-\gamma)} *|v|^{p}\right)|v|^{p-2} v=0, \quad t \in \mathbb{R}, x \in \mathbb{R}^{N}, \\
v(x, 0)=v_{0}(x) \in H^{1}\left(\mathbb{R}^{N}\right)
\end{array}\right.
$$

for $p \geq 2$ and $0<\gamma<N$. Recall $u=u(x, t)$ and $v=v(x, t)$ are complex-valued functions in the equations (4.1) and (4.2), respectively.

We remind that solutions to the equations (4.1) and (4.2), during their lifespan, conserve several quantities, including the mass, given (respectively, for NLS and gH) by

$$
M_{N L S}[u(t)] \stackrel{\text { def }}{=} \int_{\mathbb{R}^{N}}|u(x, t)|^{2} d x=M_{N L S}\left[u_{0}\right]
$$

and

$$
M_{g H}[v(t)] \stackrel{\text { def }}{=} \int_{\mathbb{R}^{N}}|v(x, t)|^{2} d x=M_{g H}\left[v_{0}\right]
$$

The energy is also conserved, which is defined for (4.1) and (4.2), respectively, by

$$
E_{N L S}[u(t)] \stackrel{\text { def }}{=} \frac{1}{2} \int_{\mathbb{R}^{N}}|\nabla u(x, t)|^{2} d x-\frac{1}{p+1} \int_{\mathbb{R}^{N}}|u(x, t)|^{p+1} d x=E_{N L S}\left[u_{0}\right]
$$


and with $Z(v) \stackrel{\text { def }}{=} \int_{\mathbb{R}^{N}}\left(|x|^{-(N-\gamma)} *|v(\cdot, t)|^{p}\right)|v(x, t)|^{p} d x$

$$
E_{g H}[v(t)] \stackrel{\text { def }}{=} \frac{1}{2} \int_{\mathbb{R}^{N}}|\nabla v(x, t)|^{2} d x-\frac{1}{2 p} Z(v)=E_{g H}\left[v_{0}\right] .
$$

Recall that the scaling in the equations (4.1) and (4.2) is as follows: if $u(x, t)$ solves (4.1), then $u^{\lambda}(x, t)=\lambda^{\frac{2}{p-1}} u\left(\lambda x, \lambda^{2} t\right)$ is also a solution to (4.1), and similarly, if $v(x, t)$ solves (4.2), then $v^{\lambda}(x, t)=\lambda^{\frac{\gamma+2}{2(p-1)}} v\left(\lambda x, \lambda^{2} t\right)$ is also a solution to (4.2). The scaleinvariant Sobolev norm is $\dot{H}^{s_{c}}$ with

$$
s_{c}=\frac{N}{2}-\frac{2}{p-1} \quad \text { for (4.1) } \quad \text { and } \quad s_{c}=\frac{N}{2}-\frac{\gamma+2}{2(p-1)} \quad \text { for (4.2). }
$$

Using Duhamel's formula, we can write (4.1) in the integral form

$$
u(t)=e^{i t \Delta} u_{0}+i \int_{0}^{t} e^{i(t-s) \Delta}|u|^{p-1} u(s) d s,
$$

and the corresponding Duhamel formulation for (4.2) is given by (2.16).

To characterize the global behavior of solutions in the NLS equation, Holmer and Roudenko in [HR07] observed that the quantities $M[u]^{1-s_{c}} E[u]^{s_{c}}$ and $\|u\|_{L^{2}}^{1-s_{c}}\|\nabla u\|_{L^{2}}^{s_{c}}$ are scale-invariant and scale as $\dot{H}^{s_{c}}$ norm with $s_{c}$ as in (4.3). In [HR08] they proved the following result, using the concentration - compactness and rigidity road map of Kenig and Merle [KM06], in the case of the focusing $3 d$ cubic NLS equation (in the radial setting)

$$
\left\{\begin{array}{l}
i u_{t}+\Delta u+|u|^{2} u=0 ; \quad t \in \mathbb{R}, \quad x \in \mathbb{R}^{3}, \\
u(x, 0)=u_{0}(x) \in H^{1}\left(\mathbb{R}^{3}\right) .
\end{array}\right.
$$

Theorem 4.1.1 Let $u_{0} \in H^{1}\left(\mathbb{R}^{3}\right)$ be radial and $u(t)$ be the corresponding solution to (4.5) in $H^{1}\left(\mathbb{R}^{3}\right)$. Suppose $M\left[u_{0}\right] E\left[u_{0}\right]<M[Q] E[Q]$. If $\left\|u_{0}\right\|_{L^{2}\left(\mathbb{R}^{3}\right)}\left\|\nabla u_{0}\right\|_{L^{2}\left(\mathbb{R}^{3}\right)}<$ $\|Q\|_{L^{2}\left(\mathbb{R}^{3}\right)}\|\nabla Q\|_{L^{2}\left(\mathbb{R}^{3}\right)}$, then the solution to $(4.5)$ is global and scatters in $H^{1}$.

Remark 4.1.2 This result is similar to part 1 of Theorem 3.1.1 in Chapter 3. Their result also contains the finite time blow-up conclusion in the case when $\left\|u_{0}\right\| L_{L^{2}}\left\|\nabla u_{0}\right\|_{L^{2}}>$ $\|Q\|_{L^{2}}\|\nabla Q\|_{L^{2}}$, however, we omit that part as it is not needed for the present Chapter. 
Remark 4.1.3 Later Duyckaerts, Holmer and Roudenko in [DHR08] extended their result to the general, non-radial setting. Since we consider only the radial case, we omit the general case.

Recently, Dodson and Murphy in [DM17] presented a simplified proof of Theorem 4.1.1 that avoids concentration-compactness route. They used a scattering criterion introduced by Tao in [Tao04], which together with the radial Sobolev embedding and virial/Morawetz estimate was sufficient to prove (in the radial setting) Theorem 4.1.1.

The purpose of this work is to generalize the method of Dodson \& Murphy [DM17] to the inter-critical range of the nonlinear Schrödinger equation (4.1) and also show that it can be applied in the case of the nonlocal potential such as in the generalized Hartree equation (4.2). Our result is a new (or an alternative) proof of the following two theorems.

Theorem 4.1.4 (Scattering in NLS, [Aro19]) Consider the NLS equation (4.1) with $N$ $>2$, and $1+\frac{4}{N}<p<1+\frac{4}{N-2}\left(0<s_{c}<1\right)$. Let $u_{0} \in H^{1}\left(\mathbb{R}^{N}\right)$ be radial and assume

$$
M\left[u_{0}\right]^{1-s_{c}} E\left[u_{0}\right]^{s_{c}}<M[Q]^{1-s_{c}} E[Q]^{s_{c}} .
$$

If

$$
\left\|u_{0}\right\|_{L^{2}\left(\mathbb{R}^{N}\right)}^{1-s_{c}}\left\|\nabla u_{0}\right\|_{L^{2}\left(\mathbb{R}^{N}\right)}^{s_{c}}<\|Q\|_{L^{2}\left(\mathbb{R}^{N}\right)}^{1-s_{c}}\|\nabla Q\|_{L^{2}\left(\mathbb{R}^{N}\right)}^{s_{c}}
$$

then the solution $u(t)$ to (4.1) is global and scatters in $H^{1}\left(\mathbb{R}^{N}\right)$.

Theorem 4.1.5 (Scattering in gH, [Aro19]) Consider the gHartree equation (4.2) with $N>2,0<\gamma<N, p \geq 2$ and $1+\frac{\gamma+2}{N}<p<1+\frac{\gamma+2}{N-2}\left(0<s_{c}<1\right)$. Let $v_{0} \in H^{1}\left(\mathbb{R}^{N}\right)$ be radial and assume

$$
M\left[v_{0}\right]^{1-s_{c}} E\left[v_{0}\right]^{s_{c}}<M[Q]^{1-s_{c}} E[Q]^{s_{c}}
$$

If

$$
\left\|v_{0}\right\|_{L^{2}\left(\mathbb{R}^{N}\right)}^{1-s_{c}}\left\|\nabla v_{0}\right\|_{L^{2}\left(\mathbb{R}^{N}\right)}^{s_{c}}<\|Q\|_{L^{2}\left(\mathbb{R}^{N}\right)}^{1-s_{c}}\|\nabla Q\|_{L^{2}\left(\mathbb{R}^{N}\right)}^{s_{c}}
$$

then the solution $v(t)$ to (4.2) is global and scatters in $H^{1}\left(\mathbb{R}^{N}\right)$. 
Remark 4.1.6 We only consider $N>2$ as we use the dispersive estimate (2.2) in (4.18), which gives the logarithmic divergence of the integral when $N=2$.

Remark 4.1.7 We do not cover the case $s=1$ (energy-critical) as this approach takes into account an a priori uniform bound on $\dot{H}^{1}$ norm of a solution in terms of the energy, and having the gap between the critical index $s$ as defined in (4.3) and $s_{c}=1$ is an essential part of the proof. (However, it would be possible to cover this case given an a priori uniform $\dot{H}^{s}$ bound and consider $s_{c}<s_{\text {.) }}$

To prove the theorems we establish Morawetz estimates for both equations in the intercritical regime by employing the radial Sobolev inequality. This implies that the potential energy escapes as $t \rightarrow \infty$, which in turn yields spreading of the mass. To obtain the scattering and conclude the proofs of Theorems 3.2 and 3.3, we generalize the scattering criterion of Tao from [Tao04] (for the 3d cubic NLS) to all inter-critical cases of NLS and also obtain the scattering criterion for the gHartree equation.

Remark 4.1.8 Theorem 4.1.5 was also proved in Theorem 3.1.1 (1) via the concentrationcompactness method in Chapter 3. Nevertheless, we show that in the radial case this new approach can also be applied in the case of the nonlocal convolution nonlinearity, i.e., for the gHartree equation.

We also note that the treatment of the gHartree case is different from the NLS case, in particular,

- the estimate of inhomogeneous term in the Duhamel formula via Strichartz estimate in Lemma 4.2.2 (using Lemma 2.3.1 to handle the convolution term), and

- most importantly, in the Morawetz estimate for gHartree, one expects (as in the NLS case) to obtain the upper bound on the potential term, $Z(v)$, which is of convolution 
type given by $Z(v)=\int_{\mathbb{R}^{N}}\left(|x|^{-(N-\gamma)} *|v(\cdot, t)|^{p}\right)|v(x, t)|^{p} d x$. It is not straightforward to rule out the concentration of mass from the potential (convolution) term in the gHartree case. Thus, when deriving the Morawetz estimate (Proposition 4.5.2), we rely on the $L_{x}^{\frac{2 N p}{N+\gamma}}$ norm. This is possible since in Lemma 4.3.4, we bound the virial from below using Sobolev inequality (thanks to the assumption $s_{c}<1$, i.e., $\left.p<\frac{N+\gamma}{N-2}\right)$ by $L_{x}^{\frac{2 N p}{N+\gamma}}$ norm

$$
\|\nabla v\|_{L^{2}\left(\mathbb{R}^{N}\right)}^{2}-\frac{s_{c}(p-1)+1}{p} Z(v) \geq \delta\|\nabla v\|_{L^{2}\left(\mathbb{R}^{N}\right)}^{2} \geq \tilde{\delta}\|v\|_{L_{x}^{\frac{2 N p}{N+\gamma}}\left(\mathbb{R}^{N}\right)}
$$

instead of using the $Z(v)$, convolution type potential term. Observing that $\frac{2 N p}{N+\gamma}>2$ allows us to obtain the mass evacuation to conclude scattering.

\subsection{Scattering criterion}

We recall the Strichartz estimate

$$
\left\|e^{i t \Delta} f_{0}\right\|_{L_{t}^{q} L_{x}^{r}\left(\mathbb{R} \times \mathbb{R}^{N}\right)} \lesssim\left\|f_{0}\right\|_{\dot{H}^{s_{c}}\left(\mathbb{R}^{N}\right)}
$$

where $(q, r)$ is an $\dot{H}^{s_{c}}$-admissible pair. We also recall the inhomogeneous version of the above estimate

$$
\|u\|_{L_{t}^{q} L_{x}^{r}\left(\left[t_{0}, \infty\right) \times \mathbb{R}^{N}\right)} \lesssim\left\|u\left(t_{0}\right)\right\|_{\dot{H}^{s}\left(\mathbb{R}^{N}\right)}+\|F(u)\|_{L_{t}^{\tilde{q}^{\prime}} L_{x}^{\tilde{r}^{\prime}}\left(\left[t_{0}, \infty\right) \times \mathbb{R}^{N}\right)},
$$

where $u$ is a solution to $i u_{t}+\Delta u=F(u)$ and $\left(\tilde{q}^{\prime}, \tilde{r}^{\prime}\right)$ is the Hölder conjugate for $(\tilde{q}, \tilde{r})$ which is an $\dot{H}^{-s_{c}}$ admissible pair with $s_{c}$ given by (4.3).

We now obtain a scattering criterion for the NLS for all $s_{c} \in(0,1)$, which generalizes Tao's scattering criterion for the $3 \mathrm{~d}$ cubic NLS for radial solutions ( Theorem 1.1 in [Tao04]). 
Lemma 4.2.1 (Scattering criterion for NLS) Consider $0<s_{c}<1$. Suppose $u$ is a radial solution to (4.1) satisfying

$$
\sup _{t \in[0, \infty)}\|u(t)\|_{H^{1}\left(\mathbb{R}^{N}\right)} \leq E \quad \text { with } \quad E>0
$$

If there exist $\varepsilon>0$ and $R>0$ depending only on $E$ such that

$$
\liminf _{t \rightarrow \infty} \int_{|x| \leq R}|u(x, t)|^{2} d x \leq \varepsilon,
$$

then $u(t)$ scatters forward in time in $H^{1}$.

Proof. Let $0<\varepsilon<1$ be a small constant and $R(\varepsilon) \gg 1$ be a large number, both to be chosen later. From (4.6), we have

$$
\left\|e^{i t \Delta} u_{0}\right\|_{L_{t}^{2(p+1)-N(p-1)} L_{x}^{p+1}\left(\mathbb{R} \times \mathbb{R}^{N}\right)} \lesssim 1 .
$$

By monotone convergence we may find a (large enough) time $T_{0}>\varepsilon^{-\frac{p-1}{2}}>1$ depending on $u$ such that

$$
\left\|e^{i t \Delta} u_{0}\right\|_{L_{t}^{2(p+1)-N(p-1)} L_{x}^{p+1}\left(\mathbb{R} \times \mathbb{R}^{N}\right)} \lesssim \varepsilon
$$

By the hypothesis, we may choose $T_{1}>T_{0}$ so that

$$
\int_{|x| \leq R}\left|u\left(x, T_{1}\right)\right|^{2} d x \lesssim \varepsilon .
$$

We denote by $\chi$ a smooth, radially symmetric function on $\mathbb{R}^{N}$ with supp $\chi \subset B(0,1)$, which equals 1 on $B(0,1 / 2)$. For any $R>0$, we define $\chi_{R}(x)=\chi(x / R)$, noting that $\chi_{R}=1$ on $B(0, R / 2)$. Then

$$
\int \chi_{R}(x)\left|u\left(x, T_{1}\right)\right|^{2} d x \lesssim \varepsilon .
$$

Multiplying (4.1) by $\bar{u}$ and adding the conjugate expression, we simply get

$$
\partial_{t}\left(|u|^{2}\right)=-2 \nabla \cdot \operatorname{Im}(\bar{u} \nabla u)
$$


Multiplying by $\chi_{R}$ and integrating by parts, yields

$$
\partial_{t} \int \chi_{R}(x)|u(x, t)|^{2} d x=2 \int \nabla \chi_{R} \cdot \operatorname{Im}(\bar{u} \nabla u)
$$

Since $\left\|\nabla \chi_{R}\right\|_{L^{\infty}(\mathbb{R})}=O(1 / R)$, we deduce from (4.8)

$$
\left.\left|\partial_{t} \int \chi_{R}(x)\right| u(x, t)\right|^{2} d x \mid \lesssim \frac{1}{R} .
$$

Set $0<\alpha<p-1$ and note that $T_{1}-\varepsilon^{-\alpha}>0$. Then for large enough $R=R(\varepsilon) \gg 0$ by (4.11) we observe that

$$
\sup _{t \in\left[T_{1}-\varepsilon^{-\alpha}, T_{1}\right]} \int \chi_{R}(x)|u(x, t)|^{2} d x=\left\|\chi_{R} u\right\|_{L_{t}^{\infty} L_{x}^{2}\left(\left[T_{1}-\varepsilon^{-\alpha}, T_{1}\right] \times \mathbb{R}^{N}\right)} \lesssim \varepsilon .
$$

We estimate the solution $u$ at time $T_{1}$. Using Duhamel's representation, we write

$$
u\left(T_{1}\right)=e^{i T_{1} \Delta} u_{0}+i \int_{0}^{T_{1}} e^{i\left(T_{1}-s\right) \Delta}\left(|u|^{p-1} u\right)(s) d s
$$

and for $t \in\left[0, T_{1}\right]$

$$
e^{i\left(t-T_{1}\right) \Delta} u\left(T_{1}\right)=e^{i t \Delta} u_{0}+F_{1}(t)+F_{2}(t)
$$

with

$$
\begin{aligned}
& F_{1}(t)=i \int_{T_{1}-\varepsilon^{-\alpha}}^{T_{1}} e^{i(t-s) \Delta}\left(|u|^{p-1} u\right)(s) d s, \\
& F_{2}(t)=i \int_{0}^{T_{1}-\varepsilon^{-\alpha}} e^{i(t-s) \Delta}\left(|u|^{p-1} u\right)(s) d s .
\end{aligned}
$$

From (4.10) we note that the contribution from the linear component in (4.14) is small. For the second term, using Minkowski's inequality, dispersive estimate (2.2), then considering characteristic function $\chi_{I_{1}}\left(t^{\prime}\right)$ and Lemma 2.3.1, we obtain

$$
\begin{aligned}
\left\|\int_{T_{1}-\varepsilon^{-\alpha}}^{T_{1}} e^{i(t-s) \Delta}\left(|u|^{p-1} u\right)(s) d s\right\| & \frac{2(p+1)(p-1)}{L_{t}^{2(p+1)-N(p-1)}} L_{x}^{p+1}\left(\left[T_{1}, \infty\right) \times \mathbb{R}^{N}\right) \\
& \lesssim\left\||u|^{p-1} u\right\|_{L_{t}^{2(p+1) p-N(p-1) p}} L_{x}^{\frac{p+1}{p}}\left(I_{1} \times \mathbb{R}^{N}\right)
\end{aligned}
$$


for $t \in I_{1}$, where $I_{1}=\left[T_{1}-\varepsilon^{-\alpha}, T_{1}\right]$. We upper bound the last term to obtain

$$
\begin{aligned}
\left\|\int_{T_{1}-\varepsilon^{-\alpha}}^{T_{1}} e^{i(t-s) \Delta}\left(|u|^{p-1} u\right)(s) d s\right\| & \frac{2(p+1)(p-1)}{2(p+1)-N(p-1)} L_{x}^{p+1}\left(\left[T_{1}, \infty\right) \times \mathbb{R}^{N}\right) \\
& \lesssim\|u\|^{p} \\
& L_{t}^{\frac{2(p+1(p-1))}{2(p+1)-N(p-1)}} L_{x}^{p+1}\left(I_{1} \times \mathbb{R}^{N}\right)
\end{aligned}
$$

Using Hölder's inequality in time, we get

$$
\begin{aligned}
\left\|\int_{T_{1}-\varepsilon^{-\alpha}}^{T_{1}} e^{i(t-s) \Delta}\left(|u|^{p-1} u\right)(s) d s\right\| & \frac{2(p+1)(p-1)}{2(p+1)-N(p-1)} L_{x}^{p+1}\left(\left[T_{1}, \infty\right) \times \mathbb{R}^{N}\right) \\
& \lesssim\left|I_{1}\right|^{\frac{2(p+1) p-N p(p-1)}{2(p+1)(p-1)}}\|u\|_{L_{t}^{\infty} L_{x}^{p+1}\left(I_{1} \times \mathbb{R}^{N}\right)}^{p} .
\end{aligned}
$$

Using Hölder's inequality, (4.12), and Lemma 2.3.3, we have

$$
\begin{aligned}
& \|u\|_{L_{t}^{\infty} L_{x}^{p+1}\left(I_{1} \times \mathbb{R}^{N}\right)}=\|u\|_{L_{t}^{\infty} L_{x}^{p+1}\left(I_{1} \times B(0, R / 2)\right)}+\|u\|_{L_{t}^{\infty} L_{x}^{p+1}\left(I_{1} \times \mathbb{R}^{N} \backslash B(0, R / 2)\right)} \\
& \lesssim\left\|\chi_{R} u\right\|_{L_{t}^{\infty} L_{x}^{2}\left(I_{1} \times \mathbb{R}^{N}\right)}^{\frac{2(p+1)-N(p-1)}{2(p+1)}}\|u\|_{L_{t}^{\infty} L_{x}^{\frac{2 N}{N-2}\left(I_{1} \times \mathbb{R}^{N}\right)}}^{\frac{N(p-1)}{2(p+1)}}+\left\|\left(1-\chi_{R}\right) u\right\|_{L_{t}^{\infty} L_{x}^{\infty}\left(I_{1} \times \mathbb{R}^{N}\right)}^{\frac{p-1}{p+1}}\|u\|_{L_{t}^{\infty} L_{x}^{2}\left(I_{1} \times \mathbb{R}^{N}\right)}^{\frac{2}{p+1}} \\
& \lesssim \varepsilon^{\frac{2(p+1)-N(p-1)}{2(p+1)}}+R^{-\frac{p-1}{p+1}} .
\end{aligned}
$$

Choosing $R>0$ such that $R^{-\frac{p-1}{p+1}} \ll \varepsilon^{\frac{2(p+1)-N(p-1)}{2(p+1)}}$, we get

$$
\|u\|_{L_{t}^{\infty} L_{x}^{p+1}\left(I_{1} \times \mathbb{R}^{N}\right)} \lesssim \varepsilon^{\frac{2(p+1)-N(p-1)}{2(p+1)}} .
$$

Therefore, we obtain

$$
\begin{aligned}
\| \int_{T_{1}-\varepsilon^{-\alpha}}^{T_{1}} e^{i(t-s) \Delta}\left(|u|^{p-1} u\right)(s) & d s \| \frac{2(p+1)(p-1)}{L_{t}^{2(p+1)-N(p-1)}} L_{x}^{p+1}\left(\left[T_{1}, \infty\right) \times \mathbb{R}^{N}\right) \\
& \lesssim \varepsilon^{\frac{2(p+1) p-N p(p-1)}{2(p+1)}}\left|I_{1}\right|^{\frac{2(p+1) p-N p(p-1)}{2(p+1)(p-1)}} \\
& \lesssim \varepsilon^{\frac{(2(p+1)-N(p-1)) p}{2(p+1)}\left(1-\frac{\alpha}{p-1}\right)} .
\end{aligned}
$$

Take $0<\beta_{1}<\frac{(2(p+1)-N(p-1)) p}{2(p+1)}\left(1-\frac{\alpha}{p-1}\right)$, then

$$
\left\|\int_{T_{1}-\varepsilon^{-\alpha}}^{T_{1}} e^{i(t-s) \Delta}\left(|u|^{p-1} u\right)(s) d s\right\|_{L_{t}^{\frac{2(p+1)(p-1)}{2(p+1)-N(p-1)}} L_{x}^{p+1}\left(\left[T_{1}, \infty\right) \times \mathbb{R}^{N}\right)} \lesssim \varepsilon^{\beta_{1}} .
$$


Recalling the definition of $F_{2}(u(t))$ from (4.14), we split it via interpolation for $1<s_{1}<$ $\frac{N}{2}$ as

$$
\begin{aligned}
& \left\|F_{2}(u)\right\| \frac{2(p+1)(p-1)}{L_{t}^{2(p+1)-N(p-1)}} L_{x}^{p+1}\left(\left[T_{1}, \infty\right) \times \mathbb{R}^{N}\right) \\
& \lesssim\left\|F_{2}(u)\right\|_{L_{t}^{q_{1}} L_{x}^{r_{1}}\left(\left[T_{1}, \infty\right) \times \mathbb{R}^{N}\right)}^{\frac{s_{1}-s_{c}}{r_{1}}}\left\|F_{2}(u)\right\|_{L_{t}^{q_{2}} L_{x}^{\infty}\left(\left[T_{1}, \infty\right) \times \mathbb{R}^{N}\right)}^{\frac{s_{c}}{s_{1}}},
\end{aligned}
$$

where, $q_{2}>\frac{2}{N-2}$ and $\left(q_{1}, r_{1}\right)$ is an $L^{2}$-admissible pair, since $2<r_{1}<\frac{2 N}{N-2}$, which follows from the fact that $N \geq 3$ and $s_{1}>1$. By the dispersive estimate (2.2) for $t \in\left[T_{1}, \infty\right)$, we bound

$$
\left\|F_{2}(u(t))\right\|_{L_{x}^{\infty}\left(\mathbb{R}^{N}\right)} \lesssim \int_{0}^{T_{1}-\varepsilon^{-\alpha}}(t-s)^{-\frac{N}{2}}\|u(s)\|_{L^{p}\left(\mathbb{R}^{N}\right)}^{p} d s .
$$

Observe that for $N>2$ and $p \geq 2$, we have $H^{1}\left(\mathbb{R}^{N}\right) \hookrightarrow L^{p}\left(\mathbb{R}^{N}\right)$, see also our Remark 4.1.6 about the restriction $N>2$, and the same is valid for $1<p<2$ from GagliardoNirenberg interpolation inequality, and thus, by (4.8) we obtain

$$
\left\|F_{2}(u(t))\right\|_{L_{t}^{q_{2}} L_{x}^{\infty}\left(\mathbb{R}^{N}\right)} \lesssim \varepsilon^{\alpha\left(\frac{N-2}{2}-\frac{1}{q_{2}}\right)},
$$

choose $q_{2}=\frac{4}{N-2 s_{1}}>\frac{4}{N-2}>\frac{2}{N-2}$ and use $2 s_{1}<N$, which gives

$$
\left\|F_{2}(u)\right\|_{L_{t}^{\frac{2 N}{N-2}} L_{x}^{\infty}\left(\left[T_{1}, \infty\right) \times \mathbb{R}^{N}\right)} \lesssim \varepsilon^{\frac{\alpha(N-2)}{2}} .
$$

Rewriting $F_{2}$ via Duhamel's formula applied on $\left[0, T_{1}-\varepsilon^{-\alpha}\right)$ ), we get

$$
F_{2}(t)=e^{i\left(t-T_{1}+\varepsilon^{-\alpha}\right) \Delta} u\left(T_{1}-\varepsilon^{-\alpha}\right)-e^{i t \Delta} u(0),
$$

and using the homogeneous Strichartz estimate (4.6) and (4.8), yields

$$
\left\|F_{2}(u)\right\|_{L_{t}^{q_{1}} L_{x}^{r_{1}}\left(\left[T_{1}, \infty\right), \mathbb{R}^{N}\right)} \lesssim\left\|u\left(T_{1}-\varepsilon^{-\alpha}\right)\right\|_{L^{2}\left(\mathbb{R}^{N}\right)}+\|u(0)\|_{L^{2}\left(\mathbb{R}^{N}\right)} \lesssim 1 .
$$

Take $0<\beta_{2}<\frac{\alpha(N-2) s_{c}}{2 s_{1}}$, then from (4.20), we estimate $F_{1}$ as

$$
\left\|F_{2}(u)\right\|_{L_{t}^{2(p+1)-N(p-1)} L_{x}^{p+1}\left(\left[T_{1}, \infty\right) \times \mathbb{R}^{N}\right)} \lesssim \varepsilon^{\beta_{2}}
$$


Putting together (4.10), (4.17), and (4.21) gives

$$
\left\|e^{i\left(t-T_{1}\right) \Delta} u\left(T_{1}\right)\right\|_{L_{t}^{\frac{2(p+1)(p-1)}{2(p+1)-N(p-1)}} L_{x}^{p+1}\left(\left[T_{1}, \infty\right) \times \mathbb{R}^{N}\right)} \lesssim \varepsilon^{\mu}
$$

where $\mu \leq \min \left\{\beta_{1}, \beta_{2}\right\}>0$.

Consider, Duhamel's formula for the solution $u(t)$ on the interval $\left[T_{1}, t\right]$ so as to pass the bound (4.22) from linear solution to the corresponding bound on non-linear solution

$$
u(t)=e^{i\left(t-T_{1}\right) \Delta} u\left(T_{1}\right)-i \int_{T_{1}}^{t} e^{i(t-s) \Delta}|u|^{p-1} u(s) d s .
$$

From (4.22) and Strichartz estimate, we observe that

$$
\|u\|_{L_{t}^{\frac{2(p+1)(p-1)}{2(p+1)-N(p-1)}} L_{x}^{p+1}\left(\left[T_{1}, \infty\right) \times \mathbb{R}^{N}\right)} \lesssim \varepsilon^{\mu}+\left\|\nabla\left(|u|^{p-1} u\right)\right\|_{L_{t}^{\frac{4(p+1)}{4(p+1-N(p-1))}} L_{x}^{\frac{p+1}{p}}\left(\left[T_{1},+\infty\right) \times \mathbb{R}^{N}\right)} .
$$

Applying the product rule and Hölder's inequality, we

$$
\begin{aligned}
& \left\|\nabla\left(|u|^{p-1} u\right)\right\| \frac{4(p+1)}{L_{t}^{\frac{4(p+1-N(p-1))}{L_{x}}} \frac{p+1}{p}\left(\left[T_{1},+\infty\right) \times \mathbb{R}^{N}\right)}
\end{aligned}
$$

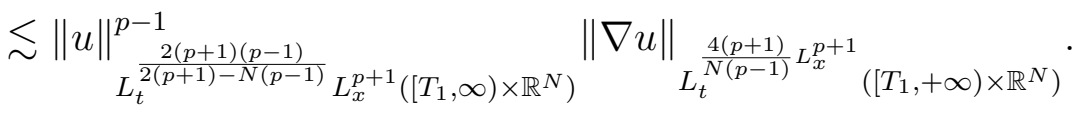

Thus, from (4.6), (4.8), (4.21) and using a standard continuity argument on the nonlinear flow, we observe that

$$
\|u\|_{L_{t}^{\frac{2(p+1)(p-1)}{2(p+1)-N(p-1)}} L_{x}^{p+1}\left(\left[T_{1}, \infty\right) \times \mathbb{R}^{N}\right)} \lesssim \varepsilon^{\mu}
$$

Now, we define

$$
u_{+} \stackrel{\text { def }}{=} e^{-i T_{1} \Delta} u\left(T_{1}\right)-i \int_{T_{1}}^{+\infty} e^{-i s \Delta} F(u(s)) d s,
$$

and since we have shown that $F(u)$ lies in $L_{t}^{\frac{4(p+1)}{4(p+1)-N(p-1)}} W_{x}^{1, p+1}\left(\left[T_{1},+\infty\right) \times \mathbb{R}^{N}\right)$, this implies that $u_{+} \in H^{1}\left(\mathbb{R}^{N}\right)$. Then from (4.23) and (4.25), we have

$$
u(t)-e^{i t \Delta} u_{+}=i \int_{t}^{+\infty} e^{i(t-s) \Delta} F(u(s)) d s
$$


for all $t \geq T_{1}$. Therefore, estimating the $H^{1}$ norm

$$
\begin{aligned}
\left\|u(t)-e^{i t \Delta} u_{+}\right\|_{H^{1}} & \lesssim\left\|\int_{t}^{+\infty} e^{i(t-s) \Delta}(1+|\nabla|) F(u(s)) d s\right\|_{S\left(L^{2}\right)} \\
& \lesssim\|(1+|\nabla|) F(u)\|_{S^{\prime}\left(L^{2} ;[t,+\infty)\right)} \\
& \lesssim\|u\|_{L_{t}^{p-1} \frac{2(p+1)(p-1)}{2(p+1)-N(p-1)} L_{x}^{p+1}\left(\left[T_{1}, \infty\right) \times \mathbb{R}^{N}\right)}^{\|\nabla u\|_{L_{t}^{\frac{4(p+1)}{N(p-1)}} L_{x}^{p+1}\left(\left[T_{1},+\infty\right) \times \mathbb{R}^{N}\right)}} .
\end{aligned}
$$

By (4.8) and (4.24) we observe that the Strichartz norm on $\left[T_{1},+\infty\right)$ for the above expression is bounded, therefore, the tail has to vanish as $t \rightarrow+\infty$. Hence,

$$
\lim _{t \rightarrow+\infty}\left\|u(t)-e^{i t \Delta} u_{+}\right\|_{H^{1}\left(\mathbb{R}^{N}\right)}=0
$$

This completes the proof.

We now prove a scattering criterion for the radial solutions to the gHartree equation (4.2).

Lemma 4.2.2 (Scattering criterion for gHartree) Consider $0<s_{c}<1$. Suppose $u$ is a radial solution to (4.2) satisfying

$$
\sup _{t \in[0, \infty)}\|v(t)\|_{H^{1}\left(\mathbb{R}^{N}\right)} \leq E
$$

If there exist constants $\varepsilon>0$ and $R>0$, depending only on $E$, such that

$$
\liminf _{t \rightarrow \infty} \int_{|x| \leq R}|v(x, t)|^{2} d x \leq \varepsilon,
$$

then $v(t)$ scatters forward in time.

Proof. The proof is similar to Lemma 4.2.1 except for the estimate for the following terms:

$$
F_{1}(t)=i \int_{T_{1}-\varepsilon^{-\alpha}}^{T_{1}} e^{i(t-s) \Delta}\left(\left(|x|^{-(N-\gamma)} *|v|^{p}\right)|v|^{p-2} v\right)(s) d s
$$

and

$$
F_{2}(t)=i \int_{0}^{T_{1}-\varepsilon^{-\alpha}} e^{i(t-s) \Delta}\left(\left(|x|^{-(N-\gamma)} *|v|^{p}\right)|v|^{p-2} v\right)(s) d s
$$


where $t \in\left[0, T_{1}\right]$. To derive the estimate of $F_{1}(t)$, we proceed as follows (using the same argument as in Lemma 4.2.1)

$$
\left\|F_{1}(v)\right\|_{L_{t}^{\frac{2 p}{1-s_{c}}} L_{x}^{\frac{2 N p}{N+\gamma}}\left(\left[T_{1}, \infty\right) \times \mathbb{R}^{N}\right)} \lesssim\left\|\left(|x|^{-(N-\gamma)} *|v|^{p}\right)|v|^{p-2} v\right\|_{L_{t}^{\frac{2 p}{(2 p-1)\left(1-s_{c}\right)}} L_{x}^{\frac{2 N p}{2 N p-N-\gamma}}\left(I_{1} \times \mathbb{R}^{N}\right)}
$$

for $t \in I_{1}=\left[T_{1}-\varepsilon^{-\alpha}, T_{1}\right]$. Using the Hölder's inequality together with the Lemma 2.3.1, we get

$$
\begin{aligned}
& \left\|\left(|x|^{-(N-\gamma)} *|v|^{p}\right)|v|^{p-2} v\right\|_{L_{t}^{(2 p-1)\left(1-s_{c}\right)}} L_{x}^{\frac{2 N p}{2 N p-N-\gamma}}\left(I_{1} \times \mathbb{R}^{N}\right)
\end{aligned}
$$

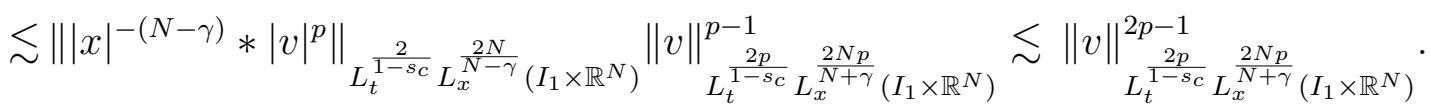

Using Hölder's inequality in time, we have

$$
\left\|F_{1}(v)\right\|_{L_{t}^{\frac{2 p}{1-s_{c}}} L_{x}^{\frac{2 N p}{N+\gamma}}\left(\left[T_{1}, \infty\right) \times \mathbb{R}^{N}\right)} \lesssim\left|I_{1}\right|^{\frac{(2 p-1)\left(1-s_{c}\right)}{2 p}}\|v\|_{L_{t}^{\infty} L_{x}^{\frac{2 N p}{N+\gamma}}\left(I_{1} \times \mathbb{R}^{N}\right)}^{2 p-1} .
$$

Using Hölder's inequality, (4.12), and Lemma 2.3.3, we have

$$
\begin{aligned}
& \|u\|_{L_{t}^{\infty} L_{x}^{\frac{2 N p}{N+\gamma}}\left(I_{1} \times \mathbb{R}^{N}\right)}=\|u\|_{L_{t}^{\infty} L_{x}^{\frac{2 N p}{N+\gamma}}\left(I_{1} \times B(0, R / 2)\right)}+\|u\|_{L_{t}^{\infty} L_{x}^{\frac{2 N p}{N+\gamma}}\left(I_{1} \times \mathbb{R}^{N} \backslash B(0, R / 2)\right)} \\
& \lesssim\left\|\chi_{R} u\right\|_{L_{t}^{\infty} L_{x}^{2}\left(I_{1} \times \mathbb{R}^{N}\right)}^{\frac{2 p+\gamma-N(p-1)}{2(p)}}\|u\|_{L_{t}^{\infty} L_{x}^{\frac{2 N}{N-2}}\left(I_{1} \times \mathbb{R}^{N}\right)}^{\frac{N(p-1)-\gamma}{N p}}+\left\|\left(1-\chi_{R}\right) u\right\|_{L_{t}^{\infty} L_{x}^{\infty}\left(I_{1} \times \mathbb{R}^{N}\right)}^{\frac{N(p-1)-\gamma}{N p}}\|u\|_{L_{t}^{\infty} L_{x}^{2}\left(I_{1} \times \mathbb{R}^{N}\right)}^{\frac{N+\gamma}{N p}} . \\
& \lesssim \varepsilon \frac{2 p+\gamma-N(p-1)}{2 p}+R^{-\frac{N(p-1)-\gamma}{N p}} .
\end{aligned}
$$

Choosing $R>0$ such that $R^{-\frac{N(p-1)-\gamma}{N p}} \ll \varepsilon^{\frac{2 p+\gamma-N(p-1)}{2 p}}$, we get

$$
\|u\|_{L_{t}^{\infty} L_{x}^{\frac{2 N p}{N+\gamma}}\left(I_{1} \times \mathbb{R}^{N}\right)} \lesssim \varepsilon^{\frac{2 p+\gamma-N(p-1)}{2 p}}
$$

Therefore, we obtain

$$
\left\|F_{1}(v)\right\|_{L_{t}^{\frac{2 p}{1-s_{c}}} L_{x}^{\frac{2 N p}{N+\gamma}}\left(\left[T_{1}, \infty\right) \times \mathbb{R}^{N}\right)} \lesssim \varepsilon^{\frac{2 p+\gamma-N(p-1)}{2 p}-\alpha \frac{(2 p-1)\left(1-s_{c}\right)}{2 p}}
$$

So, for $0<\alpha<\frac{2(p-1)}{2 p-1}$, we have that

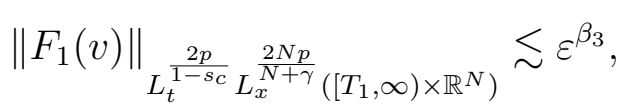


where $\beta_{3}>0$ chosen in the same way as in the previous lemma. To derive the estimate for $F_{2}(t)$, we argue in a similar fashion as in Lemma 4.2.1. By interpolation,

$$
\begin{aligned}
& \left\|F_{2}(v)\right\|_{L_{t}^{\frac{2 p}{1-s_{c}}}} L_{x}^{\frac{2 N p}{N+\gamma}}\left(\left[T_{1}, \infty\right) \times \mathbb{R}^{N}\right)
\end{aligned}
$$

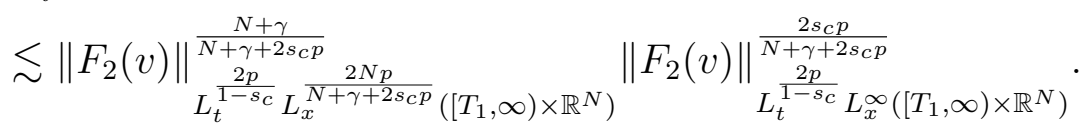

Note that $\frac{2 p}{1-s_{c}}>\frac{2}{N-2}$ and $\left(\frac{2 p}{1-s_{c}}, \frac{2 N p}{N+\gamma+2 s_{c} p}\right)$ is an $L^{2}$-admissible pair. We bound the last term above for $t \in\left[T_{1}, \infty\right)$ using the dispersive estimate (2.2) followed by Hölder's inequality and Lemma 2.3.1, that yields

$$
\left\|F_{2}(v(t))\right\|_{L_{x}^{\infty}\left(\mathbb{R}^{N}\right)} \lesssim \int_{0}^{T_{1}-\varepsilon^{\alpha}}(t-s)^{-\frac{N}{2}}\|v\|_{L^{p_{2}\left(\mathbb{R}^{N}\right)}}^{p}\|v\|_{L^{p_{1}\left(\mathbb{R}^{N}\right)}}^{p-1} d s
$$

where $p_{1}=\frac{2 N(p-1)}{N+\gamma}$ and $p_{2}=\frac{2 N p}{N+\gamma}$. Observe that for $N \geq 3$ and $2 \leq p<\frac{N+\gamma}{N-2}$, one has the embedding $H^{1}\left(\mathbb{R}^{N}\right) \hookrightarrow L^{p_{i}}\left(\mathbb{R}^{N}\right)$ with $i=1,2$, and thus, by (4.8)

$$
\left\|F_{2}(v(t))\right\|_{L_{t}^{\frac{2 p}{1-s_{c}}} L_{x}^{\infty}\left(\mathbb{R}^{N}\right)} \lesssim \varepsilon^{\alpha\left(\frac{N-2}{2}-\frac{1-s_{c}}{2 p}\right)}
$$

Next, note that

$$
\left.F_{2}(t)=e^{i\left(t-T_{1}+\varepsilon^{-\alpha}\right) \Delta} v\left(T_{1}-\varepsilon^{-\alpha}\right)-e^{i t \Delta} v(0)\right),
$$

and using the homogeneous Strichartz estimate (4.6) and (4.8), we deduce

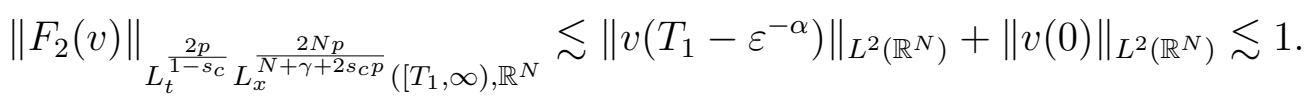

Similarly, to the step (4.21), we take $\beta_{4}>0$ so that

$$
\left\|F_{2}(v)\right\|_{L_{t}^{\frac{2 p}{1-s_{c}}} L_{x}^{\frac{2 N p}{N+\gamma}}\left(\left[T_{1}, \infty\right), \mathbb{R}^{N}\right.} \lesssim \varepsilon^{\beta_{4}}
$$

Putting together (4.10), (4.28), and (4.30) gives

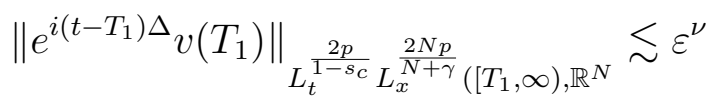


for $0<\nu \leq \min \left\{\beta_{3}, \beta_{4}\right\}$. For the bound on the nonlinear solution we again consider Duhamel's formula

$$
v(t)=e^{i\left(t-T_{1}\right) \Delta} v\left(T_{1}\right)-i \int_{T_{1}}^{t} e^{i(t-s) \Delta} F(v(s)) d s
$$

where $F(v)=\left(|x|^{-(N-\gamma)} *|v|^{p}\right)|v|^{p-2} v$. Taking $L_{t}^{\frac{2 p}{1-s_{c}}} L_{x}^{\frac{2 N p}{N+\gamma}}\left(\left[T_{1}, \infty\right), \mathbb{R}^{N}\right.$ norms, we observe from the linear evolution bound and inhomogeneous Strichartz estimate (4.7) that

$$
\|v\|_{L_{t}^{\frac{2 p}{1-s_{c}}} \frac{2 N p}{L_{x}^{N+\gamma}}\left(\left[T_{1}, \infty\right), \mathbb{R}^{N}\right.} \lesssim \varepsilon^{\nu}+\|\nabla F(v)\|_{L_{t}^{\frac{2 p-1-s_{c}(p-1)}{2 p}} L_{x}^{\frac{2 N p}{2 N p-N-\gamma}}\left(\left[T_{1}, \infty\right) \times \mathbb{R}^{N}\right)} .
$$

By Hölder's inequality, product rule and Lemma 2.3.1, we obtain

$$
\begin{aligned}
& \left\|\nabla\left(\left(|x|^{-(N-\gamma)} *|v|^{p}\right)|v|^{p-2} v\right)\right\| \frac{2 p}{L_{t}^{\frac{2 p-1-s_{c}(p-1)}{2 N p}} L_{x}^{\frac{2 N p}{2 N p-\gamma}}\left(\left[T_{1}, \infty\right) \times \mathbb{R}^{N}\right)}
\end{aligned}
$$

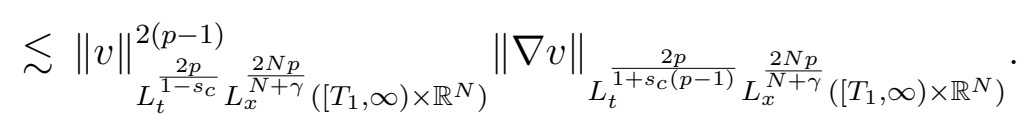

The remaining proof follows similar reasoning as in Lemma 4.2.1 and we obtain the $H^{1}$ scattering of $v(t)$.

\subsection{Variational analysis}

We consider both equations in the inter-critical regime such that $0<s_{c}<1$ with $s$ defined in (4.3), and provided $p \geq 2$ for the gHartree equation. In this case, both equations (4.1) and (4.2) admit solutions of the form $e^{i t} Q(x)$, which are global but non-scattering, where $Q$ solves in the NLS case the following nonlinear elliptic equation

$$
-Q+\Delta Q+|Q|^{p-1} Q=0
$$

and in the gHartree case $Q$ solves the Choquard equation (2.123) given by

$$
-Q+\Delta Q+\left(|x|^{-(N-\gamma)} *|Q|^{p}\right)|Q|^{p-2} Q=0
$$


The variational analysis for NLS is well known, see [HR07], [HR08], [Gue14], however, for the completeness and comparison with gHartree case we include it here. (Note that in NLS we can use the unique ground state $Q$, however, in gHartree we use the sharp constant $C_{G N}$ as $Q$ in general may not be unique. See Chapter 2, Section 2.8.)

\subsubsection{The NLS equation}

The equation (4.32) has countably many $H^{1}$ (real) solutions. Among those, there is exactly one solution of minimal mass, called the ground state, which is positive, radial, and exponentially decaying (e.g., see Berestycki and Lions [BL83a, BL83b], Kwong [Kwo89]; for a review, for example, see Tao [Tao06, Appendix B]). The ground state solution $Q$ of (4.32) optimizes the Gagliardo-Nirenberg inequality

$$
\|u\|_{L^{p+1}}^{p+1} \leq C_{G N}\|\nabla u\|_{L^{2}\left(\mathbb{R}^{N}\right)}^{\frac{N(p-1)}{2}}\|u\|_{L^{2}\left(\mathbb{R}^{N}\right)^{2}}^{2-(N-2)(p-1)},
$$

with

$$
C_{G N}=\frac{2(p+1)}{2 N-(N-2)(p+1)}\left(\frac{N(p-1)}{2(p+1)-N(p-1)}\right)^{-N(p-1) / 4}\|Q\|_{L^{2}\left(\mathbb{R}^{N}\right)}^{-(p-1)} .
$$

The Pohozaev identities for $Q$ yield

$$
\|Q\|_{L^{2}\left(\mathbb{R}^{N}\right)}^{1-s_{c}}\|\nabla Q\|_{L^{2}\left(\mathbb{R}^{N}\right)}^{s_{c}}=\left(\frac{2(p+1)}{N(p-1) C_{G N}}\right)^{\frac{1}{p-1}}
$$

and

$$
M(Q)^{1-s_{c}} E(Q)^{s_{c}}=\left(\frac{s_{c}}{N}\right)^{s_{c}}\left(\|Q\|_{L^{2}\left(\mathbb{R}^{N}\right)}^{1-s_{c}}\|\nabla Q\|_{L^{2}\left(\mathbb{R}^{N}\right)}^{s_{c}}\right)^{2}
$$

For the rest of this subsection, we suppose $u_{0} \in H^{1}\left(\mathbb{R}^{N}\right)$ and $u(t)$ solves the NLS equation (4.1).

Lemma 4.3.1 If $M\left[u_{0}\right]^{\frac{1-s_{c}}{s_{c}}} E\left[u_{0}\right]<(1-\delta) M[Q]^{\frac{1-s_{c}}{s_{c}}} E[Q]$ and
\[ \left\|u_{0}\right\|_{L^{2}\left(\mathbb{R}^{N}\right)}^{\frac{1-s_{c}}{s_{c}}}\left\|\nabla u_{0}\right\|_{L^{2}\left(\mathbb{R}^{N}\right)} \leq\|Q\|_{L^{2}\left(\mathbb{R}^{N}\right)}^{\frac{1-s_{c}}{s_{c}}}\|\nabla Q\|_{L^{2}\left(\mathbb{R}^{N}\right)}, \] 
then there exists $\delta_{1}=\delta_{1}(\delta)>0$ so that

$$
\|u(t)\|_{L^{2}\left(\mathbb{R}^{N}\right)}^{\frac{1-s_{c}}{s_{c}}}\|\nabla u(t)\|_{L^{2}\left(\mathbb{R}^{N}\right)}<\left(1-\delta_{1}\right)\|Q\|_{L^{2}\left(\mathbb{R}^{N}\right)}^{\frac{1-s_{c}}{s_{c}}}\|\nabla Q\|_{L^{2}\left(\mathbb{R}^{N}\right)}
$$

for all $t \in I$, where $u: I \times \mathbb{R}^{N} \rightarrow \mathbb{C}$ is the maximal lifespan solution to (4.1). In particular, $I=\mathbb{R}$ and $u$ is uniformly bounded in $H^{1}\left(\mathbb{R}^{N}\right)$.

Proof. While this is a simple and well-known proof, it is the core of the dichotomy and we include it for completeness. By the mass and energy conservation along with GagliardoNirenberg inequality

$$
\begin{aligned}
& (1-\delta) M[Q]^{\frac{1-s_{c}}{s_{c}}} E[Q] \geq M\left[u_{0}\right]^{\frac{1-s_{c}}{s_{c}}} E\left[u_{0}\right] \\
& \quad \geq \frac{1}{2}\left(\|u(t)\|_{L^{2}}^{\frac{1-s_{c}}{s_{c}}}\|\nabla u(t)\|_{L^{2}}\right)^{2}-\frac{1}{p+1} C_{G N}\left(\|u(t)\|_{L^{2}}^{\frac{1-s_{c}}{s_{c}}}\|\nabla u(t)\|_{L^{2}}\right)^{s_{c}(p-1)+2} .
\end{aligned}
$$

Using (4.34) and (4.35), the above estimate becomes

$1-\delta \geq \frac{N}{2 s_{c}}\left(\frac{\|u(t)\|_{L^{2}}^{\frac{1-s_{c}}{s_{c}}}\|\nabla u(t)\|_{L^{2}}}{\|Q\|_{L^{2}}^{\frac{1-s_{c}}{s_{c}}}\|\nabla Q\|_{L^{2}}}\right)^{2}-\frac{2}{s_{c}(p-1)}\left(\frac{\|u(t)\|_{L^{2}}^{\frac{1-s_{c}}{s_{c}}}\|\nabla u(t)\|_{L^{2}}}{\|Q\|_{L^{2}}^{\frac{1-s_{c}}{s_{c}}}\|\nabla Q\|_{L^{2}}}\right)^{s_{c}(p-1)+2}$.

Define $f(x)=\frac{N}{2 s_{c}} x^{2}-\frac{2}{s_{c}(p-1)} x^{s_{c}(p-1)+2}$. Since $s_{c}>0$, we always have $\operatorname{deg}(f)>2$. Therefore,

$$
f^{\prime}(x)=\frac{N}{s_{c}} x-\frac{2\left(s_{c}(p-1)+2\right)}{s_{c}(p-1)} x^{s_{c}(p-1)+1}=\frac{N}{s_{c}}\left(1-x^{s_{c}(p-1)}\right) x,
$$

which implies that $f^{\prime}(x)=0$ when $x_{0}=0$ and $x_{1}=1$. Observe that

$$
f(x) \leq 1-\delta<1=f\left(x_{1}\right)
$$

If initially we have

$$
\left\|u_{0}\right\|_{L^{2}\left(\mathbb{R}^{N}\right)}^{\frac{1-s_{c}}{s_{c}}}\left\|\nabla u_{0}\right\|_{L^{2}\left(\mathbb{R}^{N}\right)} \leq\|Q\|_{L^{2}\left(\mathbb{R}^{N}\right)}^{\frac{1-s_{c}}{s_{c}}}\|\nabla Q\|_{L^{2}\left(\mathbb{R}^{N}\right)}
$$

then by (4.36) and the continuity of $\|\nabla u(t)\|_{L^{2}\left(\mathbb{R}^{N}\right)}$ in $t$, we conclude that

$$
\left\|u_{0}\right\|_{L^{2}\left(\mathbb{R}^{N}\right)}^{\frac{1-s_{c}}{s_{c}}}\|\nabla u(t)\|_{L^{2}\left(\mathbb{R}^{N}\right)} \leq\|Q\|_{L^{2}\left(\mathbb{R}^{N}\right)}^{\frac{1-s_{c}}{s_{c}}}\|\nabla Q\|_{L^{2}\left(\mathbb{R}^{N}\right)} \text {, i.e., } x<x_{1}
$$


for all time $t \in I$. Furthermore, since the $L^{2}$-norm is conserved and invoking (4.34), we have global existence and that the $\dot{H}^{1}$-norm is uniformly bounded.

Based on the result of Lemma 4.3.1, we have that $u$ is global and uniformly bounded in $H^{1}$, moreover, there exists $\delta>0$ such that

$$
\sup _{t \in \mathbb{R}}\|u\|_{L^{2}\left(\mathbb{R}^{N}\right)}^{\frac{1-s_{c}}{s_{c}}}\|\nabla u\|_{L^{2}\left(\mathbb{R}^{N}\right)}<(1-\delta)\|Q\|_{L^{2}\left(\mathbb{R}^{N}\right)}^{\frac{1-s_{c}}{s_{c}}}\|\nabla Q\|_{L^{2}\left(\mathbb{R}^{N}\right)} .
$$

To prove Theorem 3.2 we use a virial weight in a ball around the origin of sufficiently large radius together with the coercivity to obtain a suitable lower bound. First, we need (4.37) on balls of sufficiently large radii so that we can have a necessary coercivity. Define $\chi_{R}(x)=\chi\left(\frac{x}{R}\right)$ for $R>0$, where $\chi(x) \in C_{c}^{\infty}\left(\mathbb{R}^{N}\right)$ is a smooth cutoff function on $\{|x| \leq 1\}$ with $\chi(x)=1$ for $|x| \leq \frac{1}{2}$.

Lemma 4.3.2 There exists $R_{0}=R_{0}(\delta, M(u), Q)>0$ sufficiently large so that

$$
\sup _{t \in \mathbb{R}}\left\|\chi_{R} u(t)\right\|_{L^{2}}^{1-s_{c}}\left\|\chi_{R} u(t)\right\|_{\dot{H}^{1}}^{s_{c}}<(1-\delta)\|Q\|_{L^{2}}^{1-s_{c}}\|\nabla Q\|_{L^{2}}^{s_{c}}
$$

In particular, there exists $\delta_{1}=\delta_{1}(\delta)>0$ so that

$$
\left\|\chi_{R} u(t)\right\|_{\dot{H}^{1}}^{2}-\frac{N(p-1)}{2(p+1)}\left\|\chi_{R} u(t)\right\|_{L^{p+1}}^{p+1} \geq \delta_{1}\left\|\chi_{R} u(t)\right\|_{L^{p+1}}^{p+1}
$$

uniformly for $t \in \mathbb{R}$.

Proof. For $u \in \dot{H}^{1}$, we write

$$
\|\nabla u\|_{L^{2}\left(\mathbb{R}^{N}\right)}^{2}-\frac{N(p-1)}{2(p+1)}\|u\|_{L^{p+1}\left(\mathbb{R}^{N}\right)}^{p+1}=\frac{N(p-1)}{2} E[u]-\frac{s_{c}(p-1)}{2}\|\nabla u\|_{L^{2}\left(\mathbb{R}^{N}\right)} .
$$

By the Gagliardo-Nirenberg inequality and (4.34),

$$
\begin{aligned}
E[u] & \left.\geq \frac{1}{2}\|\nabla u\|_{L^{2}\left(\mathbb{R}^{N}\right)}^{2}\left(1-\frac{2 C_{G N}}{p+1}\left(\|u\|_{L^{2}\left(\mathbb{R}^{N}\right)}^{1-s_{c}}\|\nabla u\|_{L^{2}\left(\mathbb{R}^{N}\right)}^{s_{c}}\right)\right)^{p-1}\right) \\
& \left.\geq \frac{1}{2}\|\nabla u\|_{L^{2}\left(\mathbb{R}^{N}\right)}^{2}\left(1-\frac{2 C_{G N}}{p+1}(1-\delta)\left(\|Q\|_{L^{2}\left(\mathbb{R}^{N}\right)}^{1-s_{c}}\|\nabla Q\|_{L^{2}\left(\mathbb{R}^{N}\right)}^{s_{c}}\right)\right)^{p-1}\right) \\
& =\left(\frac{s_{c}}{N}+\frac{2 \delta}{N(p-1)}\right)\|\nabla u\|_{L^{2}\left(\mathbb{R}^{N}\right)}^{2} .
\end{aligned}
$$


Therefore,

$$
\|\nabla u\|_{L^{2}\left(\mathbb{R}^{N}\right)}^{2}-\frac{N(p-1)}{2(p+1)}\|u\|_{L^{p+1}\left(\mathbb{R}^{N}\right)}^{p+1} \geq \delta\|\nabla u\|_{L^{2}\left(\mathbb{R}^{N}\right)}^{2},
$$

which implies that

$$
\|\nabla u\|_{L^{2}\left(\mathbb{R}^{N}\right)}^{2}-\frac{N(p-1)}{2(p+1)}\|u\|_{L^{p+1}\left(\mathbb{R}^{N}\right)}^{p+1} \geq \frac{N(p-1)}{2(p+1)} \frac{\delta}{1-\delta}\|u\|_{L^{p+1}\left(\mathbb{R}^{N}\right)}^{p+1},
$$

choosing $\delta_{1}=\frac{N(p-1)}{2(p+1)} \frac{\delta}{1-\delta}$ gives (4.39). To finish the proof, we need to verify (4.38). Observe that

$$
\left\|\chi_{R} u(t)\right\|_{L_{x}^{2}} \leq\|u(t)\|_{L_{x}^{2}}
$$

uniformly for $t \in \mathbb{R}$. Therefore, it is sufficient to consider the $\dot{H}^{1}$ term. We compute

$$
\begin{aligned}
\int\left|\nabla\left(\chi_{R} u\right)\right|^{2} d x & =\int \chi_{R}^{2}|\nabla u|^{2} d x+\int|u|^{2} \nabla \chi_{R} \nabla \chi_{R}+\int 2 \operatorname{Re}(\bar{u} \nabla u) \chi_{R} \nabla \chi_{R} \\
& =\int \chi_{R}^{2}|\nabla u|^{2} d x-\int|u|^{2} \chi_{R} \Delta \chi_{R}
\end{aligned}
$$

and use the following identity

$$
\int \chi_{R}^{2}|\nabla u|^{2} d x=\int\left|\nabla\left(\chi_{R} u\right)\right|^{2} d x+\int \chi_{R} \Delta\left(\chi_{R}\right)|u|^{2} d x
$$

with the definition of $\chi$ to write

$$
\left\|\nabla\left(\chi_{R} u\right)\right\|_{L^{2}}^{2} \leq\|\nabla u\|_{L^{2}}^{2}+\mathcal{O}\left(\frac{1}{R^{2}} M[u]\right)
$$

Choosing $R_{0}$ sufficiently large depending on $\delta, M[u]$ and $Q$, we get that (4.41) holds for any $R>R_{0}$, and yields the desired estimate (4.38).

\subsubsection{The gHartree equation}

The Gagliardo-Nirenberg inequality of convolution type

$$
Z(v) \leq C_{G N C}\|\nabla v\|_{L^{2}\left(\mathbb{R}^{N}\right)}^{N p-(N+\gamma)}\|v\|_{L^{2}\left(\mathbb{R}^{N}\right)}^{N+\gamma-(N-2) p}
$$


has the sharp constant $C_{G N C}$ (see discussion in Chapter 2, Section 2.8 and recall that $Q$ in gHartree may not be unique) with

$$
C_{G N C}=\frac{2 p}{N(p-1)-\gamma}\left(\frac{N+\gamma-(N-2) p}{N(p-1)-\gamma}\right)^{\frac{N(p-1)-\gamma}{2}-1}\|Q\|_{L^{2}\left(\mathbb{R}^{N}\right)}^{-2(p-1)}
$$

where ground states $Q$ solve the equation (4.33). From the value of the sharp constant $C_{G N C}$ (and Pohozaev identities), we get

$$
\|Q\|_{L^{2}\left(\mathbb{R}^{N}\right)}^{1-s_{c}}\|\nabla Q\|_{L^{2}}^{s_{c}}=\left(\frac{p\left(C_{G N C}\right)^{-1}}{s_{c}(p-1)+1}\right)^{\frac{1}{2(p-1)}}
$$

and

$$
M[Q]^{1-s_{c}} E[Q]^{s_{c}}=\left(\frac{s_{c}(p-1)}{2 s_{c}(p-1)+2}\right)^{s_{c}}\left(\|Q\|_{L^{2}\left(\mathbb{R}^{N}\right)}^{1-s_{c}}\|\nabla Q\|_{L^{2}\left(\mathbb{R}^{N}\right)}^{s_{c}}\right)^{2}
$$

For the rest of this subsection, we assume $v_{0} \in H^{1}\left(\mathbb{R}^{N}\right)$ and $v(t)$ solves the gHartree equation (4.2).

Lemma 4.3.3 If $M\left[v_{0}\right]^{\frac{1-s_{c}}{s_{c}}} E\left[v_{0}\right]<(1-\delta) M[Q]^{\frac{1-s_{c}}{s_{c}}} E[Q]$ and

$$
\left\|v_{0}\right\|_{L^{2}\left(\mathbb{R}^{N}\right)}^{\frac{1-s_{c}}{s_{c}}}\left\|\nabla v_{0}\right\|_{L^{2}\left(\mathbb{R}^{N}\right)} \leq\|Q\|_{L^{2}\left(\mathbb{R}^{N}\right)}^{\frac{1-s_{c}}{s_{c}}}\|\nabla Q\|_{L^{2}\left(\mathbb{R}^{N}\right)}
$$

then there exists $\delta_{1}=\delta_{1}(\delta)>0$ so that

$$
\|v(t)\|_{L^{2}\left(\mathbb{R}^{N}\right)}^{\frac{1-s_{c}}{s_{c}}}\|\nabla v(t)\|_{L^{2}\left(\mathbb{R}^{N}\right)}<\left(1-\delta_{1}\right)\|Q\|_{L^{2}\left(\mathbb{R}^{N}\right)}^{\frac{1-s_{c}}{s_{c}}}\|\nabla Q\|_{L^{2}\left(\mathbb{R}^{N}\right)}
$$

for all $t \in I$, where $u: I \times \mathbb{R}^{N} \rightarrow \mathbb{C}$ is the maximal lifespan solution to (4.2). In particular, $I=\mathbb{R}$ and $u$ is uniformly bounded in $H^{1}\left(\mathbb{R}^{N}\right)$.

Proof. See Chapter 3, Section 3.3, Theorem 3.3.1.

Now we prove the coercivity estimate on balls of large radii for gHartree equation. The following lemma differs from the standard approach as we lower bound the virial not by the potential term but by the $L^{\frac{2 N p}{N+\gamma}}\left(\mathbb{R}^{N}\right)$ norm. 
Lemma 4.3.4 There exists $R_{0}=R_{0}(\delta, M(v), Q)>0$ suffiiently large that for any $R>$ $R_{0}$

$$
\sup _{t \in \mathbb{R}}\left\|\chi_{R} v(t)\right\|_{L^{2}}^{1-s_{c}}\left\|\chi_{R} v(t)\right\|_{\dot{H}^{1}}^{s_{c}}<(1-\delta)\|Q\|_{L^{2}}^{1-s_{c}}\|\nabla Q\|_{L^{2}}^{s_{c}}
$$

In particular, there exists $\tilde{\delta}=\tilde{\delta}(\delta)>0$ so that

$$
\left\|\chi_{R} v(t)\right\|_{\dot{H}^{1}}^{2}-\frac{s_{c}(p-1)+1}{p} Z\left[\chi_{R} v(t)\right] \geq \tilde{\delta}\left\|\chi_{R} v(t)\right\|_{L^{\frac{2 N p}{N+\gamma}}}^{2}
$$

uniformly for $t \in \mathbb{R}$.

Proof. We write

$$
\|\nabla v\|_{L^{2}\left(\mathbb{R}^{N}\right)}^{2}-\frac{s_{c}(p-1)+1}{p} Z(v)=\left(2 s_{c}(p-1)+2\right) E[v]-s_{c}(p-1)\|\nabla v\|_{L^{2}\left(\mathbb{R}^{N}\right)}^{2} .
$$

By Gagliardo-Nirenberg inequality and (4.43),

$$
\begin{aligned}
E[v] & \geq \frac{1}{2}\|\nabla v\|_{L^{2}\left(\mathbb{R}^{N}\right)}^{2}\left(1-\frac{C_{G N C}}{p}\left(\|v\|_{L^{2}\left(\mathbb{R}^{N}\right)}^{1-s_{c}}\|\nabla v\|_{L^{2}\left(\mathbb{R}^{N}\right)}^{s_{c}}\right)^{2(p-1)}\right) \\
& \geq \frac{1}{2}\|\nabla v\|_{L^{2}\left(\mathbb{R}^{N}\right)}^{2}\left(1-\frac{C_{G N C}}{p}(1-\delta)\left(\|Q\|_{L^{2}\left(\mathbb{R}^{N}\right)}^{1-s_{c}}\|\nabla Q\|_{L^{2}\left(\mathbb{R}^{N}\right)}^{s_{c}}\right)^{2(p-1)}\right) \\
& =\left(\frac{s_{c}(p-1)}{2 s_{c}(p-1)+2}+\frac{\delta}{2 s_{c}(p-1)+2}\right)\|\nabla v\|_{L^{2}\left(\mathbb{R}^{N}\right)}^{2} .
\end{aligned}
$$

Therefore,

$$
\|\nabla v\|_{L^{2}\left(\mathbb{R}^{N}\right)}^{2}-\frac{s_{c}(p-1)+1}{p} Z(v) \geq \delta\|\nabla v\|_{L^{2}\left(\mathbb{R}^{N}\right)}^{2} .
$$

The assumption $p<\frac{N+\gamma}{N-2}$ (since $s_{c}<1$ ) implies that $\frac{2 N p}{N+\gamma}<\frac{2 N}{N-2}$ and thus, Sobolev embedding gives that $\|v\|_{L^{\frac{2 N p}{N+\gamma}}} \lesssim\|\nabla v\|_{L^{2}}$. Hence, we obtain

$$
\|\nabla v\|_{L^{2}\left(\mathbb{R}^{N}\right)}^{2}-\frac{s_{c}(p-1)+1}{p} Z(v) \geq \tilde{\delta}\|v\|_{L^{\frac{2 N p}{N+\gamma}}}^{2} .
$$

To verify (4.45) we follow the similar argument as in Lemma 4.3.2 for (4.38) which concludes the proof. 


\subsection{Proof of Theorem 4.1.4}

Suppose $u$ is a solution of (4.1) satisfying Theorem 4.1.4. Next, we recall the Morawetz identity.

Lemma 4.4.1 (Morawetz identity, NLS) Let a $: \mathbb{R}^{N} \rightarrow \mathbb{R}$ be a smooth weight. Define

$$
\mathcal{M}(t)=2 \operatorname{Im} \int \bar{u} \nabla u \cdot \nabla a d x
$$

Then

$$
\frac{d}{d t} \mathcal{M}(t)=\int-\frac{2(p-1)}{p+1}|u|^{p+1} \Delta a+|u|^{2}(-\Delta \Delta a)+4 \operatorname{Re} a_{j k} \bar{u}_{j} u_{k} d x
$$

where subscripts denote partial derivatives and repeated indices are summed.

We take $a$ to be a radial function satisfying

$$
a(x)= \begin{cases}|x|^{2} & |x|<\frac{R}{2} \\ R|x| & |x|>R .\end{cases}
$$

In the intermediate region $\frac{R}{2}<|x| \leq R$, we impose that

$$
\partial_{r} a>0, \quad \partial_{r}^{2} a \geq 0, \quad\left|\partial^{\alpha} a(x)\right| \lesssim_{\alpha} R|x|^{-\alpha+1} \quad \text { for }|\alpha| \geq 1
$$

Here, $\partial_{r}$ denotes the radial derivative, i.e., $\partial_{r} a=\nabla a \cdot \frac{x}{|x|}$. Under these conditions, the matrix $\left(a_{j k}\right)$ is non-negative. Note that for $|x| \leq \frac{R}{2}$, we have

$$
a_{j k}=2 \delta_{j k}, \quad \Delta a=2 N, \quad \Delta^{2} a=0,
$$

while for $|x|>R$, we have

$$
a_{j k}=\frac{R}{|x|}\left[\delta_{j k}-\frac{x_{j}}{|x|} \frac{x_{k}}{|x|}\right], \quad \Delta a=\frac{(N-1) R}{|x|}, \quad \Delta^{2} a=0 .
$$


Proposition 4.4.2 (Morawetz estimate, NLS) Let $T>0$. For $R \equiv R(\delta, M(u), Q)$ sufficiently large,

$$
\frac{1}{T} \int_{0}^{T} \int_{|x| \leq R}|u(x, t)|^{p+1} d x d t \lesssim u, \delta\left\{\begin{array}{ll}
\frac{R}{T}+\frac{1}{R^{\frac{(N-1)(p-1)}{2}},} & \text { if } s_{c}<\frac{1}{2} \\
\frac{R}{T}+\frac{1}{R^{2}}, & \text { if } s_{c} \geq \frac{1}{2}
\end{array} .\right.
$$

Proof. Note that by Cauchy-Schwarz, the uniform $H^{1}$ bound for $u$ and the choice of the weight $a(x)$, we have that $\sup _{t \in \mathbb{R}}|\mathcal{M}(t)| \lesssim u$. We compute

$$
\begin{aligned}
\frac{d}{d t} M(t)= & 8 \int_{|x| \leq \frac{R}{2}}|\nabla u|^{2}-\frac{N(p-1)}{2(p+1)}|u|^{p+1} d x \\
& +\int_{|x|>R}-\frac{2(N-1)(p-1)}{p+1} \frac{R}{|x|}|u|^{p+1}+\frac{4 R}{|x|}\left|\nabla_{a} u\right|^{2} d x \\
& +\int_{\frac{R}{2}<|x| \leq R} 4 \operatorname{Re} a_{j k} \bar{u}_{j} u_{k}-\frac{2(p-1)}{p+1} \frac{R}{|x|}|u|^{p+1}-\frac{R}{|x|^{3}}|u|^{2} d x
\end{aligned}
$$

where $\nabla_{a}$ denotes the angular part of the derivative. In fact, since $u$ is radial, this term is zero. We define $\chi_{R}:=\chi\left(\frac{x}{R}\right)$ for $R>0$ and write

$$
\int_{|x| \leq \frac{R}{2}} \chi_{R}^{2}|\nabla u|^{2}=\int_{|x| \leq \frac{R}{2}}\left|\nabla\left(\chi_{R} u\right)\right|^{2}+\int_{|x| \leq \frac{R}{2}} \chi_{R} \Delta\left(\chi_{R}\right)|u|^{2} .
$$

Then the Morawetz identity can be estimated as

$$
\begin{aligned}
\frac{d}{d t} \mathcal{M}(t) \geq & 8 \int_{|x| \leq \frac{R}{2}}\left|\nabla\left(\chi_{R} u\right)\right|^{2}-\frac{4 N(p-1)}{p+1} \int_{|x| \leq \frac{R}{2}}\left|\chi_{R} u\right|^{p+1}-c_{1} \int_{|x|>\frac{R}{2}} \frac{R}{|x|}|u|^{p+1} \\
& +8 \int_{|x| \leq \frac{R}{2}} \chi_{R} \Delta\left(\chi_{R}\right)|u|^{2}-\int_{\frac{R}{2}<|x| \leq R} \frac{R}{|x|^{3}}|u|^{2} d x \\
\geq & 8 \int \delta_{1}\left|\chi_{R} u(x, t)\right|^{p+1} d x-c_{1} \int_{|x|>\frac{R}{2}}|u(x, t)|^{p+1}-\frac{c_{2}}{R^{2}} M(u)
\end{aligned}
$$

where in the inequality (4.48) we have used Lemma 4.3.2 and the fact that for a fixed radius $R$ and mass $M(u)$ the terms in (4.47) is a constant multiple of $\frac{1}{R^{2}}$ and $M(u)$.

Next we apply the fundamental theorem of calculus on the interval $[0, T]$ and rearrange terms to obtain 


$$
\begin{array}{rl}
\int_{0}^{T} \int 8 \delta_{1}\left|\chi_{R} u(x, t)\right|^{p+1} & d x d t \\
& \lesssim \sup _{t \in[0, T]}|\mathcal{M}(t)|+\int_{0}^{T} \int_{|x|>R}|u(x, t)|^{p+1} d x d t+\frac{T}{R^{2}} M(u) .
\end{array}
$$

By Lemma 2.3.3 (radial Sobolev embedding), we have

$$
\begin{aligned}
\int_{|x|>R}|u(x, t)|^{p+1} d x & \lesssim \frac{1}{R^{\frac{(N-1)(p-1)}{2}}} \int|x|^{\frac{(N-1)(p-1)}{2}}|u(x, t)|^{p-1}|u(x, t)|^{2} d x \\
& \lesssim \frac{1}{R^{\frac{(N-1)(p-1)}{2}}} \|\left. u\right|_{L_{t}^{\infty} \dot{H}_{x}^{1}} ^{p-1} M(u) \\
& \lesssim \frac{1}{R^{\frac{(N-1)(p-1)}{2}}} .
\end{aligned}
$$

Therefore, we deduce that

$$
\frac{1}{T} \int_{0}^{T} \int_{|x| \leq R}|u(x, t)|^{p+1} d x d t \lesssim u, \delta \frac{R}{T}+\frac{1}{R^{\frac{(N-1)(p-1)}{2}}}+\frac{1}{R^{2}} .
$$

Observe that

$$
\begin{cases}\frac{(N-1)(p-1)}{2} \leq 2 & \text { for } s_{c} \leq \frac{1}{2} \\ \frac{(N-1)(p-1)}{2}>2 & \text { for } s_{c}>\frac{1}{2}\end{cases}
$$

Thus,

$$
\frac{1}{T} \int_{0}^{T} \int_{|x| \leq R}|u(x, t)|^{p+1} d x d t \lesssim u, \delta \begin{cases}\frac{R}{T}+\frac{1}{R^{\frac{(N-1)(p-1)}{2}},}, & \text { if } s_{c}<\frac{1}{2} \\ \frac{R}{T}+\frac{1}{R^{2}}, & \text { if } s_{c} \geq \frac{1}{2}\end{cases}
$$

as desired.

Now we prove that the potential energy of $u$ escapes to spatial infinity as $t \rightarrow \infty$.

Proposition 4.4.3 (Energy evacuation, NLS) There exists a sequence of times $t_{n} \rightarrow \infty$ and a sequence of radii $R_{n} \rightarrow \infty$ such that

$$
\lim _{n \rightarrow \infty} \int_{|x| \leq R_{n}}\left|u\left(x, t_{n}\right)\right|^{p+1} d x=0 .
$$


Proof. For $T>0$ sufficiently large with $R=T^{\frac{2}{N(p-1)-(p-3)}}$ for $s_{c}<\frac{1}{2}$ and $R=T^{1 / 3}$ for $s_{c} \geq \frac{1}{2}$ (note that $R>R_{0}$ ) and Proposition 4.4.2, we obtain for $s_{c}<\frac{1}{2}$ that

$$
\frac{1}{T} \int_{0}^{T} \int_{|x| \leq t^{\frac{2}{N(p-1)}-(p-3)}}|u(x, t)|^{p+1} d x d t \lesssim T^{-\frac{(N-1)(p-1)}{N(p-1)-(p-3)}}
$$

and

$$
\frac{1}{T} \int_{0}^{T} \int_{|x| \leq t^{1 / 3}}|u(x, t)|^{p+1} d x d t \lesssim T^{-2 / 3}
$$

for $s_{c} \geq \frac{1}{2}$. Since $u$ is global and $\|u\|_{L^{p+1}}$ is bounded, by mean value theorem there exist sequences $t_{n} \rightarrow \infty$ and $R_{n} \rightarrow \infty$ such that the energy evacuation happens on a sequence of balls as desired, i.e.,

$$
\lim _{n \rightarrow \infty} \int_{|x| \leq R_{n}}\left|u\left(x, t_{n}\right)\right|^{p+1} d x=0,
$$

which completes the proof.

Proof. (of Theorem 4.1.4) We have (by Section 4.3.1) that $u$ is global and uniformly bounded in $H^{1}$. Choose $\varepsilon$ and $R$ as in Lemma 4.2.1 with $t_{n} \rightarrow \infty$ and $R_{n} \rightarrow \infty$ as in Proposition 4.4.3. Now taking $n$ large so that $R_{n} \geq R$, Hölder's inequality gives

$$
\int_{|x| \leq R}\left|u\left(x, t_{n}\right)\right|^{2} d x \lesssim R^{\frac{N(p-1)}{p+1}}\left(\int_{|x| \leq R_{n}}\left|u\left(x, t_{n}\right)\right|^{p+1} d x\right)^{\frac{2}{p+1}} \rightarrow 0 \quad \text { as } \quad n \rightarrow \infty .
$$

Therefore, Lemma 4.2.1 implies that $u$ scatters in $H^{1}\left(\mathbb{R}^{N}\right)$ forward in time.

\subsection{Proof of Theorem 4.1.5}

We begin this section with the Morawetz identity in the gHartree case, which will be used to obtain Morawetz estimate.

Lemma 4.5.1 (Morawetz identity, gH) Let $a: \mathbb{R}^{N} \rightarrow \mathbb{R}$ be the same smooth weight as described in Section 4.4. Define

$$
\mathcal{M}_{g H}(t)=2 \operatorname{Im} \int \bar{v} \nabla v \cdot \nabla a d x .
$$


Then

$$
\begin{aligned}
\frac{d}{d t} \mathcal{M}_{g H}(t)= & \int-4\left(\frac{1}{2}-\frac{1}{p}\right)\left(|x|^{-(N-\gamma)} *|v|^{p}\right)|v|^{p} \Delta a+|v|^{2}(-\Delta \Delta a) \\
& +\int 4 \operatorname{Re} a_{j k} \bar{v}_{j} v_{k} d x-\frac{4(N-\gamma)}{p} \iint \nabla a \frac{(x-y)|v(x)|^{p}|v(y)|^{p}}{|x-y|^{N-\gamma+2}} d x d y,
\end{aligned}
$$

where subscripts denote partial derivatives and repeated indices are summed.

Proposition 4.5.2 (Morawetz estimate, gH) Let $T>0$. For $R \equiv R(\delta, M(v), Q)>0$ sufficiently large,

$$
\frac{1}{T} \int_{0}^{T}\left(\int_{|x| \leq R}|v(x, t)|^{\frac{2 N p}{N+\gamma}} d x\right)^{\frac{N+\gamma}{N p}} d t \lesssim v, \delta \begin{cases}\frac{R}{T}+\frac{1}{R^{\frac{(N-1)(N(p-1)-\gamma)}{N}}} & \text { if } s_{c}<\frac{1}{2} \\ \frac{R}{T}+\frac{1}{R^{2}} & \text { if } s_{c} \geq \frac{1}{2}\end{cases}
$$

Proof. Note that by Cauchy-Schwarz, the uniform $H^{1}$ bound for $u$ and the choice of the weight $a(x)$, we have

$$
\sup _{t \in \mathbb{R}}\left|\mathcal{M}_{g H}(t)\right| \lesssim R
$$

We recall (from Lemma 4.5.1)

$$
\begin{aligned}
\frac{d}{d t} \mathcal{M}_{g H}(t)= & \int|v|^{2}(-\Delta \Delta a)+4 \operatorname{Re} a_{j k} \bar{v}_{j} v_{k} d x \\
& -4 \int \Delta a\left(\frac{1}{2}-\frac{1}{p}\right)\left(|x|^{-(N-\gamma)} *|v|^{p}\right)|v|^{p} \\
& -\frac{4(N-\gamma)}{p} \iint \nabla a \frac{(x-y)|v(x)|^{p}|v(y)|^{p}}{|x-y|^{N-\gamma+2}} d x d y .
\end{aligned}
$$

For $|x| \leq \frac{R}{2}$, the above expression reduces to

$$
8 \int_{|x| \leq \frac{R}{2}}|\nabla v|^{2}-\int_{|x| \leq \frac{R}{2}} \frac{4(N(p-1)-\gamma)}{p}\left(|x|^{-(N-\gamma)} *|v|^{p}\right)|v|^{p} d x .
$$

In the region $\frac{R}{2}<|x| \leq R$, (4.49) yields

$$
\int_{\frac{R}{2}<|x| \leq R} 4 \operatorname{Re} a_{j k} \bar{v}_{j} v_{k}+\mathcal{O}\left(\int_{\frac{R}{2}<|x| \leq R} \frac{R}{|x|^{3}}|v|^{2}\right)
$$


and in $|x|>R$, it gives

$$
\int_{|x|>R} \frac{4 R}{|x|}\left|\nabla_{a} v\right|^{2} d x=0
$$

where $\nabla_{a}$ denotes the angular part of the derivative, which we drop, since $v$ is radial.

In the region $\frac{R}{2}<|x| \leq R,(4.50)$ yields

$$
-\int_{\frac{R}{2}<|x| \leq R} 4\left(\frac{1}{2}-\frac{1}{p}\right) \frac{R}{|x|}\left(|x|^{-(N-\gamma)} *|v|^{p}\right)|v|^{p} d x
$$

and in $|x|>R$, it gives

$$
-\int_{|x|>R} \frac{4(N-1) R}{|x|}\left(\frac{1}{2}-\frac{1}{p}\right)\left(|x|^{-(N-\gamma)} *|v|^{p}\right)|v|^{p} d x .
$$

We are left with the term in (4.51), which we write as

$$
\begin{gathered}
\frac{2(N-\gamma) c}{p} \iint_{\Omega}\left[\left(1-\frac{1}{2} \frac{R}{|x|}\right) x-\left(1-\frac{1}{2} \frac{R}{|y|}\right) y\right] \frac{(x-y)|v(x)|^{p}|v(y)|^{p}}{|x-y|^{N-\gamma+2}} d x d y \\
-\frac{4(N-\gamma)}{p} \int_{|x|>\frac{R}{2}}\left(|x|^{-(N-\gamma)} *|v|^{p}\right)|v|^{p} d x
\end{gathered}
$$

Here,

$$
\Omega=\left\{(x, y) \in \mathbb{R}^{N} \times \mathbb{R}^{N}:|x|>R / 2\right\} \cup\left\{(x, y) \in \mathbb{R}^{N} \times \mathbb{R}^{N}:|y|>R / 2\right\} .
$$

We define $\chi_{R}:=\chi\left(\frac{x}{R}\right)$ for $R>0$. Then we can write the first term in (4.52) as

$$
\int_{|x| \leq \frac{R}{2}} \chi_{R}^{2}|\nabla v|^{2}=\int_{|x| \leq \frac{R}{2}}\left|\nabla\left(\chi_{R} v\right)\right|^{2}+\int_{|x| \leq \frac{R}{2}} \chi_{R} \Delta\left(\chi_{R}\right)|v|^{2}
$$

and, thus, (4.52) can be written as

$$
\begin{aligned}
& 8 \int_{|x| \leq \frac{R}{2}}\left|\nabla\left(\chi_{R} v\right)\right|^{2}+8 \int_{|x| \leq \frac{R}{2}} \chi_{R} \Delta\left(\chi_{R}\right)|v|^{2} \\
& -\frac{4(N(p-1)-\gamma)}{p} \int_{|x| \leq \frac{R}{2}}\left(|x|^{-(N-\gamma)} *\left|\chi_{R} v\right|^{p}\right)\left|\chi_{R} v\right|^{p} d x .
\end{aligned}
$$


Adding (4.55) and (4.56), we estimate

$$
(4.55)+(4.56) \geq-\frac{4(N-1)(p-2)}{p} \int_{|x|>\frac{R}{2}}\left(|x|^{-(N-\gamma)} *|v|^{p}\right)|v|^{p} d x .
$$

Now combining (4.58) with (4.62) and putting together this with (4.60), (4.61) and (4.57), we obtain the following estimate

$$
\begin{aligned}
& \frac{d}{d t} \mathcal{M}_{g H}(t) \\
& \geq 8 \int_{|x| \leq \frac{R}{2}}\left|\chi_{R} \nabla v\right|^{2}-\frac{4(N(p-1)-\gamma)}{p} \int_{|x| \leq \frac{R}{2}}\left(|x|^{-(N-\gamma)} *\left|\chi_{R} v\right|^{p}\right)\left|\chi_{R} v\right|^{p} \\
& \quad+c_{1} \int_{\Omega}\left[\left(1-\frac{1}{2} \frac{R}{|x|}\right) x-\left(1-\frac{1}{2} \frac{R}{|y|}\right) y\right] \frac{(x-y)|v(x)|^{p}|v(y)|^{p}}{|x-y|^{N-\gamma+2}} d x d y \\
& \quad-c_{2} \int_{|x|>\frac{R}{2}}\left(|x|^{-(N-\gamma)} *|v|^{p}\right)|v|^{p}-\frac{1}{R^{2}} M[v],
\end{aligned}
$$

where $c_{1}, c_{2}>0$ are some constants. Using Lemma 4.3.4, we obtain

$$
\begin{aligned}
\frac{d}{d t} \mathcal{M}_{g H}(t) & \geq 8 \delta\left\|\chi_{R} v\right\|_{L^{\frac{2 N p}{N+\gamma}}}^{2}-c_{2} \int_{|x|>\frac{R}{2}}\left(|x|^{-(N-\gamma)} *|v|^{p}\right)|v|^{p}-\frac{1}{R^{2}} M[v] \\
+ & c_{1} \iint_{\Omega}\left[\left(1-\frac{1}{2} \frac{R}{|x|}\right) x-\left(1-\frac{1}{2} \frac{R}{|y|}\right) y\right] \frac{(x-y)|v(x)|^{p}|v(y)|^{p}}{|x-y|^{N-\gamma+2}} d x d y .
\end{aligned}
$$

Now we estimate the term

$$
\iint_{\Omega}\left(\left(1-\frac{1}{2} \frac{R}{|x|}\right) x-\left(1-\frac{1}{2} \frac{R}{|x|}\right) y\right) \frac{(x-y)|v(x)|^{p}|v(y)|^{p}}{|x-y|^{N-\gamma+2}} d x d y
$$

The key to estimate the above integral is the radial Sobolev inequaity (Lemma 2.3.3). We divide the integral in (4.63) into two regions

- Region I: In this region, we consider

$$
|x|>\frac{R}{2},|y|>\frac{R}{2}
$$

and observe that

$$
\left|\left(1-\frac{1}{2} \frac{R}{|x|}\right) x-\left(1-\frac{1}{2} \frac{R}{|y|}\right) y\right| \lesssim|x-y|
$$


then

$$
\begin{aligned}
\int_{\substack{|x|>R / 2 \\
|y|>R / 2}}\left(|x|^{-(N-\gamma)} *|v|^{p}\right)|v|^{p} d x & \lesssim\left\||x|^{-(N-\gamma)} *|v|^{p}\right\|_{L^{\frac{2 N}{N-\gamma}}}\|v\|_{L^{\frac{2 N p}{N+\gamma}}}^{p} \\
& \lesssim\|v\|_{L^{2 p}}^{2 p\left(\frac{2 N p}{N+\gamma}\right.}(\text { Lemma 2.3.1) } \\
& \lesssim \frac{1}{R^{\frac{(N-1)(N(p-1)-\gamma)}{N}}\|v\|_{L^{2}}^{\frac{N(p+1)+\gamma}{N}}\|\nabla v\|_{L^{2}}^{\frac{N(p-1)-\gamma}{N}}}
\end{aligned}
$$

$\lesssim \epsilon \quad$ (for $R$ large enough)

where in the first step we have used the Hölder's inequality and the last inequality follows from Lemma 2.3.3 (radial Sobolev inequality).

- Region II: We consider two cases:

- Case (a): $|x| \ll|y| \approx|x-y|, \quad|y|>\frac{R}{2}$ and $|x|<\frac{R}{2}$. In this case (4.63) becomes

$$
\iint \frac{1}{|x-y|^{N-\gamma}} \chi_{|y|>\frac{R}{2}}|v(y)|^{p}|v(x)|^{p} d x d y
$$

since

$$
\begin{aligned}
\mid x\left(1-\frac{R}{2} \frac{1}{|x|}\right) & -y\left(1-\frac{R}{2} \frac{1}{|y|}\right) \mid \\
& \leq|x|\left(1-\frac{R}{2} \frac{1}{|x|}\right)+|y|\left(1-\frac{R}{2} \frac{1}{|y|}\right) \\
& \lesssim|y| \approx|x-y| \quad\left(1-\frac{R}{2|y|}>1 \text { and } 1-\frac{R}{2|x|}<1\right) .
\end{aligned}
$$

Again, using Hölder's inequality, radial Sobolev inequality (Lemma 2.3.3), and Hardy-Littlewood-Sobolev inequality (Lemma 2.3.1) as in the estimate for (4.64), we bound (4.63) by

$$
\left.\frac{1}{R^{\frac{(N-1)(N(p-1)-\gamma)}{N}}}\|v\|_{L^{2}}^{\frac{N(p+1)+\gamma}{N}}\|\nabla v\|_{L^{2}}^{\frac{N(p-1)-\gamma}{N}} \lesssim \epsilon \quad \text { (for } R \text { large }\right) .
$$


- Case (b): $|y| \ll|x| \approx|x-y|, \quad|x|>\frac{R}{2}$ and $|y|<\frac{R}{2}$. This case is symmetric and treated with a similar argument as in Case (a).

Therefore, the contribution of (4.63) can be made small enough for large radius. Thus, we obtain

$$
\frac{d}{d t} \mathcal{M}_{g H}(t) \geq 8 \delta\left\|\chi_{R} v\right\|_{L^{\frac{2 N p}{N+\gamma}}}^{2}-\int_{|x|>\frac{R}{2}}\left(|x|^{-(N-\gamma)} *|v|^{p}\right)|v|^{p} d x-\frac{1}{R^{2}} M[v] .
$$

We rearrange the aboves inequality to write

$$
8 \delta\left\|\chi_{R} v\right\|_{L^{\frac{2 N p}{N+\gamma}}}^{2} \lesssim \frac{d}{d t} \mathcal{M}_{g H}(t)+\int_{|x|>\frac{R}{2}}\left(|x|^{-(N-\gamma)} *|v|^{p}\right)|v|^{p}+\frac{1}{R^{2}} M[v] .
$$

Applying the fundamental theorem of calculus to (4.65) on $[0, T]$, we obtain

$$
\begin{aligned}
8 \delta \int_{0}^{T} & \left(\int\left|\chi_{R} v(x, t)\right|^{\frac{2 N p}{N+\gamma}}\right)^{\frac{N+\gamma}{N p}} \\
& \lesssim \sup _{t \in[0, T]}\left|\mathcal{M}_{g H}(t)\right|+\int_{0}^{T} \int_{|x|>\frac{R}{2}}\left(|x|^{-(N-\gamma)} *|v|^{p}\right)|v|^{p}+\frac{T}{R^{2}} M[v]
\end{aligned}
$$

Applying the radial Sobolev inequality (Lemma 2.3.3) along with Hölder's and HardyLittlewood-Sobolev inequality (Lemma 2.3.1), we have

$$
\int_{|x|>\frac{R}{2}}\left(|x|^{-(N-\gamma)} *|v|^{p}\right)|v|^{p} d x \lesssim \frac{1}{R^{\frac{(N-1)(N(p-1)-\gamma)}{N}}} .
$$

Therefore, we obtain

$$
\frac{1}{T} \int_{0}^{T}\left(\int_{|x| \leq R}|v(x, t)|^{\frac{2 N p}{N+\gamma}} d x\right)^{\frac{N+\gamma}{N p}} d t \lesssim \frac{R}{T}+\frac{1}{R^{\frac{(N-1)(N(p-1)-\gamma)}{N}}}+\frac{1}{R^{2}} .
$$

Observe that

$$
\begin{cases}\frac{(N-1)(N(p-1)-\gamma)}{N}<2, & \text { for } s_{c}<\frac{1}{2} \\ \frac{(N-1)(N(p-1)-\gamma)}{N} \geq 2, & \text { for } s_{c} \geq \frac{1}{2}\end{cases}
$$

Thus,

$$
\frac{1}{T} \int_{0}^{T}\left(\int_{|x| \leq R}|v(x, t)|^{\frac{2 N p}{N+\gamma}} d x\right)^{\frac{N+\gamma}{N p}} d t \lesssim v, \delta \begin{cases}\frac{R}{T}+\frac{1}{R^{\frac{(N-1)(N(p-1)-\gamma)}{N}},} & \text { if } s_{c}<\frac{1}{2} \\ \frac{R}{T}+\frac{1}{R^{2}}, & \text { if } s_{c} \geq \frac{1}{2}\end{cases}
$$

as desired. 
Now we prove that the energy escapes to spatial infinity as $t \rightarrow \infty$.

Proposition 4.5.3 (Energy evacuation, gH) There exists a sequence of times $t_{n} \rightarrow \infty$ and a sequence of radii $R_{n} \rightarrow \infty$ such that

$$
\lim _{n \rightarrow \infty}\left(\int_{|x| \leq R_{n}}\left|v\left(x, t_{n}\right)\right|^{\frac{2 N p}{N+\gamma}} d x\right)^{\frac{N+\gamma}{N p}}=0 .
$$

Proof. For large $T>0$ and $R>R_{0}$ with $R=T^{\frac{N}{N+(N-1)(N(p-1)-\gamma)}}$ for $s_{c}<\frac{1}{2}$ and $R=T^{1 / 3}$ for $s_{c} \geq \frac{1}{2}$ and Proposition 4.5.2, we obtain that for $s_{c}<\frac{1}{2}$

$$
\frac{1}{T} \int_{0}^{T}\left(\int_{|x| \leq t^{\frac{N+(N-1)(N(p-1)-\gamma)}{N}}}|v(x, t)|^{\frac{2 N p}{N+\gamma}} d x\right)^{\frac{N+\gamma}{N p}} d t \lesssim T^{-\frac{(N-1)(N(p-1)-\gamma)}{N+(N-1)(N(p-1)-\gamma)}}
$$

and for $s_{c} \geq \frac{1}{2}$

$$
\frac{1}{T} \int_{0}^{T}\left(\int_{|x| \leq t^{1 / 3}}|v(x, t)|^{\frac{2 N p}{N+\gamma}} d x\right)^{\frac{N+\gamma}{N p}} d t \lesssim T^{-2 / 3}
$$

This implies that (following the similar argument as in Proposition 4.4.3) there exist sequences $t_{n} \rightarrow \infty$ and $R_{n} \rightarrow \infty$ such that the energy evacuation happens on a sequence of balls as desired.

Proof. (of Theorem 4.1.5) We have (by Section 4.3.2) that $v$ is global and uniformly bounded in $H^{1}$. Choose $\varepsilon$ and $R$ as in Lemma 4.2.2 with $t_{n} \rightarrow \infty$ and $R_{n} \rightarrow \infty$ as in Proposition 4.5.3. Now taking $n$ large so that $R_{n} \geq R$, Hölder's inequality gives

$$
\int_{|x| \leq R}\left|v\left(x, t_{n}\right)\right|^{2} d x \lesssim R^{\frac{2 s(p-1)+2}{p}}\left(\int_{|x| \leq R_{n}}\left|v\left(x, t_{n}\right)\right|^{\frac{2 N p}{N+\gamma}} d x\right)^{\frac{N+\gamma}{N p}} \rightarrow 0 \quad \text { as } \quad n \rightarrow \infty
$$

Therefore, Lemma 4.2.2 implies that $v$ scatters in $H^{1}\left(\mathbb{R}^{N}\right)$ forward in time. 


\title{
CHAPTER 5
}

\section{SINGULARITY FORMATION IN THE GHARTREE EQUATION}

\author{
If something cannot go on forever, it will stop. (Herbert Stein)
}

In this chapter we investigate the formation of singularity, which is often referred to as collapse or blow-up. It corresponds to a violent or sudden energy transfer from the larger scales to smaller ones. In particular, we are interested in understanding the solutions with finite-time existence for a variety of cases $\left(s_{c} \geq 0\right)$ in the gHartree equation (1.1). As the small data global existence is available, one may ask if the global existence can be extended for large solutions, or if there is a threshold for global existence. In Chapter 3 we showed a dichotomy for scattering vs. finite time blow-up solutions provided the initial data is in $H^{1}$; the threshold was given by a combination of the mass-energy and the gradient comparison to that of the ground state (see Chapter 3, Section 3.3, Theorem 3.3.1). For the $\dot{H}^{s_{c}}$ data, it is a more difficult question as the conserved quantities at the $\dot{H}^{s_{c}}$ level are not available (unless $s_{c}=0$ or $s_{c}=1$, note that if initial data is in $\dot{H}^{s_{c}} \cap \dot{H}^{1}$, then it stays in that regularity as the consequence of conservation laws). Nevertheless, one can still ask for a finite time blow-up criterion for any size data, which we investigate in Section 5.1 (see also [AR19]). We show that large data (although, it does not have to be "large" in the intercritical case) may blow-up in finite time. For that we give a sufficient condition for blow-up and show examples of Gaussian data with thresholds in various (energy-subcritical, critical and supercritical) cases. Such examples are important for studying the actual dynamics of finite time blow-up. For example, in [YRZ20] the dynamics of stable blow-up is investigated (including rates and profiles) for the gHartree in the mass-critical and supercritical regimes, and is compared with known blow-up dynamics of the (local) nonlinear Schrödinger equation. 
First, we give a sufficient condition for a finite time blow-up (blow-up criterion, see Theorem 5.1.1) in the gHartree equation (1.1), which follows the ideas in [HPR10, DR15, Lus02, Lus10] except that now we have to control the convolution term.

Then we use examples of Gaussian initial data to show known thresholds for global vs. finite existence and scattering in the following cases: energy-subcritical (see Figure 5.1), energy-critical (see Figure 5.2) and energy-supercritical case (Figure 5.3).

More importantly, we investigate blow-up solutions for the $L^{2}$-critical gHartree equation (1.1) with the negative energy and initial mass slightly above the ground state mass (see Theorem 5.3.2).

\subsection{Blow-up criterion}

We first recall a simple convexity argument, which demonstrates that initial datum with negative energy blows up in finite time. Define the variance, $V(t) \stackrel{\text { def }}{=}\|x u(t)\|_{L^{2}\left(\mathbb{R}^{N}\right)}^{2}$. Then, the solutions $u(t)$ of (1.1) with finite variance satisfy the following virial identities

$$
\begin{gathered}
V_{t}(t)=4 \operatorname{Im} \int_{\mathbb{R}^{N}} \bar{u} x \cdot \nabla u d x, \\
V_{t t}(t)=16 E[u]-\frac{8 s_{c}(p-1)}{p} Z(u) \equiv 16\left(s_{c}(p-1)+1\right) E[u]-8 s_{c}(p-1)\|\nabla u\|_{L^{2}}^{2} .
\end{gathered}
$$

Now for $s_{c} \geq 0$, we have

$$
V_{t t}(t)<C_{s_{c}} E[u]
$$

where $C_{s_{c}}$ is a positive constant. Integrating with respect to time, we have

$$
V(t)<\frac{C_{s_{c}}}{2} E[u] t^{2}+V_{t}(0) t+V(0) .
$$

Now, there exist three possibilities under which blow-up occurs and they are as follows:

1. $E[u]=-C^{2}<0$. This implies that $V_{t t}<0$ and $V(t)$ is concave down. Then, the blow-up occurs at some time $0<T_{*} \leq t_{0}$, where $t_{0}=\frac{V_{t}(0) \pm \sqrt{\left(V_{t}(0)\right)^{2}+2 C_{s_{c}} C^{2} V(0)}}{C_{s_{c}} C^{2}}$. 
2. $E[u]=0$ and $V_{t}(0)<0$. This implies that $V(t)$ is always decreasing and the blow-up occurs for some $0<T_{*} \leq t_{0}$, where $t_{0}=\frac{V(0)}{V_{t}(0)}$.

3. $E[u]=C^{2}>0$ and $V_{t}(0) \leq-2 \sqrt{C\left(s_{c}\right) E[u] V(0)}$. Then, the blow-up occurs for some $0<T_{*} \leq t_{0}$, where $t_{0}=\frac{-V_{t}(0) \pm \sqrt{\left(V_{t}(0)\right)^{2}-2 C_{s_{c}} E[u] V(0)}}{C s_{c} E[u]}$.

For positive energy we also have the following sufficient condition for blow-up in the generalized Hartree equation (1.1), which follows the ideas in [HPR10, DR15, Lus02, Lus10] except that now we find a bound for the convolution term

$$
Z(u)=\int_{\mathbb{R}^{N}}\left(\frac{1}{|x|^{N-\gamma}} *|u|^{p}\right)|u|^{p} d x
$$

Theorem 5.1.1 Let $u_{0} \in H^{1}$ if $s_{c} \leq 1$ and $u_{0} \in H^{s_{c}}$ if $s_{c}>1$. Assume also $V(0)<\infty$ and $E[u]>0$. The following is a sufficient condition for the blow-up in finite time for the solutions to the gHartree equation (1.1) with initial data $u_{0}$ in the mass-supercritical case $\left(s_{c}>0\right)$ :

$$
\frac{\partial_{t} V(0)}{\omega M\left[u_{0}\right]}<4 \sqrt{2} f\left(\frac{E\left[u_{0}\right] V(0)}{\left(\omega M\left[u_{0}\right]\right)^{2}}\right)
$$

where $\omega^{2}=\frac{N^{2}(N(p-2)+b-2)}{8(N(p-2)+b)}$ and the function $f$ is defined as (here, $\left.k=s_{c}(p-1)\right)$

$$
f(x)= \begin{cases}\sqrt{\frac{1}{k x^{k}}+x-\frac{1+k}{k}} & \text { if } 0<x<1 \\ -\sqrt{\frac{1}{k x^{k}}+x-\frac{1+k}{k}} & \text { if } x \geq 1\end{cases}
$$

Proof. Recalling the decomposition (4.1) from [DR15]

$$
\frac{N^{2}}{4}\|u\|_{L^{2}}^{4}+\left|\frac{V_{t}(t)}{4}\right|^{2} \leq V(t)\|\nabla u\|_{L^{2}}^{2}
$$

we obtain

$$
V_{t t}(t) \leq 16\left(s_{c}(p-1)+1\right) E[u]-2 s_{c}(p-1) \frac{N^{2}(M[u])^{2}}{V(t)}-\frac{s_{c}(p-1)}{2} \frac{\left|V_{t}(t)\right|^{2}}{V(t)}
$$


We rewrite the above by making a substitution $V(t)=B^{\frac{1}{\alpha+1}}(t)$, where $\alpha=\frac{s_{c}(p-1)}{2}=$ $\frac{N(p-2)+b-2}{4}>0$, to remove the last term and re-write (5.3) as

$$
B_{t t} \leq 16(\alpha+1)(2 \alpha+1) E[u] B^{\frac{\alpha}{\alpha+1}}-4 \alpha(\alpha+1) N^{2}(M[u])^{2} B^{\frac{\alpha-1}{\alpha+1}}
$$

Set $\gamma=4 \sqrt{2} \frac{E[u]}{\omega M[u]}$, where $\omega^{2}=\frac{N^{2} \alpha}{4(2 \alpha+1)}=\frac{N^{2}(N(p-2)+b-2)}{8(N(p-2)+b)}$ and introduce the rescaled variables $v$ and the time $s$ as follows: $s=\gamma t$ and

$$
B(t)=B_{\max } v(s), \quad \text { where } \quad B_{\max }=\left(\frac{(\omega M[u])^{2}}{E[u]}\right)^{\alpha+1} .
$$

Then, with these new variables, we obtain

$$
\frac{2}{(\alpha+1)(2 \alpha+1)} v_{s s} \leq v^{\frac{\alpha}{\alpha+1}}-v^{\frac{\alpha-1}{\alpha+1}}, \quad s \in\left[0, T^{*} / \gamma\right)
$$

and the equation (5.4) can be written as

$$
v_{s s} \leq-c \frac{\partial \widetilde{U}}{\partial v}
$$

where $c=\frac{(\alpha+1)(2 \alpha+1)}{2}$ and the potential

$$
\widetilde{U}(v)=\frac{\alpha+1}{2 \alpha} v^{\frac{2 \alpha}{\alpha+1}}-\frac{\alpha+1}{2 \alpha+1} v^{\frac{2 \alpha+1}{\alpha+1}} .
$$

In fact, for some function $g^{2}(s)>0$, we have

$$
v_{s s}=-c \frac{\partial \widetilde{U}}{\partial v}-g^{2}(s)
$$

and using the same analogy from mechanics as in [Lus10], [HPR10], [DR15], let $v(t)$ be the coordinate of the particle with the unit mass moving under two forces: $F_{1}=-c \frac{\partial \widetilde{U}}{\partial v}$ and an unknown external force $F_{2}=-g^{2}(t)<0$ (because of the sign, it pulls the particle towards the origin). The collapse occurs if the particle reaches the origin in finite time, i.e., when $v\left(t^{*}\right)=0$ for some $0<t^{*}<\infty$. If it reaches the origin without the force $F_{2}=-g^{2}(t)$, then it would also reach the origin when this force is applied, thus, leading to the following equation

$$
\frac{1}{c} v_{s s}+\frac{\partial \widetilde{U}}{\partial v}=0
$$


The energy of this particle, defined as

$$
\mathcal{E}(s)=\frac{1}{2 c} v_{s}^{2}+\widetilde{U}(v(s))
$$

is conserved. Note that the curve for $\widetilde{U}$ is increasing from the origin (for positive $v$ ) and then decreasing with the local maximum $\widetilde{U}_{\max }=\frac{\alpha+1}{2 \alpha(2 \alpha+1)}$ attained at $v=1$. Using the energy from (5.6), we obtain the blow-up conditions for (5.5) similar to Proposition 4.1 in [DR15], see also [HPR10]:

(I) $\mathcal{E}(0)<\widetilde{U}_{\max }$ and $v(0)<1$,

(II) $\mathcal{E}(0)>\widetilde{U}_{\max }$ and $v_{s}(0)<0$,

(III) $\mathcal{E}(0)=\widetilde{U}_{\max }, v_{s}(0)<0$ and $v(0)<1$.

Define $v=\widetilde{V}^{\alpha+1}$ and rewrite the energy as

$$
\mathcal{E}=\frac{\alpha+1}{2 \alpha+1} \widetilde{V}^{2 \alpha}\left(\widetilde{V}_{s}^{2}-\widetilde{V}+\frac{2 \alpha+1}{2 \alpha}\right) .
$$

Observe that

$$
\mathcal{E}<\widetilde{U}_{\max } \Longleftrightarrow \widetilde{V}_{s}^{2}<\frac{1}{2 \alpha \widetilde{V}^{2 \alpha}}+\widetilde{V}-\frac{2 \alpha+1}{2 \alpha} .
$$

Let $k=2 \alpha=s_{c}(p-1)$ and set the function

$$
f(x)=\sqrt{\frac{1}{k x^{k}}+x-\frac{1+k}{k}},
$$

then the blow-up conditions (I)-(III) with (5.7) and (5.8) are given as

$$
\widetilde{V}_{s}(0)<\left\{\begin{array}{ll}
+f(\widetilde{V}(0)) & \text { if } \widetilde{V}(0)<1 \\
-f(\widetilde{V}(0)) & \text { if } \widetilde{V}(0) \geq 1
\end{array} .\right.
$$

Substituting for $v, B_{\max }$ in $V(t)=\left(B_{\max } v\right)^{\frac{1}{\alpha+1}}$ yields

$$
V(t)=\frac{(\omega M[u])^{2}}{E[u]} \widetilde{V}\left(4 \sqrt{2} \frac{E[u]}{\omega M[u]} t\right)
$$


and therefore, we obtain

$$
\frac{V_{t}(0)}{\omega M[u]}<4 \sqrt{2} f\left(\frac{E[u] V(0)}{(\omega M[u])^{2}}\right)
$$

as claimed.

Remark. For the real-valued initial data, the expression (5.9) can be simplified to

$$
V(0)<\frac{(\omega M[u])^{2}}{E[u]}
$$

Thus, knowing how big the initial variance of the real-valued data is, yields a blow-up solution from such data. We use this in examples in the next section.

\subsection{Examples}

In this section we show examples of known thresholds in the energy-subcritical, critical and supercritical cases for the Gaussian initial data.

We note that the proof of global existence in Theorem 3.1.1 part 1 and blow-up in part 2(a) will work for $s_{c}=0$ and $s_{c}=1$. In the case $s_{c}=1$ (energy-critical gHartree), or equivalently $p=\frac{N+\gamma}{N-2}$ for $N \geq 3$, the inequality (2.126) becomes

$$
Z(u) \leq C_{G N}\|\nabla u\|_{L^{2}\left(\mathbb{R}^{N}\right)}^{\frac{2(N+\gamma)}{N-2}},
$$

and the sharp constant $C_{G N}$ for (5.11) is given by (for instance, see [DY19])

$C_{G N}=\pi^{(N-\gamma) / 2}\left(\frac{1}{N(N-2) \pi}\left(\frac{\Gamma(N)}{\Gamma(N / 2)}\right)^{\frac{\gamma+2}{N+\gamma}}\right)^{\frac{N+\gamma}{N-2}} \frac{\Gamma\left(\frac{\gamma}{2}\right)}{\Gamma\left(\frac{N+\gamma}{2}\right)}=\left(C_{N}\right)^{\frac{2(N+\gamma)}{N-2}} C(N, \gamma)$,

where $C_{N}=\frac{1}{\sqrt{N(N-2) \pi}}\left(\frac{\Gamma(N)}{\Gamma(N / 2)}\right)^{1 / N}$ is the best constant for Sobolev inequality

$$
\|u\|_{L^{\frac{2 N}{N-2}\left(\mathbb{R}^{N}\right)}} \leq C_{N}\|\nabla u\|_{L^{2}\left(\mathbb{R}^{N}\right)}
$$


and $C(N, \gamma)=\pi^{(N-\gamma) / 2} \frac{\Gamma\left(\frac{\gamma}{2}\right)}{\Gamma\left(\frac{N+\gamma}{2}\right)}\left(\frac{\Gamma(N)}{\Gamma(N / 2)}\right)^{\frac{\gamma}{N}}$ is the sharp constant in Hardy-LittlewoodSobolev inequality (see [LL01], [Lie83])

$$
\left|\int_{\mathbb{R}^{N}} \int_{\mathbb{R}^{N}} \frac{f(x) h(y)}{|x-y|^{N-\gamma}} d x d y\right| \leq C(N, \gamma)\|f\|_{L^{\frac{2 N}{N+\gamma}\left(\mathbb{R}^{N}\right)}}\|h\|_{L^{\frac{2 N}{N+\gamma}}\left(\mathbb{R}^{N}\right)} .
$$

In our examples, we use $\gamma=2$, in which case one can verify that

$$
Q(x)=\left(\frac{N(N-2)}{\pi^{N / 2}} \Gamma\left(1+\frac{N}{2}\right)\right)^{\frac{N-2}{8}} \frac{1}{\left(1+|x|^{2}\right)^{\frac{N-2}{2}}}
$$

is one of the solutions for

$$
\Delta Q+\left(|x|^{-(N-2)} *|Q|^{\frac{N+2}{N-2}}\right)|Q|^{\frac{6-N}{N-2}} Q=0
$$

where $(-\Delta)^{-1} f=I_{N-2} * f=\frac{\Gamma\left(\frac{N}{2}-1\right)}{4 \pi^{N / 2}} \frac{1}{|x|^{N-2}} * f$. In other words, the sharp constant $C_{G N}$ from (5.12) can be attained at $Q$, i.e., for $\gamma=2$, we have an equality in (5.11)

$$
Z(Q)=C_{G N}\|\nabla Q\|_{L^{2}\left(\mathbb{R}^{N}\right)}^{\frac{2(N+2)}{N-2}}
$$

Furthermore, for the function $Q$ in (5.13), multiplying the equation (5.14) by $Q$ and performing integration by parts, we have $\|\nabla Q\|_{L^{2}\left(\mathbb{R}^{N}\right)}^{2}=Z(Q)$. Thus, using this along with (5.15) we deduce that

$$
\|\nabla Q\|_{L^{2}\left(\mathbb{R}^{N}\right)}^{2}=\frac{1}{\left(C_{G N}\right)^{(N-2) / 4}}
$$

and

$$
E[Q]=\frac{2}{N+2}\|\nabla Q\|_{L^{2}\left(\mathbb{R}^{N}\right)}^{2}=\frac{2}{N+2} \frac{1}{\left(C_{G N}\right)^{(N-2) / 4}} .
$$

We next modify the definition of $\mathcal{M E}$ and $\mathcal{G}$ in (2.8.3) and write

$$
\mathcal{E}[u]=\frac{E[u]}{E[Q]} \quad \text { and } \quad \mathcal{G}[u(t)]=\frac{\|\nabla u(t)\|_{L^{2}\left(\mathbb{R}^{N}\right)}}{\|\nabla Q\|_{L^{2}\left(\mathbb{R}^{N}\right)}}
$$

where the value of $\|\nabla Q\|_{L^{2}\left(\mathbb{R}^{N}\right)}$ is determined from (5.16) via the sharp constant defined in (5.12). Now, as a consequence of the proof of Theorem 3.1.1 in Chapter 3, global existence holds in $s_{c}=1$ case along with the blow-up in finite time for finite variance. We state the following analogous result for the energy-critical case. 
Theorem 5.2.1 Let $s_{c}=1$ and $u(t)$ be the solution of $(1.1)$ with $u_{0} \in \dot{H}^{1}\left(\mathbb{R}^{N}\right)$. Assume that $\mathcal{E}\left[u_{0}\right]<1$.

1. If $\mathcal{G}\left[u_{0}\right]<1$, then the solutions exists globally in time for all $t \in \mathbb{R}$.

2. If $\mathcal{G}\left[u_{0}\right]>1$ and either $u_{0}$ is radial or $|x| u_{0} \in L^{2}\left(\mathbb{R}^{N}\right)$, then $u(t)$ blows-up in finite time.

\subsubsection{Gaussian initial data}

We are now ready to consider examples, for which we take the Gaussian initial data of the form

$$
u_{g}(x, 0)=\beta e^{-\frac{1}{2} \gamma|x|^{2}}, \quad x \in \mathbb{R}^{N}, \beta, \gamma \in(0, \infty)
$$

Then, the mass and initial variance of Gaussian data (5.18) are

$$
M\left[u_{g}\right]=\beta^{2}\left(\frac{\pi}{\gamma}\right)^{N / 2}, \quad V(0)=\frac{\beta^{2} N \pi^{N / 2}}{2 \gamma^{\frac{N}{2}+1}} .
$$

For the convenience of the energy calculation we also record

$$
\left\|\nabla u_{g}\right\|_{L^{2}\left(\mathbb{R}^{N}\right)}^{2}=\frac{N \pi^{N / 2}}{2} \frac{\beta^{2}}{\gamma^{(N-2) / 2}} .
$$

In what follows we consider mostly examples in $3 \mathrm{~d}$, with the convolution term $\frac{1}{|x|^{N-2}} * f$ as it is the fundamental solution of the Laplacian.

\section{Example 1 : Energy-subcritical case}

Consider $p=3$ in dimension $N=3$. In this case $s_{c}=\frac{1}{2}$, then (1.1) takes the form

$$
i u_{t}+\Delta u+\left(|x|^{-1} *|u|^{3}\right)|u| u=0
$$

The energy for (5.19) is

$$
E\left[u_{g}\right]=\frac{\pi^{3 / 2}}{4} \frac{\beta^{2}}{\gamma^{1 / 2}}\left(3-\frac{16 \pi}{3^{7 / 2}} \frac{\beta^{4}}{\gamma^{2}}\right) .
$$


The Pohozhaev identities are

$$
\|\nabla Q\|_{L^{2}\left(\mathbb{R}^{3}\right)}^{2}=2\|Q\|_{L^{2}\left(\mathbb{R}^{3}\right)}^{2} \quad \text { and } \quad Z(Q)=3\|Q\|_{L^{2}\left(\mathbb{R}^{3}\right)}^{2}
$$

which yields $E[Q]=\frac{1}{2} M[Q]$, where we computed (numerically) $M[Q] \approx 5.2339$ (for example, see [YRZ20]).

We obtain the following thresholds, which are schematically represented in Figure 5.1:

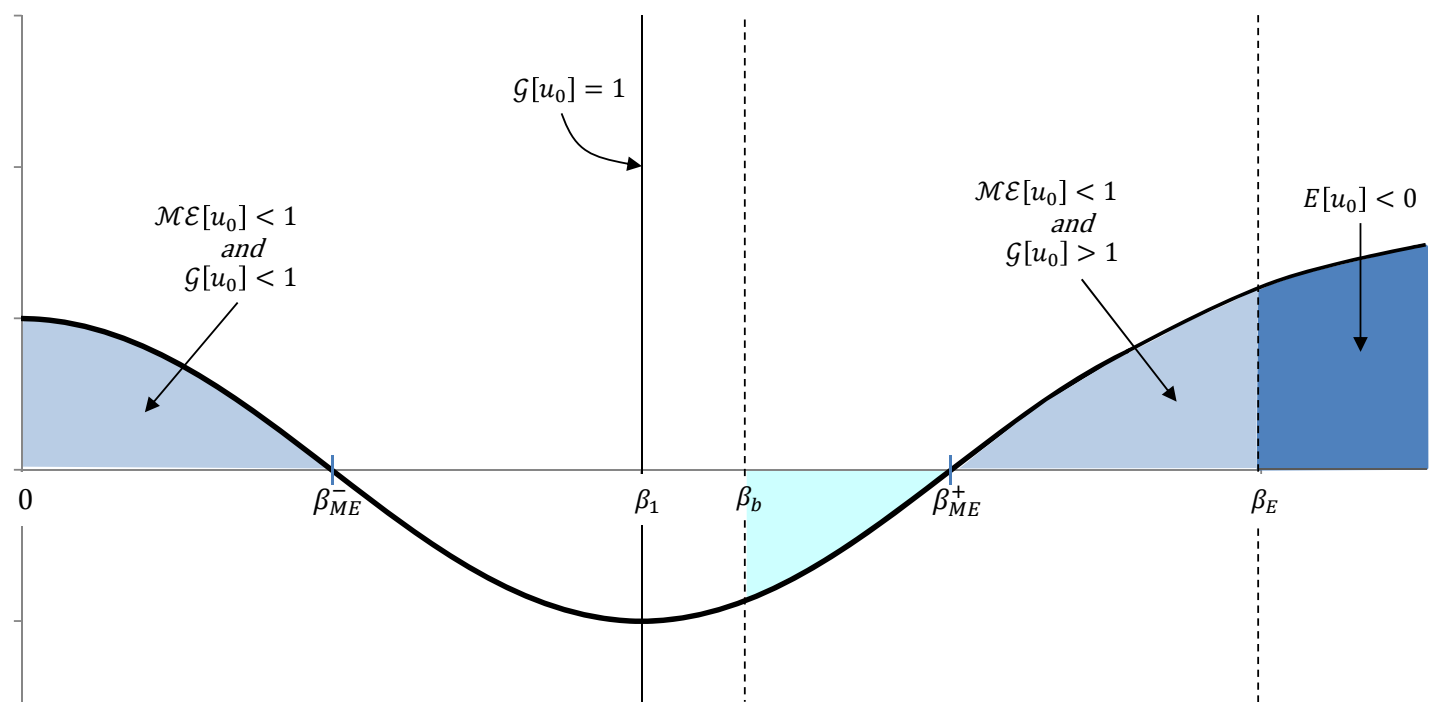

Figure 5.1: Thresholds for the Gaussian data $u_{0}(x)=\beta e^{-|x|^{2}}$ in the energy-subcritical case, see (5.20)-(5.23).

- blow-up with negative energy: $E\left[u_{g}\right]<0$ if

$$
\frac{\beta}{\sqrt{\gamma}}>\beta_{E} \equiv \frac{3^{9 / 8}}{2 \pi^{1 / 4}} \approx 1.29
$$

- blow-up criteria Theorem 5.1.1 for positive energy: condition (5.10) gives

$$
\frac{\beta}{\sqrt{\gamma}}>\beta_{b} \equiv \frac{3^{9 / 8}}{2^{5 / 4} \pi^{1 / 4}} \approx 1.08689
$$


- the mass-energy condition $\mathcal{M E}\left[u_{g}\right]<1$ in Theorem 3.1.1 yields

$$
\frac{\pi^{3} \beta^{4}}{4 \gamma^{2}}\left(3-\frac{16}{3^{7 / 2}} \frac{\beta^{4}}{\gamma^{2}}\right)<\frac{1}{2}\|Q\|_{L^{2}\left(\mathbb{R}^{3}\right)}^{4},
$$

which implies

$$
\frac{\beta}{\sqrt{\gamma}}<\beta_{M E}^{-} \approx 0.9586 \text { and } \frac{\beta}{\sqrt{\gamma}}>\beta_{M E}^{+} \approx 1.1812 .
$$

- the mass-gradient condition $\mathcal{G}\left[u_{g}\right]=1$ from Theorem 3.1.1 (useful for the separation of the mass-energy conditions above in (5.22)) gives

$$
\frac{\beta}{\sqrt{\gamma}}<\beta_{1} \equiv \frac{2^{1 / 2}}{3^{1 / 4} \pi^{3 / 4}}\|Q\|_{L^{2}\left(\mathbb{R}^{3}\right)} \approx 1.0418 .
$$

From (5.20), (5.21), (5.22) and (5.23) we conclude that (analytically proved) thresholds are: for scattering is below $\beta_{M E}^{-} \approx 0.9586$ and for blow-up is above $\beta_{b} \approx 1.08689$ (see Figure 1).

\section{Example 2 : Energy-critical case}

Consider $p=5$ in the dimension $N=3$ and write the equation

$$
i u_{t}+\Delta u+\left(|x|^{-1} *|u|^{5}\right)|u|^{3} u=0,
$$

which is energy-critical. The corresponding energy for (5.24) is

$$
E\left[u_{g}\right]=\frac{\pi^{3 / 2}}{4} \frac{\beta^{2}}{\gamma^{1 / 2}}\left(3-\frac{16 \pi}{5^{7 / 2}} \frac{\beta^{8}}{\gamma^{2}}\right) .
$$

From (5.13) and (5.14) we have that

$$
Q=\left(\frac{9}{4 \pi}\right)^{\frac{1}{8}} \frac{1}{\sqrt{1+|x|^{2}}},
$$

which solves

$$
\Delta Q+\left(\frac{1}{|x|} * Q^{5}\right) Q^{4}=0
$$


where $\frac{1}{|x|} * f=4 \pi(-\Delta)^{-1} f$ in 3 d. From (5.16), (5.17) and (5.12), we obtain

$$
\|\nabla Q\|_{L^{2}\left(\mathbb{R}^{3}\right)}^{2}=\frac{3^{3 / 2} \pi^{7 / 4}}{2^{5 / 2}} \quad \text { and } \quad E[Q]=\frac{2}{5}\|\nabla Q\|_{L^{2}\left(\mathbb{R}^{3}\right)}^{2}=\frac{3^{3 / 2} \pi^{7 / 4}}{2^{3 / 2} 5}
$$

Then

- the negative energy condition, $E\left[u_{g}\right]<0$ yields

$$
\frac{\beta}{\gamma^{1 / 4}}>\beta_{E} \equiv \frac{5^{7 / 16} 3^{1 / 8}}{2^{1 / 2} \pi^{1 / 8}} \approx 1.42161
$$

- blow-up condition (5.10) (Theorem 5.1.1) gives

$$
\frac{\beta}{\gamma^{1 / 4}}>\beta_{b} \equiv \frac{5^{5 / 16} 3^{1 / 8}}{2^{1 / 2} \pi^{1 / 8}} \approx 1.16254
$$

- the energy condition $\mathcal{E}\left[u_{g}\right]<1$ in Theorem 5.2.1 yields

$$
\frac{\pi^{3 / 2}}{4} \frac{\beta^{2}}{\gamma^{1 / 2}}\left(3-\frac{16 \pi}{5^{7 / 2}} \frac{\beta^{8}}{\gamma^{2}}\right)<\frac{3^{3 / 2} \pi^{7 / 4}}{2^{3 / 2} 5}
$$

which implies

$$
\frac{\beta}{\gamma^{1 / 4}}<\beta_{E}^{-} \approx 0.812225 \text { and } \frac{\beta}{\gamma^{1 / 4}}>\beta_{E}^{+} \approx 1.34423
$$

and the gradient condition for global existence $\mathcal{G}\left[u_{g}\right]<1$ gives

$$
\frac{\beta}{\gamma^{1 / 4}}<\beta_{1} \approx 0.902925
$$

From (5.25), (5.26), (5.27) and (5.28) we conclude that (analytically proved) thresholds are: for global existence is below $\beta_{E}^{-} \approx 0.812225$ and for blow-up is above $\beta_{b} \approx 1.16254$ (see Figure 2).

\section{Example 3 :}

For convenience, we provide one more energy-critical example in $4 \mathrm{~d}$,

$$
i u_{t}+\Delta u+\left(|x|^{-2} *|u|^{3}\right)|u| u=0
$$




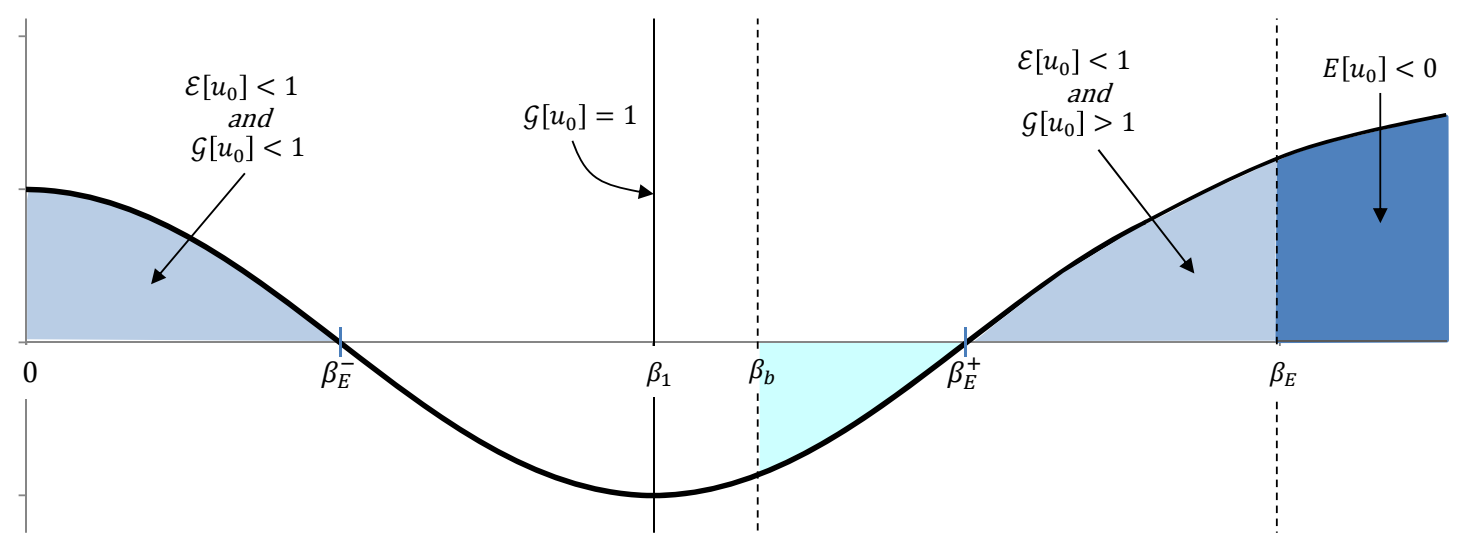

Figure 5.2: Thresholds for Gaussian data $u_{0}=\beta e^{-|x|^{2}}$ in the energy-critical case,see (5.25)-(5.28) and (5.30)-(5.33).

The energy for (5.29) is given by

$$
E\left[u_{g}\right]=\pi^{2} \frac{\beta^{2}}{\gamma}\left(1-\frac{\pi^{2}}{81} \frac{\beta^{4}}{\gamma^{2}}\right) .
$$

Again, from (5.13) and (5.14), we have that

$$
Q=\frac{2}{\sqrt{\pi}} \frac{1}{1+|x|^{2}},
$$

which solves

$$
\Delta Q+\left(\frac{1}{|x|^{2}} * Q^{3}\right) Q^{2}=0,
$$

where $\frac{1}{|x|^{2}} * f=4 \pi^{2}(-\Delta)^{-1} f$ in $4 \mathrm{~d}$. We compute from (5.16),(5.17) and (5.12)

$$
\|\nabla Q\|_{L^{2}\left(\mathbb{R}^{4}\right)}^{2}=\frac{16 \pi}{3} \quad \text { and } \quad E[Q]=\frac{1}{3}\|\nabla Q\|_{L^{2}\left(\mathbb{R}^{4}\right)}^{2}=\frac{16 \pi}{9} .
$$

Then

- the negative energy condition corresponds to

$$
\frac{\beta}{\sqrt{\gamma}}>\beta_{E} \approx 1.69257,
$$

- blow-up occurs (according to Theorem 5.1.1 condition (5.10)) when

$$
\frac{\beta}{\sqrt{\gamma}}>\beta_{b} \approx 1.28607
$$


- the energy condition $\mathcal{E}\left[u_{g}\right]<1$ in Theorem 5.2.1 gives

$$
\pi^{2} \frac{\beta^{2}}{\gamma}\left(1-\frac{\pi^{2}}{81} \frac{\beta^{4}}{\gamma^{2}}\right)<\frac{16 \pi}{9}
$$

which implies

$$
\frac{\beta}{\sqrt{\gamma}}<\beta_{E}^{-} \approx 0.768792 \text { and } \frac{\beta}{\sqrt{\gamma}}>\beta_{E}^{+} \approx 1.58845,
$$

and the gradient condition for global existence $\mathcal{G}\left[u_{g}\right]<1$ gives

$$
\frac{\beta}{\sqrt{\gamma}}<\beta_{1} \approx 0.921318
$$

From (5.30), (5.31), (5.32) and (5.33) we conclude that (analytically proved) thresholds are: for global existence is below $\beta_{E}^{-} \approx 0.768792$ and for blow-up is above $\beta_{b} \approx 1.28607$ (see Figure 2).

\section{Example 4 : Energy-supercritical case}

Finally, we consider $p=7$ in the dimension $N=3$

$$
i u_{t}+\Delta u+\left(|x|^{-1} *|u|^{7}\right)|u|^{5} u=0
$$

In this case $s_{c}=\frac{7}{6}>1$, thus, the energy-supercritical regime. The energy for (5.34) is given by

$$
E\left[u_{g}\right]=\frac{\pi^{3 / 2}}{4} \frac{\beta^{2}}{\gamma^{1 / 2}}\left(3-\frac{16 \pi}{7^{7 / 2}} \frac{\beta^{12}}{\gamma^{2}}\right) .
$$

Then, in the energy-supercritical case we have

- $E\left[u_{g}\right]<0$ if

$$
\frac{\beta}{\gamma^{1 / 6}}>\beta_{E} \equiv \frac{3^{1 / 12} 7^{7 / 24}}{2^{1 / 3} \pi^{1 / 12}} \approx 1.3946799
$$

- condition (5.10) (Theorem 5.1.1) gives

$$
\frac{\beta}{\gamma^{1 / 6}}>\beta_{b} \equiv \frac{3^{1 / 12} 7^{7 / 24}}{2^{7 / 12} \pi^{1 / 12}} \approx 1.17278
$$


Note that except for the two conditions above no other information about scattering or blow up thresholds is known in the energy-supercritical case (except for the small data shown earlier in this paper).

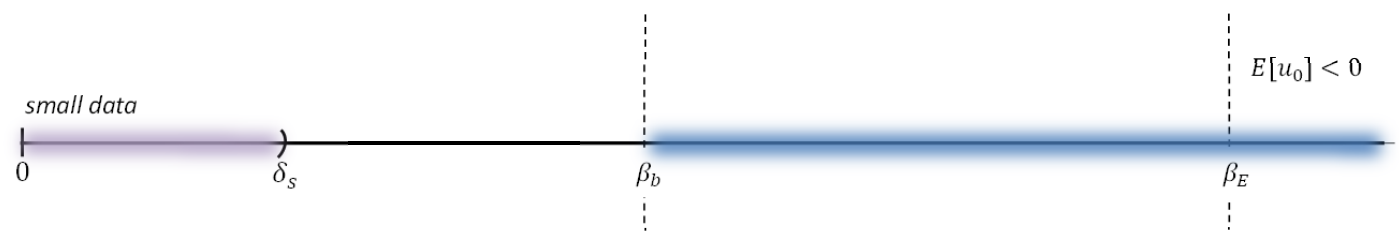

Figure 5.3: Thresholds for Gaussian data $u_{0}=\beta e^{-|x|^{2}}$ in the energy-supercritical case, see (5.35)-(5.36).

\subsection{Blow-up analysis}

Numerical simulations and asymptotic analysis in [YRZ20] show that stable blow-up dynamics in the $L^{2}$-critical gHartree equation (for $\gamma=2$ and dimensions $3 \leq N \leq 7$ ) follows the log-log regime, similar to the known results in the $L^{2}$-critical NLS equation, which had an interesting history. We mention some of it.

In the $L^{2}$-critical NLS equation, the study of stable blow-up solutions goes back to 1970's and gained an enormous amount of attention (see [YRZ18] for a review). The search of the correct blow-up rate was especially involved, since the blow-up rate $(T-t)^{-1 / 2}$, see Remark 5.3.3 below, also has a logarithmic correction term, and it was a challenging task to understand what the correction should be (it is a double logarithm, but numerically it is not possible to track double logarithm correction term). The first rigorous analytical description was done by Galina Perelman in 2001, see [Per01], in which a rigorous construction of the "log-log" blow-up solutions was shown for the $1 \mathrm{~d}$ quintic NLS equation. This was followed by a systematic study in a series of papers by Merle and Raphaël [MR03, MR04, MR05a, MR05b, MR06], obtaining a detailed description 
of the stable blow-up dynamics for solutions of the $L^{2}$-critical NLS equation with mass slightly above the mass of the ground state solution (it is still an open problem for larger masses). The proof requires certain coercivity properties on some bilinear forms, often referred to as the Spectral Property, see [MR05a, Section 4.4(D)] or [YRZ18]. In the $1 \mathrm{~d}$ case, the spectral property is proved analytically, since the ground state in the NLS equation is explicit, for example, see [MR05a, Appendix A]. In higher dimensions the available proofs are numerically-assisted (see [FMR06] for $N=2, \ldots, 5$ and [YRZ18] for $2 \leq N \leq 12$ ) due to the fact that $Q$ is not explicit as well as certain signs of the inner products are also computed numerically (and since the signs are robust to perturbations, the numerical verification is sufficient for the validity of the Spectral Property, see below Section 5.3.9).

There is very little known about the blow-up dynamics (how it happens, with what rates, profiles and other characterizations) for other dispersive equations, especially with nonlocal nonlinearity of convolution-type. We mention that understanding the blow-up dynamics for the convolution nonlinearity, as it is in the Hartree, or gHartree equation, is relevant for the development of theories for gravitational collapse of, for example, boson stars (as mentioned in Section 1.2 of Chapter 1) modeled by the equation (1.3). Fröhlich and Lenzmann [FJL07] proved the existence of finite time blow-up solutions in the pseudo-relativistic Hartree equation (1.3) in regards to the theory of gravitational collapse. Krieger, Lenzmann and Raphaël in [KLR09] studied the $4 \mathrm{~d} L^{2}$-critical Hartree equation (1.2) with a perturbed convolution kernel (destroying the pseudo-conformal invariance), obtaining the existence of minimal mass finite time blow-up solutions.

As mentioned at the beginning of this Section 5.3 we take $\gamma=2$, then the $L^{2}$-critical exponent for (1.1) is $p=1+\frac{4}{N}$, and the equation (1.1) becomes

$$
i u_{t}+\Delta u+\left(\frac{1}{|x|^{N-2}} *|u|^{1+\frac{4}{N}}\right)|u|^{\frac{4}{N}-1} u=0 .
$$


The corresponding ground state equation is

$$
-Q+\Delta Q+\left(\frac{1}{|x|^{N-2}} * Q^{1+\frac{4}{N}}\right) Q^{\frac{4}{N}}=0
$$

We point out that most of the results in the present Section 5.3 hold for the general $L^{2}$-critical gHartree equation (with $\gamma=2$ ), however, the Spectral Property (see, Theorem 5.3.21 and Remark 5.3.22 followed by Theorem 5.3.23) is only available in $\mathbb{R}^{3}$, see [ARY20]. It is an open question to prove analytically the $\log$-log blow-up dynamics in other dimensions in the $L^{2}$-critical setting of the gHartree equation, for instance, see [YRZ20, Figures 3, 4 and 6]. The following conjecture was stated in [YRZ20].

Conjecture 5.3.1 ([YRZ20]) A stable blow-up solution to the $L^{2}$-critical gHartree equation has a self-similar structure and comes with the rate

$$
\lim _{t \rightarrow T}\|\nabla u(\cdot, t)\|_{L_{x}^{2}}=\left(\frac{\ln |\ln (T-t)|}{2 \pi(T-t)}\right)^{\frac{1}{2}} \quad \text { as } \quad t \rightarrow T,
$$

known as the log-log rate. The solution blows up in a self-similar regime with profile converging to a rescaled profile $Q$, which is a ground state solution of (5.38), namely,

$$
u(x, t) \sim \frac{1}{L(t)^{\frac{d}{2}}} Q\left(\frac{x-x(t)}{L(t)}\right) e^{i \gamma(t)}
$$

with time depending parameters $L(t), x(t)$ and $\gamma(t)$, converging when $t \rightarrow T$ as follows: $x(t) \rightarrow x_{c}$ (the blow-up center), $\gamma(t) \rightarrow \gamma_{0}$ (for some $\left.\gamma_{0} \in \mathbb{R}\right)$ and $L(t) \sim\left(\frac{2 \pi(T-t)}{\ln |\ln (T-t)|}\right)^{\frac{1}{2}}$.

Thus, the stable blow-up dynamics in the $L^{2}$-critical gHartree equation is similar to the stable blow-up dynamics in the $L^{2}$-critical NLS equation.

The authors in [YRZ20] verified the Conjecture 5.3.1 (numerically) in dimensions $3 \leq$ $N \leq 7$. They showed that in the radial setting, the stable blow-up dynamics does not depend on the type of nonlinearity (i.e., local or nonlocal) in the NLS-type equations and the blow-up happens with the "log-log" rate. Moreover, the blow-up profile converges to 
the ground state solution found numerically, which is one of ground states of the equation (5.38).

We will give the proof of the above conjecture in the $3 \mathrm{~d}$ case with the one log correction rate, which is the culmination of this thesis (see also [AR20b]). The double log correction is much more involved and will be proved elsewhere. The result is as follows

Theorem 5.3.2 Let $N=3$ and consider the $L^{2}$-critical gHartree equation (5.37) with $p=\frac{7}{3}$

$$
i u_{t}+\Delta u+\left(\frac{1}{|x|} *|u|^{\frac{7}{3}}\right)|u|^{\frac{1}{3}} u=0 .
$$

Consider $u_{0} \in H^{1}\left(\mathbb{R}^{3}\right)$ such that

$$
M[Q]<M\left[u_{0}\right]<M[Q]+\alpha, \text { for some } \alpha>0,
$$

and

$$
E\left[u_{0}\right]<0, \quad \operatorname{Im}\left(\int_{\mathbb{R}^{3}} \bar{u}_{0} \nabla u_{0} d x\right)=0 .
$$

Let $u(t)$ be the corresponding solution to (5.39). Then there exist $\alpha_{0}>0$ such that for all $\alpha<\alpha_{0}$

1. there exist time depending parameters $(\lambda(t), x(t), \gamma(t)) \in \mathbb{R} \times \mathbb{R}^{3} \times \mathbb{R}$ such that

$$
u(t)=\frac{1}{\lambda(t)^{3 / 2}}(Q+\varepsilon)\left(\frac{x-x(t)}{\lambda(t)}\right) e^{i \gamma(t)},
$$

and $\|\varepsilon(t)\|_{H^{1}\left(\mathbb{R}^{3}\right)} \lesssim \sqrt{\alpha_{0}}$,

2. $u(t)$ blows up in finite time, i.e., there exists $0<T<+\infty$ such that

$$
\lim _{t \rightarrow T}\|\nabla u(t)\|_{L^{2}\left(\mathbb{R}^{3}\right)}=+\infty
$$

3. for t close to the blow-up time $T$, we have

$$
\|\nabla u(t)\|_{L^{2}\left(\mathbb{R}^{3}\right)} \leq C\left(\frac{|\ln (T-t)|}{T-t}\right)^{\frac{1}{2}},
$$

for some universal constant $C>0$. 
Remark 5.3.3 As mentioned at the start of Section 5.3, the main term in the blow-up rate found numerically is $(T-t)^{-1 / 2}$. In Theorem 5.3.2, we show that the blow-up rate (5.40) is upper bounded by $(T-t)^{-1 / 2}$ (with a correction). The lower bound by $(T-t)^{-1 / 2}$ is a consequence of the scaling invariance (see Section 2.2 of Chapter 2). More precisely, if the solution to the $L^{2}$-critical gHartree equation (5.37) with initial data $u_{0} \in H^{1}\left(\mathbb{R}^{N}\right)$ satisfies $\lim _{t \rightarrow T}\|\nabla u(t)\|_{L^{2}\left(\mathbb{R}^{N}\right)}=+\infty$ for $0<T<+\infty$, then

$$
\|\nabla u(t)\|_{L^{2}\left(\mathbb{R}^{N}\right)} \geq \frac{C_{0}}{(T-t)^{1 / 2}} \quad \text { for all } 0 \leq t<T .
$$

Indeed, suppose $u(x, t)$ is a solution to (5.37), then

$$
\tilde{u}(y, \tau)=\frac{1}{\|\nabla u(t)\|_{L^{2}\left(\mathbb{R}^{N}\right)}^{\frac{N}{2}}} u\left(\frac{y}{\|\nabla u(t)\|_{L^{2}\left(\mathbb{R}^{N}\right)}}, t+\frac{\tau}{\|\nabla u(t)\|_{L^{2}\left(\mathbb{R}^{N}\right)}^{2}}\right)
$$

is also a solution to (5.37) by scaling and time translation invariance for a fixed $t \in$ $[0, T)$. One can verify that $\|\tilde{u}(0)\|_{H^{1}\left(\mathbb{R}^{N}\right)} \leq C$, since $\|\tilde{u}(0)\|_{L^{2}\left(\mathbb{R}^{N}\right)}=\left\|u_{0}\right\|_{L^{2}\left(\mathbb{R}^{N}\right)}$ and $\|\nabla \tilde{u}(t)\|_{L^{2}\left(\mathbb{R}^{N}\right)}=1$. Thus, by the Proposition 2.6.2 ( $H^{1}$ local existence), there exists a time $\tau_{0}=\tau\left(\left\|\tilde{u}_{0}\right\|_{H^{1}}\right)>0$ such that $\tilde{u}(y, \tau)$ is defined on the interval $\left[0, \tau_{0}\right]$. As a consequence, we have

$$
t+\frac{\tau_{0}}{\|\nabla u(t)\|_{L^{2}\left(\mathbb{R}^{N}\right)}^{2}}<T
$$

which immediately yields the lower bound (5.41).

\subsubsection{Linearized equation around $Q$}

To start with the proof of Theorem 5.3.2, we decompose the solution $u(x, t)$ to (5.37) around $Q$ as follows

$$
\varepsilon(y, t)=e^{i \gamma(t)} \lambda(t)^{N / 2} u(\lambda(t) y+x(t), t)-Q(y)
$$

Note $\lambda(t)>0, x(t)$ and $\gamma(t)$ are $C^{1}$ functions to be chosen later. We rescale the time variable by $\frac{d s}{d t}=\frac{1}{\lambda(t)^{2}}$, and write $\varepsilon=\varepsilon(y, s)$, observe that in this time rescaling we have $s \in[0, \infty)$. We next study the remainder term $\varepsilon$, which we shall prove is small. 


\subsubsection{Equation for $\varepsilon$}

The following lemma gives the $\varepsilon$ equation, where

$$
\left.Q_{1} \stackrel{\text { def }}{=} \frac{d Q_{\lambda}}{d \lambda}\right|_{\lambda=1}=\frac{N}{2} Q+x \cdot \nabla Q,
$$

is the scaling generator and here, we have used $s_{c}=0 \Longleftrightarrow \frac{N}{2}=\frac{\gamma+2}{2(p-1)}$. Similarly, define

$$
Q_{2}=\frac{N}{2} Q_{1}+x \cdot \nabla Q_{1}
$$

Lemma 5.3.4 For all $s \geq 0$, we have

$$
\begin{gathered}
\left(\varepsilon_{1}\right)_{s}-L_{-} \varepsilon_{2}=\frac{\lambda_{s}}{\lambda} Q_{1}+\frac{x_{s}}{\lambda} \cdot \nabla Q+\frac{\lambda_{s}}{\lambda}\left(\varepsilon_{1}\right)_{1}+\frac{x_{s}}{\lambda} \cdot \nabla \varepsilon_{1}+\tilde{\gamma}_{s} \varepsilon_{2}-R_{2}(\varepsilon), \\
\left(\varepsilon_{2}\right)_{s}+L_{+} \varepsilon_{1}=-\tilde{\gamma}_{s} Q+\frac{\lambda_{s}}{\lambda}\left(\varepsilon_{2}\right)_{1}+\frac{x_{s}}{\lambda} \cdot \nabla \varepsilon_{2}-\tilde{\gamma}_{s} \varepsilon_{1}+R_{1}(\varepsilon),
\end{gathered}
$$

where $\tilde{\gamma}_{s}=-s-\gamma_{s}$, the operators $L_{ \pm}$are defined by

$$
\begin{aligned}
& L_{+} \varepsilon_{1}:=-\Delta \varepsilon_{1}+\varepsilon_{1}-\frac{4}{N}\left(|y|^{-(N-2)} * Q^{1+\frac{4}{N}}\right) Q^{\frac{4}{N}-1} \varepsilon_{1} \\
&-\left(1+\frac{4}{N}\right)\left(|y|^{-(N-2)} *\left(Q^{\frac{4}{N}} \varepsilon_{1}\right)\right) Q^{\frac{4}{N}}, \\
& L_{-} \varepsilon_{2}:=-\Delta \varepsilon_{2}+\varepsilon_{2}-\left(|y|^{-(N-2)} * Q^{1+\frac{4}{N}}\right) Q^{\frac{4}{N}-1} \varepsilon_{2},
\end{aligned}
$$

and the remainder terms $R_{1}, R_{2}$ are given by

$$
\begin{aligned}
R_{1}(\varepsilon) & =\left(|y|^{-(N-2)} *|Q+\varepsilon|^{1+\frac{4}{N}}\right)|Q+\varepsilon|^{\frac{4}{N}-1}\left(Q+\varepsilon_{1}\right) \\
& -\left(|y|^{-(N-2)} * Q^{1+\frac{4}{N}}\right) Q^{\frac{4}{N}-1} Q-\frac{4}{N}\left(|y|^{-(N-2)} * Q^{1+\frac{4}{N}}\right) Q^{\frac{4}{N}-1} \varepsilon_{1} \\
& -\left(1+\frac{4}{N}\right)\left(|y|^{-(N-2)} *\left(Q^{\frac{4}{N}} \varepsilon_{1}\right)\right) Q^{\frac{4}{N}} \\
R_{2}(\varepsilon)= & \left(|y|^{-(N-2)} *|Q+\varepsilon|^{1+\frac{4}{N}}\right)|Q+\varepsilon|^{\frac{4}{N}-1} \varepsilon_{2}-\left(|y|^{-(N-2)} * Q^{1+\frac{4}{N}}\right) Q^{\frac{4}{N}-1} \varepsilon_{2} .
\end{aligned}
$$

Proof. This is a direct computation; substituting (5.42) into (5.37) and writing the equations for real and imaginary parts of $\varepsilon$ gives the desired result. 


\subsubsection{Useful properties of $Q$}

For the equation (1.1), we recall the convolution-type Gagliardo-Nirenberg inequality (2.126) (discussed previously in Section 2.8 of Chapter 2)

$$
Z(u) \equiv \int_{\mathbb{R}^{N}} \int_{\mathbb{R}^{N}} \frac{|u(x)|^{p}|u(y)|^{p}}{|x-y|^{b}} d x d y \leq C_{G N}\|\nabla u\|_{L^{2}\left(\mathbb{R}^{N}\right)}^{2 s_{c}(p-1)+2}\|u\|_{L^{2}\left(\mathbb{R}^{N}\right)}^{2\left(1-s_{c}(p-1)\right.},
$$

where the sharp constant $C_{G N}$ (see Section 2.8 of present thesis or Section 4 in [AR20a] for details) is given by

$$
C_{G N}=\frac{2 p}{N(p-1)-\gamma}\left(\frac{N+\gamma-(N-2) p}{N(p-1)-\gamma}\right)^{\frac{N(p-1)-\gamma}{2}-1}\|Q\|_{L^{2}\left(\mathbb{R}^{N}\right)}^{-2(p-1)} .
$$

In the $L^{2}$-critical case, $s_{c}=0$ with $b=N-2$, the inequality (2.126) becomes

$$
\int_{\mathbb{R}^{N}} \int_{\mathbb{R}^{N}} \frac{|u(x)|^{1+\frac{4}{N}}|u(y)|^{1+\frac{4}{N}}}{|x-y|^{N-2}} d x d y \leq C_{G N}\|\nabla u\|_{L^{2}\left(\mathbb{R}^{N}\right)}^{2}\|u\|_{L^{2}\left(\mathbb{R}^{N}\right)}^{\frac{8}{N}} .
$$

We recall (again from Section 2.8 of the present thesis) that the sharp constant $C_{G N}$ from (5.50) is attained at (any ground state) $Q$, which solve (5.38). The existence, decay asymptotics (exponential decay for $p>2$ ) and other properties of ground state solutions $Q$ are investigated by Moroz and Van Schaftingen in [MVS13]. Also recall that the uniqueness is only known in the standard Hartree case $p=2, \gamma=2$ and $2<N<6$, see Section 2.8 of the present thesis (also for $p=2+\epsilon, \gamma=2$ in dimension $N=3$, see [Xia16]). Therefore, for the purpose of proving the Theorem 5.3.2, we take a ground state solution $Q$. We mention that solving (2.123) with $\gamma=2, N>2$ and $p<\frac{N-2}{N+2}\left(s_{c}<1\right)$, or (5.38) when $s_{c}=0$ numerically suggests that there exists a unique ground state solution to (2.123), or the $L^{2}$-critical ground state equation (5.37) (iterations always converge to the same $Q$, see [YRZ20, Appendix]).

The following algebraic identities hold from the symmetries of (5.37):

- phase symmetry relates to $L_{-} Q=0$, 
- translation invariance gives $L_{+}(\nabla Q)=0$,

- scaling symmetry generates $L_{+}\left(Q_{1}\right)=-2 Q$,

- Galilean invariance produces $L_{-}(x Q)=-2 \nabla Q$,

- pseudo-conformal symmetry is associated with $L_{-}\left(|x|^{2} Q\right)=-4 Q_{1}$.

An immediate adaptation of an argument in [Wei85, Appendix B] (since all the above algebraic identities are similar to the NLS case) leads to an additional relation corresponding to an extra direction in the generalized null-space associated with the last relation above, $L_{+} \rho=-|x|^{2} Q$.

\subsubsection{Spectral properties of $L_{+}$and $L_{-}$in $N=3$}

While most of the analysis works in any dimension, it is the spectral properties, which make things challenging to consider in a general setting. Thus, the results of the present subsection are proved for the dimension $N=3$. We start with the following statement.

Lemma 5.3.5 The operator $L_{+}$has exactly one negative eigenvalue.

Proof. First we observe that

$$
\left(Q, L_{+} Q\right)=-\frac{8}{3} Z(Q)<0,
$$

where $Z$ is defined in (2.125). Recalling the min-max principle [RS78, Theorem XIII.1], we have that the operator $L_{+}$has at least one negative eigenvalue. From Section 2.8 of Chapter 2 (see also [AR20a], or [MVS13]), we also know that $Q$ is a minimizer of the Gagliardo-Nirenberg functional given by

$$
J(u)=\frac{\|\nabla u\|_{L^{2}\left(\mathbb{R}^{3}\right)}^{2}\|u\|_{L^{2}\left(\mathbb{R}^{3}\right)}^{\frac{8}{2}}}{Z(u)}
$$


for $u \in H^{1}\left(\mathbb{R}^{3}\right)$ with $u \not \equiv 0$ (note that this holds for other dimensions). Therefore,

$$
\left.\frac{d^{2}}{d \epsilon^{2}}\right|_{\epsilon=0} J(Q+\epsilon h) \geq 0, \quad \forall h \in C^{\infty}\left(\mathbb{R}^{3}\right) .
$$

By a direct (and tedious) computation the previous statement is equivalent to

$$
\left(L_{+} h_{1}, h_{1}\right) \geq \frac{14}{3}\left(\int Q h_{1}\right)^{2}-\frac{16}{3} \int Q h_{1} \int\left(|x|^{-1} * Q^{\frac{7}{3}}\right) Q^{\frac{4}{3}} h_{1}
$$

and

$$
\left(L_{-} h_{2}, h_{2}\right) \geq 0
$$

Therefore,

$$
\left(L_{+} h_{1}, h_{1}\right) \geq 0 \quad \text { if } \quad h_{1} \perp\left(|x|^{-1} * Q^{\frac{7}{3}}\right) Q^{\frac{4}{3}}
$$

(implying that $h_{1} \perp(-\Delta Q+Q)$ ). Let $\mu_{j}$ is the $j$ th eigenvalue of $L_{+}$. Then again using the min-max principle, one can deduce that there is exactly one function $\phi_{1}$ in the orthogonal space (one dimensional subspace of $L^{2}$ ) such that

$$
\mu_{2}\left(L_{+}\right)=\sup _{\phi_{1}}\left\{\inf _{h_{1} \perp \phi_{1} ;\left\|h_{1}\right\|_{L^{2}}=1}\left(h_{1}, L_{+} h_{1}\right)\right\} \geq 0,
$$

since $L_{+}$is nonnegative for $\phi_{1}=\left(|x|^{-1} * Q^{\frac{7}{3}}\right) Q^{\frac{4}{3}}$. Thus, 0 is the second eigenvalue, since we know from a direct computation that $L_{+} \nabla Q=0$. Therefore, there can be at most one negative eigenvalue. Combining this with the starting observation, we conclude that $L_{+}$ has exactly one negative eigenvalue.

We follow the approach of Weinstein [Wei85, Proposition 2.8] and introduce some essential ingredients, which will be required to prove the main result of this section regarding the null-spaces of $L_{+}$and $L_{-}$. We first write the Laplacian in $3 \mathrm{~d}$ in spherical coordinates as

$$
\Delta f=\frac{\partial^{2} f}{\partial r^{2}}+\frac{2}{r} \frac{\partial f}{\partial r}+\frac{1}{r^{2}} \Delta_{\mathbb{S}^{2}} f
$$

where

$$
\Delta_{\mathbb{S}^{2}} f=\frac{1}{\sin \theta} \frac{\partial f}{\partial \theta}\left(\sin \theta \frac{\partial f}{\partial \theta}\right)+\frac{1}{\sin ^{2} \theta} \frac{\partial^{2} f}{\partial \phi^{2}} .
$$


It is well-known that the (homogeneous) harmonic functions on $\mathbb{R}^{3}$, i.e., solutions of the Laplace equation can be found by separation of variables. Thus, we write $f(r, \theta, \phi)=$ $r^{l} g(\theta, \phi)$ and obtain that

$$
\Delta f=r^{l-2}\left(l(l+1) g+\Delta_{\mathbb{S}^{2}} g\right)
$$

Hence,

$$
\Delta f=0 \quad \text { if and only if } \quad \Delta_{\mathbb{S}^{2}} g=-l(l+1) g
$$

i.e., $g$ is an eigenfunction of $\Delta_{\mathbb{S}^{2}}$ for the eigenvalue $-l(l+1)$. Hence, Laplacian in spherical coordinates can be expressed as

$$
\Delta_{(l)}=\frac{\partial^{2}}{\partial r^{2}}+\frac{2}{r} \frac{\partial}{\partial r}-\frac{l(l+1)}{r^{2}} .
$$

Therefore, decomposing any solution $\eta \in L^{2}\left(\mathbb{R}^{3}\right)$ of $L_{+} \eta=0$ via spherical harmonics, we have

$$
\eta=\sum_{l=0}^{\infty} \sum_{m=-l}^{l} \eta_{l, m}(r) Y_{l, m}(\omega)
$$

where $x=r \omega$ with $r=|x|$ and $\omega \in \mathbb{S}^{2}$. Here, $Y_{l, m}$ denotes the spherical harmonics, which is the eigenfunction corresponding to the eigenvalue $-l(l+1)$ of the spherical Laplacian $\Delta_{\mathbb{S}^{2}}$. We now recall the multipole expansion (following the representation in [Len09])

$$
\frac{1}{\left|x-x^{\prime}\right|}=4 \pi \sum_{l=0}^{\infty} \sum_{m=-l}^{l} \frac{1}{2 l+1} \frac{r_{<}^{l}}{r_{>}^{l+1}} Y_{l, m}(\omega) Y_{l, m}^{*}\left(\omega^{\prime}\right)
$$

where $r_{\text {min }}=\min \left(|x|,\left|x^{\prime}\right|\right)$ and $r_{\max }=\max \left(|x|,\left|x^{\prime}\right|\right)$. Then with $\eta$ given by (5.55), we have

$$
L_{+} \eta=0 \Longleftrightarrow L_{+,(l)} \eta_{l, m}=0, \quad \text { for } l=0,1,2, \ldots \text { and } m=-l, \ldots,+l,
$$

where

$$
\left(L_{+,(l)} f\right)(r)=\left(-\Delta_{(l)}+1\right) f(r)+\left(V_{(l)} f\right)(r)
$$


with $-\Delta_{(l)}$ given by $(5.54)$ and

$$
\begin{aligned}
& \left(V_{(l)} f\right)(r)=-\frac{4}{3} V(r) f(r)-\frac{28 \pi}{3(2 l+1)}\left(W_{(l)} f\right)(r) \\
& \stackrel{\text { def }}{=}-\frac{4}{3}\left(|x|^{-1} * Q^{\frac{7}{3}}\right)(r) Q^{\frac{1}{3}}(r) f(r)-\frac{28 \pi}{3(2 l+1)}\left(\int_{0}^{\infty} \frac{r_{\min }^{l}}{r_{\max }^{l+1}} Q^{\frac{4}{3}}(s) f(s) s^{2} d s\right) Q^{\frac{4}{3}}(r),
\end{aligned}
$$

here, $r_{\min }=\min (r, s)$ and $r_{\max }=\max (r, s)$. Now we show that each $L_{+,(l)}$ for $l \geq 1$ satisfies Perron-Frobenius property. This result was proved by Lenzmann for pseudorelativistic Hartree equations (see [Len09, Lemma 7]). We next prove this for our 3d gHartree case.

Lemma 5.3.6 For each $l \geq 1$, the operator $L_{+,(l)}$ is essentially self-adjoint on $C_{0}^{\infty}\left(\mathbb{R}^{+}\right) \subset$ $L^{2}\left(\mathbb{R}_{+}\right)$in spherical coordinates and bounded below. Moreover, each $L_{+,(l)}$ satisfies the Perron-Frobenius property, i.e., if $e_{0,(l)}$ denotes the lowest eigenvalue of $L_{+,(l)}$, then $e_{0,(l)}$ is simple and the corresponding eigenfunction $\phi_{0,(l)}(r)$ is strictly positive.

Proof. Observe that from (5.53), we have that $\left.L_{+,(l)}\right|_{Q^{\perp}} \geq 0$. Thus, $L_{+,(l)}$ is bounded below. We also have from [RS72] (Appendix to X.1, Theorem X.10 and Example 4) that $-\Delta_{(l)}\left(\right.$ defined in (5.54)) is essentially self-adjoint on $C_{0}^{\infty}\left(\mathbb{R}^{+}\right)$provided $l(l+1) / r^{2} \geq$ $3 / 4 r^{2}$ (which is true since $l \geq 1$ ). We now show that the Coulomb potential $|x|^{-1}$ can be written as $|x|^{-1}=g_{1}(x)+g_{2}(x)$ with $g_{1} \in L^{2}$ and $g_{2} \in L^{\infty}$. We split the Coulomb potential into two pieces, the part living inside the unit ball and the part outside. Observe that $\left\|g_{2}\right\|_{L^{\infty}\left(\mathbb{R}^{3}\right)} \leq C(\epsilon)$ for some arbitrary $0<\epsilon<1$, since $\frac{1}{|x|}$ is bounded by 1 outside the unit ball and

$$
\int_{|x| \leq 1} \frac{1}{|x|^{2}} d x=4 \pi \int_{0}^{1} \frac{1}{r^{2}} r^{2} d r=4 \pi, \quad \text { inside the unit ball. }
$$

Thus, using Young's inequality in the first inequality below and Gagliardo-Nirenberg interpolation inequality, i.e., $\|f\|_{L^{\infty}\left(\mathbb{R}^{3}\right)} \leq C_{*}\|(-\Delta) f\|_{L^{2}\left(\mathbb{R}^{3}\right)}^{3 / 4}\|f\|_{L^{2}\left(\mathbb{R}^{3}\right)}^{1 / 4}$ in the second in- 
equality along with the fact that $\left\|g_{2}\right\|_{L^{\infty}} \leq C(\epsilon)$, we have

$$
\begin{aligned}
\|V f\|_{L^{2}} & =\left\|\left(|x|^{-1} * Q^{\frac{7}{3}}\right) Q^{\frac{1}{3}} f\right\|_{L^{2}}=\left\|\left(g_{1} * Q^{\frac{7}{3}}\right) Q^{\frac{1}{3}} f\right\|_{L^{2}}+\left\|\left(g_{2} * Q^{\frac{7}{3}}\right) Q^{\frac{1}{3}} f\right\|_{L^{2}} \\
& \leq\left\|g_{1}\right\|_{L^{2}}\|Q\|_{L^{14 / 5}}^{7 / 3}\|Q\|_{L^{2}}^{1 / 3}\|f\|_{L^{\infty}}+\left\|g_{2}\right\|_{L^{\infty}}\|Q\|_{L^{14 / 5}}^{7 / 3}\|Q\|_{L^{2}}^{1 / 3}\|f\|_{L^{2}} \\
& \leq C_{Q}\left(4 \pi C_{*}\|(-\Delta) f\|_{L^{2}}^{3 / 4}\|f\|_{L^{2}}^{1 / 4}+C(\epsilon)\|f\|_{L^{2}}\right) \\
& \leq C_{Q}\left(3 \pi C_{*}\|(-\Delta) f\|_{L^{2}}+\left(\pi C_{*}+C(\epsilon)\right)\|f\|_{L^{2}}\right),
\end{aligned}
$$

where in the last inequality we have used that

$$
\|(-\Delta) f\|_{L^{2}}^{3 / 4}\|f\|_{L^{2}}^{1 / 4} \leq \frac{3}{4}\|(-\Delta) f\|_{L^{2}}+\frac{1}{4}\|f\|_{L^{2}} .
$$

Thus, $V f$ is $-\Delta$ bounded for $0<C_{Q} C_{*}<\frac{1}{3 \pi}$. Now, we prove a similar fact for the second potential in (5.46),

$$
\begin{aligned}
\|\left(|x|^{-1} *\right. & \left.\left(Q^{\frac{4}{3}} f\right)\right) Q^{\frac{4}{3}}\left\|_{L^{2}}=\right\|\left(g_{1} *\left(Q^{\frac{4}{3}} f\right)\right) Q^{\frac{4}{3}}\left\|_{L^{2}}+\right\|\left(g_{2} *\left(Q^{\frac{4}{3}} f\right)\right) Q^{\frac{4}{3}} \|_{L^{2}} \\
& \leq\left\|g_{1}\right\|_{L^{2}}\|Q\|_{L^{8 / 3}}^{8 / 3}\|f\|_{L^{\infty}}+\left\|g_{2}\right\|_{L^{\infty}}\|Q\|_{L^{8 / 3}}^{8 / 3}\|f\|_{L^{2}} \\
& \leq \tilde{C}_{Q}\left(4 \pi C_{*}\|-\Delta f\|_{L^{2}}^{3 / 4}\|f\|_{L^{2}}^{1 / 4}+C(\epsilon)\|f\|_{L^{2}}\right) \\
& \leq \tilde{C}_{Q}\left(3 \pi C_{*}\|-\Delta f\|_{L^{2}}+\left(\pi C_{*}+C(\epsilon)\right)\|f\|_{L^{2}}\right) .
\end{aligned}
$$

Hence, invoking the Kato-Rellich theorem, see [RS72, Theorem X.12 and Theorem X.15], proves that $L_{+}$(defined in (5.46)) is essentially self-adjoint on $C_{0}^{\infty}\left(\mathbb{R}^{+}\right)$. This implies from the equivalence of $L_{+}$and $L_{+,(l)}\left(\right.$ see (5.57)) that $L_{+,(l)}($ defined in (5.58)) is also essentially self-adjoint on $C_{0}^{\infty}\left(\mathbb{R}^{+}\right)$.

To prove the Perron-Frobenius property of $L_{+,(l)}$, we first recall that an operator $T$ is called positivity improving if $T f$ is strictly positive whenever $f$ is positive. We also recall (see expression (7-15) in [Len09]) that $\left(-\Delta_{(l)}+\mu\right)^{-1}$ is positivity improving on $L^{2}\left(\mathbb{R}^{+}, r^{2} d r\right)$ for all $\mu>0$. Next, since $Q$ is positive, we also have that $-V_{(l)}$ (defined in (5.59)) is positivity improving on $L^{2}\left(\mathbb{R}^{+}, r^{2} d r\right)$. Therefore, we have

$$
\left(L_{+,(l)}+\mu\right)^{-1}=\left(-\Delta_{(l)}+1+\mu\right)^{-1}\left(1+V_{(l)}\left(-\Delta_{(l)}+1+\mu\right)^{-1}\right)^{-1} .
$$


Next, recall the generalization of geometric series given by

$$
(\mathrm{Id}-T)^{-1}=\sum_{k=0}^{\infty} T^{k}
$$

where $T$ is a bounded linear operator and Id is the identity operator. Using this generalization, we write

$$
\left(L_{+,(l)}+\mu\right)^{-1}=\left(-\Delta_{(l)}+1+\mu\right)^{-1} \sum_{k=0}^{\infty}\left(-V_{(l)}\left(-\Delta_{(l)}+1+\mu\right)^{-1}\right)^{k} .
$$

Since $V_{(l)}$ is bounded, we deduce that the convergence of above series is guaranteed provided $\mu \gg 1$. Using (5.60) along with positivity improving property of $\left(-\Delta_{(l)}+1+\mu\right)^{-1}$ and $-V_{(l)}$, we conclude that $\left(L_{+,(l)}+\mu\right)^{-1}$ must also be positivity improving.

Now let $e_{0,(l)}$ be the lowest eigenvalue of $L_{+,(l)}$. Observe that $\left(L_{+,(l)}+\mu\right)^{-1}$ is bounded, self-adjoint and positivity improving with $\left(e_{0,(l)}+\mu\right)^{-1}$ being the largest eigenvalue. Hence, by [RS78, Theorem XIII.43 part (a)], $\left(e_{0,(l)}+\mu\right)^{-1}$ is simple and the corresponding eigenfunction $\phi_{0,(l)}$ is strictly positive. This completes the proof of Lemma 5.3.6.

We may now prove the following proposition. We mention that the statement below is conditional on the uniqueness of ground state $Q$.

Proposition 5.3.7 Suppose $N=3$, then

1. The null-space of self-adjoint operator $L_{+}$is spanned by the vectors $\partial_{x_{j}} Q$, where $1 \leq j \leq 3$

2. $L_{-}$is a nonnegative self-adjoint operator and the null-space of $L_{-}$is spanned by $Q$

Proof. $\quad$ 1. Observe that $L_{+}\left(Q_{x_{j}}\right)=0$ and $Q_{x_{j}}=Q^{\prime}(r) \frac{x_{j}}{r}$, which shows that $L_{+(1)} Q^{\prime}=$ 0 . Thus, from the monotonic properties of $Q$, we deduce that $Q^{\prime}<0$ is an eigenfunction of self-adjoint operator $L_{+,(1)}$ that does not change its sign. In fact, Lemma 
5.3.6 implies that $Q^{\prime}=-\phi_{0,(1)}$, where $\phi_{0,(l)}$ is strictly positive with the corresponding eigenvalue $e_{0,(1)}=0$. Suppose $e_{0,(l)}$ be the lowest eigenvalue with the associated eigenfunction $\phi_{0,(l)}$ for $L_{+,(l)}$. Here, $l \geq 2$ is fixed. Now we notice (using (5.54), (5.58) and (5.59)) that

$$
\begin{aligned}
L_{+,(l)} \phi_{0,(l)}= & L_{+,(1)} \phi_{0,(l)}+\frac{l(l+1)-2}{r^{2}} \phi_{0,(l)} \\
& +\frac{28 \pi}{3}\left(\int_{0}^{\infty}\left(\frac{1}{3} \frac{r_{\text {min }}}{r_{\text {max }}^{2}}-\frac{1}{2 l+1} \frac{r_{\text {min }}^{l}}{r_{\text {max }}^{l+1}}\right) Q^{\frac{4}{3}}(s) \phi_{0,(l)}(s) s^{2} d s\right) Q^{4 / 3}(r) \\
\geq & \frac{l(l+1)-2}{r^{2}} \phi_{0,(l)} \\
+ & \frac{28 \pi}{3}\left(\int_{0}^{\infty} \frac{1}{3} \frac{r_{\min }}{r_{\text {max }}^{2}}\left(1-\frac{3}{2 l+1}\left(\frac{r_{\text {min }}}{r_{\text {max }}}\right)^{l-1}\right) Q^{\frac{4}{3}}(s) \phi_{0,(l)}(s) s^{2} d s\right) Q^{4 / 3}(r),
\end{aligned}
$$

where we have used the fact that $L_{+,(1)} \geq 0$. Using the strict positivity of $Q$ along with the strict positivity of $\phi_{0,(l)}$ (from Lemma 5.3.6), we have that (5.62) $>0$ for $l \geq 2$ and $\frac{r_{\min }}{r_{\max }}<1$. Using the strict positivity of $\phi_{0,(l)}$ along with the fact that $l \geq 2$, we also have that $(5.61)>0$. Hence, $L_{+,(l)}>0$ for $l \geq 2$. Now we are left with the case $l=0$, for which we need to prove that $L_{+,(0)} f=0$ implies $f \equiv 0$. This can be deduced by a similar argument as in [LMR08, Lemma 2.1] (also see, [Mar02]), since the potential terms in $L_{+,(0)}$ still decay exponentially (in our case unlike the standard Hartree there are $Q^{1 / 3}$ and $Q^{4 / 3}$ outside the convolution, where $Q(x)=O\left(|x|^{-1 / 2} e^{-|x|}\right)$ as $\left.|x| \rightarrow \infty\right)$. This completes the proof of part (1).

2. We know from (5.52) that $L_{-}$is nonnegative. Observe that $L_{-} Q=0$ is equivalent to the ground state equation (5.38) in $\mathbb{R}^{3}$. As mentioned in Section 5.3.3 (also see Section 2.8 of Chapter 2) that we do not have analytic uniqueness of $Q$, thus, assuming a conditional uniqueness of $Q$ (since numerically solving (5.38) produces a unique ground state solution, see [YRZ20, Appendix]), completes the proof of part (2).

The proof of Proposition 5.3.7 is now complete. 
Next, before proceeding further we show the following

Lemma 5.3.8 $\left(\partial_{x_{k}} Q,\left(Q_{1}+Q_{2}\right)\right)=0$.

Proof. We fix $k=1$. Now observe that $Q\left(x_{1}, x_{2}, \ldots, x_{N}\right)=Q\left(-x_{1}, x_{2}, \ldots, x_{N}\right)$ and

$$
\partial_{x_{1}} Q\left(x_{1}, x_{2}, \ldots, x_{N}\right)=-\partial_{x_{1}} Q\left(-x_{1}, x_{2}, \ldots, x_{N}\right)
$$

Therefore, we compute

$$
\begin{aligned}
& \int \partial_{x_{1}} Q\left(Q_{1}+Q_{2}\right)=\left(\frac{N}{2}+1\right) \int\left(\partial_{x_{1}} Q\right) Q_{1}+\int\left(\partial_{x_{1}} Q\right) x \cdot \nabla Q_{1} \\
& =\frac{N}{2}\left(\frac{N}{2}+1\right) \int\left(\partial_{x_{1}} Q\right) Q+(N+1) \int\left(\partial_{x_{1}} Q\right) x \cdot \nabla Q+\int\left(\partial_{x_{1}} Q\right) x \cdot \nabla(x \cdot \nabla Q) .
\end{aligned}
$$

Note that the first integral on the right in the above expression is equal to 0 by (5.63). The second integral on the right in the above expression is also equal to 0 by the following computation

$$
\begin{aligned}
\int\left(\partial_{x_{1}} Q\right) x \cdot \nabla Q= & \int \partial_{x_{1}} Q\left(x_{1} \partial_{x_{1}} Q+x_{2} \partial_{x_{2}} Q+\ldots+x_{N} \partial_{x_{N}} Q\right) \\
= & \int \partial_{x_{1}} Q\left(-x_{1}, x_{2}, \ldots, x_{N}\right)\left(-x_{1} \partial_{x_{1}} Q\left(-x_{1}, x_{2}, \ldots, x_{N}\right)\right) \\
& +\int \partial_{x_{1}} Q\left(-x_{1}, x_{2}, \ldots, x_{N}\right)\left(x_{2} \partial_{x_{2}} Q\left(-x_{1}, x_{2}, \ldots, x_{N}\right)\right)+\ldots \\
& \ldots+\int \partial_{x_{1}} Q\left(-x_{1}, x_{2}, \ldots, x_{N}\right)\left(x_{N} \partial_{x_{N}} Q\left(-x_{1}, x_{2}, \ldots, x_{N}\right)\right) \\
= & -\int \partial_{x_{1}} Q\left(x_{1}, x_{2}, \ldots, x_{N}\right)\left(x_{1} \partial_{x_{1}} Q\left(x_{1}, x_{2}, \ldots, x_{N}\right)\right) \\
& -\int \partial_{x_{1}} Q\left(x_{1}, x_{2}, \ldots, x_{N}\right)\left(x_{2} \partial_{x_{2}} Q\left(x_{1}, x_{2}, \ldots, x_{N}\right)\right)-\ldots \\
& \ldots-\int \partial_{x_{1}} Q\left(x_{1}, x_{2}, \ldots, x_{N}\right)\left(x_{N} \partial_{x_{N}} Q\left(x_{1}, x_{2}, \ldots, x_{N}\right)\right) \\
= & -\int\left(\partial_{x_{1}} Q\right) x \cdot \nabla Q
\end{aligned}
$$

which implies that $\int\left(\partial_{x_{1}} Q\right) x \cdot \nabla Q=0$. Now we treat the third and last integral

$$
\int\left(\partial_{x_{1}} Q\right) x \cdot \nabla(x \cdot \nabla Q)=\int\left(\partial_{x_{1}} Q\right) \sum_{i, j}\left(x_{i} \partial_{x_{i}}\left(x_{j}\right) \partial_{x_{j}} Q+x_{i} x_{j} \partial_{x_{i} x_{j}} Q\right)
$$


Using the fact that $\partial_{x_{i}}\left(x_{j}\right)=0$ for $i \neq j$, first integral on the right reduces to

$$
\int\left(\partial_{x_{1}} Q\right) \sum_{\substack{i=1 \\ i=j}}^{N}\left(x_{i} \partial_{x_{i}} Q\right)=\int\left(\partial_{x_{1}} Q\right)(x \cdot \nabla Q)=0
$$

where the last equality follows for the previous calculation. Lastly,

$$
\int\left(\partial_{x_{1}} Q\right) \sum_{i, j}\left(x_{i} x_{j} \partial_{x_{i} x_{j}} Q\right)=\int\left(\partial_{x_{1}} Q\right) \sum_{\substack{i=1 \\ i=j}}^{N}\left(x_{i}^{2} \partial_{x_{i}}^{2} Q\right)+\int\left(\partial_{x_{1}} Q\right) \sum_{i \neq j}\left(x_{i} x_{j} \partial_{x_{i} x_{j}} Q\right) .
$$

First integral on the right is equal to zero, since $\left(\partial_{x_{1}} Q\right)$ is negative after the change of variable but $x_{i}^{2} \partial_{x_{i}}^{2} Q$ is positive. Thus, we are left to evaluate

$$
\begin{aligned}
\int\left(\partial_{x_{1}} Q\right) \sum_{i \neq j}\left(x_{i} x_{j} \partial_{x_{i} x_{j}} Q\right)= & \int\left(\partial_{x_{1}} Q\right) \sum_{\substack{i=2 \\
i \neq j \\
j=1}}^{N}\left(x_{i} x_{1} \partial_{x_{i} x_{1}} Q\right)+\int\left(\partial_{x_{1}} Q\right) \sum_{\substack{j=2 \\
i \neq j \\
i=1}}^{N}\left(x_{1} x_{j} \partial_{x_{1} x_{j}} Q\right) \\
& +\int\left(\partial_{x_{1}} Q\right) \sum_{i, j=2}^{N}\left(x_{i} x_{j} \partial_{x_{i} x_{j}} Q\right)
\end{aligned}
$$

All the terms on the right are odd terms, which implies that $\int\left(\partial_{x_{1}} Q\right) \sum_{i \neq j}\left(x_{i} x_{j} \partial_{x_{i} x_{j}} Q\right)=$ 0 , and thus, $\int\left(\partial_{x_{1}} Q\right) x \cdot \nabla(x \cdot \nabla Q)=0$. This completes the proof of lemma.

Now we prove that under specific set of orthogonality conditions, the quadratic form generated by $L_{+}$and $L_{-}$is positive-definite.

Proposition 5.3.9 (I) There exists a constant $c_{1}>0$ such that for any radial function $h_{1} \in H^{\perp}$, we have

$$
\left(L_{+} h_{1}, h_{1}\right) \geq c_{1}\left\|h_{1}\right\|_{L^{2}\left(\mathbb{R}^{N}\right)}^{2},
$$

where $H^{\perp}=\left\{h_{1} \in H^{1}:\left(h_{1}, Q\right)=\left(h_{1}, x_{j} Q\right)=\left(h_{1},|x|^{2} Q\right)=0, j=1, \ldots, N\right\}$.

(II) There exists a constant $c_{2}>0$ such that for any radial function $h_{2} \in H_{\perp}$, we have

$$
\left(L_{-} h_{2}, h_{2}\right) \geq c_{2}\left\|h_{2}\right\|_{L^{2}\left(\mathbb{R}^{N}\right)}^{2},
$$

where $H_{\perp}=\left\{h_{2} \in H^{1}:\left(h_{2}, Q\right)=0\right\}$. 
Proof. Recall that the unique minimizer of (5.51) is attained at a ground state $Q$, and therefore,

$$
\left.\frac{d^{2}}{d \epsilon^{2}}\right|_{\epsilon=0} J(Q+\epsilon h) \geq 0, \quad \forall h \in C^{\infty}\left(\mathbb{R}^{N}\right),
$$

is equivalent to (for some constants $A, B>0$ )

$$
\left(L_{+} h_{1}, h_{1}\right) \geq A\left(\int Q h_{1}\right)^{2}-B \int Q h_{1} \int\left(|x|^{-(N-2)} * Q^{1+\frac{4}{N}}\right) Q^{\frac{4}{N}} h_{1}
$$

and

$$
\left(L_{-} h_{2}, h_{2}\right) \geq 0
$$

(I) By (5.66), we have that $\left(L_{+} h_{1}, h_{1}\right) \geq 0$ if $h_{1} \perp Q$.

Let $\eta=\left\{\left(L_{+} h_{1}, h_{1}\right):\left\|h_{1}\right\|_{L^{2}}=1,\left(h_{1}, Q\right)=\left(h_{1}, x_{j} Q\right)=\left(h_{1},|x|^{2} Q\right)=0\right\}$; $j=1, \ldots, N$, thus, $\eta \geq 0$. We argue by contradiction and assume that $\eta=0$. Consider $h_{1}^{*}$ satisfying

(i) $\left(L_{+} h_{1}^{*}, h_{1}^{*}\right)=0$

(ii) $\left(L_{+}-l\right) h_{1}^{*}=\beta_{0} Q+\beta_{j} x_{j} Q+\beta_{N+1}|x|^{2} Q ; j=1, \ldots, N$

(iii) $\left\|h_{1}\right\|_{L^{2}}=1$ with $\left(h_{1}, Q\right)=\left(h_{1}, x_{j} Q\right)=0 ; j=1, \ldots, N$.

Taking the scalar product of (ii) with $h_{1}^{*}$, we deduce from (iii) and (i) that $\left(L_{+} h_{1}^{*}, h_{1}^{*}\right)$ $=l=0$. Next, taking the scalar product of (ii) with $Q_{1}$, integrating by parts, and using $\left(Q, Q_{1}\right)=0$ and $\left(x_{j} Q, Q_{1}\right)=0$ (see Lemma 5.3.8) along with the fact that $L_{+} Q_{1}=-2 Q$, we have

$$
0=2\left(h_{1}^{*}, Q\right)=\beta_{N+1} \int|x|^{2} Q^{2},
$$

since $\int|x|^{2} Q^{2} \neq 0$, we find that $\beta_{N+1}=0$. Now taking the scalar product with $Q_{x_{j}}$ and using $\left(Q, Q_{x_{j}}\right)=0$ along with the fact that $Q_{x_{j}} \in \operatorname{ker} L_{+}$, we have

$$
0=\left(h_{1}^{*}, L_{+} Q_{x_{j}}\right)=\beta_{j} \int x_{j} Q Q_{x_{j}}
$$


since $\int x_{j} Q Q_{x_{j}}=\frac{1}{2} \int x_{j}\left(Q^{2}\right)_{x_{j}}=-\frac{1}{2} \int Q^{2} \neq 0$, we deduce that $\beta_{j}=0$ for $j=$ $1, \ldots, N$. Finally, taking the inner product with $\rho$ and recalling that $L_{+} \rho=-|x|^{2} Q$, we get

$$
0=-\left(h_{1}^{*},|x|^{2} Q\right)=\left(h_{1}^{*}, L_{+} \rho\right)=\beta_{0} \int \rho Q,
$$

since $\int \rho Q=-\frac{1}{2} \int \rho L_{+} Q_{1}=-\frac{1}{2} \int\left(L_{+} \rho\right) Q_{1}=\frac{1}{2} \int|x|^{2} Q Q_{1}=-\frac{1}{2} \int|x|^{2} Q^{2} \neq 0$, yielding $\beta_{0}=0$. This implies that $h_{1}^{*}=0$, which provides a contradiction, since $\left\|h_{1}^{*}\right\|_{L^{2}}=1$. Hence, $\eta>0$ and this completes the proof of (I).

(II) The proof is similar to the previous argument and follows easily by taking the inner product of $L_{-} h_{2}^{*}=\nu Q$ with $Q$. We omit the details.

This concludes the proof of Proposition 5.3.9.

Although Proposition 5.3.9 shows that eliminating certain directions would make the bilinear form $(L h, h)$ positive, the directions for $L_{+}$are not suitable for our analysis. An alternative is to consider the direction corresponding to the negative eigenvalue of $L_{+}$along with the kernel of $L_{+}$. We use the positivity of $L_{+}$under these (alternative) directions to prove the smallness of $\varepsilon$ in $H^{1}$ norm (see Lemma 5.3.15).

Lemma 5.3.10 Let $\chi_{0}$ be the positive radially symmetric eigenfunction associated to the only negative eigenvalue $-_{-}$(with $\left.e_{-}>0\right)$. Then, there exists $c_{3}>0$ such that for any $h \in H^{1}$ satisfying

$$
\left(h, \chi_{0}\right)=\left(h, Q_{x_{j}}\right)=0, \quad j=1, \ldots, N
$$

we have

$$
\left(L_{+} h, h\right) \geq c_{3}\|h\|_{L^{2}\left(\mathbb{R}^{N}\right)}^{2} .
$$

Proof. The result follows from Claim 5.3.5, which says that $L_{+}$has only one negative eigenvalue and Proposition 5.3.7, which gives us the kernel. Eliminating the negative direction (corresponding to negative eigenvalue) and kernel (corresponding to 0 eigenvalue) yields the desired result. 


\subsubsection{Conservation laws for $\varepsilon$}

Here, we return to a general setting in dimension $N$ and start with proving the mass and energy conservation for $\varepsilon$. Define

$$
\alpha_{0}=2 \int_{\mathbb{R}^{N}} Q(y) \varepsilon_{1}(y, 0) d y+\int_{\mathbb{R}^{N}}|\varepsilon(y, 0)|^{2} d y\left(=\int_{\mathbb{R}^{N}}\left|u_{0}\right|^{2}-\int_{\mathbb{R}^{N}} Q^{2}\right) .
$$

Lemma 5.3.11 For any $s \geq 0$, we have

1. Mass for $\varepsilon: M[\varepsilon(s)]=\alpha_{0}$.

2. Energy conservation for $\varepsilon: E[Q+\varepsilon(s)]=\lambda^{2}(s) E\left[u_{0}\right]$.

3. Energy linearization:

$$
E[Q+\varepsilon]+\left(\int_{\mathbb{R}^{N}} Q \varepsilon_{1}+\frac{1}{2} \int_{\mathbb{R}^{N}}|\varepsilon|^{2}\right)=\frac{1}{2}(L \varepsilon, \varepsilon)-\frac{N}{2(N+4)} \int_{\mathbb{R}^{N}} F(\varepsilon),
$$

where $(L \varepsilon, \varepsilon)=\left(L_{+} \varepsilon, \varepsilon\right)+\left(L_{-} \varepsilon, \varepsilon\right)$ and $F(\varepsilon)$ is given by

$$
\begin{aligned}
F(\varepsilon)= & \left(|y|^{-(N-2)} *|Q+\varepsilon|^{1+\frac{4}{N}}\right)|Q+\varepsilon|^{1+\frac{4}{N}}-\left(|y|^{-(N-2)} * Q^{1+\frac{4}{N}}\right) Q^{1+\frac{4}{N}} \\
& -2\left(1+\frac{4}{N}\right)\left(|y|^{-(N-2)} *|Q|^{1+\frac{4}{N}}\right)|Q|^{\frac{4}{N}} \varepsilon_{1} \\
& -\left(1+\frac{4}{N}\right)^{2}\left(|y|^{-(N-2)} *|Q|^{\frac{4}{N}} \varepsilon_{1}\right)|Q|^{\frac{4}{N}} \varepsilon_{1} \\
& -\left(1+\frac{4}{N}\right)\left(|y|^{-(N-2)} *|Q|^{1+\frac{4}{N}}\right)|Q|^{\frac{4}{N}-1}\left(\frac{4}{N} \varepsilon_{1}^{2}+\varepsilon_{2}^{2}\right)
\end{aligned}
$$

which is cubic in $\varepsilon$.

Proof. Let $v(y, s)=Q(y)+\varepsilon(y, s)$.

1. We have by the scaling invariance of $L^{2}$ norm and by conservation of mass

$$
\begin{aligned}
\int|v(y, s)|^{2} d y=\int \lambda^{N}(t)|u(\lambda(t) y+x(t), t)|^{2} d y & =\int|u(x, t)|^{2} d x \\
& =M[u(t)]=M[u(0)] .
\end{aligned}
$$


On the other hand, we obtain

$$
\begin{gathered}
\int|Q(y)+\varepsilon(y, s)|^{2} d y=\int|v(y, s)|^{2} d y \\
\int Q^{2}(y) d y+2 \int Q(y) \varepsilon_{1}(y, s) d y+\int|\varepsilon(y, s)|^{2} d y=\int\left|u_{0}(x)\right|^{2} d x \\
=\int Q^{2}(y) d y+2 \int Q(y) \varepsilon_{1}(y, 0) d y+\int|\varepsilon(y, 0)|^{2} d y
\end{gathered}
$$

thus,

$$
\begin{aligned}
M[\varepsilon(s)] & =2 \int Q(y) \varepsilon_{1}(y, s) d y+\int|\varepsilon(y, s)|^{2} d y \\
& =2 \int Q(y) \varepsilon_{1}(y, 0) d y+\int|\varepsilon(y, 0)|^{2} d y=\alpha_{0} .
\end{aligned}
$$

2. We have

$$
\begin{aligned}
E[Q & +\varepsilon(s)]=E[v(s)] \\
& =\frac{\lambda^{N+2}}{2} \int|\nabla u(\lambda(t) y+x(t), t)|^{2} d y \\
& -\frac{N \lambda^{N+4}}{2(N+4)} \iint \frac{\left|u\left(\lambda(t) y^{\prime}+x(t), t\right)\right|^{1+\frac{4}{N}}|u(\lambda(t) y+x(t), t)|^{1+\frac{4}{N}}}{\left|y-y^{\prime}\right|^{N-2}} d y d y^{\prime} \\
& =\frac{\lambda^{N+2}}{2 \lambda^{N}} \int|\nabla u(x, t)|^{2} d x-\frac{N \lambda^{N+4}}{2(N+4)} \frac{\lambda^{N-2}}{\lambda^{2 N}} \iint \frac{|u(y, t)|^{1+\frac{4}{N}}|u(x, t)|^{1+\frac{4}{N}}}{|x-y|^{N-2}} d x d y \\
& =\lambda^{2} E[u(t)]=\lambda^{2} E\left[u_{0}\right] .
\end{aligned}
$$

3. We have

$$
\begin{aligned}
E[Q+\varepsilon]= & \frac{1}{2} \int|\nabla Q+\nabla \varepsilon|^{2} d y \\
& +\frac{N}{2(N+4)} \iint \frac{\left|(Q+\varepsilon)\left(y^{\prime}\right)\right|^{1+\frac{4}{N}}|(Q+\varepsilon)(y)|^{1+\frac{4}{N}}}{\left|y-y^{\prime}\right|^{N-2}} d y d y^{\prime}
\end{aligned}
$$

Expanding $|Q+\varepsilon|^{1+\frac{4}{N}}$ and collecting the terms of zeroth, first and second order of $\epsilon$, we obtain 


$$
\begin{aligned}
E[Q+\varepsilon]= & E[Q]+\frac{1}{2} \int|\nabla \varepsilon|^{2}+\int \nabla Q \nabla \varepsilon_{1}+\int|\varepsilon|^{2}-\int|\varepsilon|^{2} \\
& -\frac{N}{2(N+4)} \int F(\varepsilon)-\int\left(|y|^{-(N-2)} * Q^{1+\frac{4}{N}}\right) Q^{\frac{4}{N}} \varepsilon_{1} \\
& -\frac{1}{2} \int\left(1+\frac{4}{N}\right)\left(|y|^{-(N-2)} * Q^{\frac{4}{N}} \varepsilon_{1}\right) Q^{\frac{4}{N}} \varepsilon_{1} \\
& -\frac{1}{2} \int\left(|y|^{-(N-2)} * Q^{1+\frac{4}{N}}\right) Q^{\frac{4}{N}-1}\left(\frac{4}{N} \varepsilon_{1}^{2}+\varepsilon_{2}^{2}\right),
\end{aligned}
$$

where $F(\varepsilon)$ is given by (5.71). Integrating by parts in the third term on the righthand side and using (5.38) along with (5.46) and (5.47) yields the desired result.

This completes the proof of Lemma 5.3.11.

\subsubsection{Choice of modulation parameters}

The following lemma is based on the variational property of $Q$ and shows the proximity of the solution to $Q$ up to scaling, phase and translation factors.

Lemma 5.3.12 Let $u \in H^{1}\left(\mathbb{R}^{N}\right)$ and $\alpha_{u}=\int_{\mathbb{R}^{N}}|u|^{2}-\int_{\mathbb{R}^{N}} Q^{2}$. There exists a $\delta_{1}>0$ such that the following is true. Assume that $E[u]<0$ and $\alpha_{u}<\delta_{1}$, then there exist parameters $x_{0} \in \mathbb{R}^{N}$ and $\gamma_{0} \in \mathbb{R}$ such that

$$
\left\|Q-e^{i \gamma_{0}} \lambda_{0}^{N / 2} u\left(\lambda_{0}\left(x+x_{0}\right)\right)\right\|_{H^{1}\left(\mathbb{R}^{N}\right)}<\delta\left(M_{u}\right)
$$

with $\lambda_{0}=\|\nabla Q\|_{L^{2}\left(\mathbb{R}^{N}\right)} /\|\nabla u\|_{L^{2}\left(\mathbb{R}^{N}\right)}$.

Proof. Recalling the variational structure of the function $Q$ associated with the GagliardoNirenberg inequality of convolution type (2.126) (see Lemma 2.8.1), we have that for $u \in H^{1}\left(\mathbb{R}^{N}\right)$

$$
E[u]=0, \quad \int_{\mathbb{R}^{N}}|u|^{2}=\int_{\mathbb{R}^{N}}|Q|^{2}, \quad \int_{\mathbb{R}^{N}}|\nabla u|^{2}=\int_{\mathbb{R}^{N}}|\nabla Q|^{2}
$$


is equivalent to $u=Q\left(\cdot+x_{0}\right)$ for some $x_{0} \in \mathbb{R}^{N}$. Let $u \in H^{1}(\mathbb{C})$ be such that $E[u]=0$ and $\int_{\mathbb{R}^{N}}|u|^{2}=\int_{\mathbb{R}^{N}}|Q|^{2}$. Thus, $|u| \in H^{1}\left(\mathbb{R}^{N}\right)$ satisfies $\int_{\mathbb{R}^{N}}(\nabla|u|)^{2} \leq \int_{\mathbb{R}^{N}}|\nabla u|^{2}$ such that $E[|u|] \leq E[u]=0$. On the other hand, from Gagliardo-Nirenberg inequality of convolution type (2.126) (for the function $|u|$ ), we have $E[|u|] \geq 0$. Therefore, $E[|u|]=$ $E[u]=0$ and $|u|=\lambda_{0}^{\frac{N}{2}} Q\left(\lambda_{0}\left(\cdot+x_{0}\right)\right)$ for some parameters $\lambda_{0}>0$ and $x_{0} \in \mathbb{R}^{N}$. This implies that $u$ does not vanish and we may write $u=|u| e^{i \theta(x)}$ and $\int_{\mathbb{R}^{N}}|\nabla u|^{2}=$ $\int_{\mathbb{R}^{N}}(\nabla|u|)^{2}+\int_{\mathbb{R}^{N}}|u|^{2}(\nabla \theta(x))^{2}$. We deduce from $E[|u|]=E[u]$ that $\theta(x)$ is constant. This means that if $u \in H^{1}(\mathbb{C})$ such that $E[u]=0$ and $\int_{\mathbb{R}^{N}}|u|^{2}=\int_{\mathbb{R}^{N}}|Q|^{2}$, then $u=$ $e^{i \gamma_{0}} \lambda_{0}^{\frac{N}{2}} Q\left(\lambda_{0}\left(\cdot+x_{0}\right)\right)$ for some parameters $\lambda_{0}>0, \gamma_{0} \in \mathbb{R}$ and $x_{0} \in \mathbb{R}^{N}$. Arguing by contradiction, we assume that there is a sequence $u_{n} \in H^{1}\left(\mathbb{R}^{N}\right)$ such that $E\left(u_{n}\right) \leq 0$ and $\int_{\mathbb{R}^{N}}\left|u_{n}\right|^{2} \rightarrow \int_{\mathbb{R}^{N}}|Q|^{2}$ as $n \rightarrow \infty$. Consider $v_{n}=\lambda_{n}^{\frac{N}{2}} u_{n}\left(\lambda_{n} x\right)$, where $\lambda_{n}=\frac{\|\nabla Q\|_{L^{2}}}{\left\|\nabla u_{n}\right\|_{L^{2}}}$. We have that $v_{n}$ satisfies

$$
\int_{\mathbb{R}^{N}}\left|v_{n}\right|^{2} \rightarrow \int_{\mathbb{R}^{N}}|Q|^{2}, \quad \int_{\mathbb{R}^{N}}\left|\nabla v_{n}\right|^{2}=\int_{\mathbb{R}^{N}}|\nabla Q|^{2}, \quad \text { and } \quad E\left[v_{n}\right]<0 .
$$

And, from Gagliardo-Nirenberg inequality (2.126),

$$
E\left[v_{n}\right] \geq \frac{1}{2}\left(\int_{\mathbb{R}^{N}}\left|\nabla v_{n}\right|^{2}\right)\left(1-\left(\frac{\int_{\mathbb{R}^{N}}\left|v_{n}\right|^{2}}{\int_{\mathbb{R}^{N}}|Q|^{2}}\right)^{\frac{8}{N}}\right),
$$

which implies that $E\left[v_{n}\right] \rightarrow 0$. Adapting the concentration compactness principle (see [Lio84a, Lemma III.1]), there exists $x_{n} \in \mathbb{R}^{N}$ and $\gamma_{n} \in \mathbb{R}^{N}$ such that $e^{i \gamma_{n}} v_{n}\left(x+x_{n}\right) \rightarrow Q$ strongly in $H^{1}$ (see again [Lio84a, Theorem III.1]) as desired.

For $u \in H^{1}, \lambda_{1}>0, \gamma_{1} \in \mathbb{R}$ and $x_{1} \in \mathbb{R}^{N}$, we now define the following decomposition of the solution

$$
\varepsilon_{\lambda_{1}, \gamma_{1}, x_{1}}(y)=e^{i \gamma_{1}} \lambda_{1}^{N / 2} u\left(\lambda_{1} y+x_{1}\right)-Q(y)
$$

such that $\varepsilon$ satisfies particular set of orthogonality conditions. For $\alpha>0$, let

$$
U_{\alpha}=\left\{u \in H^{1}:\|u-Q\|_{H^{1}} \leq \alpha\right\} .
$$


Lemma 5.3.13 There exists $\bar{\alpha}>0$ and a unique $C^{1}$ map

$$
\left(\lambda_{1}, \gamma_{1}, x_{1}\right): U_{\bar{\alpha}} \rightarrow(1-\bar{\lambda}, 1+\bar{\lambda}) \times \mathbb{R} \times \mathbb{R}^{N}
$$

such that if $u \in U_{\bar{\alpha}}$ and $\varepsilon_{\lambda_{1}, \gamma_{1}, x_{1}}(y)$ is given by (5.73), then

$$
\left(\varepsilon_{\lambda_{1}, \gamma_{1}, x_{1}}\right)_{1} \perp y_{j} Q, \quad\left(\varepsilon_{\lambda_{1}, \gamma_{1}, x_{1}}\right)_{1} \perp Q_{1}+Q_{2} \quad \text { and } \quad\left(\varepsilon_{\lambda_{1}, \gamma_{1}, x_{1}}\right)_{2} \perp Q_{2}
$$

where $j=1, \ldots, N$ and $\varepsilon_{\lambda_{1}, \gamma_{1}, x_{1}}=\left(\varepsilon_{\lambda_{1}, \gamma_{1}, x_{1}}\right)_{1}+i\left(\varepsilon_{\lambda_{1}, \gamma_{1}, x_{1}}\right)_{2}$. Moreover, if $u \in U_{\alpha}$, with $0<\alpha<\bar{\alpha}$, then

$$
\left\|\varepsilon_{\lambda_{1}, \gamma_{1}, x_{1}}\right\|_{H^{1}}+\left|\lambda_{1}-1\right|+\left|\gamma_{1}\right|+\left|x_{1}\right| \leq C_{1} \alpha
$$

Proof. Let $\varepsilon_{\lambda_{1}, \gamma_{1}, x_{1}}$ be as in (5.73). Differentiating (5.73), we have

$$
\begin{gathered}
\left.\frac{\partial \varepsilon_{\lambda_{1}, \gamma_{1}, x_{1}}}{\partial\left(x_{1}\right)_{i}}\right|_{\lambda_{1}=1, \gamma_{1}=0, x_{1}=0}=u_{y_{j}}(y), \quad j=1, \ldots, N, \\
\left.\frac{\partial \varepsilon_{\lambda_{1}, \gamma_{1}, x_{1}}}{\partial \lambda_{1}}\right|_{\lambda_{1}=1, \gamma_{1}=0, x_{1}=0}=\frac{N}{2} u(y)+y \cdot \nabla u(y)=u_{1}
\end{gathered}
$$

and

$$
\left.\frac{\partial \varepsilon_{\lambda_{1}, \gamma_{1}, x_{1}}}{\partial \gamma_{1}}\right|_{\lambda_{1}=1, \gamma_{1}=0, x_{1}=0}=i u(y)
$$

We define the following functionals

$$
\begin{gathered}
\rho_{\lambda_{1}, \gamma_{1}, x_{1}}^{i}(u)=\int\left(\varepsilon_{\lambda_{1}, \gamma_{1}, x_{1}}\right)_{1} y_{j} Q, \quad 1 \leq j \leq N \\
\rho_{\lambda_{1}, \gamma_{1}, x_{1}}^{N+1}(u)=\int\left(\varepsilon_{\lambda_{1}, \gamma_{1}, x_{1}}\right)_{1}\left(Q_{1}+Q_{2}\right)
\end{gathered}
$$

and

$$
\rho_{\lambda_{1}, \gamma_{1}, x_{1}}^{N+2}(u)=\int\left(\varepsilon_{\lambda_{1}, \gamma_{1}, x_{1}}\right)_{2} Q_{2}
$$


From (5.77)-(5.79), the first column of the Jacobian matrix is given by

$$
\begin{aligned}
\left.\frac{\partial \rho_{\lambda_{1}, \gamma_{1}, x_{1}}^{1}(u)}{\partial\left(x_{1}\right)_{1}}\right|_{\lambda_{1}=1, \gamma_{1}=0, x_{1}=0, u=Q} & =\int Q_{y_{1}} y_{1} Q=-\frac{1}{2} \int Q^{2} \\
\left.\frac{\partial \rho_{\lambda_{1}, \gamma_{1}, x_{1}}^{2}(u)}{\partial\left(x_{1}\right)_{1}}\right|_{\lambda_{1}=1, \gamma_{1}=0, x_{1}=0, u=Q} & =\int Q_{y_{1}} y_{2} Q=0 \\
\vdots & =\int Q_{y_{1}} y_{N} Q=0 \\
\left.\frac{\partial \rho_{\lambda_{1}, \gamma_{1}, x_{1}}^{N}(u)}{\partial\left(x_{1}\right)_{1}}\right|_{\lambda_{1}=1, \gamma_{1}=0, x_{1}=0, u=Q} & =\int Q_{y_{1}}\left(Q_{1}+Q_{2}\right)=0 \\
\left.\frac{\partial \rho_{\lambda_{1}, \gamma_{1}, x_{1}}^{N+1}(u)}{\partial\left(x_{1}\right)_{1}}\right|_{\lambda_{1}=1, \gamma_{1}=0, x_{1}=0, u=Q} & =\int 0\left(Q_{2}\right)=0 \\
\left.\frac{\partial \rho_{\lambda_{1}, \gamma_{1}, x_{1}}^{N+2}(u)}{\partial\left(x_{1}\right)_{1}}\right|_{\lambda_{1}=1, \gamma_{1}=0, x_{1}=0, u=Q} &
\end{aligned}
$$

the second column will be

$$
\begin{aligned}
\left.\frac{\partial \rho_{\lambda_{1}, \gamma_{1}, x_{1}}^{1}(u)}{\partial\left(x_{1}\right)_{2}}\right|_{\lambda_{1}=1, \gamma_{1}=0, x_{1}=0, u=Q} & =\int Q_{y_{2}} y_{1} Q=0 \\
\left.\frac{\partial \rho_{\lambda_{1}, \gamma_{1}, x_{1}}^{2}(u)}{\partial\left(x_{1}\right)_{2}}\right|_{\lambda_{1}=1, \gamma_{1}=0, x_{1}=0, u=Q} & =\int Q_{y_{2}} y_{2} Q=-\frac{1}{2} \int Q^{2} \\
\vdots & =\int Q_{y_{2}} y_{N} Q=0 \\
\left.\frac{\partial \rho_{\lambda_{1}, \gamma_{1}, x_{1}}^{N}(u)}{\partial\left(x_{1}\right)_{2}}\right|_{\lambda_{1}=1, \gamma_{1}=0, x_{1}=0, u=Q} & =\int Q_{y_{2}}\left(Q_{1}+Q_{2}\right)=0 \\
\left.\frac{\partial \rho_{\lambda_{1}, \gamma_{1}, x_{1}}^{N+1}(u)}{\partial\left(x_{1}\right)_{2}}\right|_{\lambda_{1}=1, \gamma_{1}=0, x_{1}=0, u=Q} & =\int 0\left(Q_{2}\right)=0 \\
\left.\frac{\partial \rho_{\lambda_{1}, \gamma_{1}, x_{1}}^{N+2}(u)}{\partial\left(x_{1}\right)_{2}}\right|_{\lambda_{1}=1, \gamma_{1}=0, x_{1}=0, u=Q} & =\int
\end{aligned}
$$


similarly, the Nth column is

$$
\begin{aligned}
\left.\frac{\partial \rho_{\lambda_{1}, \gamma_{1}, x_{1}}^{1}(u)}{\partial\left(x_{1}\right)_{N}}\right|_{\lambda_{1}=1, \gamma_{1}=0, x_{1}=0, u=Q} & =\int Q_{y_{N}} y_{1} Q=0 ; \\
\left.\frac{\partial \rho_{\lambda_{1}, \gamma_{1}, x_{1}}^{2}(u)}{\partial\left(x_{1}\right)_{2}}\right|_{\lambda_{1}=1, \gamma_{1}=0, x_{1}=0, u=Q} & =\int Q_{y_{N}} y_{2} Q=0 ; \\
\vdots & =\int Q_{y_{2}} y_{N} Q=-\frac{1}{2} \int Q^{2} ; \\
\left.\frac{\partial \rho_{\lambda_{1}, \gamma_{1}, x_{1}}^{N}(u)}{\partial\left(x_{1}\right)_{N}}\right|_{\lambda_{1}=1, \gamma_{1}=0, x_{1}=0, u=Q} & =\int Q_{y_{N}}\left(Q_{1}+Q_{2}\right)=0 ; \\
\left.\frac{\partial \rho_{\lambda_{1}, \gamma_{1}, x_{1}}^{N+1}(u)}{\partial\left(x_{1}\right)_{N}}\right|_{\lambda_{1}=1, \gamma_{1}=0, x_{1}=0, u=Q} & =\int 0\left(Q_{2}\right)=0 ; \\
\left.\frac{\partial \rho_{\lambda_{1}, \gamma_{1}, x_{1}}^{N+2}(u)}{\partial\left(x_{1}\right)_{N}}\right|_{\lambda_{1}=1, \gamma_{1}=0, x_{1}=0, u=Q} & =\int
\end{aligned}
$$

the $(\mathrm{N}+1)$ th column

$$
\begin{aligned}
\left.\frac{\partial \rho_{\lambda_{1}, \gamma_{1}, x_{1}}^{1}(u)}{\partial \lambda_{1}}\right|_{\lambda_{1}=1, \gamma_{1}=0, x_{1}=0, u=Q} & =\int Q_{1} y_{1} Q=0 ; \\
\left.\frac{\partial \rho_{\lambda_{1}, \gamma_{1}, x_{1}}^{2}(u)}{\partial \lambda_{1}}\right|_{\lambda_{1}=1, \gamma_{1}=0, x_{1}=0, u=Q} & =\int Q_{1} y_{2} Q=0 ; \\
& =\int Q_{1} y_{N} Q=0 ; \\
\left.\frac{\partial \rho_{\lambda_{1}, \gamma_{1}, x_{1}}^{N}(u)}{\partial \lambda_{1}}\right|_{\lambda_{1}=1, \gamma_{1}=0, x_{1}=0, u=Q} & =\int Q_{1}\left(Q_{1}+Q_{2}\right)=\int Q_{1}^{2} ; \\
\left.\frac{\partial \rho_{\lambda_{1}, \gamma_{1}, x_{1}}^{N+1}(u)}{\partial \lambda_{1}}\right|_{\lambda_{1}=1, \gamma_{1}=0, x_{1}=0, u=Q} & =\int 0\left(Q_{2}\right)=0 ;
\end{aligned}
$$


finally, the $(\mathrm{N}+2)$ th column

$$
\begin{aligned}
\left.\frac{\partial \rho_{\lambda_{1}, \gamma_{1}, x_{1}}^{1}(u)}{\partial \gamma_{1}}\right|_{\lambda_{1}=1, \gamma_{1}=0, x_{1}=0, u=Q} & =\int 0 y_{1} Q=0 \\
\left.\frac{\partial \rho_{\lambda_{1}, \gamma_{1}, x_{1}}^{2}(u)}{\partial \gamma_{1}}\right|_{\lambda_{1}=1, \gamma_{1}=0, x_{1}=0, u=Q} & =\int 0 y_{2} Q=0 \\
\vdots & =\int 0 y_{N} Q=0 \\
\left.\frac{\partial \rho_{\lambda_{1}, \gamma_{1}, x_{1}}^{N}(u)}{\partial \gamma_{1}}\right|_{\lambda_{1}=1, \gamma_{1}=0, x_{1}=0, u=Q} & =\int 0\left(Q_{1}+Q_{2}\right)=0 \\
\left.\frac{\partial \rho_{\lambda_{1}, \gamma_{1}, x_{1}}^{N+1}(u)}{\partial \gamma_{1}}\right|_{\lambda_{1}=1, \gamma_{1}=0, x_{1}=0, u=Q} & =\int Q\left(Q_{2}\right)=-\int Q_{1}^{2} . \\
\left.\frac{\partial \rho_{\lambda_{1}, \gamma_{1}, x_{1}}^{N+2}(u)}{\partial \gamma_{1}}\right|_{\lambda_{1}=1, \gamma_{1}=0, x_{1}=0, u=Q} & =\int
\end{aligned}
$$

Note that at $\lambda_{1}=1, \gamma_{1}=0, x_{1}=0, u=Q$,

$$
\left(\rho_{\lambda_{1}, \gamma_{1}, x_{1}}^{1}(u), \rho_{\lambda_{1}, \gamma_{1}, x_{1}}^{2}(u), \ldots, \rho_{\lambda_{1}, \gamma_{1}, x_{1}}^{N}(u), \rho_{\lambda_{1}, \gamma_{1}, x_{1}}^{N+1}(u), \rho_{\lambda_{1}, \gamma_{1}, x_{1}}^{N+2}(u)\right)=(0,0, \ldots, 0,0,0)
$$

and the Jacobian matrix is nonzero, thus, we can apply the Implicit Function theorem to obtain the existence of $\bar{\alpha}>0$, a neighborhood $V$ of $(1,0,0)$ in $\mathbb{R}^{N+2}$ and a unique $C^{1}$ $\operatorname{map}\left(\lambda_{1}, \gamma_{1}, x_{1}\right):\left\{u \in H^{1}:\|u-Q\|_{H^{1}}<\bar{\alpha}\right\} \rightarrow V$ such that orthogonality conditions (5.75) are satisfied.

Note that since $\left(\lambda_{1}, \gamma_{1}, x_{1}\right)$ is sufficiently close to $(1,0,0)$, there exists a $C_{1}>0$ such that if $\|u-Q\|_{H^{1}}<\alpha<\bar{\beta}$, then $\left|\lambda_{1}-1\right|+\left|\gamma_{1}\right|+\left|x_{1}\right| \leq C_{1} \alpha$. Moreover, by the expression of $\varepsilon_{\lambda_{1}, \gamma_{1}, x_{1}}$ in (5.73), we also get $\left\|\varepsilon_{\lambda_{1}, \gamma_{1}, x_{1}}\right\|_{H^{1}} \leq C_{1} \alpha$, for some $C_{1}>0$.

Assume that $u(t) \in U_{\bar{\alpha}}$ for all time $u(t)$ is defined, and thus, by energy conservation, we have $E[u(t)]=E\left[u_{0}\right]<0$ for all time $u(t)$ is defined. Observe that since one can always assume a smaller $\delta_{1}$ by the continuity of $u(t)$ with respect to $t$ on a closed time interval before the blow-up time such that $\alpha_{0}<\delta_{1}$ for all time $u(t)$ is defined, and therefore, by Lemma 5.3.12 there exists $x_{0}(t) \in \mathbb{R}^{N}$ and $\gamma_{0}(t) \in \mathbb{R}$ such that

$$
\left\|Q-e^{i \gamma_{0}(t)} \lambda_{0}(t)^{N / 2} u\left(\lambda_{0}(t)\left(x+x_{0}(t)\right)\right)\right\|_{H^{1}\left(\mathbb{R}^{N}\right)}<\delta\left(\alpha_{0}\right),
$$


where $\lambda_{0}(t)=\|\nabla Q\|_{L^{2}\left(\mathbb{R}^{N}\right)} /\|\nabla u(t)\|_{L^{2}\left(\mathbb{R}^{N}\right)}$. Taking $\delta_{2}>0$ such that $\alpha_{0}<\delta_{2}$ and $\delta\left(\alpha_{0}\right)<\delta\left(\delta_{2}\right)<\bar{\alpha}$. Then, for all time $u(t)$ is defined, we have

$$
\left\|Q-e^{i \gamma_{0}(t)} \lambda_{0}(t)^{N / 2} u\left(\lambda_{0}(t)\left(x+x_{0}(t)\right)\right)\right\|_{H^{1}\left(\mathbb{R}^{N}\right)}<\bar{\alpha}
$$

Thus, applying Lemma 5.3.13, we choose the parameters $\lambda(t)>0, x(t) \in \mathbb{R}^{N}$ and $\gamma(t) \in \mathbb{R}$ close to $\lambda_{0}(t), x_{0}(t)$ and $x_{0}(t)$ such that

$$
\varepsilon(y, t)=\varepsilon_{\lambda(t), x(t), \gamma(t)}=e^{i \gamma(t)} \lambda^{\frac{N}{2}}(t) u(\lambda(t) y+x(t), t)-Q(y),
$$

where $\varepsilon=\varepsilon_{1}+i \varepsilon_{2}$ satisfy

$$
\left(\varepsilon_{1}, y_{i} Q\right)=\left(\varepsilon_{1}, Q_{1}+Q_{2}\right)=0 \quad \text { and } \quad\left(\varepsilon_{2}, Q_{2}\right)=0
$$

Moreover,

$$
\|\varepsilon(t)\|_{H^{1}} \leq \delta\left(\alpha_{0}\right), \quad \text { where } \quad \delta\left(\alpha_{0}\right) \rightarrow 0 \quad \text { as } \quad \alpha_{0} \rightarrow 0
$$

\subsubsection{Control of parameters}

Next, we need to understand how various parameters are controlled and related to each other. We begin with the following lemma.

Lemma 5.3.14 Let $P(y)$ is any polynomial and if $\|\varepsilon(s)\|_{H^{1}} \leq 1$ then

1. First order terms are controlled by

$$
\left|\left(\varepsilon_{1,2}, P(y) \frac{d^{k}}{d y^{k}} Q(y)\right)\right| \leq C(P, k)\left(\int_{\mathbb{R}^{N}}|\nabla \varepsilon|^{2}\right)^{1 / 2},
$$

where $0 \leq k \leq 3$.

2. Second order terms are controlled by

$$
\left|\left(R(\varepsilon), P(y) \frac{d^{k}}{d y^{k}} Q(y)\right)\right| \leq C(P, k)\left(\int_{\mathbb{R}^{N}}|\nabla \varepsilon|^{2}\right),
$$

where $R(\varepsilon)=R_{1}(\varepsilon)+i R_{2}(\varepsilon)$ and $R_{1}(\varepsilon), R_{2}(\varepsilon)$ is given by (5.48), (5.49), respectively. 


\section{Third order terms are controlled by}

$$
\int_{\mathbb{R}^{N}}|F(\varepsilon)|+\left|\left(\tilde{R}_{1}(\varepsilon), P(y) \frac{d^{k}}{d y^{k}} Q(y)\right)\right| \leq C\|\varepsilon\|_{H^{1}}\left(\int_{\mathbb{R}^{N}}|\nabla \varepsilon|^{2}\right),
$$

where $F(\varepsilon)$ is given by (5.71) and

$$
\begin{aligned}
\tilde{R}_{1}(\varepsilon)= & R_{1}(\varepsilon)-\left(|y|^{-(N-2)} * Q^{\frac{4}{N}} \varepsilon_{1}\right) Q^{\frac{4}{N}-1} \varepsilon_{1} \\
& +\left(|y|^{-(N-2)} * Q^{\frac{4}{N}-1} \varepsilon_{1}^{2}\right) Q^{\frac{4}{N}}+\left(|y|^{-(N-2)} * Q^{1+\frac{4}{N}}\right) Q^{\frac{4}{N}-2} \varepsilon_{1}^{2} .
\end{aligned}
$$

is the cubic part of $R_{1}(\varepsilon)$.

Proof. Since the function $Q$ and all of its derivatives decays exponentially at $+\infty$ and $-\infty$, i.e., $\left|P(y) \frac{d^{k}}{d y^{k}} Q(y)\right| \leq C(P, k) e^{-|y|}$, we have the following controls

1. Using Sobolev inequality $\|\varepsilon\|_{L^{\frac{2 N}{N-2}}} \leq C\|\nabla \varepsilon\|_{L^{2}}$, first order terms are estimated as

$$
\left|\left(\varepsilon_{1,2}, P(y) \frac{d^{k}}{d y^{k}} Q(y)\right)\right| \leq C(P, k)\|\varepsilon\|_{L^{\frac{2 N}{N-2}}} \leq C(P, k)\|\nabla \varepsilon\|_{L^{2}} .
$$

2. Control of second order terms: we consider the sets

$$
E_{x}=\left\{\varepsilon(x) \in H^{1}: Q(x) \gg \varepsilon(x)\right\} \quad \text { and } \quad E_{y}=\left\{\varepsilon(y) \in H^{1}: Q(y) \gg \varepsilon(y)\right\},
$$

and since the behavior of $Q$ and its derivative is similar (exponentially decaying), we write for simplicity the inner product in (2) as follows

$$
\begin{aligned}
& \int R(\varepsilon) Q d x=\iint_{E_{x} \cup E_{y}} \frac{|(Q+\varepsilon)(y)|^{1+\frac{4}{N}}|(Q+\varepsilon)(x)|^{\frac{4}{N}-1}(Q+\varepsilon)(x) Q(x)}{|x-y|^{N-2}} d x d y \\
& +\iint_{E_{x}^{c} \cup E_{y}} \frac{|(Q+\varepsilon)(y)|^{1+\frac{4}{N}}|(Q+\varepsilon)(x)|^{\frac{4}{N}-1}(Q+\varepsilon)(x) Q(x)}{|x-y|^{N-2}} d x d y \\
& +\iint_{E_{x} \cup E_{y}^{c}} \frac{|(Q+\varepsilon)(y)|^{1+\frac{4}{N}}|(Q+\varepsilon)(x)|^{\frac{4}{N}-1}(Q+\varepsilon)(x) Q(x)}{|x-y|^{N-2}} d x d y \\
& +\iint_{E_{x}^{c} \cup E_{y}^{c}} \frac{|(Q+\varepsilon)(y)|^{1+\frac{4}{N}}|(Q+\varepsilon)(x)|^{\frac{4}{N}-1}(Q+\varepsilon)(x) Q(x)}{|x-y|^{N-2}} d x d y \\
& \quad-\iint \frac{Q^{1+\frac{4}{N}}(y) Q^{1+\frac{4}{N}}(x)}{|x-y|^{N-2}} d x d y-\iint \frac{Q^{1+\frac{4}{N}}(y) Q^{\frac{4}{N}}(x) \varepsilon(x)}{|x-y|^{N-2}} d x d y \\
& \quad-\iint \frac{Q^{\frac{4}{N}}(y) \varepsilon(y) Q^{1+\frac{4}{N}}(x)}{|x-y|^{N-2}} d x d y .
\end{aligned}
$$


We first consider the term on $E_{x} \cup E_{y}$ and expand $|Q+\varepsilon|^{1+\frac{4}{N}}$ and $|Q+\varepsilon|^{\frac{4}{N}-1}$ to obtain

$$
\begin{aligned}
\int_{E_{x} \cup E_{y}} R(\varepsilon) Q(x) d x \lesssim & \iint \frac{Q^{1+\frac{4}{N}}(y) Q^{\frac{4}{N}-1}(x)|\varepsilon(x)|^{2}}{|x-y|^{N-2}} d x d y \\
& +\iint \frac{Q^{\frac{4}{N}}(y)|\varepsilon(y)| Q^{\frac{4}{N}}(x)|\varepsilon(x)|}{|x-y|^{N-2}} d x d y
\end{aligned}
$$

For the terms on $E_{x}^{c} \cup E_{y}$, we only expand $|Q+\varepsilon|^{1+\frac{4}{N}}$ and use the fact that $|Q+\varepsilon| \leq$ $2 \varepsilon$ on $E_{x}^{c}$, we get

$$
\int_{E_{x}^{c} \cup E_{y}} R(\varepsilon) Q(x) d x \lesssim \iint \frac{Q^{1+\frac{4}{N}}(y)|\varepsilon(x)|^{1+\frac{4}{N}}}{|x-y|^{N-2}} d x d y .
$$

Next, the set $E_{x} \cup E_{y}^{c}$ is symmetric to the previous one and treated similarly (expanding the term $|Q+\varepsilon|^{\frac{4}{N}-1}$ and using the triangle inequality on the other term), we obtain

$$
\int_{E_{x} \cup E_{y}^{c}} R(\varepsilon) Q(x) d x \lesssim \iint \frac{Q^{1+\frac{4}{N}}(x)|\varepsilon(y)|^{1+\frac{4}{N}}}{|x-y|^{N-2}} d x d y .
$$

Finally, we observe (by triangle inequality) that the term on $E_{x}^{c} \cup E_{y}^{c}$ is of higher order. Therefore, from (5.83), (5.84) and (5.85), we have

$$
\begin{aligned}
\left|\int R(\varepsilon) Q d x\right| \lesssim \mid \iint \frac{Q^{1+\frac{4}{N}}(y) Q^{\frac{4}{N}}-1}{|x-y|^{N-2}|\varepsilon(x)|^{2}} \\
|x d y| \\
+\left|\iint \frac{Q^{\frac{4}{N}}(y)|\varepsilon(y)| Q^{\frac{4}{N}}(x)|\varepsilon(x)|}{|x-y|^{N-2}} d x d y\right| \\
+\left|\iint \frac{Q^{1+\frac{4}{N}}(y)|\varepsilon(x)|^{1+\frac{4}{N}}}{|x-y|^{N-2}} d x d y\right| .
\end{aligned}
$$

Using Hölder's and Hardy-Littlewood-Sobolev inequalities, we obtain

$$
\begin{aligned}
\left|\int R(\varepsilon) Q d x\right| \lesssim & \|Q\|_{L^{\frac{2(N+4)}{N+2}}}^{1+\frac{4}{N}}\|Q\|_{L^{\infty}}^{\frac{4}{N}-1}\|\varepsilon\|_{L^{\frac{4 N}{N+2}}}^{2} \\
& +\|Q\|_{L^{2}}^{\frac{8}{N}}\|\varepsilon\|_{L^{\frac{2 N}{N-2}}}^{2}+\|Q\|_{L^{\frac{2(N+4)}{N+2}}}^{1+\frac{4}{N}}\|\varepsilon\|_{L^{\frac{2(N+4)}{N+2}}}^{1+\frac{4}{N}} \\
& \lesssim\|\nabla \varepsilon\|_{L^{2}}^{2}+\|\nabla \varepsilon\|_{L^{2}}^{2}+\|\nabla \varepsilon\|_{L^{2}}^{1+\frac{4}{N}} \lesssim\|\nabla \varepsilon\|_{L^{2}}^{2}
\end{aligned}
$$


where in the second inequality, we have used the fact that $2<\frac{2(N+4)}{N+2}<\frac{2 N}{N-2}$ and for $2<N<6,2<\frac{4 N}{N+2}<\frac{2 N}{N-2}$.

3. Third order terms are also controlled using the similar approach as above, thus, we have

$$
\begin{aligned}
&\left|\int F(\varepsilon) d x+\int \tilde{R}_{1}(\varepsilon) Q d x\right| \lesssim\left|\iint \frac{Q^{1+\frac{4}{N}}(y)|\varepsilon(x)|^{1+\frac{4}{N}}}{|x-y|^{N-2}} d x d y\right| \\
&+\left|\iint \frac{Q^{\frac{4}{N}}(y)|\varepsilon(y)| Q^{\frac{4}{N}-1}(x)|\varepsilon(x)|^{2}}{|x-y|^{N-2}} d x d y\right| \\
& \lesssim\|Q\|_{L^{\frac{2(N+4)}{N+2}}}^{1+\frac{4}{N}}\|\varepsilon\|_{L^{\frac{2(N+4)}{N+2}}}^{1+\frac{4}{N}}+\|Q\|_{L^{2}}^{\frac{4}{N}}\|\varepsilon\|_{L^{\frac{2 N}{N-2}}}\|Q\|_{L^{\infty}}^{\frac{4}{N}-1}\|\varepsilon\|_{L^{\frac{4 N}{N+2}}}^{2} \\
& \lesssim\left(\|\nabla \varepsilon\|_{L^{2}}^{\frac{4}{N}-1}+\|\nabla \varepsilon\|_{L^{2}}\right)\|\nabla \varepsilon\|_{L^{2}}^{2} \lesssim\|\varepsilon\|_{H^{1}}^{\frac{4}{N}-1}\|\nabla \varepsilon\|_{L^{2}}^{2},
\end{aligned}
$$

where in the last step we have used $\|\varepsilon\|_{H^{1}} \leq 1$ implicitly.

This concludes the proof of Lemma 5.3.14.

Next, we establish the control on $\|\varepsilon(s)\|_{H^{1}}$.

Lemma 5.3.15 There exists $\delta_{3}>0$ such that if $\|\varepsilon(s)\|_{H^{1}} \leq \delta\left(\alpha_{0}\right)$ for $\alpha_{0}<\delta_{3}$ and $\varepsilon(s)$ satisfies (5.75) from Lemma 5.3.13 for all $s \geq 0$, then there exists a universal constant $C_{3}>0$ such that

$$
\|\varepsilon(s)\|_{H^{1}} \leq C_{3} \sqrt{\alpha_{0}}
$$

where $\alpha_{0}$ is given by (5.69).

Proof. From (5.70), we have

$$
(L \varepsilon(s), \varepsilon(s))=2 E[Q+\varepsilon(s)]+M_{0}+\frac{N}{N+4} \int F(\varepsilon(s)) .
$$


We estimate using the computation in Lemma 5.3.14

$$
\begin{aligned}
\int|F(\varepsilon(s))| \lesssim & \left|\iint \frac{Q^{1+\frac{4}{N}}\left(y^{\prime}, s\right)|\varepsilon(y, s)|^{1+\frac{4}{N}}}{\left|y-y^{\prime}\right|^{N-2}} d y d y^{\prime}\right| \\
& +\left|\iint \frac{Q^{\frac{4}{N}}\left(y^{\prime}, s\right)\left|\varepsilon\left(y^{\prime}, s\right)\right| Q^{\frac{4}{N}-1}(y, s)|\varepsilon(y, s)|^{2}}{\left|y-y^{\prime}\right|^{N-2}} d y d y^{\prime}\right| \\
& \lesssim\left\|\left(|y|^{-(N-2)} * Q^{1+\frac{4}{N}}\right)\right\|_{L^{\infty}}\|\varepsilon(s)\|_{L^{2}}^{2}\|\varepsilon(s)\|_{L^{\infty}}^{\frac{4}{N}-1} \\
& +\left\|\left(|y|^{-(N-2)} * Q^{\frac{4}{N}} \varepsilon\right)\right\|_{L^{\infty}}\|Q\|_{L^{\infty}}^{\frac{4}{N}-1}\|\varepsilon(s)\|_{L^{2}}^{2} .
\end{aligned}
$$

Using Hardy-Littlewood-Sobolev and the fact that $\|\varepsilon(s)\|_{L^{\infty}} \lesssim\|\varepsilon(s)\|_{H^{1}} \leq 1$, we get

$$
\int|F(\varepsilon(s))| \lesssim\|\varepsilon(s)\|_{H^{1}}^{\frac{4}{N}-1}\|\varepsilon(s)\|_{L^{2}}^{2}
$$

Therefore, there exists a universal constant $C_{2}>0$ such that using Lemma 5.3.11 part (2), (5.87) can be estimated as

$$
(L \varepsilon(s), \varepsilon(s)) \leq M_{0}+C_{2}\|\varepsilon(s)\|_{H^{1}}^{\frac{4}{N}-1}\|\varepsilon(s)\|_{L^{2}}^{2} .
$$

Now, we consider a translated function

$$
\tilde{\varepsilon}(s)=\varepsilon(s)-a Q_{x_{j}}-b Q_{1}-i c Q
$$

where $\varepsilon(s)=\varepsilon_{1}(s)+i \varepsilon_{2}(s)$ satisfies Lemma 5.3.13 and $\tilde{\varepsilon}(s)=\tilde{\varepsilon}_{1}(s)+i \tilde{\varepsilon}_{2}(s)$. We wish to compare the $L^{2}$ norm of $\varepsilon(s)$ with the $L^{2}$ norm of $\tilde{\varepsilon}(s)$. Taking the scalar product of the real part of (5.90) with $\chi_{0}$ (eigenfunction corresponding to the only negative eigenvalue of $L_{+}$, defined in Lemma 5.3.10), we have

$$
\left(\tilde{\varepsilon}_{1}(s), \chi_{0}\right)=0 \quad \text { with } \quad b=\frac{e_{-}\left(\varepsilon_{1}(s), \chi_{0}\right)}{2\left(Q, \chi_{0}\right)}
$$

where $e_{-}$is the only negative eigenvalue of $L_{+}$. Here, we have used the fact that $\left(Q_{x_{j}}, \chi_{0}\right)=$ 0 and $\left(Q_{1}, \chi_{0}\right)=\frac{2}{e_{-}}\left(Q, \chi_{0}\right)$. Now, taking the scalar product of the real part of (5.90) with $Q_{x_{j}}$, we obtain

$$
\left(\tilde{\varepsilon}_{1}(s), Q_{x_{j}}\right)=0 \quad \text { with } \quad a=\frac{\left(\varepsilon_{1}(s), Q_{x_{j}}\right)}{\left\|Q_{x_{j}}\right\|_{L^{2}}^{2}}
$$


where we have used $\left(Q_{x_{j}}, Q_{1}+Q_{2}\right)=0$. Similarly, taking the scalar product of the imaginary part of (5.90) with $Q$, we get

$$
\left(\tilde{\varepsilon}_{2}(s), Q\right)=0 \quad \text { with } \quad c=\frac{\left(\varepsilon_{2}(s), Q\right)}{\|Q\|_{L^{2}}^{2}} .
$$

Next, we use the orthogonality conditions (5.75) from Lemma 5.3.13 to get another set of values for $a, b$ and $c$. Taking the scalar product of the real part of (5.90) with $x_{j} Q$ and

$$
\text { using }\left(\varepsilon_{1}(s), x_{j} Q\right)=0, \quad \text { we have } \quad a=\frac{2\left(\tilde{\varepsilon}(s), x_{j} Q\right)}{\|Q\|_{L^{2}}^{2}} .
$$

Next, taking the scalar product of real part of (5.90) with $Q_{1}+Q_{2}$ and

$$
\text { using }\left(\varepsilon_{1}(s), Q_{1}+Q_{2}\right)=0, \quad \text { we have } \quad b=-\frac{\left(\tilde{\varepsilon}_{1}(s), Q_{1}+Q_{2}\right)}{\left\|Q_{1}\right\|_{L^{2}}^{2}} \text {. }
$$

Lastly, taking the scalar product of (5.90) with $Q_{2}$ and from orthogonality condition

$$
\text { using }\left(\varepsilon_{2}, Q_{2}\right)=0, \quad \text { we have } \quad c=\frac{\left(\tilde{\varepsilon}_{2}(s), Q_{2}\right)}{\left\|Q_{1}\right\|_{L^{2}}^{2}} \text {. }
$$

Therefore, using the two set of values of $a, b$ and $c$, we obtain

$$
A_{1}(\varepsilon, \varepsilon) \leq(\tilde{\varepsilon}, \tilde{\varepsilon}) \leq B_{1}(\varepsilon, \varepsilon)
$$

for some constants $A_{1}, B_{1}>0$. We also record that

$$
\left(L_{+} \tilde{\varepsilon}_{1}(s), \tilde{\varepsilon}_{1}(s)=\left(L_{+} \varepsilon_{1}(s), \varepsilon_{1}(s)+4 b\left(\varepsilon_{1}(s), Q\right), \quad\left(\tilde{\varepsilon}_{1}(s), Q\right)=\left(\varepsilon_{1}(s), Q\right),\right.\right.
$$

and

$$
\left(L_{-} \tilde{\varepsilon}_{2}(s), \tilde{\varepsilon}_{2}(s)\right)=\left(L_{-} \varepsilon_{2}(s), \varepsilon_{2}(s)\right)
$$

Thus, using (5.91), (5.92), (5.89) along with (5.65) and (5.68), we get

$$
\begin{aligned}
\left(\varepsilon(s), \varepsilon(s) \leq \frac{1}{A_{1}}(\tilde{\varepsilon}(s), \tilde{\varepsilon}(s)\right. & \leq(L \tilde{\varepsilon}(s), \tilde{\varepsilon}(s) \\
& \leq \alpha_{0}+4|b|\left|\left(\varepsilon_{1}(s), Q\right)\right|+C_{2}\|\varepsilon(s)\|_{H^{1}}^{\frac{4}{N}-1}\|\varepsilon(s)\|_{L^{2}}^{2}
\end{aligned}
$$


We note that $|b| \leq \tilde{c}\|\varepsilon(s)\|_{H^{1}}$ (from the expressions of $b$ ) and $2\left|\left(\varepsilon_{1}(s), Q\right)\right| \leq \alpha_{0}+$ $\|\varepsilon(s)\|_{L^{2}}^{2}$ by (5.88). Therefore,

$$
(\varepsilon(s), \varepsilon(s)) \leq\left(1+2 \tilde{c}\|\varepsilon(s)\|_{H^{1}}\right) \alpha_{0}+\left(2 \tilde{c}\|\varepsilon(s)\|_{H^{1}}+C_{2}\|\varepsilon(s)\|_{H^{1}}^{\frac{4}{N}-1}\right)\|\varepsilon(s)\|_{L^{2}}^{2}
$$

Now, by the hypothesis there exists $\delta_{3}>0$ such that if $\|\varepsilon(s)\|_{H^{1}}<\delta\left(\alpha_{0}\right)$ for $\alpha_{0}<\delta_{3}$, then $\tilde{c}\|\varepsilon\|_{H^{1}} \leq 1 / 8$ and $C_{2}\|\varepsilon(s)\|_{H^{1}}^{\frac{4}{N}-1} \leq 1 / 2$. Thus, we can take the last term on righthand side of (5.93) to the left-hand side and write

$$
(\varepsilon(s), \varepsilon(s)) \leq 2 \alpha_{0}
$$

Now we use (5.46) (expression of $L_{+}$) to write

$$
\begin{aligned}
\|\varepsilon(s)\|_{H^{1}}^{2}= & \int|\varepsilon(s)|^{2}+\int|\nabla \varepsilon(s)|^{2} \\
= & (L \varepsilon(s), \varepsilon(s))+\frac{4}{N} \iint \frac{Q^{1+\frac{4}{N}}\left(y^{\prime}, s\right) Q^{\frac{4}{N}-1}(y, s)|\varepsilon(y, s)|^{2}}{\left|y-y^{\prime}\right|^{N-2}} d y d y^{\prime} \\
& +\frac{N+4}{N} \iint \frac{Q^{\frac{4}{N}}\left(y^{\prime}, s\right)\left|\varepsilon\left(y^{\prime}, s\right)\right| Q^{\frac{4}{N}}(y, s)|\varepsilon(y, s)|}{\left|y-y^{\prime}\right|^{N-2}} d y d y^{\prime}
\end{aligned}
$$

From (5.94), we get

$$
\begin{aligned}
\|\varepsilon(s)\|_{H^{1}}^{2} & \leq \alpha_{0}+C_{2}\|\varepsilon(s)\|_{H^{1}}^{\frac{4}{N}-1}\|\varepsilon(s)\|_{L^{2}}^{2}+C\left(N,\|Q\|_{L^{\infty}}\right)\|\varepsilon(s)\|_{L^{2}}^{2} \\
& \leq\left(1+2 C_{2}\|\varepsilon(s)\|_{H^{1}}^{\frac{4}{N}-1}+2 C\left(N,\|Q\|_{L^{\infty}}\right)\right) \alpha_{0} \\
& \leq\left(2+2 C\left(N,\|Q\|_{L^{\infty}}\right)\right) \alpha_{0}
\end{aligned}
$$

setting $C_{3}=\sqrt{2+2 C\left(N,\|Q\|_{L^{\infty}}\right)}$, we conclude the proof.

We are now able to prove the following properties of modulation parameters.

Lemma 5.3.16 There exists $\delta_{4}>0$ such that if $\alpha_{0}<\delta_{4}, \lambda(s), \gamma(s)$ and $x(s)$ are $C^{1}$ functions of $s$ with the following properties: 
1. Equation of $\lambda_{s}, \gamma_{s}$ and $x_{s}$ :

$$
\begin{aligned}
\frac{\lambda_{s}}{\lambda} \int \varepsilon_{1}, y \cdot \nabla\left(y_{i} Q\right)- & \tilde{\gamma}_{s} \int \varepsilon_{2} y_{i} Q+\frac{\left(x_{i}\right)_{s}}{\lambda}\left(\frac{1}{2}\|Q\|_{L^{2}}^{2}+\int \varepsilon_{1}\left(Q+y_{i} Q_{y_{i}}\right)\right) \\
& +\left.\frac{\left(x_{j}\right)_{s}}{\lambda} \int \varepsilon_{1} y_{i} Q_{y_{j}}\right|_{i \neq j}=-2 \int \varepsilon_{2}, Q_{y_{i}}-\int R_{2}(\epsilon) y_{i} Q
\end{aligned}
$$

$$
\begin{aligned}
\frac{\lambda_{s}}{\lambda}\left(\left\|Q_{1}\right\|_{L^{2}}^{2}-\int \varepsilon_{1}\left(Q_{1}\right.\right. & \left.\left.+Q_{2}\right)_{1}\right)+\tilde{\gamma}_{s} \int \varepsilon_{2} Q_{1}-\frac{x_{s}}{\lambda} \int \varepsilon_{1} \nabla\left(Q_{1}+Q_{2}\right) \\
& =-\int \varepsilon_{2} L_{-}\left(Q_{1}\right)-\int \varepsilon_{2} L_{-}\left(Q_{2}\right)-\int R_{2}(\varepsilon)\left(Q_{1}+Q_{2}\right),
\end{aligned}
$$

and

$$
\begin{aligned}
-\frac{\lambda_{s}}{\lambda} \int \varepsilon_{2}\left(Q_{2}\right)_{1}+\tilde{\gamma}_{s}\left(\left\|Q_{1}\right\|_{L^{2}}^{2}+\right. & \left.\int \varepsilon_{1} Q_{2}\right)-\frac{x_{s}}{\lambda} \int \varepsilon_{2} \nabla Q_{2} \\
& =\int \varepsilon_{1} L_{+}\left(Q_{2}\right)-\int R_{1}(\varepsilon) Q_{2}
\end{aligned}
$$

2. Estimates on $\lambda_{s}, \gamma_{s}$ and $x_{s}$ :

$$
\left|\frac{\lambda_{s}}{\lambda}\right|+\left|\tilde{\gamma}_{s}\right|+\left|\frac{x_{s}}{\lambda}\right| \leq C_{4}\left(\int_{\mathbb{R}^{N}}|\nabla \varepsilon|^{2}\right)^{1 / 2} .
$$

Proof. 1. We take the inner product of (5.44) with $y_{i} Q$, integrate by parts and use first orthogonality condition from (5.75), $L_{-}\left(y_{i} Q\right)=-2 Q_{y_{i}}$ along with $\int Q_{1}\left(y_{i} Q\right)=0$ to obtain, after rearranging, the equation (5.95). Now taking the inner product of (5.44) with $Q_{1}+Q_{2}$, integrating by parts, using second and third orthogonality condition from (5.75) along with the relations $\int Q_{1} Q_{2}=0, \int \nabla Q\left(Q_{1}+Q_{2}\right)=0$, we obtain, after rearranging, the equation (5.96). Finally, taking the inner product of (5.45) with $Q_{2}$, integrating by parts, using third orthogonality condition from (5.75) along with $\int Q Q_{2}=-\left\|Q_{1}\right\|_{L^{2}}^{2}$ and rearranging, we obtain the equation (5.97). 
2. To prove (5.98), we observe that the leading order terms in (5.95) is $\|Q\|_{L^{2}}^{2},(5.96)$ and (5.97) is $\left\|Q_{1}\right\|_{L^{2}}^{2}$ ensuring that the coefficient matrix associated to the variables $\frac{\lambda_{s}}{\lambda}, \frac{x_{s}}{\lambda}$ and $\gamma_{s}$ is non-zero. Thus, using (5.81) along with Lemma 5.3.14 part (1), one can solve the system of equations given by (5.95)-(5.97) and obtain a universal constant $C_{4}>0$ (depending on the powers of $Q$ and its partial derivatives) such that (5.98) holds.

The proof of Lemma 5.3.16 is now complete.

The last result in this section is the estimates due to the conservation laws:

Lemma 5.3.17 We have for all $s \geq 0$,

1. the estimate from the energy conservation:

$$
\left|\left(\varepsilon_{1}(s), Q\right)\right| \leq C_{5}\left(\int_{\mathbb{R}^{N}}|\nabla \varepsilon(s)|^{2}\right)+\lambda^{2}(s)\left|E_{0}\right|,
$$

2. the estimate from the conservation of momentum:

$$
\left|\left(\varepsilon_{2}(s), \nabla Q\right)\right| \leq C_{6}\|\varepsilon(s)\|_{H^{1}}\left(\int_{\mathbb{R}^{N}}|\nabla \varepsilon(s)|^{2}\right)^{1 / 2} .
$$

Proof. 1. From Lemma 5.3.11 part (2) and (5.70) in part (3), we have

$$
\begin{aligned}
(L \varepsilon, \varepsilon) & =2\left(\int_{\mathbb{R}^{N}} Q \varepsilon_{1}+\frac{1}{2} \int_{\mathbb{R}^{N}}|\varepsilon|^{2}\right)-2 \lambda^{2}\left|E_{0}\right|+\frac{N}{N+4} \int_{\mathbb{R}^{N}} F(\varepsilon) \\
& \leq M_{0}-2 \lambda^{2}\left|E_{0}\right|+C\|\varepsilon\|_{H^{1}}^{\frac{4}{N}}-1\|\nabla \varepsilon\|_{L^{2}}^{2}
\end{aligned}
$$

where in the last inequality we have used (5.69) and Lemma 5.3.14 part (3). Thus, realizing that the leading terms on the right-hand side of the below expression are of second order, we get

$$
\begin{aligned}
\left|\int \varepsilon_{1} Q\right| & =\left|\frac{1}{2}\left((L \varepsilon, \varepsilon)-\int|\varepsilon|^{2}-\frac{N}{2(N+4)} \int F(\varepsilon)\right)\right|+\lambda^{2}\left|E_{0}\right| \\
& \leq C_{5} \int|\nabla \varepsilon|^{2}+\lambda^{2}(s)\left|E_{0}\right|
\end{aligned}
$$

as desired. 
2. We rewrite $\operatorname{Im}\left(\int \bar{u}_{0} \nabla u_{0}\right)=0$ in $\varepsilon$ variable to obtain

$$
\left|\int \varepsilon_{2} \nabla Q\right| \leq C_{6}\|\varepsilon\|_{L^{2}}\|\nabla \varepsilon\|_{L^{2}}
$$

so that with (5.76), we deduce (5.100).

This completes the proof od Lemma 5.3.17.

\subsubsection{The local virial estimate}

Here, we derive the virial identity in $\varepsilon$ variable. Recall that if $\int|x|^{2}\left|u_{0}(x)\right|^{2} d x<+\infty$, one can write the virial identity given by

$$
\frac{d}{d t} \int|x|^{2}|u(x, t)|^{2} d x=4 \operatorname{Im}\left(\int \bar{u} x \cdot \nabla u\right)=-16\left|E\left[u_{0}\right]\right| t+C .
$$

Thus, virial identity for $\varepsilon$ is given by calculating the time derivative (in $s$ ) of the quantity $\Psi(\varepsilon(s))=\operatorname{Im}\left(\int \bar{\varepsilon} x \cdot \nabla \varepsilon\right)(s)$. In particular, evaluating

$$
\Psi(u(t))=\operatorname{Im}\left(\int(\bar{\varepsilon}+Q) y \cdot \nabla(\varepsilon+Q)\right)=-4\left|E\left[u_{0}\right]\right| t+\frac{C}{4},
$$

we observe that

$$
\Psi(\varepsilon(s))-2 \int \varepsilon_{2} Q_{1}=-4\left|E\left[u_{0}\right]\right| t+\frac{C}{4} .
$$

Taking the derivative of above expression with respect to $s$ and using $\frac{d t}{d s}=\lambda^{2}(s)$, we get

$$
(\Psi(\varepsilon))_{s}(s)=2\left(\varepsilon_{2}, Q_{1}\right)_{s}(s)-4 \lambda^{s}(s)\left|E\left[u_{0}\right]\right|
$$

Thus, for the virial identity in $\varepsilon$ we compute $\left(\varepsilon_{2}, Q_{1}\right)_{s}$ and obtain the following:

Lemma 5.3.18 (Local virial identity) Let $u_{0} \in H^{1}$ such that it satisfies the assumptions of Theorem 5.3.2. Then

$$
\left(\varepsilon_{2}, Q_{1}\right)_{s}=H(\varepsilon, \varepsilon)+2 \lambda^{2}\left|E_{0}\right|-\tilde{\gamma}_{s}\left(\varepsilon_{1}, Q_{1}\right)-\frac{\lambda_{s}}{\lambda}\left(\varepsilon_{2}, Q_{2}\right)-\frac{x_{s}}{\lambda}\left(\varepsilon_{2}, \nabla\left(Q_{1}\right)\right)+G(\varepsilon),
$$


where

$$
G(\varepsilon)=+\left(\tilde{R}_{1}(\varepsilon), Q_{1}\right)-\frac{N}{2(N+4)} \int F(\varepsilon)
$$

with $\tilde{R}_{1}(\varepsilon)$ given by (5.82) is cubic in $\varepsilon$, and

$$
\begin{aligned}
H(\varepsilon, \varepsilon) & =H_{1}\left(\varepsilon_{1}, \varepsilon_{1}\right)+H_{2}\left(\varepsilon_{2}, \varepsilon_{2}\right) \\
& =\left(\mathcal{L}_{1} \varepsilon_{1}, \varepsilon_{1}\right)+\left(\mathcal{L}_{2} \varepsilon_{2}, \varepsilon_{2}\right),
\end{aligned}
$$

where

$$
\mathcal{L}_{1}=-\Delta+\frac{4}{N} V_{2}+\left(1+\frac{4}{N}\right)\left(|y|^{-(N-2)} * Q^{\frac{4}{N}}(\cdot)\right)\left(Q^{\frac{4}{N}}+y \cdot \nabla Q^{\frac{4}{N}}\right)
$$

and

$$
\mathcal{L}_{2}=-\Delta+V_{2}
$$

where

$$
V_{2}=\frac{1}{2}\left(|y|^{-(N-2)} *\left(2 Q^{1+\frac{4}{N}}+\tilde{y} \cdot \nabla Q^{1+\frac{4}{N}}\right)\right) Q^{\frac{4}{N}-1}+\frac{1}{2}\left(|y|^{-N-2} * Q^{1+\frac{4}{N}}\right)\left(y \cdot \nabla Q^{\frac{4}{N}-1}\right) .
$$

Proof. Taking the inner product of (5.45) with $Q_{1}$ and using $L_{+}\left(Q_{1}\right)=-2 Q$ with the fact that $\left(Q, Q_{1}\right)=0$, we obtain, after performing integration by parts,

$$
\left(\varepsilon_{2}, Q_{1}\right)_{s}=2\left(\varepsilon_{1}, Q\right)-\tilde{\gamma}_{s}\left(\varepsilon_{1}, Q_{1}\right)-\frac{\lambda_{s}}{\lambda}\left(\varepsilon_{2}, Q_{2}\right)-\frac{x_{s}}{\lambda}\left(\varepsilon_{2}, \nabla\left(Q_{1}\right)\right)+\left(R_{1}(\varepsilon), Q_{1}\right) .
$$

Substituting $2\left(\varepsilon_{1}, Q\right)$ from (5.70) into the above expression, we get

$$
\begin{aligned}
\left(\varepsilon_{2}, Q_{1}\right)_{s}= & \int|\nabla \varepsilon|^{2}-\int\left(|y|^{-(N-2)} *\left(Q^{1+\frac{4}{N}}\right)\right) Q^{\frac{4}{N}-1}\left(\frac{4}{N} \varepsilon_{1}^{2}+\varepsilon_{2}^{2}\right) \\
& -\left(1+\frac{4}{N}\right) \int\left(|y|^{-(N-2)} *\left(Q^{\frac{4}{N}} \varepsilon_{1}\right)\right) Q^{\frac{4}{N}} \varepsilon_{1}+2 \lambda^{2}\left|E_{0}\right|-\frac{N}{2(N+4)} \int F(\varepsilon) \\
& -\tilde{\gamma}_{s}\left(\varepsilon_{1}, Q_{1}\right)-\frac{\lambda_{s}}{\lambda}\left(\varepsilon_{2}, Q_{2}\right)-\frac{x_{s}}{\lambda}\left(\varepsilon_{2}, \nabla\left(Q_{1}\right)\right)+\left(R_{1}(\varepsilon), Q_{1}\right)
\end{aligned}
$$


Now, we use (5.48) to collect all the second order terms in $\varepsilon$ and write

$$
\begin{aligned}
R_{1}(\varepsilon)= & \frac{2}{N}\left(1+\frac{4}{N}\right) \int\left(|y|^{-(N-2)} *\left(Q^{\frac{4}{N}-1} \varepsilon_{1}^{2}\right)\right) Q^{\frac{4}{N}} \\
& +\frac{1}{2}\left(1+\frac{4}{N}\right) \int\left(|y|^{-(N-2)} *\left(Q^{\frac{4}{N}-1} \varepsilon_{2}^{2}\right)\right) Q^{\frac{4}{N}} \\
& +\frac{4}{N}\left(1+\frac{4}{N}\right) \int\left(|y|^{-(N-2)} *\left(Q^{\frac{4}{N}} \varepsilon_{1}\right)\right) Q^{\frac{4}{N}-1} \varepsilon_{1} \\
& +\frac{2}{N}\left(\frac{4}{N}-1\right) \int\left(|y|^{-(N-2)} *\left(Q^{1+\frac{4}{N}}\right)\right) Q^{\frac{4}{N}-2} \varepsilon_{1}^{2} \\
& +\frac{1}{2}\left(\frac{4}{N}-1\right) \int\left(|y|^{-(N-2)} *\left(Q^{1+\frac{4}{N}}\right)\right) Q^{\frac{4}{N}-2} \varepsilon_{2}^{2}+\tilde{R}_{1}(\varepsilon),
\end{aligned}
$$

where $\tilde{R}_{1}(\varepsilon)$ is given by (5.82) and is cubic in $\varepsilon$. Observe that $F(\varepsilon)$ is also cubic in $\varepsilon$ and so we define

$$
G(\varepsilon)=\left(\tilde{R}_{1}(\varepsilon), Q_{1}\right)-\frac{N}{2(N+4)} \int F(\varepsilon) .
$$

Thus, computing the inner product of second order terms from $R_{1}(\varepsilon)$ with $Q_{1}$ and using the symmetry of convolution, we get

$$
\begin{aligned}
\left(R_{1}(\varepsilon), Q_{1}\right)= & 2 \int\left(|y|^{-(N-2)} * Q^{1+\frac{4}{N}}\right) Q^{\frac{4}{N}-1}\left(\frac{4}{N} \varepsilon_{1}^{2}+\varepsilon_{2}^{2}\right) \\
& +2\left(1+\frac{4}{N}\right) \int\left(|y|^{-(N-2)} *\left(Q^{\frac{4}{N}} \varepsilon_{1}\right)\right) Q^{\frac{4}{N}} \varepsilon_{1} \\
& +\frac{1}{2} \int\left(|y|^{-(N-2)} *\left(y \cdot \nabla Q^{1+\frac{4}{N}}\right)\right) Q^{\frac{4}{N}-1}\left(\frac{4}{N} \varepsilon_{1}^{2}+\varepsilon_{2}^{2}\right) \\
& +\frac{1}{2} \int\left(|y|^{-(N-2)} * Q^{1+\frac{4}{N}}\right)\left(y \cdot \nabla Q^{\frac{4}{N}-1}\right)\left(\frac{4}{N} \varepsilon_{1}^{2}+\varepsilon_{2}^{2}\right) \\
& +\left(1+\frac{4}{N}\right) \int\left(|y|^{-(N-2)} *\left(Q^{\frac{4}{N}} \varepsilon_{1}\right)\right)\left(y \cdot \nabla Q^{\frac{4}{N}}\right) \varepsilon_{1} .
\end{aligned}
$$

Substituting (5.112) along with (5.111) into (5.110) yields the desired result.

Remark 5.3.19 We mention that one can verify the following two identities by direct calculation (recall that $f_{1}$ is the scaling generator defined in (5.43))

$$
\tilde{\mathcal{L}}_{1} f=\frac{1}{2}\left(L_{+}\left(f_{1}\right)-\left(L_{+} f\right)_{1}\right) \quad \text { and } \quad \mathcal{L}_{2} f=\frac{1}{2}\left(L_{-}\left(f_{1}\right)-\left(L_{-} f\right)_{1}\right)
$$


where $\mathcal{L}_{2}$ is given by (5.108) and

$$
\begin{aligned}
\tilde{\mathcal{L}}_{1} f= & \frac{4}{N} \mathcal{L}_{2} f+\frac{1}{2}\left(1+\frac{4}{N}\right)\left(|y|^{-1} *\left(Q^{\frac{4}{N}} f\right)\right)\left(y \cdot \nabla Q^{\frac{4}{N}}+2 Q^{\frac{4}{N}}\right) \\
& +\frac{1}{2}\left(1+\frac{4}{N}\right)\left(|y|^{-1} *\left(\tilde{y} \cdot \nabla Q^{\frac{4}{N}}\right) f\right) Q^{\frac{4}{N}} .
\end{aligned}
$$

Observe that we have $\tilde{\mathcal{L}}_{1}$ (obtained by direct computation, which we used in [ARY20] to obtain the Spectral Property) in place of $\mathcal{L}_{1}$ (obtained in Lemma 5.3.18) in the first identity. However, the magic happens under the inner product setting

$$
\begin{aligned}
\left(\tilde{\mathcal{L}}_{1} f, f\right)= & \frac{4}{N}\left(\mathcal{L}_{2} f, f\right)+\frac{1}{2}\left(1+\frac{4}{N}\right)\left(\left[\left(|y|^{-1} *\left(Q^{\frac{4}{N}} f\right)\right)\left(y \cdot \nabla Q^{\frac{4}{N}}+2 Q^{\frac{4}{N}}\right)\right], f\right) \\
& +\frac{1}{2}\left(1+\frac{4}{N}\right) \int\left(|y|^{-1} *\left(\tilde{y} \cdot \nabla Q^{\frac{4}{N}}\right) f\right) Q^{\frac{4}{N}} f
\end{aligned}
$$

which can be re-written as

$$
\begin{aligned}
\left(\tilde{\mathcal{L}}_{1} f, f\right)= & \frac{4}{N}\left(\mathcal{L}_{2} f, f\right)+\left(1+\frac{4}{N}\right)\left(\left[\left(|y|^{-1} *\left(Q^{\frac{4}{N}} f\right)\right)\left(y \cdot \nabla Q^{\frac{4}{N}}+Q^{\frac{4}{N}}\right)\right], f\right) \\
& +\frac{1}{2}\left(1+\frac{4}{N}\right) \int\left(|y|^{-1} *\left(\tilde{y} \cdot \nabla Q^{\frac{4}{N}}\right) f\right) Q^{\frac{4}{N}} f \\
& -\frac{1}{2}\left(1+\frac{4}{N}\right) \int\left(|y|^{-1} *\left(Q^{\frac{4}{N}} f\right)\right) y \cdot \nabla Q^{\frac{4}{N}} f \\
= & \frac{4}{N}\left(\mathcal{L}_{2} f, f\right)+\left(1+\frac{4}{N}\right)\left(\left[\left(|y|^{-1} *\left(Q^{\frac{4}{N}} f\right)\right)\left(y \cdot \nabla Q^{\frac{4}{N}}+2 Q^{\frac{4}{N}}\right)\right], f\right) \\
= & \left(\mathcal{L}_{1} f, f\right) .
\end{aligned}
$$

For our analysis, we will use $\mathcal{L}_{1}$, since it is the same as $\tilde{\mathcal{L}}_{1}$ under the inner product.

The next step would be to show coercivity of the bilinear form $H$ and then proceed with the bounds on $\left(\varepsilon_{2}, Q_{1}\right)_{s}$, which will allow us to obtain the blow-up rate with the $\log$ correction.

This is a point, where the Spectral Property is needed to prove Theorem 5.3.2, which we discuss next. This will explain why we only consider the $3 \mathrm{~d}$ case, . 


\subsubsection{Spectral property}

We start with the definition of Spectral Property.

Definition 5.3.20 (Spectral Property, see [ARY20]) Let $N>2$. Given the two real Schrödinger operators $L_{1,2}$ defined by (5.107) and (5.108). Let the real quadratic form for $\mathbf{z}=(u, v)^{T} \in H_{r}^{1} \times H_{r}^{1}$ with radial symmetry be

$$
\mathcal{B}(\mathbf{z}, \mathbf{z})=\mathcal{B}_{1}(u, u)+\mathcal{B}_{2}(v, v)
$$

The system is said to satisfy a spectral property with radial symmetric assumption on the subspace $\mathcal{U} \in H_{r}^{1} \times H_{r}^{1}$, if there exists a universal constant $\delta_{0}>0$ such that $\forall \mathbf{z} \in \mathcal{U}$,

$$
\mathcal{B}(\mathbf{z}, \mathbf{z}) \geq \delta_{0} \int|\nabla \mathbf{z}|^{2}+e^{-|\mathbf{y}|}|\mathbf{z}|^{2} d \mathbf{y}
$$

Then, the following results were established in [ARY20] via the numerically-assisted proof.

Theorem 5.3.21 If we treat the dimension $N$ as a parameter, since we are under the radial symmetric assumption, we have the following results:

1. Let the dimensions $\alpha_{1} \leq N \leq \alpha_{2}$ and assume the subspace $\mathcal{U} \subset L_{r}^{2} \times L_{r}^{2}$ with the orthogonal conditions

$$
\langle f, Q\rangle=0, \quad\left\langle f, Q_{1}\right\rangle=0 ; \quad\left\langle g, Q_{1}\right\rangle=0, \quad\left\langle g, Q_{2}\right\rangle=0
$$

Then, the spectral property holds for $(f, g)^{T} \in \mathcal{U}$ with $\alpha_{1} \approx 2.02$ and $\alpha_{2} \approx 2.6$.

2. Let the dimensions $\alpha_{3} \leq N \leq \alpha_{4}$ and assume the subspace $\mathcal{U} \subset L_{r}^{2} \times L_{r}^{2}$ with the orthogonal conditions

$$
\langle f, Q\rangle=0, \quad\left\langle f, Q_{1}\right\rangle=0 ; \quad\left\langle g, Q_{1}\right\rangle=0, \quad\left\langle g, Q_{2}\right\rangle=0
$$

Then, the spectral property holds for $(f, g)^{T} \in \mathcal{U}$ with $\alpha_{3} \approx 2.7$ and $\alpha_{4} \approx 3.1$. 
Remark 5.3.22 Observe that in the above Theorem the only acceptable integer is $N=3$ (between $\alpha_{3}$ and $\alpha_{4}$ ). For the purpose of the analytical proof later (specifically, to ensure the positivity of $H_{1}\left(\varepsilon_{1}, \varepsilon_{1}\right)$ in Proposition 5.3.25), we need a modified version of the above spectral property to incorporate the span of $Q_{1}$, which we state next, see [ARY20].

Theorem 5.3.23 The spectral property holds for the $3 d$ generalized Hartree equation for $(f, g)^{T} \in \mathcal{U} \subset L^{2} \times L^{2}$ in the space orthogonal to the spans

$$
\langle f, Q\rangle=0, \quad\left\langle f, Q_{1}+\alpha Q_{2}\right\rangle=0 ; \quad\left\langle g, Q_{1}\right\rangle=0, \quad\left\langle g, Q_{2}\right\rangle=0,
$$

with $\alpha$ in the range $\alpha<\alpha_{1}^{*}$ or $\alpha>\alpha_{2}^{*}$, where $\alpha_{1}^{*} \approx-0.44601$ and $\alpha_{2}^{*} \approx 0.69022$.

Theorem 5.3.23 actually holds for $2.8 \leq N \leq 3.1$ with slightly different values of $\alpha_{1}^{*}$ and $\alpha_{2}^{*}$ depending on the value of $N$. We point that the $3 \mathrm{~d}$ case is of the most interest (as this is the only integer dimension that fits the above spectral property).

Remark 5.3.24 Note that the reason that we cannot consider the case $N=4$ is due to the fact that in $4 d$ the potentials in Definition 5.3.20 decay as $\frac{C}{|x|^{2}}$ with a large constant $C$ (computed numerically), which leads to infinitely many negative eigenvalues, and thus, we would get infinitely many directions (or orthogonal conditions) to deal with, see [RS78].

We now reformulate the spectral property in terms of the quadratic form $H$ in $3 d$ from Lemma 5.3.18.

Proposition 5.3.25 Let $N=3$. Consider two real-valued operators $L_{1}$ and $L_{2}$ given by

$$
\mathcal{L}_{1}=-\Delta+\frac{4}{3} V_{2}+\frac{7}{3}\left(|y|^{-1} * Q^{\frac{4}{3}}(\cdot)\right)\left(Q^{\frac{4}{3}}+y \cdot \nabla Q^{\frac{4}{3}}\right) \quad \text { and } \quad \mathcal{L}_{2}=-\Delta+V_{2}
$$

with

$$
V_{2}=\frac{1}{2}\left(|y|^{-1} *\left(2 Q^{\frac{7}{3}}+\tilde{y} \cdot \nabla Q^{\frac{7}{3}}\right)\right) Q^{\frac{1}{3}}+\frac{1}{2}\left(|y|^{-1} * Q^{\frac{7}{3}}\right)\left(y \cdot \nabla Q^{\frac{1}{3}}\right) .
$$


The associated real-valued quadratic form $H(\tilde{\varepsilon}, \tilde{\varepsilon})$ for $\tilde{\varepsilon}=\tilde{\varepsilon}_{1}+i \tilde{\varepsilon}_{2} \in H^{1}\left(\mathbb{R}^{3}\right)$ be given by (5.105) (equivalently by (5.106)). Then there exists a universal constant $\tilde{\delta}_{0}>0$ such that for any $\tilde{\varepsilon} \in H^{1}\left(\mathbb{R}^{3}\right)$, the quadratic form $H$ is positive, or more precisely,

$$
H(\tilde{\varepsilon}, \tilde{\varepsilon}) \geq \tilde{\delta}_{0}\left(\int|\nabla \tilde{\varepsilon}|^{2}\right)
$$

provided

$$
\left(\tilde{\varepsilon}_{1}, Q\right)=\left(\tilde{\varepsilon}_{1}, Q_{1}+Q_{2}\right)=0 \quad \text { and } \quad\left(\tilde{\varepsilon}_{2}, Q_{1}\right)=\left(\tilde{\varepsilon}_{2}, Q_{2}\right)=0
$$

We fix the dimension $N=3$ for further analysis and use the above spectral property to demonstrate why the choice of the orthogonality condition $Q_{1}+Q_{2}$ comes into play. Let $\varepsilon \in H^{1}$ with $\left(\varepsilon_{1}, y_{i} Q\right)=\left(\varepsilon_{1}, Q_{1}+Q_{2}\right)=\left(\varepsilon_{2}, Q_{2}\right)=0$ (i.e., $\varepsilon$ satisfies Lemma 5.3.13). We set

$$
\tilde{\varepsilon}=\varepsilon-a Q-b Q_{1}-i c Q_{1} .
$$

Observe that $\left(\tilde{\varepsilon}_{1}, y_{i} Q\right)=\left(\tilde{\varepsilon}_{2}, Q_{2}\right)=0$. Also, $\left(\tilde{\varepsilon}_{1}, Q\right)=0$ and $\left(\tilde{\varepsilon}_{1}, Q_{1}+Q_{2}\right)=0$ with

$$
a=\frac{\left(\varepsilon_{1}, Q\right)}{\|Q\|_{L^{2}}^{2}}=b
$$

Similarly, $\left(\tilde{\varepsilon}_{2}, Q_{1}\right)=0$ with $c=\frac{\left(\varepsilon_{2}, Q_{1}\right)}{\left\|Q_{1}\right\|_{L^{2}}^{2}}$. Hence, $\tilde{\varepsilon}$ satisfies Proposition 5.3.25 along with Lemma 5.3.13.

We then evaluate

$$
\begin{aligned}
H(\varepsilon, \varepsilon)= & H(\tilde{\varepsilon}, \tilde{\varepsilon})+2 a\left(\tilde{\varepsilon}_{1}, \mathcal{L}_{1} Q\right)+2 b\left(\tilde{\varepsilon}_{1}, \mathcal{L}_{1} Q_{1}\right)+a^{2} H_{1}(Q, Q)+b^{2} H_{1}\left(Q_{1}, Q_{1}\right) \\
& +2 a b\left(\mathcal{L}_{1} Q, Q_{1}\right)+2 c\left(\tilde{\varepsilon}_{2}, \mathcal{L}_{2} Q_{1}\right)+c^{2} H_{2}\left(Q_{1}, Q_{1}\right) \\
\geq & \tilde{\delta}_{0} \int|\nabla \tilde{\varepsilon}|^{2}-C\left(a^{2}+c^{2}\right) \\
\geq & \delta_{0} \int|\nabla \varepsilon|^{2}-\frac{1}{\delta_{0}}\left(\left(\varepsilon_{1}, Q\right)^{2}+\left(\varepsilon_{2}, Q_{1}\right)^{2}\right)
\end{aligned}
$$

for some fixed universal constant $\delta_{1}>0$ small enough. Here, we have used the fact that $H_{1}(Q, Q)<0, H_{1}\left(Q_{1}, Q_{1}\right)=0, H_{2}\left(Q_{1}, Q_{1}\right)<0$ and $\left(\mathcal{L}_{1} Q, Q_{1}\right)<0$ along with Lemma 5.3.14. 


\subsubsection{The local virial inequality: Application of orthogonality con- ditions}

We now justify our choice of the orthogonality conditions (5.75), which allows us to cancel some oscillatory terms and provide a suitable control for other terms. In particular, we have the following lemma.

Lemma 5.3.26 There exists a universal constant $\delta_{5}>0$ such that for $\alpha_{0}<\delta_{5}$ small enough, we have for all s:

$$
\left(\varepsilon_{2}, Q_{1}\right)_{s} \geq \frac{\delta_{5}}{2}\left(\int|\nabla \varepsilon|^{2}\right)+2 \lambda^{2}\left|E_{0}\right|-\frac{2}{\delta_{5}}\left(\left(\varepsilon_{1}, Q\right)^{2}+\left(\varepsilon_{2}, Q_{1}\right)^{2}\right) .
$$

Proof. The choice of the orthogonality condition $\left(\varepsilon_{2}, Q_{2}\right)=0$ in (5.75) guarantees that the scalar product term associated with the scaling parameter in (5.103) vanishes, i.e.,

$$
\frac{\lambda_{s}}{\lambda}\left(\varepsilon_{2}, Q_{2}\right)=0
$$

The orthogonality condition $\left(\varepsilon_{1}, y_{i} Q\right)=0$ in (5.75) ensures the following control on the scalar product associated with the translation parameter in (5.103) (see (63) in [MR05a])

$$
\left|\frac{x_{s}}{\lambda}\left(\left(\varepsilon_{2}, \nabla\left(Q_{1}\right)\right)\right)\right| \leq C \delta\left(\alpha_{0}\right)\left(\int|\nabla \varepsilon|^{2}\right)
$$

Recall that we have modified the second orthogonality condition in (5.75) (see also Proposition 5.3.25) to facilitate the spectral analysis for $\mathcal{L}_{1}$. This introduces an oscillatory integral in the virial estimate (which vanishes in the work of Merle-Rapahël [MR03], [MR05a]). To handle this term, which is associated with the phase parameter in (5.103), we take the inner product of (5.45) with $Q_{2}$ and multiply the resultant expression with $\left(\varepsilon_{1}, Q_{1}\right)$

$$
\tilde{\gamma}_{s}\left(\varepsilon_{1}, Q_{1}\right)=\frac{1}{\left\|Q_{1}\right\|_{L^{2}}^{2}}\left(\varepsilon_{1}, L_{+} Q_{2}\right)\left(\varepsilon_{1}, Q_{1}\right)+G_{1}(\varepsilon)
$$


where

$G_{1}(\varepsilon)=\frac{1}{\left\|Q_{1}\right\|_{L^{2}}^{2}}\left(\varepsilon_{1}, Q_{1}\right)\left(\tilde{\gamma}_{s}\left(\varepsilon_{1}, Q_{2}\right)-\frac{\lambda_{s}}{\lambda}\left(\left(\varepsilon_{2}\right)_{1}, Q_{2}\right)-\frac{x_{s}}{\lambda}\left(\nabla \varepsilon_{2}, Q_{2}\right)-\left(R_{1}(\varepsilon), Q_{2}\right)\right)$

is cubic in $\varepsilon$. Thus, using (5.119), (5.120) and (5.121) along with Lemma 5.3.14, we rewrite (5.103) as

$$
\begin{aligned}
\left(\varepsilon_{2}, Q_{1}\right)_{s} \geq H(\varepsilon, \varepsilon) & +2 \lambda^{2}\left|E_{0}\right|-C \delta\left(M_{0}\right)\left(\int|\nabla \varepsilon|^{2}\right) \\
& -\frac{1}{\left\|Q_{1}\right\|_{L^{2}}^{2}}\left(\varepsilon_{1}, L_{+} Q_{2}\right)\left(\varepsilon_{1}, Q_{1}\right) .
\end{aligned}
$$

Now suppose $\mathcal{B}_{1}$ be the bilinear form associated with $\mathcal{H}_{1}$, where

$$
\mathcal{H}_{1}\left(\varepsilon_{1}, \varepsilon_{1}\right)=H_{1}\left(\varepsilon_{1}, \varepsilon_{1}\right)-\frac{1}{\left\|Q_{1}\right\|_{L^{2}}^{2}}\left(\varepsilon_{1}, L_{+} Q_{2}\right)\left(\varepsilon_{1}, Q_{1}\right)
$$

is the quadratic form on $\varepsilon_{1}$. Therefore, we get

$$
\begin{aligned}
\mathcal{B}_{1}\left(\varepsilon_{1}, Q_{1}\right) & =\left(\varepsilon_{1}, \mathcal{L}_{1}\left(Q_{1}\right)\right)-\frac{1}{2\left\|Q_{1}\right\|_{L^{2}}^{2}}\left(\varepsilon_{1},\left(\left(Q_{1}, Q_{1}\right) L_{+}\left(Q_{2}\right)+\left(Q_{1}, L_{+}\left(Q_{2}\right)\right) Q_{1}\right)\right) \\
& =\left(\varepsilon_{1}, \mathcal{L}_{1}\left(Q_{1}\right)\right)-\frac{1}{2}\left(\varepsilon_{1}, L_{+}\left(Q_{2}\right)+2 Q_{1}\right)=0
\end{aligned}
$$

where the last equality follows from the fact that

$$
\mathcal{L}_{1}\left(Q_{1}\right)=\frac{1}{2} L_{+}\left(Q_{2}\right)+Q_{1}
$$

Let $\varepsilon_{1} \in H^{1}$ such that $\left(\varepsilon_{1}, Q_{1}+Q_{2}\right)=\left(\varepsilon_{1}, y_{i} Q\right)=0$. Set $\tilde{\varepsilon}_{1}=\varepsilon_{1}+\tilde{b} Q_{1}$ with $\tilde{b}=-\frac{\left(\varepsilon_{1}, Q_{1}\right)}{\left\|Q_{1}\right\|_{L^{2}}^{2}}$ so that $\left(\tilde{\varepsilon}_{1}, Q_{1}\right)=0$ and $\left(\varepsilon_{1}, y_{i} Q\right)=0$. Now using $\left(\varepsilon_{1}, Q_{1}+Q_{2}\right)=0$, we also have that $\tilde{b}=\frac{\left(\tilde{\varepsilon}_{1}, Q_{2}\right)}{\left\|Q_{1}\right\|_{L^{2}}^{2}}$. Therefore, for some universal constant $A_{2}, B_{2}>0$, we have

$$
A_{2}\left(\nabla \varepsilon_{1}, \nabla \varepsilon_{1}\right) \leq\left(\nabla \tilde{\varepsilon}_{1}, \nabla \tilde{\varepsilon}_{1}\right) \leq B_{2}\left(\nabla \varepsilon_{1}, \nabla \varepsilon_{1}\right)
$$

Moreover, observe that

$$
\mathcal{H}_{1}\left(\varepsilon_{1}, \varepsilon_{1}\right)=\mathcal{H}_{1}\left(\tilde{\varepsilon}_{1}-\tilde{b} Q_{1}, \tilde{\varepsilon}_{1}-\tilde{b} Q_{1}\right)=\mathcal{H}_{1}\left(\tilde{\varepsilon}_{1}, \tilde{\varepsilon}_{1}\right)=H_{1}\left(\tilde{\varepsilon}_{1}, \tilde{\varepsilon}_{1}\right)
$$


where the second equality follows from the fact that $\mathcal{B}_{1}\left(\varepsilon_{1}, Q_{1}\right)=0$ and $\mathcal{H}_{1}\left(Q_{1}, Q_{1}\right)=0$ and the last equality follows from $\left(\tilde{\varepsilon}_{1}, Q_{1}\right)=0$. Thus, from the spectral property of $H$, we obtain

$$
\mathcal{H}_{1}\left(\varepsilon_{1}, \varepsilon_{1}\right)=H_{1}\left(\tilde{\varepsilon}_{1}, \tilde{\varepsilon}_{1}\right) \geq \tilde{\delta}_{0} \int\left|\nabla \tilde{\varepsilon}_{1}\right|^{2} \geq \delta_{0} \int\left|\nabla \varepsilon_{1}\right|^{2}
$$

Substituting (5.123) along with (5.117) into (5.122), we obtain

$$
\left(\varepsilon_{2}, Q_{1}\right)_{s}=\left(\delta_{0}-C \delta\left(M_{0}\right)\right)\left(\int|\nabla \varepsilon|^{2}\right)+2 \lambda^{2}\left|E_{0}\right|-\frac{1}{\delta_{0}}\left(\left(\varepsilon_{1}, Q\right)^{2}+\left(\varepsilon_{2}, Q_{1}\right)^{2}\right) .
$$

For $\alpha_{0}$ small enough such that $C \delta\left(\alpha_{0}\right)<\delta_{0}=\frac{\delta_{5}}{2}$, (5.124) implies (5.118) as desired.

\subsubsection{Refined virial inequality}

We obtained (5.118) from (5.103) using the orthogonality conditions (5.75) in Lemma 5.3.13, Proposition 5.3.25 and (5.117). Thus, we would need to understand the role two scalar product terms, appearing in (5.118), play in the analysis. The following lemma, similar to the NLS case takes care of the $\left(\varepsilon_{1}, Q\right)$ term.

Lemma 5.3.27 There exists a universal constant $\delta_{0}>0$ and $\delta_{6}$ such that for $\alpha_{0}<\delta_{6}$ small enough, we have

1. for all $s \in \mathbb{R}$,

$$
\left[\left(1+\frac{1}{4 \delta_{0}}\left(\varepsilon_{1}, Q\right)\right)\left(\varepsilon_{2}, Q_{1}\right)\right]_{s} \geq \delta_{0}\left(\int|\nabla \varepsilon|^{2}\right)+2 \lambda^{2}\left|E_{0}\right|-\frac{1}{2 \delta_{0}}\left(\varepsilon_{2}, Q_{1}\right)^{2},
$$

2. and for all $s_{2} \geq s_{1}$,

$$
\begin{aligned}
{\left[\left(1+\frac{1}{4 \delta_{0}}\left(\varepsilon_{1}, Q\right)(s)\right)\left(\varepsilon_{2}, Q_{1}\right)(s)\right]_{s_{1}}^{s_{2}} \geq } & \delta_{0} \int_{s_{1}}^{s_{2}}\left(\int|\nabla \varepsilon|^{2}\right)+2 \int_{s_{1}}^{s_{2}} \lambda^{2}\left|E_{0}\right| \\
& -\frac{1}{2 \delta_{0}} \int_{s_{1}}^{s_{2}}\left(\varepsilon_{2}, Q_{1}\right)^{2}
\end{aligned}
$$


Proof. 1. Taking the inner product of (5.44) with $Q$ and using $L_{-}(Q)=0$ along with $\left(Q, Q_{1}\right)=0$, we get

$$
\left(\varepsilon_{1}, Q\right)_{s}=-\frac{\lambda_{s}}{\lambda}\left(\varepsilon_{1}, Q_{1}\right)-\frac{x_{s}}{\lambda}\left(\varepsilon_{1}, \nabla Q\right)+\tilde{\gamma}_{s}\left(\varepsilon_{2}, Q\right)-\left(R_{2}(\varepsilon), Q\right) .
$$

Recall (5.109),

$$
\left(\varepsilon_{2}, Q_{1}\right)_{s}=2\left(\varepsilon_{1}, Q\right)-\tilde{\gamma}_{s}\left(\varepsilon_{1}, Q_{1}\right)-\frac{\lambda_{s}}{\lambda}\left(\varepsilon_{2}, Q_{2}\right)-\frac{x_{s}}{\lambda}\left(\varepsilon_{2}, \nabla\left(Q_{1}\right)\right)+\left(R_{1}(\varepsilon), Q_{1}\right) .
$$

Adding the above expressions and using (5.76), (5.100), (5.98), we estimate

$$
\left|\left(\varepsilon_{1}, Q\right)_{s}\right|+\left|\left(\varepsilon_{2}, Q_{1}\right)_{s}-2\left(\varepsilon_{1}, Q\right)\right| \leq C\left(\int|\nabla \varepsilon|^{2}\right) .
$$

Multiplying the above inequality with $\left(\varepsilon_{1}, Q\right)$ and using triangle inequality along with $\left(\varepsilon_{1}, Q\right) \lesssim \delta\left(\alpha_{0}\right)$, we write

$$
\begin{gathered}
\left|\left(\varepsilon_{1}, Q\right)_{s}\left(\varepsilon_{1}, Q\right)+\left(\varepsilon_{2}, Q_{1}\right)_{s}\left(\varepsilon_{1}, Q\right)-2\left(\varepsilon_{1}, Q\right)^{2}\right| \leq C \delta\left(\alpha_{0}\right)\left(\int|\nabla \varepsilon|^{2}\right) \\
\left|\left[\left(\varepsilon_{1}, Q\right)\left(\varepsilon_{2}, Q Q_{1}\right)\right]_{s}-2\left(\varepsilon_{1}, Q\right)^{2}-\left(\varepsilon_{1}, Q\right)_{s}\left(\left(\varepsilon_{2}, Q_{1}\right)-\left(\varepsilon_{1}, Q\right)\right)\right| \\
\leq C \delta\left(\alpha_{0}\right)\left(\int|\nabla \varepsilon|^{2}\right) .
\end{gathered}
$$

Since $\left|\left(\varepsilon_{2}, Q_{1}\right)\right| \lesssim \delta\left(\alpha_{0}\right)$ and $\left|\left(\varepsilon_{1}, Q\right)\right| \lesssim \delta\left(\alpha_{0}\right)$, the above estimate can be rewritten as

$$
\left|\left[\left(\varepsilon_{1}, Q\right)\left(\varepsilon_{2}, Q_{1}\right)\right]_{s}-2\left(\varepsilon_{1}, Q\right)^{2}\right| \leq C \delta\left(\alpha_{0}\right)\left(\int|\nabla \varepsilon|^{2}\right) .
$$

Inserting this relation into (5.118),

$$
\begin{aligned}
\left(\varepsilon_{2}, Q_{1}\right)_{s}+\frac{1}{\delta_{1}}\left[\left(\varepsilon_{1}, Q\right)\left(\varepsilon_{2}, Q_{1}\right)\right]_{s} \geq & \left(\frac{\delta_{1}}{2}-\frac{C \delta\left(\alpha_{0}\right)}{\delta_{1}}\right)\left(\int|\nabla \varepsilon|^{2}\right) \\
& +2 \lambda^{2}\left|E_{0}\right|-\frac{2}{\delta_{1}}\left(\varepsilon_{2}, Q_{1}\right)^{2} .
\end{aligned}
$$

For $\delta_{0}=\frac{\delta_{1}}{4}$ and $\alpha_{0}$ small enough such that $C \delta\left(\alpha_{0}\right) \leq \delta_{0}$, we obtain the desired estimate (5.125). 
2. Integrating (5.125) on the time interval $\left[s_{1}, s_{2}\right]$ yields (5.126).

This concludes the proof of Lemma 5.3.27.

We record that similar to NLS the virial dispersive estimate (5.125) is derived from the virial identity $(5.101)$ in the variable $u(x, t)$. We then inject decomposition

$$
\varepsilon(y, t)=e^{i \gamma(t)} \lambda^{\frac{3}{2}}(t) u(\lambda(t) y+x(t), t)-Q(y)
$$

into the virial identity and observe that through this transformation it translates into the scalar product involving $\varepsilon$, namely $\left(\varepsilon_{2}, Q_{1}\right)$. This relation provides a connection between the linear term and the quadratic term.

\subsubsection{Monotonicity: Control on scaling parameter}

Here we exhibit the maximum principle type property which gives the sign structure of the quantity $\left(\varepsilon_{2}, Q_{1}\right)$. We achieve this by injecting the virial relation (5.126) into the equation for the scaling parameter, this in turn yields an equation involving scaling parameter $\lambda$ of the form

$$
\frac{\lambda_{s}}{\lambda} \sim-\left(\varepsilon_{2}, Q_{1}\right)
$$

Lemma 5.3.28 Let $\delta_{7}>0$ such that for $\alpha_{0}<\delta_{7}$, there exists a unique $s_{0} \in \mathbb{R}$ such that

1. For all $s<s_{0},\left(\varepsilon_{2}, Q_{1}\right)(s)<0$, for all $s>s_{0},\left(\varepsilon_{2}, Q_{1}\right)(s)>0$ and $\left(\varepsilon_{2}, Q_{1}\right)\left(s_{0}\right)=$ 0 .

2. Moreover, for all $s_{2} \geq s_{1} \geq s_{0}$,

$$
\begin{aligned}
& 3 \int_{s_{1}}^{s_{2}}\left(\varepsilon_{2}, Q_{1}\right)-C\left(\delta_{0}\right) \delta\left(\alpha_{0}\right) \leq-\| y \|_{L^{2}}^{2} \ln \left(\frac{\lambda\left(s_{2}\right)}{\lambda\left(s_{1}\right)}\right) \\
& \leq 5 \int_{s_{1}}^{s_{2}}\left(\varepsilon_{2}, Q_{1}\right)+C\left(\delta_{0}\right) \delta\left(\alpha_{0}\right)
\end{aligned}
$$

and

$$
\lambda\left(s_{2}\right)<2 \lambda\left(s_{1}\right)
$$


Proof. First we assume $\alpha_{0}$ small enough such that

$$
\frac{1}{2} \leq 1+\frac{1}{4 \delta_{0}}\left(\varepsilon_{1}, Q\right) \leq \frac{3}{2}
$$

We claim that for all $s_{2} \geq s_{1}$,

$$
\left|4 \int_{s_{1}}^{s_{2}}\left(\varepsilon_{2}, Q_{1}\right)+\|x Q\|_{L^{2}}^{2} \ln \left(\frac{\lambda\left(s_{2}\right)}{\lambda\left(s_{1}\right)}\right)\right| \leq C \delta\left(\alpha_{0}\right)+\int_{s_{1}}^{s_{2}}\left|\left(\varepsilon_{2}, Q_{1}\right)\right|+\int_{s_{1}}^{s_{2}}\left|\left(\varepsilon_{1}, Q_{2}\right)\right| .
$$

Taking the inner product of (5.44) with the well-localized function $|x|^{2} Q$ and using the relation $L_{-}\left(|x|^{2} Q\right)=-4 Q_{1}$, we get

$$
\begin{aligned}
\left(\varepsilon_{1},|x|^{2} Q\right)_{s}+4\left(\varepsilon_{2}, Q_{1}\right)+\frac{\lambda_{s}}{\lambda}\|x Q\|_{L^{2}}^{2}=- & \frac{\lambda_{s}}{\lambda}\left(\varepsilon_{1},\left(|\mathbf{x}|^{2} Q\right)_{1}\right)-\frac{x_{s}}{\lambda}\left(\varepsilon_{1}, \nabla\left(|x|^{2} Q\right)\right) \\
& +\tilde{\gamma}_{s}\left(\varepsilon_{2},|x|^{2} Q\right)-\left(R_{2}(\varepsilon),|x|^{2} Q\right) .
\end{aligned}
$$

Using (5.98), we estimate

$$
\left|\left(\varepsilon_{1},|x|^{2} Q\right)_{s}+4\left(\varepsilon_{2}, Q_{1}\right)+\frac{\lambda_{s}}{\lambda}\|x Q\|_{L^{2}}^{2}\right| \leq C\left(\int|\nabla \varepsilon|^{2}\right) .
$$

Integrating above inequality on the time interval $\left[s_{1}, s_{2}\right]$, we obtain

$$
\left|4 \int_{s_{1}}^{s_{2}}\left(\varepsilon_{2}, Q_{1}\right)+\|x Q\|_{L^{2}}^{2} \ln \left(\frac{\lambda\left(s_{2}\right)}{\lambda\left(s_{1}\right)}\right)\right| \leq C \delta\left(\alpha_{0}\right)+C \int_{s_{1}}^{s_{2}}\left(\int|\nabla \varepsilon|^{2}\right) .
$$

Now, using (5.126)

$$
\begin{aligned}
\delta_{0} \int_{s_{1}}^{s_{2}}\left(\int|\nabla \varepsilon|^{2}\right) & \leq \frac{3}{2}\left|\left(\varepsilon_{2}, Q_{1}\right)\left(s_{2}\right)-\left(\varepsilon_{2}, Q_{1}\right)\left(s_{1}\right)\right|-2 \int_{s_{1}}^{s_{2}} \lambda^{2}\left|E_{0}\right| \\
& +\frac{1}{2 \delta_{0}} \int_{s_{1}}^{s_{2}}\left(\varepsilon_{2}, Q_{1}\right)^{2}+\frac{1}{2 \delta_{0}} \int_{s_{1}}^{s_{2}}\left(\varepsilon_{1}, Q_{2}\right)^{2} \\
\leq \frac{3}{2}\left|\left(\varepsilon_{2}, Q_{1}\right)\right|\left(s_{2}\right) & +\frac{3}{2}\left|\left(\varepsilon_{2}, Q_{1}\right)\left(s_{1}\right)\right|+\frac{1}{2 \delta_{0}} \int_{s_{1}}^{s_{2}}\left(\varepsilon_{2}, Q_{1}\right)^{2}+\frac{1}{2 \delta_{0}} \int_{s_{1}}^{s_{2}}\left(\varepsilon_{1}, Q_{2}\right)^{2} \\
& \leq C \delta\left(\alpha_{0}\right)+\frac{1}{2 \delta_{0}} \int_{s_{1}}^{s_{2}}\left(\varepsilon_{2}, Q_{1}\right)^{2}+\frac{1}{2 \delta_{0}} \int_{s_{1}}^{s_{2}}\left(\varepsilon_{1}, Q_{2}\right)^{2}
\end{aligned}
$$


for $\alpha_{0}$ small enough, we estimate from (5.132)

$$
\begin{aligned}
\left|4 \int_{s_{1}}^{s_{2}}\left(\varepsilon_{2}, Q_{1}\right)+\|x Q\|_{L^{2}}^{2} \ln \left(\frac{\lambda\left(s_{2}\right)}{\lambda\left(s_{1}\right)}\right)\right| \leq & C \delta\left(\alpha_{0}\right)+C\left(\delta_{0}\right) \int_{s_{1}}^{s_{2}}\left(\varepsilon_{2}, Q_{1}\right)^{2} \\
& +C\left(\delta_{0}\right) \int_{s_{1}}^{s_{2}}\left(\varepsilon_{1}, Q_{2}\right)^{2} \\
\leq & C \delta\left(\alpha_{0}\right)+\int_{s_{1}}^{s_{2}}\left|\left(\varepsilon_{2}, Q_{1}\right)\right|+\int_{s_{1}}^{s_{2}}\left|\left(\varepsilon_{1}, Q_{2}\right)\right|,
\end{aligned}
$$

which concludes the proof of Claim (5.131).

1. We now claim that as a consequence of (5.125): if for some $s_{2} \in \mathbb{R},\left(\varepsilon_{2}, Q_{1}\right)\left(s_{2}\right)=$ 0 , then $\left(\varepsilon_{2}, Q_{1}\right)_{s}\left(s_{2}\right)>0$. Arguing by contradiction, we assume that for some $s_{2} \in \mathbb{R},\left(\varepsilon_{2}, Q_{1}\right)\left(s_{2}\right)=0$ and $\left(\varepsilon_{2}, Q_{1}\right)_{s}\left(s_{2}\right) \leq 0$. Then

$$
\left(\left(1+\frac{1}{4 \delta_{0}}\left(\varepsilon_{1}, Q\right)\right)\left(\varepsilon_{2}, Q_{1}\right)\right)_{s}\left(s_{2}\right) \leq 0 .
$$

Inserting this into (5.125) gives

$$
\left(\int|\nabla \varepsilon|^{2}\right)\left(s_{2}\right) \leq 0
$$

which implies $\varepsilon\left(s_{2}\right)=0$. This contradicts (5.99), since $\lambda^{2}(s)\left|E_{0}\right|>0$ for all $s$. Therefore, $\left(\varepsilon_{2}, Q_{1}\right)$ (a function of time) may vanish at most once in $\mathbb{R}$ at some point $s_{0}$, then is strictly positive at the right of this point and negative at its left.

We now want to prove that such a time $s_{0}$ must exist. Assume that

$$
\forall s \in \mathbb{R},\left(\varepsilon_{2}, Q_{1}\right)(s)<0
$$

We try to invoke a contradiction to $(5.133)$ by observing asymptotic properties of the solution as $s \rightarrow+\infty$. Inserting (5.133) into (5.131), we get

$$
\begin{aligned}
-4 \int_{0}^{s}\left(\varepsilon_{2}, Q_{1}\right)-\|x Q\|_{L^{2}}^{2} \ln & \left(\frac{\lambda(s)}{\lambda(0)}\right) \leq\left|4 \int_{0}^{s}\left(\varepsilon_{2}, Q_{1}\right)+\|x Q\|_{L^{2}}^{2} \ln \left(\frac{\lambda(s)}{\lambda(0)}\right)\right| \\
& \leq C\left(\delta_{0}\right) \delta\left(\alpha_{0}\right)+\int_{0}^{s}\left|\left(\varepsilon_{2}, Q_{1}\right)\right|+\int_{0}^{s}\left|\left(\varepsilon_{1}, Q_{2}\right)\right| \\
& \leq C\left(\delta_{0}\right) \delta\left(\alpha_{0}\right)-\int_{0}^{s}\left(\varepsilon_{2}, Q_{1}\right)+\int_{0}^{s}\left|\left(\varepsilon_{1}, Q_{2}\right)\right|
\end{aligned}
$$


Thus, for all $s \geq 0$,

$$
-\|x Q\|_{L^{2}}^{2} \ln \left(\frac{\lambda(s)}{\lambda(0)}\right) \leq C\left(\delta_{0}\right) \delta\left(\alpha_{0}\right)+3 \int_{0}^{s}\left(\varepsilon_{2}, Q_{1}\right)+\int_{0}^{s}\left|\left(\varepsilon_{1}, Q_{2}\right)\right| .
$$

Now suppose $\int_{0}^{+\infty}\left(\varepsilon_{2}, Q_{1}\right)=-\infty$, then the above inequality implies that

$$
\lim _{s \rightarrow+\infty} \lambda(s)=+\infty
$$

From (5.76), we get

$$
\lim _{t \rightarrow T}\|\nabla u(t)\|_{L^{2}}^{2}=0 .
$$

This leads to a contradiction by the Gagliardo-Nirenberg inequality to the fact that $E\left(u_{0}\right)<0$. Thus, we have that

$$
\left|\int_{0}^{+\infty}\left(\varepsilon_{2}, Q_{1}\right)\right|<+\infty
$$

Again, by (5.131)

$$
\forall s>0, \quad 0<\lambda_{1} \leq \lambda(s) \leq \lambda_{2}
$$

From (5.109), we deduce that $\left|\left(\varepsilon_{2}, Q_{1}\right)_{s}\right|<C$ uniformly in $s$. Combining this with (5.133) and (5.134), we obtain

$$
\left(\varepsilon_{2}, Q_{1}\right)(s) \rightarrow 0 \text { as } s \rightarrow+\infty
$$

Consider the dispersive relation (5.125)

$$
\left(\left(1+\frac{1}{4 \delta_{0}}\left(\varepsilon_{1}, Q\right)\right)\left(\varepsilon_{2}, Q_{1}\right)\right)_{s} \geq \delta_{0}\left(\int|\nabla \varepsilon|^{2}\right)-\frac{1}{2 \delta_{0}}\left(\varepsilon_{2}, Q_{1}\right)^{2}--\frac{1}{2 \delta_{0}}\left(\varepsilon_{1}, Q_{2}\right)^{2} .
$$

Since the left-hand side of the above relation is a time derivative of a uniformly bounded function in time, then from (5.136), for some sequence $\tilde{s}_{n} \rightarrow+\infty$, we have

$$
\lim _{n \rightarrow+\infty}\left(\int|\nabla \varepsilon|^{2}\right)\left(\tilde{s}_{n}\right)=0
$$


Now, from (5.99)

$$
\begin{aligned}
\lambda^{2}\left|E_{0}\right| & \leq C\left(\int|\nabla \varepsilon|^{2}\right)^{\frac{3}{2}}+\left|\left(\varepsilon_{1}, Q\right)\right| \\
& \leq C\left(\int|\nabla \varepsilon|^{2}\right)^{\frac{1}{2}}
\end{aligned}
$$

where the last inequality follows from (5.76) and the control on linear term. This implies that $\lambda\left(s_{n}\right) \rightarrow 0$ as $s_{n} \rightarrow+\infty$, which contardicts (5.135). In a similar fashion, observing asymptotic properties of solution as $s \rightarrow-\infty$, it can be proved that $\forall s \in \mathbb{R},\left(\varepsilon_{2}, Q_{1}\right)(s)>0$ leads to a contradiction.

2. Since $\left(\varepsilon_{2}, Q_{1}\right)(s)>0$ for $s>s_{0}$, (5.128) follows directly from (5.131).

To prove (5.129), we argue by contradiction. Assume that $s_{0} \leq s_{1}<s_{2}$, such that $\lambda\left(s_{2}\right)>2 \lambda\left(s_{1}\right)$. Then from (5.128), we have

$$
\|x Q\|_{L^{2}}^{2} \ln \left(\frac{\lambda\left(s_{2}\right)}{\lambda\left(s_{1}\right)}\right)-C\left(\delta_{0}\right) \delta\left(\alpha_{0}\right) \leq-3 \int_{s_{1}}^{s_{2}}\left(\varepsilon_{2}, Q_{1}\right)<0
$$

which for $\alpha_{0}$ small enough implies

$$
\|x Q\|_{L^{2}}^{2} \ln (2)<0
$$

a contradiction.

The proof of Lemma 5.3.28 is now complete.

\subsubsection{Finite time blow-up and preliminary control (upper bound) on blow-up rate}

In this section, we conclude the proof of Theorem 5.3.2 by using the estimates proved in the previous section. Similar to the NLS case, as a consequence of both the monotonicity 
of the scaling parameter and the negative energy constraint, we get a result of a finite or infinite time blow-up, i.e.,

$$
\lim _{s \rightarrow+\infty} \lambda(s)=0 ; \quad \lambda(s) \sim \frac{1}{\|\nabla u(s)\|_{L^{2}}} .
$$

We then use a refined version of the almost monotonicity of the scaling parameter, which will give us a weaker upper bound on the blow-up rate. Then we use a refined version of the virial inequality (5.125) to prove the desired bound as claimed in the statement of Theorem 5.3.2.

We prove the following proposition:

Proposition 5.3.29 Suppose $u_{0} \in H^{1}$ such that it satisfies the assumptions of Theorem 5.3.2 and $u(t)$ be the corresponding solution to (5.37), then $u(t)$ blows up in finite time $0<T<+\infty$, and for $t$ close to the blow-up time $T$,

$$
\|\nabla u(t)\|_{L^{2}} \leq \frac{C}{\sqrt{|E[u(0)]|}(T-t)} .
$$

The proof is based on the refined version of the almost monotonicity of the scaling parameter. Let $\delta_{0}$ and a fixed constant $C\left(\delta_{0}\right)$ be same as in the estimates (5.125) and (5.126), respectively. Consider a fix constant $c_{0}>1$ such that

$$
0<\ln \left(c_{0}\right)<\frac{\delta_{0}}{5\|x Q\|_{L^{2}}^{2}},
$$

and let $\alpha_{8}$ in Proposition 5.3.31 is small enough such that

$$
\frac{2}{\|x Q\|_{L^{2}}^{2}} C\left(\delta_{0}\right) \sqrt{\alpha_{8}} \leq \ln \left(c_{0}\right) .
$$

Lemma 5.3.30 Let $s_{2} \geq \tilde{s}_{0}$ and $s \in\left[s_{1}, s_{2}\right]$, then

$$
\frac{1}{2} \lambda\left(s_{2}\right) \leq \lambda(s) \leq \lambda\left(s_{2}\right)
$$

and

$$
\left(\varepsilon_{2}, Q_{1}\right)(s) \leq 4\left(\varepsilon_{2}, Q_{1}\right)\left(s_{2}\right)
$$


Proof. Let $s_{2} \geq \tilde{s}_{0}$. Then by using the almost monotonicity of the scaling parameter (5.129) $\forall s \leq s_{2}$, we obtain $\lambda\left(s_{2}\right) \leq 2 \lambda(s) \leq 2 c_{0} \lambda\left(s_{2}\right)$, which proves (5.142). To prove (5.143), let

$$
K=\sup _{s \in\left[s_{1}, s_{2}\right]} \frac{\left(\varepsilon_{2}, Q_{1}\right)(s)}{\left(\varepsilon_{2}, Q_{1}\right)\left(s_{2}\right)}>0 .
$$

Fixing $s \in\left[s_{1}, s_{2}\right]$ and applying (5.126) with (5.130) on $\left[s, s_{2}\right]$, we get

$$
\frac{1}{2}\left(\varepsilon_{2}, Q_{1}\right)(s) \leq \frac{3}{2}\left(\varepsilon_{2}, Q_{1}\right)\left(s_{2}\right)+\frac{1}{2 \delta_{0}} \int_{s}^{s_{2}}\left(\varepsilon_{2}, Q_{1}\right)^{2} .
$$

From the definition of $K$ and Proposition 5.3.28 part (1), we have that $\forall s \in\left[s_{1}, s_{2}\right]$

$$
\left(\varepsilon_{2}, Q_{1}\right)(s) \leq\left(\varepsilon_{2}, Q_{1}\right)\left(s_{2}\right)\left(3+\frac{K}{\delta_{0}} \int_{s}^{s_{2}}\left(\varepsilon_{2}, Q_{1}\right)\right) .
$$

Taking the supremum over $s \in\left[s_{1}, s_{2}\right]$, we obtain

$$
K \leq 3+\frac{K}{\delta_{0}} \int_{s}^{s_{2}}\left(\varepsilon_{2}, Q_{1}\right) .
$$

Applying (5.128) with (5.141) and (5.142) on the interval $\left[s, s_{2}\right]$, we get

$$
\begin{aligned}
3 \int_{s}^{s_{2}}\left(\varepsilon_{2}, Q_{1}\right) & \leq C\left(\delta_{0}\right) \sqrt{\alpha_{0}}-\|x Q\|_{L^{2}}^{2} \ln \left(\frac{\lambda\left(s_{2}\right)}{\lambda\left(s_{1}\right)}\right) \\
& \leq \frac{1}{2}\|x Q\|_{L^{2}}^{2} \ln \left(c_{0}\right)+\|x Q\|_{L^{2}}^{2} \ln \left(c_{0}\right)=\frac{3}{2}\|x Q\|_{L^{2}}^{2} \ln \left(c_{0}\right),
\end{aligned}
$$

thus, by (5.140)

$$
K=3+\frac{K}{2 \delta_{0}}\|x Q\|_{L^{2}}^{2} \ln \left(c_{0}\right) \leq 3+\frac{1}{10} K,
$$

which implies that $K \leq 4$ and this proves (5.143).

This lemma allows us establish a new link between $\left(\varepsilon_{2}, Q_{1}\right)$ and $\lambda(s)$. More accurately, we have the following control on the scaling parameter in terms of $\left(\varepsilon_{2}, Q_{1}\right)$.

Lemma 5.3.31 There exists a universal constant $C_{7}$ and $\delta_{8}>0$ such that for $\alpha_{0}<\delta_{8}$, there exists $\tilde{s}_{0} \geq 0$ so that for all $s \geq \tilde{s}_{0}$, we have

$$
|E[u(0)]| \lambda^{2}(s) \leq C_{7}\left(\left(\varepsilon_{2}, Q_{1}\right)(s)\right)^{2} .
$$


Proof. First we observe from (5.140) and (5.141) we have that (5.136) holds true with $B=\left(\frac{60}{\|x Q\|_{L^{2}}^{2} \ln \left(c_{0}\right)}+\frac{8}{\delta_{0}}\right)$. The finite time blow-up result: $\lambda(s) \rightarrow 0$ as $s \rightarrow+\infty$ implies that there exists $\tilde{s}_{0} \geq 0$ such that for all $s_{2} \geq \tilde{s}_{0}$, there exists $s_{1}\left(s_{2}\right) \in\left(0, s_{2}\right)$ such that

$$
\lambda\left(s_{1}\right)=c_{0} \lambda\left(s_{2}\right) \text { and } \forall s \in\left[s_{1}, s_{2}\right], \lambda(s) \leq c_{0} \lambda\left(s_{2}\right)
$$

Let $s_{2} \geq \tilde{s}_{0}$ and the constant $C_{7}$ is such that on an interval $\left[s_{1}, s_{2}\right]$, we have

$$
E[u(0)] \lambda^{2}(s) \geq C_{7}\left(\varepsilon_{2}, Q_{1}\right)^{2}\left(s_{2}\right)
$$

We re-write (5.126) as

$$
\left(\left(1+\frac{1}{4 \delta_{0}}\left(\varepsilon_{1}, Q\right)\right)\left(\varepsilon_{2}, Q_{1}\right)\right)_{s}(s) \geq 2 \lambda^{2}(s)|E[u(0)]|-\frac{1}{2 \delta_{0}}\left(\varepsilon_{2}, Q_{1}\right)^{2}(s) .
$$

Using Lemma 5.3.30 and (5.146), we obtain for $s \in\left[s_{1}, s_{2}\right]$

$$
\begin{aligned}
2 \lambda^{2}(s)\left|E_{0}\right|-\frac{1}{2 \delta_{0}}\left(\varepsilon_{2}, Q_{1}\right)^{2}(s) & \geq 2 E[u(0)] \frac{\lambda^{2}\left(s_{2}\right)}{4}-\frac{16}{2 \delta_{0}}\left(\varepsilon_{2}, Q_{1}\right)^{2}\left(s_{2}\right) \\
& \geq\left(\frac{B}{2}-\frac{8}{\delta_{0}}\right)\left(\varepsilon_{2}, Q_{1}\right)^{2}\left(s_{2}\right) .
\end{aligned}
$$

Observe that if $\frac{B}{2}-\frac{8}{\delta_{0}} \leq 0$, then we are done. Otherwise, integrating (5.125) on the interval $\left[s_{1}, s_{2}\right]$ and using the above inequality, we obtain

$$
\frac{3}{2}\left(\varepsilon_{2}, Q_{1}\right)^{2}\left(s_{2}\right)-\left(\frac{B}{2}-\frac{8}{\delta_{0}}\right)\left(s_{2}-s_{1}\right)\left(\varepsilon_{2}, Q_{1}\right)^{2}\left(s_{2}\right) \geq \frac{1}{2}\left(\varepsilon_{2}, Q_{1}\right)^{2}\left(s_{1}\right) \geq 0
$$

which implies that

$$
\frac{3}{2}>\left(\frac{B}{2}-\frac{8}{\delta_{0}}\right)\left(s_{2}-s_{1}\right)\left(\varepsilon_{2}, Q_{1}\right)\left(s_{2}\right)
$$

Now we recall (5.128) and use (5.141) with (5.145) to get

$$
\begin{array}{r}
\|x Q\|_{L^{2}}^{2} \ln \left(c_{0}\right)=-\|x Q\|_{L^{2}}^{2} \ln \left(\frac{\lambda\left(s_{2}\right)}{\lambda\left(s_{1}\right)}\right) \leq 5 \int_{s_{1}}^{s_{2}}\left(\varepsilon_{2}, Q_{1}\right)+C\left(\delta_{0}\right) \sqrt{\alpha_{0}} \\
\leq 5 \int_{s_{1}}^{s_{2}}\left(\varepsilon_{2}, Q_{1}\right)+\frac{1}{2}\|x Q\|_{L^{2}}^{2} \ln \left(c_{0}\right)
\end{array}
$$


which implies

$$
\|x Q\|_{L^{2}}^{2} \ln \left(c_{0}\right) \leq 10 \int_{s_{1}}^{s_{2}}\left(\varepsilon_{2}, Q_{1}\right) .
$$

Using (5.143), the last inequality becomes

$$
\|x Q\|_{L^{2}}^{2} \ln \left(c_{0}\right) \leq 40\left(s_{2}-s_{1}\right)\left(\varepsilon_{2}, Q_{1}\right)\left(s_{2}\right) .
$$

Applying this estimate to (5.147), we obtain

$$
\frac{3}{2} \geq \frac{1}{40}\left(\frac{B}{2}-\frac{8}{\delta_{0}}\right)\|x Q\|_{L^{2}}^{2},
$$

which finally implies

$$
B \leq 2\left(\frac{60}{\|x Q\|_{L^{2}}^{2} \ln \left(c_{0}\right)}+\frac{8}{\delta_{0}}\right),
$$

thus, concluding the proof of Proposition 5.3.31.

We now use Lemma 5.3.31 to prove Proposition 5.3.29.

Proof. (of Proposition 5.3.29.) Using (5.138), we consider a sequence of times $t_{n}$ such that

$$
\lambda\left(t_{n}\right)=2^{-n}
$$

and let $s_{n}=s\left(t_{n}\right)$ be the corresponding sequence. Let $\tilde{t}_{0}$ be such that by Proposition 5.3.31, we have $s\left(\tilde{t}_{0}\right)=\tilde{s}_{0}$. Note that we may assume $n \geq \tilde{n}_{0}$ such that $t_{n} \geq \tilde{t}_{0}$. Also, by (5.129), we have that $0<t_{n}<t_{n+1}$, and so $0<s_{n}<s_{n+1}$. Moreover, $t_{n} \rightarrow T$, and (5.129) yields that $\forall s \in\left[s_{n}, s_{n+1}\right]$,

$$
2^{-(n+2)} \leq \lambda(s) \leq 2^{-(n-1)}
$$

For $n$ large enough, using Proposition 5.3.28 part (1) we write (5.144) $\forall s \in\left[s_{n}, s_{n+1}\right]$,

$$
0<\lambda(s) \leq \sqrt{\frac{C_{7}}{|E[u(0)]|}}\left(\varepsilon_{2}, Q_{1}\right)(s),
$$


integrating the above relation for the interval $\left[s_{n}, s_{n+1}\right]$

$$
\int_{s_{n}}^{s_{n+1}} \lambda(s) d s \leq \sqrt{\frac{C_{7}}{|E[u(0)]|}} \int_{s_{n}}^{s_{n+1}}\left(\varepsilon_{2}, Q_{1}\right)(s) d s .
$$

We use (5.128), which implies that for $\alpha_{0}<\alpha^{*}$ small enough, we have

$$
3 \int_{s_{n}}^{s_{n+1}}\left(\varepsilon_{2}, Q_{1}\right)(s) d s \leq C\left(\delta_{0}\right) \delta\left(\alpha_{0}\right)+\|y Q\|_{L^{2}}^{2} \ln 2 \leq 3\|y Q\|_{L^{2}}^{2} \ln 2 .
$$

Thus,

$$
\int_{s_{n}}^{s_{n+1}} \lambda(s) d s \leq \sqrt{\frac{C_{7}}{|E[u(0)]|}}\|y Q\|_{L^{2}}^{2} \ln 2 .
$$

Changing the variables using $\frac{d s}{d t}=\frac{1}{\lambda^{2}(s)}$ and estimating the above inequality using (5.129) and (5.149), we get

$$
\begin{aligned}
\sqrt{\frac{C_{7}}{|E[u(0)]|}}\|x Q\|_{L^{2}}^{2} \ln 2 & \geq \int_{s_{n}}^{s_{n+1}} \frac{1}{\lambda(s)} \lambda^{2}(s) d s \\
& \geq 2^{n-1} \int_{t_{n}}^{t_{n+1}} d t=2^{n-1}\left(t_{n+1}-t_{n}\right) .
\end{aligned}
$$

Thus, for $n \geq n_{0}$ (using $\ln 2<\frac{3}{4}$ )

$$
t_{n+1}-t_{n} \leq \frac{C}{\sqrt{\left|E\left[u_{0}\right]\right|}} 2^{-(n+1)}
$$

Summing this inequality in $n$ gives

$$
T=\lim _{n \rightarrow+\infty} t_{n+1}=\sum_{n}\left(t_{n+1}-t_{n}\right) \leq \sum_{n} 2^{-(n+1)}<+\infty,
$$

which proves that the blow-up occurs in finite time. Furthermore, for $n$ large the summation also gives the following estimate

$$
T-t_{n} \leq \frac{C}{\sqrt{\left|E\left[u_{0}\right]\right|}} \lambda\left(t_{n+1}\right) .
$$

Let $T>t>t_{n_{0}}$ so that $t_{n} \leq t \leq t_{n+1}$ for some $n$, then the above estimate combined with (5.129) yields

$$
T-t \leq T-t_{n} \leq \frac{C}{\sqrt{\left|E\left[u_{0}\right]\right|}} \lambda\left(t_{n+1}\right)<2 \frac{C}{\sqrt{\left|E\left[u_{0}\right]\right|}} \lambda(t) .
$$

Recall that $\lambda(s) \sim \frac{1}{\|\nabla u(s)\|_{L^{2}}}$, thus, (5.150) implies (5.139), this concludes the Proof of Proposition 5.3.29. 


\subsubsection{Refined upper bound on the blow-up rate.}

Here, we prove the upper bound on the blow-up rate as stated in Theorem 5.3.2, i.e.,

$$
\|\nabla u(t)\|_{L^{2}} \leq C\left(\frac{|\ln (T-t)|}{T-t}\right)^{\frac{1}{2}} .
$$

In what follows, we assume that $\lambda(s) \rightarrow 0$ as $s \rightarrow+\infty$, i.e., blow-up in finite time occurs. Let $\varepsilon=\varepsilon_{1}+i \varepsilon_{2}$ be a solution of

$$
\left\{\begin{array}{l}
\left(\varepsilon_{1}\right)_{s}-L_{-} \varepsilon_{2}=\sigma(s) Q_{1}+\rho(s) \nabla Q, \\
\left(\varepsilon_{2}\right)_{s}-L_{+} \varepsilon_{2}=\beta(s) Q,
\end{array}\right.
$$

where $\sigma(s), \rho(s)$ and $\beta(s)$ are some parameters. Based on the linear Liouville Theorem in [MM00] (see Theorem 3) we hope to prove from (5.125) that the space of uniformly bounded solutions in time of the linear equation that satisfy orthogonality conditions (5.75) is in fact one dimensional and generated by the stationary solution

$$
\varepsilon=i W \quad \text { with } \quad W=|y|^{2} Q+\mu Q
$$

where $\mu$ is such that

$$
\left(W, Q_{2}\right)=0 \Longrightarrow \mu=\frac{1}{\left\|Q_{1}\right\|_{L^{2}}^{2}}\left(|y|^{2} Q, Q_{2}\right) .
$$

We now prove the refined version of the virial inequality (5.125).

Proposition 5.3.32 Let $\tilde{\varepsilon}=\varepsilon+i \frac{\left(\varepsilon_{2}, Q_{1}\right)}{\|y Q\|_{L^{2}}^{2}} W$. There exist universal constants $\tilde{\delta}_{0}>0$ and $C_{8}>0$ along with $\delta_{9}>0$ such that for $\alpha_{0}<\delta_{9}$, there exists $\tilde{s}_{1}$ such that for all $s \geq \tilde{s}_{1}$,

$$
\left(\left(1+\frac{1}{\|y Q\|_{L^{2}}^{2}}\left(\varepsilon_{1}, W_{1}\right)\right)\left(\varepsilon_{2}, Q_{1}\right)\right)_{s}+C_{8}\left(\varepsilon_{2}, Q_{1}\right)^{3} \geq \tilde{\delta}_{0}\left(\int|\nabla \varepsilon|^{2}\right)+\lambda^{2}\left|E_{0}\right| .
$$

Proof. The argument follows [MR05a], Proposition 7 proof verbatim, except for an extra term, $\tilde{\gamma}_{s}\left(\varepsilon_{1}, Q_{1}\right)$, which as mentioned before is a result of modified orthogonality condition (see second condition in (5.75)). To estimate this term we follow the strategy used in Lemma 5.3.26 to prove (5.118). 
Now we use Proposition 5.3.32 to refine the estimate (5.144).

Proposition 5.3.33 There exists a universal constants $C_{10}>0$ and $\delta_{10}>0$ such that for $\alpha_{0}<\delta_{10}$, there exists $\tilde{s}_{4}$ such that for all $s \geq \tilde{s}_{4}$,

$$
\lambda^{2}(s) \leq \exp \left(-\frac{C_{10}}{\left(\varepsilon_{2}, Q_{1}\right)(s)}\right), \quad \text { or equivalently, } \quad\left(\varepsilon_{2}, Q_{1}\right)(s) \geq \frac{C_{10}}{|\ln (\lambda(s))|}
$$

Proof. From Lemma 5.3.28 we have that $\left(\varepsilon_{2}, Q_{1}\right)(s)>0$ for $s>0$. Let the function

$$
f(s)=\left(1+\frac{1}{\|y Q\|_{L^{2}}^{2}}\left(\varepsilon_{1}, W_{1}\right)\right)\left(\varepsilon_{2}, Q_{1}\right)
$$

satisfy

$$
\frac{1}{2}\left(\varepsilon_{2}, Q_{1}\right) \leq f(s) \leq 2\left(\varepsilon_{2}, Q_{1}\right)
$$

for $\alpha_{0}$ small enough, i.e., remains positively bounded, and thus, does not vanish for $s>0$. The estimate (5.153) can then be analyzed as a differential inequality

$$
f_{s}+C_{8} f^{3} \geq 0
$$

Integrating this inequality for $\left[s, \tilde{s}_{1}\right]$, where $\tilde{s}_{1}$ as in Proposition 5.3.32 and using (5.155), we obtain

$$
2\left(\varepsilon_{2}, Q_{1}\right) \geq f(s) \geq \frac{C_{8}}{s^{1 / 2}}
$$

for $s \geq \tilde{s}_{2}$. We now invoke the inequality (5.128) on the time interval $\left[\tilde{s}_{2}, s\right]$,

$$
3 \int_{\tilde{s}_{2}}^{s}\left(\varepsilon_{2}, Q_{1}\right) \leq-\|y Q\|_{L^{2}}^{2} \ln \left(\frac{\lambda(s)}{\lambda\left(\tilde{s}_{2}\right)}\right)+C\left(\delta_{0}\right) \delta\left(M_{0}\right) \leq-\frac{1}{2}\|y Q\|_{L^{2}}^{2} \ln \left(\frac{\lambda(s)}{\lambda\left(\tilde{s}_{2}\right)}\right) .
$$

Using (5.156) for $s \geq \tilde{s}_{3}$ large enough, we write the above inequality as

$$
C_{9}\left(s^{1 / 2}-\tilde{s}_{2}^{1 / 2}\right) \leq \int_{\tilde{s}_{2}}^{s}\left(\varepsilon_{2}, Q_{1}\right) \leq-\ln \left(\frac{\lambda(s)}{\lambda\left(\tilde{s}_{2}\right)}\right)
$$

i.e.,

$$
C_{9} s^{1 / 2} \leq-\ln (\lambda(s))=|\ln (\lambda(s))|
$$


for some universal constant $C_{9}>0$ and $s \geq \tilde{s}_{4}$. Now from (5.156), we conclude that

$$
|\ln (\lambda(s))| \geq C_{9} s^{1 / 2} \geq \frac{C_{10}}{\left(\varepsilon_{2}, Q_{1}\right)(s)}
$$

as desired.

\subsubsection{Climax: Log speed}

Proof of Theorem 5.3.2. Again consider a sequence of times $t_{n}$ such that $\lambda\left(t_{n}\right)=2^{-n}$ and $s_{n}=s\left(t_{n}\right)$ be the corresponding sequence. Let $\tilde{t}_{4}$ be such that $s\left(\tilde{t}_{4}\right)=\tilde{s}_{4}$ as in Proposition 5.3.33. We may assume $n \geq n_{4}$ such that $t_{n} \geq \tilde{t}_{4}$. Also, note that $t_{n} \rightarrow T$, where $T$ is the blow-up time. Recall from (5.129) that for all $s_{n} \leq s \leq s_{n+1}$, we have $2^{-(n+2)} \leq \lambda(s) \leq 2^{-(n-1)}$. Then from (5.128), the relation $\frac{d s}{d t}=\frac{1}{\lambda^{2}}$, and estimate (5.154), for all $n \geq n_{4}$,

$$
C \geq \int_{s_{n}}^{s_{n+1}}\left(\varepsilon_{2}, Q_{1}\right) d s \geq \int_{s_{n}}^{s_{n+1}} \frac{C d s}{|\ln (\lambda(s))|}=\int_{t_{n}}^{t_{n+1}} \frac{C d t}{\lambda^{2}(t)|\ln (\lambda(t))|}
$$

which gives that $C \lambda^{2}\left(t_{n}\right)\left|\ln \left(\lambda\left(t_{n}\right)\right)\right| \geq t_{n+1}-t_{n}$. Using the definition of the sequence $t_{n}$ and summing the above inequality in $n$, we get

$$
\begin{aligned}
C\left(T-t_{n}\right) & \leq \sum_{k \geq n} 2^{-2 k} k=\sum_{n \leq k \leq 2 n} 2^{-2 k} k+\sum_{k \geq 2 n} 2^{-2 k} k \\
& \leq C 2^{-2 n} n+2^{-4 n} n \sum_{\tilde{k} \geq 0} 2^{-2 \tilde{k}}\left(2+\frac{\tilde{k}}{n}\right) \\
& \leq C 2^{-2 n} n+C 2^{-4 n} n \leq C 2^{-2 n} n \leq C \lambda^{2}\left(t_{n}\right)\left|\ln \left(\lambda\left(t_{n}\right)\right)\right|
\end{aligned}
$$

Since $t \geq \tilde{t}_{4}$, for some $n \geq n_{4}, t \in\left[t_{n}, t_{n+1}\right]$, and from $\frac{1}{4} \lambda\left(t_{n}\right)=\frac{1}{2} \lambda\left(t_{n+1}\right) \leq \lambda(t)$, we get

$$
\lambda^{2}(t)|\ln (\lambda(t))| \geq C \lambda^{2}\left(t_{n}\right)\left|\ln \left(\lambda\left(t_{n}\right)\right)\right| \geq C\left(T-t_{n}\right) \geq C(T-t)
$$


Observe that the function $f(x)=x^{2}|\ln (x)|$ is non-decreasing near $x=0$, and for $t$ close to $T$, we have

$$
f\left(\frac{C \sqrt{T-t}}{|\ln (T-t)|^{\frac{1}{2}}}\right)=C(T-t)\left(1-C \frac{\ln (|\ln (T-t)|)}{|\ln (T-t)|}\right) \leq C(T-t)
$$

such that for some universal constant $C>0$ we obtain that

$$
f(\lambda(t)) \geq f\left(\frac{C \sqrt{T-t}}{|\ln (T-t)|^{\frac{1}{2}}}\right)
$$

i.e.,

$$
\lambda(t) \geq \frac{C \sqrt{T-t}}{|\ln (T-t)|^{\frac{1}{2}}},
$$

implying (5.40) and this concludes the proof of Theorem 5.3.2.
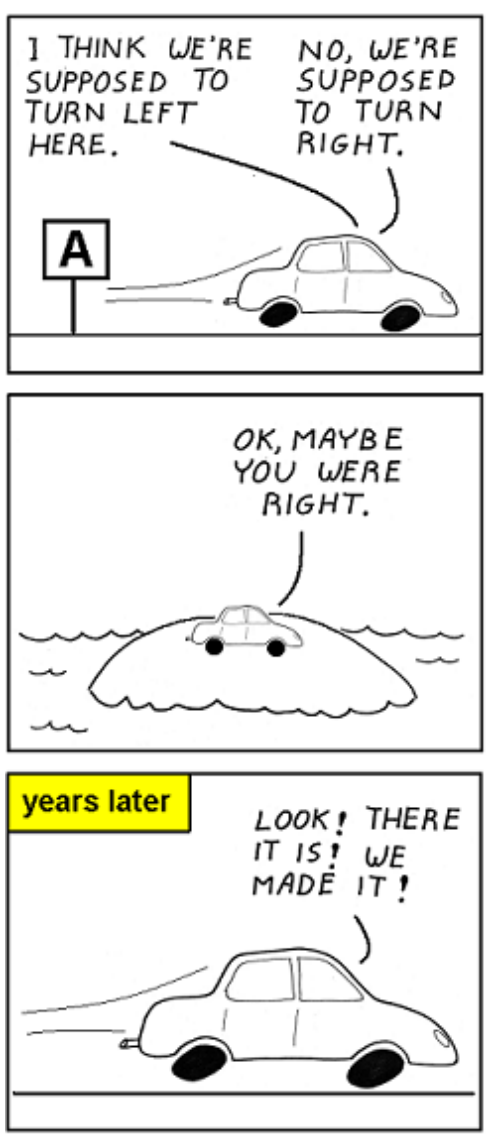
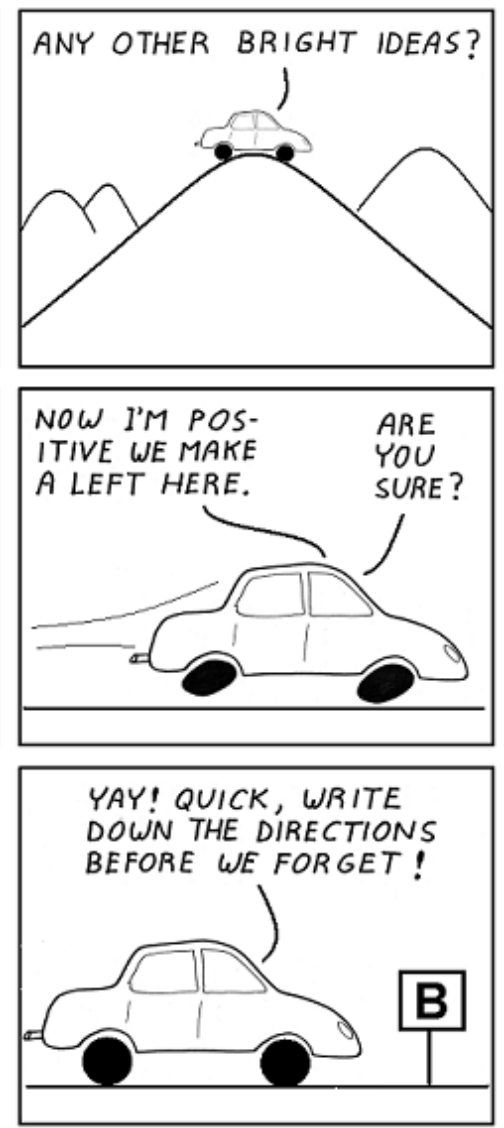

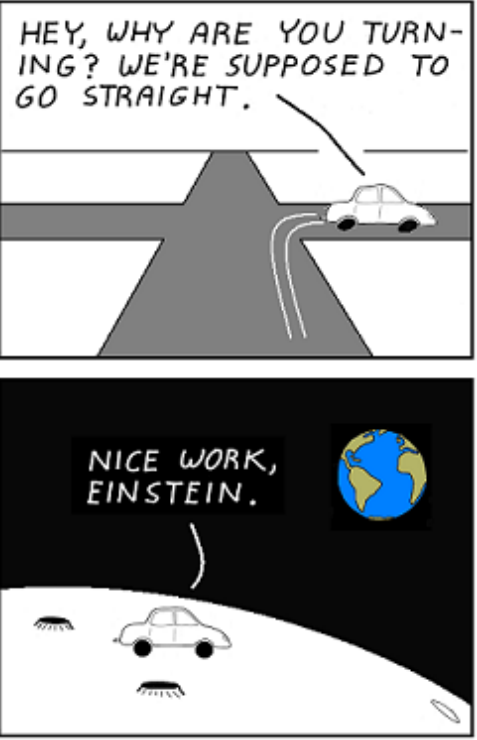

From A

Turn left on Ricci Street

Turn right on Hamilton Ave

$B$ is on your left

This is how most mathematical proofs are written. 


\section{CHAPTER 6}

\section{CONCLUSIONS AND FUTURE RESEARCH}

\section{If I have seen further it is by standing on the shoulders of Giants. (Issac Newton)}

We started the mathematical exposition in the second chapter with the well-posedness theory for the generalized Hartree equation. That showed that it is locally well-posed in $H^{s}\left(\mathbb{R}^{N}\right)$ for $s \geq \max \left(s_{c}, 0\right)$, where $s_{c}$ is the scale-invariant quantity. However, the local well-posedness can become very weak (or can even break down) when the nonlinearity becomes very rough in comparison to the regularity $s$. Thus, it would be interesting to investigate the local well-posedness for $1<p<2$.

In the second chapter, we also investigated the existence and uniqueness of ground state solutions $Q$, where $Q$ solves the nonlinear Choquard or Choquard-Pekar equation (2.123) in the intercritical regime, i.e., $0<s_{c}<1$, which are positive, vanishing at infinity solutions, which are radial. However, the uniqueness proof exists only when $p=2$ and $\gamma=2$ with any (reasonable) $N$. Therefore, it is important and challenging to develop a more general strategy to cover the other cases, i.e., $p \geq 2$ and other possible values of $0<\gamma<N$

In the next two chapters we studied the global behavior of $H^{1}\left(\mathbb{R}^{N}\right)$ solutions to the generalized Hartree equation and showed the scattering behavior. We first employed the well-known concentration-compactness and rigidity method of Kenig and Merle in Chapter 3 and gave an alternative proof of scattering in Chapter 4 for both nonlinear Schrödinger and generalized Hartree equations in the radial setting in the inter-critical regime, following the approach of Dodson and Murphy. It would be an interesting project to study the scattering behavior for the generalized Hartree equation in the nonradial setting avoiding the concentration-compactness route.

In Chapter 5, we go into the world of singularities (phenomenon of blow-up or collapse). First, we give a sufficient condition for finite-time blow-up (blow-up criterion) 
in the generalized Hartree equation. We then gave examples of the various thresholds (established in Chapters 3 and 4 together with the blow-up criterion from Chapter 5) in variety of cases (energy-subcritical, critical and supercritical) for Gaussian data.

Lastly, we give a rigorous analysis of stable singularity formations in the mass-critical generalized Hartree equation in dimension 3. We show that the solution blows-up with a self-similar profile at the rate with one $\log$ corrections. This is the first step in proving Conjecture 5.3.1, which says that the stable blow-up rate is with "log-log" corrections (known as the "log-log" regime), see [YRZ20, Conjecture 1]. The next challenging project would be to upgrade the one log correction to "log-log".

An additional interesting and challenging future project is to give a similar detailed and rigorous description of "log-log" blow-up solutions for the standard Hartree equation in dimension 4, i.e., $p=2$ and $\gamma=2$. 


\section{BIBLIOGRAPHY}

[AN13] Takafumi Akahori and Hayato Nawa. Blowup and scattering problems for the nonlinear Schrödinger equations. Kyoto J. Math., 53(3):629-672, 2013.

[AR19] Anudeep K. Arora and Svetlana Roudenko. On well-posedness and blow-up in the generalized Hartree equation. submitted, arXiv: 1910.01085, 2019.

[AR20a] Anudeep K. Arora and Svetlana Roudenko. Global behavior of solutions to the focusing generalized Hartree equation. Michigan Math J., forthcoming, 2020 .

[AR20b] Anudeep. K. Arora and Svetlana Roudenko. Stable blow-up dynamics in the mass-critical generalized Hartree equation. preprint, 2020.

[Aro19] Anudeep K. Arora. Scattering of radial data in the focusing NLS and generalized Hartree equations. Discrete Contin. Dyn. Syst. Series-A, 39(11):66436668, 2019.

[ARY20] Anudeep. K. Arora, Svetlana Roudenko, and Kai Yang. The spectral property for the critical generalized Hartree equation. preprint, 2020.

[BEG ${ }^{+}$02] Claude Bardos, Laszlo Erdős, François Golse, Norbert Mauser, and HorngTzer Yau. Derivation of the Schrödinger-Poisson equation from the quantum N-body problem. C. R. Math. Acad. Sci. Paris, 334(6):515-520, 2002.

[BGM00] Claude Bardos, François Golse, and Norbert J. Mauser. Weak coupling limit of the $N$-particle Schrödinger equation. Methods Appl. Anal., 7(2):275-293, 2000 .

[BL83a] H. Berestycki and P.-L. Lions. Nonlinear scalar field equations. I. Existence of a ground state. Arch. Rational Mech. Anal., 82(4):313-345, 1983.

[BL83b] H. Berestycki and P.-L. Lions. Nonlinear scalar field equations. II. Existence of infinitely many solutions. Arch. Rational Mech. Anal., 82(4):347-375, 1983.

[Bok] Stanislav Bokser. Vector - Blue realistic laser beam background. Laser rays iolated on black background. Modern style abstract. Bright shiny lasers pattern. Vector illustration. Image ID 124109022. Royalty Free Stock Photos. https: / / www.123rf.com/photo_124109022_stock-vector- 
blue-realistic-laser-beam-background-laser-rays-

iolated-on-black-background-modern-style-abstractbr.html.

[Caz03] Thierry Cazenave. Semilinear Schrödinger equations, volume 10 of Courant Lecture Notes in Mathematics. New York University, Courant Institute of Mathematical Sciences, New York; American Mathematical Society, Providence, RI, 2003.

[CFH11] Thierry Cazenave, Daoyuan Fang, and Zheng Han. Continuous dependence for NLS in fractional order spaces. Ann. Inst. H. Poincaré Anal. Non Linéaire, 28(1):135-147, 2011.

[CG04] Giuseppe Maria Coclite and Vladimir Georgiev. Solitary waves for MaxwellSchrödinger equations. Electron. J. Differential Equations, pages No. 94, 31, 2004.

[CU] Catalyst University. Development of Quantum Mechanics: Blackbody Radiation \& UV Catastrophe. YouTube. 25 April 2018. https:// www.youtube.com/watch?v=jw1w5ijeyvg.

[CW91] F. M. Christ and M. I. Weinstein. Dispersion of small amplitude solutions of the generalized Korteweg-de Vries equation. J. Funct. Anal., 100(1):87-109, 1991.

[DHR08] Thomas Duyckaerts, Justin Holmer, and Svetlana Roudenko. Scattering for the non-radial 3D cubic nonlinear Schrödinger equation. Math. Res. Lett., 15(6):1233-1250, 2008.

[DK98] D. L. Durfee and Wolfgang Ketterle. Experimental studies of Bose-Einstein condensation. Optics express, 2 8:299-313, 1998.

[DM17] Benjamin Dodson and Jason Murphy. A new proof of scattering below the ground state for the 3D radial focusing cubic NLS. Proc. Amer. Math. Soc., 145(11):4859-4867, 2017.

[DR15] Thomas Duyckaerts and Svetlana Roudenko. Going beyond the threshold: scattering and blow-up in the focusing NLS equation. Comm. Math. Phys., 334(3):1573-1615, 2015. 
[DY19] Lele Du and Minbo Yang. Uniqueness and nondegeneracy of solutions for a critical nonlocal equationn. Discrete Contin. Dyn. Syst. Series - A, 39(10):5847-5866, 2019.

[FGO18] Kazumasa Fujiwara, Vladimir Georgiev, and Tohru Ozawa. Higher order fractional Leibniz rule. J. Fourier Anal. Appl., 24(3):650-665, 2018.

[FGS07] Jürg Fröhlich, Sandro Graffi, and Simon Schwarz. Mean-field- and classical limit of many-body Schrödinger dynamics for bosons. Comm. Math. Phys., 271(3):681-697, 2007.

[FJL07] J. Fröhlich, B. L. G. Jonsson, and E. Lenzmann. Effective dynamics for boson stars. Nonlinearity, 20(5):1031-1075, 2007.

[FL04] Jürg Fröhlich and Enno Lenzmann. Mean-field limit of quantum Bose gases and nonlinear Hartree equation. In Séminaire: Équations aux Dérivées Partielles. 2003-2004, Sémin. Équ. Dériv. Partielles, pages Exp. No. XIX, 26. École Polytech., Palaiseau, 2004.

[Fla] Wallpaper Flare. HD wallpaper: landscape photography of body of water, ocean, beach, Los Angeles. https://www.wallpaperflare.com/ landscape-photography-of-body-of-water-oceanbeach-los-angeles-wallpaper-eaytb.

[FMR06] Gadi Fibich, Frank Merle, and Pierre Raphaël. Proof of a spectral property related to the singularity formation for the $L^{2}$ critical nonlinear Schrödinger equation. Phys. D, 220(1):1-13, 2006.

[fon] fonimak. Speed or Location. toonpool. 2 february 2018. https://www.toonpool.com/cartoons/Speed\%20or\% 20Location_308537.

[Fos05] Damiano Foschi. Inhomogeneous Strichartz estimates. J. Hyperbolic Differ. Equ., 2(1):1-24, 2005.

[FTY00] Jürg Fröhlich, Tai-Peng Tsai, and Horng-Tzer Yau. On a classical limit of quantum theory and the non-linear Hartree equation. Geom. Funct. Anal., (Special Volume, Part I):57-78, 2000. GAFA 2000 (Tel Aviv, 1999).

[FTY02] Jürg Fröhlich, Tai-Peng Tsai, and Horng-Tzer Yau. On the point-particle (Newtonian) limit of the non-linear Hartree equation. Comm. Math. Phys., 225(2):223-274, 2002. 
[FXC11] DaoYuan Fang, Jian Xie, and Thierry Cazenave. Scattering for the focusing energy-subcritical nonlinear Schrödinger equation. Sci. China Math., 54(10):2037-2062, 2011.

[GO93] J. Ginibre and T. Ozawa. Long range scattering for nonlinear Schrödinger and Hartree equations in space dimension $n \geq 2$. Comm. Math. Phys., 151(3):619-645, 1993.

[Gue14] Cristi Darley Guevara. Global behavior of finite energy solutions to the $d$ dimensional focusing nonlinear Schrödinger equation. Appl. Math. Res. Express. AMRX, (2):177-243, 2014.

[GV80] Jean Ginibre and Giorgio Velo. On a class of nonlinear Schrödinger equations with nonlocal interaction. Math. Z., 170(2):109-136, 1980.

[GV00a] J. Ginibre and G. Velo. Long range scattering and modified wave operators for some Hartree type equations. I. Rev. Math. Phys., 12(3):361-429, 2000.

[GV00b] J. Ginibre and G. Velo. Long range scattering and modified wave operators for some Hartree type equations. II. Ann. Henri Poincaré, 1(4):753-800, 2000 .

[GV00c] J. Ginibre and G. Velo. Scattering theory in the energy space for a class of Hartree equations. In Nonlinear wave equations (Providence, RI, 1998), volume 263 of Contemp. Math., pages 29-60. Amer. Math. Soc., Providence, RI, 2000.

[GV01] J. Ginibre and G. Velo. Long range scattering and modified wave operators for some Hartree type equations. III. Gevrey spaces and low dimensions. $J$. Differential Equations, 175(2):415-501, 2001.

[Hep74] Klaus Hepp. The classical limit for quantum mechanical correlation functions. Comm. Math. Phys., 35:265-277, 1974.

[HNO98] Nakao Hayashi, Pavel I. Naumkin, and Tohru Ozawa. Scattering theory for the Hartree equation. SIAM J. Math. Anal., 29(5):1256-1267, 1998.

[Hoo] Marlowe Hood. Scientists set to unveil first picture of a black hole. 6 april 2019. https://phys.org/news/2019-04-scientistsunveil-picture-black-hole.html. 
[HPR10] Justin Holmer, Rodrigo Platte, and Svetlana Roudenko. Blow-up criteria for the 3D cubic nonlinear Schrödinger equation. Nonlinearity, 23(4):977-1030, 2010.

[HR07] Justin Holmer and Svetlana Roudenko. On blow-up solutions to the 3D cubic nonlinear Schrödinger equation. Appl. Math. Res. Express. AMRX, (1):Art. ID abm004, 31, 2007.

[HR08] Justin Holmer and Svetlana Roudenko. A sharp condition for scattering of the radial 3D cubic nonlinear Schrödinger equation. Comm. Math. Phys., 282(2):435-467, 2008.

[HR10] Justin Holmer and Svetlana Roudenko. Divergence of infinite-variance nonradial solutions to the 3D NLS equation. Comm. Partial Differential Equations, 35(5):878-905, 2010.

[Hra] Josh Hrala. Astronomers Discover The Biggest Object in the Universe so far - the BOSS Great Wall. SPACE. Sciencealert. 13 march 2016. https://www.sciencealert.com/astronomers-declarethe-boss-great-wall-the-biggest-thing-in-everfound-in-the-universe.

[HT87] Nakao Hayashi and Yoshio Tsutsumi. Scattering theory for Hartree type equations. Ann. Inst. H. Poincaré Phys. Théor., 46(2):187-213, 1987.

[Ima] Ezume Images. Close colorful atomic particle background science 3D illustration. Shutterstock. 17 October 2017.

[KLR09] Joachim Krieger, Enno Lenzmann, and Pierre Raphäl. On stability of pseudo-conformal blowup for $L^{2}$-critical Hartree NLS. Ann. Henri Poincaré, 10(6):1159-1205, 2009.

[KM06] Carlos E. Kenig and Frank Merle. Global well-posedness, scattering and blow-up for the energy-critical, focusing, non-linear Schrödinger equation in the radial case. Invent. Math., 166(3):645-675, 2006.

[KT98] Markus Keel and Terence Tao. Endpoint Strichartz estimates. Amer. J. Math., 120(5):955-980, 1998.

[KV10] Rowan Killip and Monica Visan. Energy-supercritical NLS: critical $\dot{H}^{s}$ bounds imply scattering. Comm. Partial Differential Equations, 35(6):945$987,2010$. 
[Kwo89] Man Kam Kwong. Uniqueness of positive solutions of $\Delta u-u+u^{p}=0$ in $\mathbf{R}^{n}$. Arch. Rational Mech. Anal., 105(3):243-266, 1989.

[lad] ladyfirst. "Dream Interpretation: Rainbow." What it mean to dream about a rainbow?. 4 january 2020. https://www.lady-first.me/ article/dream-interpretation-rainbow,11604.html.

[Len09] Enno Lenzmann. Uniqueness of ground states for pseudorelativistic Hartree equations. Anal. PDE, 2(1):1-27, 2009.

[Lie83] Elliott H. Lieb. Sharp constants in the Hardy-Littlewood-Sobolev and related inequalities. Ann. of Math. (2), 118(2):349-374, 1983.

[Lie03] Elliott H. Lieb. The stability of matter and quantum electrodynamics. Milan J. Math., 71:199-217, 2003.

[Lie77] Elliott H. Lieb. Existence and uniqueness of the minimizing solution of Choquard's nonlinear equation. Studies in Appl. Math., 57(2):93-105, 1976/77.

[Lio80] P.-L. Lions. The Choquard equation and related questions. Nonlinear Anal., 4(6):1063-1072, 1980.

[Lio84a] P.-L. Lions. The concentration-compactness principle in the calculus of variations. The locally compact case. I. Ann. Inst. H. Poincaré Anal. Non Linéaire, 1(2):109-145, 1984.

[Lio84b] P.-L. Lions. The concentration-compactness principle in the calculus of variations. The locally compact case. II. Ann. Inst. H. Poincaré Anal. Non Linéaire, 1(4):223-283, 1984.

[LL01] Elliott H. Lieb and Michael Loss. Analysis, volume 14 of Graduate Studies in Mathematics. American Mathematical Society, Providence, RI, second edition, 2001.

[LMR08] Mohammed Lemou, Florian Méhats, and Pierre Raphaël. Structure of the linearized gravitational Vlasov-Poisson system close to a polytropic ground state. SIAM J. Math. Anal., 39(6):1711-1739, 2008.

[LP09] Felipe Linares and Gustavo Ponce. Introduction to nonlinear dispersive equations. Universitext. Springer, New York, 2009. 
[LT84] Elliott H. Lieb and Walter E. Thirring. Gravitational collapse in quantum mechanics with relativistic kinetic energy. Ann. Physics, 155(2):494-512, 1984.

[Lus02] Pavel Lushnikov. Collapse of Bose-Einstein condensates with dipole-dipole interactions. Physical Review A, 66, 2002.

[Lus10] Pavel Lushnikov. Collapse and stable self-trapping for bose-einstein condensates with $1 / r^{b}$ type attractive interatomic interaction potential. Physical Review A, 82, 2010.

[LY87] Elliott H. Lieb and Horng-Tzer Yau. The Chandrasekhar theory of stellar collapse as the limit of quantum mechanics. Comm. Math. Phys., 112(1):147$174,1987$.

[Mar02] Mihai Mariş. Existence of nonstationary bubbles in higher dimensions. $J$. Math. Pures Appl. (9), 81(12):1207-1239, 2002.

[mbSP] Michael Dubson (mods by S. Pollock). A brief history of modern physics and the development of the Schrödinger equation . 15 august 2008. https://physicscourses.colorado.edu/phys3220/ phys3220_fa08/notes/notes/.

[MM00] Yvan Martel and Frank Merle. A Liouville theorem for the critical generalized Korteweg-de Vries equation. J. Math. Pures Appl. (9), 79(4):339-425, 2000 .

[MPT98] Irene M. Moroz, Roger Penrose, and Paul Tod. Spherically-symmetric solutions of the Schrödinger-Newton equations. Classical Quantum Gravity, 15(9):2733-2742, 1998.

[MR03] F. Merle and P. Raphael. Sharp upper bound on the blow-up rate for the critical nonlinear Schrödinger equation. Geom. Funct. Anal., 13(3):591-642, 2003.

[MR04] Frank Merle and Pierre Raphael. On universality of blow-up profile for $L^{2}$ critical nonlinear Schrödinger equation. Invent. Math., 156(3):565-672, 2004.

[MR05a] Frank Merle and Pierre Raphael. The blow-up dynamic and upper bound on the blow-up rate for critical nonlinear Schrödinger equation. Ann. of Math. (2), 161(1):157-222, 2005. 
[MR05b] Frank Merle and Pierre Raphael. Profiles and quantization of the blow up mass for critical nonlinear Schrödinger equation. Comm. Math. Phys., 253(3):675-704, 2005.

[MR06] Frank Merle and Pierre Raphael. On a sharp lower bound on the blow-up rate for the $L^{2}$ critical nonlinear Schrödinger equation. J. Amer. Math. Soc., 19(1):37-90, 2006.

[MVS13] Vitaly Moroz and Jean Van Schaftingen. Groundstates of nonlinear Choquard equations: existence, qualitative properties and decay asymptotics. J. Funct. Anal., 265(2):153-184, 2013.

[MVS17] Vitaly Moroz and Jean Van Schaftingen. A guide to the Choquard equation. J. Fixed Point Theory Appl., 19(1):773-813, 2017.

[Nig] Gauri Nigudkar. The atomic structure wave-particle duality, de Broglies thesis. 5 november 2016. https://madoverchemistry.com/2016/ 11/05/30-atomic-structure-wave-particle-dualitydebroglies-thesis/.

[OGKA00] D. O’Dell, S. Giovanazzi, G. Kurizki, and V. M. Akulin. Bose Condensates with $1 / r$ Interatomic Attraction: Electromagnetically Induced Gravity. Phys. Rev. Lett., 84:5687-5690, 2000.

[Orn17] Stephen Ornes. Core Concept: How Bose-Einstein condensates keep revealing weird physics. Proceedings of National Academy of Sciences, 114(23):5766-5768, 2017.

[Pek54] S. I. Pekar. Untersuchung über die elektronentheorie der kristalle. Comm. Math. Phys., 1954.

[Per01] Galina Perelman. On the formation of singularities in solutions of the critical nonlinear Schrödinger equation. Ann. Henri Poincaré, 2(4):605-673, 2001.

[PLQ $\left.{ }^{+} 15\right] \quad$ L. Piazza, T. T. A. Lummen, E. Quinonez, Y. Murooka, B. W. Reed, B. Barwick, and F. Carbone. Simultaneous observation of the quantization and the interference pattern of a plasmonic near-field. Nature Communications, 2015.

[RS72] Michael Reed and Barry Simon. Methods of modern mathematical physics. I. Functional analysis. Academic Press, New York-London, 1972. 
[RS78] Michael Reed and Barry Simon. Methods of modern mathematical physics. IV. Analysis of operators. Academic Press [Harcourt Brace Jovanovich, Publishers], New York-London, 1978.

[Spo80] Herbert Spohn. Kinetic equations from Hamiltonian dynamics: Markovian limits. Rev. Modern Phys., 52(3):569-615, 1980.

[Ste70] Elias M. Stein. Singular integrals and differentiability properties of functions. Princeton Mathematical Series, No. 30. Princeton University Press, Princeton, N.J., 1970.

[Str77a] Walter A. Strauss. Existence of solitary waves in higher dimensions. Comm. Math. Phys., 55(2):149-162, 1977.

[Str77b] Robert S. Strichartz. Restrictions of Fourier transforms to quadratic surfaces and decay of solutions of wave equations. Duke Math. J., 44(3):705-714, 1977.

[SWM10] M. Saffman, T. G. Walker, and K. Molmer. Quantum information with Rydberg atoms. Reviews of Modern Physics, 82, 2010.

[Tao04] Terence Tao. On the asymptotic behavior of large radial data for a focusing non-linear Schrödinger equation. Dyn. Partial Differ. Equ., 1(1):1-48, 2004.

[Tao06] Terence Tao. Nonlinear dispersive equations, volume 106 of CBMS Regional Conference Series in Mathematics. Published for the Conference Board of the Mathematical Sciences, Washington, DC; by the American Mathematical Society, Providence, RI, 2006. Local and global analysis.

[Vis06] Monica Visan. The defocusing energy-critical nonlinear Schroedinger equation in dimensions five and higher. ProQuest LLC, Ann Arbor, MI, 2006. Thesis (Ph.D.)-University of California, Los Angeles.

[Wal] WallpapaerAccess. Solar system hd wallpapers. https: / / wallpaperaccess.com/solar-system-hd.

[Wei85] Michael I. Weinstein. Modulational stability of ground states of nonlinear Schrödinger equations. SIAM J. Math. Anal., 16(3):472-491, 1985.

[Wei83] Michael I. Weinstein. Nonlinear Schrödinger equations and sharp interpolation estimates. Comm. Math. Phys., 87(4):567-576, 1982/83. 
[WID] Elizabeth A. Weaver II and Hilary H. Doyle. How does the brain work?" Dana Foundation. 11 august 2019. https://www.dana.org/ article/how-does-the-brain-work/.

[Xia16] Chang-Lin Xiang. Uniqueness and nondegeneracy of ground states for Choquard equations in three dimensions. Calc. Var. Partial Differential Equations, 55(6):Art. 134, 25, 2016.

[YRZ18] Kai Yang, Svetlana Roudenko, and Yanxiang Zhao. Blow-up dynamics and spectral property in the $L^{2}$-critical nonlinear Schrödinger equation in high dimensions. Nonlinearity, 31(9):4354-4392, 2018.

[YRZ19] Kai Yang, Svetlana Roudenko, and Yanxiang Zhao. Blow-up dynamics in the mass super-critical NLS equations. Physica D Nonlinear Phenomena, 396:47-69, 2019.

[YRZ20] K. Yang, S. Roudenko, and Y. Zhao. Stable blow-up dynamics in the $L^{2}$ critical and $L^{2}$-supercritical generalized Hartree equation. Stud Appl Math., pages $1-48,2020$.

[Zur] University Of Zurich. Erwin Schrödinger Nobel Prize in Physics 1933. 18 december 2015. www.nobelpreis.uzh.ch/en_schroedinger.html. 
VITA

\section{ANUDEEP KUMAR ARORA}

February 16, 1984

2006

2012
Born, India

B.E., Mechanical Engineering IET, DAVV University

Indore, India

M.S., Mathematical Sciences

Florida International University

Miami, Florida

\section{PUBLICATIONS AND PREPRINTS}

Arora, Anudeep Kumar and Dodson, Benjamin and Murphy, Jason, (2020). Scattering below the ground state for the $2 d$ radial nonlinear Schrödinger equation. Proc. Amer. Math. Soc., 148(4):1653-1663.

Arora, Anudeep. K., (2019). Scattering of radial data in the focusing NLS and generalized Hartree equations. Discrete Contin. Dyn. Syst. Series-A, 39(11):6643-6668.

Arora, Anudeep K. and Roudenko, S., (2019) Global behavior of solutions to the focusing generalized Hartree equation. Michigan Math J., forthcoming, arXiv: 1904.05339.

Arora, Anudeep K. and Roudenko, S., (2019) On well-posedness and blow-up in the generalized Hartree equation. Submitted, arXiv: 1910.01085.

Arora, Anudeep K. and Roudenko, S. and Yang, K., (2020) Spectral property for the generalized Hartree equation. preprint.

Arora, Anudeep K. and Roudenko, S., (2020) Stable blow-up dynamics in the masscritical generalized Hartree equation. preprint. 